\title{
Direct Synthesis of Chiral NH Lactams via Ru-catalyzed Asymmetric Reductive Amination/Cyclization Cascade of Keto-Acids/Esters
}

\author{
Yongjie Shi, ${ }^{\#, \uparrow}$ Xuefeng Tan, ${ }^{\#, \dagger}$ Shuang Gao, ${ }^{\#, \dagger}$ Yao Zhang, ${ }^{\dagger}$ Jingxin Wang, ${ }^{\dagger}$ Xumu Zhang, ${ }^{\dagger}$ and Qin Yin ${ }^{*},, \sharp$ \\ †Shenzhen Grubbs Institute and Department of Chemistry, Southern University of Science and Technology, \\ Shenzhen 518055, China \\ ‡Academy for Advanced Interdisciplinary Studies, Southern University of Science and Technology, Shenzhen \\ 518000, China \\ $\S D e p a r t m e n t$ of Chemistry, State Key Laboratory of Synthetic Chemistry, the University of Hong Kong, Pokfulam \\ Road, Hong Kong (P. R. China)
}

yinq@sustech.edu.cn

Supporting Information

\section{Contents}

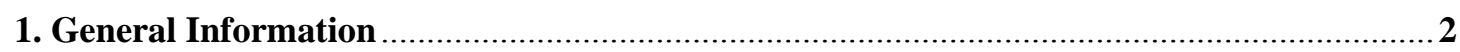

2. General Procedures for the Synthesis of Substrates ........................................................

3. Ru-catalyzed Asymmetric Reductive Amination/Cyclization Cascade of Keto-Acids/Esters 26

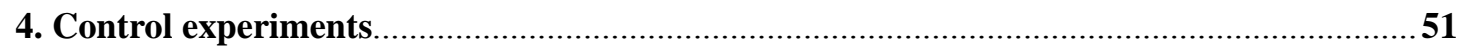

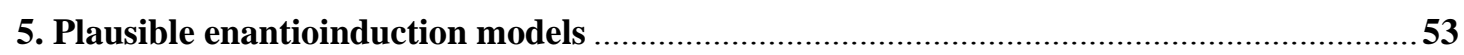

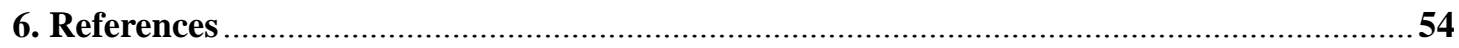

7. NMR spectra

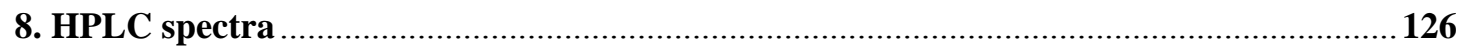




\section{I . General Information}

Unless otherwise mentioned, all experiments were carried out under an atmosphere of argon in a glovebox or using standard Schlenk techniques. Solvents were dried with standard procedures and degassed with $\mathrm{N}_{2}$. Flash column chromatography was performed using Tsingdao silica gel (60, particle size 300-400 mesh). NMR spectra were recorded on a Bruker DPX 400 spectrometer at $400 \mathrm{MHz}$ for ${ }^{1} \mathrm{H}$ NMR, $101 \mathrm{MHz}$ for ${ }^{13} \mathrm{C}$ NMR or a Bruker DPX 500 spectrometer at $500 \mathrm{MHz}$ for ${ }^{1} \mathrm{H} \mathrm{NMR}, 126 \mathrm{MHz}$ for ${ }^{13} \mathrm{C} \mathrm{NMR}$ in $\mathrm{CDCl}_{3}$ or $\mathrm{CD}_{3} \mathrm{OD}$ with tetramethylsilane (TMS) as internal standard. Chemical shifts are reported in ppm and coupling constants are given in Hz. Chemical shifts were reported relative to TMS (0.00 ppm) for ${ }^{1} \mathrm{H}$ NMR and relative to $\mathrm{CDCl}_{3}(77.00 \mathrm{ppm})$ or $\mathrm{CD}_{3} \mathrm{OD}(49.00 \mathrm{ppm})$ for ${ }^{13} \mathrm{C} \mathrm{NMR}$. GC analysis was carried out on Angilent 1200 Series instrument using achiral capillary columns. Substrates $\mathbf{1} \mathbf{a e}, \mathbf{3 a}, \mathbf{5 g}, \mathbf{5} \mathbf{j}$ and $\mathbf{3 i}$ were purchased from commercial suppliers and used without further purification. High resolution mass spectra (HRMS) were obtained on Thermo Scientific Q Exactive hybrid quadrupole-Orbitrap mass spectrometer. PE refers to petroleum ether, EA refers to ethyl acetate, TFE refers to 2,2,2-trifluoroethanol, and MTBE refers to methyl tert-butyl ether.

\section{General Procedures for the Synthesis of Substrates}

\subsection{General Procedure A:}

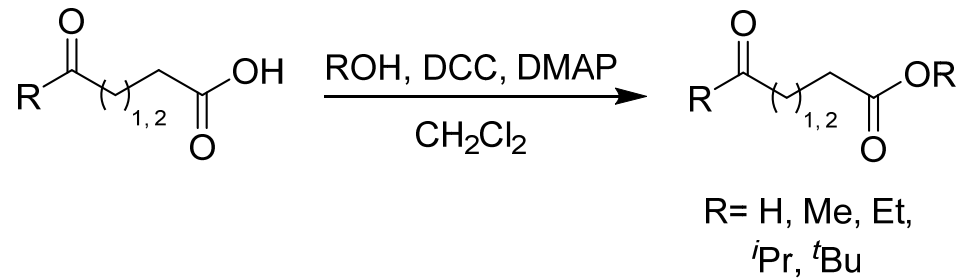

The keto-acid (20 mmol) was dissolved in $20 \mathrm{~mL} \mathrm{DCM}$, followed by successively addition of the corresponding alcohol $(60$ mmol $)$ and DMAP (4-Dimethylaminopyridine, $1.95 \mathrm{~g}, 16 \mathrm{mmol})$. DCC (N,N'-Dicyclohexylcarbodiimide, $22 \mathrm{mmol}$ ) was then added at $0{ }^{\circ} \mathrm{C}$. The resulting mixture was stirred at room 
temperature for $24 \mathrm{~h}$. Upon completion, the reaction mixture was filtered over a small silica gel column. The filtrate was collected and concentrated under reduced pressure. The residue was subjected to column chromatography on silica gel (eluent: EA/Hexane $=5-10 \%)$ to afford the corresponding ester (68-96\% yield).

\subsection{General Procedure B:}

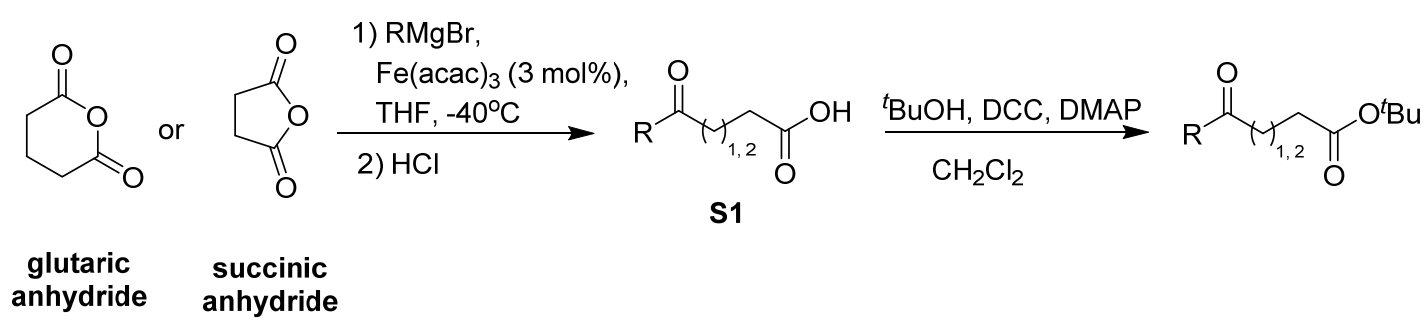

General procedure B was implemented following a reported method with modification. $^{[1]}$

Under Argon, succinic anhydride $(20.0 \mathrm{mmol})$ or glutaric anhydride $(20.0 \mathrm{mmol})$, $\mathrm{Fe}(\mathrm{acac})_{3}(212 \mathrm{mg}, 0.6 \mathrm{mmol})$ were dissolved in $24 \mathrm{~mL}$ of THF. The mixture was cooled to $-40{ }^{\circ} \mathrm{C}$. A solution of the corresponding Grignard reagent $(20 \mathrm{mmol})$ in $\mathrm{THF}$ was added dropwise over 40 minutes. The resulting mixture was then warmed to room temperature and stirred overnight. Upon completion, the reaction was quenched by slowly addition of $80 \mathrm{~mL} \mathrm{HCl}_{\mathrm{aq}}$ solution $(2 \mathrm{M}$ ) and the organic layer was extracted with $\mathrm{Et}_{2} \mathrm{O}(3 \times 40 \mathrm{~mL})$. The combined organic layer was washed with $1 \mathrm{M} \mathrm{NaOH}_{\mathrm{aq}}$ solution $(3 \times 30 \mathrm{~mL})$. The organic layer was discarded and the combined aqueous phases were acidified with $2 \mathrm{M} \mathrm{HCl}_{\mathrm{aq}}$ solution until the $\mathrm{PH}$ value was $c a$. 1 . The mixture was then extracted with $\mathrm{Et}_{2} \mathrm{O}(3 \times 40 \mathrm{~mL})$, and the combined organic layer was dried over anhydrous $\mathrm{NaSO}_{4}$. The solvent was removed under reduced pressure, and the resulting crude keto-acid S1 was directly used in the next step without further purification.

The keto-acid S1 obtained from the last step was dissolved in $20 \mathrm{~mL}$ DCM, followed by successively addition of tert-butyl alcohol (3 equiv) and DMAP (4-Dimethylaminopyridine, 0.8 equiv). DCC (N,N'-Dicyclohexylcarbodiimide, 1.1 euiqv) was then added at $0{ }^{\circ} \mathrm{C}$. The resulting mixture was warmed to room 
temperature and stirred for $24 \mathrm{~h}$. Upon completion, the reaction mixture was filtered over celite, and the organic layer was collected and concentrated under reduced pressure. The residue was subjected to column chromatography on silica gel (eluent: EA/Hexane $=5-10 \%)$ to afford the corresponding easter $(10-78 \%$ yields overall two steps).

\subsection{General Procedure C:}

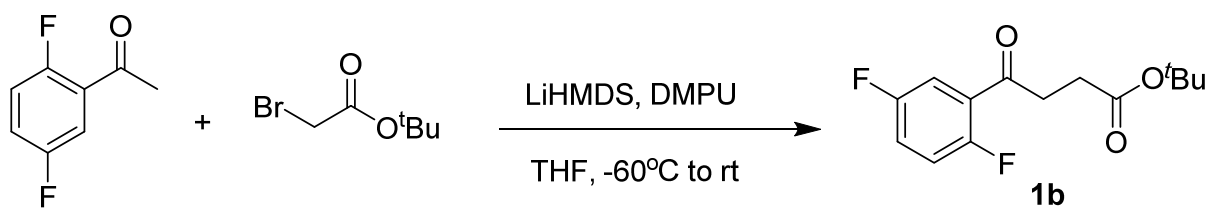

1-(2,5-difluorophenyl)ethan-1-one (4.68 g, $30 \mathrm{mmol})$ was dissolved in $75 \mathrm{~mL}$ THF and $23 \mathrm{~mL}$ DMPU (N, N'-Dimethylpropyleneurea), LiHMDS (1.3M in THF, $23.1 \mathrm{~mL}, 30 \mathrm{mmol}$ ) was then added dropwise at $-60{ }^{\circ} \mathrm{C}$. After stirring for $10 \mathrm{~min}$, tert-butyl 2-bromoacetate $(8.78 \mathrm{~g}, 45 \mathrm{mmol})$ was added quickly at the same temperature, and the resulting mixture was continuously stirred for another $10 \mathrm{~min}$. The reaction was warmed to room temperature and stirred for $4 \mathrm{~h}$ before quenching with saturated aqueous $\mathrm{NH}_{4} \mathrm{Cl}$. The mixture was diluted with water, then extracted with MTBE $(3 \times 100 \mathrm{~mL})$. The combined organic layer was dried over anhydrous $\mathrm{NaSO}_{4}$. The solvent was removed under reduced pressure and the residue was subjected to column chromatography on silica gel (eluent: EA/Hexane=2\%) to afford $1 \mathbf{b}(2.84 \mathrm{~g}, 32 \%$ yield $)$.

\subsection{General Procedure D:}
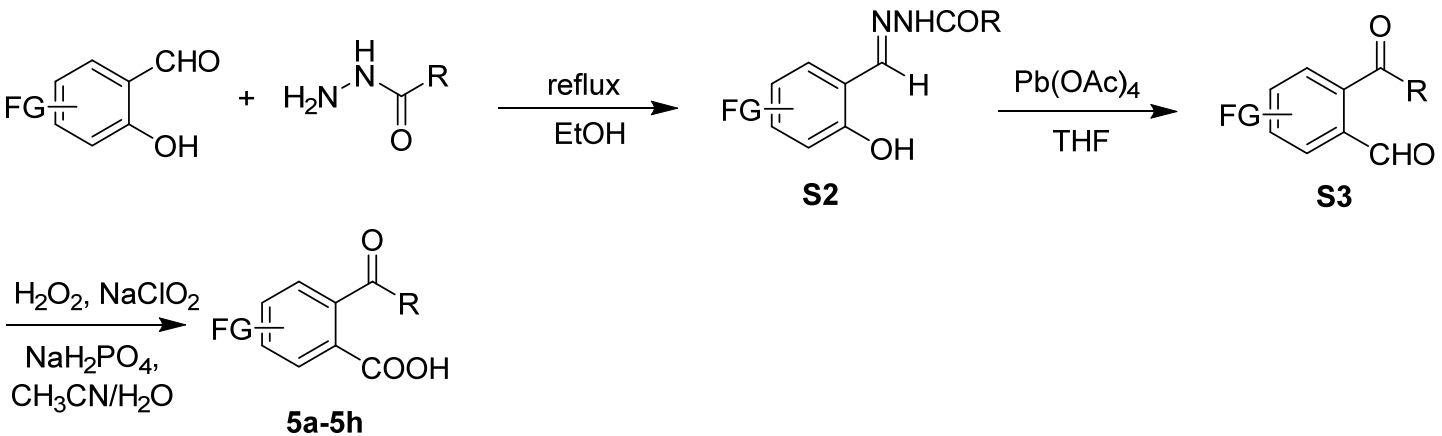
General procedure D was implemented following reported methods with modification. ${ }^{[2][3]}$

Acethydrazide $(10 \mathrm{mmol})$ was added to a solution of the salicylaldehyde derivative $(10 \mathrm{mmol})$ in $\mathrm{EtOH}(50 \mathrm{~mL})$. The reaction was heated to reflux for $12 \mathrm{~h}$. Upon completion, the mixture was cooled to room temperature, concentrated and washed with PE. The crude S2 was directly used in the next step without purification.

Hydrazide S2 (20 mmol) was dissolved in $100 \mathrm{~mL} \mathrm{THF}$, and $\mathrm{Pb}(\mathrm{OAc})_{4}(9.76 \mathrm{~g}$, $22 \mathrm{mmol}$ ) was added in portions while stirring. The reaction was stirred at room temperature for $2 \mathrm{~h}$. The resulting solid was filtered through a short column of celite, and the filtrate was collected and evaporated under reduced pressure. The residue was dissolved in $300 \mathrm{~mL}$ of EA and washed with water $(2 \times 50 \mathrm{~mL})$. The combined organic phases were successively washed with saturated aqueous $\mathrm{NaHCO}_{3}$ and brine. The combined organic layers were dried over anhydrous $\mathrm{Na}_{2} \mathrm{SO}_{4}$, filtered and evaporated. The residue was subjected to column chromatography on silica gel (eluent: EA/Hexane $=10 \%$ ) to afford S3.

$\mathrm{NaH}_{2} \mathrm{PO}_{4}(480 \mathrm{mg}, 3.09 \mathrm{mmol}), \mathrm{H}_{2} \mathrm{O}_{2}\left(30 \%\right.$ in $\left.\mathrm{H}_{2} \mathrm{O}, 2.2 \mathrm{~mL}\right)$ and $\mathrm{NaClO}_{2}(3.24 \mathrm{~g}$, $36 \mathrm{mmol})$ were successively added to a solution of S3 $(9 \mathrm{mmol})$ in $\mathrm{MeCN}(90 \mathrm{~mL})$ and water $(7.5 \mathrm{~mL})$ at $0{ }^{\circ} \mathrm{C}$. The mixture was vigorously stirred at $0{ }^{\circ} \mathrm{C}$ for $1 \mathrm{~h}$ and then diluted with EA $(120 \mathrm{~mL})$ and water $(75 \mathrm{~mL})$. The organic layer was separated, and the aqueous layer was extracted by EA $(3 \times 50 \mathrm{~mL})$. The combined organic layer was washed with water and brine, dried over anhydrous $\mathrm{Na}_{2} \mathrm{SO}_{4}$, filtered and evaporated. The resulting acid is generally pure enough to use without further purification unless other notification (33-47\% overall yield).

Notification: Dependent on the substituent on the benzene ring, free acid 5 may exist in equilibrium with its intramolecular cyclization hemiketal at $\mathrm{rt}^{[4]}$<smiles>[R]C(=O)c1ccc(F)cc1C(=O)O</smiles> 
Synthesis of 5ab.

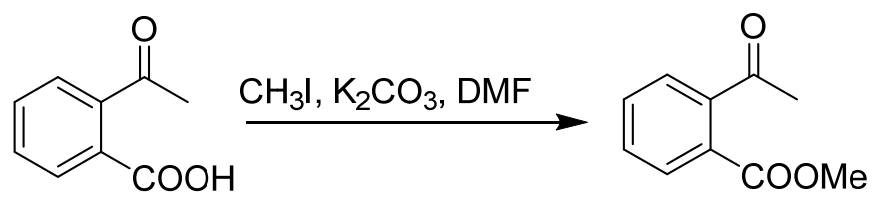

$5 a$

$5 \mathrm{ab}$

2-Propionylbenzoic acid (5a, 1.0g, $6 \mathrm{mmol}), \mathrm{K}_{2} \mathrm{CO}_{3}(1.3 \mathrm{~g}, 9 \mathrm{mmol})$ were dissolved in $5 \mathrm{~mL}$ DMF. $\mathrm{CH}_{3} \mathrm{I}(2.6 \mathrm{~g}, 18 \mathrm{mmol})$ in $4 \mathrm{~mL}$ DMF was added over $10 \mathrm{~min}$ and then stirred for $3 \mathrm{~h}$ at room temperature. Upon completion, DMF was removed under vacuum. The residue was dissolved in DCM (60 mL), and washed with water for 3 times $(3 \times 10 \mathrm{~mL})$. The organic layer was dried over anhydrous $\mathrm{Na}_{2} \mathrm{SO}_{4}$, filtered and evaporated. The residue was subjected to column chromatography on silica gel (eluent: EA/Hexane $=10 \%$ ) to afford 5 ba in $92 \%$ yield.

\subsection{General Procedure E:}<smiles>CC1(C)CC(=O)c2ccccc21</smiles>

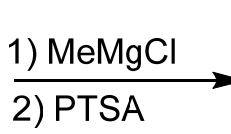<smiles>CC1=CC(C)(C)c2ccccc21</smiles>

S4<smiles>O=C1CCCc2ccccc21</smiles><smiles>CC1=CCCc2ccccc21</smiles>

S4'<smiles>CC(=O)c1cc(C(C)(C)C(=O)O)ccc1C(C)(C)C(=O)O</smiles>

$5 \mathrm{~h}$

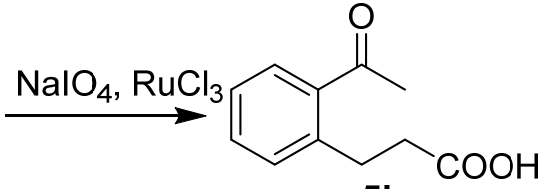

$5 \mathbf{i}$

General procedure E was implemented following a reported method with modification. ${ }^{[5]}$

Benzo-fused ketone (20 mmol) was dissolved in $40 \mathrm{~mL}$ THF, followed by slow addition of $10 \mathrm{~mL} \mathrm{MeMgCl}\left(3 \mathrm{M}\right.$ in THF) at $0{ }^{\circ} \mathrm{C}$. The reaction was warmed to room temperature and stirred for $2 \mathrm{~h}$. The reaction was quenched by saturated aqueous $\mathrm{NH}_{4} \mathrm{Cl}$, diluted with water, and then extracted with EA $(3 \times 50 \mathrm{~mL})$. The combined organic layers were dried over anhydrous $\mathrm{NaSO}_{4}$, filtrated and evaporated under reduced pressure. The residue was dissolved in $50 \mathrm{~mL} \mathrm{MeOH}$, followed by slow addition of PTSA ( $p$-toluenesulfonic acid, $1 \mathrm{mmol}$ ). The resulting mixture was heated 
to reflux for $3 \mathrm{~h}$. The reaction was then cooled to room temperature, and the solvent was removed under vacuum. The residue was subjected to column chromatography on silica gel (eluent: EA/Hexane $=10 \%$ ) to afford S4 or S4'.

S4 or S4' $(16.8 \mathrm{mmol})$ and $\mathrm{NaIO}_{4}(84 \mathrm{mmol})$ were dissolved in $100 \mathrm{~mL}$ mixed solvent $\left(\mathrm{CCl}_{4} / \mathrm{MeCN} / \mathrm{H}_{2} \mathrm{O}=3 / 3 / 4\right.$ and stirred at $\mathrm{rt}$ for $15 \mathrm{~min} . \mathrm{RuCl}_{3}(0.42 \mathrm{mmol} \mathrm{x} 2)$ was then added every $30 \mathrm{~min}$. Upon completion, the reaction mixture was filtered through a short column of celite. The filtrate was washed with $1 \mathrm{M} \mathrm{NaOH}_{\mathrm{aq}}$ solution $(3 \times 50 \mathrm{~mL})$. The organic layer was discarded and the combined aqueous basic phase was acidified with $12 \mathrm{M} \mathrm{HCl}_{\mathrm{aq}}$ solution until the $\mathrm{PH}$ value reached $c a$. 1 . The mixture was then extracted with EA $(3 \times 40 \mathrm{~mL})$, and the combined organic layers were dried over anhydrous $\mathrm{NaSO}_{4}$, filtered and evaporated under reduced pressure. The resulting acid 5 h ( $42 \%$ yield) or $5 \mathbf{i}$ (34\% yield) was pure enough to use and analyze.

\subsection{Characterization data of substrates}

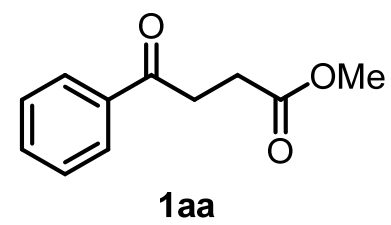

Chemical Formula: $\mathrm{C}_{11} \mathrm{H}_{12} \mathrm{O}_{3}$

Exact Mass: 192.0786

\section{Methyl 4-oxo-4-phenylbutanoate (1aa)}

Followed by General procedure A. Light yellow oil, 3.68 g, 96\% yield, obtained by the purification with flash column chromatography on silica gel (EtOAc/petroleum ether 1:19-1:9). ${ }^{1} \mathrm{H}$ NMR (400 MHz, $\left.\mathrm{CDCl}_{3}\right) \delta 7.99(\mathrm{~d}, J=7.7 \mathrm{~Hz}, 2 \mathrm{H}), 7.57$ (t, $J=$ $7.4 \mathrm{~Hz}, 1 \mathrm{H}), 7.47$ (t, $J=7.5 \mathrm{~Hz}, 2 \mathrm{H}), 3.71(\mathrm{~s}, 3 \mathrm{H}), 3.33$ (t, $J=6.6 \mathrm{~Hz}, 2 \mathrm{H}), 2.77$ (t, $J=$ $6.6 \mathrm{~Hz}, 2 \mathrm{H}) .{ }^{13} \mathrm{C}\left\{{ }^{1} \mathrm{H}\right\} \mathrm{NMR}\left(101 \mathrm{MHz}, \mathrm{CDCl}_{3}\right) \delta 198.0,173.3,136.4,133.2,128.6$, 128.0, 51.8, 33.3, 27.9. HRMS (ESI) m/z: $[\mathrm{M}+\mathrm{H}]^{+}$Calcd for $\mathrm{C}_{11} \mathrm{H}_{13} \mathrm{O}_{3}{ }^{+}$193.0859; Found 193.0860. 
<smiles>CCOC(=O)CCC(=O)c1ccccc1</smiles>

Chemical Formula: $\mathrm{C}_{12} \mathrm{H}_{14} \mathrm{O}_{3}$

Exact Mass: 206.0943

\section{Ethyl 4-oxo-4-phenylbutanoate (1ab)}

Followed by General procedure A. Light yellow oil, 3.91g, 95\% yield, obtained by the purification with flash column chromatography on silica gel (EtOAc/petroleum ether 1:19-1:9). ${ }^{1} \mathrm{H}$ NMR (400 MHz, Chloroform-d) $\delta 7.99$ (d, J=8.5 Hz, 2H), 7.57 (t, $J=7.4 \mathrm{~Hz}, 1 \mathrm{H}), 7.47(\mathrm{t}, J=7.6 \mathrm{~Hz}, 2 \mathrm{H}), 4.16(\mathrm{q}, J=7.2 \mathrm{~Hz}, 2 \mathrm{H}), 3.32(\mathrm{t}, J=6.6 \mathrm{~Hz}$, 2H), $2.76(\mathrm{t}, J=6.7 \mathrm{~Hz}, 2 \mathrm{H}), 1.27(\mathrm{t}, J=7.1 \mathrm{~Hz}, 3 \mathrm{H}) .{ }^{13} \mathrm{C}\left\{{ }^{1} \mathrm{H}\right\}$ NMR (101 MHz, Chloroform-d) $\delta 198.1,172.9,136.5,133.2,128.6,128.0,60.6,33.4,28.3,14.2$. HRMS (ESI) m/z: [M+H] $]^{+}$Calcd for $\mathrm{C}_{12} \mathrm{H}_{15} \mathrm{O}_{3}{ }^{+}$207.1016; Found 207.1016.<smiles>CCCOC(=O)CCC(=O)c1ccccc1</smiles>

$1 \mathrm{ac}$

Chemical Formula: $\mathrm{C}_{13} \mathrm{H}_{16} \mathrm{O}_{3}$

Exact Mass: 220.1099

\section{Isopropyl 4-oxo-4-phenylbutanoate (1ac)}

Followed by General procedure A. Light yellow oil, 3.96 g, 90\% yield, obtained by the purification with flash column chromatography on silica gel (EtOAc/petroleum ether 1:19-1:9). ${ }^{1} \mathrm{H}$ NMR (400 MHz, $\left.\mathrm{CDCl}_{3}\right) \delta 7.99(\mathrm{~d}, J=7.7 \mathrm{~Hz}, 2 \mathrm{H}), 7.57$ (t, $J=$ $7.3 \mathrm{~Hz}, 1 \mathrm{H}), 7.47(\mathrm{t}, J=7.6 \mathrm{~Hz}, 2 \mathrm{H}), \delta 5.10-4.96$ (hept, $J=6.2 \mathrm{~Hz}, 1 \mathrm{H}), 3.31(\mathrm{t}, J=$ $6.6 \mathrm{~Hz}, 2 \mathrm{H}), 2.73(\mathrm{t}, J=6.5 \mathrm{~Hz}, 2 \mathrm{H}), 1.25(\mathrm{~d}, J=6.2 \mathrm{~Hz}, 6 \mathrm{H}) .{ }^{13} \mathrm{C}\left\{{ }^{1} \mathrm{H}\right\} \mathrm{NMR}(101$ MHz, Chloroform-d) $\delta$ 197.0, 172.1, 136.3, 132.9, 128.3, 127.7, 67.6, 33.1, 28.3, 21.5. HRMS (ESI) m/z: [M+H] ${ }^{+}$Calcd for $\mathrm{C}_{13} \mathrm{H}_{17} \mathrm{O}_{3}{ }^{+}$221.1172; Found 221.1173. 


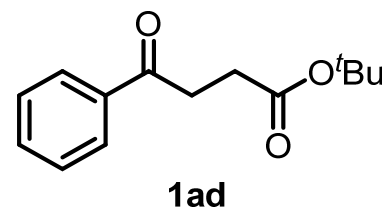

Chemical Formula: $\mathrm{C}_{14} \mathrm{H}_{18} \mathrm{O}_{3}$

Exact Mass: 234.1256

\section{Tert-butyl 4-oxo-4-phenylbutanoate (1ad)}

Followed by General procedure A. Light yellow oil, 3.98 g, 82\% yield, obtained by the purification with flash column chromatography on silica gel (EtOAc/petroleum ether 1:19-1:9). ${ }^{1} \mathrm{H}$ NMR (400 MHz, $\left.\mathrm{CDCl}_{3}\right) \delta 7.98(\mathrm{~d}, J=7.2 \mathrm{~Hz}, 2 \mathrm{H}), 7.56(\mathrm{t}, J=$ $7.4 \mathrm{~Hz}, 1 \mathrm{H}), 7.47$ (d, J = 7.8 Hz, 2H), 3.26 (t, $J=6.7 \mathrm{~Hz}, 2 \mathrm{H}), 2.68$ (t, $J=6.7 \mathrm{~Hz}, 2 \mathrm{H})$, 1.45 (s, 9H). ${ }^{13} \mathrm{C}\left\{{ }^{1} \mathrm{H}\right\}$ NMR (101 MHz, Chloroform-d) $\delta 198.3,172.1,136.7,133.1$, 128.5, 128.0, 80.5, 33.4, 29.4, 28.0. HRMS (ESI), m/z: $[\mathrm{M}+\mathrm{H}]^{+}$, Calcd for $\mathrm{C}_{14} \mathrm{H}_{19} \mathrm{O}_{3}{ }^{+}$ 235.1329; Found: 235.1327.<smiles>CCCCC(=O)CCC(=O)c1cc(F)ccc1F</smiles>

$1 \mathrm{~b}$

Chemical Formula: $\mathrm{C}_{14} \mathrm{H}_{16} \mathrm{~F}_{2} \mathrm{O}_{3}$

Exact Mass: 270.1068

\section{Tert-butyl 4-(2,5-difluorophenyl)-4-oxobutanoate (1b)}

Followed by General procedure C. Light yellow oil, 2.56 g, 32\% yield, obtained by the purification with flash column chromatography on silica gel (EtOAc/petroleum ether 1:19-1:9). ${ }^{1} \mathrm{H}$ NMR (600 MHz, $\left.\mathrm{CDCl}_{3}\right) \delta$ 7.61-7.51 (m, 1H), 7.25-7.18 (m, 1H), 7.17-7.08 (m, 1H), 3.22-3.26(m, 2H), $2.67(\mathrm{t}, J=6.4 \mathrm{~Hz}, 2 \mathrm{H}), 1.45(\mathrm{~s}, 9 \mathrm{H}) .{ }^{13} \mathrm{C}\left\{{ }^{1} \mathrm{H}\right\}$ NMR (151 MHz, Chloroform-d) $\delta 195.3\left(\mathrm{~d}, J_{\mathrm{CF}}=4.6 \mathrm{~Hz}\right), 171.7,158.5\left(\mathrm{dd}, J_{\mathrm{CF}}=\right.$ 244.6, 2.1 Hz), $158.0\left(\mathrm{dd}, J_{\mathrm{CF}}=250.7,2.1 \mathrm{~Hz}\right), 126.2\left(\mathrm{dd}, J_{\mathrm{CF}}=15.9,6.3 \mathrm{~Hz}\right), 121.2$ $\left(\mathrm{dd}, J_{\mathrm{CF}}=24.6,9.4 \mathrm{~Hz}\right), 118.1\left(\mathrm{dd}, J_{\mathrm{CF}}=27.2,7.9 \mathrm{~Hz}\right), 116.4\left(\mathrm{dd}, J_{\mathrm{CF}}=25.1,3.3 \mathrm{~Hz}\right)$, 80.5, $38.2\left(\mathrm{~d}, J_{\mathrm{CF}}=8.7 \mathrm{~Hz}\right), 29.26\left(\mathrm{~d}, J_{\mathrm{CF}}=2.2 \mathrm{~Hz}\right), 27.93 . \mathrm{HRMS}(\mathrm{ESI}) \mathrm{m} / \mathrm{z}$ : $[\mathrm{M}+\mathrm{Na}]^{+}$Calcd for $\mathrm{C}_{14} \mathrm{H}_{16} \mathrm{~F}_{2} \mathrm{NaO}_{3}{ }^{+}$293.0960; Found 293.0961 . 
<smiles>CCCCOC(=O)CCC(=O)c1cccc(F)c1</smiles>

Chemical Formula: $\mathrm{C}_{14} \mathrm{H}_{17} \mathrm{FO}_{3}$

Exact Mass: 252.1162

\section{Tert-butyl 4-(3-fluorophenyl)-4-oxobutanoate (1c)}

Followed by General procedure A. Light yellow oil, 3.42 g, 68\% yield, obtained by the purification with flash column chromatography on silica gel (EtOAc/petroleum ether 1:19-1:9). ${ }^{1} \mathrm{H}$ NMR (400 MHz, $\left.\mathrm{CDCl}_{3}\right) \delta 7.81-7.73(\mathrm{~m}, 1 \mathrm{H}), 7.66(\mathrm{~m}, 1 \mathrm{H})$, $7.45 \mathrm{~m}, 1 \mathrm{H}), 7.26(\mathrm{~m}, 1 \mathrm{H}), 3.23$ (t, $J=6.6 \mathrm{~Hz}, 2 \mathrm{H}), 2.69$ (t, $J=6.6 \mathrm{~Hz}, 2 \mathrm{H}), 1.45$ (s, 9H). ${ }^{13} \mathrm{C}\left\{{ }^{1} \mathrm{H}\right\}$ NMR (101 MHz, Chloroform-d) $\delta 197.0\left(\mathrm{~d}, J_{\mathrm{CF}}=2.1 \mathrm{~Hz}\right), 171.9,162.8$ $\left(\mathrm{d}, J_{\mathrm{CF}}=247.8 \mathrm{~Hz}\right), 138.7\left(\mathrm{~d}, J_{\mathrm{CF}}=6.2 \mathrm{~Hz}\right), 130.2\left(\mathrm{~d}, J_{\mathrm{CF}}=7.6 \mathrm{~Hz}\right), 123.7\left(\mathrm{~d}, J_{\mathrm{CF}}=\right.$ $3.1 \mathrm{~Hz}), 120.0\left(\mathrm{~d}, J_{\mathrm{CF}}=21.5 \mathrm{~Hz}\right), 114.7\left(\mathrm{~d}, J_{\mathrm{CF}}=22.2 \mathrm{~Hz}\right), 80.6,33.5,29.2,28.0$. HRMS (ESI) m/z: [M+Na] $]^{+}$Calcd for $\mathrm{C}_{14} \mathrm{H}_{17} \mathrm{FNaO}_{3}{ }^{+}$275.1054; Found 275.1053.<smiles>CCCCOC(=O)CCC(=O)c1ccc(F)cc1</smiles>

$1 d$

Chemical Formula: $\mathrm{C}_{14} \mathrm{H}_{17} \mathrm{FO}_{3}$

Exact Mass: 252.1162

\section{Tert-butyl 4-(4-fluorophenyl)-4-oxobutanoate (1d)}

Followed by General procedure B. White solid, $806 \mathrm{mg}, 16 \%$ yield, obtained by the purification with flash column chromatography on silica gel (EtOAc/petroleum ether 1:19-1:9). ${ }^{1} \mathrm{H}$ NMR (400 MHz, $\mathrm{CDCl}_{3}$ ) $\delta$ 8.06-7.96 (m, 2H), 7.18-7.08 (m, 2H), 3.23 $(\mathrm{t}, J=6.6 \mathrm{~Hz}, 2 \mathrm{H}), 2.68(\mathrm{t}, J=6.6 \mathrm{~Hz}, 2 \mathrm{H}), 1.45(\mathrm{~s}, 9 \mathrm{H}) .{ }^{13} \mathrm{C}\left\{{ }^{1} \mathrm{H}\right\} \mathrm{NMR}(101 \mathrm{MHz}$, Chloroform-d) $\delta 196.7,172.0,165.7\left(\mathrm{~d}, J_{\mathrm{CF}}=254.6 \mathrm{~Hz}\right), 133.1\left(\mathrm{~d}, J_{\mathrm{CF}}=3.1 \mathrm{~Hz}\right)$, $130.6\left(\mathrm{~d}, J_{\mathrm{CF}}=9.3 \mathrm{~Hz}\right), 115.6\left(\mathrm{~d}, J_{\mathrm{CF}}=21.9 \mathrm{~Hz}\right), 80.6,33.3,29.4,28.0$. HRMS (ESI) $\mathrm{m} / \mathrm{z}:[\mathrm{M}+\mathrm{Na}]^{+}$, Calcd for $\mathrm{C}_{14} \mathrm{H}_{17} \mathrm{FNaO}_{3}{ }^{+}$275.1054; Found 275.1054 . 
<smiles>CCCCC(=O)CCC(=O)c1ccc(Cl)cc1</smiles>

Chemical Formula: $\mathrm{C}_{14} \mathrm{H}_{17} \mathrm{ClO}_{3}$

Exact Mass: 268.0866

\section{Tert-butyl 4-(4-chlorophenyl)-4-oxobutanoate (1e)}

Followed by General procedure A. Yellowish solid, $4.28 \mathrm{~g}, 80 \%$ yield, obtained by the purification with flash column chromatography on silica gel (EtOAc/petroleum ether 1:19-1:9). ${ }^{1} \mathrm{H}$ NMR (400 MHz, $\left.\mathrm{CDCl}_{3}\right) \delta$ 7.96-7.87 (m, 2H), 7.47-7.37 (m, 2H), $3.22(\mathrm{t}, J=6.7 \mathrm{~Hz}, 2 \mathrm{H}), 2.68(\mathrm{t}, J=6.7 \mathrm{~Hz}, 2 \mathrm{H}), 1.45(\mathrm{~s}, 9 \mathrm{H}) .{ }^{13} \mathrm{C}\left\{{ }^{1} \mathrm{H}\right\}$ NMR $(101$ MHz, Chloroform-d) $\delta 197.1,171.9,139.5,135.0,129.4,128.8,80.6,33.4,29.3,28.0$. HRMS (ESI) m/z: [M+Na] $]^{+}$, Calcd for $\mathrm{C}_{14} \mathrm{H}_{17} \mathrm{ClNaO}_{3}{ }^{+}$291.0758; Found: 291.0760.<smiles>CCCOC(=O)CCC(=O)c1ccc(Br)cc1</smiles>

Chemical Formula: $\mathrm{C}_{14} \mathrm{H}_{17} \mathrm{BrO}_{3}$

Exact Mass: 312.0361

\section{Tert-butyl 4-(4-bromophenyl)-4-oxobutanoate (1f)}

Followed by General procedure A. Yellowish solid, 1.50 g, 24\% yield, obtained by the purification with flash column chromatography on silica gel (EtOAc/petroleum ether 1:19-1:9). ${ }^{1} \mathrm{H}$ NMR (400 MHz, $\left.\mathrm{CDCl}_{3}\right) \delta$ 7.88-7.81 (m, 2H), 7.63-7.56 (m, 2H), $3.21(\mathrm{t}, J=6.6 \mathrm{~Hz}, 2 \mathrm{H}), 2.68(\mathrm{t}, J=6.6 \mathrm{~Hz}, 2 \mathrm{H}), 1.45(\mathrm{~s}, 9 \mathrm{H}) .{ }^{13} \mathrm{C}\left\{{ }^{1} \mathrm{H}\right\}$ NMR $(101$ MHz, Chloroform-d) $\delta$ 197.3, 171.9, 135.3, 131.8, 129.5, 128.2, 80.6, 33.3, 29.2, 28.0. HRMS (ESI) m/z: $\left([\mathrm{M}+\mathrm{Na}]^{+}\right)$Calcd for $\mathrm{C}_{14} \mathrm{H}_{17} \mathrm{BrNaO}_{3}{ }^{+}$335.0253; Found 335.0255. 
<smiles>CCCCOC(=O)CCC(=O)c1ccccc1C</smiles>

$1 \mathrm{~g}$

Chemical Formula: $\mathrm{C}_{15} \mathrm{H}_{20} \mathrm{O}_{3}$

Exact Mass: 248.1412

\section{Tert-butyl 4-oxo-4-(o-tolyl)butanoate (1g)}

Followed by General procedure B. Light yellow oil, $1.19 \mathrm{~g}, 24 \%$ yield, obtained by the purification with flash column chromatography on silica gel (EtOAc/petroleum ether 1:19-1:9). ${ }^{1} \mathrm{H}$ NMR (400 MHz, $\left.\mathrm{CDCl}_{3}\right) \delta$ 7.73-7.66 (m, 1H), 7.40-7.31 (m, 1H), 7.30-7.19 (m, 2H), 3.15 (t, $J=6.6 \mathrm{~Hz}, 2 \mathrm{H}), 2.65$ (t, $J=6.6 \mathrm{~Hz}, 2 \mathrm{H}), 2.48$ (s, 3H), 1.45 (s, 9H). ${ }^{13} \mathrm{C}\left\{{ }^{1} \mathrm{H}\right\}$ NMR (101 MHz, Chloroform-d) $\delta$ 202.2, 172.0, 137.9, 137.7, 131.8, 131.1, 128.3, 125.6, 80.4, 36.2, 29.6, 28.0, 21.1. HRMS (ESI) m/z: $[\mathrm{M}+\mathrm{Na}]^{+}$Calcd for $\mathrm{C}_{15} \mathrm{H}_{20} \mathrm{NaO}_{3}{ }^{+}$271.1305; Found 271.1305.<smiles>CCCCC(=O)CCC(=O)c1cccc(C)c1</smiles>

$1 \mathrm{~h}$

Chemical Formula: $\mathrm{C}_{15} \mathrm{H}_{20} \mathrm{O}_{3}$

Exact Mass: 248.1412

\section{Tert-butyl 4-oxo-4-(m-tolyl)butanoate (1h)}

Followed by General procedure B. Light yellow oil, $3.87 \mathrm{~g}, 78 \%$ yield, obtained by the purification with flash column chromatography on silica gel (EtOAc/petroleum ether 1:19-1:9). ${ }^{1} \mathrm{H}$ NMR (400 MHz, $\left.\mathrm{CDCl}_{3}\right) \delta$ 7.82-7.73 (m, 2H), 7.40-7.29 (m, 2H), $3.24(\mathrm{t}, J=6.7 \mathrm{~Hz}, 2 \mathrm{H}), 2.67(\mathrm{t}, J=6.7 \mathrm{~Hz}, 2 \mathrm{H}), 2.40(\mathrm{~s}, 3 \mathrm{H}), 1.45(\mathrm{~s}, 9 \mathrm{H}) .{ }^{13} \mathrm{C}\left\{{ }^{1} \mathrm{H}\right\}$ NMR (101 MHz, Chloroform-d) $\delta$ 198.4, 172.1, 138.3, 136.7, 133.8, 128.5, 128.4, 125.2, 80.5, 33.5, 29.4, 28.0, 21.3. HRMS (ESI) m/z: $[\mathrm{M}+\mathrm{Na}]^{+}$Calcd for $\mathrm{C}_{15} \mathrm{H}_{20} \mathrm{NaO}_{3}{ }^{+} 271.1305 ; 271.1305$. 
<smiles>CCCCC(=O)CCC(=O)c1ccc(C)cc1</smiles>

$1 \mathrm{i}$

Chemical Formula: $\mathrm{C}_{15} \mathrm{H}_{20} \mathrm{O}_{3}$

Exact Mass: 248.1412

\section{Tert-butyl 4-oxo-4-(p-tolyl)butanoate (1i)}

Followed by General procedure B.Yellowish solid, $2.78 \mathrm{~g}, 56 \%$ yield, obtained by the purification with flash column chromatography on silica gel (EtOAc/petroleum ether 1:19-1:9). ${ }^{1} \mathrm{H}$ NMR (400 MHz, $\left.\mathrm{CDCl}_{3}\right) \delta 7.88(\mathrm{~d}, J=8.1 \mathrm{~Hz}, 2 \mathrm{H}), 7.25(\mathrm{~d}, J=$ $8.0 \mathrm{~Hz}, 2 \mathrm{H}), 3.23(\mathrm{t}, J=6.7 \mathrm{~Hz}, 2 \mathrm{H}), 2.67(\mathrm{t}, J=6.7 \mathrm{~Hz}, 2 \mathrm{H}), 2.40(\mathrm{~s}, 3 \mathrm{H}), 1.45$ (s, 9H). ${ }^{13} \mathrm{C}\left\{{ }^{1} \mathrm{H}\right\}$ NMR (101 MHz, Chloroform-d) $\delta 197.9,172.2,143.8,134.2,129.2$, 128.1, 80.5, 33.3, 29.4, 28.0, 21.6. HRMS (ESI) m/z: $271.1304[\mathrm{M}+\mathrm{Na}]^{+}$Calcd for $\mathrm{C}_{15} \mathrm{H}_{20} \mathrm{NaO}_{3}{ }^{+}$271.1305; Found 271.1305.<smiles>CCCCC(=O)OCCC(=O)c1ccc(Br)cc1</smiles>

$1 \mathrm{j}$

Chemical Formula: $\mathrm{C}_{18} \mathrm{H}_{26} \mathrm{O}_{3}$

Exact Mass: 290.1882

\section{Tert-butyl 4-(4-(tert-butyl)phenyl)-4-oxobutanoate (1j)}

Followed by General procedure B. Light yellow oil, $2.32 \mathrm{~g}, 40 \%$ yield, obtained by the purification with flash column chromatography on silica gel (EtOAc/petroleum ether 1:19-1:9). ${ }^{1} \mathrm{H}$ NMR (400 MHz, $\left.\mathrm{CDCl}_{3}\right) \delta$ 7.95-7.90 (m, 2H), 7.50-7.44 (m, 2H), $3.24(\mathrm{t}, J=6.7 \mathrm{~Hz}, 2 \mathrm{H}), 2.67(\mathrm{t}, J=6.7 \mathrm{~Hz}, 2 \mathrm{H}), 1.45(\mathrm{~s}, 9 \mathrm{H}), 1.34(\mathrm{~s}, 9 \mathrm{H}) .{ }^{13} \mathrm{C}\left\{{ }^{1} \mathrm{H}\right\}$ NMR (101 MHz, Chloroform-d) $\delta 197.9,172.2,156.7,134.1,127.9,125.4,80.4,35.0$, 33.3, 31.0, 29.5, 28.0. HRMS (ESI), m/z: $[\mathrm{M}+\mathrm{Na}]^{+}$Calcd for $\mathrm{C}_{18} \mathrm{H}_{26} \mathrm{NaO}_{3}{ }^{+}$313.1774; Found 313.1775. 
<smiles>CCCCC(=O)CCC(=O)c1ccc(OC)cc1</smiles>

$1 \mathrm{k}$

Chemical Formula: $\mathrm{C}_{15} \mathrm{H}_{20} \mathrm{O}_{4}$

Exact Mass: 264.1362

\section{Tert-butyl 4-(4-methoxyphenyl)-4-oxobutanoate (1k)}

Followed by General procedure B. Yellowish solid, 1.74 g, 33\% yield, obtained by the purification with flash column chromatography on silica gel (EtOAc/petroleum ether 1:19-1:9). ${ }^{1} \mathrm{H}$ NMR (400 MHz, $\left.\mathrm{CDCl}_{3}\right) \delta$ 8.01-7.92 (m, 2), 6.98-6.89 (m, 2H), $3.87(\mathrm{~s}, 3 \mathrm{H}), 3.21(\mathrm{t}, J=6.7 \mathrm{~Hz}, 2 \mathrm{H}), 2.67(\mathrm{t}, J=6.7 \mathrm{~Hz}, 2 \mathrm{H}), 1.45(\mathrm{~s}, 9 \mathrm{H}) .{ }^{13} \mathrm{C}\left\{{ }^{1} \mathrm{H}\right\}$ NMR (101 MHz, Chloroform-d) $\delta 196.8,172.3,163.4,130.2,129.8,113.6,80.5,55.4$, 33.0, 29.5, 28.0. HRMS (ESI) $\mathrm{m} / \mathrm{z}$ : $[\mathrm{M}+\mathrm{H}]^{+}$Calcd for $\mathrm{C}_{15} \mathrm{H}_{21} \mathrm{O}_{4}{ }^{+}$: 265.1434; Found 265.1433 .<smiles>CCCCC(=O)CCC(=O)c1ccc(-c2ccccc2)cc1</smiles>

Chemical Formula: $\mathrm{C}_{20} \mathrm{H}_{22} \mathrm{O}_{3}$

Exact Mass: 310.1569

\section{Tert-butyl 4-([1,1'-biphenyl]-4-yl)-4-oxobutanoate (1l)}

Followed by General procedure A. White solid, $1.05 \mathrm{~g}, 17 \%$ yield, obtained by the purification with flash column chromatography on silica gel (EtOAc/petroleum ether 1:19-1:9). ${ }^{1} \mathrm{H}$ NMR (400 MHz, Chloroform-d) $\delta$ 8.10-8.02 (m, 2H), 7.72-7.58 (m, 4H), 7.51-7.37 (m, 3H), $3.29(\mathrm{t}, J=6.7 \mathrm{~Hz}, 2 \mathrm{H}), 2.71(\mathrm{t}, J=6.7 \mathrm{~Hz}, 2 \mathrm{H}), 1.46(\mathrm{~s}, 9 \mathrm{H})$. ${ }^{13} \mathrm{C}\left\{{ }^{1} \mathrm{H}\right\}$ NMR (101 MHz, Chloroform-d) $\delta$ 197.9, 172.2, 145.7, 139.8, 135.4, 128.9, 128.6, 128.2, 127.2, 127.2, 80.6, 33.5, 29.5, 28.1. RMS (ESI) m/z: $[\mathrm{M}+\mathrm{Na}]^{+}$Calcd for $\mathrm{C}_{20} \mathrm{H}_{22} \mathrm{NaO}_{3}{ }^{+}$333.1461; Found 303.1452. 


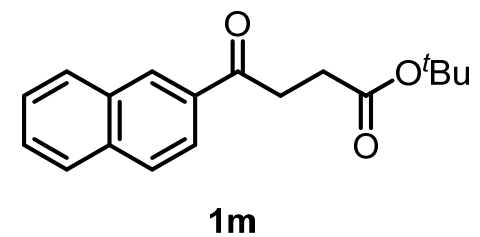

Chemical Formula: $\mathrm{C}_{18} \mathrm{H}_{20} \mathrm{O}_{3}$

Exact Mass: 284.1412

\section{Tert-butyl 4-(naphthalen-2-yl)-4-oxobutanoate (1m)}

Followed by General procedure B. Yellow solid, $568 \mathrm{mg}, 10 \%$ yield, obtained by the purification with flash column chromatography on silica gel (EtOAc/petroleum ether 1:19-1:9). ${ }^{1} \mathrm{H}$ NMR (400 MHz, Chloroform-d) $\delta 8.50(\mathrm{~s}, 1 \mathrm{H}), 8.07-8.00(\mathrm{~m}, 1 \mathrm{H})$, 7.98-7.91 (m, 1H), 7.91-7.82 (m, 2H), 7.63-7.49 (m, 2H), $3.39(\mathrm{t}, J=6.7 \mathrm{~Hz}, 2 \mathrm{H})$, $2.74(\mathrm{t}, J=6.7 \mathrm{~Hz}, 2 \mathrm{H}), 1.47$ (s, 9H). ${ }^{13} \mathrm{C}\left\{{ }^{1} \mathrm{H}\right\}$ NMR (101 MHz, Chloroform-d) $\delta$ $198.2,172.2,135.5,134.0,132.4,129.6,129.5,128.4,127.7,126.7,123.7,80.6,33.5$, 29.5, 28.0. HRMS (ESI) m/z: [M+Na] $]^{+}$Calcd for $\mathrm{C}_{18} \mathrm{H}_{20} \mathrm{NaO}_{3}{ }^{+}$307.1305; Found 307.1307 .<smiles>CCCCC(=O)CCC(C)=O</smiles>

Chemical Formula: $\mathrm{C}_{9} \mathrm{H}_{16} \mathrm{O}_{3}$

Exact Mass: 172.1099

\section{Tert-butyl 4-oxopentanoate (1n)}

Followed by General procedure A. Colorless oil, $2.13 \mathrm{~g}, 62 \%$ yield, obtained by the purification with flash column chromatography on silica gel (EtOAc/petroleum ether 1:19-1:9). ${ }^{1} \mathrm{H}$ NMR (400 MHz, Chloroform-d) $\delta 2.70(\mathrm{t}, J=6.6 \mathrm{~Hz}, 2 \mathrm{H}), 2.49(\mathrm{t}, J=$ $6.5 \mathrm{~Hz}, 2 \mathrm{H}), 2.19(\mathrm{~s}, 3 \mathrm{H}), 1.44(\mathrm{~s}, 9 \mathrm{H}) .{ }^{13} \mathrm{C}\left\{{ }^{1} \mathrm{H}\right\}$ NMR (101 MHz, Chloroform-d) $\delta$ 206.71, 171.81, 80.35, 37.90, 29.71, 29.03. HRMS (ESI) m/z: [M+Na] Calcd for $\mathrm{C}_{9} \mathrm{H}_{16} \mathrm{NO}_{3} \mathrm{Na}^{+}$195.0992; Found 195.0994. 
<smiles>O=C(O)CCCC(=O)c1ccccc1</smiles>

3aa

Chemical Formula: $\mathrm{C}_{11} \mathrm{H}_{12} \mathrm{O}_{3}$

Exact Mass: 192.0786

\section{5-oxo-5-phenylpentanoic acid (3aa)}

Commercially available and used as received. White solid.<smiles>COC(=O)CCCC(=O)c1ccccc1</smiles>

$3 a b$

Chemical Formula: $\mathrm{C}_{12} \mathrm{H}_{14} \mathrm{O}_{3}$

Exact Mass: 206.0943

\section{Methyl 5-oxo-5-phenylpentanoate (3ab)}

Followed by General procedure A. Colorless oil, $3.71 \mathrm{~g}, 90 \%$ yield, obtained by the purification with flash column chromatography on silica gel (EtOAc/petroleum ether 1:19-1:9). ${ }^{1} \mathrm{H}$ NMR (400 MHz, Chloroform-d) $\delta 7.97$ (d, $\left.J=7.4 \mathrm{~Hz}, 2 \mathrm{H}\right), 7.56$ (t, $J=$ $7.4 \mathrm{~Hz}, 1 \mathrm{H}), 7.46$ (t, $J=7.4 \mathrm{~Hz}, 2 \mathrm{H}), 3.68(\mathrm{~s}, 3 \mathrm{H}), 3.06(\mathrm{t}, J=7.2 \mathrm{~Hz}, 2 \mathrm{H}), 2.46$ (t, $J=$ 7.2 Hz, 2H), 2.16-1.98 (m, 2H). ${ }^{13} \mathrm{C}\left\{{ }^{1} \mathrm{H}\right\}$ NMR (101 MHz, Chloroform-d) $\delta 199.3$, 173.6, 136.7, 133.0, 128.5, 127.9, 51.5, 37.4, 33.0, 19.2. HRMS (ESI) m/z: $[\mathrm{M}+\mathrm{H}]^{+}$ Calcd for $\mathrm{C}_{12} \mathrm{H}_{15} \mathrm{O}_{3}{ }^{+}$207.1016; Found 207.1016.<smiles>CCCOC(=O)CCCC(=O)c1ccccc1</smiles>

Chemical Formula: $\mathrm{C}_{14} \mathrm{H}_{18} \mathrm{O}_{3}$

Exact Mass: 234.1256

\section{Isopropyl 5-oxo-5-phenylpentanoate (3ac)}

Followed by General procedure A. Colorless oil, $4.21 \mathrm{~g}, 90 \%$ yield, obtained by the purification with flash column chromatography on silica gel (EtOAc/petroleum ether 
1:19-1:9). ${ }^{1} \mathrm{H}$ NMR (400 MHz, Chloroform-d) $\delta 7.96(\mathrm{~d}, J=7.4 \mathrm{~Hz}, 2 \mathrm{H}), 7.55$ (t, $J=$ $7.4 \mathrm{~Hz}, 1 \mathrm{H}), 7.45$ (t, $J=7.4 \mathrm{~Hz}, 2 \mathrm{H}), 5.14-4.91$ (hept, $J=5.0 \mathrm{~Hz}, 1 \mathrm{H}$ ), 3.04 (t, $J=7.2$ Hz, 2H), 2.40 (t, $J=7.2 \mathrm{~Hz}, 2 \mathrm{H}), 2.13-1.97(\mathrm{~m}, 2 \mathrm{H}), 1.23(\mathrm{~d}, J=6.3 \mathrm{~Hz}, 6 \mathrm{H})$. ${ }^{13} \mathrm{C}\left\{{ }^{1} \mathrm{H}\right\}$ NMR (101 MHz, Chloroform-d) $\delta$ 199.2, 172.5, 136.6, 132.8, 128.4, 127.8, 67.4, 37.2, 33.5, 21.6, 19.3. HRMS (ESI), m/z: $[\mathrm{M}+\mathrm{H}]^{+}$Calcd for $\mathrm{C}_{14} \mathrm{H}_{19} \mathrm{O}_{3}{ }^{+}$ 235.1329; Found 235.1329.<smiles>CCCCC(=O)CCCC(=O)c1ccccc1</smiles>

3a

Chemical Formula: $\mathrm{C}_{15} \mathrm{H}_{20} \mathrm{O}_{3}$

Exact Mass: 248.1412

\section{Tert-butyl 5-oxo-5-phenylpentanoate (3a)}

Followed by General procedure A. Colorless oil, 3.52 g, 71\% yield, obtained by the purification with flash column chromatography on silica gel (EtOAc/petroleum ether 1:19-1:9). ${ }^{1} \mathrm{H}$ NMR (400 MHz, Chloroform-d) $\delta$ 8.01-7.92 (m, 2H), 7.59-7.41 (m, 3H), $3.03(\mathrm{t}, J=7.2 \mathrm{~Hz}, 2 \mathrm{H}), 2.34(\mathrm{t}, J=7.2 \mathrm{~Hz}, 2 \mathrm{H}), 2.08-1.98(\mathrm{~m}, 2 \mathrm{H}), 1.45(\mathrm{~s}, 9 \mathrm{H})$. ${ }^{13} \mathrm{C}\left\{{ }^{1} \mathrm{H}\right\}$ NMR (101 MHz, Chloroform-d) $\delta$ 199.2, 172.3, 136.7, 132.8, 128.4, 127.8, 80.0, 37.3, 34.4, 27.9, 19.4. HRMS (ESI) m/z: $[\mathrm{M}+\mathrm{Na}]^{+}$Calcd for $\mathrm{C}_{15} \mathrm{H}_{20} \mathrm{NaO}_{3}{ }^{+}$ 271.1305; Found 271.1305.<smiles>CCCCC(=O)CCCC(=O)c1ccccc1C</smiles>

3b

Chemical Formula: $\mathrm{C}_{16} \mathrm{H}_{22} \mathrm{O}_{3}$

Exact Mass: 262.1569

\section{Tert-butyl 5-oxo-5-(o-tolyl)pentanoate (3b)}

Followed by General procedure B. Colorless oil, $3.20 \mathrm{~g}, 61 \%$ yield, obtained by the purification with flash column chromatography on silica gel (EtOAc/petroleum ether 1:19-1:9). ${ }^{1} \mathrm{H}$ NMR (400 MHz, Chloroform-d) $\delta 7.63$ (d, $\left.J=7.9 \mathrm{~Hz}, 1 \mathrm{H}\right), 7.40-7.32$ 
(m, 1H), 7.27-7.20 (m, 2H), $2.95(\mathrm{t}, J=7.2 \mathrm{~Hz}, 2 \mathrm{H}), 2.49(\mathrm{~s}, 3 \mathrm{H}), 2.32(\mathrm{t}, J=7.2 \mathrm{~Hz}$ 2H), 2.06-1.95 (m, 2H), $1.44(\mathrm{~s}, 9 \mathrm{H}) .{ }^{13} \mathrm{C}\left\{{ }^{1} \mathrm{H}\right\}$ NMR (101 MHz, Chloroform-d) $\delta$ 203.6, 172.5, 137.9, 131.8, 131.1, 128.3, 125.6, 80.2, 40.3, 34.6, 28.0, 21.2, 19.7. HRMS (ESI) m/z: [M+Na] ${ }^{+}$Calcd for $\mathrm{C}_{16} \mathrm{H}_{22} \mathrm{NaO}_{3}{ }^{+}$285.1461; Found 285.1462.<smiles>CCCCC(=O)OCCCC(=O)c1cccc(C)c1</smiles>

Chemical Formula: $\mathrm{C}_{16} \mathrm{H}_{22} \mathrm{O}_{3}$

Exact Mass: 262.1569

\section{Tert-butyl 5-oxo-5-(m-tolyl)pentanoate (3c)}

Followed by General procedure B. Pale yellow oil, 2.36 g, 45\% yield, obtained by the purification with flash column chromatography on silica gel (EtOAc/petroleum ether 1:19-1:9). ${ }^{1} \mathrm{H}$ NMR (400 MHz, Chloroform-d) $\delta$ 7.79-7.73 (m, 2H), 7.40-7.31 (m, 2H), 3.02 (t, $J=7.2 \mathrm{~Hz}, 2 \mathrm{H}), 2.41(\mathrm{~s}, 3 \mathrm{H}), 2.34(\mathrm{t}, J=7.2 \mathrm{~Hz}, 2 \mathrm{H}), 2.07-1.98$ (m, 2H), 1.45 (s, 9H). ${ }^{13} \mathrm{C}\left\{{ }^{1} \mathrm{H}\right\}$ NMR (101 MHz, Chloroform-d) $\delta$ 199.7, 172.6, 138.3, 136.8, 133.7, 128.5, 128.4, 125.2, 80.2, 37.5, 34.6, 28.0, 21.3, 19.6. HRMS (ESI), m/z: $[\mathrm{M}+\mathrm{Na}]^{+}$Calcd for $\mathrm{C}_{16} \mathrm{H}_{22} \mathrm{NaO}_{3}{ }^{+} 285.1461$; Found 285.1463.<smiles>CCCCC(=O)OCCCC(=O)c1ccc(C)cc1</smiles>

3d

Chemical Formula: $\mathrm{C}_{16} \mathrm{H}_{22} \mathrm{O}_{3}$

Exact Mass: 262.1569

\section{Tert-butyl 5-oxo-5-(p-tolyl)pentanoate (3d)}

Followed by General procedure B. White solid, $3.25 \mathrm{~g}, 62 \%$ yield, obtained by the purification with flash column chromatography on silica gel (EtOAc/petroleum ether 1:19-1:9). ${ }^{1} \mathrm{H}$ NMR (400 MHz, Chloroform-d) $\delta 7.86$ (d, $\left.J=7.9 \mathrm{~Hz}, 2 \mathrm{H}\right), 7.25$ (d, $J=$ $7.9 \mathrm{~Hz}, 2 \mathrm{H}), 3.00$ (t, J = 7.2 Hz, 2H), 2.41 (s, 3H), 2.34 (t, J=7.2 Hz, 2H), $2.08-$ 1.97 (m, 2H), 1.45 (s, 9H). ${ }^{13} \mathrm{C}$ NMR (101 MHz, Chloroform-d) $\delta$ 199.3, 172.7, 143.8, 
134.4, 129.3, 128.2, 80.3, 37.4, $\left\{{ }^{1} \mathrm{H}\right\} 34.8,28.1,21.7,19.8$. HRMS (ESI) $\mathrm{m} / \mathrm{z}$ $[\mathrm{M}+\mathrm{Na}]^{+}$Calcd for $\mathrm{C}_{16} \mathrm{H}_{22} \mathrm{NaO}_{3}{ }^{+} 285.1461$; Found 285.1462.<smiles>CCCCC(=O)CCCC(=O)c1ccc(OC)cc1</smiles>

$3 e$

Chemical Formula: $\mathrm{C}_{16} \mathrm{H}_{22} \mathrm{O}_{4}$

Exact Mass: 278.1518

\section{Tert-butyl 5-(4-methoxyphenyl)-5-oxopentanoate (3e)}

Followed by General procedure B. White solid, $3.28 \mathrm{~g}$, 59\% yield, obtained by the purification with flash column chromatography on silica gel (EtOAc/petroleum ether 1:19-1:9). ${ }^{1} \mathrm{H}$ NMR (400 MHz, Chloroform-d) $\delta 7.94$ (d, $\left.J=9.0 \mathrm{~Hz}, 2 \mathrm{H}\right), 6.93$ (d, $J=$ $9.0 \mathrm{~Hz}, 2 \mathrm{H}), 3.86(\mathrm{~s}, 3 \mathrm{H}), 2.98(\mathrm{t}, J=7.3 \mathrm{~Hz}, 2 \mathrm{H}), 2.33$ (t, $J=7.2 \mathrm{~Hz}, 2 \mathrm{H}), 2.00$ (q, $J$ $=7.3 \mathrm{~Hz}, 2 \mathrm{H}), 1.45(\mathrm{~s}, 9 \mathrm{H}) .{ }^{13} \mathrm{C}\left\{{ }^{1} \mathrm{H}\right\}$ NMR (101 MHz, Chloroform-d) $\delta$ 198.1, 172.5, 163.3, 130.2, 129.8, 113.6, 80.1, 55.3, 37.0, 34.6, 28.0, 19.8. HRMS (ESI) m/z: $[\mathrm{M}+\mathrm{H}]^{+}$Calcd for $\mathrm{C}_{16} \mathrm{H}_{23} \mathrm{O}_{4}{ }^{+}$279.1591; Found 279.1592.<smiles>CCCCC(=O)OCCCC(=O)c1ccc(F)cc1</smiles>

Chemical Formula: $\mathrm{C}_{15} \mathrm{H}_{19} \mathrm{FO}_{3}$

Exact Mass: 266.1318

\section{Tert-butyl 5-(4-fluorophenyl)-5-oxopentanoate (3f)}

Followed by General procedure A. White solid, 3.19 g, $60 \%$ yield, obtained by the purification with flash column chromatography on silica gel (EtOAc/petroleum ether 1:19-1:9). ${ }^{1} \mathrm{H}$ NMR (400 MHz, Chloroform-d) $\delta 8.07$ - 7.88 (m, 2H), 7.20 - 7.07 (m, 2H), $3.01(\mathrm{t}, J=7.2 \mathrm{~Hz}, 2 \mathrm{H}), 2.34(\mathrm{t}, J=7.2 \mathrm{~Hz}, 2 \mathrm{H}), 2.12-1.95(\mathrm{~m}, 2 \mathrm{H}), 1.45$ (s, 9H). ${ }^{13} \mathrm{C}\left\{{ }^{1} \mathrm{H}\right\}$ NMR (101 MHz, Chloroform-d) $\delta$ 198.0, 172.5, $165.7\left(\mathrm{~d}, J_{\mathrm{CF}}=254.5\right.$ $\mathrm{Hz}), 133.3\left(\mathrm{~d}, J_{\mathrm{CF}}=3.0 \mathrm{~Hz}\right), 130.6\left(\mathrm{~d}, J_{\mathrm{CF}}=9.3 \mathrm{~Hz}\right), 115.6\left(\mathrm{~d}, J_{\mathrm{CF}}=21.8 \mathrm{~Hz}\right), 80.3$, 37.4, 34.6, 28.1, 19.6. HRMS (ESI) $\mathrm{m} / \mathrm{z}[\mathrm{M}+\mathrm{Na}]^{+} \mathrm{Calcd}$ for $\mathrm{C}_{15} \mathrm{H}_{19} \mathrm{FNaO}_{3}{ }^{+}$289.1210; 
<smiles>CCCCC(=O)CCCC(=O)c1cc(OC)cc(OC)c1</smiles>

Chemical Formula: $\mathrm{C}_{17} \mathrm{H}_{24} \mathrm{O}_{5}$

Exact Mass: 308.1624

\section{Tert-butyl 5-(3,5-dimethoxyphenyl)-5-oxopentanoate (3g)}

Followed by General procedure B. Light yellow oil, 3.02 g, 49\% yield, obtained by the purification with flash column chromatography on silica gel (EtOAc/petroleum ether 1:19-1:9). ${ }^{1} \mathrm{H}$ NMR (400 MHz, Chloroform-d) $\delta 7.10$ (d, $\left.J=2.3 \mathrm{~Hz}, 2 \mathrm{H}\right), 6.64$ (t, $J=2.3 \mathrm{~Hz}, 1 \mathrm{H}), 3.83$ (s, 6H), 2.99 (t, $J=7.2 \mathrm{~Hz}, 2 \mathrm{H}), 2.33$ (t, $J=7.2 \mathrm{~Hz}, 2 \mathrm{H}), 2.08-$ $1.94(\mathrm{~m}, 2 \mathrm{H}), 1.45$ (s, 9H). ${ }^{13} \mathrm{C}\left\{{ }^{1} \mathrm{H}\right\} \mathrm{NMR}(101 \mathrm{MHz}$, Chloroform-d) $\delta$ 199.3, 172.6, $160.8,138.8,105.8,105.3,80.3,55.6,37.6,34.6,28.1,19.7$. HRMS (ESI) m/z $[\mathrm{M}+\mathrm{H}]^{+}$Calcd for $\mathrm{C}_{17} \mathrm{H}_{25} \mathrm{O}_{5}{ }^{+}$309.1697; Found 309.1697.<smiles>CCCCC(=O)OCCCC(=O)c1ccc(F)c(F)c1</smiles>

3h

Chemical Formula: $\mathrm{C}_{15} \mathrm{H}_{18} \mathrm{~F}_{2} \mathrm{O}_{3}$

Exact Mass: 284.1224

\section{Tert-butyl 5-(3,4-difluorophenyl)-5-oxopentanoate (3h)}

Followed by General procedure B. White solid, $2.50 \mathrm{~g}$, 44\% yield, obtained by the purification with flash column chromatography on silica gel (EtOAc/petroleum ether 1:19-1:9). ${ }^{1} \mathrm{H}$ NMR (600 MHz, Chloroform-d) $\delta 7.83$ - $7.78(\mathrm{~m}, 1 \mathrm{H}), 7.77$ - 7.73 (m, 1H), $7.27-7.21(\mathrm{~m}, 1 \mathrm{H}), 2.99$ (t, J = 7.2 Hz, 2H), 2.34 (t, J = 7.1 Hz, 2H), 2.02 (p, J $=7.2 \mathrm{~Hz}, 2 \mathrm{H}), 1.45(\mathrm{~s}, 9 \mathrm{H}) .{ }^{13} \mathrm{C}\left\{{ }^{1} \mathrm{H}\right\}$ NMR (151 MHz, Chloroform-d) $\delta$ 197.0, 172.5, $153.6\left(\mathrm{dd}, J_{\mathrm{CF}}=256.7,12.8 \mathrm{~Hz}\right), 150.4\left(\mathrm{dd}, J_{\mathrm{CF}}=250.7,12.8 \mathrm{~Hz}\right), 133.9\left(\mathrm{t}, J_{\mathrm{CF}}=3.8\right.$ $\mathrm{Hz}), 125.0\left(\mathrm{dd}, J_{\mathrm{CF}}=7.4,3.6 \mathrm{~Hz}\right), 117.5\left(\mathrm{~d}, J_{\mathrm{CF}}=17.8 \mathrm{~Hz}\right), 117.3\left(\mathrm{dd}, J_{\mathrm{CF}}=18.0,1.4\right.$ $\mathrm{Hz}), \quad 80.4,37.4, \quad 34.4,28.1$, 19.5. HRMS (ESI) $\mathrm{m} / \mathrm{z}[\mathrm{M}+\mathrm{Na}]^{+}$Calcd for 
<smiles>O=C(O)CCCCC(=O)c1ccccc1</smiles>

$3 \mathbf{i}$

Chemical Formula: $\mathrm{C}_{12} \mathrm{H}_{14} \mathrm{O}_{3}$

Exact Mass: 206.0943

\section{6-oxo-6-phenylhexanoic acid (3i)}

Commercially available and used as received. White solid.<smiles>CC(=O)c1ccccc1C(=O)O</smiles>

Chemical Formula: $\mathrm{C}_{9} \mathrm{H}_{8} \mathrm{O}_{3}$

Exact Mass: 164.0473

\section{2-acetylbenzoic acid (5aa)}

Commercially available and used as received. White solid.<smiles>COC(=O)c1ccccc1C(C)=O</smiles>

5 ab

Chemical Formula: $\mathrm{C}_{10} \mathrm{H}_{10} \mathrm{O}_{3}$

Exact Mass: 178.0630

\section{Methyl 2-acetylbenzoate (5ab)}

Colorless oil, $982 \mathrm{mg}, 92 \%$ yield, obtained by the purification with flash column chromatography on silica gel (EtOAc/petroleum ether 1:9). 1H NMR (400 MHz, Chloroform-d) $\delta 7.85(\mathrm{~d}, \mathrm{~J}=8.0 \mathrm{~Hz}, 1 \mathrm{H}), 7.60-7.48(\mathrm{~m}, 2 \mathrm{H}), 7.43$ (d, J = 7.6 Hz, 1H), $3.90(\mathrm{~s}, 3 \mathrm{H}), 2.55$ (s, 3H). HRMS (ESI), m/z $179.0703\left([\mathrm{M}+\mathrm{H}]^{+}\right)$, Calcd for $\mathrm{C}_{10} \mathrm{H}_{11} \mathrm{O}_{3}{ }^{+}: 179.0703$. The ${ }^{1} \mathrm{H}$ NMR data is consistent with that reported. ${ }^{[6]}$ 
<smiles>CCC(=O)c1ccccc1C(=O)O</smiles>

$5 b$

Chemical Formula: $\mathrm{C}_{10} \mathrm{H}_{10} \mathrm{O}_{3}$

Exact Mass: 178.0630

\section{2-propionylbenzoic acid (5b)}

Followed by General procedure D, recrystallized from EA/Hex. White solid, $673 \mathrm{mg}$, $42 \%$ yield. ${ }^{1} \mathrm{H}$ NMR $(600 \mathrm{MHz}$, Chloroform- $d) \delta 7.82(\mathrm{~d}, J=7.2 \mathrm{~Hz}, 1 \mathrm{H}), 7.71(\mathrm{t}, J=$ $7.1 \mathrm{~Hz}, 1 \mathrm{H}), 7.63-7.50(\mathrm{~m}, 2 \mathrm{H}), 2.19$ (d, $J=54.7 \mathrm{~Hz}, 2 \mathrm{H}), 0.90(\mathrm{t}, J=7.0 \mathrm{~Hz}, 3 \mathrm{H})$. ${ }^{13} \mathrm{C}\left\{{ }^{1} \mathrm{H}\right\}$ NMR (151 MHz, Chloroform-d) $\delta$ 168.6, 148.5, 134.6, 130.6, 126.9, 125.5, 122.3, 108.0, 32.0, 7.7. HRMS (ESI) m/z: $[\mathrm{M}+\mathrm{H}]^{+}$Calcd for $\mathrm{C}_{10} \mathrm{H}_{11} \mathrm{O}_{3}{ }^{+}$179.0703; Found 179.0703.<smiles>CC(=O)c1ccc(Br)cc1C(=O)O</smiles>

$5 c$

Chemical Formula: $\mathrm{C}_{9} \mathrm{H}_{7} \mathrm{BrO}_{3}$

Exact Mass: 241.9579

\section{2-acetyl-5-bromobenzoic acid (5c)}

Followed by General procedure D. Yellow solid, $2.27 \mathrm{~g}, 47 \%$ yield. HRMS (ESI) $\mathrm{m} / \mathrm{z}$ : $[\mathrm{M}+\mathrm{H}]^{+}$Calcd for $\mathrm{C}_{9} \mathrm{H}_{8} \mathrm{BrO}_{3}{ }^{+}$242.9651; Found 242.9652. The NMR of 5c is messy, possibly due to a fast equilibrium between $\mathbf{5 c}$ and $5 c^{\prime}$ at $r$. Therefore, its structure was determined by converting it to the corresponding methyl ester $\mathbf{5} \mathbf{c b}$. However, the catalytic reaction was performed with $\mathbf{5 c}$.<smiles>CC(=O)c1ccc(Br)cc1C(=O)O</smiles>

$5 c$

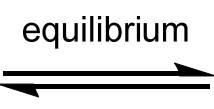<smiles>CC1(O)OC(=O)c2cc(Br)ccc21</smiles>

$5 c^{\prime}$ 
<smiles>COC(=O)c1cc(Br)ccc1C(C)=O</smiles>

$5 \mathrm{cb}$

Chemical Formula: $\mathrm{C}_{10} \mathrm{H}_{9} \mathrm{BrO}_{3}$

Exact Mass: 255.9735

\section{methyl 2-acetyl-5-bromobenzoate (5cb)}

${ }^{1} \mathrm{H}$ NMR (400 MHz, Chloroform-d) $\delta 7.97$ (d, J = 1.9 Hz, 1H), 7.70 (dd, J = 8.2, 1.9 $\mathrm{Hz}, 1 \mathrm{H}), 7.32(\mathrm{~d}, \mathrm{~J}=8.2 \mathrm{~Hz}, 1 \mathrm{H}), 3.91(\mathrm{~s}, 3 \mathrm{H}), 2.53(\mathrm{~s}, 3 \mathrm{H}) .{ }^{13} \mathrm{C}\left\{{ }^{1} \mathrm{H}\right\}$ NMR (101 MHz, Chloroform-d) $\delta$ 201.47, 166.21, 140.94, 134.86, 132.61, 130.81, 128.16, 124.39, 52.85, 29.73. HRMS (ESI) $\mathrm{m} / \mathrm{z}$ : $[\mathrm{M}+\mathrm{H}]^{+}$Calcd for $\mathrm{C}_{10} \mathrm{H}_{10} \mathrm{BrO}_{3}{ }^{+}:$256.9808; Found 256.9801 .<smiles>COc1ccc(C(=O)O)c(C(C)=O)c1</smiles>

5d

Chemical Formula: $\mathrm{C}_{10} \mathrm{H}_{10} \mathrm{O}_{4}$

Exact Mass: 194.0579

\section{2-acetyl-4-methoxybenzoic acid (5d)}

Followed by General procedure D, recrystallized from EA/Hex. White solid, $699 \mathrm{mg}$, $40 \%$ yield. ${ }^{1} \mathrm{H}$ NMR (600 MHz, Chloroform- $d$ ) $\delta 7.69$ (d, $\left.J=8.4 \mathrm{~Hz}, 1 \mathrm{H}\right), 7.07-6.91$ (m, 2H), $3.91(\mathrm{~s}, 3 \mathrm{H}), 1.86(\mathrm{~s}, 3 \mathrm{H}) .{ }^{13} \mathrm{C}\left\{{ }^{1} \mathrm{H}\right\}$ NMR (151 MHz, Chloroform-d) $\delta 168.3$, 165.2, 152.6, 127.1, 118.2, 117.7, 106.0, 105.0, 55.9, 26.0. HRMS (ESI), m/z: $[\mathrm{M}+\mathrm{H}]^{+}$Calcd for $\mathrm{C}_{10} \mathrm{H}_{11} \mathrm{O}_{4}{ }^{+}$195.0652; Found 195.0654. 
<smiles>CC(=O)c1ccc(C)cc1C(=O)O</smiles>

$5 e$

Chemical Formula: $\mathrm{C}_{10} \mathrm{H}_{10} \mathrm{O}_{3}$

Exact Mass: 178.0630

\section{2-acetyl-5-methylbenzoic acid (5e)}

Followed by General procedure D. White solid, $561 \mathrm{mg}, 35 \%$ yield. ${ }^{1} \mathrm{H}$ NMR (400 $\mathrm{MHz}$, Chloroform-d) $\delta 7.65$ (s, 1H), $7.56-7.38$ (m, 2H), $4.68(\mathrm{~s}, 1 \mathrm{H}), 2.47$ (s, 3H), $2.01(\mathrm{~s}, 3 \mathrm{H}) .{ }^{13} \mathrm{C}\left\{{ }^{1} \mathrm{H}\right\}$ NMR (101 MHz, Methanol-d 4$) \delta 170.7,149.4,142.1,136.9$, 127.8, 125.9, 123.1, 108.0, 26.4, 21.2. HRMS (ESI) m/z: [M+H] $]^{+}$Calcd for $\mathrm{C}_{10} \mathrm{H}_{11} \mathrm{O}_{3}{ }^{+}$179.0703; Found 179.0704 .<smiles>CC(=O)c1cc(C)ccc1C(=O)O</smiles>

$5 f$

Chemical Formula: $\mathrm{C}_{10} \mathrm{H}_{10} \mathrm{O}_{3}$

Exact Mass: 178.0630

\section{2-acetyl-4-methylbenzoic acid (5f)}

Followed by General procedure D. White solid, $529 \mathrm{mg}, 33 \%$ yield. ${ }^{1} \mathrm{H}$ NMR (400 MHz, Chloroform-d) $\delta 7.69$ (s, 1H), 7.35 (d, J=7.7 Hz, 2H), 4.41 (br, 1H), 2.49 (s, $3 \mathrm{H}), 1.87$ (s, 3H). ${ }^{13} \mathrm{C}\left\{{ }^{1} \mathrm{H}\right\}$ NMR (101 MHz, Chloroform-d) $\delta$ 168.7, 150.2, 146.1, 131.5, 125.3, 123.5, 122.4, 105.7, 26.0, 22.0. HRMS (ESI) m/z: $[\mathrm{M}+\mathrm{H}]^{+}$Calcd for $\mathrm{C}_{10} \mathrm{H}_{11} \mathrm{O}_{3}{ }^{+}$179.0703; Found 179.0702.<smiles>CC(=O)c1cccnc1C(=O)OC(C)C</smiles>

$5 \mathbf{g}$

Chemical Formula: $\mathrm{C}_{11} \mathrm{H}_{13} \mathrm{NO}_{3}$

Exact Mass: 207.0895 


\section{Isopropyl 3-acetylpicolinate (5g)}

Brown liquid. Commercially available and used as received.<smiles>CC(=O)c1ccccc1C(C)(C)C(=O)O</smiles>

$5 \mathrm{~h}$

Chemical Formula: $\mathrm{C}_{12} \mathrm{H}_{14} \mathrm{O}_{3}$

Exact Mass: 206.0943

\section{2-(2-acetylphenyl)-2-methylpropanoic acid (5h)}

Followed by General procedure E. Yellow solid, 1.73 g, 42\% yield. ${ }^{1} \mathrm{H}$ NMR (400 MHz, Chloroform-d) $\delta 7.52$ (dd, $J=7.7,1.5 \mathrm{~Hz}, 1 \mathrm{H}), 7.44(\mathrm{~m}, 2 \mathrm{H}), 7.32-7.15(\mathrm{~m}$, 1H), 5.19 (br, 1H), 2.36 (s, 3H), 1.59 (s, 6H). ${ }^{13} \mathrm{C}$ NMR (101 MHz, Chloroform-d) $\delta$ 180.8, 143.6, 137.1, 130.7, 127.4, 126.5, 126.1, 45.5, 29.8, 28.7. HRMS (ESI) m/z: $[\mathrm{M}+\mathrm{H}]^{+}$Calcd for $\mathrm{C}_{12} \mathrm{H}_{15} \mathrm{O}_{3}{ }^{+}$207.1016; Found 207.1017.

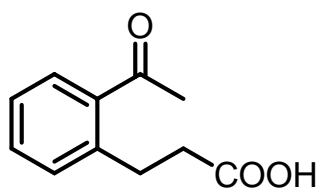

$5 \mathbf{i}$

Chemical Formula: $\mathrm{C}_{11} \mathrm{H}_{12} \mathrm{O}_{3}$

Exact Mass: 192.0786

\section{3-(2-acetylphenyl)propanoic acid (5i)}

Followed by General procedure E. White solid, $1.31 \mathrm{~g}, 34 \%$ yield. ${ }^{1} \mathrm{H}$ NMR (400 MHz, Methanol-d4) $\delta 7.80(\mathrm{~d}, J=8.0 \mathrm{~Hz}, 1 \mathrm{H}), 7.44(\mathrm{t}, J=7.5 \mathrm{~Hz}, 1 \mathrm{H}), 7.39-7.27$ $(\mathrm{m}, 2 \mathrm{H}), 3.10(\mathrm{t}, \mathrm{J}=7.7 \mathrm{~Hz}, 2 \mathrm{H}), 2.65-2.52(\mathrm{~m}, 5 \mathrm{H}) .{ }^{13} \mathrm{C}\left\{{ }^{1} \mathrm{H}\right\}$ NMR (101 MHz, Methanol-d4) $\delta$ 204.2, 176.7, 142.1, 139.0, 132.9, 132.4, 130.8, 127.6, 36.7, 30.4, 29.7. HRMS (ESI) m/z: [M+H] $]^{+}$Calcd for $\mathrm{C}_{11} \mathrm{H}_{13} \mathrm{O}_{3}{ }^{+}$193.0859; Found 193.0860. 
<smiles>O=C(O)c1ccccc1C(=O)c1ccccc1</smiles>

5j

Chemical Formula: $\mathrm{C}_{14} \mathrm{H}_{10} \mathrm{O}_{3}$

Exact Mass: 226.0630

\section{2-benzoylbenzoic acid (5j)}

Commercially available and used as received.

\section{Ru-catalyzed Asymmetric Reductive Amination/Cyclization Cascade of Keto-Acids/Esters}

\subsection{Synthesis of $\operatorname{Ru}(\mathrm{OAc})_{2}(\mathrm{~L} 2)^{[7][8]}$}

(R)-DTBM-C3 ${ }^{*}$-TunePhos (L2, $\left.59 \mathrm{mg}, 0.05 \mathrm{mmol}\right)$ and $\left[\mathrm{RuCl}_{2} \text { (benzene) }\right]_{2}(12.5 \mathrm{mg}$, $0.025 \mathrm{mmol}$ ) were placed in a Schlenk tube, followed by addition of $N, N$-Dimethylformamide $(2 \mathrm{~mL})$. The resulting solution was stirred at $100^{\circ} \mathrm{C}$ for $1 \mathrm{~h}$. The reaction was cooled to room temperature, then a solution of $\mathrm{NaOAc}(1 \mathrm{mmol})$ in methanol (3 mL, degassed) was added in one portion. The reaction was stirred for 20 minutes followed by adding water (degassed, $8 \mathrm{~mL}$ ). After vigorously stirring for 5 minutes, the precipitated yellow solids were filtered, washed with water for three times, and then dried under vacuum to give a lightly yellow solid which was used directly as catalyst. Other $\mathrm{Ru}(\mathrm{OAc})_{2}$ complexes were prepared accordingly.

3.2. General Procedure F: In a glovebox, $\mathrm{Ru}(\mathrm{OAc})_{2}(\mathrm{~L} 2)(2.8 \mathrm{mg}, 0.002 \mathrm{mmol})$, substrate $(0.2 \mathrm{mmol})$, ammonium salt $(30.8 \mathrm{mg}, 0.4 \mathrm{mmol})$ and TFE $(0.4 \mathrm{ml})$ were successively added to a $5 \mathrm{~mL}$ vial equipped with a magnetic stirring bar. The mixture was then transferred to a stain-less autoclave and purged by three cycles of pressurization/venting with $\mathrm{H}_{2}$. The required $\mathrm{H}_{2}$ pressure $(50 \mathrm{~atm})$ was then installed and the autoclave was placed in an oil bath preheated to $90{ }^{\circ} \mathrm{C}$. The autoclave was 
cooled down to room temperature after $24 \mathrm{~h}$ and the pressure was slowly released in the hood. The reaction was quenched by $\mathrm{H}_{2} \mathrm{O}(1 \mathrm{~mL})$ and extracted with ethyl ether $(1$ $\mathrm{mL} \times 3$ ). The combined organic phase was dried over anhydrous $\mathrm{Na}_{2} \mathrm{SO}_{4}$, filtered and evaporated under reduced pressure. The residue was subjected to column chromatography on silica gel (eluent: EA/Hexane $=30 \%$ ) to afford desired products.

\subsection{General Procedure G (Scale-up reactions):}

In a glovebox, $\mathrm{Ru}(\mathrm{OAc})_{2}(\mathbf{L} 2)(11.2 \mathrm{mg}, 0.008 \mathrm{mmol})$, substrate (4 mmol), ammonium salt $(616 \mathrm{mg}, 8 \mathrm{mmol})$ and TFE $(8 \mathrm{ml})$ were successively added to a $20 \mathrm{~mL}$ vial equipped with a magnetic stirring bar. The mixture was then transferred to a stain-less autoclave and purged by three cycles of pressurization/venting with $\mathrm{H}_{2}$. The required $\mathrm{H}_{2}$ pressure (50 atm) was then installed and the autoclave was placed in an oil bath preheated to $90{ }^{\circ} \mathrm{C}$. The autoclave was cooled down to room temperature after $24 \mathrm{~h}$ and the pressure was slowly released in the hood. The reaction was quenched by $\mathrm{H}_{2} \mathrm{O}$ $(10 \mathrm{~mL})$ and extracted with ethyl ether $(10 \mathrm{~mL} \times 3)$. The combined organic phase was dried over anhydrous $\mathrm{Na}_{2} \mathrm{SO}_{4}$, filtered and evaporated under reduced pressure. The residue was subjected to column chromatography on silica gel (eluent: EA/Hexane = $30 \%)$ to afford desired products.

\subsection{Details of condition optimization}

Table S1. Evaluation of the solvents 
<smiles>COC(=O)CCC(=O)c1ccccc1</smiles>

$1 a$

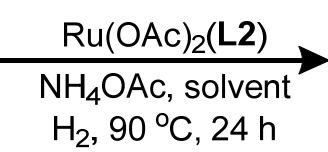

$\mathrm{H}_{2}, 90^{\circ} \mathrm{C}, 24 \mathrm{~h}$

\begin{tabular}{cccc}
\hline Entry $^{\mathrm{a}}$ & Solvent & conversion $(\%)^{\mathrm{b}}$ & ee $(\%)^{\mathrm{c}}$ \\
\hline 1 & MeOH & Messy & 20 \\
2 & EtOH & Messy & 48 \\
3 & iPrOH & Messy & -- \\
4 & TFE & $>99$ & 77 \\
5 & HFIP & $>99$ & 57 \\
6 & DCM & low & -- \\
7 & toluene & low & -- \\
8 & THF & low & - \\
\hline
\end{tabular}

[a] Reaction conditions: $1 \mathrm{a}(0.2 \mathrm{mmol}), \mathrm{NH}_{4} \mathrm{OAc}(0.4 \mathrm{mmol})$, $[\mathrm{Ru}](1 \mathrm{~mol} \%)$, solvent $(0.4 \mathrm{~mL}), \mathrm{H}_{2}(50 \mathrm{bar}), 90{ }^{\circ} \mathrm{C}, 24 \mathrm{~h}$. TFE = trifluoroethanol. [b] Determined by ${ }^{1} \mathrm{H}$ NMR analysis. [c] The ee values were determined by HPLC using chiral columns.

Table S2. Evaluation of the equivalent of ammonium salts

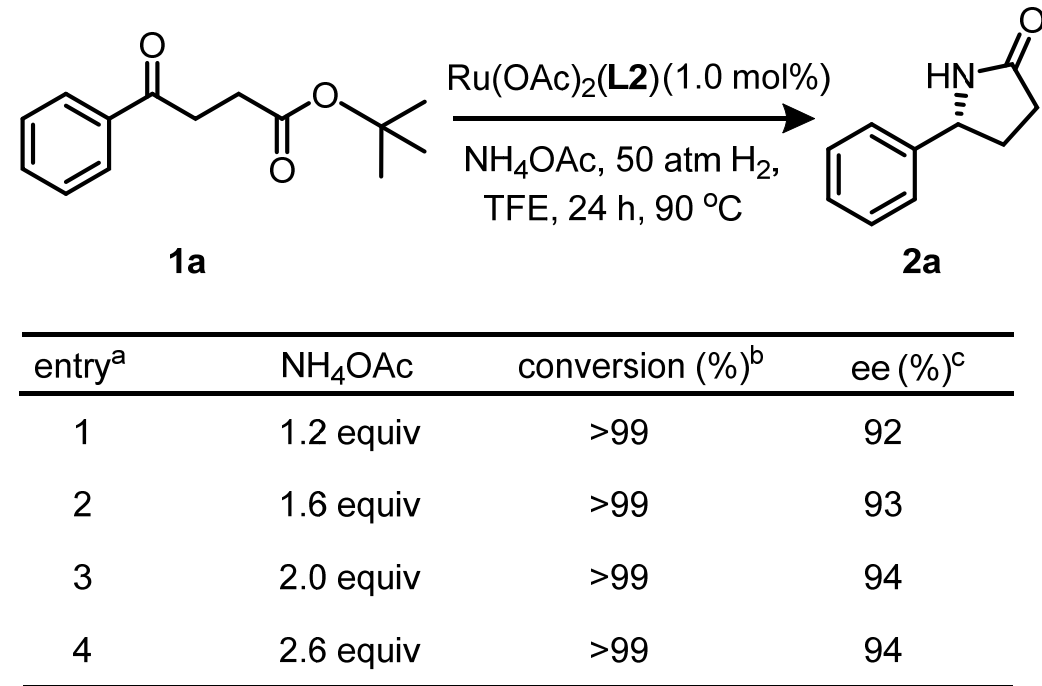

[a] Reaction conditions: $1 \mathrm{a}(0.2 \mathrm{mmol}), \mathrm{NH}_{4} \mathrm{OAc},[\mathrm{Ru}](1 \mathrm{~mol} \%)$, TFE $(0.4 \mathrm{~mL}), \mathrm{H}_{2}$ (50 bar), $90^{\circ} \mathrm{C}, 24 \mathrm{~h}$. [b] Determined by ${ }^{1} \mathrm{H}$ NMR analysis. [c] The ee values were determined by HPLC using chiral columns.

Table S3. Evaluation of reaction temperature 


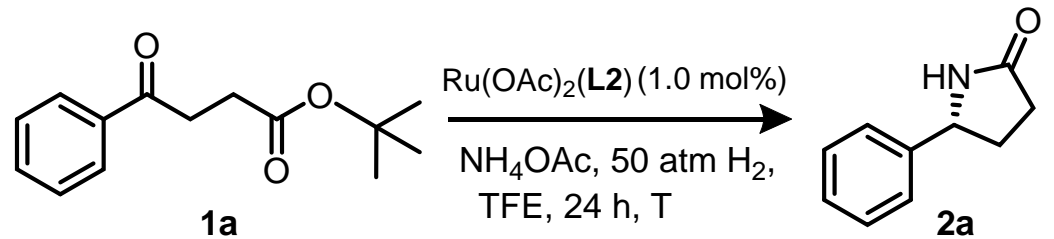

\begin{tabular}{cccc}
\hline entry $^{\mathrm{a}}$ & $\mathrm{T}\left({ }^{\mathrm{O}} \mathrm{C}\right)$ & ${\text { conversion }(\%)^{\mathrm{b}}}$ & ee (\%) \\
\hline 1 & 80 & 92 & 93 \\
2 & 90 & $>99$ & 94 \\
3 & 100 & $>99$ & 90 \\
\hline
\end{tabular}

[a] Reaction conditions: $1 \mathbf{a}(0.2 \mathrm{mmol}), \mathrm{NH}_{4} \mathrm{OAc}(0.4 \mathrm{mmol}),[\mathrm{Ru}]$ (1 mol\%), TFE (0.4 mL), $\mathrm{H}_{2}$ (50 bar), $24 \mathrm{~h}$. [b] Determined by ${ }^{1} \mathrm{H}$ NMR analysis. [c] The ee values were determined by HPLC using chiral columns.

Table S4. Evaluation of hydrogen pressure

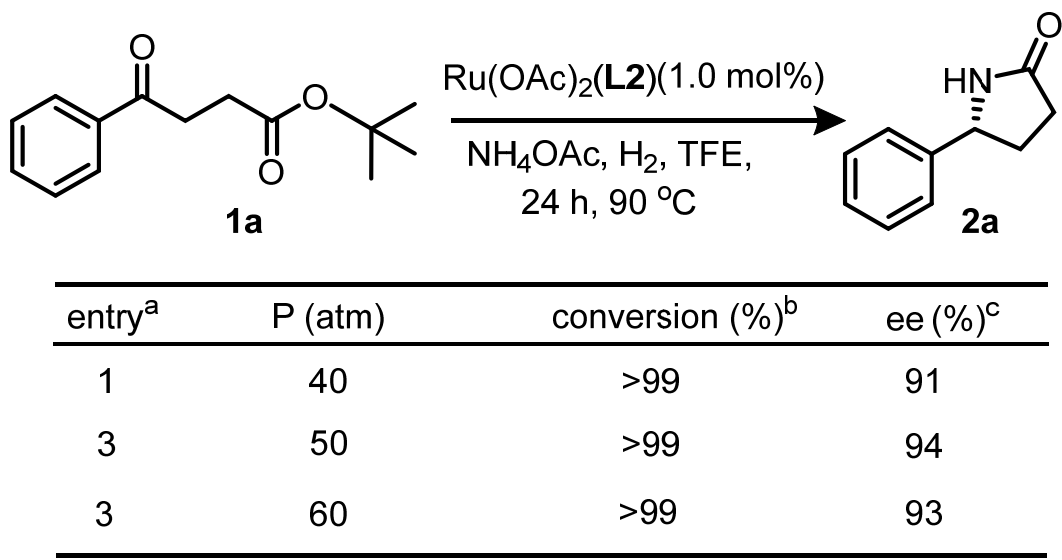

[a] Reaction conditions: $1 \mathrm{a}(0.2 \mathrm{mmol}), \mathrm{NH}_{4} \mathrm{OAc}(0.4 \mathrm{mmol})$, [Ru] (1 mol\%), TFE (0.4 mL), $\mathrm{H}_{2}(50 \mathrm{bar}), 90^{\circ} \mathrm{C}, 24 \mathrm{~h}$. [b] Determined by ${ }^{1} \mathrm{H}$ NMR analysis. [c] The ee values were determined by HPLC using chiral columns.

\subsection{Characterization data of Chiral NH Lactams}<smiles>O=C1CCC(c2ccccc2)N1</smiles>

$2 a$

Chemical Formula: $\mathrm{C}_{10} \mathrm{H}_{11} \mathrm{NO}$

Exact Mass: 161.0841 


\section{(R)-5-phenylpyrrolidin-2-one (2a)}

White solid, $30.9 \mathrm{mg}, 96 \%$ yield, $94 \%$ ee, $[\alpha]^{25}=40.9\left(\mathrm{c}=0.6, \mathrm{CHCl}_{3}\right)$, obtained by the purification with flash column chromatography on silica gel (EtOAc/petroleum ether 3:7). ${ }^{1} \mathrm{H}$ NMR (600 MHz, Chloroform-d) $\delta 7.39$ - 7.34 (m, 2H), 7.32 - 7.28 (m, $3 \mathrm{H}), 6.43(\mathrm{br}, 1 \mathrm{H}), 4.75(\mathrm{t}, J=7.1 \mathrm{~Hz}, 1 \mathrm{H}), 2.61-2.53(\mathrm{~m}, 1 \mathrm{H}), 2.50-2.36(\mathrm{~m}, 2 \mathrm{H})$, $2.02-1.93(\mathrm{~m}, 1 \mathrm{H}) .{ }^{13} \mathrm{C}\left\{{ }^{1} \mathrm{H}\right\} \mathrm{NMR}(151 \mathrm{MHz}$, Chloroform-d) $\delta 178.5,142.5,128.9$, 127.9, 125.6, 58.1, 31.3, 30.1. HRMS (ESI) m/z: $[\mathrm{M}+\mathrm{H}]^{+}$Calcd for $\mathrm{C}_{10} \mathrm{H}_{12} \mathrm{NO}^{+}$ 162.0913; Found 162.0913. HPLC: Chiracel IA Column (250 mm); detected at 210 nm; $n$-hexane $/ i$-propanol $=90 / 10$; flow $=0.8 \mathrm{~mL} / \mathrm{min}$; Retention time: $12.7 \mathrm{~min}$ (minor), 13.9 min (major). The absolute configuration of 2a was determined by comparison of its optical rotation with that reported. ${ }^{[9]}$ The absolute configuration of 2b-2n was determined by comparing with $2 \mathbf{a}$.

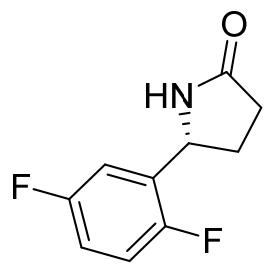

$2 \mathrm{~b}$

Chemical Formula: $\mathrm{C}_{10} \mathrm{H}_{9} \mathrm{~F}_{2} \mathrm{NO}$

Exact Mass: 197.0652

\section{(R)-5-(2,5-difluorophenyl)pyrrolidin-2-one (2b)}

White solid, $31.9 \mathrm{mg}, 81 \%$ yield, $87 \%$ ee, on $0.2 \mathrm{mmol}$ scale; $323 \mathrm{mg}$, $82 \%$ yield, $87 \%$ ee, on $2.0 \mathrm{mmol}$ scale( $68 \%$ yield, $>99 \%$ ee after recrystallization from EtOH), $[\alpha]^{25}$ $=40.2\left(\mathrm{c}=0.5, \mathrm{CHCl}_{3}\right)$, obtained by the purification with flash column chromatography on silica gel (EtOAc/petroleum ether 3:7). ${ }^{1} \mathrm{H}$ NMR (600 MHz, Chloroform-d) $\delta 7.10-7.00(\mathrm{~m}, 2 \mathrm{H}), 7.00-6.91(\mathrm{~m}, 1 \mathrm{H}), 6.03(\mathrm{~s}, 1 \mathrm{H}), 5.04(\mathrm{t}, J=$ $6.9 \mathrm{~Hz}, 1 \mathrm{H}), 2.71-2.63(\mathrm{~m}, 1 \mathrm{H}), 2.52-2.38(\mathrm{~m}, 2 \mathrm{H}), 2.06-1.96(\mathrm{~m}, 1 \mathrm{H}) .{ }^{13} \mathrm{C}\left\{{ }^{1} \mathrm{H}\right\}$ NMR (101 MHz, Chloroform-d) $\delta 178.7,159.0$ (dd, $\left.J_{C F}=243.6,2.3 \mathrm{~Hz}\right), 155.9$ (dd, $\left.J_{C F}=242.4,2.6 \mathrm{~Hz}\right), 131.4\left(\mathrm{dd}, J_{C F}=15.7,6.8 \mathrm{~Hz}\right), 116.8\left(\mathrm{dd}, J_{C F}=24.3,8.6 \mathrm{~Hz}\right)$, 
$115.5\left(\mathrm{dd}, J_{C F}=24.2,8.6 \mathrm{~Hz}\right), 113.0\left(\mathrm{dd}, J_{C F}=25.2,4.6 \mathrm{~Hz}\right), 51.6\left(\mathrm{~d}, J_{C F}=3.5 \mathrm{~Hz}\right)$, 29.7, 29.3. HRMS (ESI) $\mathrm{m} / \mathrm{z}$ : $[\mathrm{M}+\mathrm{H}]^{+}$Calcd for $\mathrm{C}_{10} \mathrm{H}_{10} \mathrm{~F}_{2} \mathrm{NO}^{+}$: 198.0725; Found 198.0726. HPLC: Chiracel IA Column $(250 \mathrm{~mm})$; detected at $210 \mathrm{~nm}$; n-hexane / i-propanol $=90 / 10$; flow $=0.8 \mathrm{~mL} / \mathrm{min}$; Retention time: $12.4 \mathrm{~min}$ (major), $14.2 \mathrm{~min}$ (minor).

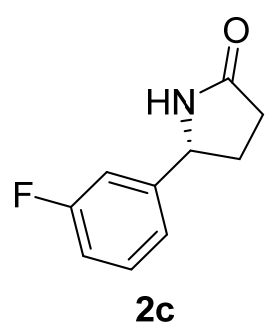

Chemical Formula: $\mathrm{C}_{10} \mathrm{H}_{10} \mathrm{FNO}$

Exact Mass: 179.0746

\section{(R)-5-(3-fluorophenyl)pyrrolidin-2-one (2c)}

White solid, $31.2 \mathrm{mg}, 87 \%$ yield, $91 \%$ ee, $[\alpha]^{26}=46.8\left(\mathrm{c}=0.5, \mathrm{CHCl}_{3}\right)$, obtained by the purification with flash column chromatography on silica gel (EtOAc/petroleum ether 3:7). ${ }^{1} \mathrm{H}$ NMR (400 MHz, Chloroform-d) $\delta 7.39-7.29(\mathrm{~m}, 1 \mathrm{H}), 7.08(\mathrm{~d}, J=7.7$ $\mathrm{Hz}, 1 \mathrm{H}), 7.04-6.96(\mathrm{~m}, 2 \mathrm{H}), 6.02(\mathrm{br}, 1 \mathrm{H}), 4.76(\mathrm{t}, J=7.1 \mathrm{~Hz}, 1 \mathrm{H}), 2.65-2.55(\mathrm{~m}$, 1H), $2.54-2.37(\mathrm{~m}, 2 \mathrm{H}), 2.04-1.88(\mathrm{~m}, 1 \mathrm{H}) .{ }^{13} \mathrm{C}\left\{{ }^{1} \mathrm{H}\right\}$ NMR (101 MHz, Chloroform-d) $\delta 178.3,163.2(\mathrm{~d}, J=247.2 \mathrm{~Hz}), 145.2(\mathrm{~d}, J=6.6 \mathrm{~Hz}), 130.6(\mathrm{~d}, J=$ $8.3 \mathrm{~Hz}), 121.2(\mathrm{~d}, J=2.9 \mathrm{~Hz}), 114.9$ (d, $J=21.1 \mathrm{~Hz}), 112.6$ (d, $J=22.0 \mathrm{~Hz}), 57.5$ (d, $J=1.9 \mathrm{~Hz}), 31.3,30.0$. HRMS (ESI) $\mathrm{m} / \mathrm{z}:[\mathrm{M}+\mathrm{H}]^{+}$Calcd for $\mathrm{C}_{10} \mathrm{H}_{11} \mathrm{FNO}^{+}$180.0819; Found 180.0819. HPLC: Chiracel AD-3 Column $(250 \mathrm{~mm})$; detected at $210 \mathrm{~nm}$; n-hexane $/$ i-propanol $=90 / 10$; flow $=0.8 \mathrm{~mL} / \mathrm{min}$; Retention time: $11.6 \mathrm{~min}$ (minor), 12.6 min (major). 


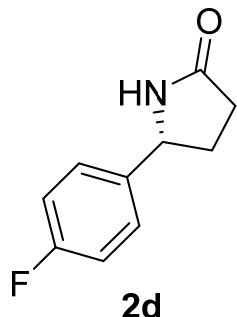

Chemical Formula: $\mathrm{C}_{10} \mathrm{H}_{10} \mathrm{FNO}$

Exact Mass: 179.0746

\section{(R)-5-(4-fluorophenyl)pyrrolidin-2-one (2d)}

White solid, $34.0 \mathrm{mg}, 95 \%$ yield, $90 \%$ ee, $[\alpha]^{26}{ }_{\mathrm{D}}=52.5\left(\mathrm{c}=0.5, \mathrm{CHCl}_{3}\right)$, obtained by the purification with flash column chromatography on silica gel (EtOAc/petroleum ether 3:7). ${ }^{1} \mathrm{H}$ NMR (400 MHz, Chloroform-d) $\delta 7.30-7.24(\mathrm{~m}, 2 \mathrm{H}), 7.10-7.01(\mathrm{~m}$, 2H), 6.69 (br, 1H), $4.75(\mathrm{t}, J=7.1 \mathrm{~Hz}, 1 \mathrm{H}), 2.60-2.51(\mathrm{~m}, 1 \mathrm{H}), 2.50-2.35(\mathrm{~m}, 2 \mathrm{H})$, 1.97-1.88 (m, 1H). ${ }^{13} \mathrm{C}\left\{{ }^{1} \mathrm{H}\right\}$ NMR $\left(101 \mathrm{MHz}\right.$, Chloroform-d) $\delta 178.6,162.3\left(\mathrm{~d}, J_{C F}\right.$ $=246.3 \mathrm{~Hz}), 138.2\left(\mathrm{~d}, J_{C F}=3.2 \mathrm{~Hz}\right), 127.3\left(\mathrm{~d}, J_{C F}=8.2 \mathrm{~Hz}\right), 115.7\left(\mathrm{~d}, J_{C F}=21.6 \mathrm{~Hz}\right)$, 57.5, 31.4, 30.3. HRMS (ESI) m/z: $[\mathrm{M}+\mathrm{H}]^{+}$Calcd for $\mathrm{C}_{10} \mathrm{H}_{11} \mathrm{FNO}^{+}$180.0819; Found 180.0820. HPLC: Chiracel IA Column $(250 \mathrm{~mm})$; detected at $210 \mathrm{~nm}$; n-hexane / i-propanol $=90 / 10$; flow $=0.8 \mathrm{~mL} / \mathrm{min}$; Retention time: $13.0 \mathrm{~min}$ (minor), $13.7 \mathrm{~min}$ (major).

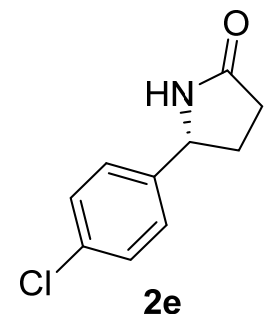

Chemical Formula: $\mathrm{C}_{10} \mathrm{H}_{10} \mathrm{CINO}$

Exact Mass: 195.0451

\section{(R)-5-(4-chlorophenyl)pyrrolidin-2-one (2e)}

White solid, $35.9 \mathrm{mg}, 92 \%$ yield, $85 \%$ ee, $[\alpha]^{25}=47.7\left(\mathrm{c}=0.5, \mathrm{CHCl}_{3}\right)$, obtained by the purification with flash column chromatography on silica gel (EtOAc/petroleum ether 3:7). ${ }^{1} \mathrm{H}$ NMR (400 MHz, Chloroform-d) $\delta 7.38$ - 7.31 (m, 2H), 7.26 - 7.20 (m, 2H), $6.11(\mathrm{~s}, 1 \mathrm{H}), 4.74(\mathrm{t}, J=7.1 \mathrm{~Hz}, 1 \mathrm{H}), 2.64-2.52(\mathrm{~m}, 1 \mathrm{H}), 2.52-2.36(\mathrm{~m}, 2 \mathrm{H})$, 
$2.00-1.86(\mathrm{~m}, 1 \mathrm{H}) .{ }^{13} \mathrm{C}\left\{{ }^{1} \mathrm{H}\right\} \mathrm{NMR}(101 \mathrm{MHz}$, Chloroform- $d) \delta$ 178.3, 141.0, 133.7, 129.1, 127.0, 57.4, 31.4, 30.1. HRMS (ESI) m/z: $[\mathrm{M}+\mathrm{H}]^{+}$Calcd for $\mathrm{C}_{10} \mathrm{H}_{11} \mathrm{ClNO}^{+}$ 196.0524; Found 196.0525. HPLC: Chiracel IA Column (250 mm); detected at 210 $\mathrm{nm}$; n-hexane $/$ i-propanol $=90 / 10$; flow $=0.8 \mathrm{~mL} / \mathrm{min}$; Retention time: $14.1 \mathrm{~min}$ (major), $15.3 \mathrm{~min}$ (minor).

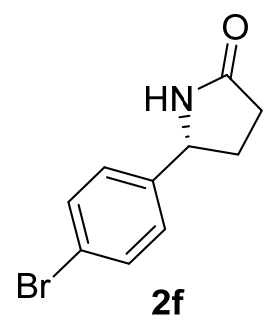

Chemical Formula: $\mathrm{C}_{10} \mathrm{H}_{10} \mathrm{BrNO}$

Exact Mass: 238.9946

\section{(R)-5-(4-bromophenyl)pyrrolidin-2-one (2f)}

White solid, $44.5 \mathrm{mg}, 93 \%$ yield, $86 \%$ ee, $[\alpha]^{25}=28.0\left(\mathrm{c}=0.5, \mathrm{CHCl}_{3}\right)$, obtained by the purification with flash column chromatography on silica gel (EtOAc/petroleum ether 3:7). ${ }^{1} \mathrm{H}$ NMR (400 MHz, Chloroform-d) $\delta 7.53(\mathrm{~d}, J=8.5 \mathrm{~Hz}, 2 \mathrm{H}), 7.22(\mathrm{~d}, J=$ $8.4 \mathrm{~Hz}, 2 \mathrm{H}), 5.47(\mathrm{t}, J=6.4 \mathrm{~Hz}, 1 \mathrm{H}), 2.71-2.62(\mathrm{~m}, 3 \mathrm{H}), 2.19-2.07(\mathrm{~m}, 1 \mathrm{H})$. ${ }^{13} \mathrm{C}\left\{{ }^{1} \mathrm{H}\right\}$ NMR (101 MHz, Chloroform-d) $\delta$ 176.5, 138.4, 131.9, 126.9, 122.4, 80.4, 30.9, 28.8. HRMS (ESI) m/z: $[\mathrm{M}+\mathrm{H}]^{+}$Calcd for $\mathrm{C}_{10} \mathrm{H}_{11} \mathrm{BrNO}^{+}$240.0019; Found 240.0019. HPLC: Chiracel IA Column (250 mm); detected at $210 \mathrm{~nm}$; n-hexane / i-propanol $=90 / 10$; flow $=0.8 \mathrm{~mL} / \mathrm{min}$; Retention time: $14.8 \mathrm{~min}$ (major), $16.3 \mathrm{~min}$ (minor).

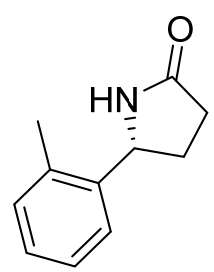

$2 \mathrm{~g}$

Chemical Formula: $\mathrm{C}_{11} \mathrm{H}_{13} \mathrm{NO}$

Exact Mass: 175.0997 


\section{(R)-5-(o-tolyl)pyrrolidin-2-one (2g)}

White solid, $17.9 \mathrm{mg}, 51 \%$ yield, $90 \%$ ee, $[\alpha]^{26}{ }_{\mathrm{D}}=121.2\left(\mathrm{c}=0.5, \mathrm{CHCl}_{3}\right)$, obtained by the purification with flash column chromatography on silica gel (EtOAc/petroleum ether 3:7). ${ }^{1} \mathrm{H}$ NMR (400 MHz, Chloroform-d) $\delta 7.37$ - 7.31 (m, 1H), 7.26 - 7.15 (m, $3 \mathrm{H}), 5.78(\mathrm{~s}, 1 \mathrm{H}), 5.00(\mathrm{t}, J=6.4 \mathrm{~Hz}, 1 \mathrm{H}), 2.70-2.58(\mathrm{~m}, 1 \mathrm{H}), 2.53-2.38(\mathrm{~m}, 2 \mathrm{H})$, 2.35 (s, 3H), $1.94-1.84(\mathrm{~m}, 1 \mathrm{H}) .{ }^{13} \mathrm{C}\left\{{ }^{1} \mathrm{H}\right\}$ NMR (101 MHz, Chloroform-d) $\delta$ 178.4, 140.4, 134.4, 130.8, 127.5, 126.6, 124.0, 54.4 29.8, 29.6, 19.0. HRMS (ESI) m/z: $[\mathrm{M}+\mathrm{H}]^{+}$Calcd for $\mathrm{C}_{11} \mathrm{H}_{14} \mathrm{NO}^{+}$176.1070; Found 176.1071. HPLC: Chiracel IA Column $(250 \mathrm{~mm})$; detected at $210 \mathrm{~nm}$; n-hexane $/$ i-propanol $=90 / 10$; flow $=0.8$ $\mathrm{mL} / \mathrm{min}$; Retention time: $11.5 \mathrm{~min}$ (minor), $13.2 \mathrm{~min}$ (major).

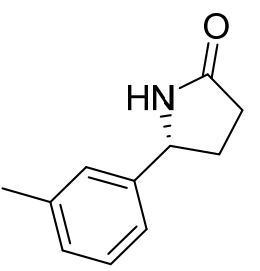

$2 \mathrm{~h}$

Chemical Formula: $\mathrm{C}_{11} \mathrm{H}_{13} \mathrm{NO}$

Exact Mass: 175.0997

\section{(R)-5-(m-tolyl)pyrrolidin-2-one (2h)}

White solid, $27.0 \mathrm{mg}, 77 \%$ yield, $91 \%$ ee, $[\alpha]^{24}=30.1\left(\mathrm{c}=0.5, \mathrm{CHCl}_{3}\right)$, obtained by the purification with flash column chromatography on silica gel (EtOAc/petroleum ether 3:7). ${ }^{1} \mathrm{H}$ NMR (600 MHz, Chloroform-d) $\delta 7.28-7.25$ (m, 1H), 7.10 (q, $J=7.9$ $\mathrm{Hz}, 3 \mathrm{H}), 6.02(\mathrm{~s}, 1 \mathrm{H}), 4.72(\mathrm{t}, J=7.1 \mathrm{~Hz}, 1 \mathrm{H}), 2.59-2.53(\mathrm{~m}, 1 \mathrm{H}), 2.52-2.38(\mathrm{~m}, 2 \mathrm{H})$, $2.36(\mathrm{~s}, 3 \mathrm{H}), 2.01-1.94(\mathrm{~m}, 1 \mathrm{H}) .{ }^{13} \mathrm{C}\left\{{ }^{1} \mathrm{H}\right\}$ NMR (151 MHz, Chloroform-d) $\delta$ 178.4, 142.4, 138.7, 128.8, 128.7, 126.3, 122.7, 58.0, 31.4, 30.2, 21.4. HRMS (ESI) m/z: $[\mathrm{M}+\mathrm{H}]^{+}$Calcd for $\mathrm{C}_{11} \mathrm{H}_{14} \mathrm{NO}^{+}$176.1070; Found 176.1071. HPLC: Chiracel IA Column $(250 \mathrm{~mm})$; detected at $210 \mathrm{~nm}$; n-hexane $/$ i-propanol $=90 / 10$; flow $=0.8$ $\mathrm{mL} / \mathrm{min}$; Retention time: $10.7 \mathrm{~min}$ (minor), $12.1 \mathrm{~min}$ (major). 


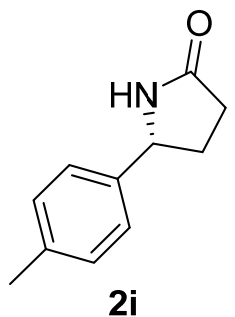

Chemical Formula: $\mathrm{C}_{11} \mathrm{H}_{13} \mathrm{NO}$

Exact Mass: 175.0997

\section{(R)-5-(p-tolyl)pyrrolidin-2-one (2i)}

White solid, $30.5 \mathrm{mg}, 87 \%$ yield, $90 \%$ ee, $[\alpha]^{25}=36.6\left(\mathrm{c}=0.5, \mathrm{CHCl}_{3}\right)$, obtained by the purification with flash column chromatography on silica gel (EtOAc/petroleum ether 3:7). ${ }^{1} \mathrm{H}$ NMR (600 MHz, Chloroform-d) $\delta 7.18$ (br, 4H), 5.84 (s, 1H), 4.72 (t, J $=7.2 \mathrm{~Hz}, 1 \mathrm{H}), 2.57-2.54(\mathrm{~m}, 1 \mathrm{H}), 2.51-2.38(\mathrm{~m}, 2 \mathrm{H}), 2.35(\mathrm{~s}, 3 \mathrm{H}), 2.01-1.92(\mathrm{~m}$, 1H). ${ }^{13} \mathrm{C}\left\{{ }^{1} \mathrm{H}\right\}$ NMR $(151 \mathrm{MHz}$, Chloroform-d) $\delta 178.2,139.4,137.8,129.6,125.6$, 57.8, 31.5, 30.3, 21.1. HRMS (ESI) m/z: $[\mathrm{M}+\mathrm{H}]^{+}$Calcd for $\mathrm{C}_{11} \mathrm{H}_{14} \mathrm{NO}^{+}$176.1070; Found 176.1070. HPLC: Chiracel IA Column $(250 \mathrm{~mm})$; detected at $210 \mathrm{~nm}$; n-hexane $/$ i-propanol $=90 / 10$; flow $=0.8 \mathrm{~mL} / \mathrm{min}$; Retention time: $13.0 \mathrm{~min}$ (minor), 13.7 min (major).

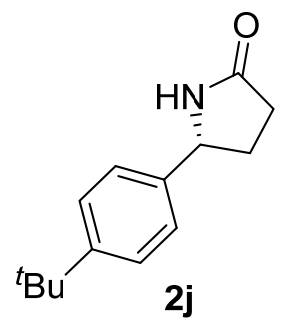

Chemical Formula: $\mathrm{C}_{14} \mathrm{H}_{19} \mathrm{NO}$

Exact Mass: 217.1467

\section{(R)-5-(4-(tert-butyl)phenyl)pyrrolidin-2-one (2j)}

White solid, $40.8 \mathrm{mg}, 94 \%$ yield, $95 \%$ ee, $[\alpha]^{25}=25.4\left(\mathrm{c}=0.5, \mathrm{CHCl}_{3}\right)$, obtained by the purification with flash column chromatography on silica gel (EtOAc/petroleum ether 3:7). ${ }^{1} \mathrm{H}$ NMR (400 MHz, Chloroform-d) $\delta 7.40(\mathrm{~d}, J=8.4 \mathrm{~Hz}, 2 \mathrm{H}), 7.23(\mathrm{~d}, \mathrm{~J}=$ $8.3 \mathrm{~Hz}, 2 \mathrm{H}), 5.08(\mathrm{br}, 1 \mathrm{H}), 4.73(\mathrm{t}, \mathrm{J}=7.2 \mathrm{~Hz}, 1 \mathrm{H}), 2.61-2.37(\mathrm{~m}, 3 \mathrm{H}), 2.05-1.94$ (m, 1H), 1.32 (s, 9H). ${ }^{13} \mathrm{C}\left\{{ }^{1} \mathrm{H}\right\}$ NMR (101 MHz, Chloroform-d) $\delta 178.2,151.1,139.3$, 
125.8, 125.4, 57.8, 34.6, 31.4, 31.3, 30.3. HRMS (ESI) m/z: $[\mathrm{M}+\mathrm{H}]^{+}$Calcd for $\mathrm{C}_{14} \mathrm{H}_{20} \mathrm{NO}^{+}$218.1539; Found 218.1514. HPLC: Chiracel IA Column (250 mm); detected at $210 \mathrm{~nm}$; n-hexane $/$ i-propanol $=90 / 10$; flow $=0.8 \mathrm{~mL} / \mathrm{min}$; Retention time: 10.9 min (minor), 12.1 min (major).

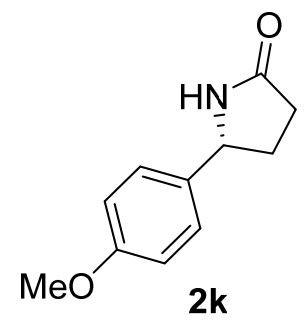

Chemical Formula: $\mathrm{C}_{11} \mathrm{H}_{13} \mathrm{NO}_{2}$

Exact Mass: 191.0946

\section{(R)-5-(4-methoxyphenyl)pyrrolidin-2-one (2k)}

White solid, $36.3 \mathrm{mg}, 95 \%$ yield, $85 \%$ ee, $[\alpha]^{25}=19.7\left(\mathrm{c}=0.93, \mathrm{CHCl}_{3}\right)$, obtained by the purification with flash column chromatography on silica gel (EtOAc/petroleum ether 3:7). ${ }^{1} \mathrm{H}$ NMR (400 MHz, Chloroform-d) $\delta 7.25-7.18(\mathrm{~m}, 2 \mathrm{H}), 6.93-6.86(\mathrm{~m}$, 2H), $6.30(\mathrm{~s}, 1 \mathrm{H}), 4.70(\mathrm{t}, J=7.2 \mathrm{~Hz}, 1 \mathrm{H}), 3.80(\mathrm{~s}, 3 \mathrm{H}), 2.58-2.34(\mathrm{~m}, 3 \mathrm{H}), 2.00-$ $1.90(\mathrm{~m}, 1 \mathrm{H}) .{ }^{13} \mathrm{C}\left\{{ }^{1} \mathrm{H}\right\}$ NMR (101 MHz, Chloroform-d) $\delta 178.4,159.3,134.4,126.9$, 114.2, 57.6, 55.3, 31.5, 30.4. HRMS (ESI) m/z: $[\mathrm{M}+\mathrm{H}]^{+}$Calcd for $\mathrm{C}_{11} \mathrm{H}_{14} \mathrm{NO}_{2}{ }^{+}$ 192.1019; Found 192.1020. HPLC: Chiracel IA Column (250 mm); detected at 210 nm; n-hexane $/$ i-propanol $=90 / 10$; flow $=0.8 \mathrm{~mL} / \mathrm{min}$; Retention time: $18.6 \mathrm{~min}$ (minor), 19.8 min (major).

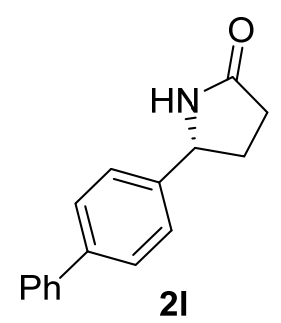

Chemical Formula: $\mathrm{C}_{16} \mathrm{H}_{15} \mathrm{NO}$

Exact Mass: 237.1154

(R)-5-([1,1'-biphenyl]-4-yl)pyrrolidin-2-one (2l) 
White solid, $43.16 \mathrm{mg}, 91 \%$ yield, $89 \%$ ee, $[\alpha]^{24}=6.2\left(\mathrm{c}=0.5, \mathrm{CHCl}_{3}\right)$, obtained by the purification with flash column chromatography on silica gel (EtOAc/petroleum ether 3:7). ${ }^{1} \mathrm{H}$ NMR (400 MHz, Chloroform-d) $\delta 7.62-7.55$ (m, 4H), 7.45 (t, $J=7.5$ Hz, 2H), 7.37 (dd, J = 7.8, 2.4 Hz, 3H), 5.89 (s, 1H), 4.81 (t, J=7.1 Hz, 1H), $2.70-$ $2.40(\mathrm{~m}, 3 \mathrm{H}), 2.09-1.97(\mathrm{~m}, 1 \mathrm{H})$. HRMS (ESI) m/z: $[\mathrm{M}+\mathrm{H}]^{+}$Calcd for $\mathrm{C}_{16} \mathrm{H}_{16} \mathrm{NO}^{+}$ 238.1226; Found 238.1226. ${ }^{13} \mathrm{C}\left\{{ }^{1} \mathrm{H}\right\}$ NMR (101 MHz, Chloroform-d) $\delta$ 178.3, $141.4,141.1,140.5,128.8,127.7,127.5,127.1,126.1,57.8,31.5,30.2$. HPLC: Chiracel IE Column (250 mm); detected at $210 \mathrm{~nm}$; n-hexane / i-propanol = 85/15; flow $=0.8 \mathrm{~mL} / \mathrm{min}$; Retention time: $30.2 \mathrm{~min}$ (major), $32.9 \mathrm{~min}$ (minor).

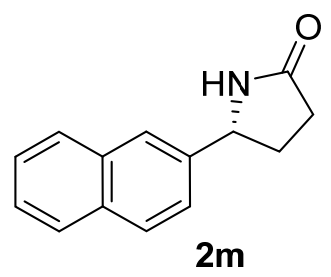

Chemical Formula: $\mathrm{C}_{14} \mathrm{H}_{13} \mathrm{NO}$

Exact Mass: 211.0997

\section{(R)-5-(naphthalen-2-yl)pyrrolidin-2-one (2m)}

White solid, $32.9 \mathrm{mg}, 78 \%$ yield, $92 \%$ ee, $[\alpha]^{27}=17.2\left(\mathrm{c}=0.5, \mathrm{CHCl}_{3}\right)$, obtained by the purification with flash column chromatography on silica gel (EtOAc/petroleum ether 3:7). ${ }^{1} \mathrm{H}$ NMR (600 MHz, Chloroform-d) $\delta 7.91-7.80(\mathrm{~m}, 3 \mathrm{H}), 7.73(\mathrm{~s}, 1 \mathrm{H})$, $7.55-7.46(\mathrm{~m}, 2 \mathrm{H}), 7.40(\mathrm{~d}, J=8.5 \mathrm{~Hz}, 1 \mathrm{H}), 6.23(\mathrm{~s}, 1 \mathrm{H}), 4.91(\mathrm{t}, J=7.1 \mathrm{~Hz}, 1 \mathrm{H})$, $2.69-2.60(\mathrm{~m}, 1 \mathrm{H}), 2.58-2.41(\mathrm{~m}, 2 \mathrm{H}), 2.12-2.02(\mathrm{~m}, 1 \mathrm{H}) .{ }^{13} \mathrm{C}\left\{{ }^{1} \mathrm{H}\right\}$ NMR $(151$ MHz, Chloroform-d) $\delta 178.4,139.7,133.2,133.0,129.0,127.8,127.7,126.5,126.2$, 124.3, 123.6, 58.1, 31.2, 30.1. HRMS (ESI) m/z: $[\mathrm{M}+\mathrm{H}]^{+}$Calcd for $\mathrm{C}_{14} \mathrm{H}_{14} \mathrm{NO}^{+}$ 212.1070; Found 212.1070. HPLC: Chiracel AD-3 Column (250 mm); detected at 210 $\mathrm{nm}$; n-hexane $/$ i-propanol $=90 / 10$; flow $=0.8 \mathrm{~mL} / \mathrm{min}$; Retention time: $14.4 \mathrm{~min}$ (minor), 15.6 min (major). 


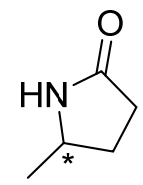

2n

Chemical Formula: $\mathrm{C}_{5} \mathrm{H}_{9} \mathrm{NO}$

Exact Mass: 99.0684

\section{5-methylpyrrolidin-2-one (2n)}

Light yellow oil, $8.1 \mathrm{mg}, 41 \%$ yield, $50 \%$ ee, $[\alpha]^{22}=21.0\left(\mathrm{c}=0.5, \mathrm{CHCl}_{3}\right)$, obtained by the purification with flash column chromatography on silica gel (EtOAc/petroleum ether 3:7). ${ }^{1} \mathrm{H}$ NMR (400 MHz, Chloroform-d) $\delta 6.50$ (s, 1H), 3.79 (h, $\left.J=6.4 \mathrm{~Hz}, 1 \mathrm{H}\right)$, $2.42-2.21(\mathrm{~m}, 3 \mathrm{H}), 1.71-1.60(\mathrm{~m}, 1 \mathrm{H}), 1.23(\mathrm{~d}, J=6.3 \mathrm{~Hz}, 3 \mathrm{H}) .{ }^{13} \mathrm{C}\left\{{ }^{1} \mathrm{H}\right\} \mathrm{NMR}$ $(101 \mathrm{MHz}$, Chloroform- $d) \delta 178.30,50.04,30.53,29.13,22.16$. Chiracel AD-3 Column $(250 \mathrm{~mm})$; detected at $210 \mathrm{~nm}$; n-hexane $/$ i-propanol $=90 / 10$; flow $=0.8$ $\mathrm{mL} / \mathrm{min}$; Retention time: $9.3 \mathrm{~min}$ (major), $10.4 \mathrm{~min}$ (minor). The NMR data is consistent with that reported. ${ }^{[10]}$<smiles>O=C1CCC[C@H](c2ccccc2)N1</smiles>

Chemical Formula: $\mathrm{C}_{11} \mathrm{H}_{13} \mathrm{NO}$

Exact Mass: 175.0997

\section{(R)-6-phenylpiperidin-2-one (4a)}

White solid, $32.9 \mathrm{mg}$, 94\% yield, 94\% ee, on $0.2 \mathrm{mmol} \mathrm{scale;} 595 \mathrm{mg}$, $85 \%$ yield, $91 \%$ ee, on $4.0 \mathrm{mmol}$ scale, $[\alpha]_{\mathrm{D}}^{25}=61.7\left(\mathrm{c}=1.0, \mathrm{CHCl}_{3}\right)$, obtained by the purification with flash column chromatography on silica gel (EtOAc/petroleum ether 3:7). ${ }^{1} \mathrm{H}$ NMR (400 MHz, Chloroform-d) $\delta 7.37$ (m $J=7.8,6.5,1.2 \mathrm{~Hz}, 2 \mathrm{H}), 7.33-7.25$ (m, 3H), $6.01(\mathrm{~s}, 1 \mathrm{H}), 4.60-4.49(\mathrm{~m}, 1 \mathrm{H}), 2.53-2.36(\mathrm{~m}, 2 \mathrm{H}), 2.17-2.06(\mathrm{~m}, 1 \mathrm{H}), 1.96-$ $1.86(\mathrm{~m}, 1 \mathrm{H}), 1.86-1.73(\mathrm{~m}, 1 \mathrm{H}), 1.73-1.62(\mathrm{~m}, 1 \mathrm{H}) .{ }^{13} \mathrm{C}\left\{{ }^{1} \mathrm{H}\right\}$ NMR $(101 \mathrm{MHz}$ Chloroform- $d$ ) $\delta 172.3,142.5,128.8,127.9,126.0,57.7,32.1,31.2,19.6$. HRMS (ESI) 
m/z: $[\mathrm{M}+\mathrm{H}]^{+}$Calcd for $\mathrm{C}_{11} \mathrm{H}_{14} \mathrm{NO}^{+}$176.1070; Found 176.1070. HPLC: Chiracel IA Column $(250 \mathrm{~mm})$; detected at $210 \mathrm{~nm}$; n-hexane $/$ i-propanol $=90 / 10$; flow $=0.8$ $\mathrm{mL} / \mathrm{min}$; Retention time: 18.9 min (major), 22.0 min (minor). The absolute configuration of 4a was identified by comparison of its physical and spectroscopic data with the ones reported in the literature. ${ }^{[9]}$ The absolute configuration of $\mathbf{4 b} \mathbf{b} \mathbf{4 h}$ was determined by comparing with $\mathbf{4 a}$.<smiles>Cc1ccccc1C1CCCC(=O)N1</smiles>

4b

Chemical Formula: $\mathrm{C}_{12} \mathrm{H}_{15} \mathrm{NO}$

Exact Mass: 189.1154

\section{(R)-6-(o-tolyl)piperidin-2-one (4b)}

White solid, $34.4 \mathrm{mg}, 91 \%$ yield, $91 \%$ ee, $[\alpha]^{25}=44.4\left(\mathrm{c}=1.0, \mathrm{CHCl}_{3}\right)$, obtained by the purification with flash column chromatography on silica gel (EtOAc/petroleum ether 3:7). ${ }^{1} \mathrm{H}$ NMR (600 MHz, Chloroform-d) $\delta 7.35$ (d, $\left.J=7.6 \mathrm{~Hz}, 1 \mathrm{H}\right)$, 7.26-7.23 (m, 1H), 7.21-7.19 (m, 1H), $7.16(\mathrm{~d}, J=7.5 \mathrm{~Hz}, 1 \mathrm{H}), 5.80(\mathrm{~s}, 1 \mathrm{H}), 4.80$ (dd, $J=8.9$, $4.8 \mathrm{~Hz}, 1 \mathrm{H}), 2.54-2.41(\mathrm{~m}, 2 \mathrm{H}), 2.35(\mathrm{~s}, 3 \mathrm{H}), 2.17-2.07(\mathrm{~m}, 1 \mathrm{H}), 1.97-1.88(\mathrm{~m}$, 1H), 1.84-1.77 (m, 1H), 1.64-1.58 (m, 1H). ${ }^{13} \mathrm{C}\left\{{ }^{1} \mathrm{H}\right\}$ NMR (151 MHz, Chloroform-d) $\delta 172.5,140.3,134.4,130.8,127.6,126.6,125.6,54.0,31.4,30.0,19.6,18.9$. HRMS (ESI) m/z: [M+H] $]^{+}$Calcd for $\mathrm{C}_{12} \mathrm{H}_{16} \mathrm{NO}^{+}$190.1226; Found 190.1227. HPLC: Chiracel OD-3 Column (250 mm); detected at $210 \mathrm{~nm}$; n-hexane / i-propanol =90/10; flow = $0.8 \mathrm{~mL} / \mathrm{min}$; Retention time: $10.7 \mathrm{~min}$ (major), $21.9 \mathrm{~min}$ (minor).<smiles>Cc1cccc([C@H]2CCCC(=O)N2)c1</smiles>

Chemical Formula: $\mathrm{C}_{12} \mathrm{H}_{15} \mathrm{NO}$

Exact Mass: 189.1154 


\section{(R)-6-(m-tolyl)piperidin-2-one (4c)}

White solid, $35.2 \mathrm{mg}, 93 \%$ yield, $91 \%$ ee, $[\alpha]^{25}=37.6\left(\mathrm{c}=1.0, \mathrm{CHCl}_{3}\right)$, obtained by the purification with flash column chromatography on silica gel (EtOAc/petroleum ether 3:7). ${ }^{1} \mathrm{H}$ NMR (600 MHz, Chloroform-d) $\delta 7.28$ - $7.23(\mathrm{~m}, 1 \mathrm{H}), 7.13$ - $7.06(\mathrm{~m}$, 3H), $5.87(\mathrm{~s}, 1 \mathrm{H}), 4.51(\mathrm{dd}, J=9.3,4.6 \mathrm{~Hz}, 1 \mathrm{H}), 2.51-2.40(\mathrm{~m}, 2 \mathrm{H}), 2.36(\mathrm{~s}, 3 \mathrm{H}), 2.14$ - $2.06(\mathrm{~m}, 1 \mathrm{H}), 1.95-1.89(\mathrm{~m}, 1 \mathrm{H}), 1.83-1.74(\mathrm{~m}, 1 \mathrm{H}), 1.71-1.63(\mathrm{~m}, 1 \mathrm{H}) .{ }^{13} \mathrm{C}\left\{{ }^{1} \mathrm{H}\right\}$ NMR (151 MHz, Chloroform-d) $\delta$ 172.3, 142.5, 138.6, 128.7, 128.7, 126.7, 123.1, 57.8, 32.2, 31.3, 21.4, 19.7. HRMS (ESI) $\mathrm{m} / \mathrm{z}$ : $[\mathrm{M}+\mathrm{H}]^{+}$Calcd for $\mathrm{C}_{12} \mathrm{H}_{16} \mathrm{NO}^{+}$ 190.1226; Found 190.1228. HPLC: Chiracel AD-3 Column (250 mm); detected at 210 $\mathrm{nm}$; $\mathrm{n}$-hexane $/ \mathrm{i}$-propanol $=90 / 10$; flow $=0.8 \mathrm{~mL} / \mathrm{min}$; Retention time: $16.2 \mathrm{~min}$ (minor), $17.7 \mathrm{~min}$ (major).

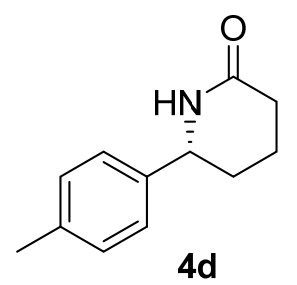

Chemical Formula: $\mathrm{C}_{12} \mathrm{H}_{15} \mathrm{NO}$

Exact Mass: 189.1154

\section{(R)-6-(p-tolyl)piperidin-2-one (4d)}

White solid, $35.9 \mathrm{mg}, 95 \%$ yield, $90 \%$ ee, $[\alpha]^{25}=35.0\left(\mathrm{c}=1.0, \mathrm{CHCl}_{3}\right)$, obtained by the purification with flash column chromatography on silica gel (EtOAc/petroleum ether 3:7). ${ }^{1} \mathrm{H}$ NMR (600 MHz, Chloroform-d) $\delta 7.18$ (br, 4H), 5.85 (s, 1H), 4.51 (dd, $J=9.4,4.6 \mathrm{~Hz}, 1 \mathrm{H}), 2.51-2.39(\mathrm{~m}, 2 \mathrm{H}), 2.35(\mathrm{~s}, 3 \mathrm{H}), 2.13-2.06(\mathrm{~m}, 1 \mathrm{H}), 1.94-1.90$ $(\mathrm{m}, 1 \mathrm{H}), 1.83-1.76(\mathrm{~m}, 1 \mathrm{H}), 1.68-1.61(\mathrm{~m}, 1 \mathrm{H}) .{ }^{13} \mathrm{C}\left\{{ }^{1} \mathrm{H}\right\}$ NMR $(151 \mathrm{MHz}$, Chloroform-d) $\delta 172.3,139.5,137.7,129.5,126.0,57.6,32.2,31.3,21.0,19.8$. HRMS (ESI) m/z: [M+H] $]^{+}$Calcd for $\mathrm{C}_{12} \mathrm{H}_{16} \mathrm{NO}^{+}$190.1226; Found 190.1228. HPLC: Chiracel IA Column (250 mm); detected at $210 \mathrm{~nm}$; n-hexane / i-propanol = 90/10; flow $=0.8 \mathrm{~mL} / \mathrm{min}$; Retention time: $16.7 \mathrm{~min}$ (major), $21.6 \mathrm{~min}$ (minor). 
<smiles>COc1ccc([C@H]2CCCC(=O)N2)cc1</smiles>

Chemical Formula: $\mathrm{C}_{12} \mathrm{H}_{15} \mathrm{NO}_{2}$

Exact Mass: 205.1103

\section{(R)-6-(4-methoxyphenyl)piperidin-2-one.}

White solid, $35.3 \mathrm{mg}, 86 \%$ yield, $90 \%$ ee, $[\alpha]^{25}=39.6\left(\mathrm{c}=1.0, \mathrm{CHCl}_{3}\right)$, obtained by the purification with flash column chromatography on silica gel (EtOAc/petroleum ether 3:7). ${ }^{1} \mathrm{H}$ NMR (600 MHz, Chloroform-d) $\delta 7.21$ (d, $\left.J=8.4 \mathrm{~Hz}, 2 \mathrm{H}\right), 6.89$ (d, $J=$ $8.5 \mathrm{~Hz}, 2 \mathrm{H}), 5.80(\mathrm{~s}, 1 \mathrm{H}), 4.49(\mathrm{dd}, J=9.3,4.4 \mathrm{~Hz}, 1 \mathrm{H}), 3.81(\mathrm{~s}, 3 \mathrm{H}), 2.51-2.39(\mathrm{~m}$, 2H), 2.10-2.05 (m, 1H), 1.83-1.75 (m, 1H), $1.83-1.75(\mathrm{~m}, 1 \mathrm{H}), 1.68-1.61(\mathrm{~m}, 1 \mathrm{H})$. ${ }^{13} \mathrm{C}\left\{{ }^{1} \mathrm{H}\right\}$ NMR (151 MHz, Chloroform-d) $\delta 172.3,159.3,134.5,127.3,114.2,57.4$, 55.3, 32.3, 31.2, 19.8. HRMS (ESI) $\mathrm{m} / \mathrm{z}[\mathrm{M}+\mathrm{H}]^{+}$Calcd for $\mathrm{C}_{12} \mathrm{H}_{16} \mathrm{NO}_{2}{ }^{+}$206.1176; Found 206.1177. HPLC: Chiracel IA Column $(250 \mathrm{~mm})$; detected at $210 \mathrm{~nm}$; n-hexane $/$ i-propanol $=90 / 10$; flow $=0.8 \mathrm{~mL} / \mathrm{min}$; Retention time: $25.6 \mathrm{~min}$ (major), $29.6 \min$ (minor).<smiles>O=C1CCC[C@H](c2ccc(F)cc2)N1</smiles>

Chemical Formula: $\mathrm{C}_{11} \mathrm{H}_{12} \mathrm{FNO}$

Exact Mass: 193.0903

\section{(R)-6-(4-fluorophenyl)piperidin-2-one (4f)}

White solid, $34.9 \mathrm{mg}, 91 \%$ yield, $90 \%$ ee, $[\alpha]^{25}=50.5\left(\mathrm{c}=1.0, \mathrm{CHCl}_{3}\right)$, obtained by the purification with flash column chromatography on silica gel (EtOAc/petroleum ether 3:7). ${ }^{1} \mathrm{H}$ NMR (600 MHz, Chloroform-d) $\delta 7.27(\mathrm{~m}, 2 \mathrm{H}), 7.06(\mathrm{t}, J=8.4 \mathrm{~Hz}$, 2H), $5.91(\mathrm{~s}, 1 \mathrm{H}), 4.58-4.50(\mathrm{~m}, 1 \mathrm{H}), 2.53-2.38(\mathrm{~m}, 2 \mathrm{H}), 2.14-2.06(\mathrm{~m}, 1 \mathrm{H}), 1.96$ $-1.88(\mathrm{~m}, 1 \mathrm{H}), 1.85-1.76(\mathrm{~m}, 1 \mathrm{H}), 1.69-1.60(\mathrm{~m}, 1 \mathrm{H}) .{ }^{13} \mathrm{C}\left\{{ }^{1} \mathrm{H}\right\}$ NMR $(151 \mathrm{MHz}$, Chloroform-d) $\delta 172.3,162.3\left(\mathrm{~d}, J_{C F}=246.4 \mathrm{~Hz}\right), 138.3\left(\mathrm{~d}, J_{C F}=3.2 \mathrm{~Hz}\right), 127.7(\mathrm{~d}$, 
$\left.J_{C F}=8.2 \mathrm{~Hz}\right), 115.7\left(\mathrm{~d}, J_{C F}=21.6 \mathrm{~Hz}\right), 57.2,32.3,31.2,19.6 . \mathrm{HRMS}(\mathrm{ESI}) \mathrm{m} / \mathrm{z}$ : $[\mathrm{M}+\mathrm{H}]^{+}$Calcd for $\mathrm{C}_{11} \mathrm{H}_{13} \mathrm{FNO}^{+}$194.0976; Found 194.0978. HPLC: Chiracel IA Column $(250 \mathrm{~mm})$; detected at $210 \mathrm{~nm}$; $\mathrm{n}$-hexane $/$ i-propanol $=90 / 10$; flow $=0.8$ $\mathrm{mL} / \mathrm{min}$; Retention time: $17.1 \mathrm{~min}$ (major), $22.6 \mathrm{~min}$ (minor).<smiles>COc1cc(OC)cc([C@H]2CCCC(=O)N2)c1</smiles>

Chemical Formula: $\mathrm{C}_{13} \mathrm{H}_{17} \mathrm{NO}_{3}$

Exact Mass: 235.1208

\section{(R)-6-(3,5-dimethoxyphenyl)piperidin-2-one (4g)}

White solid, $42.8 \mathrm{mg}, 91 \%$ yield, $92 \%$ ee, $[\alpha]^{25}=36.1\left(\mathrm{c}=1.0, \mathrm{CHCl}_{3}\right)$, obtained by the purification with flash column chromatography on silica gel (EtOAc/petroleum ether 3:7). ${ }^{1} \mathrm{H}$ NMR (400 MHz, Chloroform-d) $\delta 6.46-6.41(\mathrm{~m}, 2 \mathrm{H}), 6.39(\mathrm{t}, \mathrm{J}=2.3$ $\mathrm{Hz}, 1 \mathrm{H}), 5.89(\mathrm{~s}, 1 \mathrm{H}), 4.48(\mathrm{dd}, \mathrm{J}=9.2,4.6 \mathrm{~Hz}, 1 \mathrm{H}), 3.79(\mathrm{~s}, 6 \mathrm{H}), 2.54-2.37(\mathrm{~m}, 2 \mathrm{H})$, $2.15-2.05(\mathrm{~m}, 1 \mathrm{H}), 1.98-1.88(\mathrm{~m}, 1 \mathrm{H}), 1.86-1.75(\mathrm{~m}, 1 \mathrm{H}), 1.71-1.62(\mathrm{~m}, 1 \mathrm{H})$. ${ }^{13} \mathrm{C}\left\{{ }^{1} \mathrm{H}\right\}$ NMR (101 MHz, Chloroform-d) $\delta 172.3,161.2,145.0,104.0,99.6,57.9$, 55.4, 32.0, 31.3, 19.8. HRMS (ESI) m/z: $[\mathrm{M}+\mathrm{H}]^{+}$Calcd for $\mathrm{C}_{13} \mathrm{H}_{18} \mathrm{NO}_{3}{ }^{+}$236.1281; Found 236.1282. HPLC: Chiracel OJ-3 Column $(250 \mathrm{~mm})$; detected at $210 \mathrm{~nm}$; n-hexane $/$ i-propanol $=85 / 5$; flow $=0.8 \mathrm{~mL} / \mathrm{min}$; Retention time: $22.9 \mathrm{~min}$ (minor), $27.0 \mathrm{~min}$ (major).<smiles>O=C1CCC[C@H](c2ccc(F)c(F)c2)N1</smiles>

Chemical Formula: $\mathrm{C}_{11} \mathrm{H}_{11} \mathrm{~F}_{2} \mathrm{NO}$

Exact Mass: 211.0809 


\section{(R)-6-(3,4-difluorophenyl)piperidin-2-one (4h)}

White solid, $37.6 \mathrm{mg}, 89 \%$ yield, $91 \%$ ee, $[\alpha]^{25}=43.2\left(\mathrm{c}=1.0, \mathrm{CHCl}_{3}\right)$, obtained by the purification with flash column chromatography on silica gel (EtOAc/petroleum ether 3:7). ${ }^{1} \mathrm{H}$ NMR (400 MHz, Chloroform-d) $\delta 7.21$ - 7.07 (m, 2H), 7.07 - 6.98 (m, $1 \mathrm{H}), 6.23(\mathrm{~s}, 1 \mathrm{H}), 4.54(\mathrm{dd}, \mathrm{J}=9.0,4.7 \mathrm{~Hz}, 1 \mathrm{H}), 2.53-2.35(\mathrm{~m}, 2 \mathrm{H}), 2.16$ - $2.06(\mathrm{~m}$, 1H), $1.96-1.85(\mathrm{~m}, 1 \mathrm{H}), 1.85-1.73(\mathrm{~m}, 1 \mathrm{H}), 1.70-1.58(\mathrm{~m}, 1 \mathrm{H}) .{ }^{13} \mathrm{C}\left\{{ }^{1} \mathrm{H}\right\} \mathrm{NMR}$ (101 MHz, Chloroform-d) $\delta 172.33,150.5\left(\mathrm{dd}, J_{C F}=250.5,13.1 \mathrm{~Hz}\right), 149.8\left(\mathrm{dd}, J_{C F}\right.$ $=249.5,13.1 \mathrm{~Hz}), 139.61\left(\mathrm{dd}, J_{C F}=15.4,18.4 \mathrm{~Hz}\right), 121.98\left(\mathrm{dd}, J_{C F}=6.4,3.6 \mathrm{~Hz}\right)$, $117.62\left(\mathrm{~d}, J_{C F}=17.4 \mathrm{~Hz}\right), 115.16\left(\mathrm{~d}, J_{C F}=18.1 \mathrm{~Hz}\right), 56.75\left(\mathrm{~d}, J_{C F}=1.4 \mathrm{~Hz}\right), 32.04$, 31.19, 19.36. HRMS (ESI) m/z: $[\mathrm{M}+\mathrm{H}]^{+}$Calcd for $\mathrm{C}_{11} \mathrm{H}_{12} \mathrm{~F}_{2} \mathrm{NO}^{+}$212.0881; Found 212.0884. HPLC: Chiracel ODH Column (250 mm); detected at $210 \mathrm{~nm}$; n-hexane / i-propanol $=90 / 10$; flow $=0.8 \mathrm{~mL} / \mathrm{min}$; Retention time: $19.9 \mathrm{~min}$ (major), $24.9 \mathrm{~min}$ (minor).<smiles>O=C1CCCC[C@H](c2ccccc2)N1</smiles>

Chemical Formula: $\mathrm{C}_{12} \mathrm{H}_{15} \mathrm{NO}$

Exact Mass: 189.1154

\section{7-phenylazepan-2-one (4i)}

White solid, $15.9 \mathrm{mg}, 42 \%$ yield, $90 \%$ ee, $[\alpha]^{25}=25.7\left(\mathrm{c}=0.7, \mathrm{CHCl}_{3}\right)$, obtained by the purification with flash column chromatography on silica gel (EtOAc/petroleum ether 3:7). ${ }^{1} \mathrm{H}$ NMR (600 MHz, Chloroform-d) $\delta 7.37$ (t, J=7.5 Hz, 2H), 7.32 (t, J = $7.3 \mathrm{~Hz}, 3 \mathrm{H}), 5.68(\mathrm{~s}, 1 \mathrm{H}), 4.46$ (q, J = 10.0, $3.9 \mathrm{~Hz}, 1 \mathrm{H}), 2.65-2.52(\mathrm{~m}, 2 \mathrm{H}), 2.08$ (dd, $\mathrm{J}=11.1,3.8 \mathrm{~Hz}, 1 \mathrm{H}), 2.02-1.97(\mathrm{~m}, 1 \mathrm{H}), 1.97-1.88(\mathrm{~m}, 2 \mathrm{H}), 1.72-1.61(\mathrm{~m}, 2 \mathrm{H})$. ${ }^{13} \mathrm{C}\left\{{ }^{1} \mathrm{H}\right\}$ NMR (101 MHz, Chloroform-d) $\delta$ 177.2, 142.4, 129.1, 128.1, 126.2, 58.7, 37.1, 37.1, 29.9, 23.1. HRMS (ESI) m/z: $[\mathrm{M}+\mathrm{H}]^{+}$Calcd for $\mathrm{C}_{12} \mathrm{H}_{16} \mathrm{NO}^{+}$190.1226; Found 190.1226. HPLC: Chiracel IA Column $(250 \mathrm{~mm})$; detected at $210 \mathrm{~nm}$; n-hexane $/$ i-propanol $=90 / 10$; flow $=0.8 \mathrm{~mL} / \mathrm{min}$; Retention time: $12.6 \mathrm{~min}$ (major), 
14.0 min (minor). The absolute configuration of $\mathbf{4 a}$ was identified by comparison of its physical and spectroscopic data with the one reported in the literature. ${ }^{[1]}$<smiles>CC1NC(=O)c2ccccc21</smiles>

6a

Chemical Formula: $\mathrm{C}_{9} \mathrm{H}_{9} \mathrm{NO}$

Exact Mass: 147.0684

\section{(R)-3-methylisoindolin-1-one (6a)}

White solid, $26.8 \mathrm{mg}, 91 \%$ yield, $97 \%$ ee, $[\alpha]^{26}{ }_{\mathrm{D}}=14.5\left(\mathrm{c}=0.5, \mathrm{CHCl}_{3}\right)$, obtained by the purification with flash column chromatography on silica gel (EtOAc/petroleum ether 3:7). ${ }^{1} \mathrm{H}$ NMR (400 MHz, Chloroform-d) $\delta 7.92$ (br, 1H), 7.85 (d, $J=7.5 \mathrm{~Hz}$, 1H), $7.57(\mathrm{t}, J=7.5,1.2 \mathrm{~Hz}, 1 \mathrm{H}), 7.50-7.41(\mathrm{~m}, 2 \mathrm{H}), 4.72$ (q, $J=6.7 \mathrm{~Hz}, 1 \mathrm{H}), 1.52$ $(\mathrm{d}, J=6.8 \mathrm{~Hz}, 3 \mathrm{H}) .{ }^{13} \mathrm{C}\left\{{ }^{1} \mathrm{H}\right\} \mathrm{NMR}(101 \mathrm{MHz}$, Chloroform-d $) \delta 171.1,148.9,131.8$, 131.6, 128.0, 123.6, 122.2, 52.6, 20.2. HPLC: Chiracel OD-3 Column (250 mm); detected at $210 \mathrm{~nm}$; n-hexane $/$ i-propanol $=90 / 10$; flow $=0.8 \mathrm{~mL} / \mathrm{min}$; Retention time: $9.6 \mathrm{~min}$ (minor), $10.4 \mathrm{~min}$ (major). The absolute configuration of $\mathbf{6 a}$ was identified by comparison of its physical and spectroscopic data with the one reported in the literature. ${ }^{[11]}$ The absolute configuration of $\mathbf{6 c - 6} \mathbf{- 6}$ was determined by comparing with $\mathbf{4 a}$.<smiles>CCC1NC(=O)c2ccccc21</smiles>

6b

Chemical Formula: $\mathrm{C}_{10} \mathrm{H}_{11} \mathrm{NO}$

Exact Mass: 161.0841

\section{(R)-3-ethylisoindolin-1-one (6b)}

White solid, $29.6 \mathrm{mg}, 92 \%$ yield, $96 \%$ ee, $[\alpha]^{25}=42.2\left(\mathrm{c}=0.5, \mathrm{CHCl}_{3}\right)$, obtained by the purification with flash column chromatography on silica gel (EtOAc/petroleum ether 3:7). ${ }^{1} \mathrm{H}$ NMR (400 MHz, Chloroform-d) $\delta 7.86(\mathrm{~d}, J=7.5 \mathrm{~Hz}, 1 \mathrm{H}), 7.64-7.53$ 
(m, 2H), $7.51-7.38(\mathrm{~m}, 2 \mathrm{H}), 4.61$ (q, $J=6.4,4.4 \mathrm{~Hz} 1 \mathrm{H}), 2.10-1.97$ (m, 1H), 1.79 $1.66(\mathrm{~m}, 1 \mathrm{H}), 0.97(\mathrm{t}, J=7.4 \mathrm{~Hz}, 3 \mathrm{H}) .{ }^{13} \mathrm{C}\left\{{ }^{1} \mathrm{H}\right\} \mathrm{NMR}(101 \mathrm{MHz}$, Chloroform-d) $\delta$ 171.3, 147.4, 132.1, 131.7, 128.0, 123.7, 122.4, 58.0, 27.3, 9.5. HRMS (ESI) m/z: $[\mathrm{M}+\mathrm{H}]^{+}$Calcd for $\mathrm{C}_{10} \mathrm{H}_{12} \mathrm{NO}^{+}$162.0913; Found 162.0914. HPLC: Chiracel IA Column $(250 \mathrm{~mm})$; detected at $210 \mathrm{~nm}$; n-hexane $/$ i-propanol $=90 / 10$; flow $=0.8$ $\mathrm{mL} / \mathrm{min}$; Retention time: $10.4 \mathrm{~min}$ (major), $11.3 \mathrm{~min}$ (minor). The absolute configuration of $\mathbf{6 b}$ was identified by comparison of its physical and spectroscopic data with the one reported in the literature. ${ }^{[11]}$<smiles>CC1NC(=O)c2cc(Br)ccc21</smiles>

6c

Chemical Formula: $\mathrm{C}_{9} \mathrm{H}_{8} \mathrm{BrNO}$

Exact Mass: 224.9789

\section{(R)-6-bromo-3-methylisoindolin-1-one (6c)}

White solid, $42.7 \mathrm{mg}$, 95\% yield, $97 \%$ ee, on $0.2 \mathrm{mmol} \mathrm{scale;} 864 \mathrm{mg}$, 95\% yield, 96\% ee, on $4.0 \mathrm{mmol}$ scale, $[\alpha]_{\mathrm{D}}^{26}=20.1\left(\mathrm{c}=0.5, \mathrm{CHCl}_{3}\right)$, obtained by the purification with flash column chromatography on silica gel (EtOAc/petroleum ether 3:7). ${ }^{1} \mathrm{H}$ NMR (600 MHz, Chloroform-d) $\delta 7.97(\mathrm{~s}, 1 \mathrm{H}), 7.82(\mathrm{~s}, 1 \mathrm{H}), 7.69$ (dd, J = 8.0, 1.8 Hz, 1H), $7.32(\mathrm{~d}, \mathrm{~J}=8.0 \mathrm{~Hz}, 1 \mathrm{H}), 4.68(\mathrm{q}, \mathrm{J}=6.8 \mathrm{~Hz}, 1 \mathrm{H}), 1.51(\mathrm{~d}, \mathrm{~J}=6.8 \mathrm{~Hz}, 3 \mathrm{H}) .{ }^{13} \mathrm{C}\left\{{ }^{1} \mathrm{H}\right\}$ NMR (151 MHz, Chloroform-d) $\delta 169.5,147.5,134.8,133.7,126.9,123.9,122.1$, 52.4, 20.1. HRMS (ESI) m/z: $[\mathrm{M}+\mathrm{H}]^{+}$Calcd for $\mathrm{C}_{9} \mathrm{H}_{9} \mathrm{BrNO}^{+}$225.9862; Found 225.9863. HPLC: Chiracel IA Column $(250 \mathrm{~mm})$; detected at $210 \mathrm{~nm}$; n-hexane / i-propanol $=90 / 10$; flow $=0.8 \mathrm{~mL} / \mathrm{min}$; Retention time: $10.5 \mathrm{~min}$ (major), $11.7 \mathrm{~min}$ (minor). 
<smiles>COc1ccc2c(c1)C(C)NC2=O</smiles>

Chemical Formula: $\mathrm{C}_{10} \mathrm{H}_{11} \mathrm{NO}_{2}$

Exact Mass: 177.0790

\section{(R)-5-methoxy-3-methylisoindolin-1-one (6d)}

White solid, $29.4 \mathrm{mg}, 83 \%$ yield, $96 \%$ ee, $[\alpha]^{25}=50.5\left(\mathrm{c}=0.5, \mathrm{CHCl}_{3}\right)$, obtained by the purification with flash column chromatography on silica gel (EtOAc/petroleum ether 3:7). ${ }^{1} \mathrm{H}$ NMR (400 MHz, Chloroform-d) $\delta 7.76(\mathrm{~d}, J=8.4 \mathrm{~Hz}, 1 \mathrm{H}), 7.70$ (br, 1H), $6.98(\mathrm{~m}, 1 \mathrm{H}), 6.90(\mathrm{~d}, J=2.1 \mathrm{~Hz}, 1 \mathrm{H}), 4.65(\mathrm{q}, J=6.7 \mathrm{~Hz}, 1 \mathrm{H}), 3.88(\mathrm{~s}, 3 \mathrm{H})$, $1.50(\mathrm{~d}, J=6.8 \mathrm{~Hz}, 3 \mathrm{H}) .{ }^{13} \mathrm{C}\left\{{ }^{1} \mathrm{H}\right\}$ NMR (101 MHz, Chloroform-d) $\delta$ 170.9, 163.0, 151.3, 125.0, 124.2, 114.5, 107.0, 55.6, 52.3, 20.4. HRMS (ESI) m/z: 178.0862 $[\mathrm{M}+\mathrm{H}]^{+}$Calcd for $\mathrm{C}_{10} \mathrm{H}_{12} \mathrm{NO}_{2}{ }^{+}$178.0863; Found 225.9863. HPLC: Chiracel AD-3 Column $(250 \mathrm{~mm})$; detected at $210 \mathrm{~nm}$; n-hexane $/$ i-propanol $=90 / 10$; flow $=0.8$ $\mathrm{mL} / \mathrm{min}$; Retention time: $17.8 \mathrm{~min}$ (minor), $21.4 \mathrm{~min}$ (major).<smiles>Cc1ccc2c(c1)C(=O)NC2C</smiles>

6e

Chemical Formula: $\mathrm{C}_{10} \mathrm{H}_{11} \mathrm{NO}$

Exact Mass: 161.0841

\section{(R)-3,6-dimethylisoindolin-1-one (6e)}

White solid, $29.6 \mathrm{mg}, 92 \%$ yield, $92 \%$ ee, $[\alpha]^{25}=18.3\left(\mathrm{c}=0.5, \mathrm{CHCl}_{3}\right)$, obtained by the purification with flash column chromatography on silica gel (EtOAc/petroleum ether 3:7). ${ }^{1} \mathrm{H}$ NMR (400 MHz, Chloroform-d) $\delta 7.65$ (s, 1H), 7.49 (s, 1H), 7.38 (d, J $=8.5 \mathrm{~Hz}, 1 \mathrm{H}), 7.31(\mathrm{~d}, \mathrm{~J}=7.7 \mathrm{~Hz}, 1 \mathrm{H}), 4.67(\mathrm{q}, \mathrm{J}=6.7 \mathrm{~Hz}, 1 \mathrm{H}), 2.45(\mathrm{~s}, 3 \mathrm{H}), 1.49(\mathrm{~d}$, $\mathrm{J}=6.7 \mathrm{~Hz}, 3 \mathrm{H}) .{ }^{13} \mathrm{C}\left\{{ }^{1} \mathrm{H}\right\} \mathrm{NMR}(101 \mathrm{MHz}$, Chloroform-d) $\delta$ 171.1, 146.2, 138.0, 132.8, 131.7, 123.9, 121.9, 52.4, 21.3, 20.4. HRMS (ESI) m/z: $[\mathrm{M}+\mathrm{H}]^{+}$Calcd for $\mathrm{C}_{10} \mathrm{H}_{12} \mathrm{NO}^{+}$162.0913; Found 162.0915. HPLC: Chiracel IA Column (250 mm); detected at $210 \mathrm{~nm}$; n-hexane $/$ i-propanol $=90 / 10$; flow $=0.8 \mathrm{~mL} / \mathrm{min}$; Retention 
time: $9.9 \min$ (major), $10.9 \min$ (minor).<smiles>Cc1ccc2c(c1)C(C)NC2=O</smiles>

6f

Chemical Formula: $\mathrm{C}_{10} \mathrm{H}_{11} \mathrm{NO}$

Exact Mass: 161.0841

\section{(R)-3,5-dimethylisoindolin-1-one (6f)}

White solid, 29.6mg, 92\% yield, $96 \%$ ee, $[\alpha]^{25.5}=42.6\left(\mathrm{c}=0.5, \mathrm{CHCl}_{3}\right)$, obtained by the purification with flash column chromatography on silica gel (EtOAc/petroleum ether 3:7). ${ }^{1} \mathrm{H}$ NMR (400 MHz, Chloroform-d) $\delta 7.72(\mathrm{~d}, J=7.8 \mathrm{~Hz}, 1 \mathrm{H}), 7.38$ (br, 1H), 7.27 (d, $J=7.7 \mathrm{~Hz}, 1 \mathrm{H}), 7.23(\mathrm{~s}, 1 \mathrm{H}), 4.65(\mathrm{q}, J=6.7 \mathrm{~Hz}, 1 \mathrm{H}), 2.47$ (s, 3H), 1.49 $(\mathrm{d}, J=6.7 \mathrm{~Hz}, 3 \mathrm{H}) .{ }^{13} \mathrm{C}\left\{{ }^{1} \mathrm{H}\right\} \mathrm{NMR}(101 \mathrm{MHz}$, Chloroform-d) $\delta 171.0,149.3,142.5$, 129.1, 123.5, 122.7, 52.3, 21.9, 20.3. HRMS (ESI) $\mathrm{m} / \mathrm{z}[\mathrm{M}+\mathrm{H}]^{+}$Calcd for $\mathrm{C}_{10} \mathrm{H}_{12} \mathrm{NO}^{+}$ 162.0913; Found 162.0915. HPLC: Chiracel IA Column (250 mm); detected at 210 $\mathrm{nm}$; $\mathrm{n}$-hexane $/$ i-propanol $=90 / 10 ;$ flow $=0.8 \mathrm{~mL} / \mathrm{min}$; Retention time: $13.2 \mathrm{~min}$ (minor), $14.4 \mathrm{~min}$ (major).<smiles>CC1NC(=O)c2ncccc21</smiles>

$6 \mathrm{~g}$

Chemical Formula: $\mathrm{C}_{8} \mathrm{H}_{8} \mathrm{~N}_{2} \mathrm{O}$

Exact Mass: 148.0637

\section{(R)-5-methyl-5,6-dihydro-7H-pyrrolo[3,4-b]pyridin-7-one (6g)}

Obtained from the corresponding isopropyl ester $5 \mathrm{~g}$. White solid, $24.0 \mathrm{mg}, 81 \%$ yield, $91 \%$ ee, $[\alpha]_{D}^{25}=18.6\left(\mathrm{c}=0.5, \mathrm{CHCl}_{3}\right)$, obtained by the purification with flash column chromatography on silica gel (EtOAc/petroleum ether 3:7). ${ }^{1} \mathrm{H}$ NMR (400 MHz, Chloroform-d) $\delta 8.84-8.77(\mathrm{~m}, 1 \mathrm{H}), 8.24(\mathrm{~d}, \mathrm{~J}=58.8 \mathrm{~Hz}, 1 \mathrm{H}), 7.86-7.79(\mathrm{~m}, 1 \mathrm{H})$, $7.47(\mathrm{dd}, \mathrm{J}=7.7,4.8 \mathrm{~Hz}, 1 \mathrm{H}), 4.76(\mathrm{q}, \mathrm{J}=6.8 \mathrm{~Hz}, 1 \mathrm{H}), 1.56(\mathrm{~d}, \mathrm{~J}=6.8 \mathrm{~Hz}, 3 \mathrm{H})$. ${ }^{13} \mathrm{C}\left\{{ }^{1} \mathrm{H}\right\}$ NMR (101 MHz, Chloroform-d) $\delta 168.9,150.9,149.9,142.3,130.6,125.5$, 
50.6, 19.9. HRMS (ESI) m/z: $[\mathrm{M}+\mathrm{H}]^{+}$Calcd for $\mathrm{C}_{8} \mathrm{H}_{9} \mathrm{~N}_{2} \mathrm{O}^{+}$149.0709; Found 149.0710. HPLC: Chiracel OJ-3 Column (250 mm); detected at $210 \mathrm{~nm}$; n-hexane / i-propanol $=90 / 10$; flow $=0.8 \mathrm{~mL} / \mathrm{min}$; Retention time: $21.2 \mathrm{~min}$ (minor), $23.3 \mathrm{~min}$ (major).

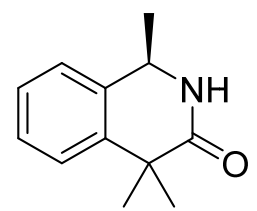

6h

Chemical Formula: $\mathrm{C}_{12} \mathrm{H}_{15} \mathrm{NO}$

Exact Mass: 189.1154

\section{(R)-1,4,4-trimethyl-1,4-dihydroisoquinolin-3(2H)-one (6h)}

White solid, $34.0 \mathrm{mg}, 90 \%$ yield, $94 \%$ ee, $[\alpha]^{25}=17.6\left(\mathrm{c}=0.5, \mathrm{CHCl}_{3}\right)$, obtained by the purification with flash column chromatography on silica gel (EtOAc/petroleum ether 3:7). ${ }^{1} \mathrm{H}$ NMR (600 MHz, Chloroform-d) $\delta 7.38(\mathrm{~d}, \mathrm{~J}=7.8 \mathrm{~Hz}, 1 \mathrm{H}), 7.31$ (t, J = $7.5 \mathrm{~Hz}, 1 \mathrm{H}), 7.27-7.23(\mathrm{~m}, 1 \mathrm{H}), 7.17$ (d, J = 7.6 Hz, 1H), 6.66 (s, 1H), 4.73 (q, J = $6.1 \mathrm{~Hz}, 1 \mathrm{H}), 1.59(\mathrm{~s}, 3 \mathrm{H}), 1.56(\mathrm{~d}, \mathrm{~J}=6.7 \mathrm{~Hz}, 3 \mathrm{H}), 1.54(\mathrm{~s}, 3 \mathrm{H}) .{ }^{13} \mathrm{C}\left\{{ }^{1} \mathrm{H}\right\}$ NMR $(101$ MHz, Chloroform-d) $\delta 176.4,140.7,134.3,127.7,126.4,125.7,125.0,50.1,41.3$, 28.7, 27.2, 25.0. HRMS (ESI) m/z: $[\mathrm{M}+\mathrm{H}]^{+}$Calcd for $\mathrm{C}_{12} \mathrm{H}_{16} \mathrm{NO}^{+} 190.1226$; Found 190.1228. HPLC: Chiracel IE Column (250 mm); detected at $210 \mathrm{~nm}$; n-hexane / i-propanol $=90 / 10$; flow $=0.8 \mathrm{~mL} / \mathrm{min}$; Retention time: $16.7 \mathrm{~min}$ (minor), $17.6 \mathrm{~min}$ (major).

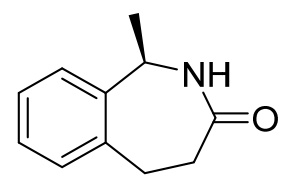

$6 \mathbf{i}$

Chemical Formula: $\mathrm{C}_{11} \mathrm{H}_{13} \mathrm{NO}$

Exact Mass: 175.0997

\section{(R)-1-methyl-1,2,4,5-tetrahydro-3H-benzo[c]azepin-3-one (6i)}

White solid, $18.2 \mathrm{mg}, 52 \%$ yield, $90 \%$ ee, $[\alpha]^{25}=-41.5\left(\mathrm{c}=0.4, \mathrm{CHCl}_{3}\right)$, obtained by the purification with flash column chromatography on silica gel (EtOAc/petroleum 
ether 3:7). ${ }^{1} \mathrm{H}$ NMR (400 MHz, Chloroform-d) $\delta 7.30-7.21(\mathrm{~m}, 4 \mathrm{H}), 5.83(\mathrm{~s}, 1 \mathrm{H})$, $4.99-4.82(\mathrm{~m}, 1 \mathrm{H}), 3.36-3.20(\mathrm{~m}, 1 \mathrm{H}), 3.00-2.79(\mathrm{~m}, 2 \mathrm{H}), 2.64-2.48(\mathrm{~m}, 1 \mathrm{H})$, $1.64(\mathrm{~d}, \mathrm{~J}=6.9 \mathrm{~Hz}, 3 \mathrm{H}) .{ }^{13} \mathrm{C}\left\{{ }^{1} \mathrm{H}\right\} \mathrm{NMR}(101 \mathrm{MHz}$, Chloroform-d) $\delta 173.5,140.3$, 139.9, 129.0, 128.2, 126.8, 123.9, 47.7, 35.4, 28.3, 19.1. HRMS (ESI) m/z: $[\mathrm{M}+\mathrm{H}]^{+}$ Calcd for $\mathrm{C}_{11} \mathrm{H}_{14} \mathrm{NO}^{+}$176.1070; Found 176.1071. HPLC: Chiracel IA Column (250 $\mathrm{mm}$ ); detected at $210 \mathrm{~nm}$; $\mathrm{n}$-hexane $/$ i-propanol $=90 / 10$; flow $=0.8 \mathrm{~mL} / \mathrm{min}$; Retention time: 20.4 min (minor), 23.5 min (major). The absolute configuration of $\mathbf{6 i}$ was identified by comparison of the physical and spectroscopic data with the similar ones reported in the literature. ${ }^{[12]}$<smiles>O=C1NC(c2ccccc2)c2ccccc21</smiles>

6j

Chemical Formula: $\mathrm{C}_{14} \mathrm{H}_{11} \mathrm{NO}$

Exact Mass: 209.0841

\section{(R)-3-phenylisoindolin-1-one (6j)}

White solid, $22.2 \mathrm{mg}, 53 \%$ yield, $88 \%$ ee, $[\alpha]^{25}=-65.9\left(\mathrm{c}=0.5, \mathrm{CHCl}_{3}\right)$, obtained by the purification with flash column chromatography on silica gel (EtOAc/petroleum ether 3:7). ${ }^{1} \mathrm{H}$ NMR (400 MHz, DMSO-d $) \delta 9.07$ (s, 1H), $7.71(\mathrm{~d}, J=7.2 \mathrm{~Hz}, 1 \mathrm{H})$, $7.56-7.46(\mathrm{~m}, 2 \mathrm{H}), 7.39-7.34(\mathrm{~m}, 2 \mathrm{H}), 7.33-7.27(\mathrm{~m}, 4 \mathrm{H}), 5.73(\mathrm{~s}, 1 \mathrm{H}) .{ }^{13} \mathrm{C}\left\{{ }^{1} \mathrm{H}\right\}$ NMR (151 MHz, DMSO-d 6 ) $\delta$ 169.76, 148.23, 139.71, 131.93, 131.43, 128.86, 128.20, 127.97, 126.63, 123.57, 122.96, 59.58. HPLC: Chiracel AD-3 Column (250 $\mathrm{mm}$ ); detected at $210 \mathrm{~nm}$; $\mathrm{n}$-hexane $/$ i-propanol $=90 / 10$; flow $=0.8 \mathrm{~mL} / \mathrm{min}$; Retention time: 13.0 min (major), 21.9 min (minor). The NMR data is consistent with that reported. ${ }^{[13]}$ The absolute configuration of $\mathbf{6 j}$ was identified by comparison of the physical and spectroscopic data with the similar ones reported in the literature. ${ }^{[14]}$

\subsection{Reduction of $\mathbf{2 b}$}




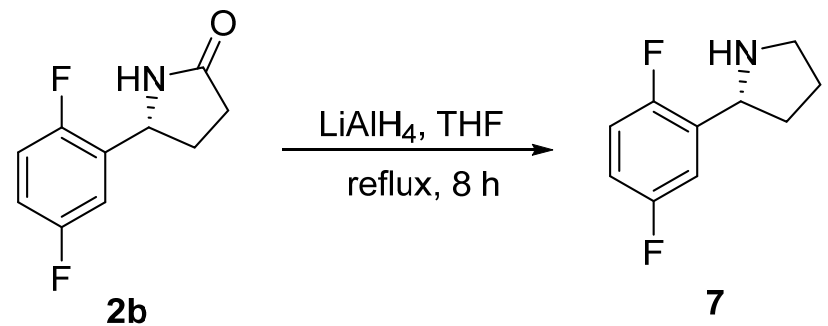

A suspension of $\mathrm{LiAlH}_{4}$ in $5 \mathrm{~mL}$ THF was heated to reflux. 2b $(97 \mathrm{mg}, 0.5$ mmol, $>99 \%$ ee) in $1 \mathrm{~mL}$ THF was added slowly. The resulting mixture was continued to stir for 8 hours under reflux. The reaction was cooled to $0{ }^{\circ} \mathrm{C}$ and quenched by successive addition of $\mathrm{H}_{2} \mathrm{O}, \mathrm{NaOH}(15 \%$, aq. $)$, and $\mathrm{H}_{2} \mathrm{O}(19 \mu \mathrm{L} / 19 \mu \mathrm{L}$ /57 $\mu \mathrm{L}, 1: 1: 3$ ). The solid was filterted and washed with $\mathrm{CH}_{2} \mathrm{Cl}_{2}$. The filtrate was dried over anhydrous $\mathrm{Na}_{2} \mathrm{SO}_{4}$, and then concentrated under vacuum. The residue was purified with column chromatography on silica gel (eluent: $\mathrm{DCM} / \mathrm{MeOH} / \mathrm{Et}_{2} \mathrm{NH}=$ 97:2:1) to afford 7 as yellow oil.<smiles>Fc1ccc(F)c(C2CCCN2)c1</smiles>

Chemical Formula: $\mathrm{C}_{10} \mathrm{H}_{11} \mathrm{~F}_{2} \mathrm{~N}$

Exact Mass: 183.0860

\section{(R)-2-(2,5-difluorophenyl)pyrrolidine (7)}

Yellow oil. $77.8 \mathrm{mg}, 85 \%$ yield, $[\alpha]^{25}=37.8\left(\mathrm{c}=0.1, \mathrm{CHCl}_{3}\right) .{ }^{1} \mathrm{H}$ NMR $(600 \mathrm{MHz}$, Chloroform-d) $\delta 7.26-7.22(\mathrm{~m}, 1 \mathrm{H}), 6.96-6.92(\mathrm{~m}, 1 \mathrm{H}), 6.88-6.83(\mathrm{~m}, 1 \mathrm{H}), 4.40$ $(\mathrm{t}, \mathrm{J}=7.6 \mathrm{~Hz}, 1 \mathrm{H}), 3.22-3.14(\mathrm{~m}, 1 \mathrm{H}), 3.10-3.01(\mathrm{~m}, 1 \mathrm{H}), 2.29-2.23(\mathrm{~m}, 1 \mathrm{H})$, $2.08(\mathrm{br}, 1 \mathrm{H}), 1.94-1.80(\mathrm{~m}, 2 \mathrm{H}), 1.66-1.59(\mathrm{~m}, 1 \mathrm{H})$. The ${ }^{1} \mathrm{H}$ NMR data is consistent with that reported, $[\alpha]^{20}{ }_{\mathrm{D}}=38.4\left(\mathrm{c}=0.1, \mathrm{CHCl}_{3}\right){ }^{[15]}$ 


\section{Control Experiments}

Plausible reaction pathways:

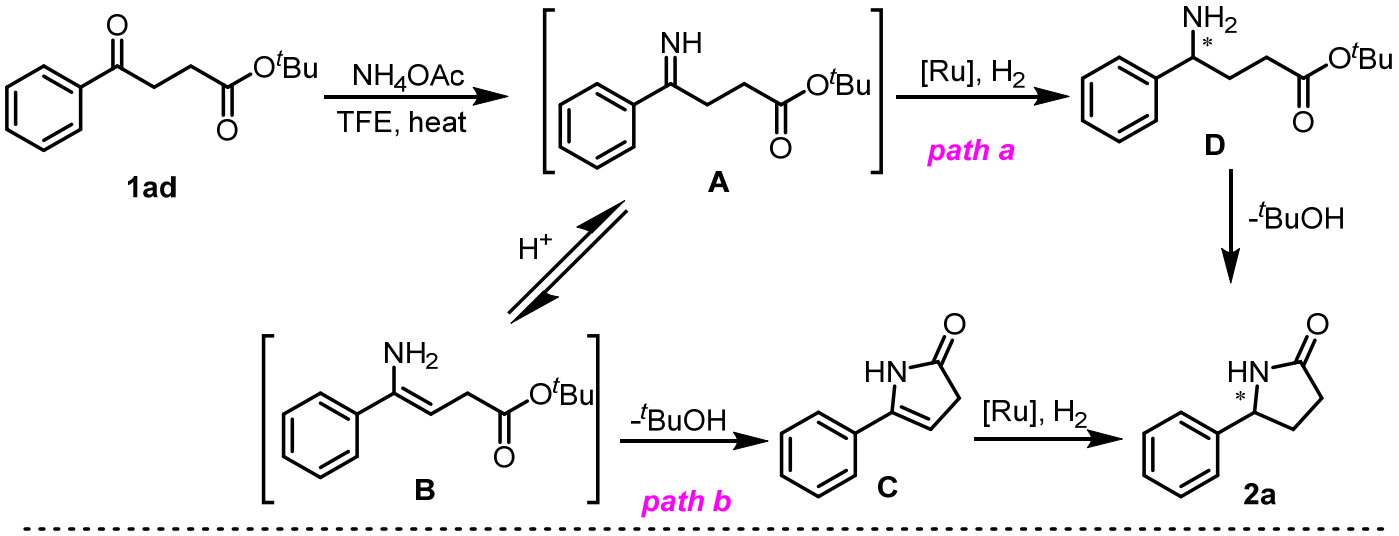

Control experiments:<smiles>O=C1CC=C(c2ccccc2)N1</smiles>

C

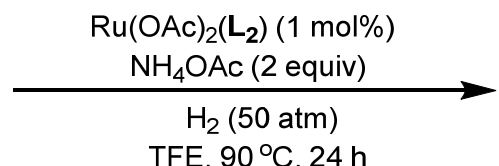

TFE, $90^{\circ} \mathrm{C}, 24 \mathrm{~h}$

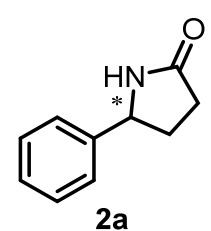

$10 \%$ yield, rac

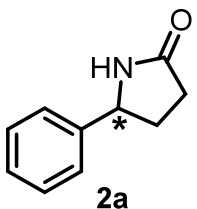

without $\mathrm{NH}_{4} \mathrm{OAc}, 50 \%$ yield, $0 \%$ ee with 2 equiv $\mathrm{NH}_{4} \mathrm{OAc}, 95 \%$ yield, $16 \%$ ee

Independent synthesis of $\mathbf{C}^{[16]}$<smiles>NC(=O)CCCC(=O)O</smiles><smiles>O=C1CC=C(c2ccccc2)N1</smiles>

4-Oxo-4-phenylbutanoic acid 1ae (1.41 g, 7.9 mmol) and $\mathrm{NH}_{4} \mathrm{OAc}(1.53 \mathrm{~g}, 19.8$ mmol) were dissolved in $20 \mathrm{~mL}$ of acetic anhydride. The resulting mixture was heated to $100^{\circ} \mathrm{C}$ for $3 \mathrm{~h}$ and then cooled to room temperature. The reaction was slowly quenched with saturated aqueous $\mathrm{NaHCO}_{3}$. The mixture was diluted with water, and extracted with EA $(3 \times 30 \mathrm{~mL})$. The combined organic layers were dried over anhydrous $\mathrm{NaSO}_{4}$, filtered and evaporated under reduced pressure. The residue was subjected to column chromatography on silica gel (eluent: EA/Hexane $=5 \%$ ) to afford C $(0.89 \mathrm{~g}, 70 \%$ yield $)$. 
<smiles>O=C1CC=C(c2ccccc2)N1</smiles>

Chemical Formula: $\mathrm{C}_{10} \mathrm{H}_{9} \mathrm{NO}$

Exact Mass: 159.0684

\section{5-phenyl-1,3-dihydro-2H-pyrrol-2-one}

${ }^{1} \mathrm{H}$ NMR (400 MHz, Chloroform-d) $\delta 7.56(\mathrm{dd}, J=7.7,2.0 \mathrm{~Hz}, 2 \mathrm{H}), 7.40-7.31(\mathrm{~m}$, $3 \mathrm{H}), 5.73(\mathrm{t}, J=2.8 \mathrm{~Hz}, 1 \mathrm{H}), 3.33(\mathrm{~s}, 2 \mathrm{H}) .{ }^{13} \mathrm{C}\left\{{ }^{1} \mathrm{H}\right\}$ NMR (101 MHz, Chloroform-d) $\delta$ $175.64,153.47,129.26,128.39,128.12,124.39,97.67,34.30$.

(4-Oxo-4-phenylbutanamide) $\mathbf{E}$ is synthesized according to a reported literature. ${ }^{[17]}$<smiles>NC(=O)CCC(=O)c1ccccc1</smiles>

E

Chemical Formula: $\mathrm{C}_{10} \mathrm{H}_{11} \mathrm{NO}_{2}$ Exact Mass: 177.0790

\section{4-Oxo-4-phenylbutanamide (E)}

Pale solid. ${ }^{1} \mathrm{H}$ NMR (400 MHz, Methanol-d4) $\delta 8.03-7.97$ (m, 2H), 7.60 (t, J=7.4 $\mathrm{Hz}, 1 \mathrm{H}), 7.49$ (t, $J=7.6 \mathrm{~Hz}, 2 \mathrm{H}), 3.34(\mathrm{t}, J=6.7 \mathrm{~Hz}, 2 \mathrm{H}), 2.63(\mathrm{t}, J=6.7 \mathrm{~Hz}, 2 \mathrm{H})$.

Following General Procedure F, product 2a was obtained in $10 \%$ yield as a racemic form from compound $\mathbf{C}$ (eq 1 ).

Following General Procedure F, in the absence of $\mathrm{NH}_{4} \mathrm{OAc}$, product 2a was obtained in 50\% yield as a racemic form from compound $\mathbf{E}$ (eq 2).

Following General Procedure $\mathbf{F}$, in the prsence of 2 equiv of $\mathrm{NH}_{4} \mathrm{OAc}$, product 2a was obtained in $95 \%$ yield and $16 \%$ ee from compound $\mathbf{E}$ (eq2). 


\section{V . Plausible enantioinduction models}

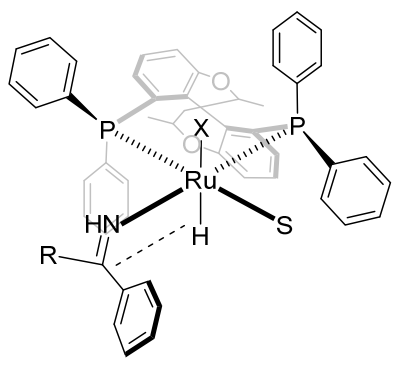

(R)-product favored

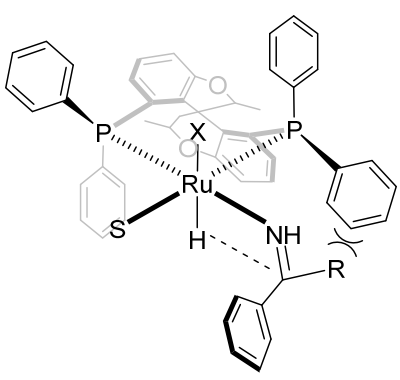

(S)-product

We tentatively propose enantioinduction models for this reaction, which are depicted as above. We speculate the ester group in the substrate will not coordinate to the $\mathrm{Ru}$ center otherwise it will form a seven- or eight-membered ring transition state which is energetically unfavorable. The steric repulsion between the tethered ester moiety and the phenyl group on $\mathrm{P}$ atom results in the energy difference between two transition states, which is consistent with the fact that the enantioselectivity of $\mathbf{2 a}$ is increasing along with the bulkiness of ester moiety increasing. Therefore, the $R$-configuration product is dominated. 


\section{I. References}

1. D. Guijarro, Ó. Pablo, and M. Yus. J. Org. Chem., 2013, 3647-3654

2. D. H. T. Phan, B. Kim, and V. M. Dong. J. Am. Chem. Soc., 2009, 15608-9.

3. J. Petrignet, S. I. Ngi, M. Abarbri, J. Thibonnet. Tetrahedron Lett., 2014, 982-984.

4. D. J. Rao, D. Rajaniverma, P.V. Prasannakumar, V. Seetaramauah, Y. Ramakrishna. Journal of Molecular Structure., 2019, 482-489.

5. Y. Katayama, T. Nagasawa, H. Takahashi. J. Photopolym. Sci. Tec., 2010, 467-471.

6. B. Lu, M. Zhao, G. Ding, X. Xie, L. Jiang, V. R.-Vidal, and Z. Zhang. ChemCatChem., 2017, 3989-3996

7. H. Takaya, T. Ohta, S. Inoue, M. Tokunaga, M. Kitamura, and R. Noyori. Organic Syntheses, 1995, 74-85.

8. X. Tan, S. Gao, W. Zeng, S. Xin, Q. Yin, and X. Zhang. J. Am. Chem. Soc., 2018, 2024-2027.

9. M. N. Cheemala, and P. Knochel. Org. Lett., 2007, 3089-92.

10. H. Wang, Y. Park, Z. Bai, S. Chang, G. He, and G. Chen. J. Am. Chem. Soc., 2019, 7194-7201.

11. M.-W. Chen, Q.-A. Chen, Y. Duan, Z.-S. Ye and Yong-Gui Zhou. Chem. Commun., 2012, 1698-700.

12. D. Dumoulin, S. Lebrun, E. Deniau, A. Couture, and Pierre Grandclaudon. Eur. J. Org. Chem., 2009, 3741-3752.

13. T. Lundrigan, E. N. Welsh, T. Hynes, C.-Hung T., M. R. Adams, K. R. Roy, K. N. Robertson, and A. W. H. Speed. J. Am. Chem. Soc., 2019, 14083-14088.

14. W. Zhou, Y.-X. Zhang, X.-D. Nie, C.-M. Si, X. Sun, and B.-G. Wei. J. Org. Chem., 2018, 9879-9889.

15. Y. Zhang, L. He, and L. Shi. Tetrahedron Lett., 2018, 1592-1595.

16. A. Y. Egorova, V. A. Sedavkina, and Z. Y. Timofeeva. Chemistry of Heterocyclic Compounds, 2001, 550.

17. Mengmeng Zhao, Wanfang Li, Xiaoming Li, Kai Ren, Xiaoming Tao, Xiaomin Xie, Tahar Ayad, Virginie Ratovelomanana-Vidal, and Zhaoguo Zhang. J. Org. Chem., 2014, 6164-6171. 


\section{NMR Spectra}

${ }^{1} \mathrm{H}$ NMR (400 MHz, Chloroform-d) of compound 1aa

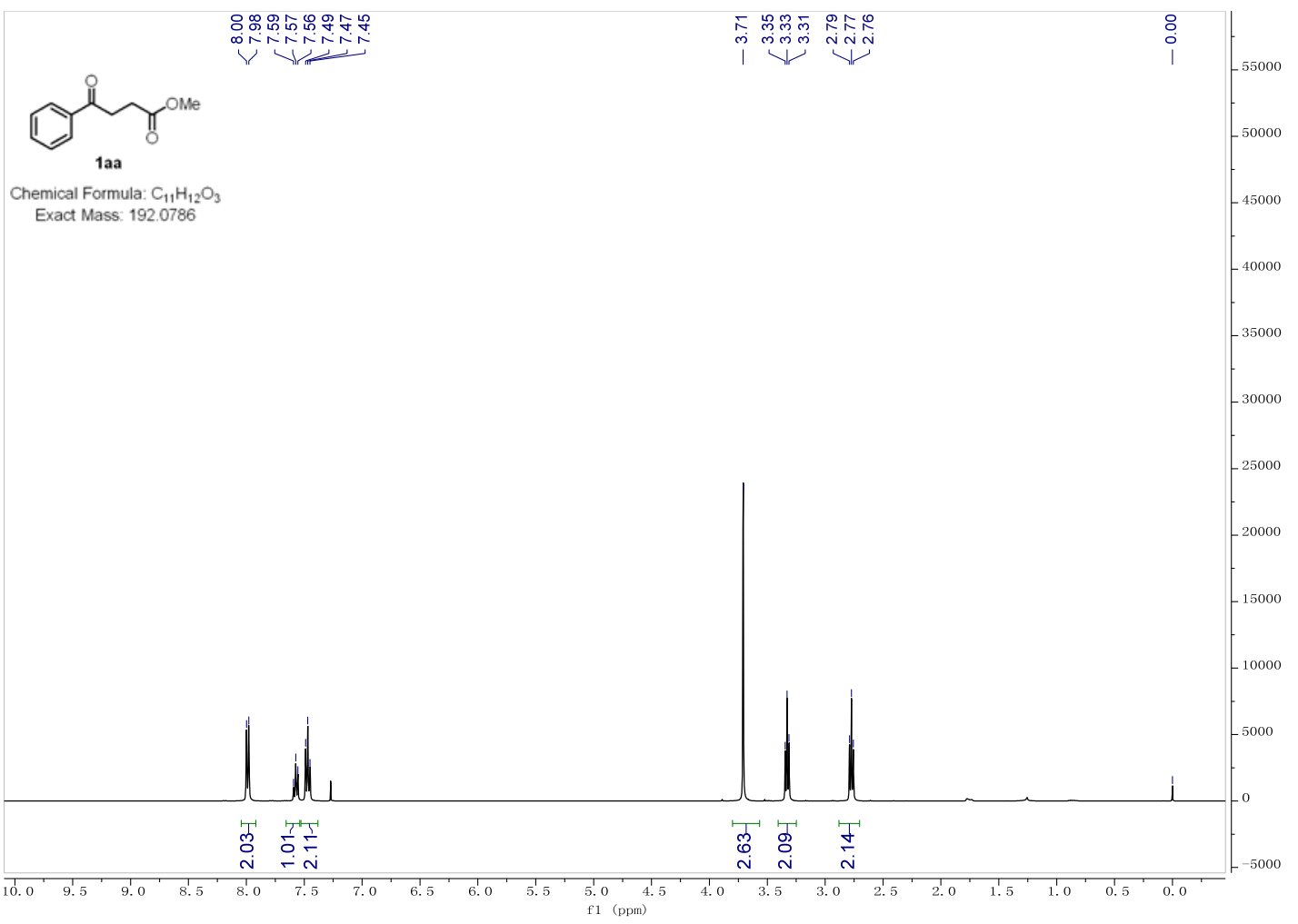

${ }^{13} \mathrm{C}\left\{{ }^{1} \mathrm{H}\right\}$ NMR (101 MHz, Chloroform-d) of compound 1aa
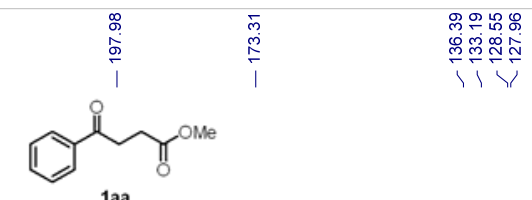

Chemical Formula: $\mathrm{C}_{11} \mathrm{H}_{12} \mathrm{O}_{3}$

Exact Mass: 192.0786

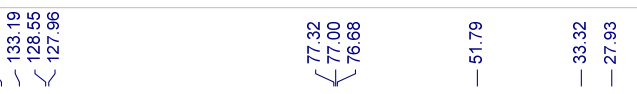

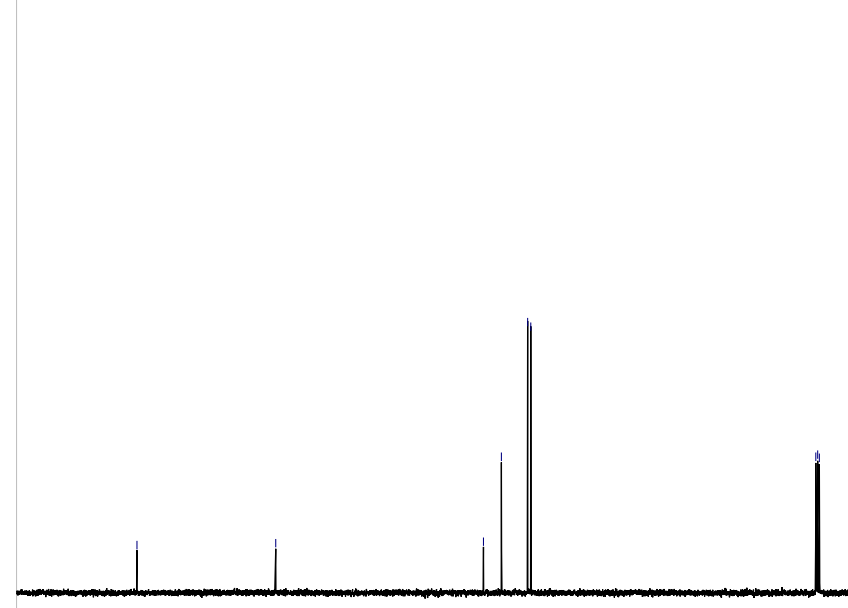


${ }^{1}$ H NMR (400 MHz, Chloroform-d) of compound 1ab

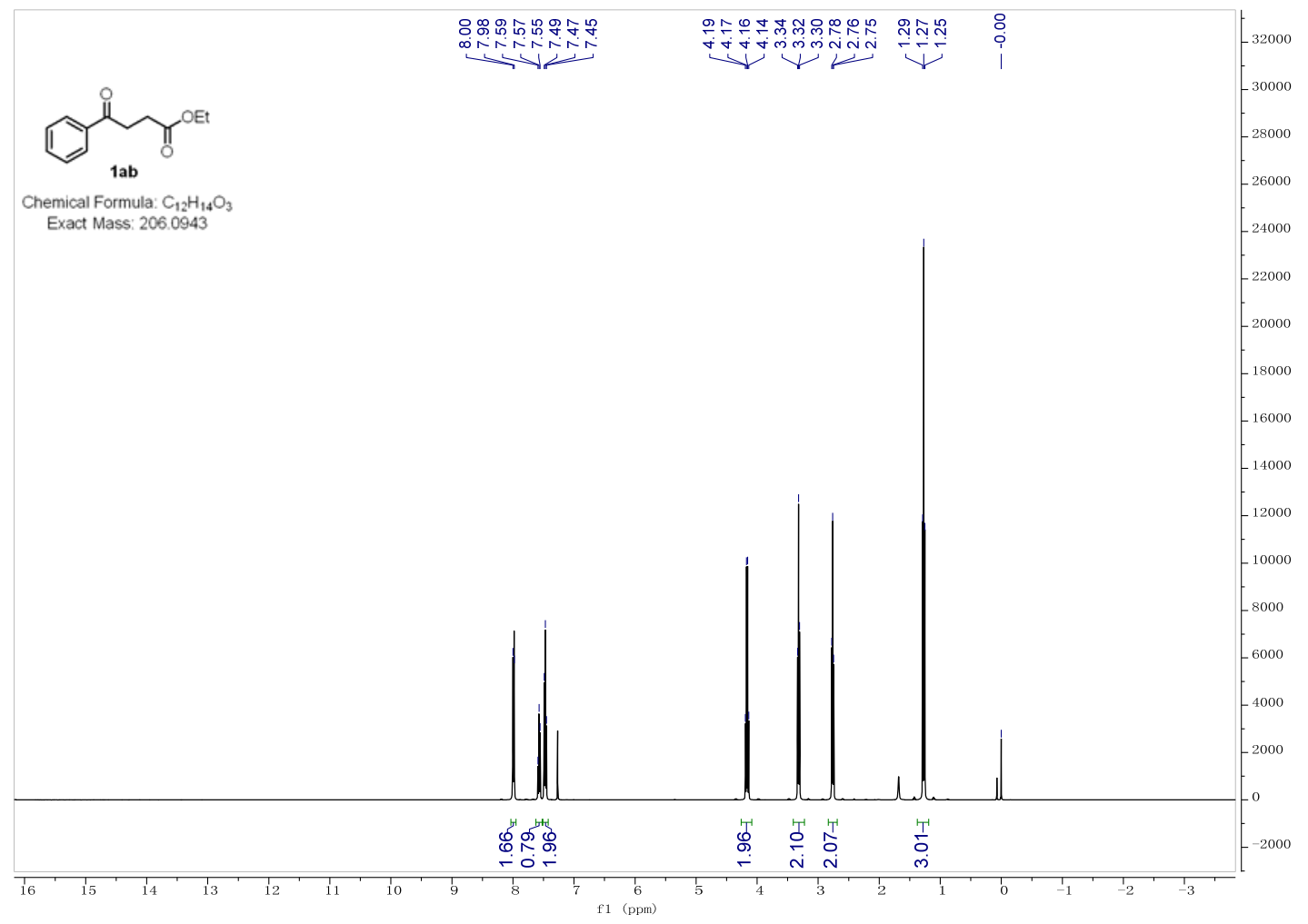

${ }^{13} \mathrm{C}\left\{{ }^{1} \mathrm{H}\right\}$ NMR (101 MHz, Chloroform-d) of compound 1ab

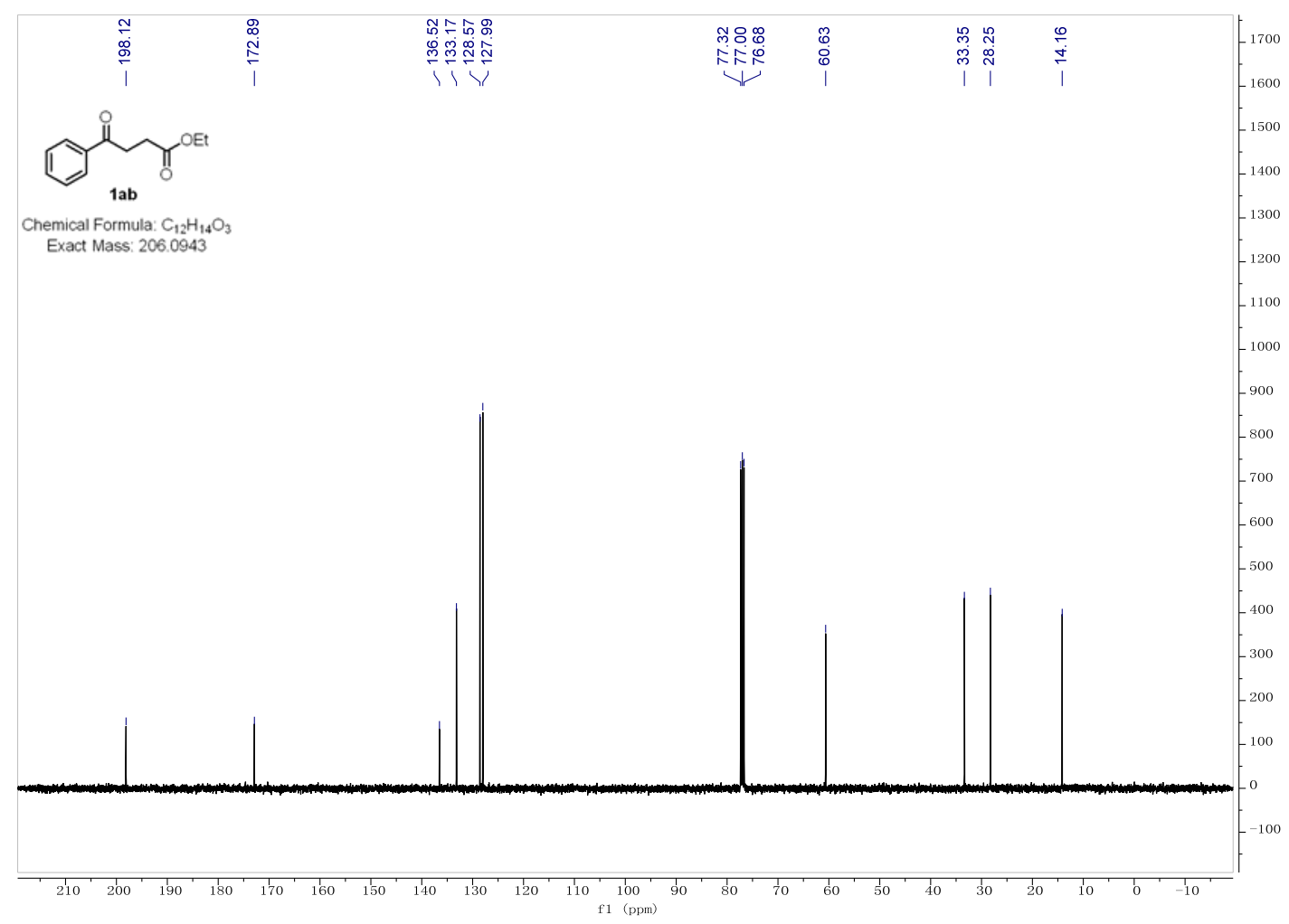


${ }^{1}$ H NMR (400 MHz, Chloroform-d) of compound 1ac

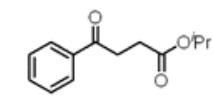

1ac

Chemical Formula: $\mathrm{C}_{13} \mathrm{H}_{16} \mathrm{O}$

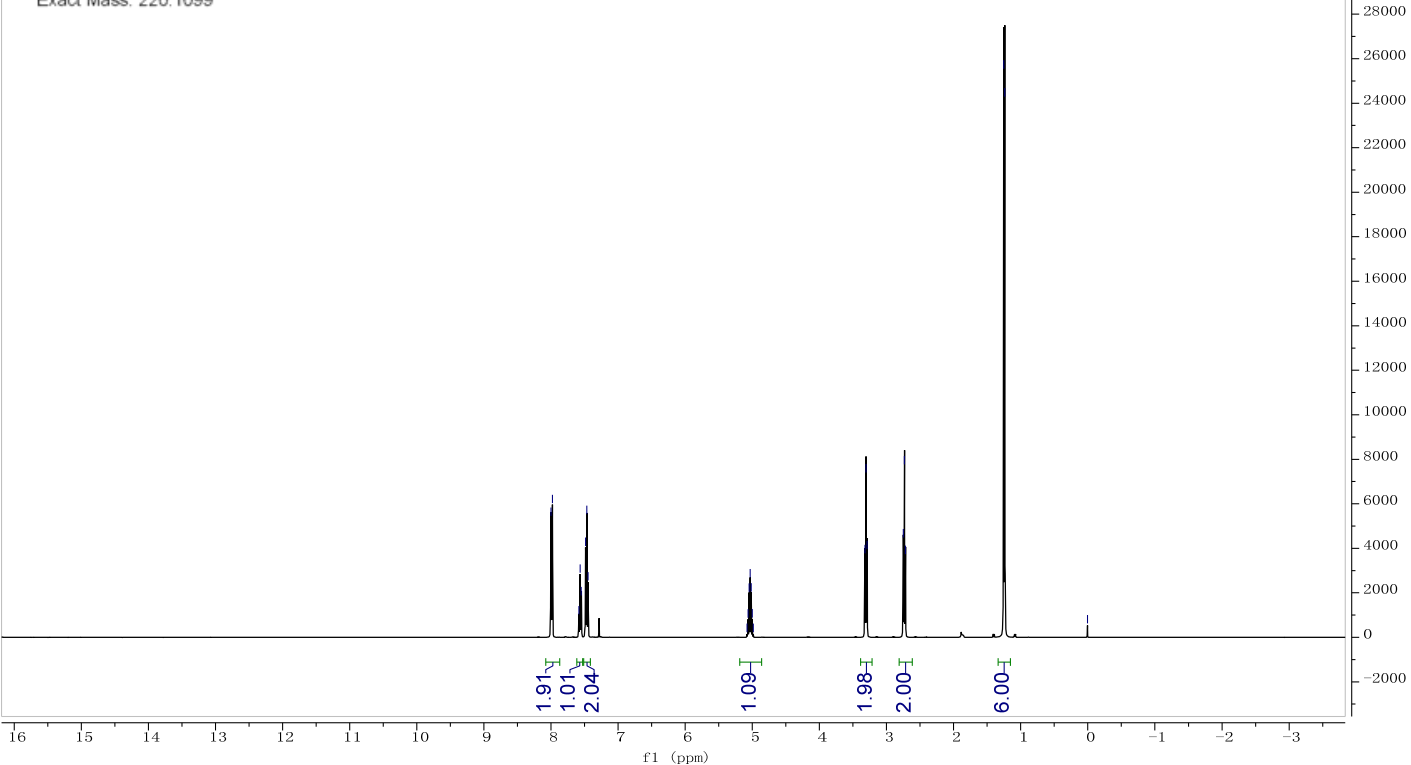

${ }^{13} \mathrm{C}\left\{{ }^{1} \mathrm{H}\right\}$ NMR (101MHz, Chloroform-d) of compound 1ac

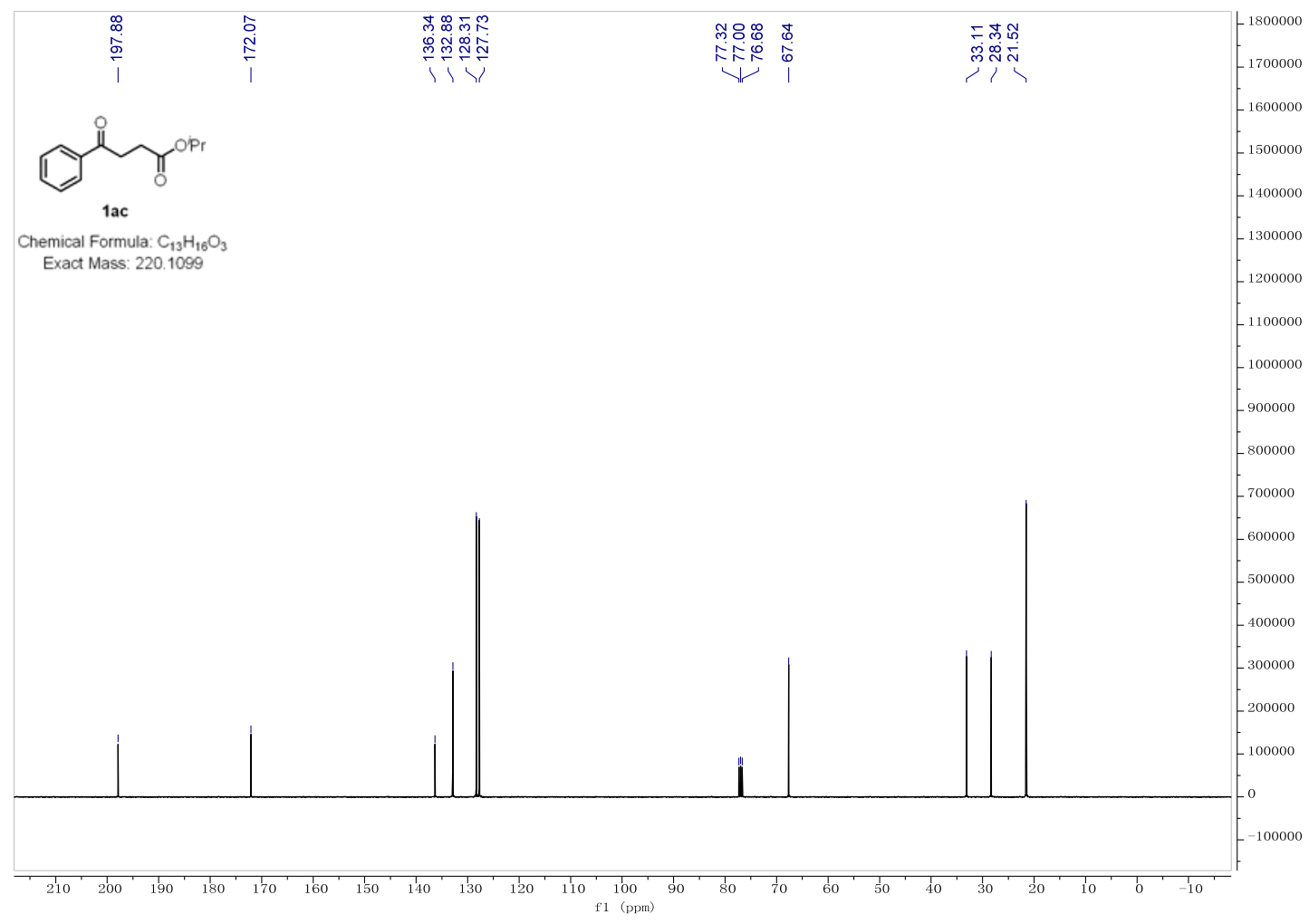


${ }^{1}$ H NMR (400 MHz, Chloroform-d) of compound 1ad

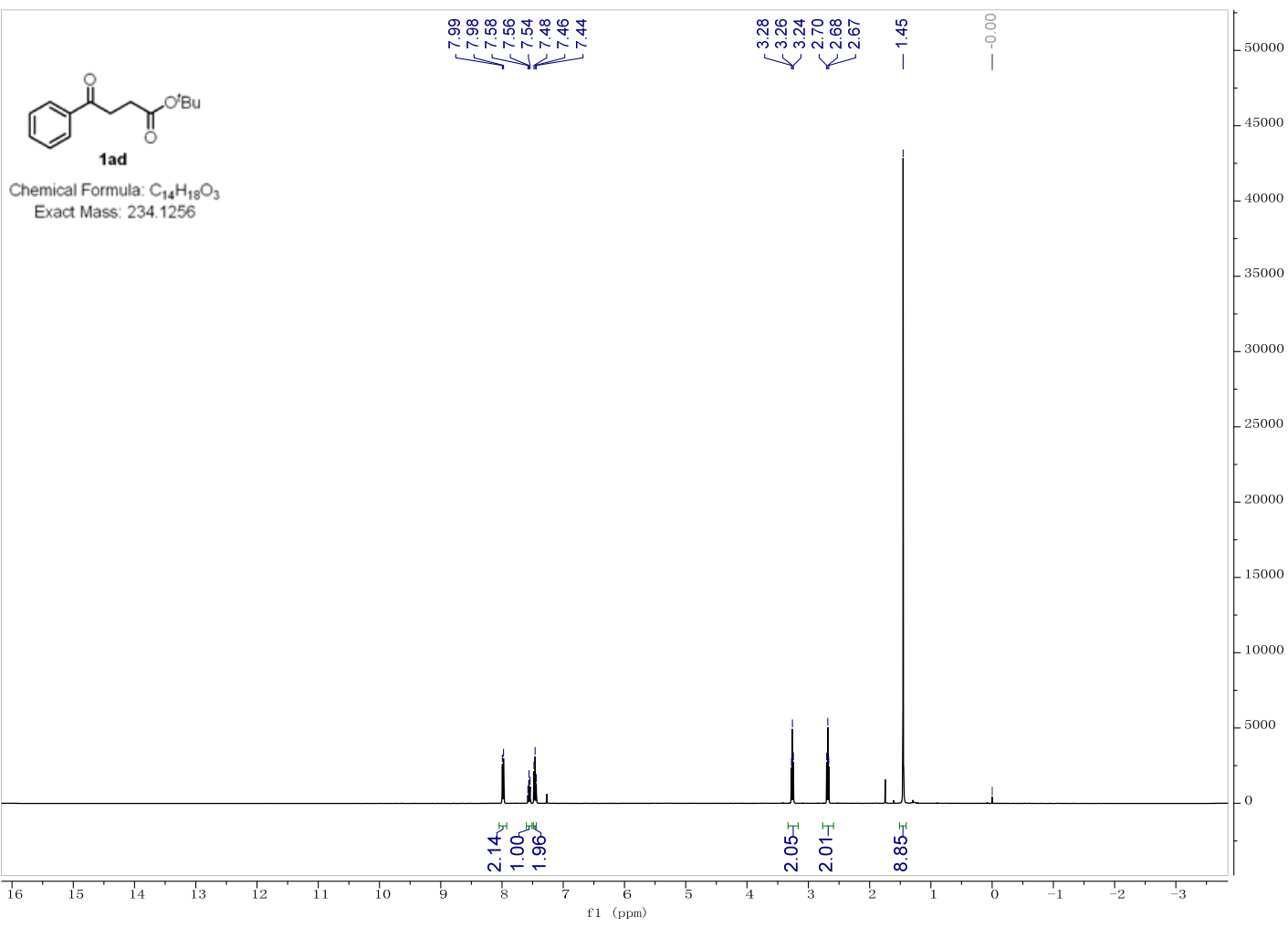

${ }^{13} \mathrm{C}\left\{{ }^{1} \mathrm{H}\right\}$ NMR (101 MHz, Chloroform-d) of compound 1ad

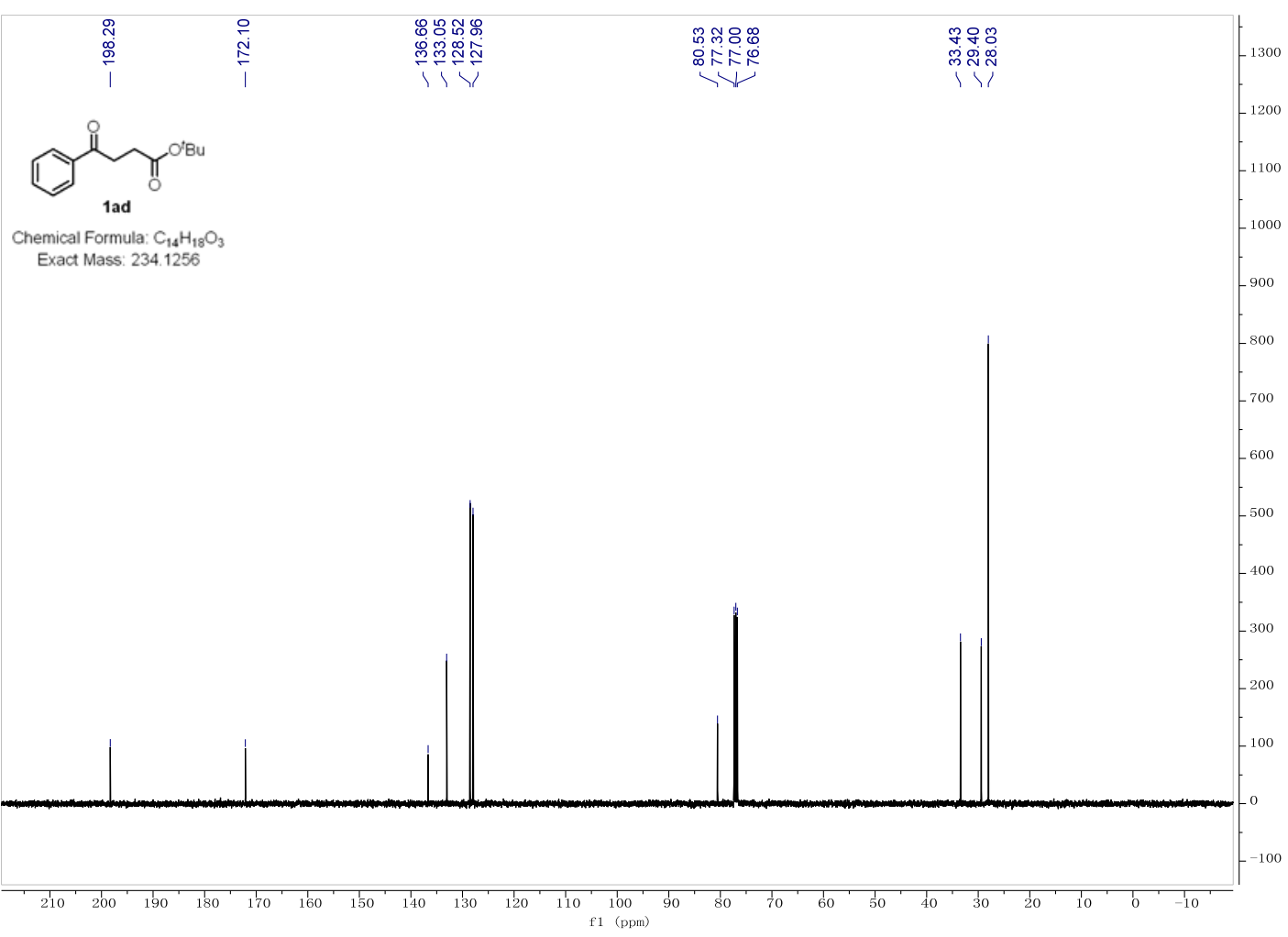


${ }^{1} \mathrm{H}$ NMR (600 MHz, Chloroform-d) of compound 1b

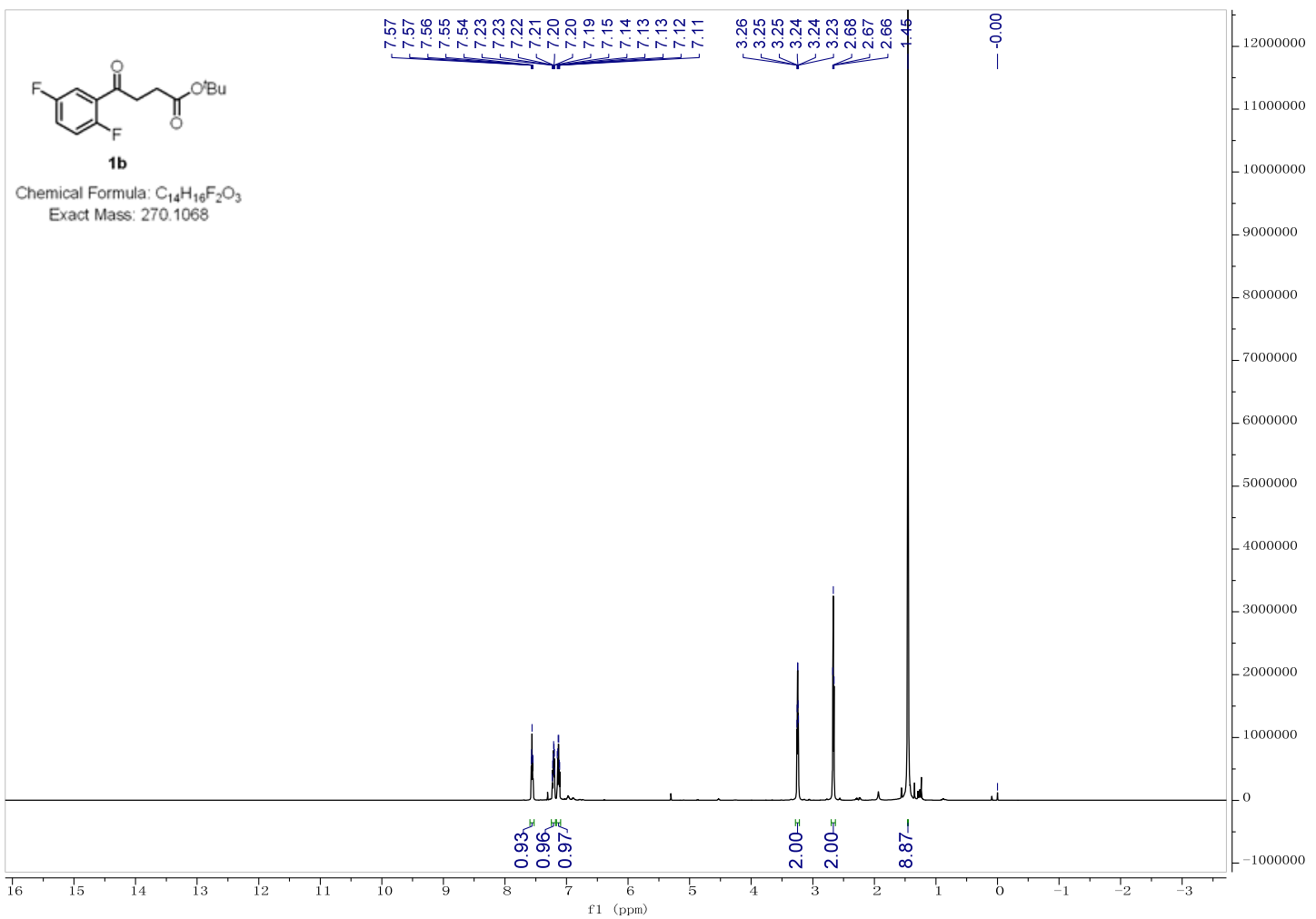

${ }^{13} \mathrm{C}\left\{{ }^{1} \mathrm{H}\right\}$ NMR (151 MHz, Chloroform-d) of compound $\mathbf{1 b}$

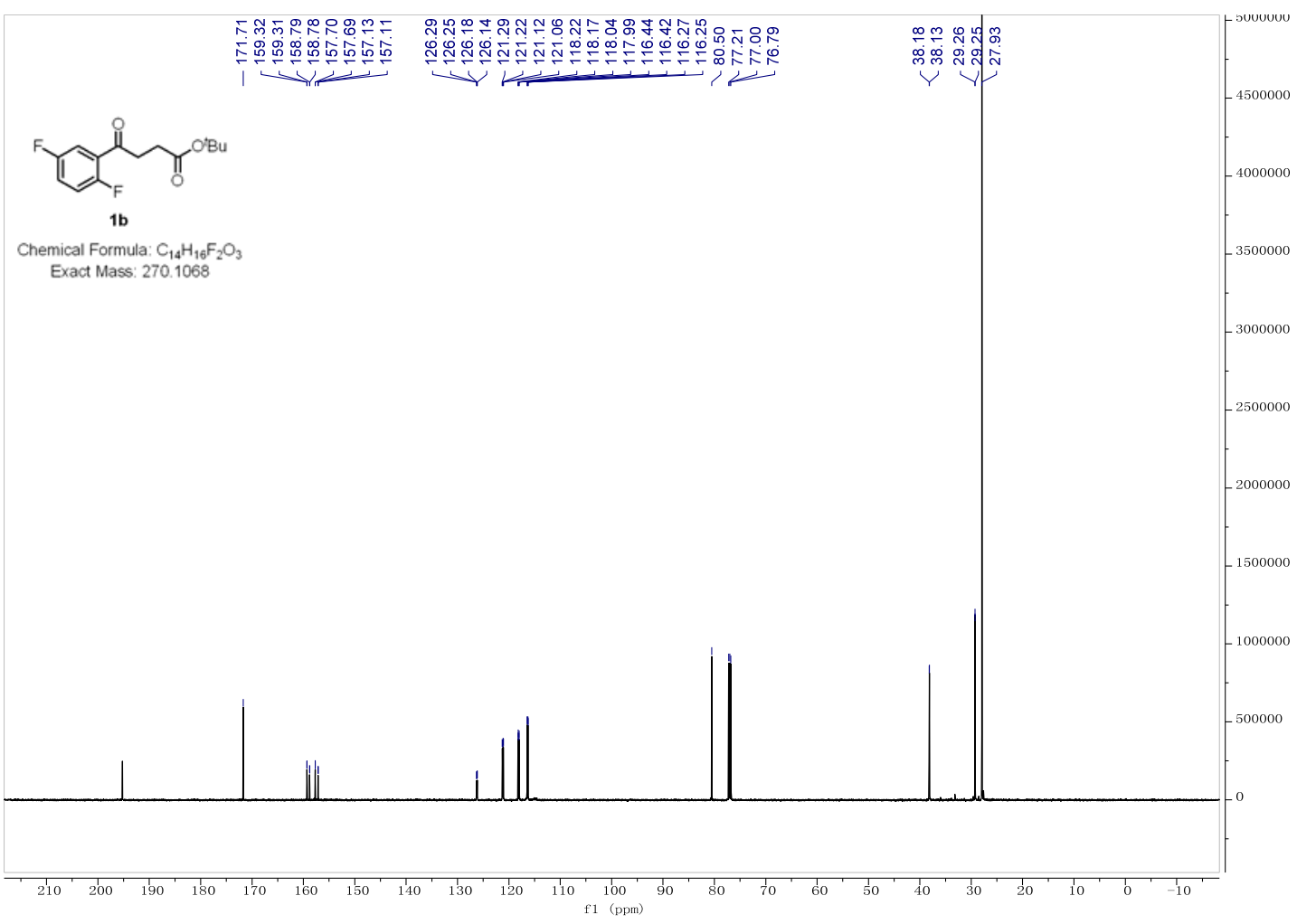


${ }^{1}$ H NMR (400 MHz, Chloroform-d) of compound 1c

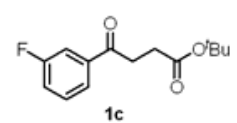

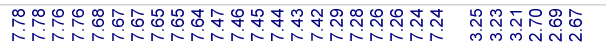

Chemical Formula: $\mathrm{C}_{14} \mathrm{H}_{17} \mathrm{FO}$

Exact Mass: 252.1162

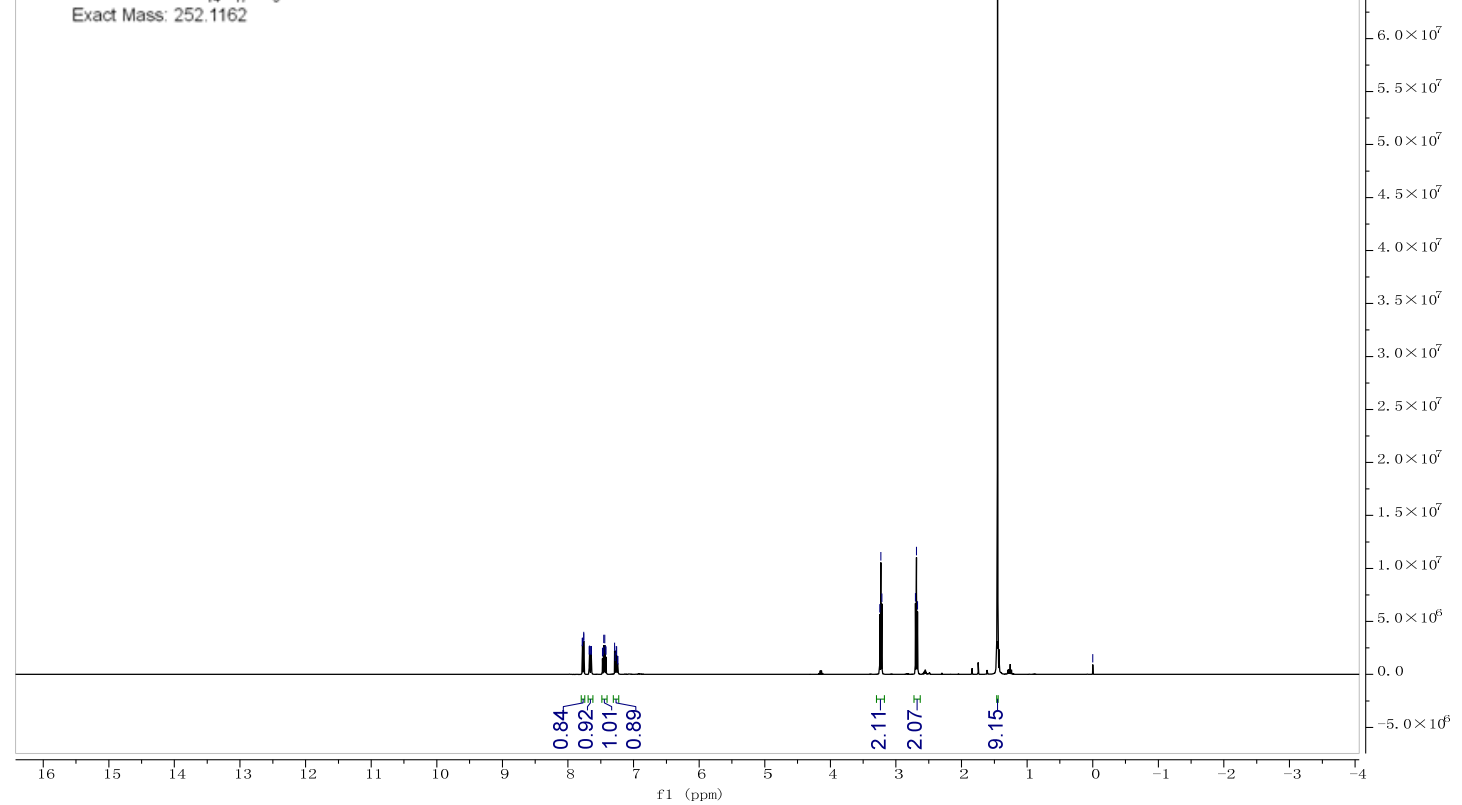

${ }^{13} \mathrm{C}\left\{{ }^{1} \mathrm{H}\right\}$ NMR (101 MHz, Chloroform-d) of compound 1c

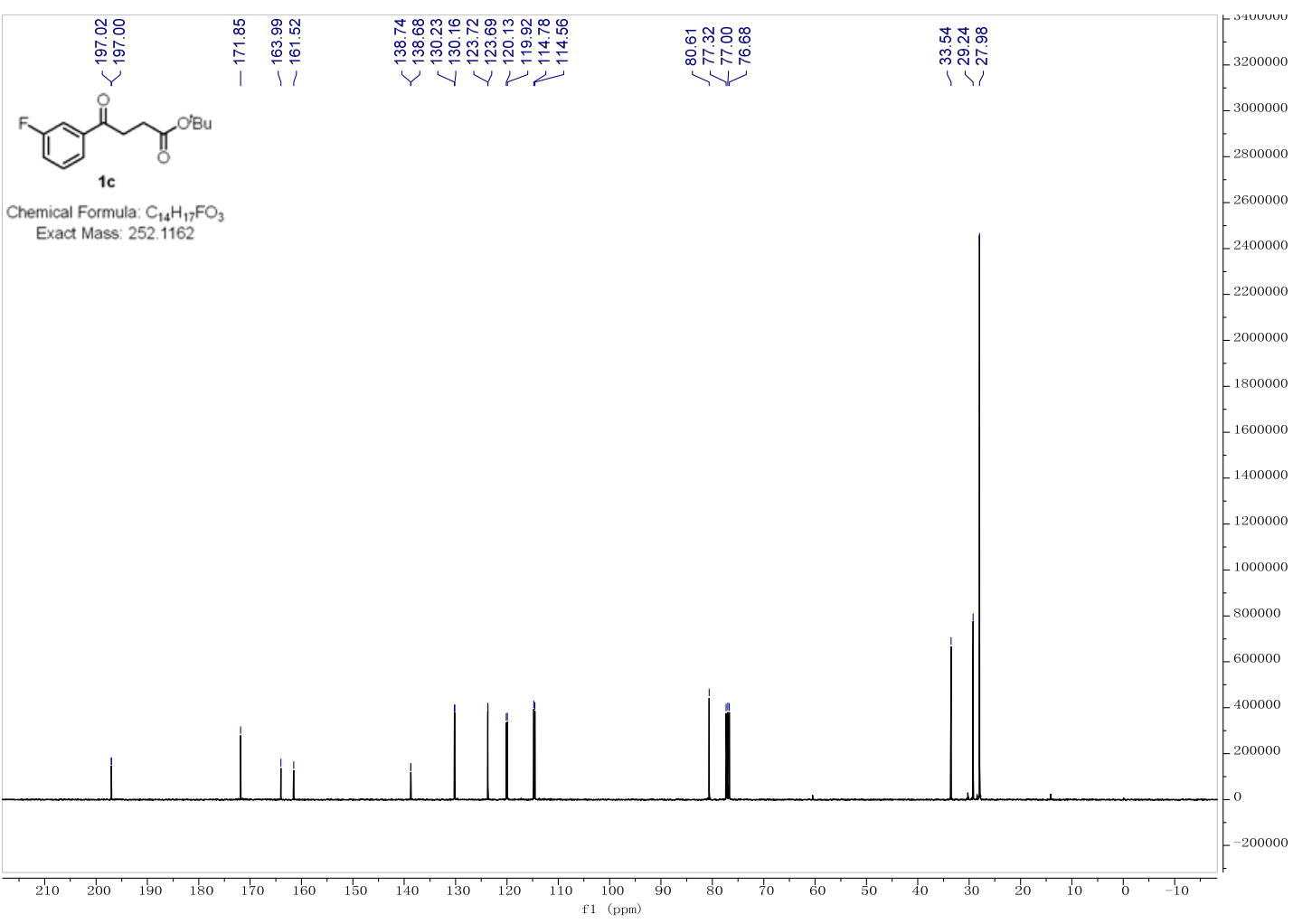


${ }^{1}$ H NMR (400 MHz, Chloroform-d) of compound 1d

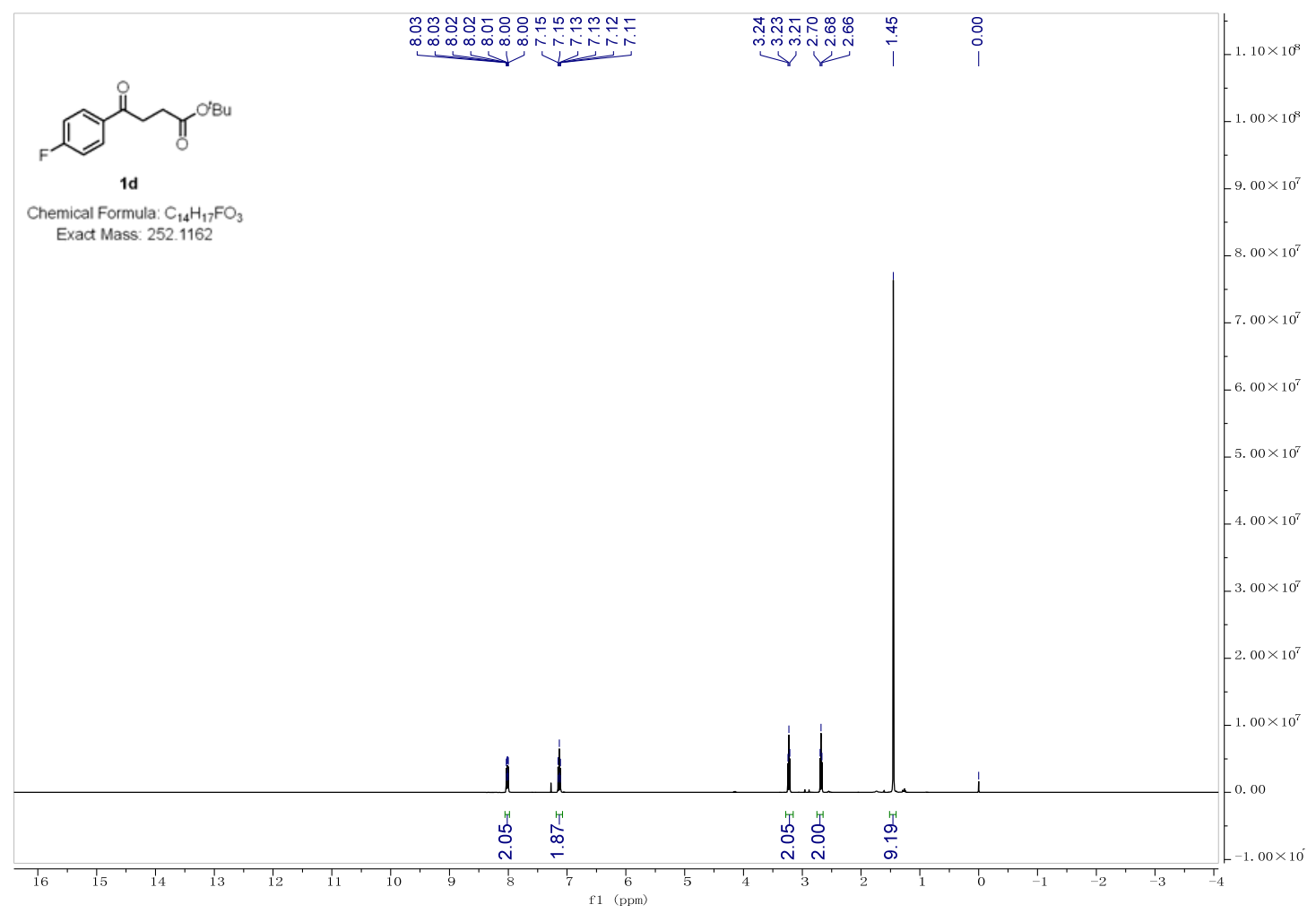

${ }^{13} \mathrm{C}\left\{{ }^{1} \mathrm{H}\right\}$ NMR (101 MHz, Chloroform-d) of compound 1d

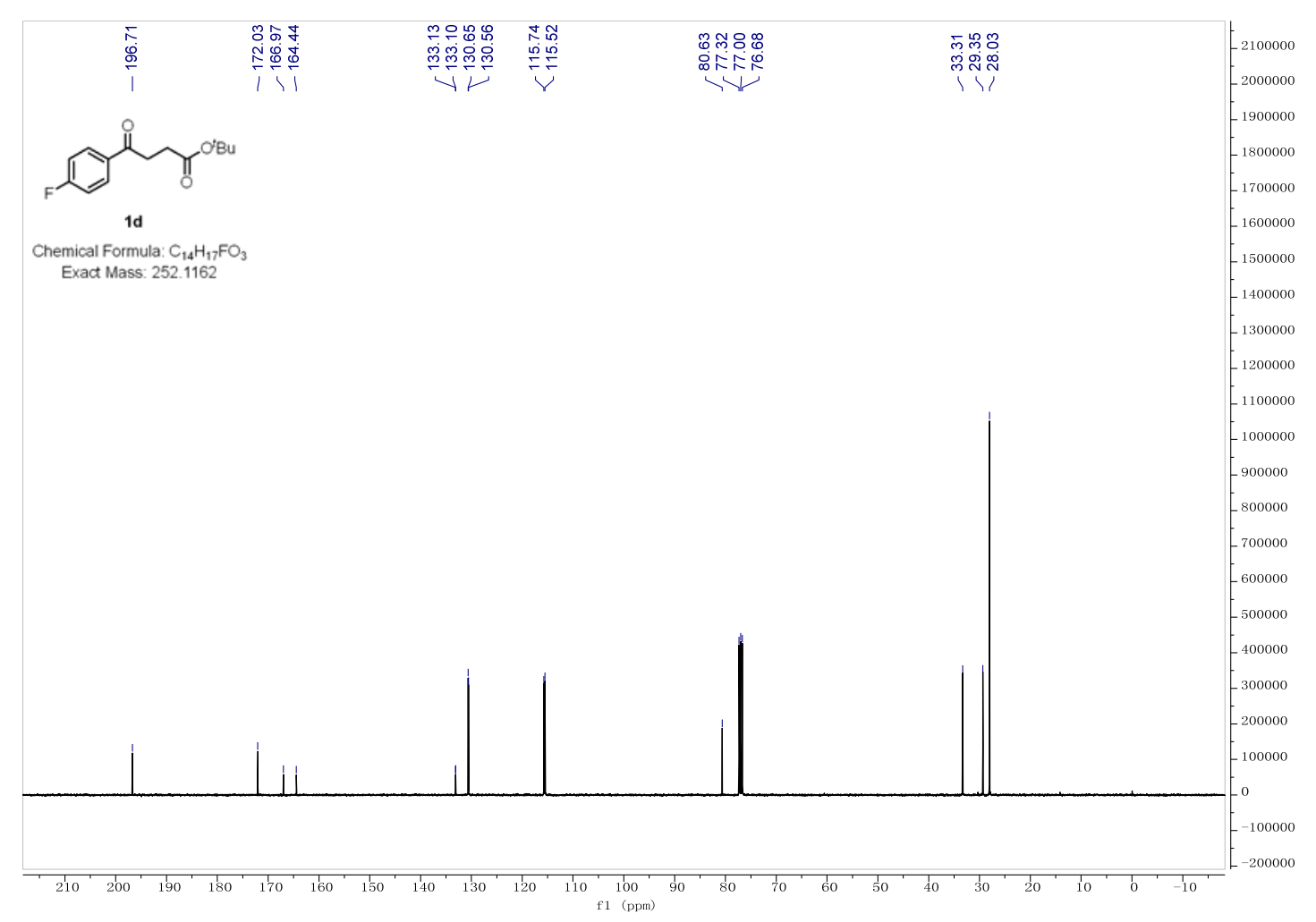


${ }^{1}$ H NMR (400 MHz, Chloroform-d) of compound $\mathbf{1 e}$

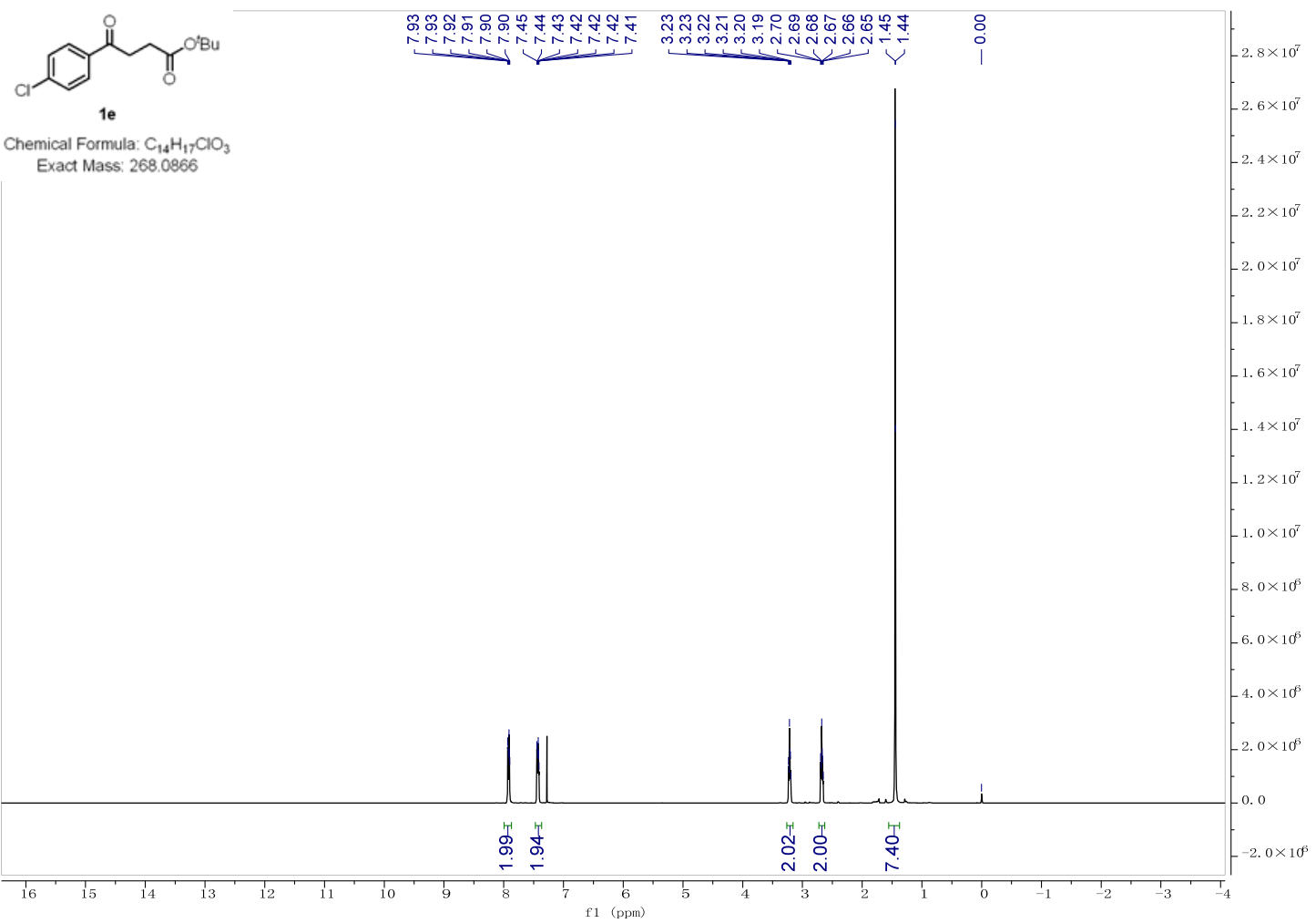

${ }^{13} \mathrm{C}\left\{{ }^{1} \mathrm{H}\right\}$ NMR (101 MHz, Chloroform-d) of compound 1e
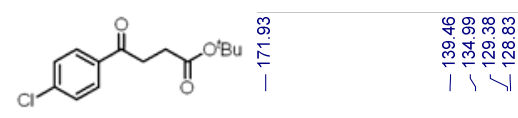

贷

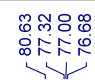

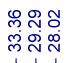

Chemical Formula: $\mathrm{C}_{14} \mathrm{H}_{17} \mathrm{ClO}_{3}$

Exact Mass: 268.0866

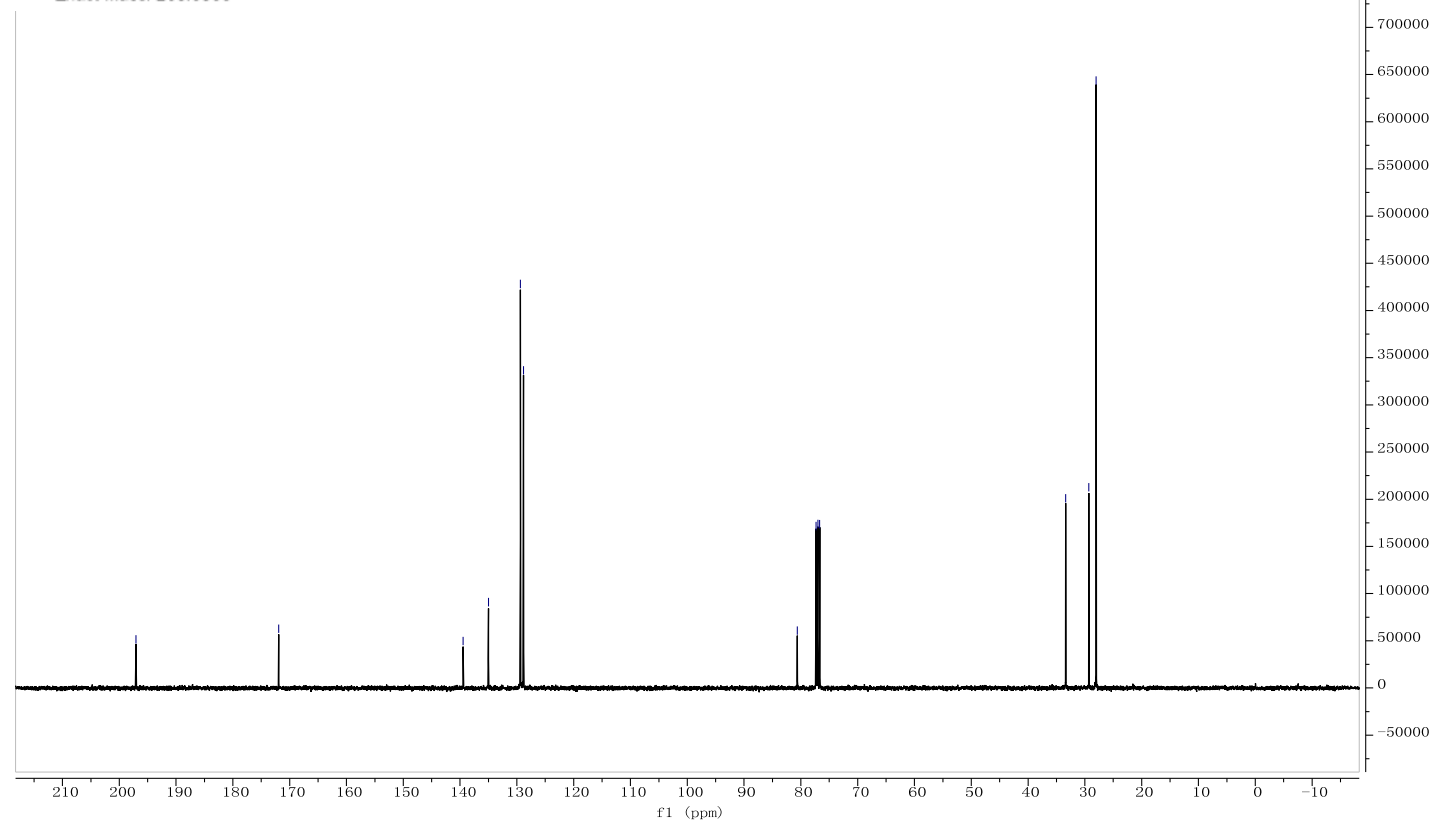


${ }^{1}$ H NMR (400 MHz, Chloroform-d) of compound $\mathbf{1 f}$

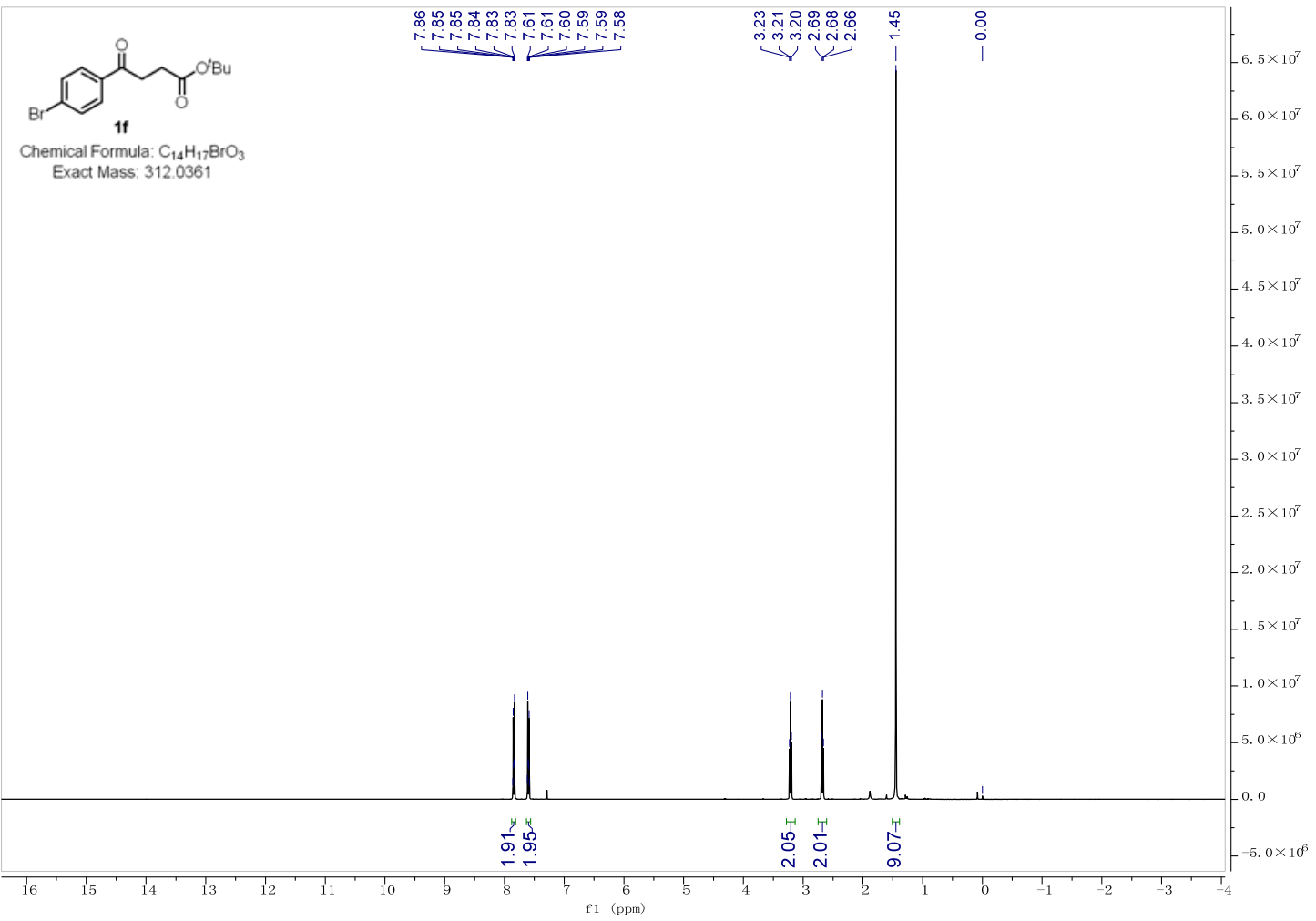

${ }^{13} \mathrm{C}\left\{{ }^{1} \mathrm{H}\right\}$ NMR (101 MHz, Chloroform-d) of compound $\mathbf{1 f}$

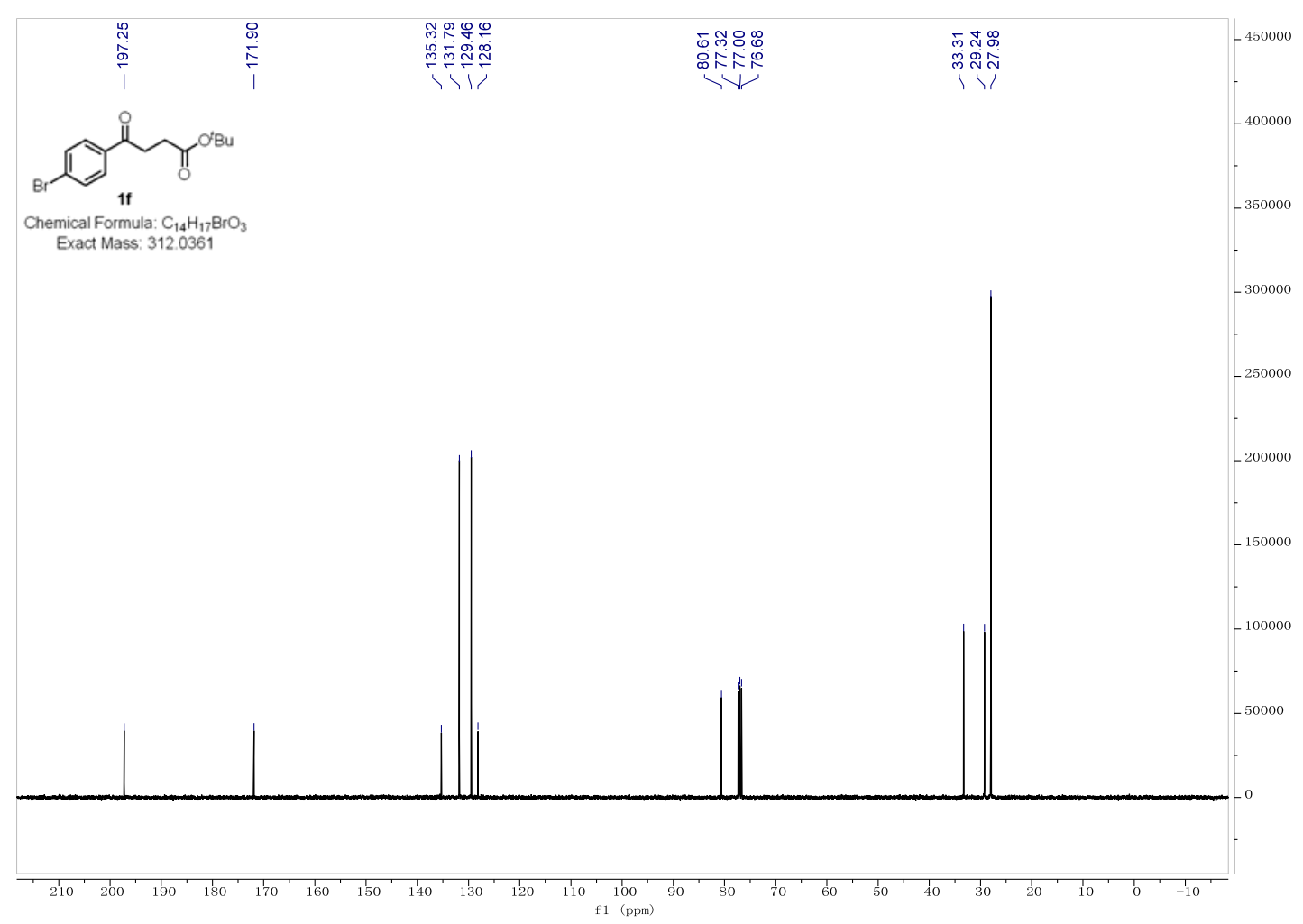


${ }^{1} \mathrm{H}$ NMR (400 MHz, Chloroform-d) of compound 1 g

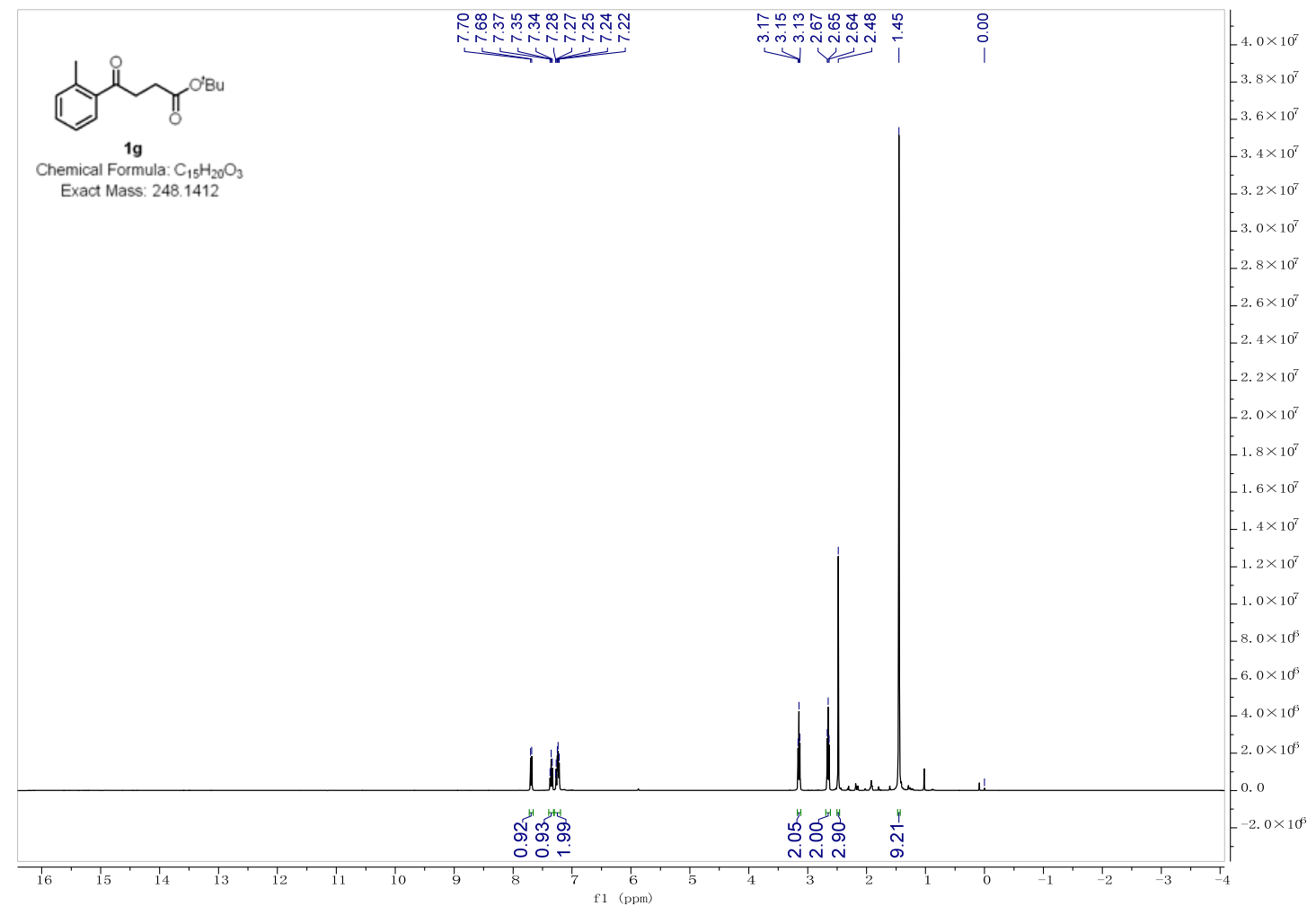

${ }^{13} \mathrm{C}\left\{{ }^{1} \mathrm{H}\right\}$ NMR (101 MHz, Chloroform-d) of compound $\mathbf{1 g}$

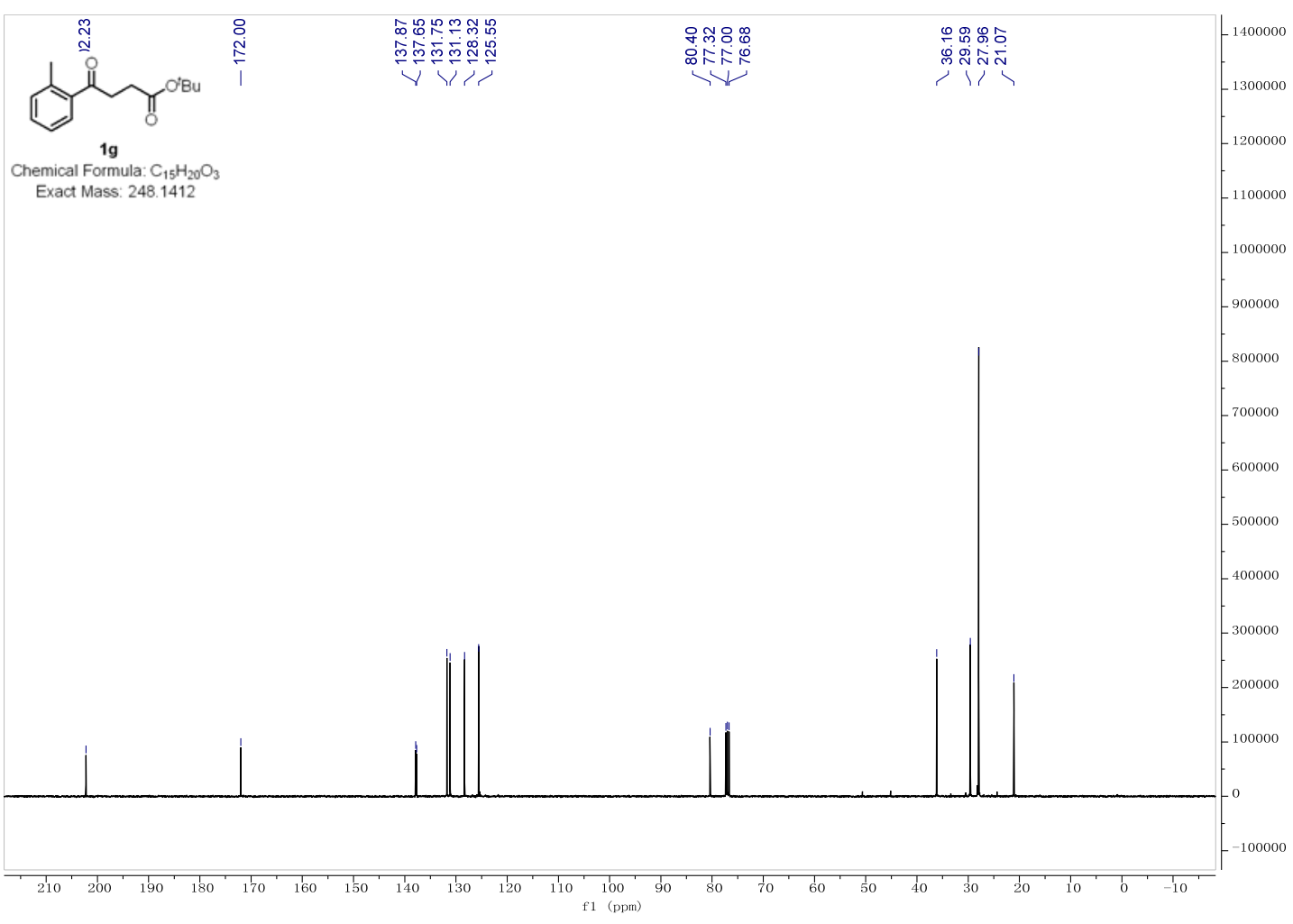


${ }^{1}$ H NMR (400 MHz, Chloroform-d) of compound $\mathbf{1 h}$

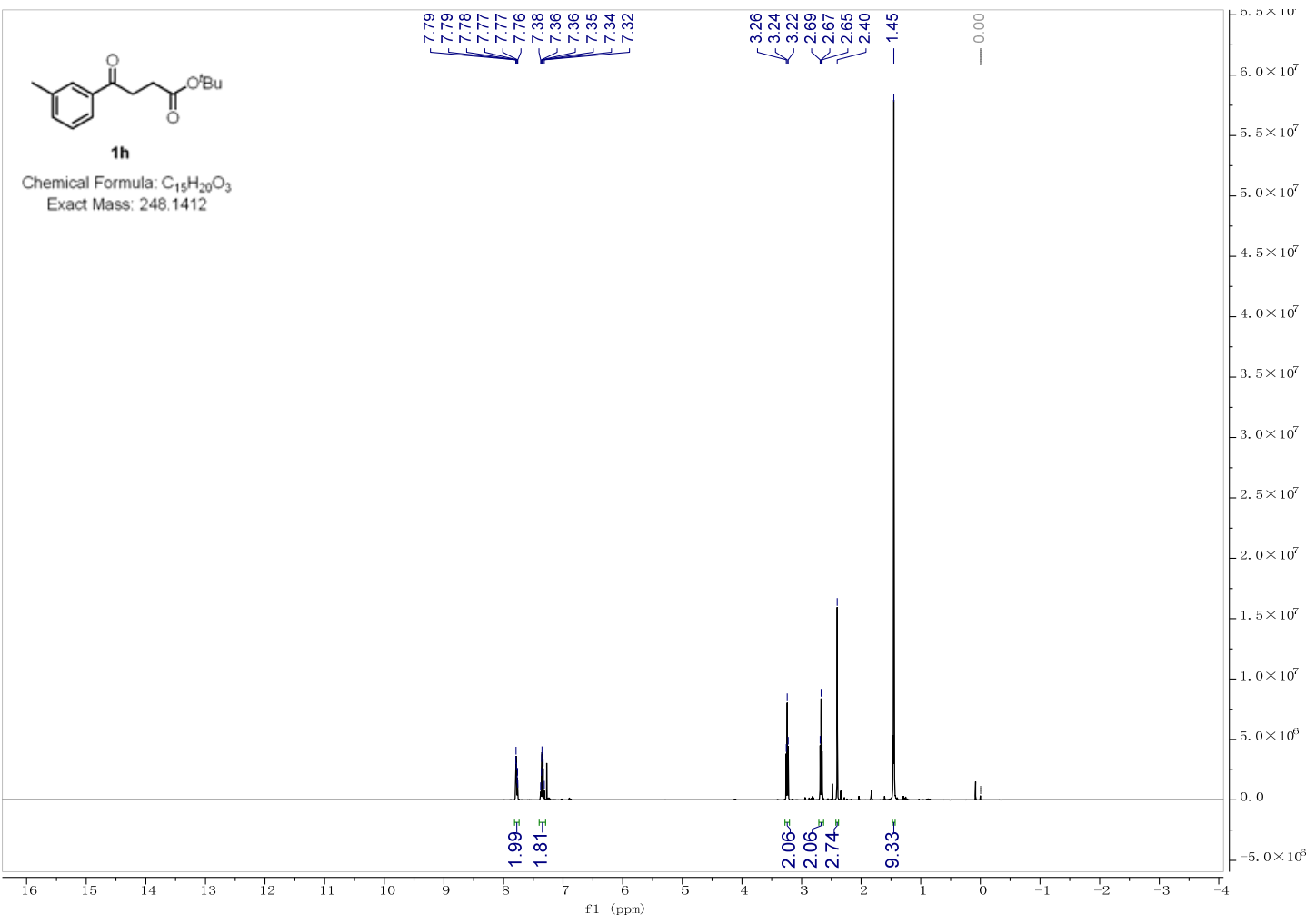

${ }^{13} \mathrm{C}\left\{{ }^{1} \mathrm{H}\right\}$ NMR (101 MHz, Chloroform-d) of compound $\mathbf{1 h}$

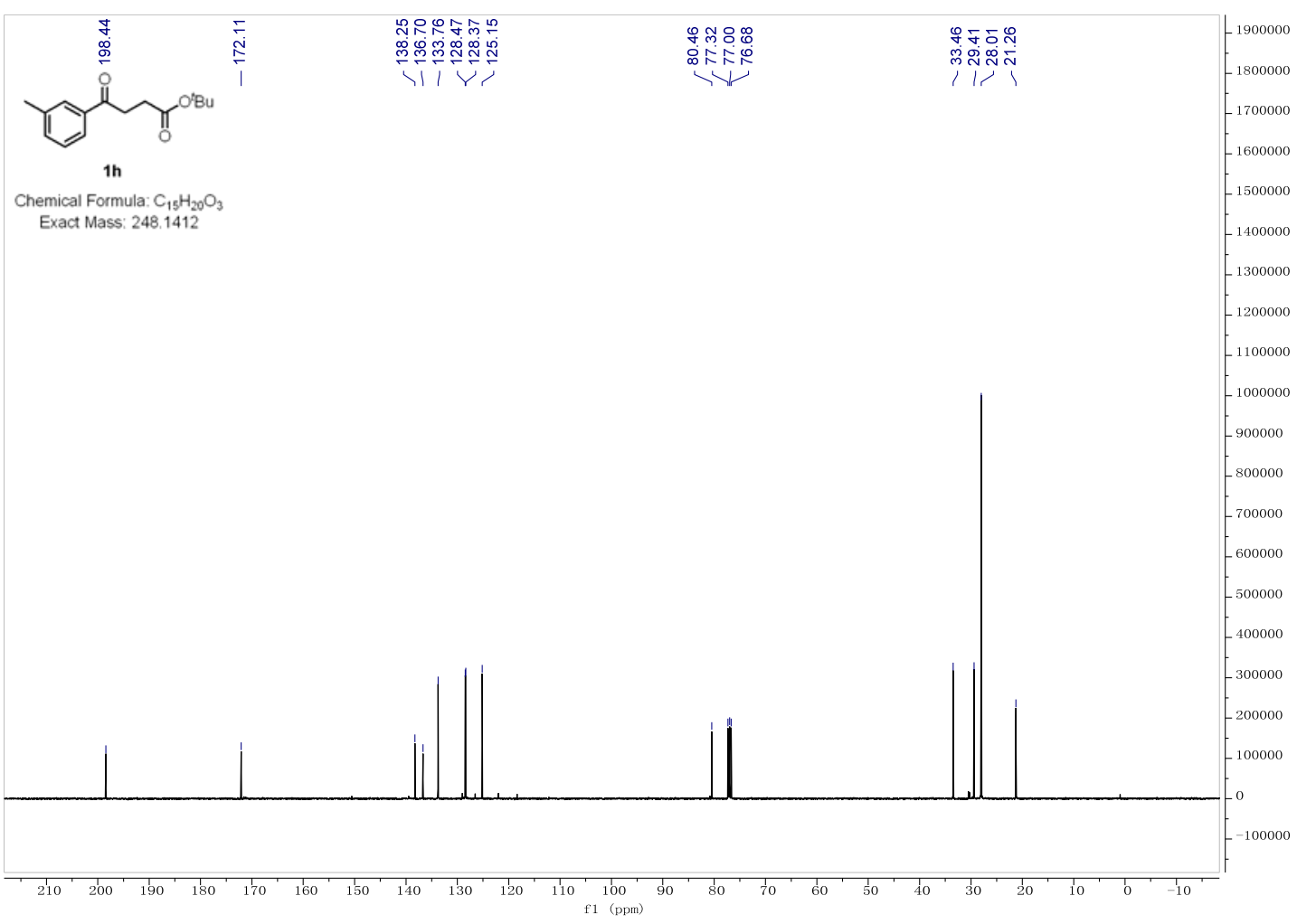


${ }^{1}$ H NMR (400 MHz, Chloroform-d) of compound 1i

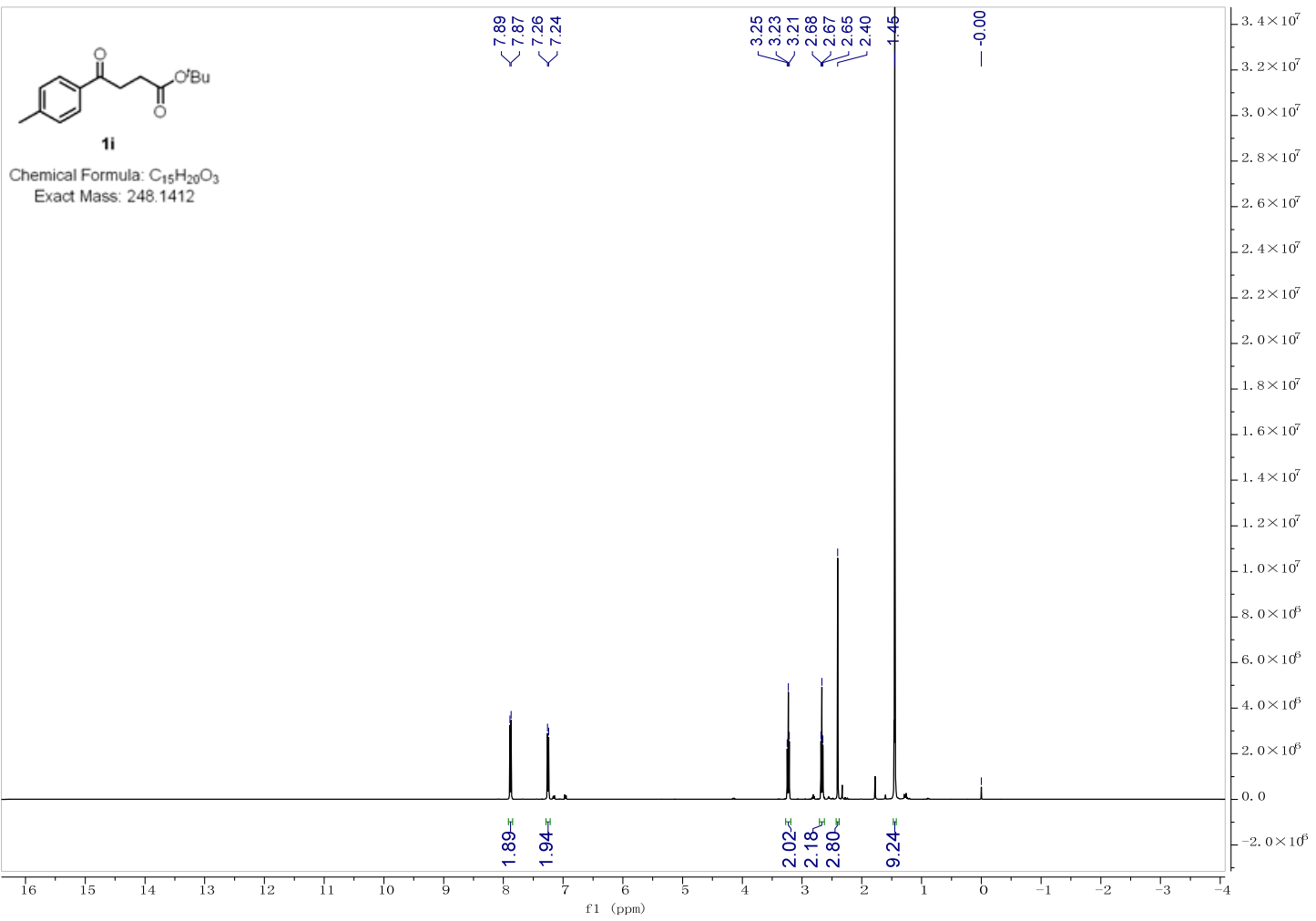

${ }^{1} \mathrm{H}\left\{{ }^{1} \mathrm{H}\right\}$ NMR (101 MHz, Chloroform-d) of compound $\mathbf{1 h}$

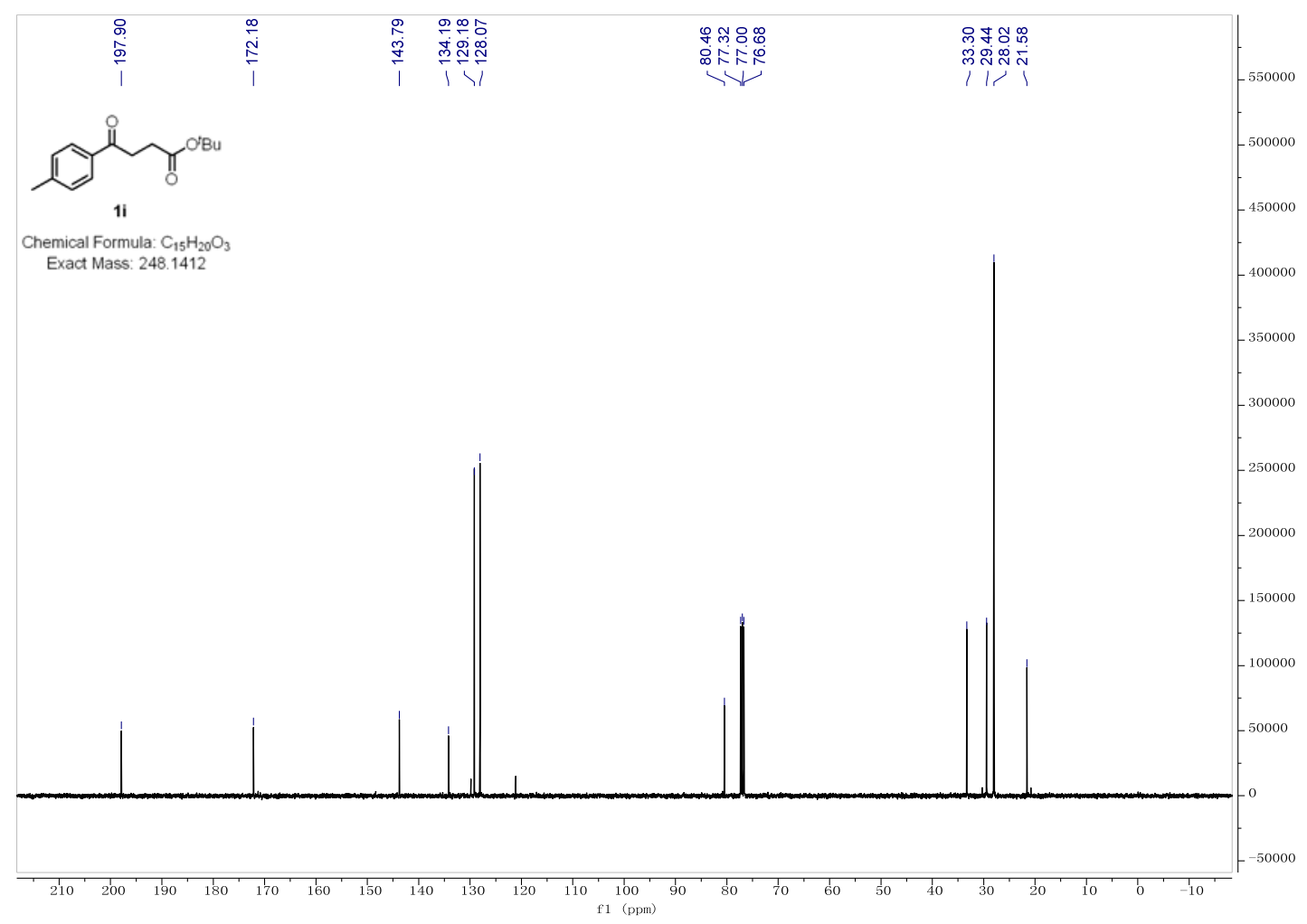


${ }^{1}$ H NMR (400 MHz, Chloroform-d) of compound $\mathbf{1 j}$

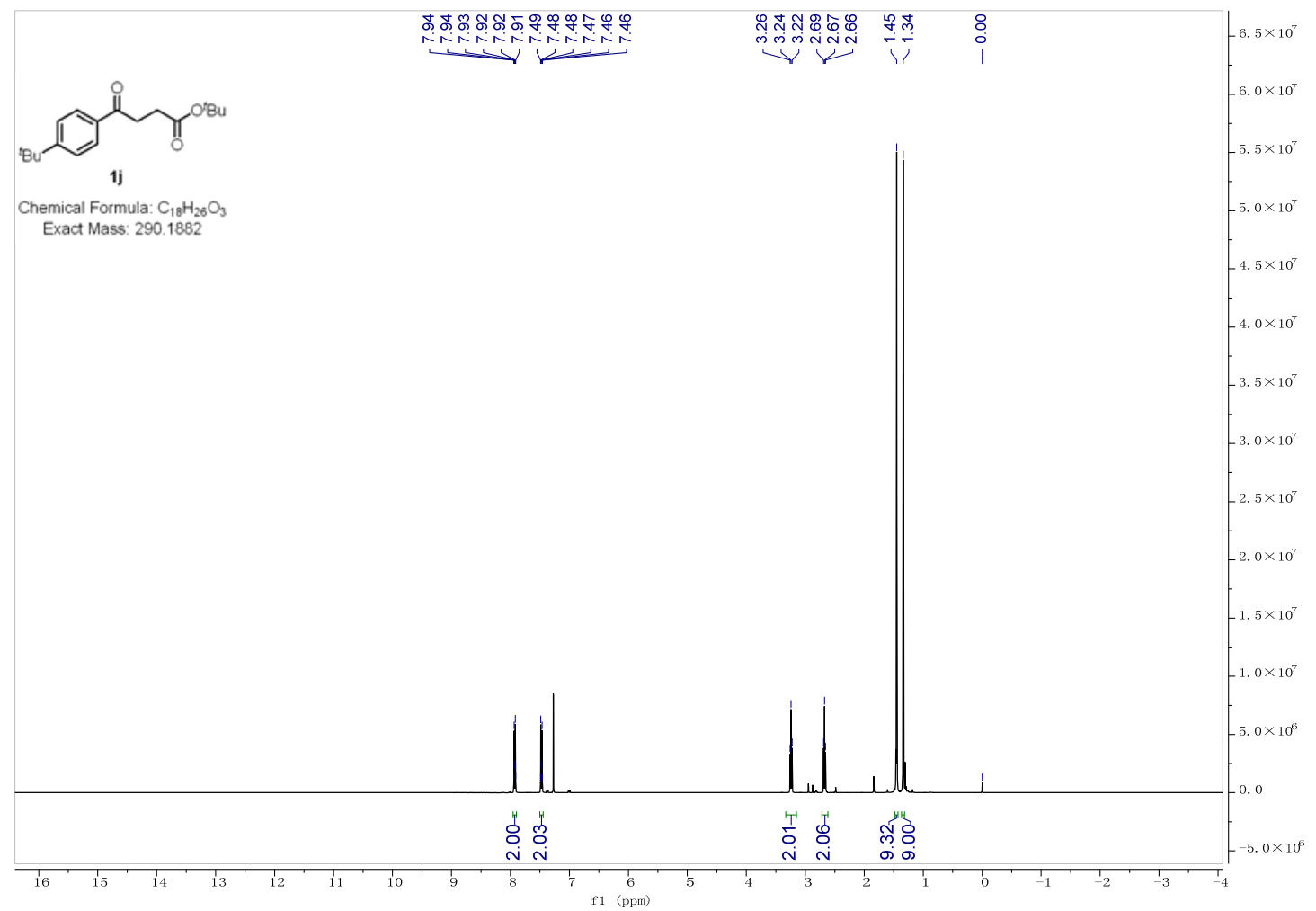

${ }^{13} \mathrm{C}\left\{{ }^{1} \mathrm{H}\right\}$ NMR (101 MHz, Chloroform-d) of compound $\mathbf{1 j}$

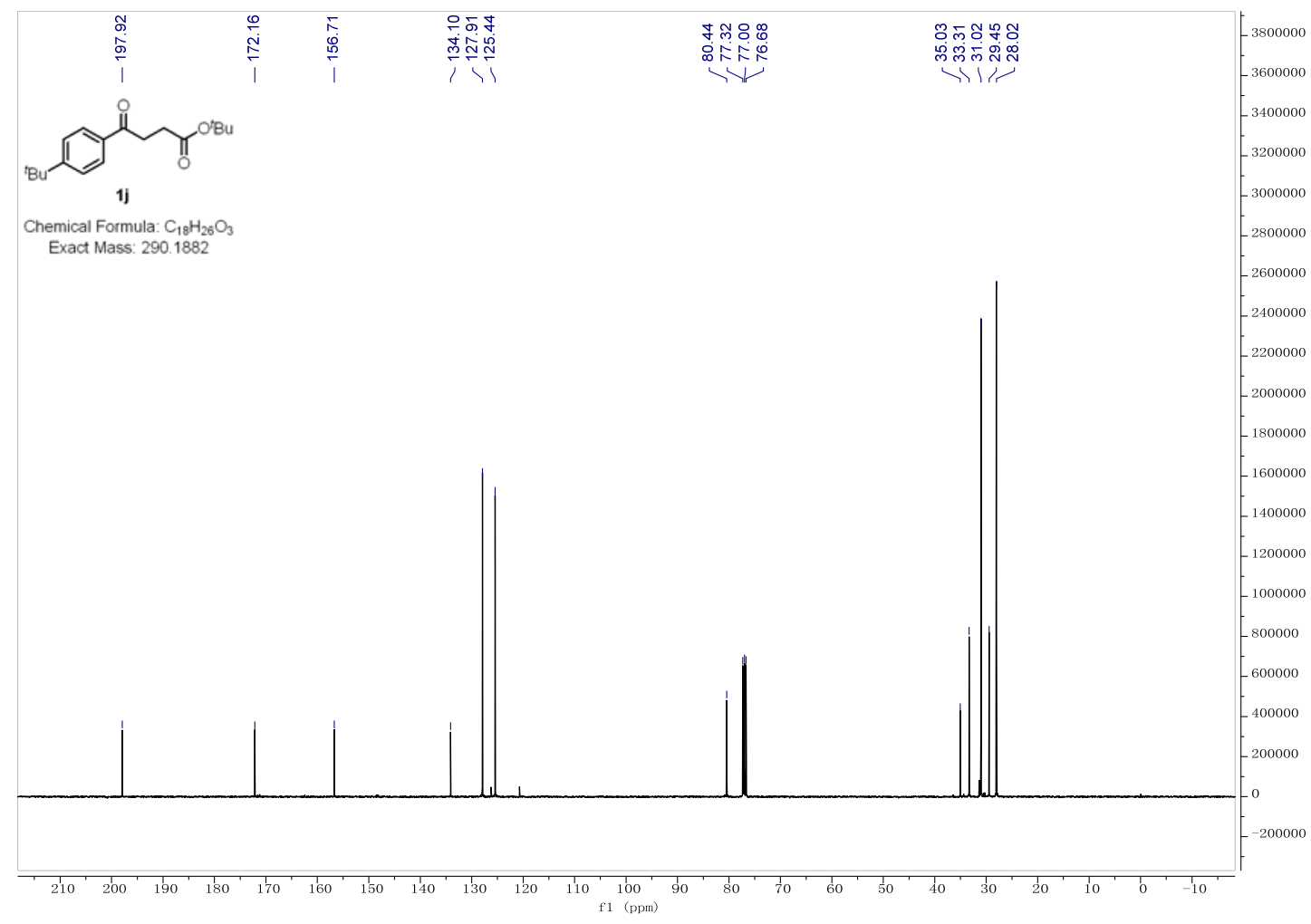


${ }^{1}$ H NMR (400 MHz, Chloroform-d) of compound 1k

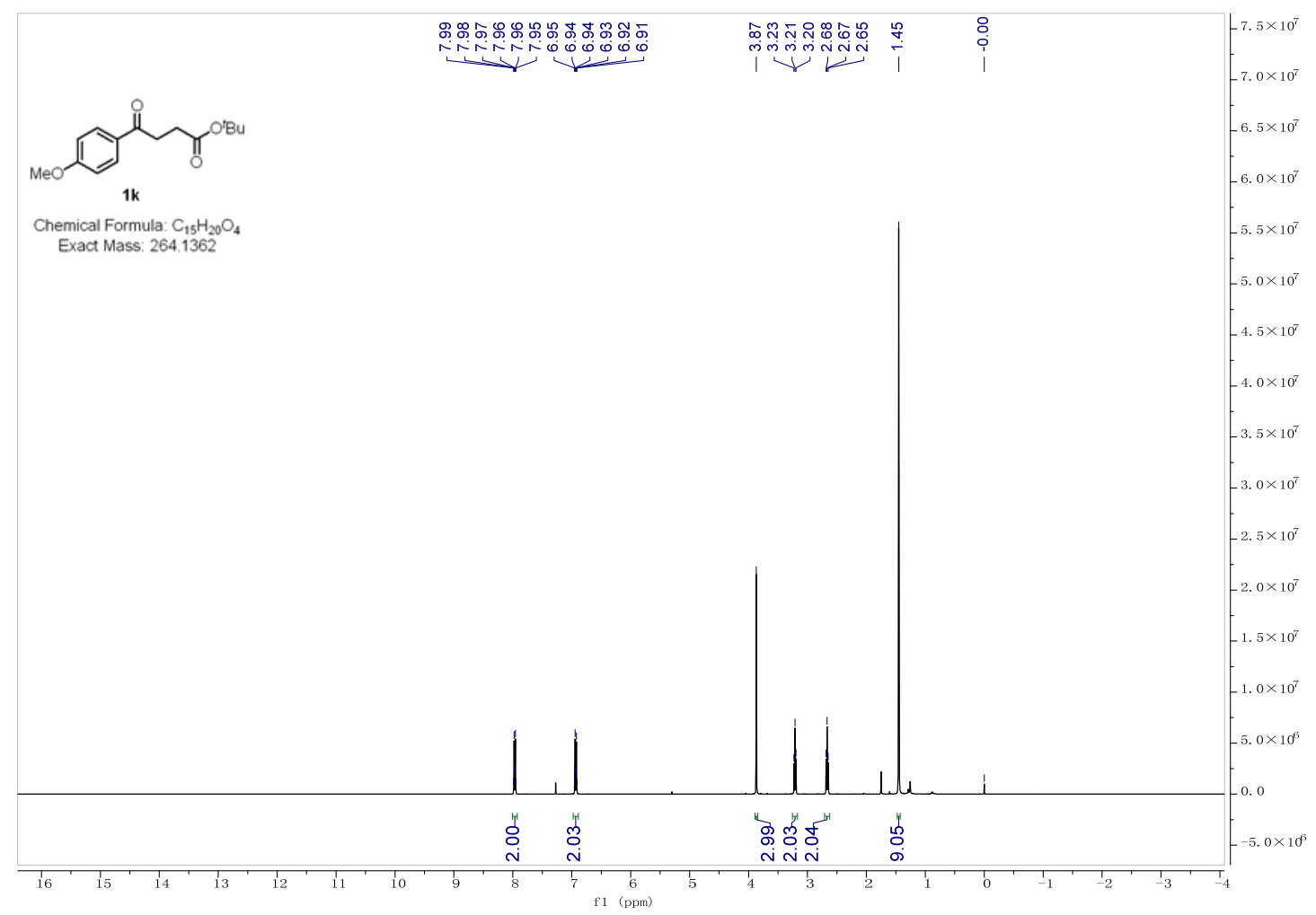

${ }^{13} \mathrm{C}\left\{{ }^{1} \mathrm{H}\right\}$ NMR (101 MHz, Chloroform-d) of compound $\mathbf{1 k}$

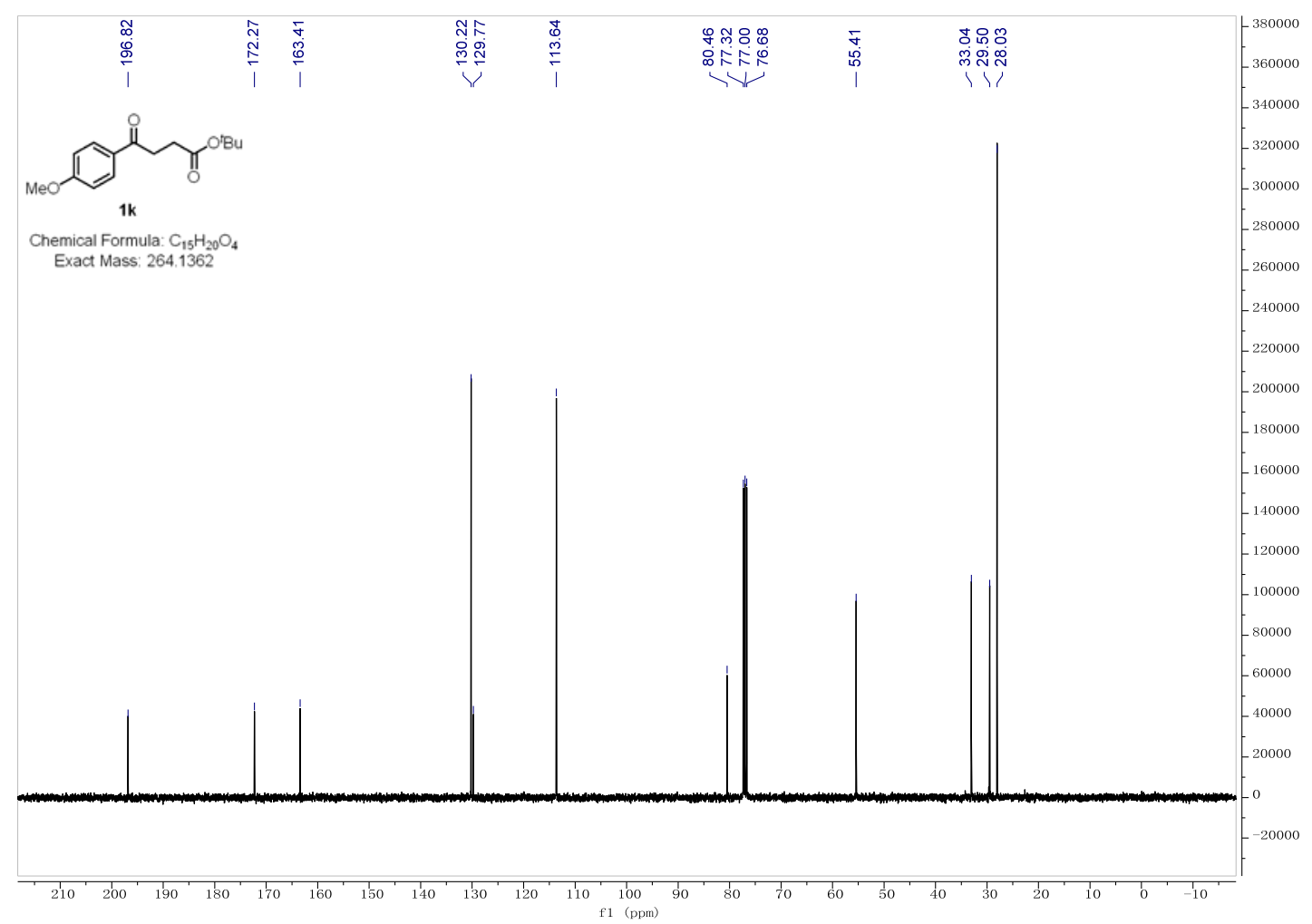


${ }^{1}$ H NMR (400 MHz, Chloroform-d) of compound 1 l

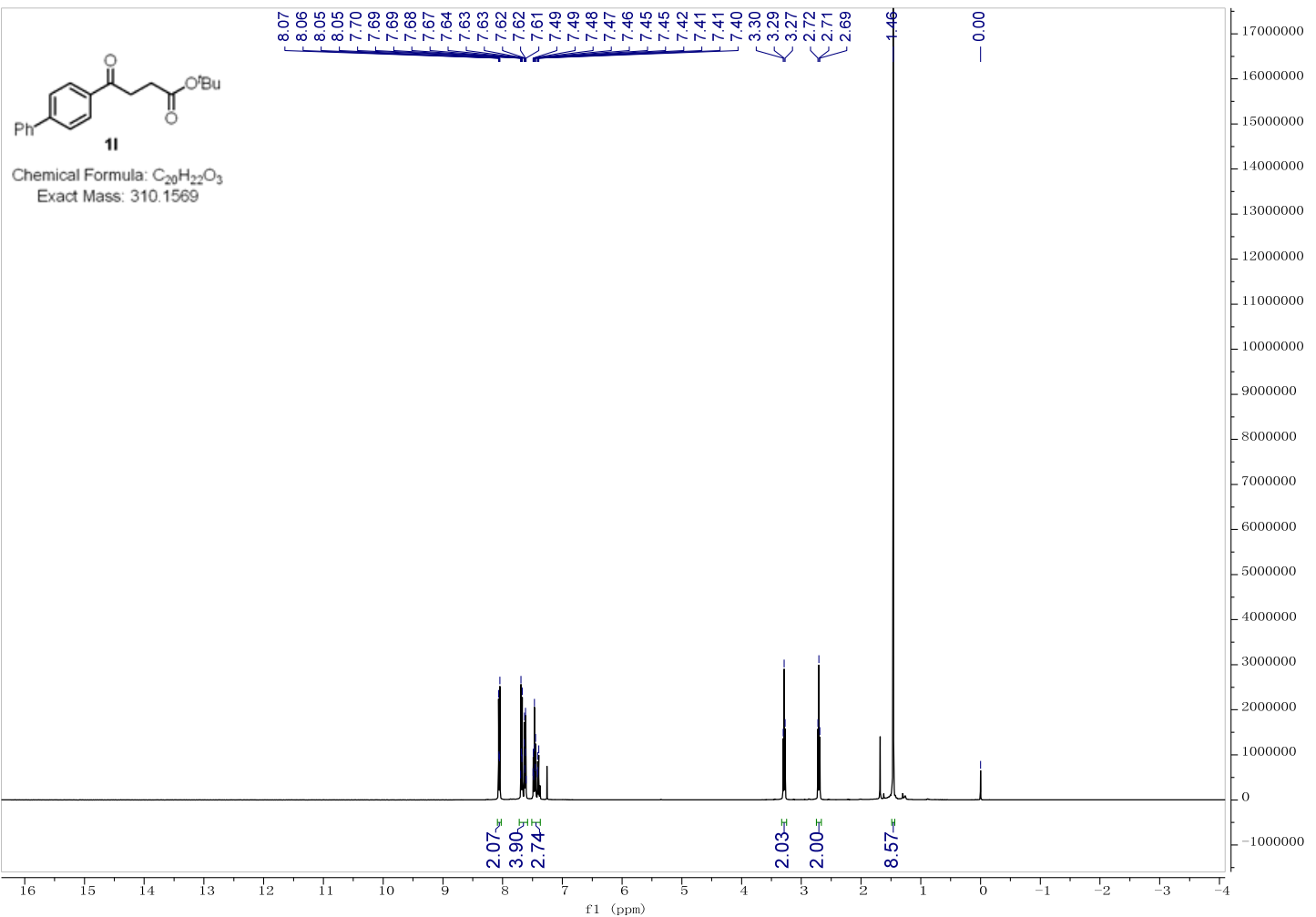

${ }^{13} \mathrm{C}\left\{{ }^{1} \mathrm{H}\right\}$ NMR (101 MHz, Chloroform-d) of compound $\mathbf{1 l}$

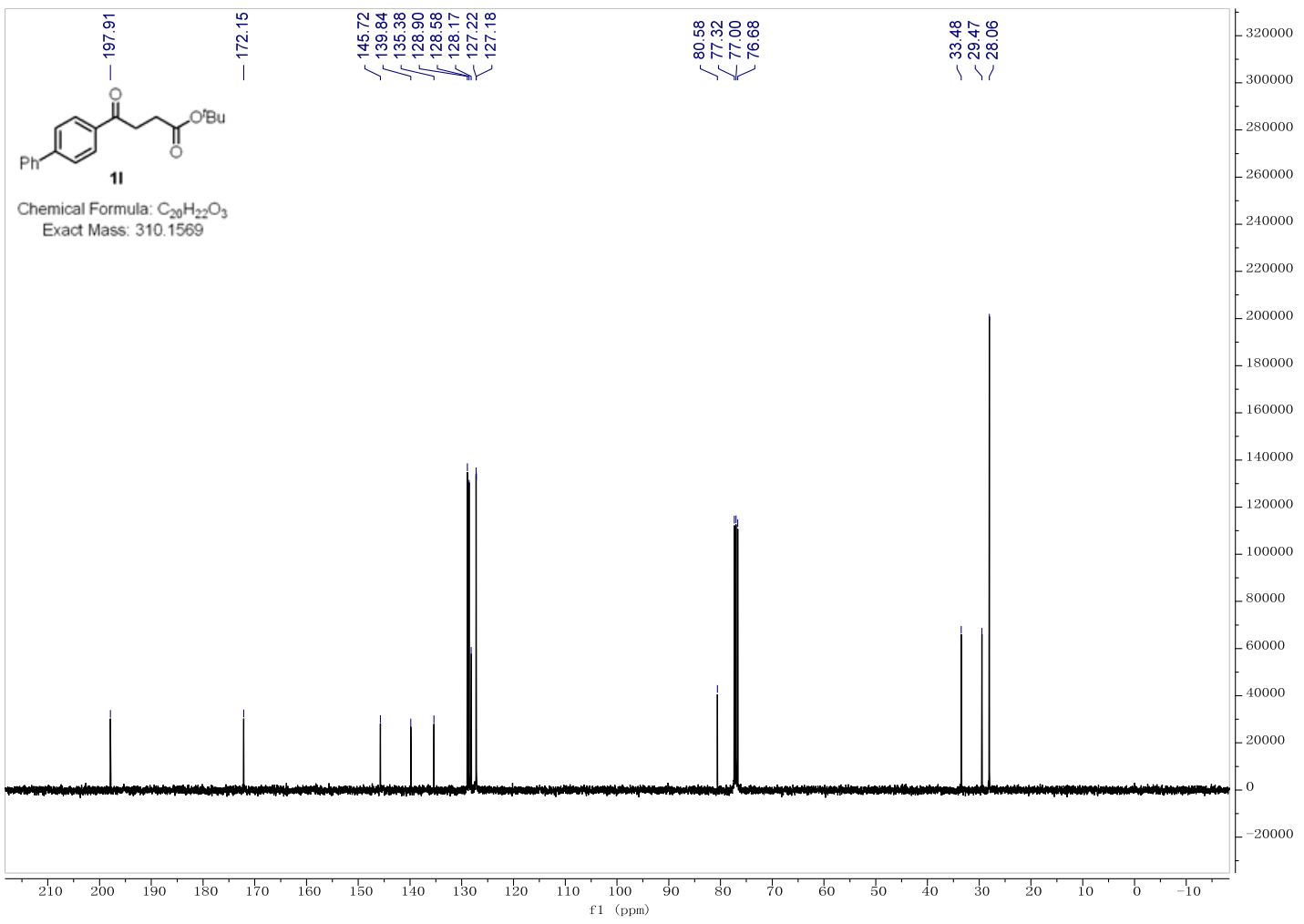


${ }^{1}$ H NMR (400 MHz, Chloroform-d) of compound 1m

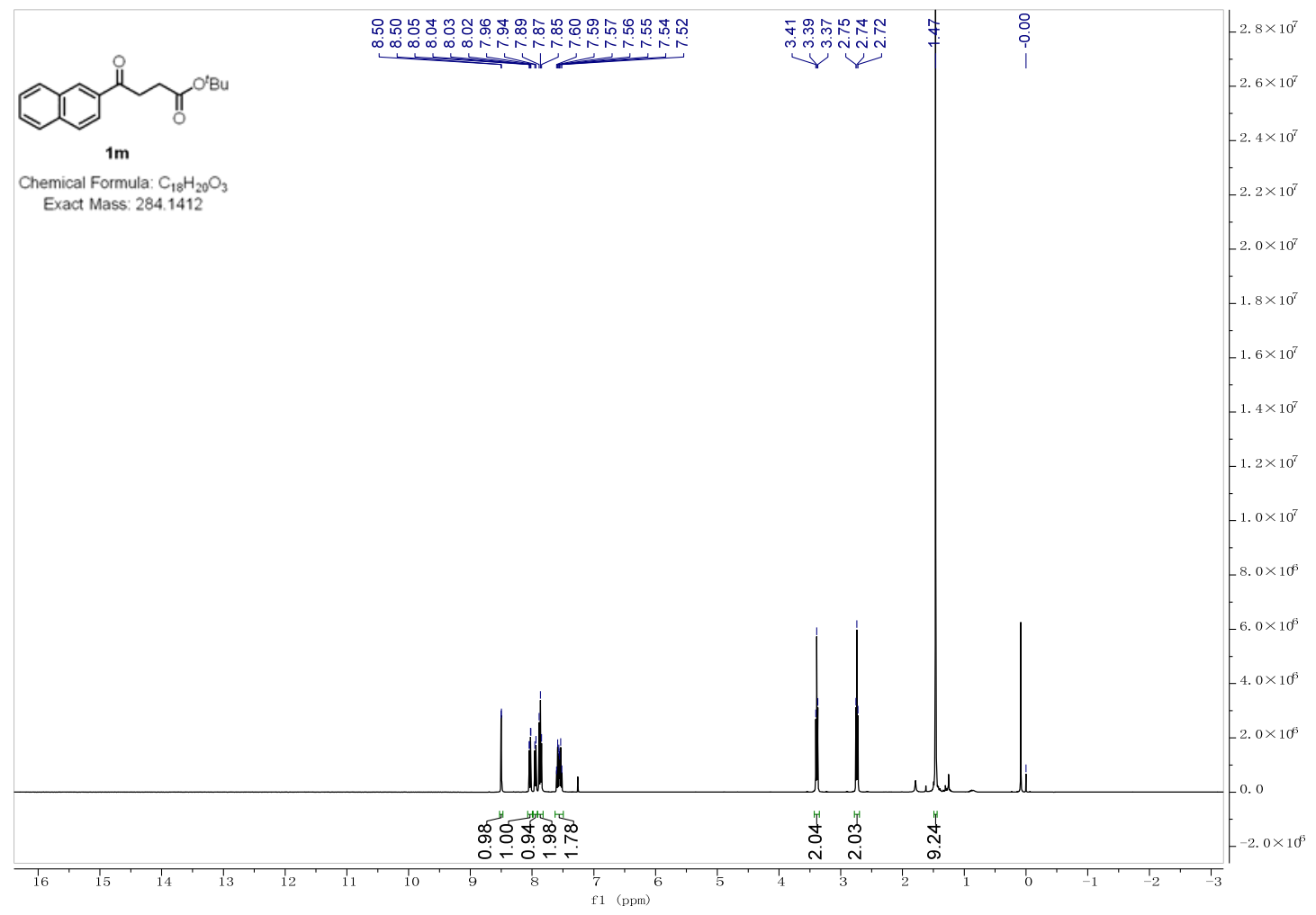

${ }^{13} \mathrm{C}\left\{{ }^{1} \mathrm{H}\right\}$ NMR (101 MHz, Chloroform-d) of compound $\mathbf{1 m}$

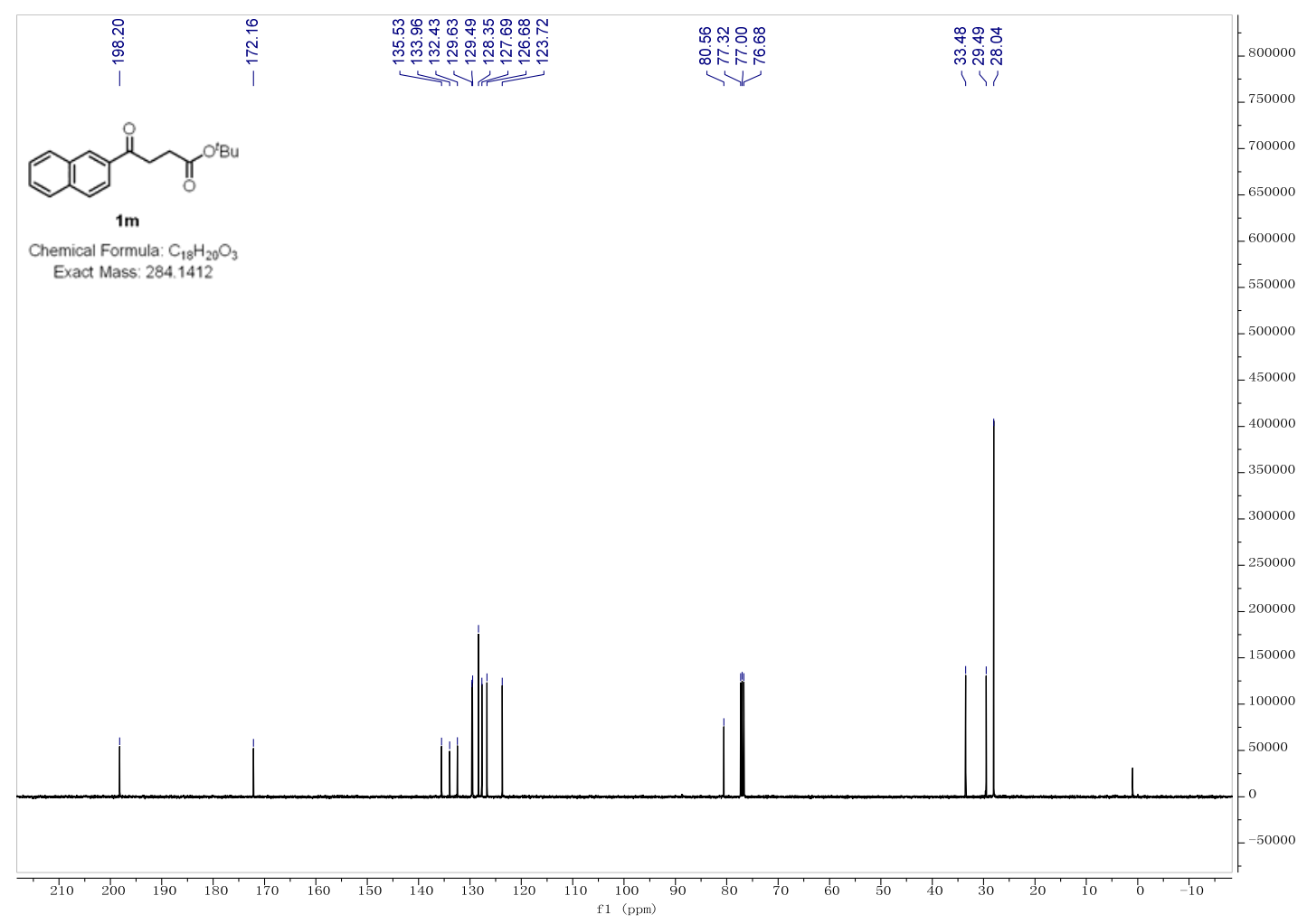


${ }^{1}$ H NMR (400 MHz, Chloroform-d) of compound 1n

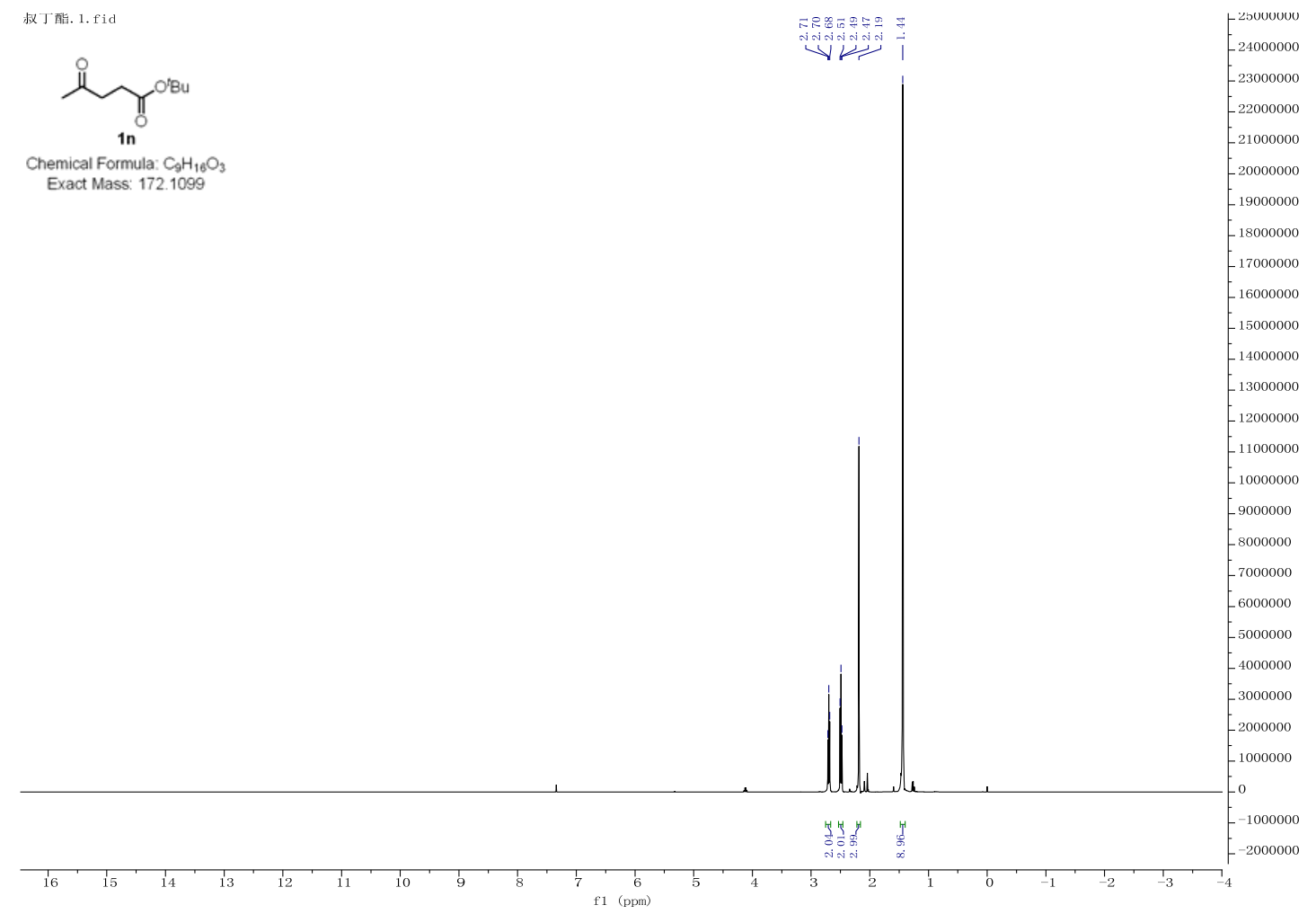

${ }^{13} \mathrm{C}\left\{{ }^{1} \mathrm{H}\right\}$ NMR (101 MHz, Chloroform-d) of compound 1m

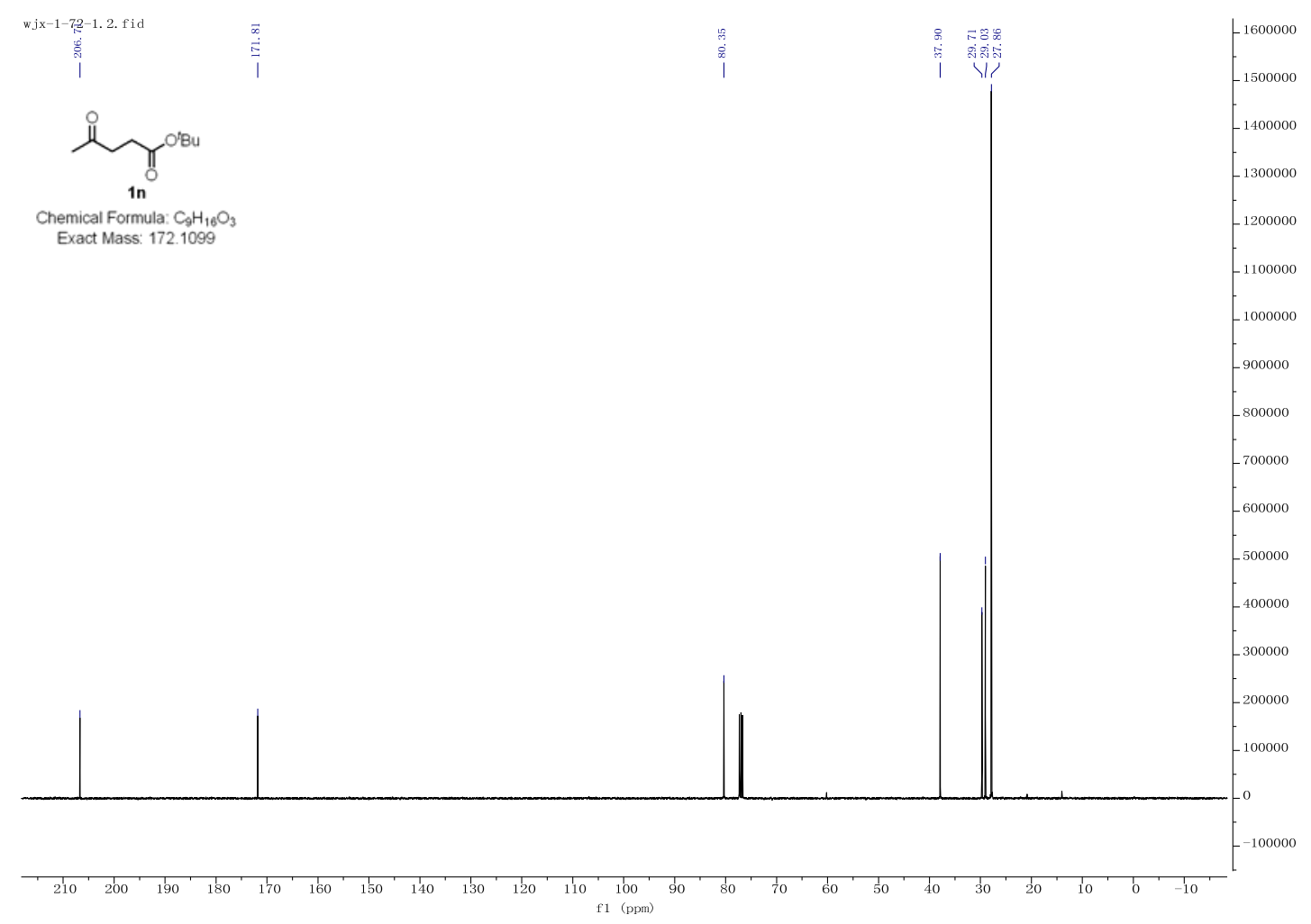


${ }^{1}$ H NMR (400 MHz, Chloroform-d) of compound 3ab

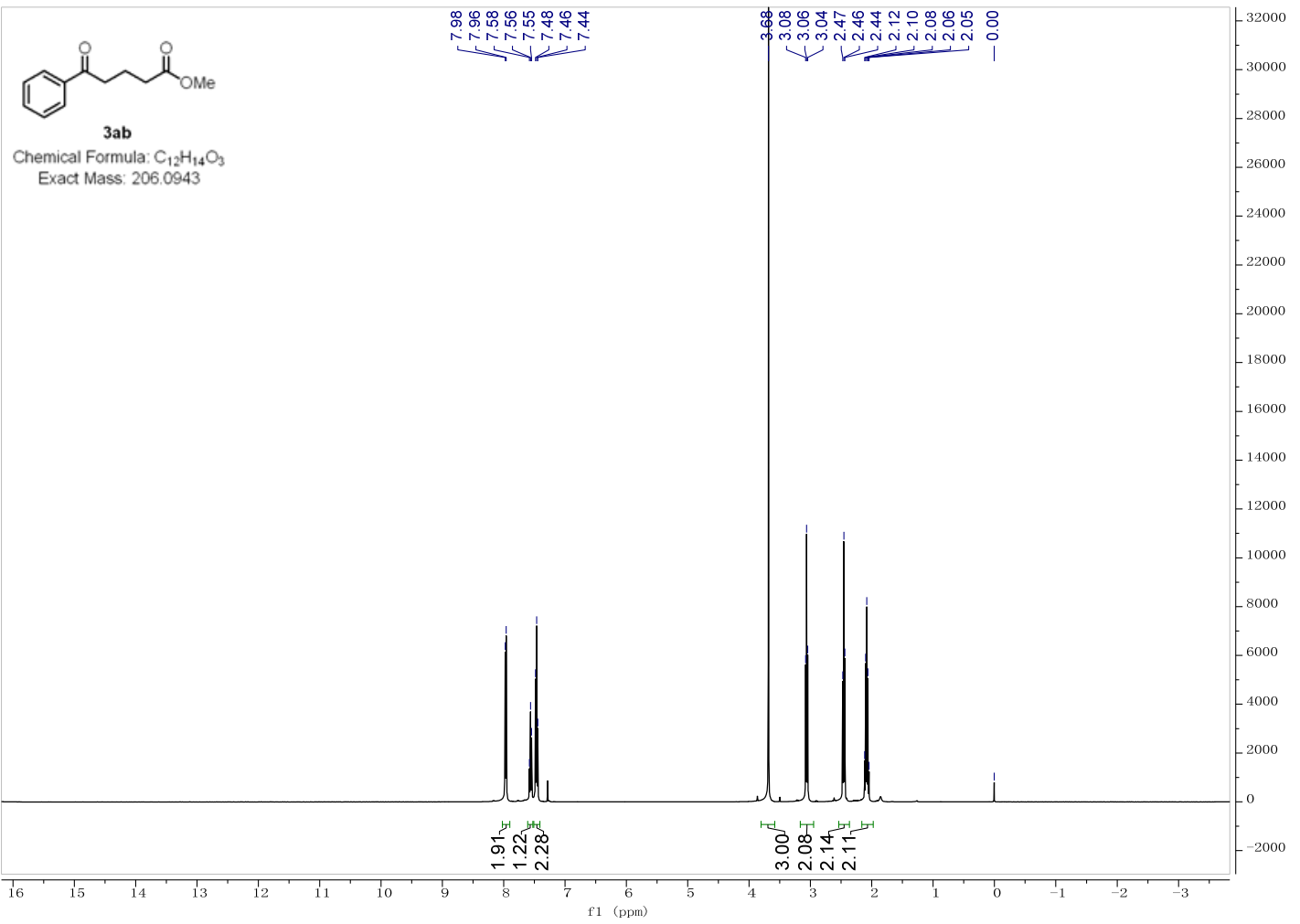

${ }^{13} \mathrm{C}\left\{{ }^{1} \mathrm{H}\right\}$ NMR (101 MHz, Chloroform-d) of compound 3ab

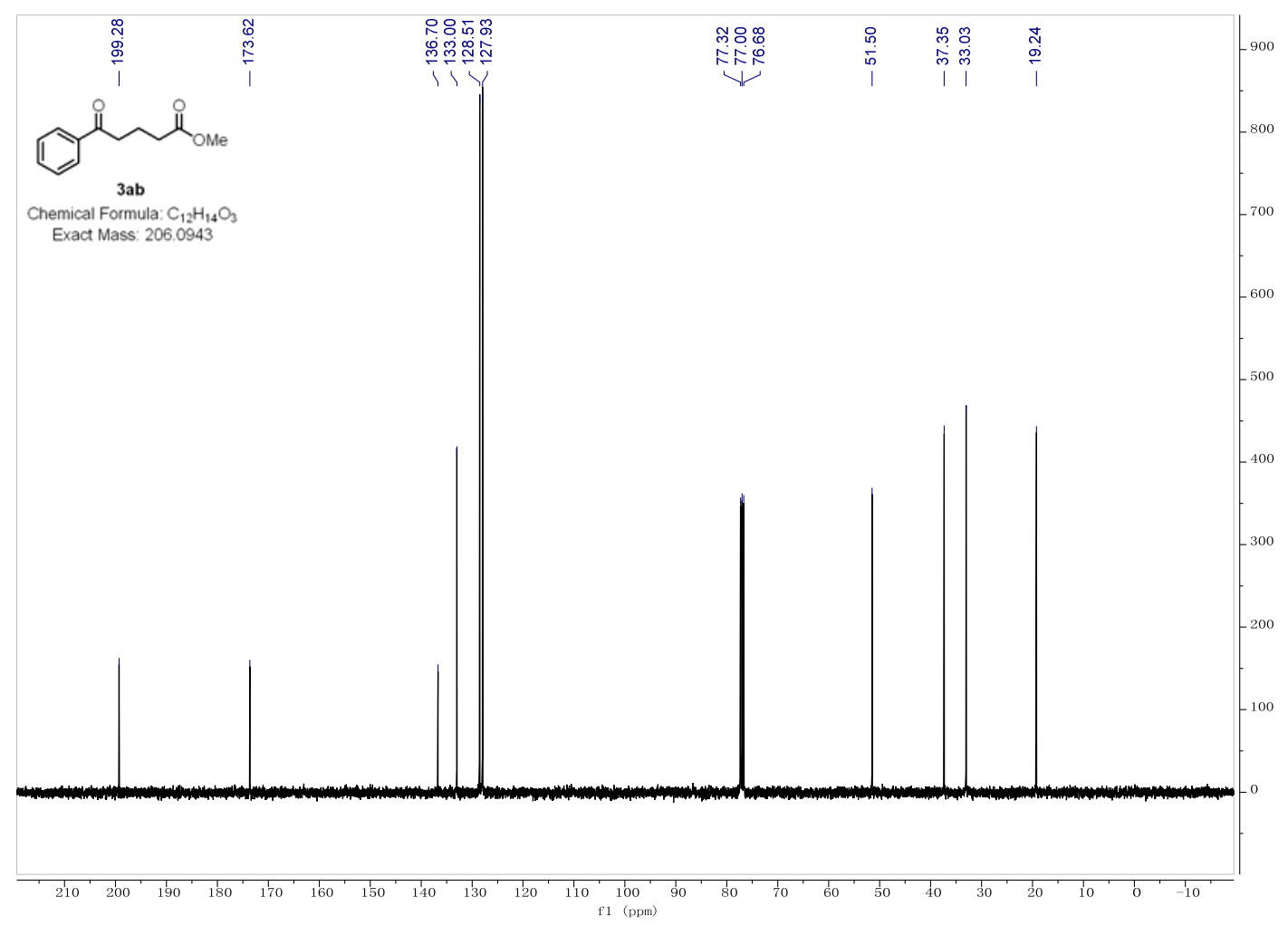


${ }^{1} \mathrm{H}$ NMR (400 MHz, Chloroform-d) of compound 3ac

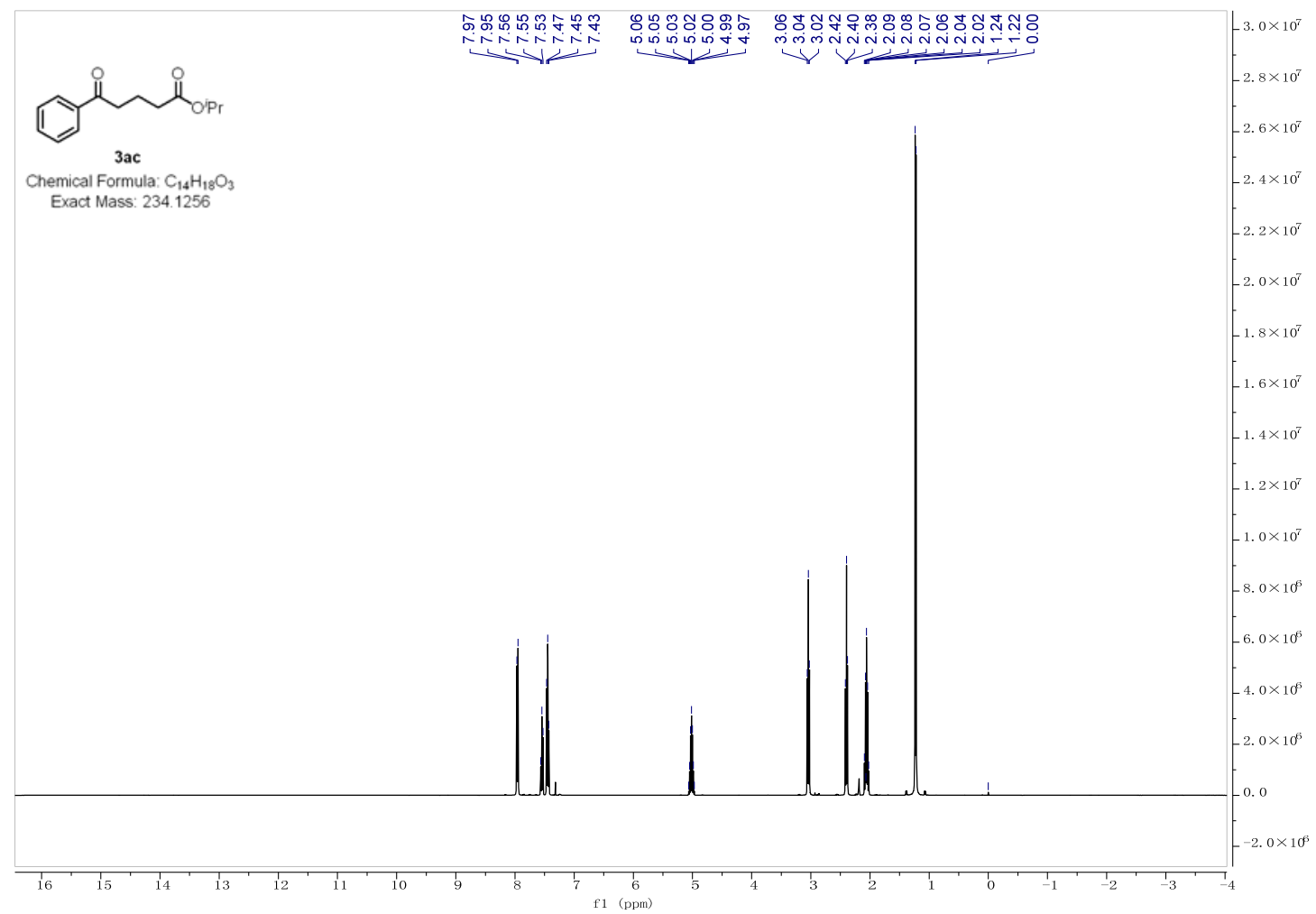

${ }^{13} \mathrm{C}\left\{{ }^{1} \mathrm{H}\right\}$ NMR (101 MHz, Chloroform-d) of compound 3ac

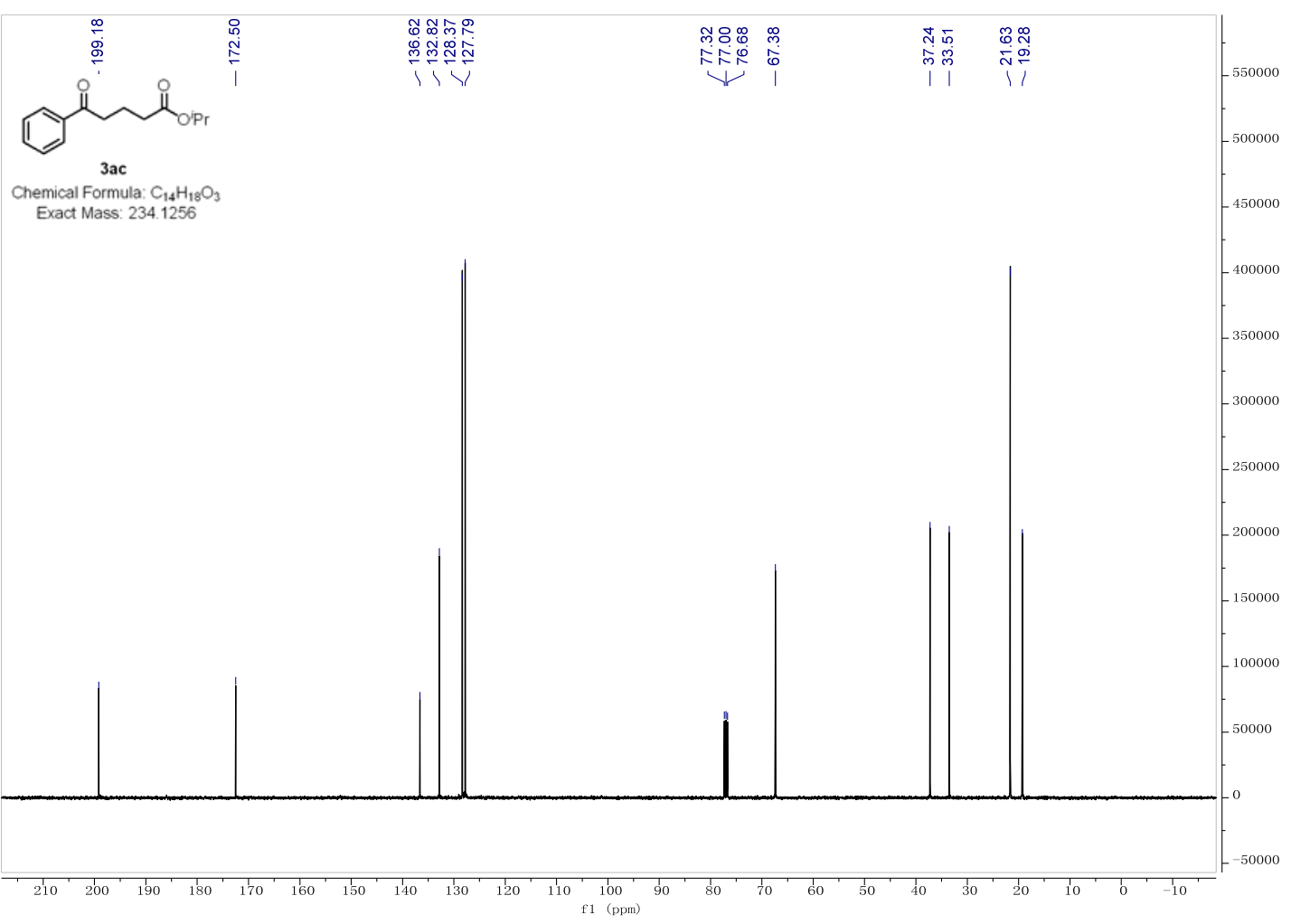


${ }^{1}$ H NMR (400 MHz, Chloroform-d) of compound 3a

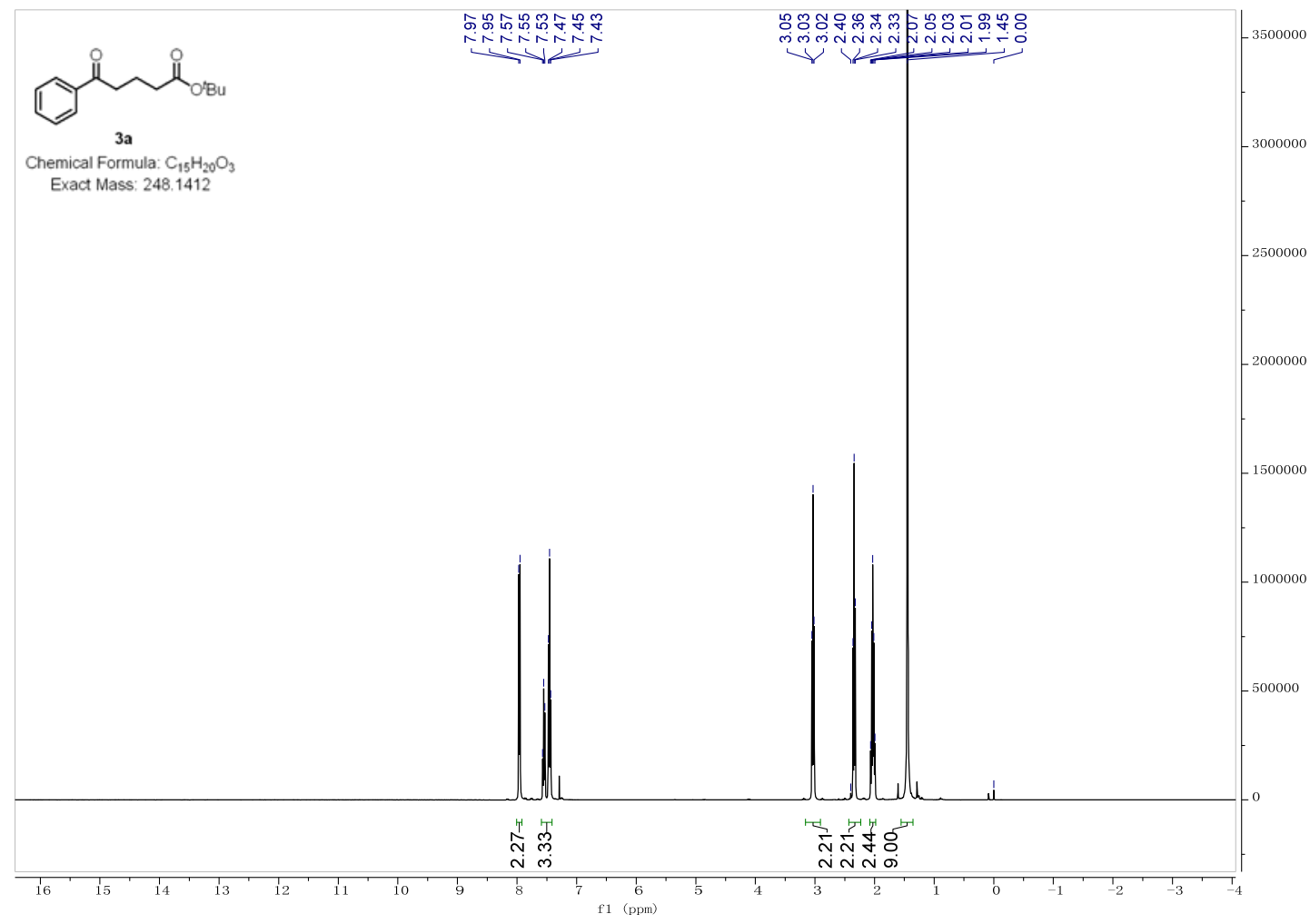

${ }^{13} \mathrm{C}\left\{{ }^{1} \mathrm{H}\right\}$ NMR (101 MHz, Chloroform-d) of compound 3a

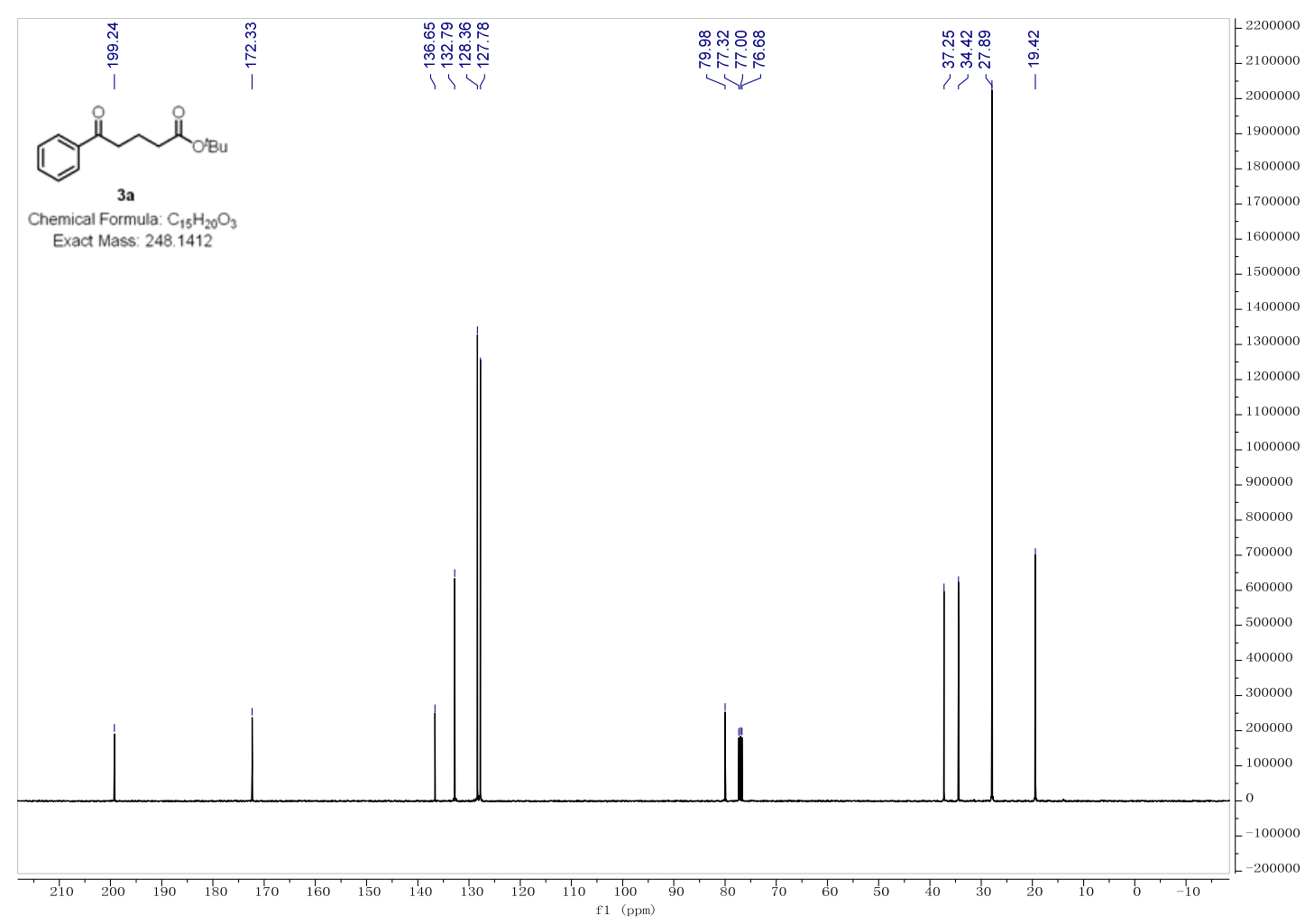


${ }^{1}$ H NMR (400 MHz, Chloroform-d) of compound $3 \mathbf{b}$

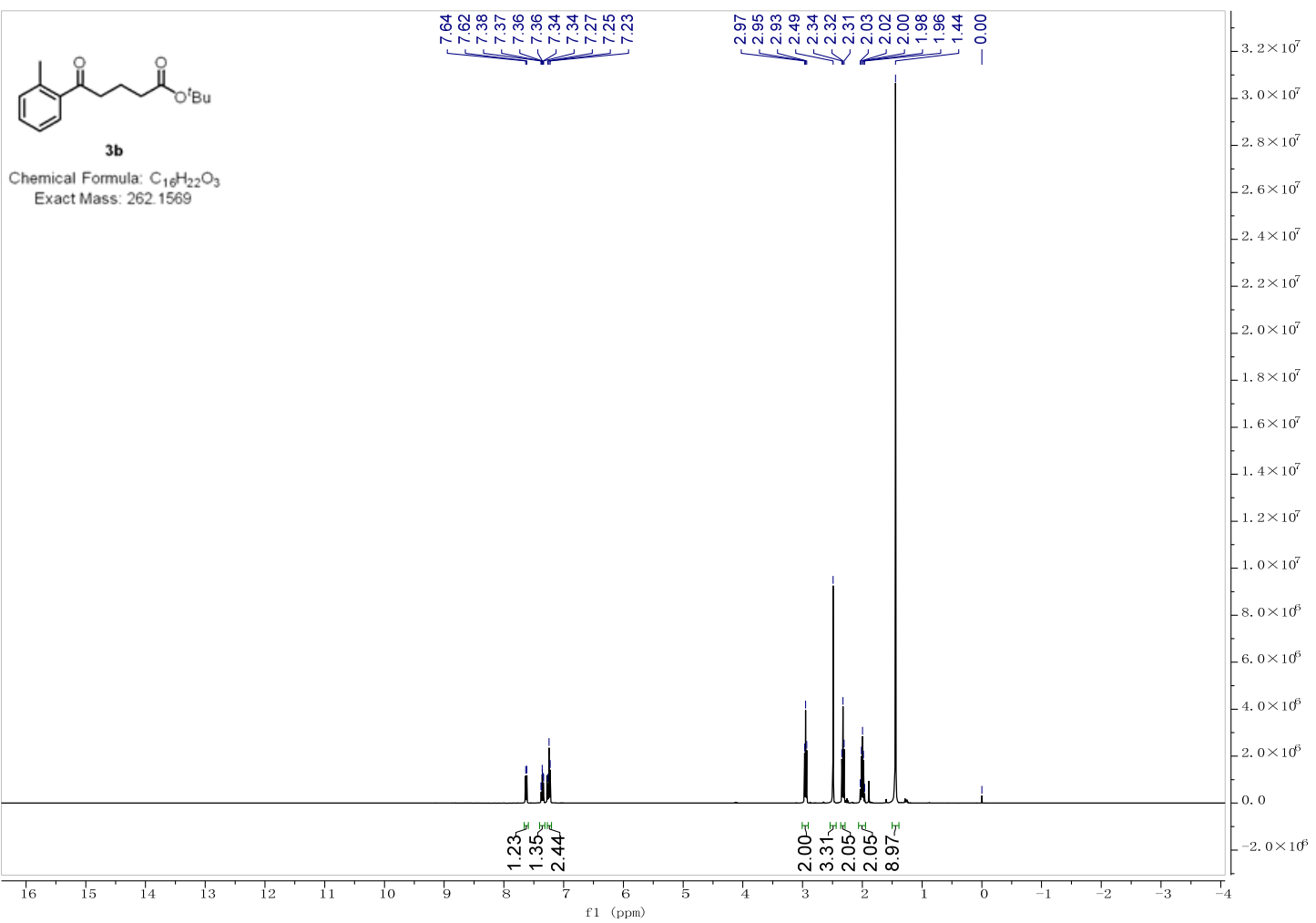

${ }^{13} \mathrm{C}\left\{{ }^{1} \mathrm{H}\right\}$ NMR (101 MHz, Chloroform-d) of compound $\mathbf{3 b}$

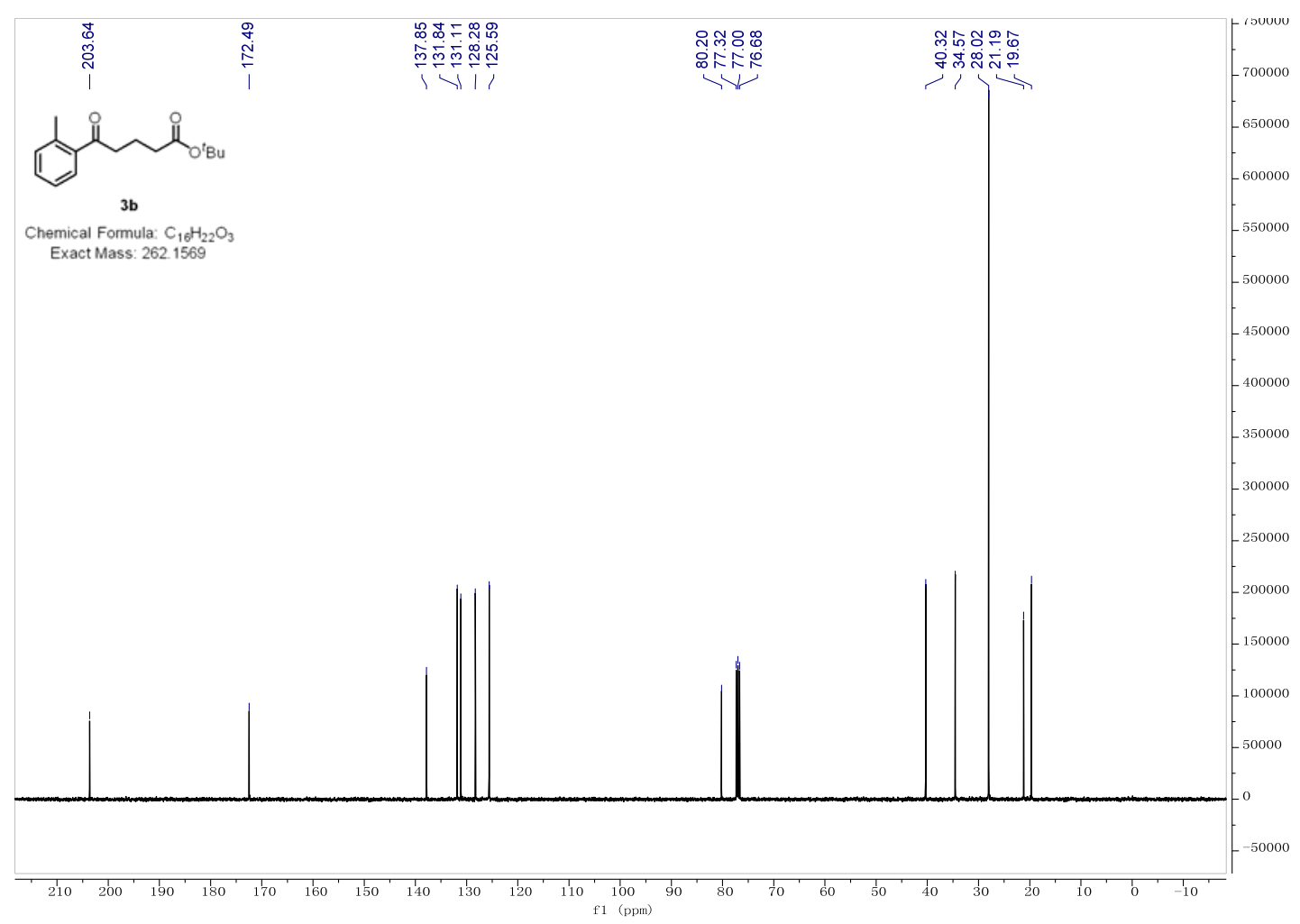


${ }^{1}$ H NMR (400 MHz, Chloroform-d) of compound 3c

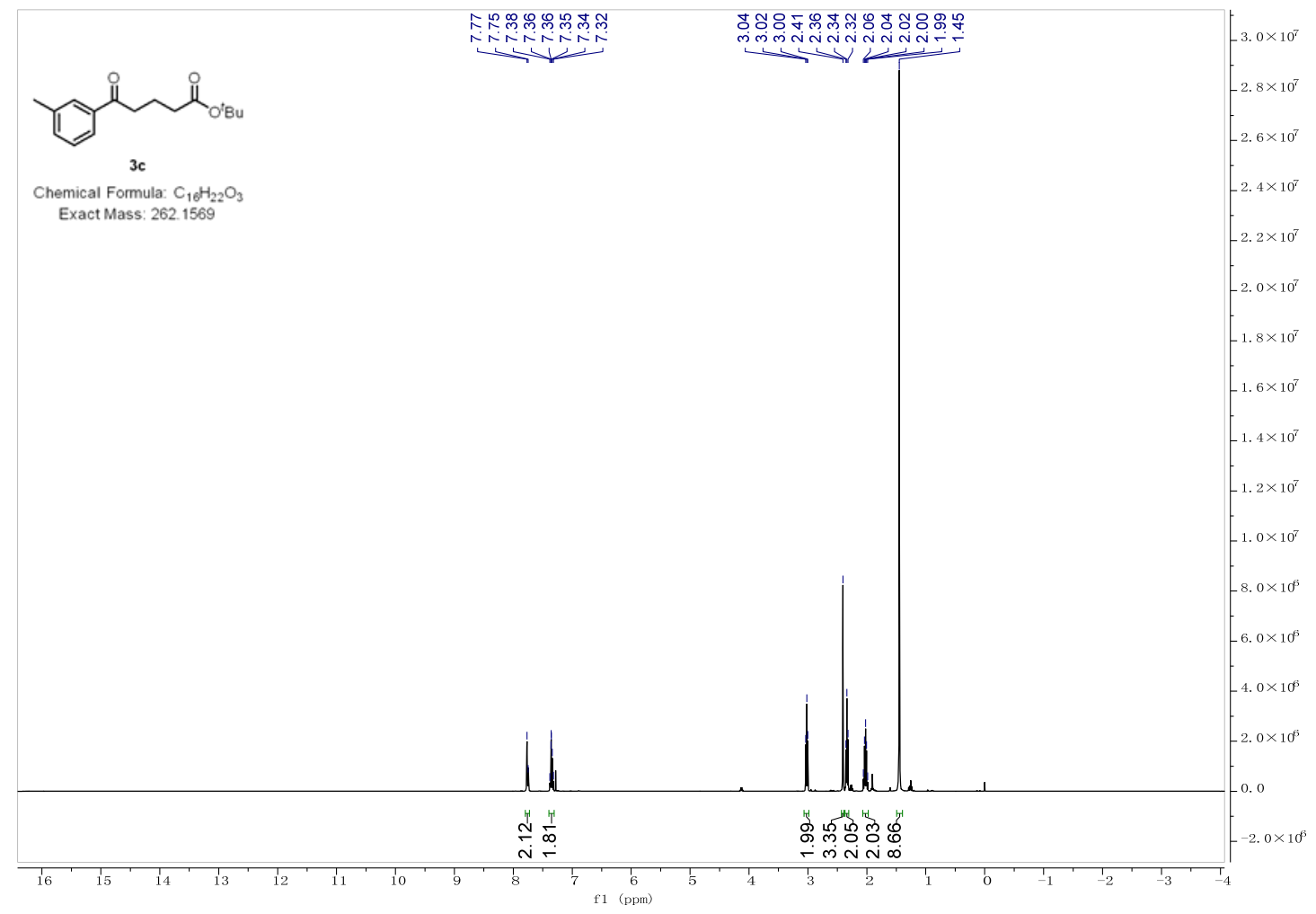

${ }^{13} \mathrm{C}\left\{{ }^{1} \mathrm{H}\right\}$ NMR (101 MHz, Chloroform-d) of compound 3c

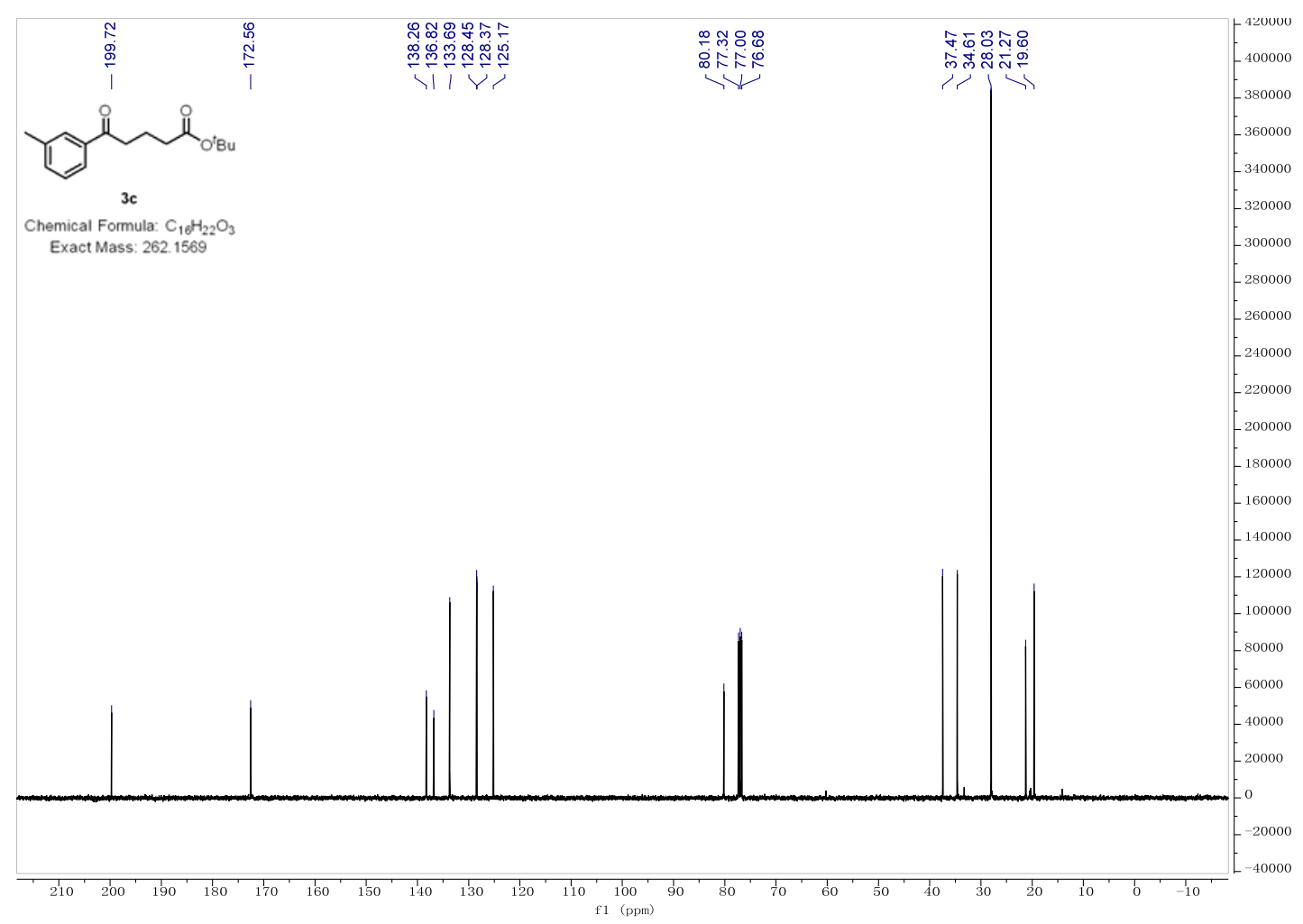


${ }^{1}$ H NMR (400 MHz, Chloroform-d) of compound 3d

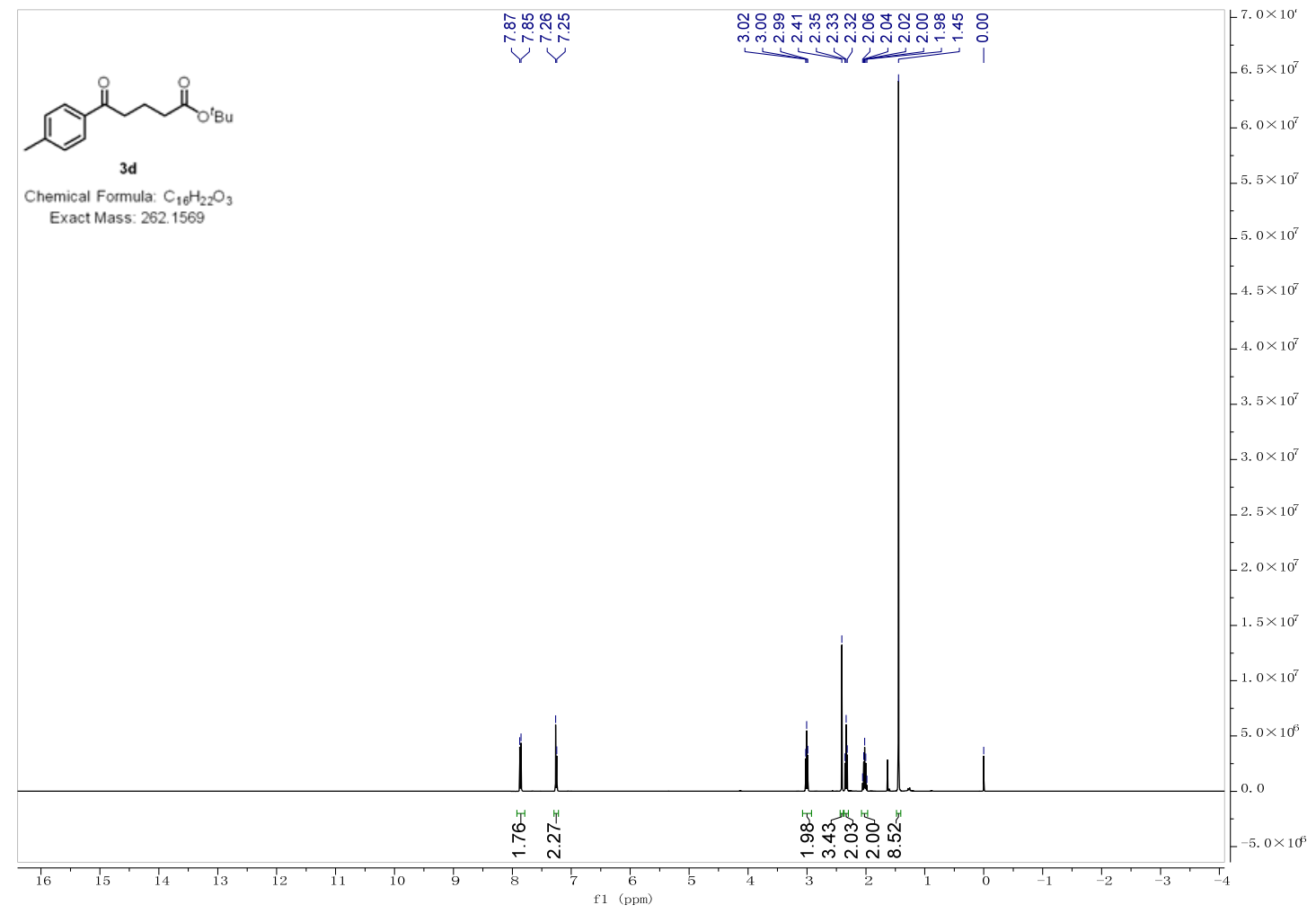

${ }^{13} \mathrm{C}\left\{{ }^{1} \mathrm{H}\right\}$ NMR (101 MHz, Chloroform-d) of compound 3d

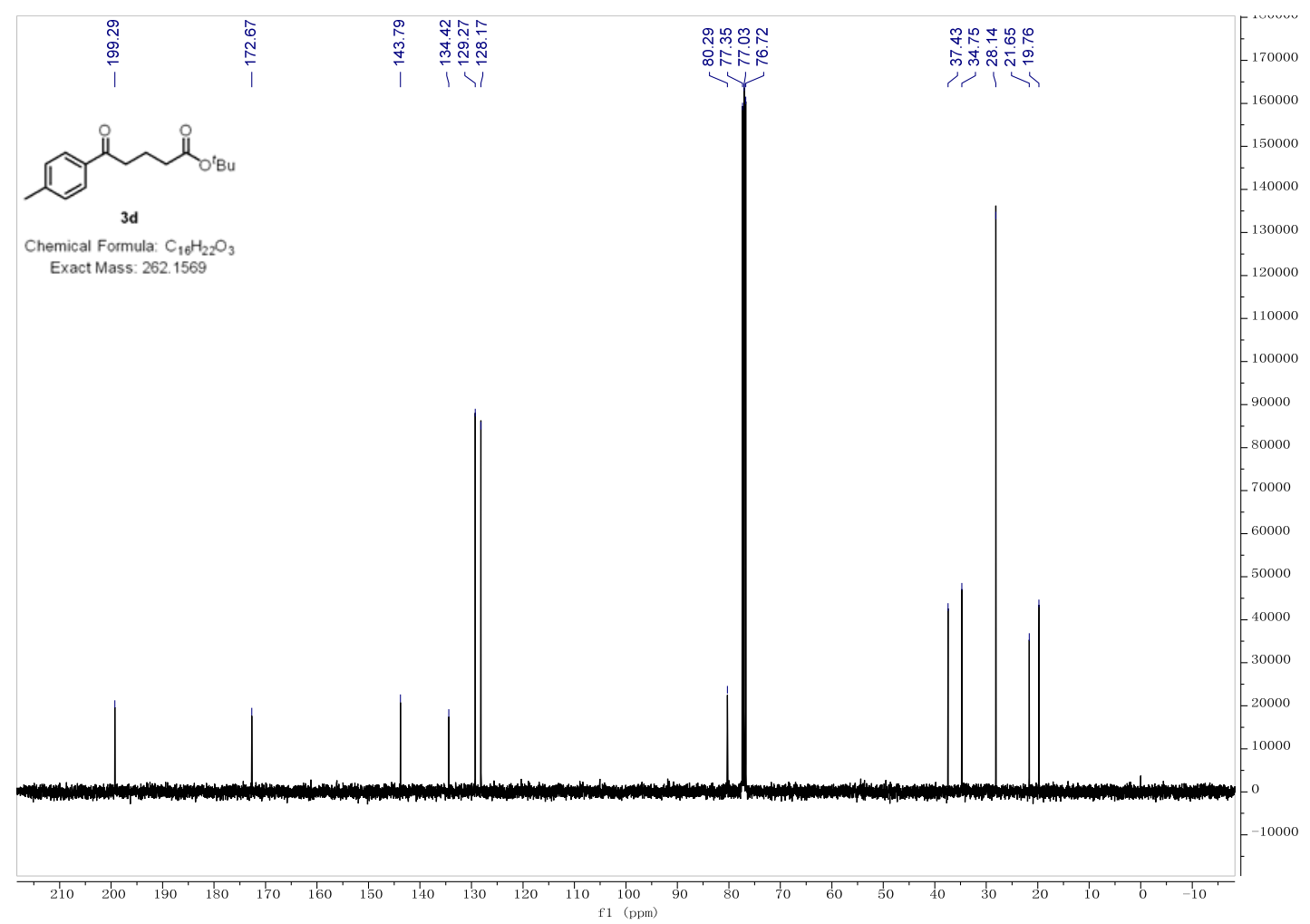


${ }^{1} \mathrm{H}$ NMR (400 MHz, Chloroform-d) of compound $3 \mathbf{e}$

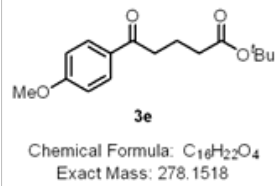

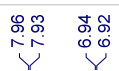

@

Exact Mass: 278.1518

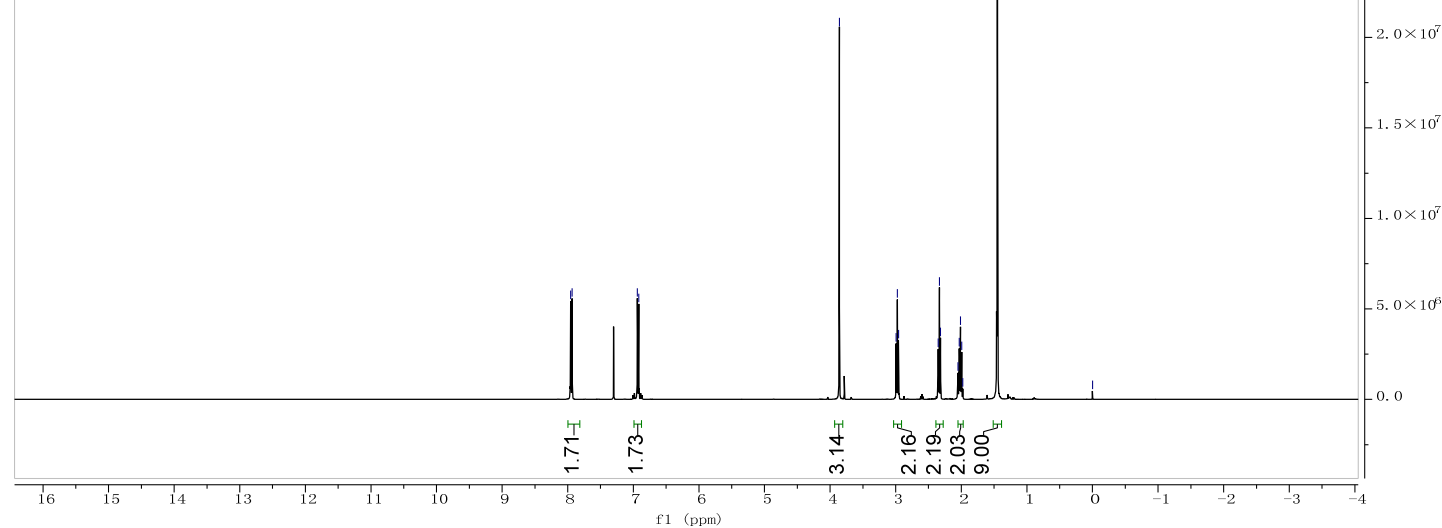

${ }^{13} \mathrm{C}\left\{{ }^{1} \mathrm{H}\right\}$ NMR (101 MHz, Chloroform-d) of compound 3e

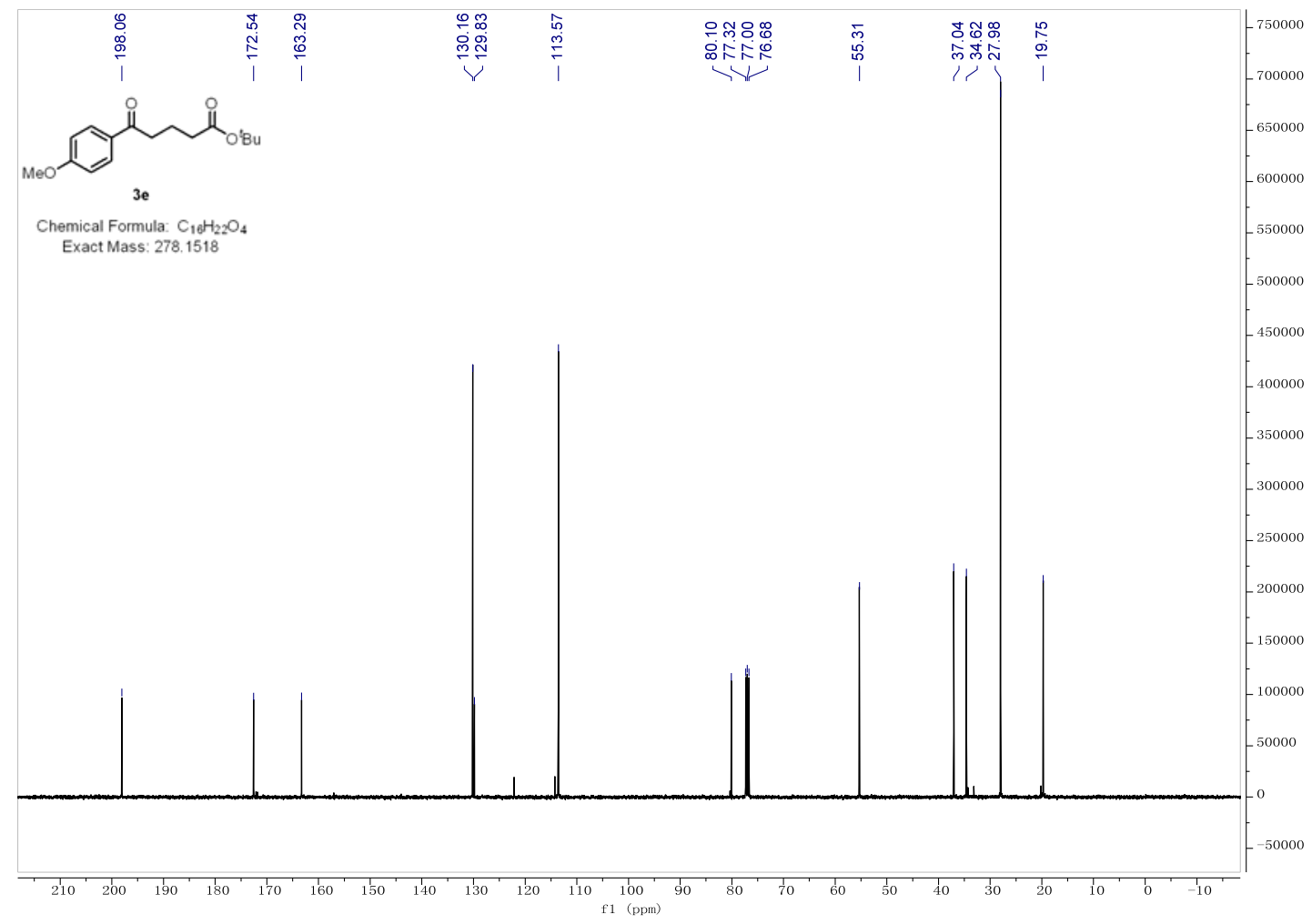


${ }^{1}$ H NMR (400 MHz, Chloroform-d) of compound 3f

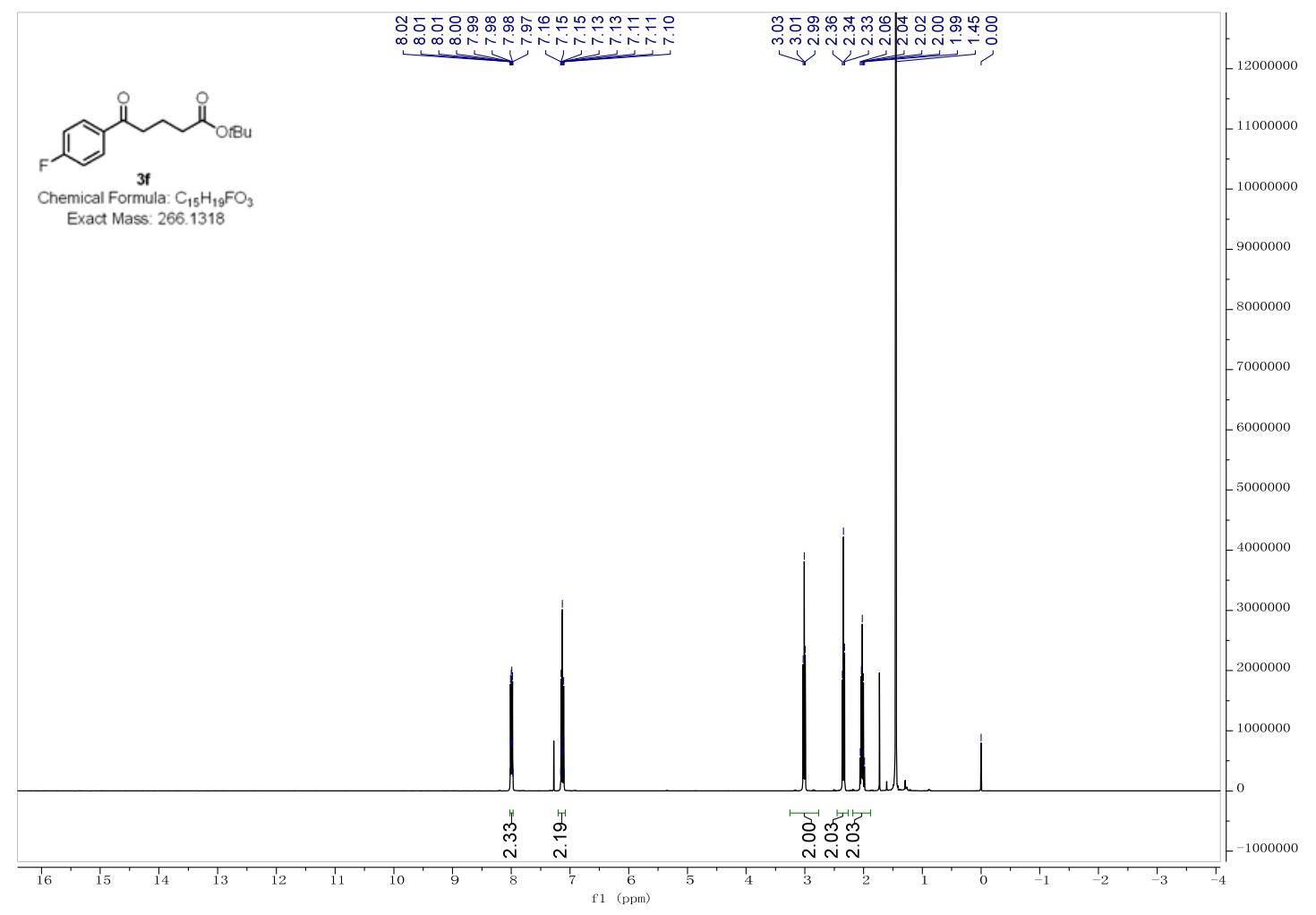

${ }^{13} \mathrm{C}\left\{{ }^{1} \mathrm{H}\right\}$ NMR (101 MHz, Chloroform-d) of compound $3 \mathbf{f}$

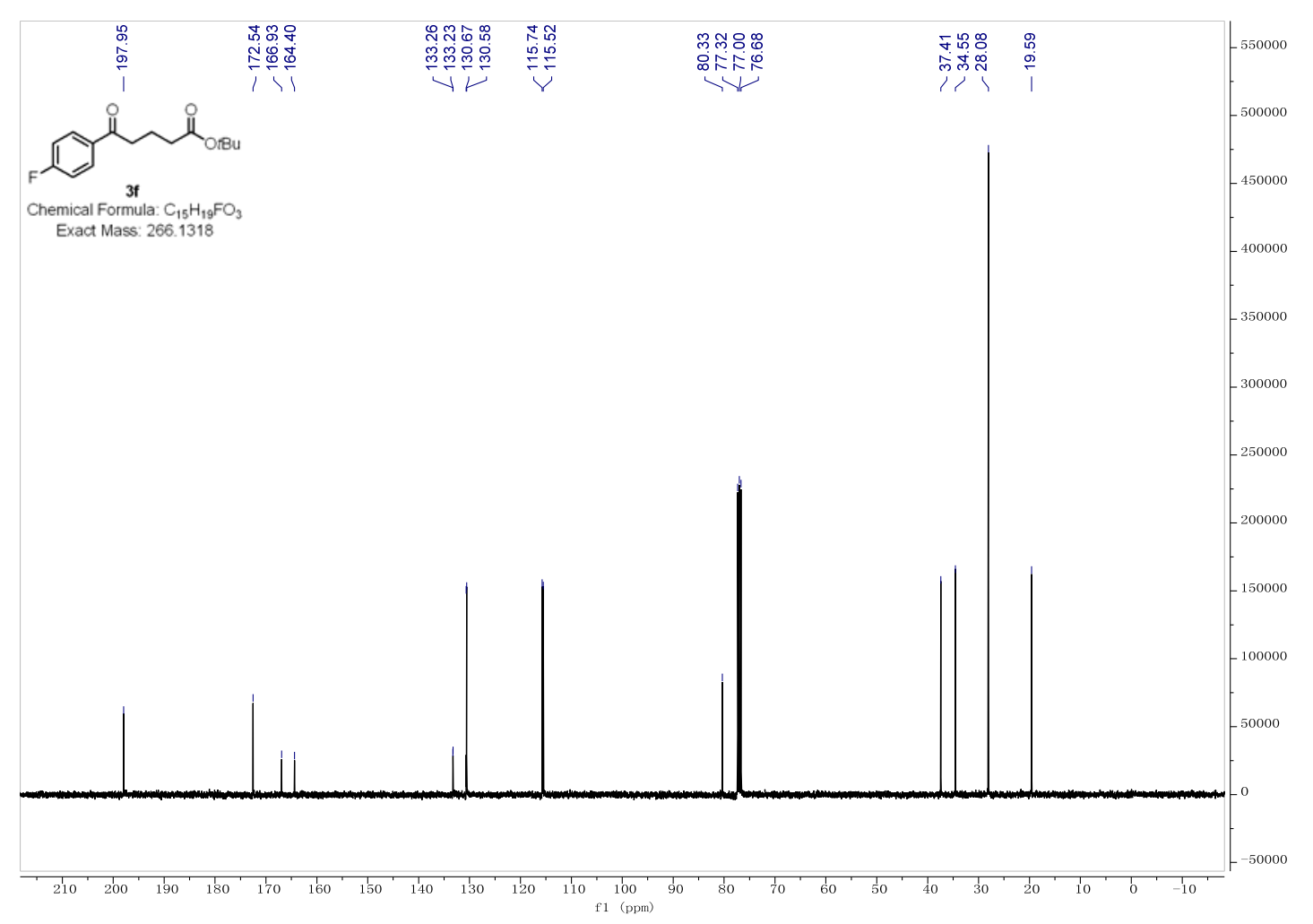


${ }^{1}$ H NMR (400 MHz, Chloroform-d) of compound 3g

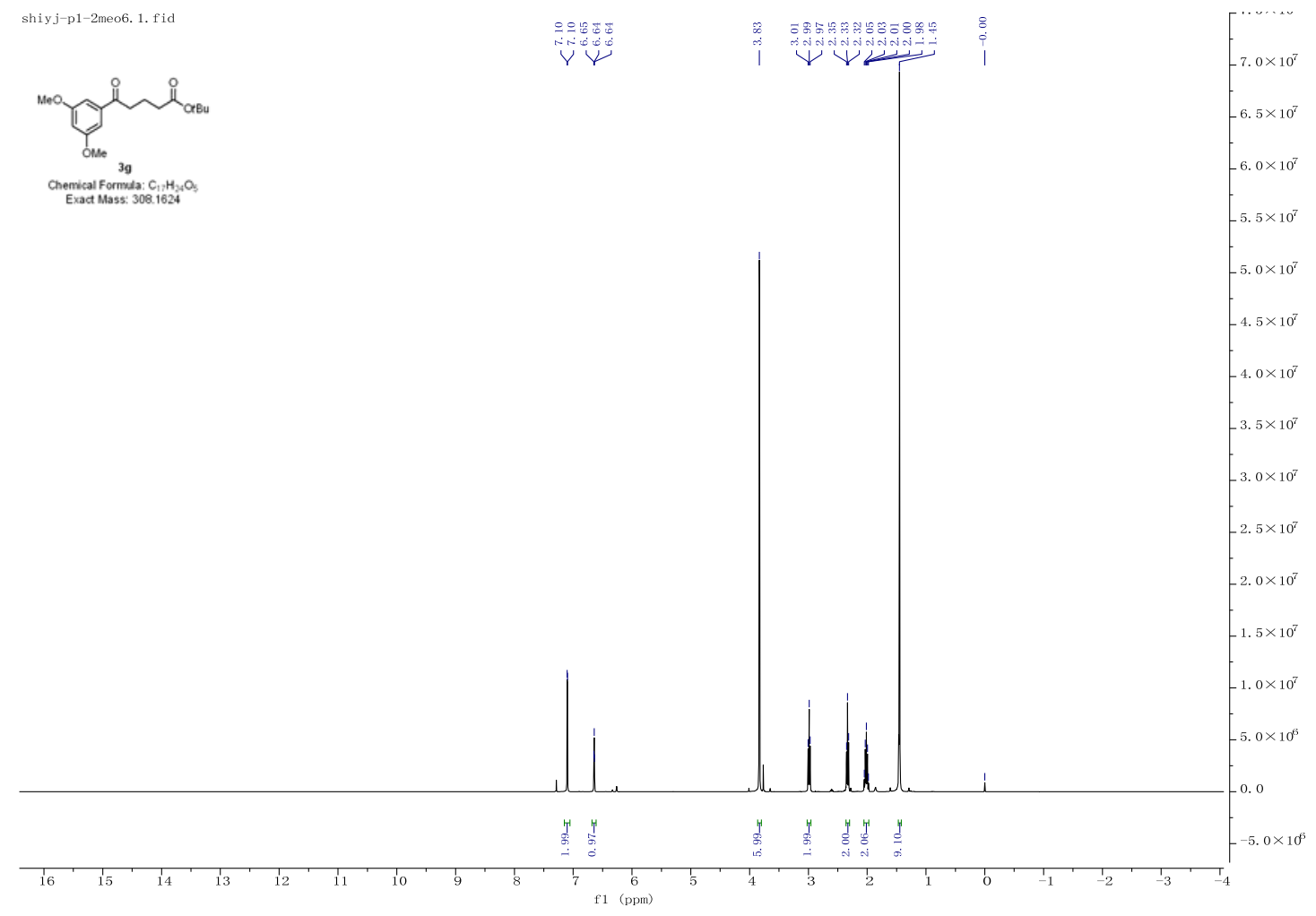

${ }^{13} \mathrm{C}\left\{{ }^{1} \mathrm{H}\right\}$ NMR (101 MHz, Chloroform-d) of compound 3g

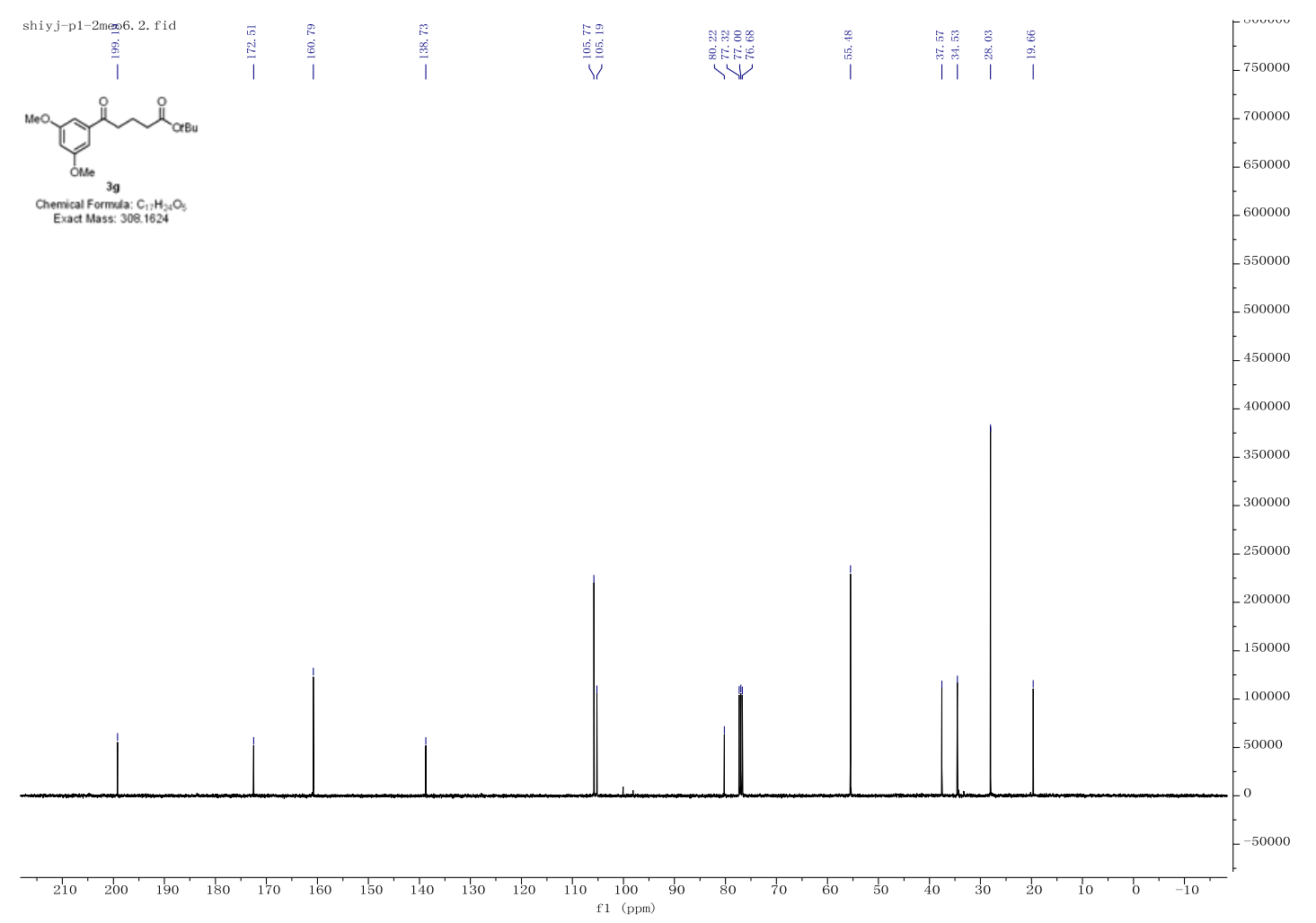


${ }^{1}$ H NMR (600 MHz, Chloroform-d) of compound 3h

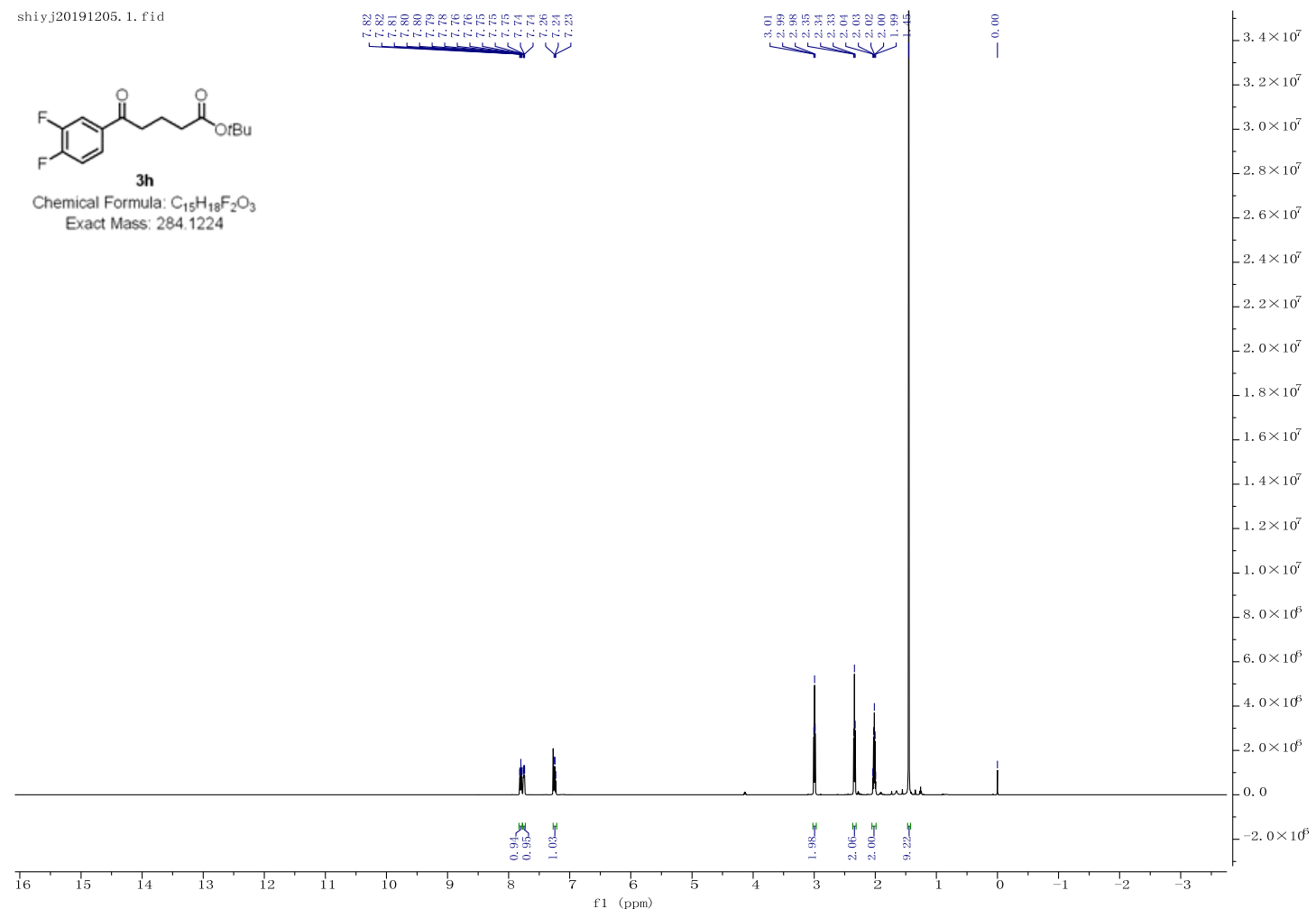

${ }^{13} \mathrm{C}$ NMR (151 MHz, Chloroform-d) of compound 3h

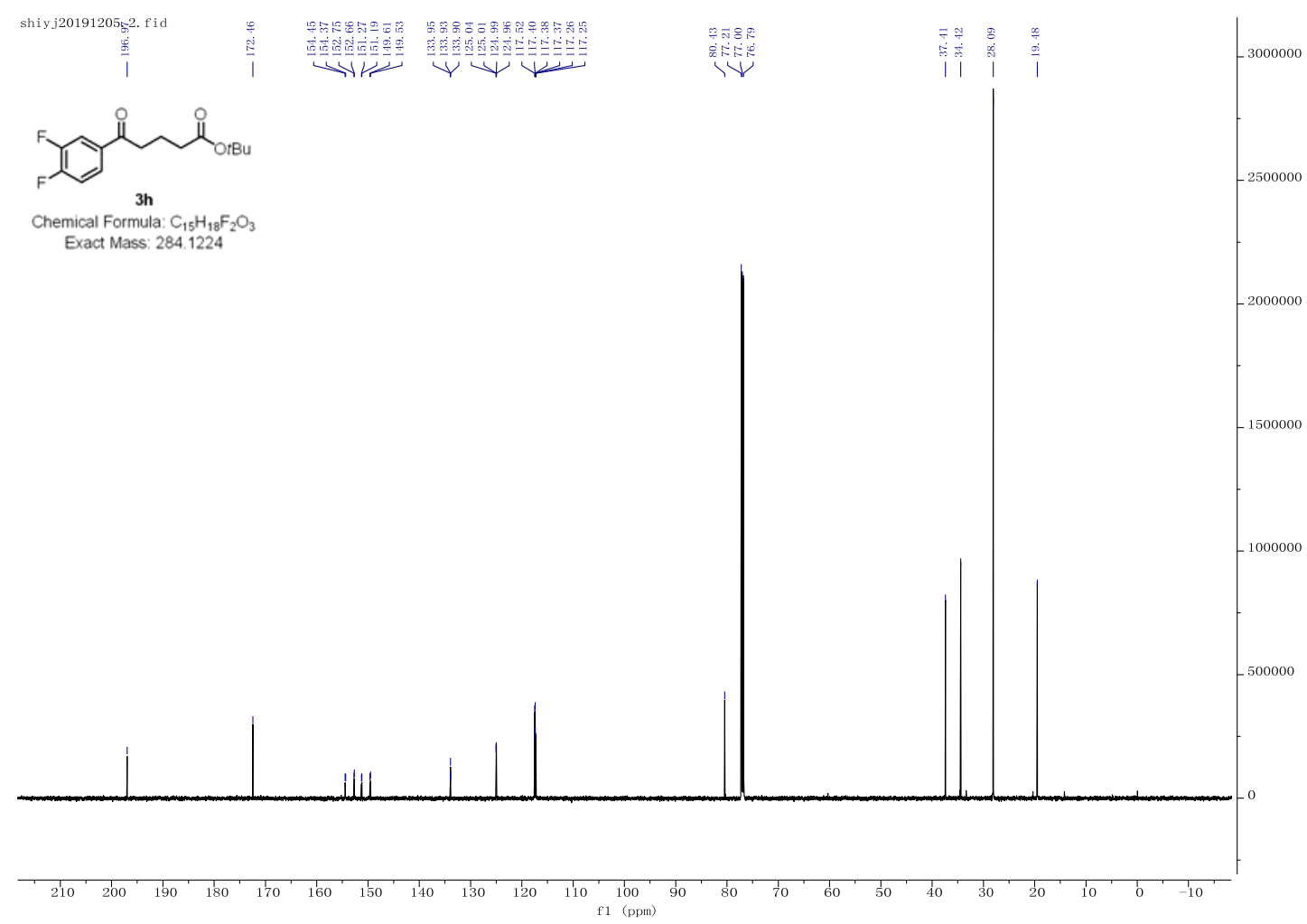


${ }^{1}$ H NMR (400 MHz, Chloroform-d) of compound 5ab

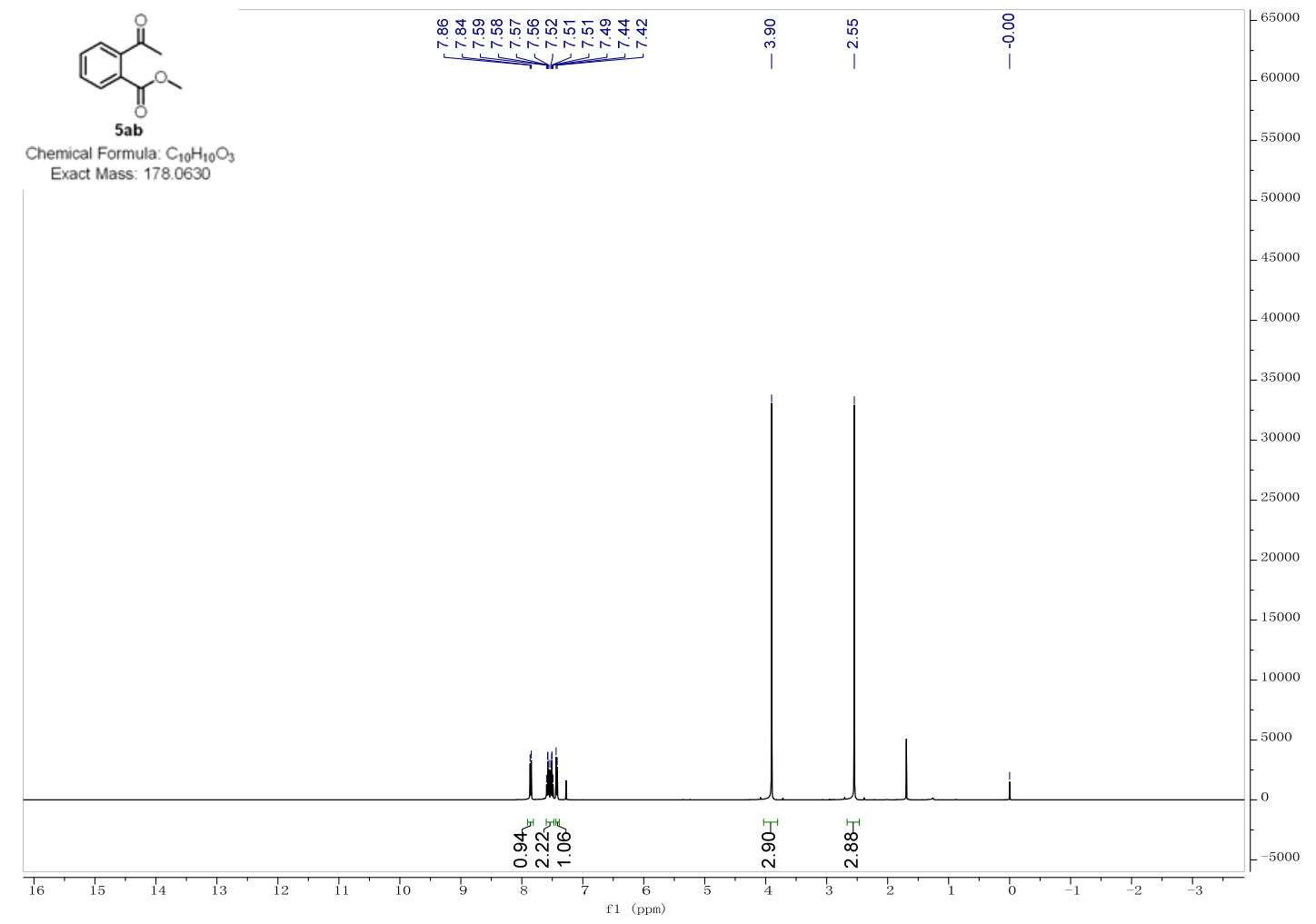


${ }^{1} \mathrm{H}$ NMR (600 MHz, Chloroform-d) of compound 5b

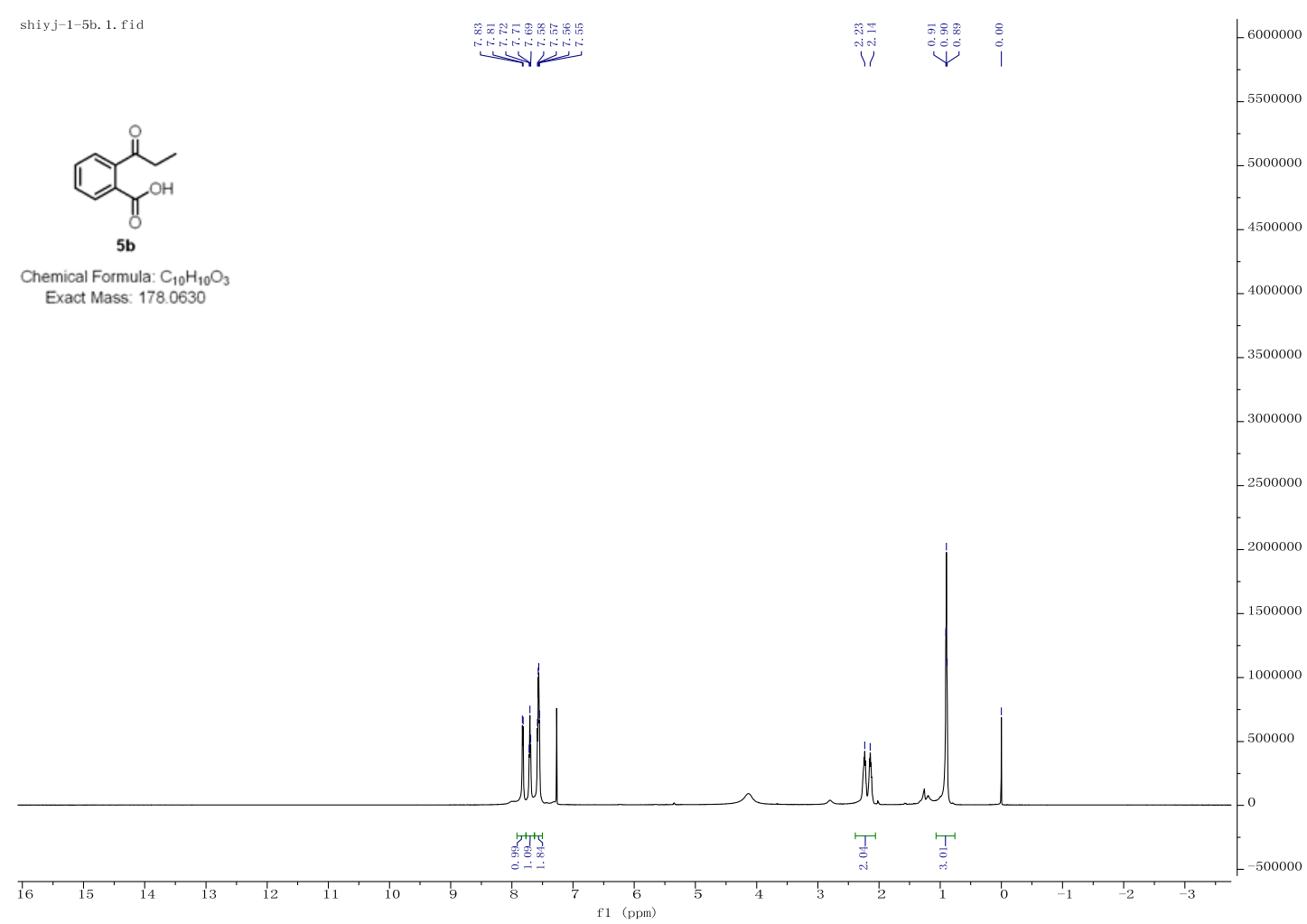

${ }^{13} \mathrm{C}\left\{{ }^{1} \mathrm{H}\right\}$ NMR (151 MHz, Chloroform-d) of compound 5b

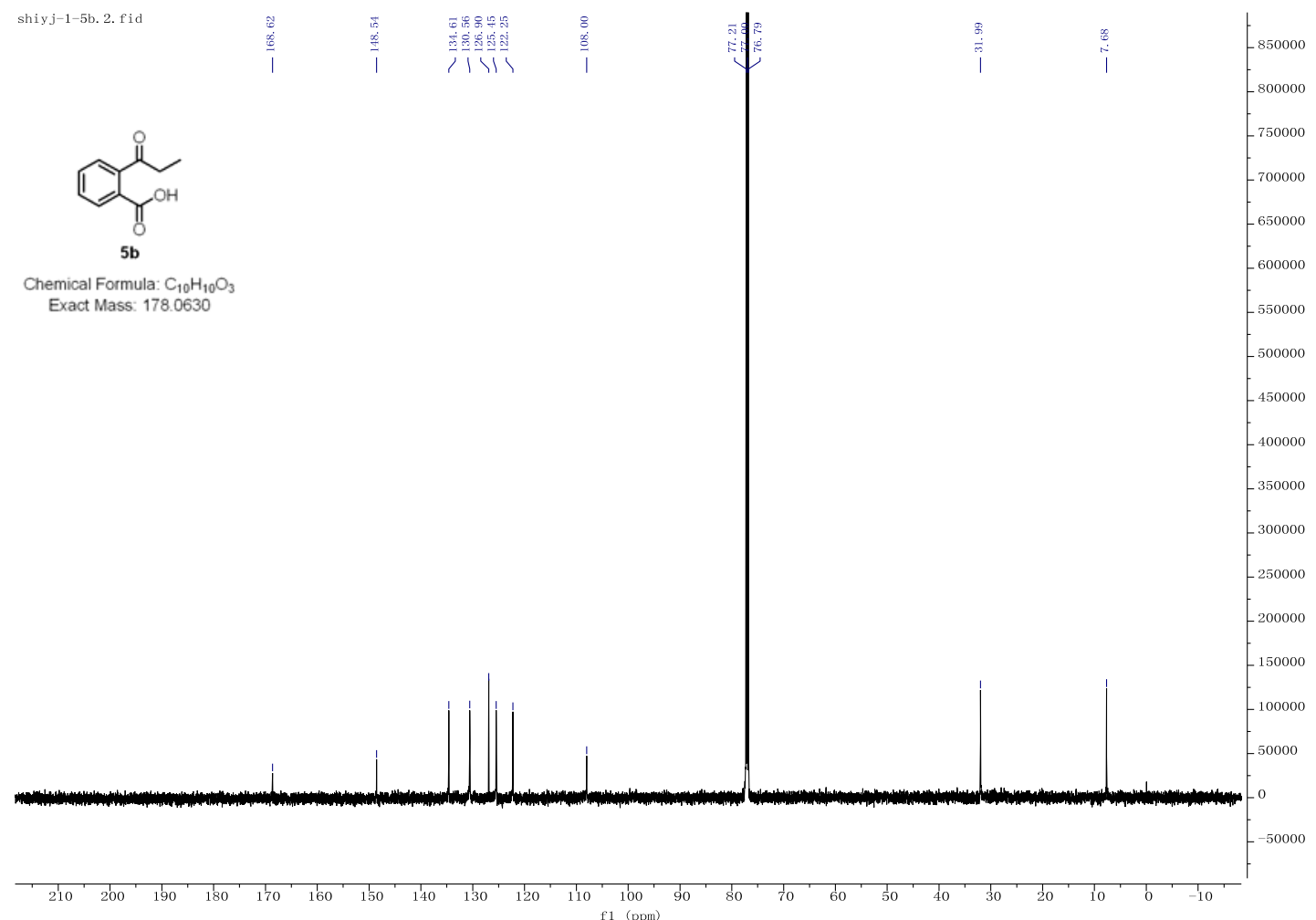


${ }^{1}$ H NMR (400 MHz, Chloroformed) of compound 5 cb

shiyj-p1-5cb. 1. fid

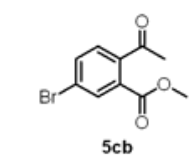

Chemical Formula: $\mathrm{C}_{10} \mathrm{H}_{9} \mathrm{BrO}_{3}$

$$
\text { Exact Mass: } 255.9735
$$

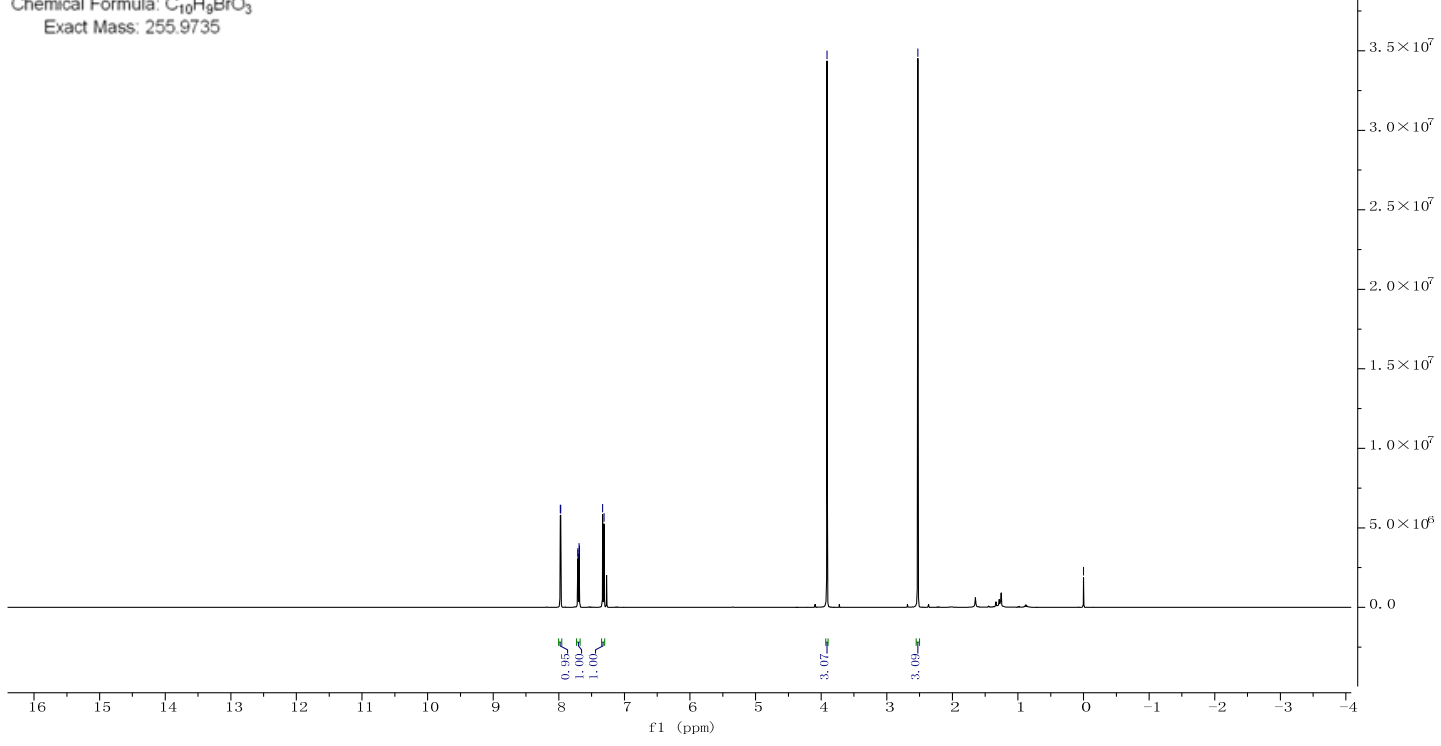

${ }^{13} \mathrm{C}\left\{{ }^{1} \mathrm{H}\right\}$ NMR (101 MHz, Chloroformed) of compound 5cb

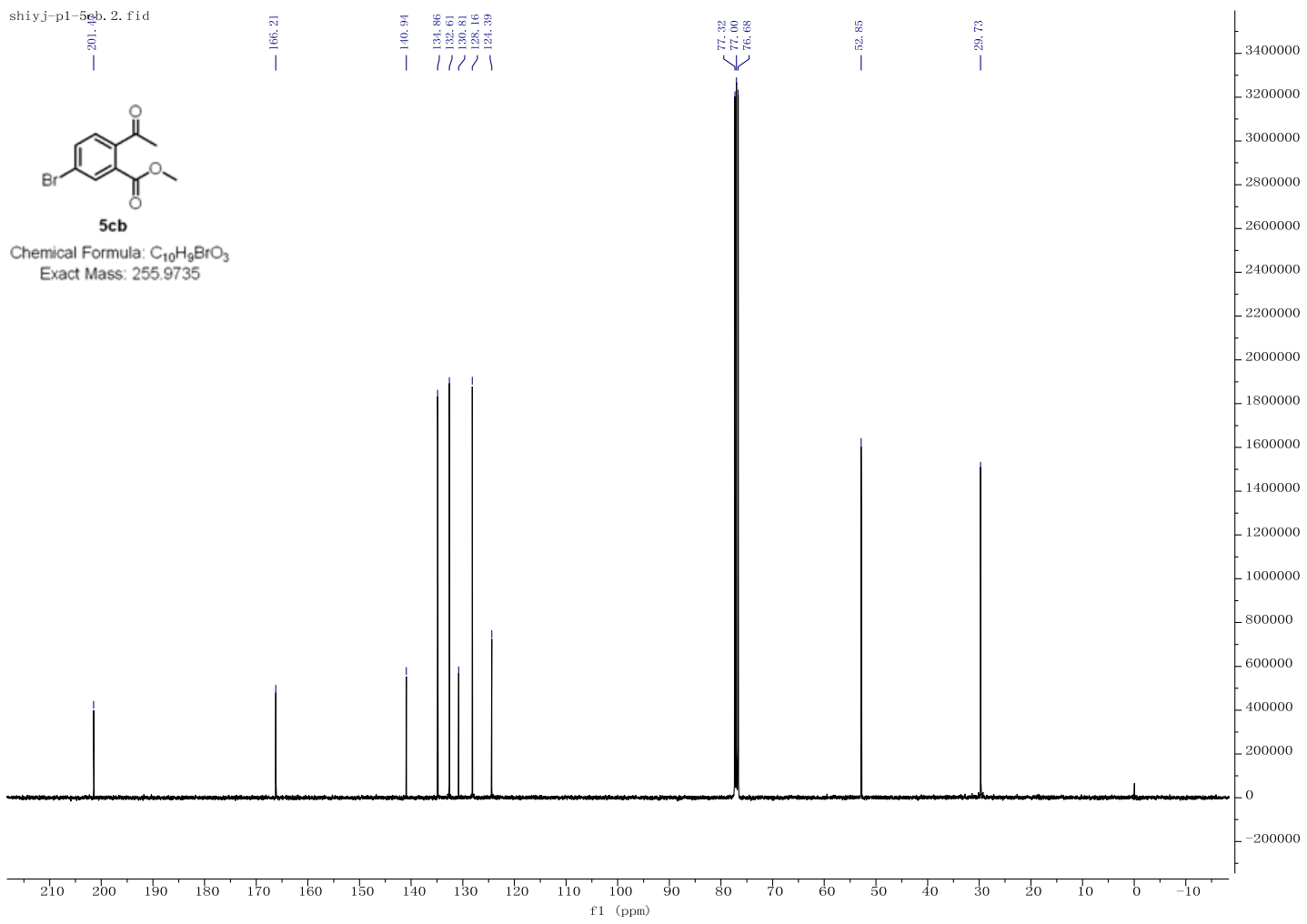

84 
${ }^{1}$ H NMR (600 MHz, Chloroform-d) of compound 5d

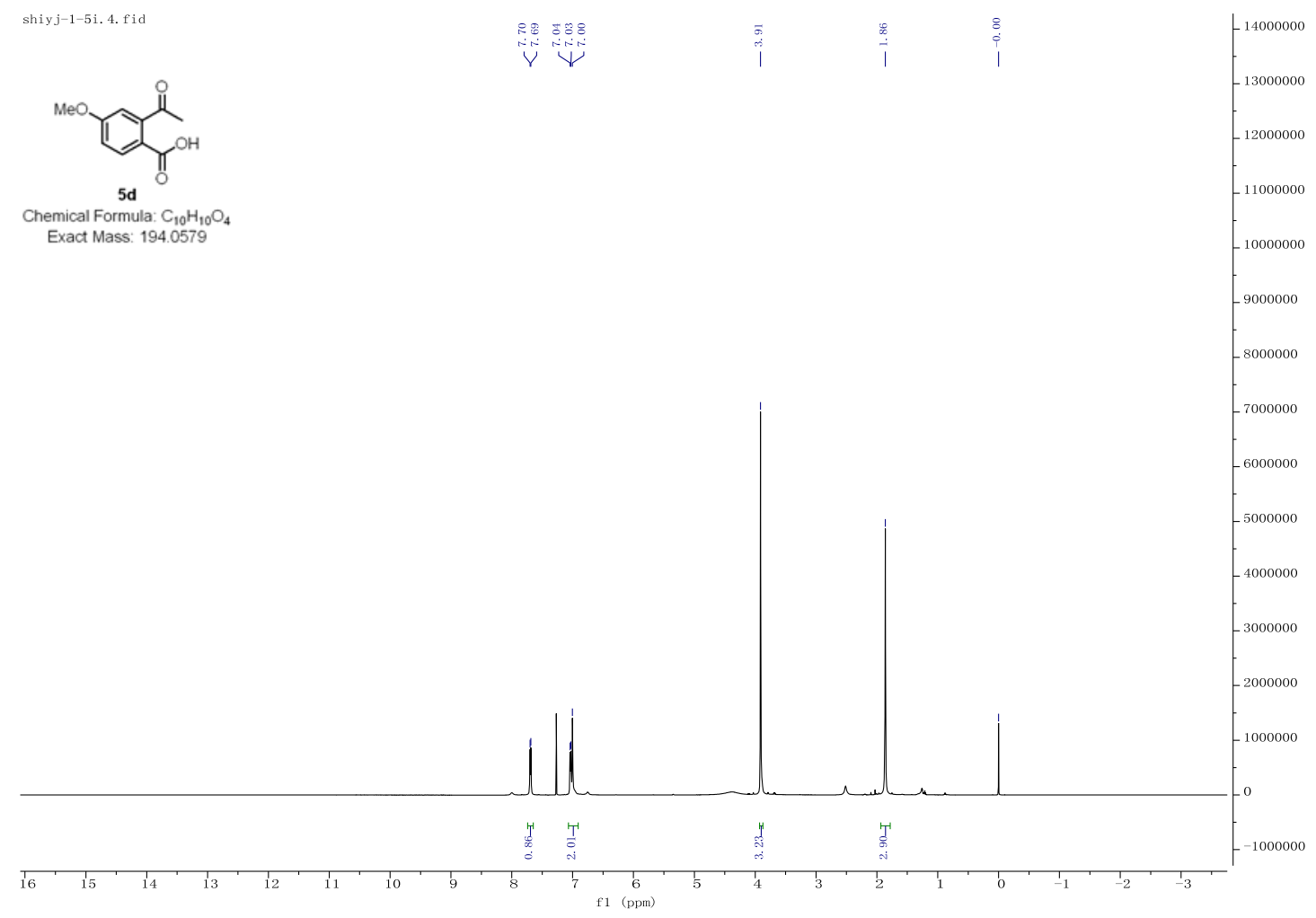

${ }^{13} \mathrm{C}\left\{{ }^{1} \mathrm{H}\right\}$ NMR (151 MHz, Chloroform-d) of compound 5d

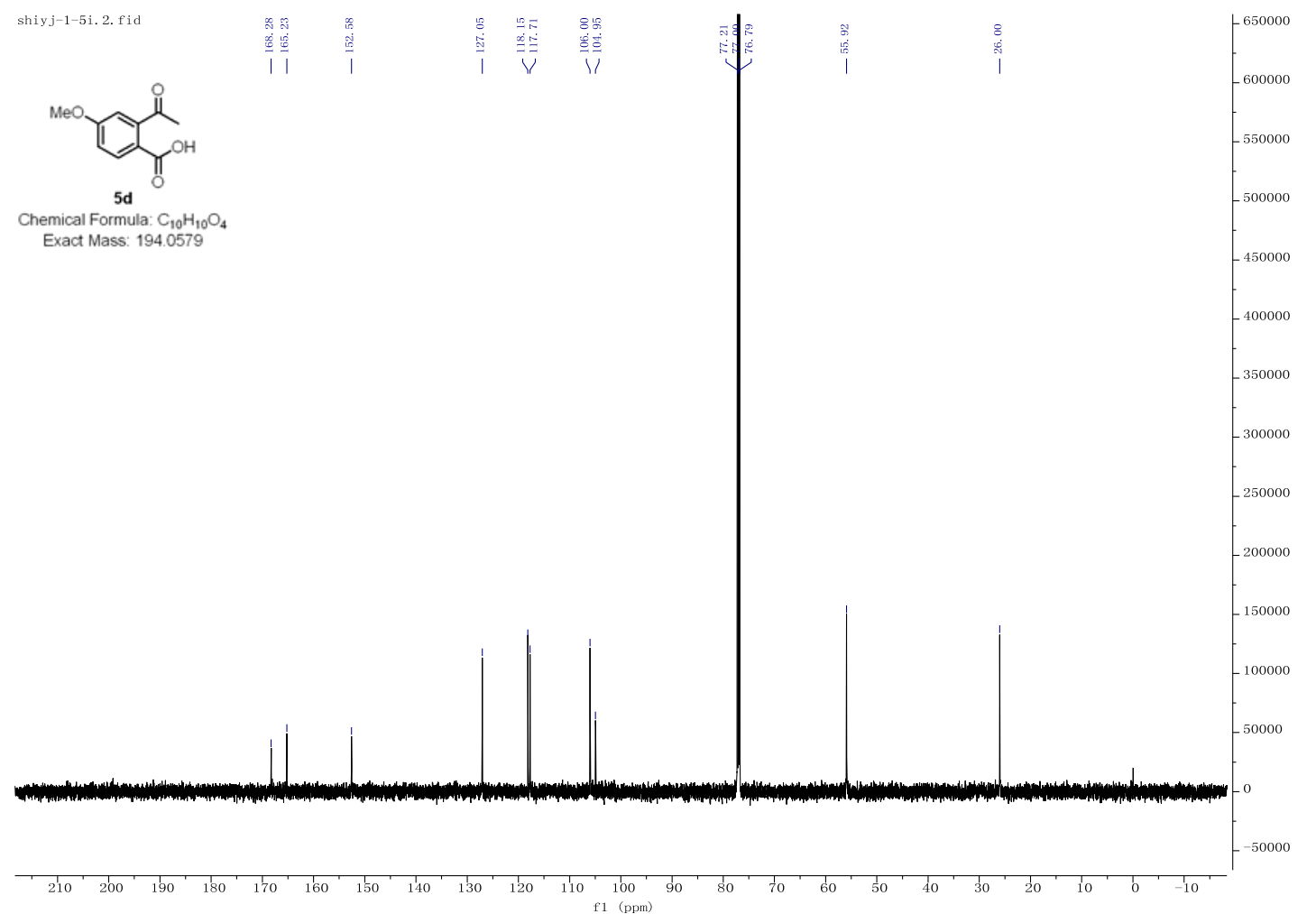


${ }^{1} \mathrm{H}$ NMR (600 MHz, Methanol-d4) of compound $5 \mathbf{e}$

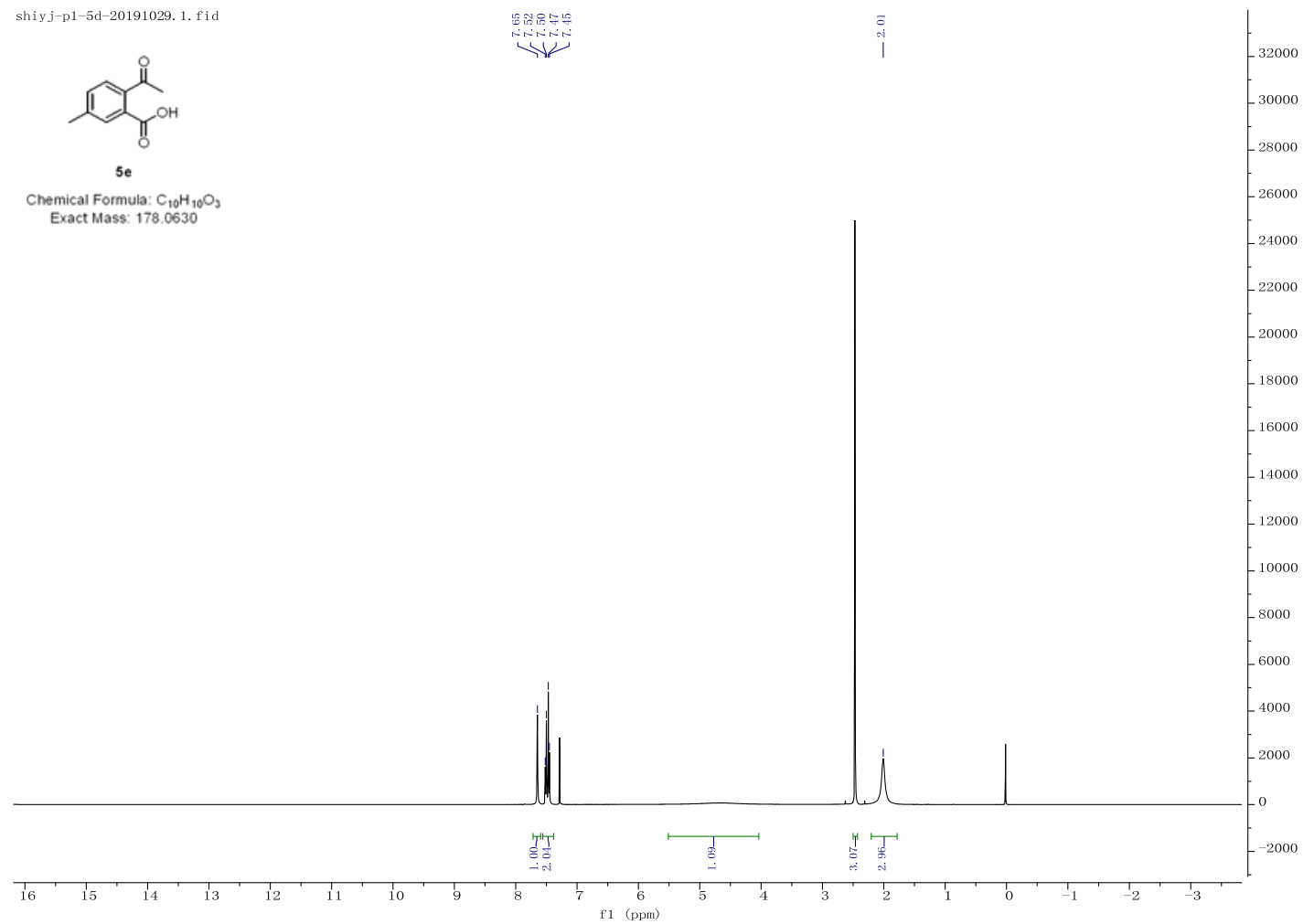

${ }^{13} \mathrm{C}\left\{{ }^{1} \mathrm{H}\right\}$ NMR (101 MHz, Methanol-d4) of compound 5e

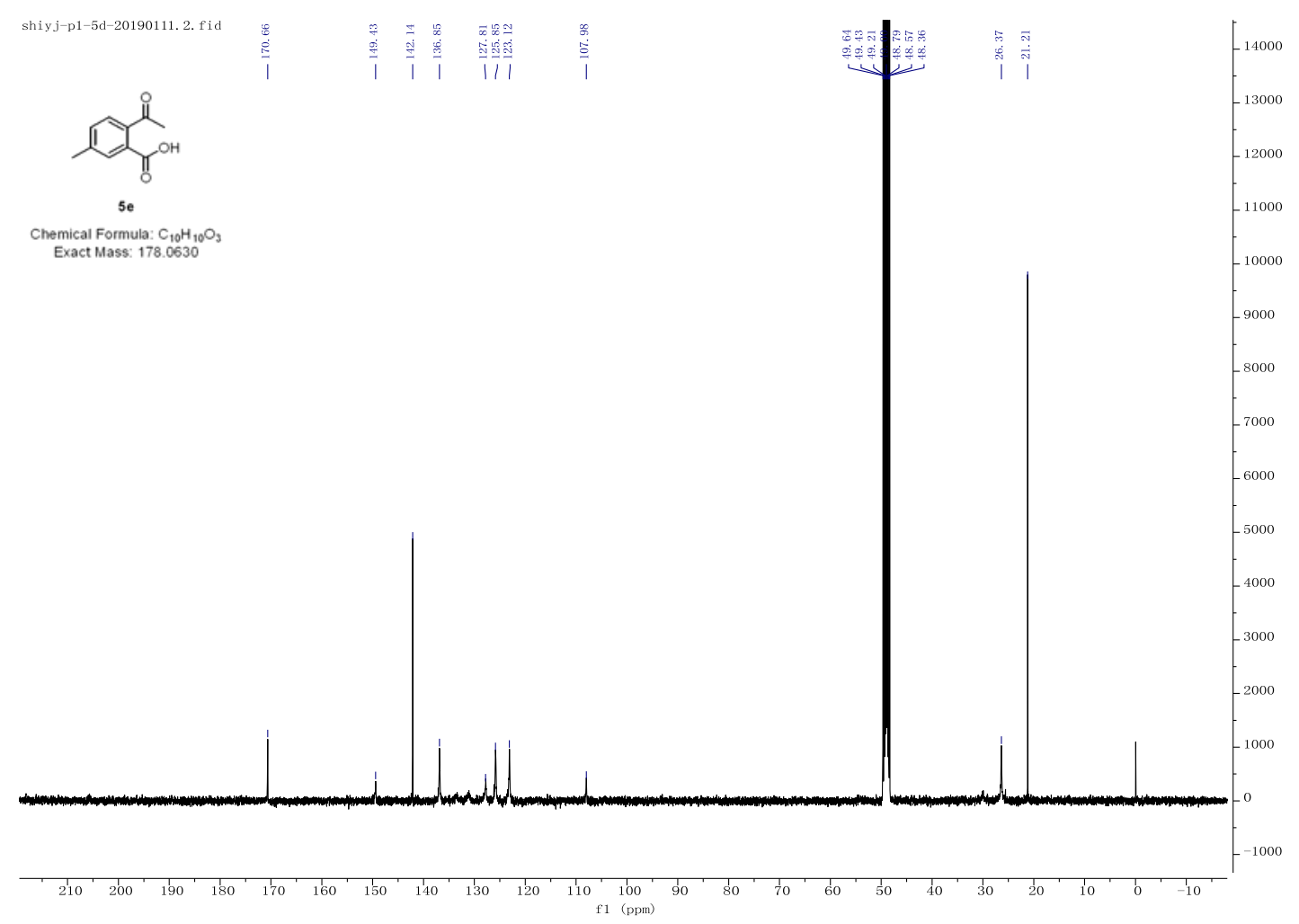


${ }^{1}$ H NMR (400 MHz, Chloroform-d) of compound $5 f$

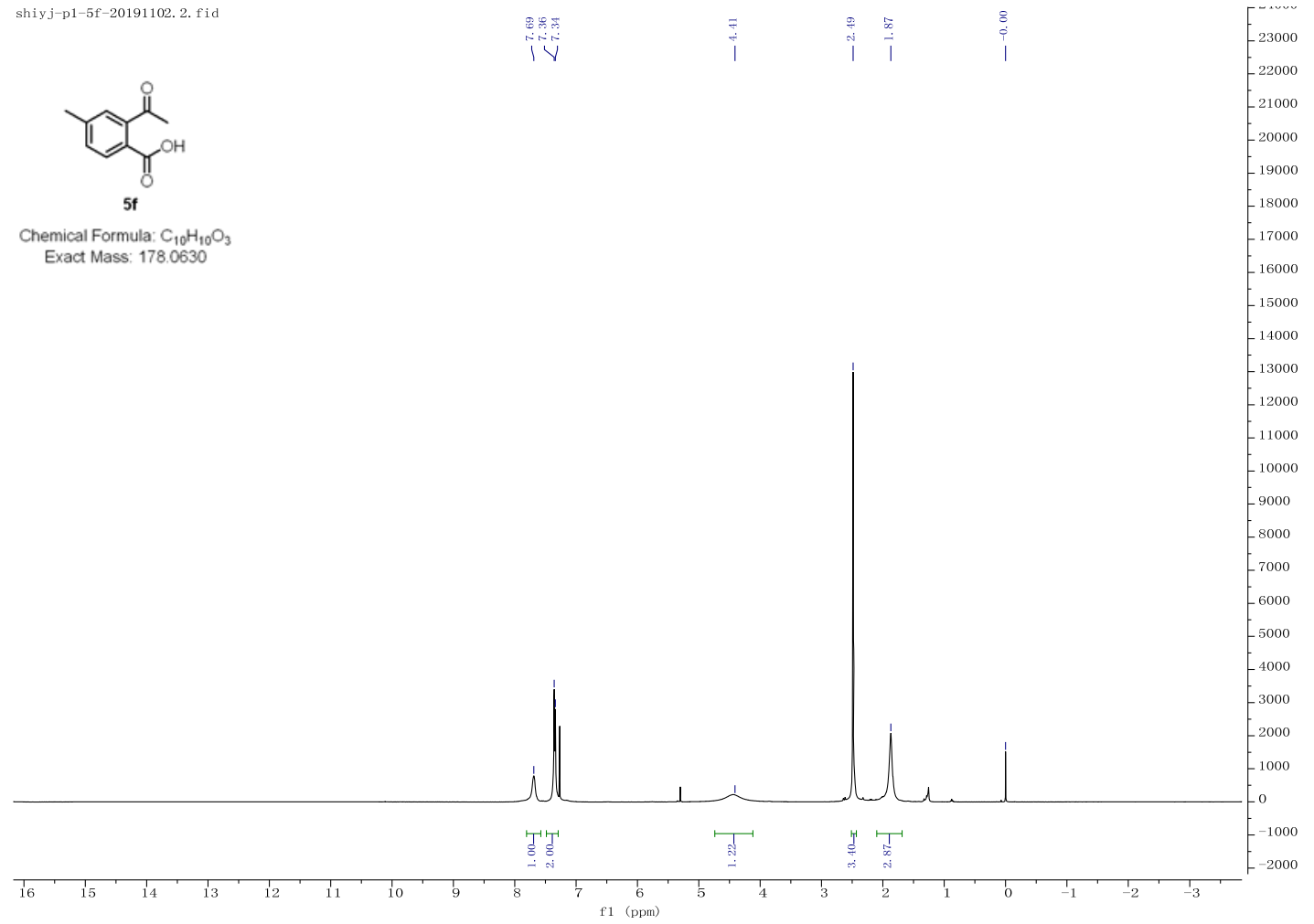

${ }^{13} \mathrm{C}\left\{{ }^{1} \mathrm{H}\right\}$ NMR (151 MHz, Chloroform-d) of compound $\mathbf{5 b}$

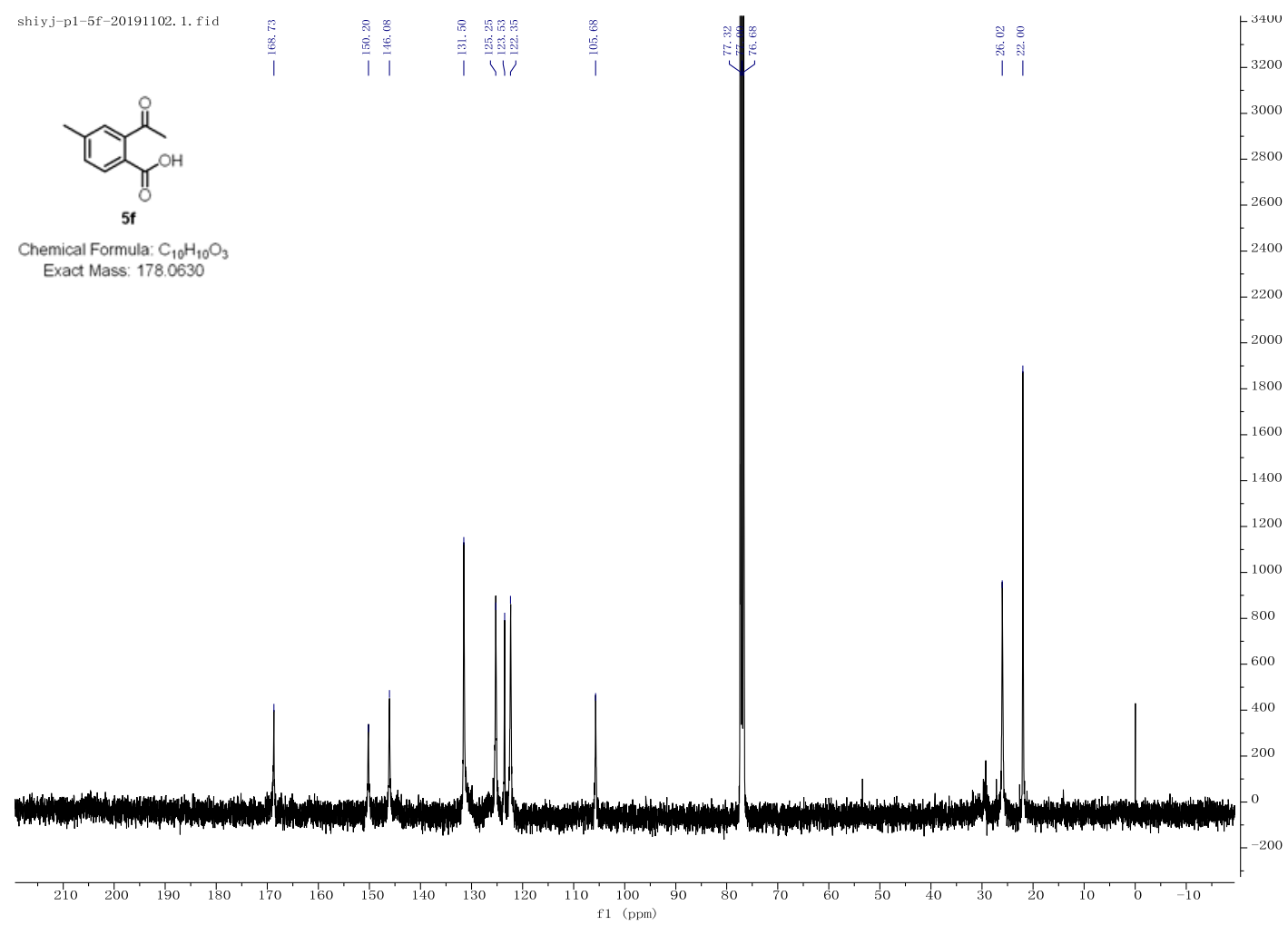


${ }^{1}$ H NMR (600 MHz, Chloroform-d) of compound $\mathbf{5 h}$

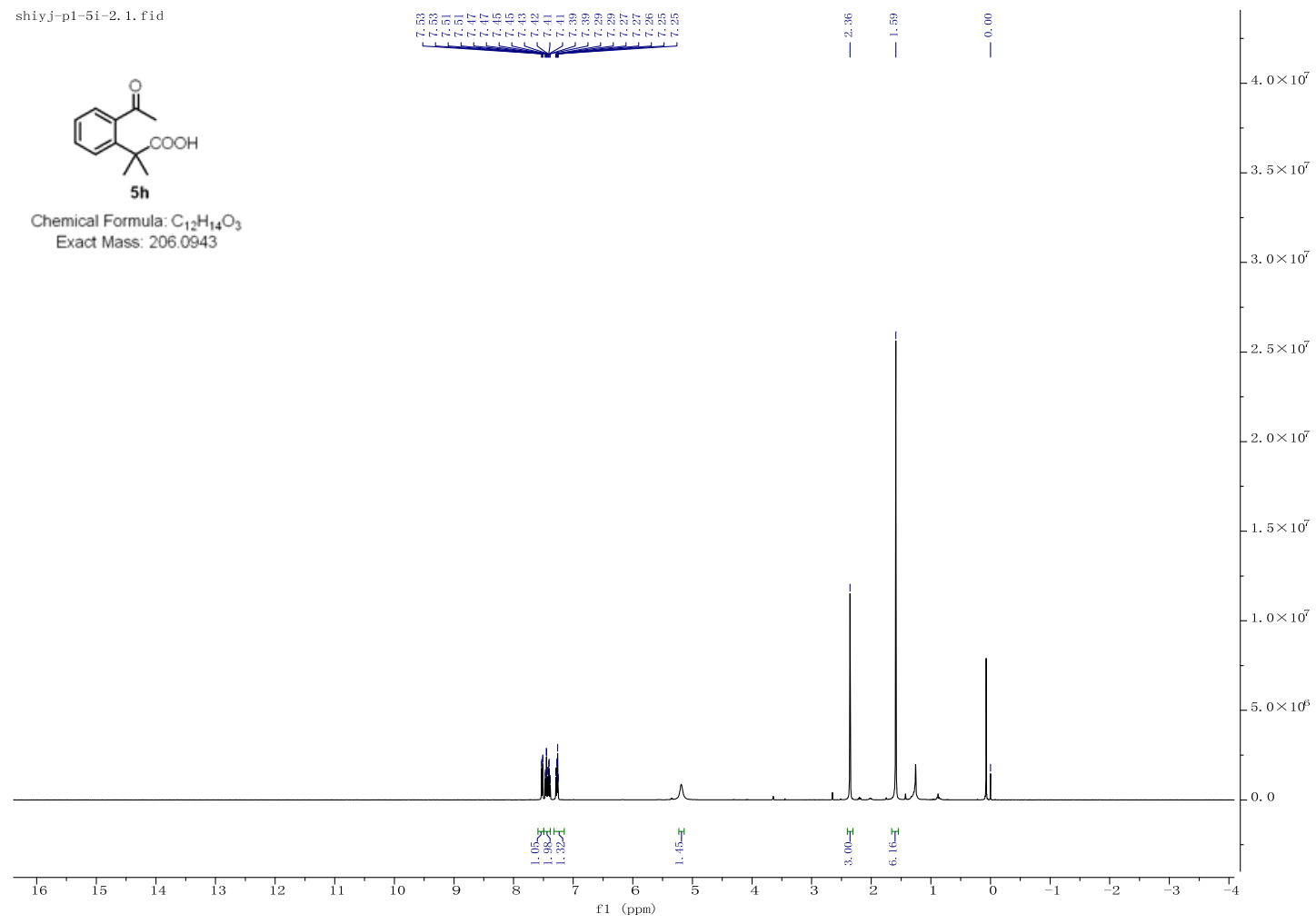

${ }^{13} \mathrm{C}\left\{{ }^{1} \mathrm{H}\right\}$ NMR (101 MHz, Chloroform-d) of compound $\mathbf{5 b}$

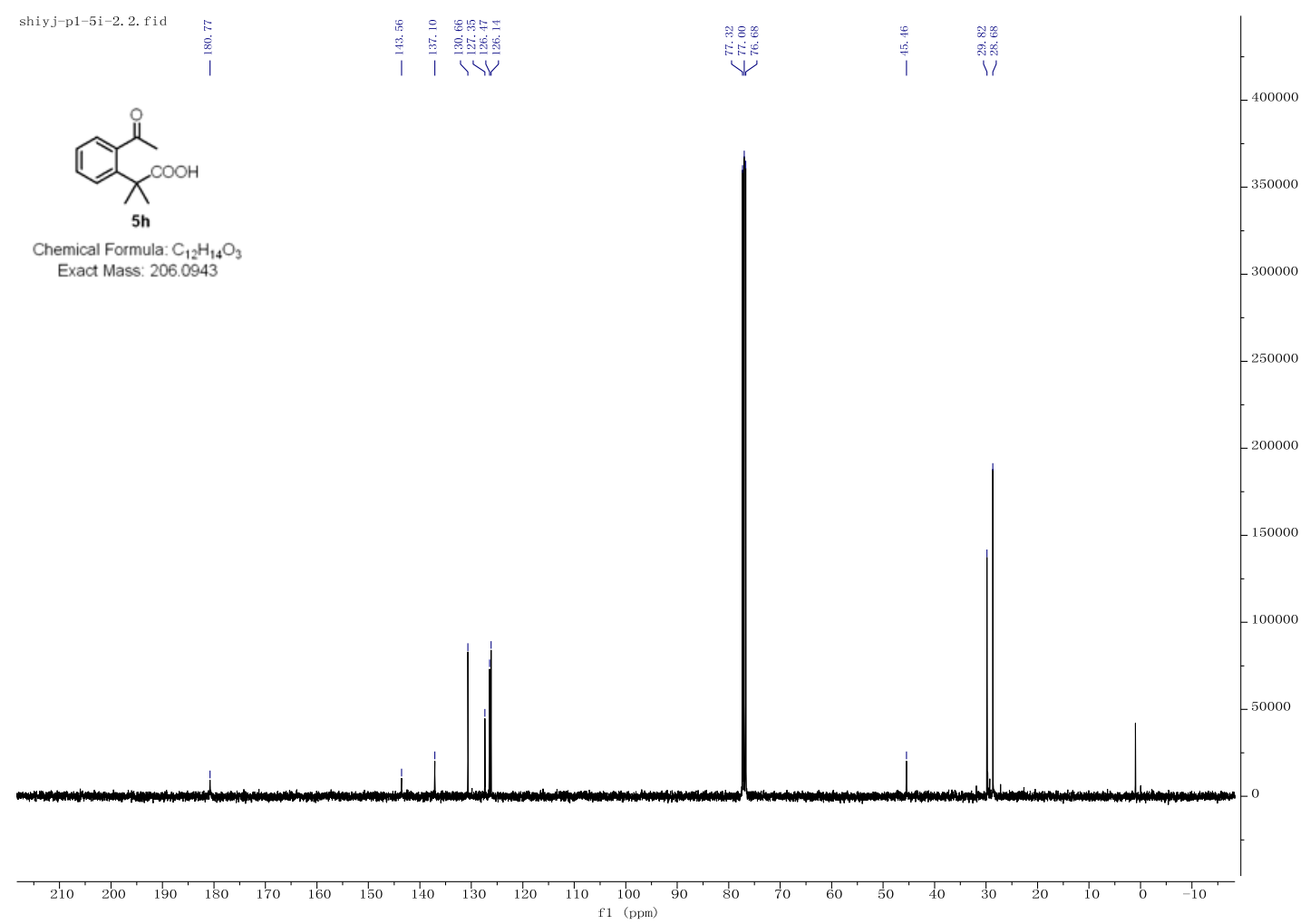


${ }^{1} \mathrm{H}$ NMR (400 MHz, Methanol-d4) of compound 5i

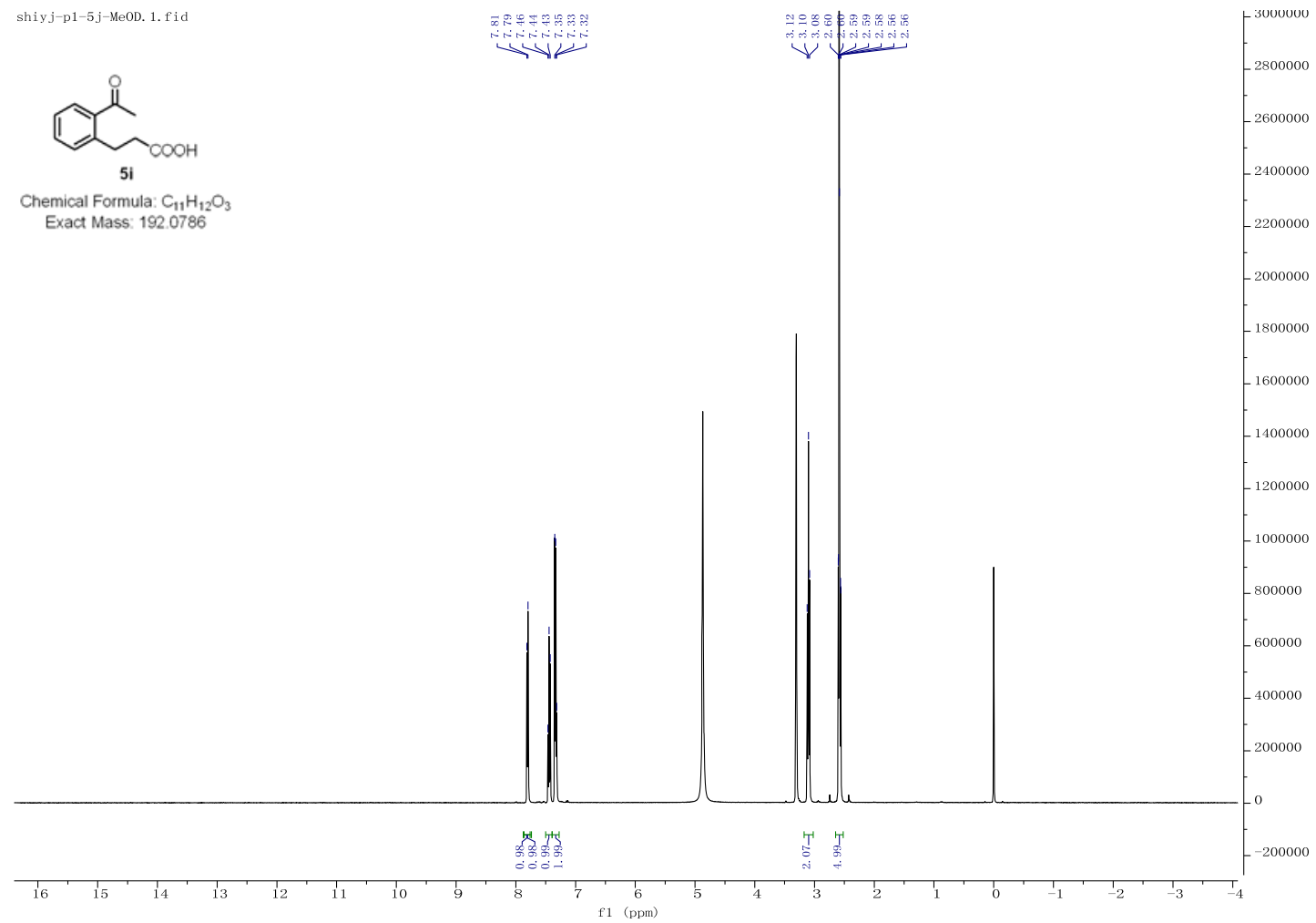

${ }^{13} \mathrm{C}\left\{{ }^{1} \mathrm{H}\right\}$ NMR (101 MHz, Methanol-d4) of compound 5i

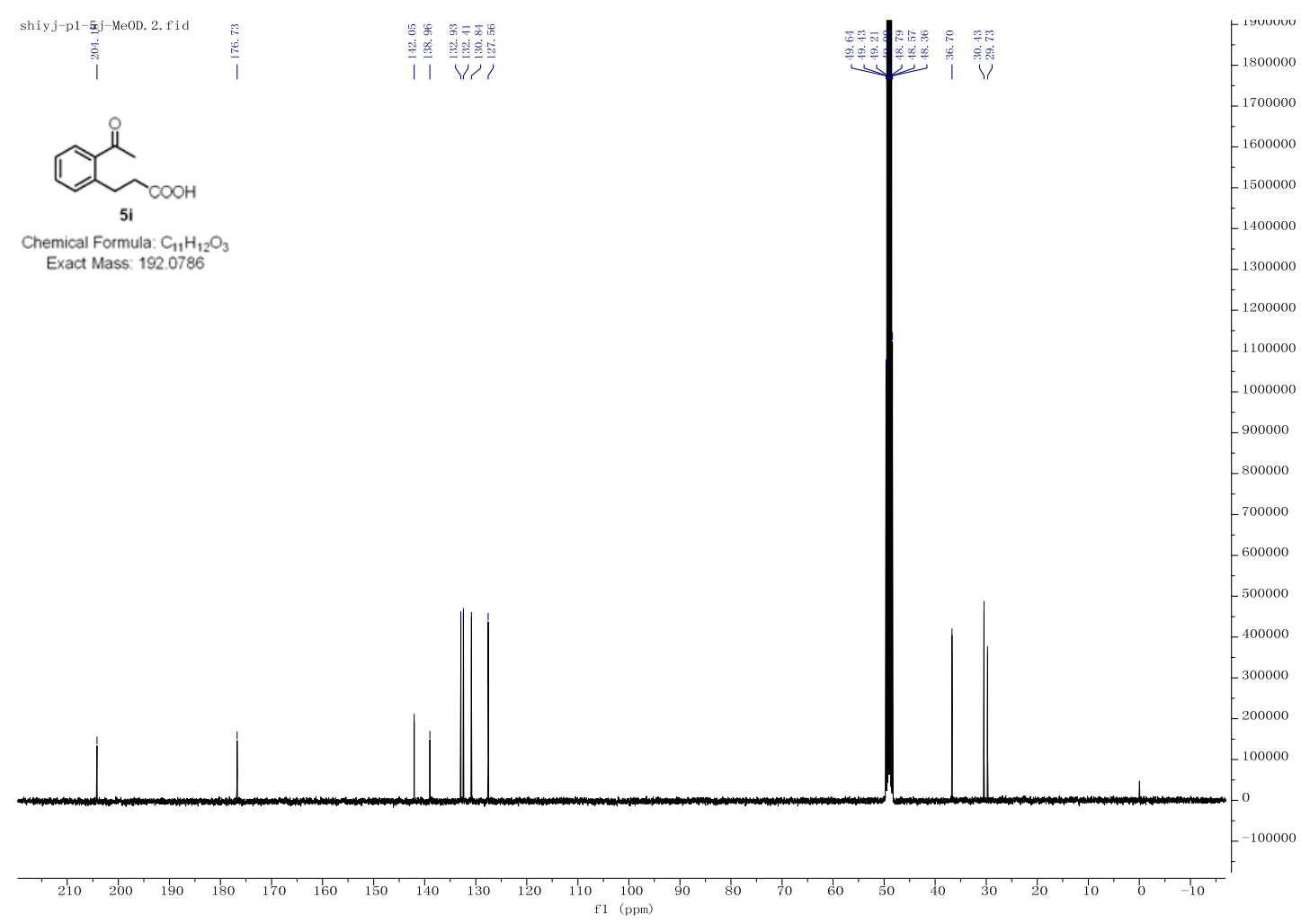


${ }^{1} \mathrm{H}$ NMR (600 MHz, Chloroform-d) of compound 2a

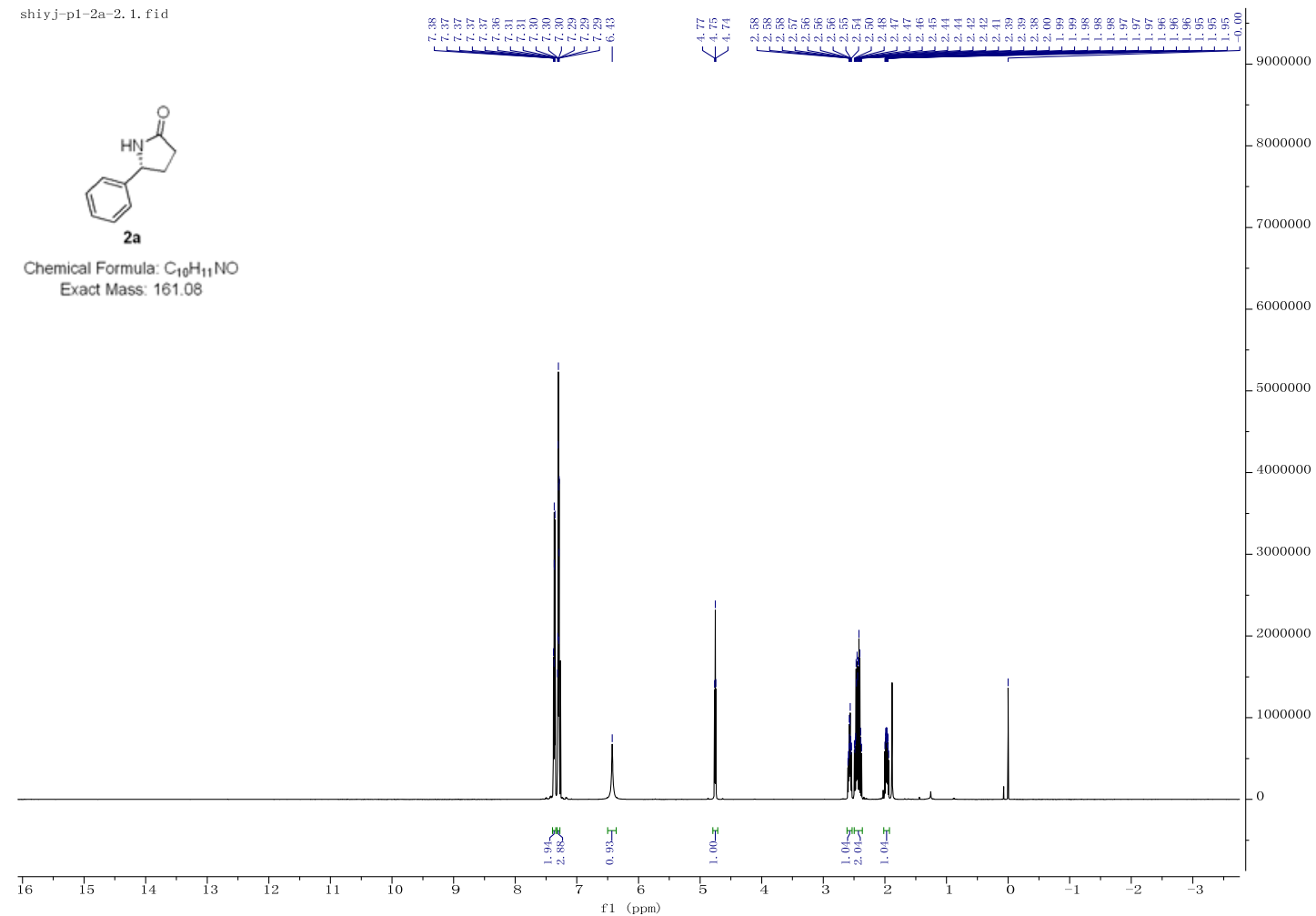

${ }^{13} \mathrm{C}\left\{{ }^{1} \mathrm{H}\right\}$ NMR (101 MHz, Chloroform-d) of compound 2a

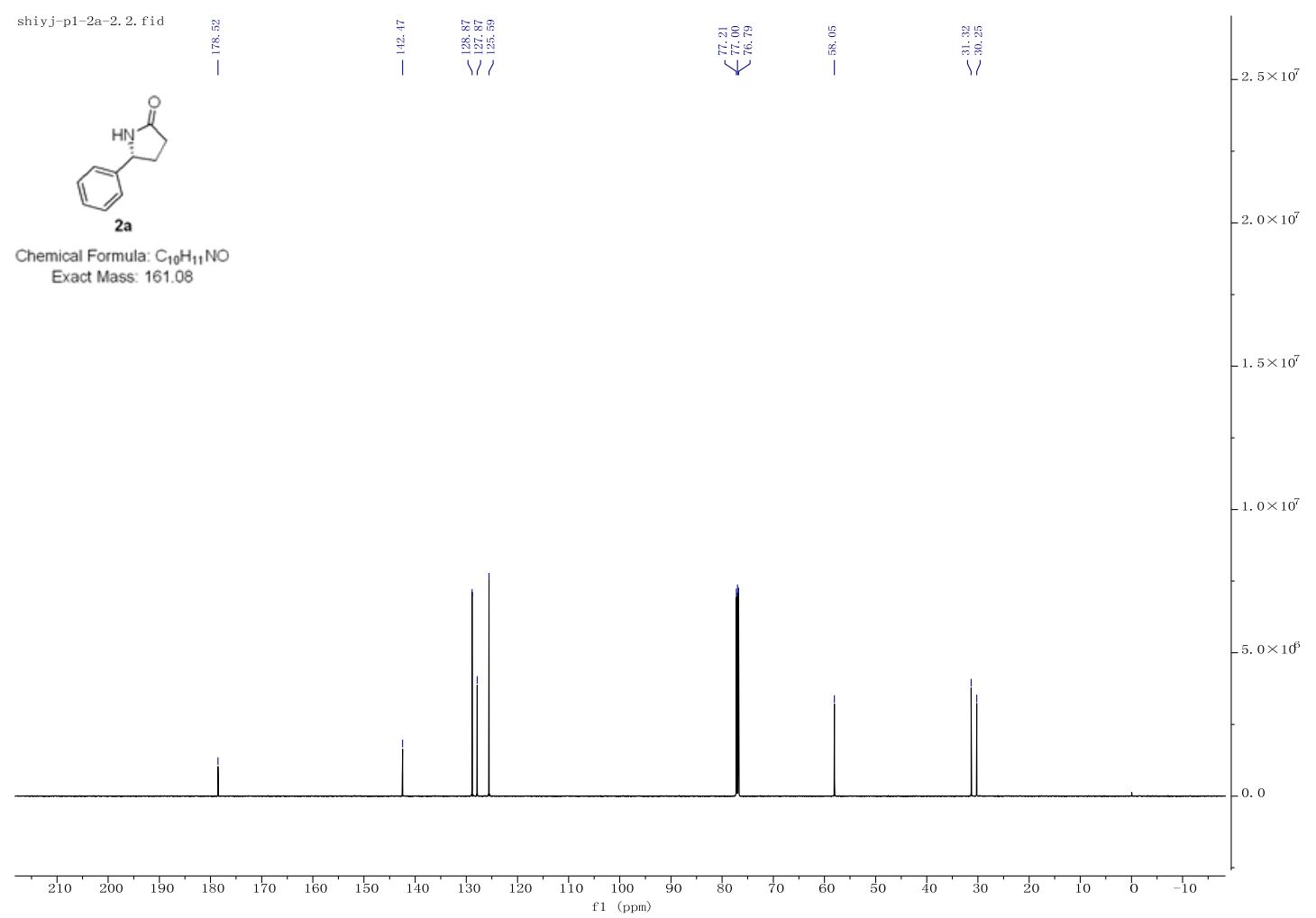


${ }^{1} \mathrm{H}$ NMR (600 MHz, Chloroform-d) of compound 2b

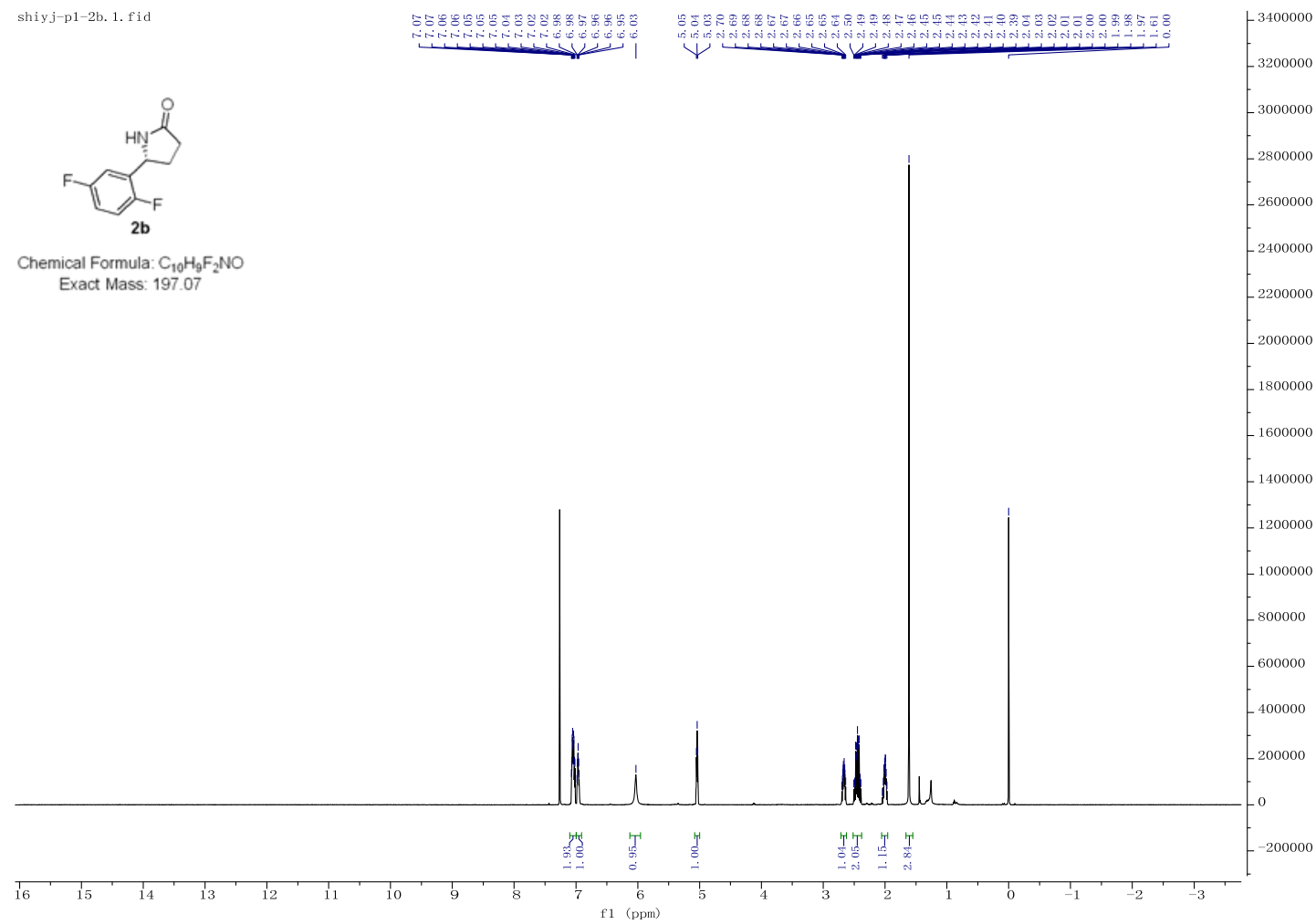

${ }^{13} \mathrm{C}\left\{{ }^{1} \mathrm{H}\right\}$ NMR (101 MHz, Chloroform-d) of compound $\mathbf{2 b}$

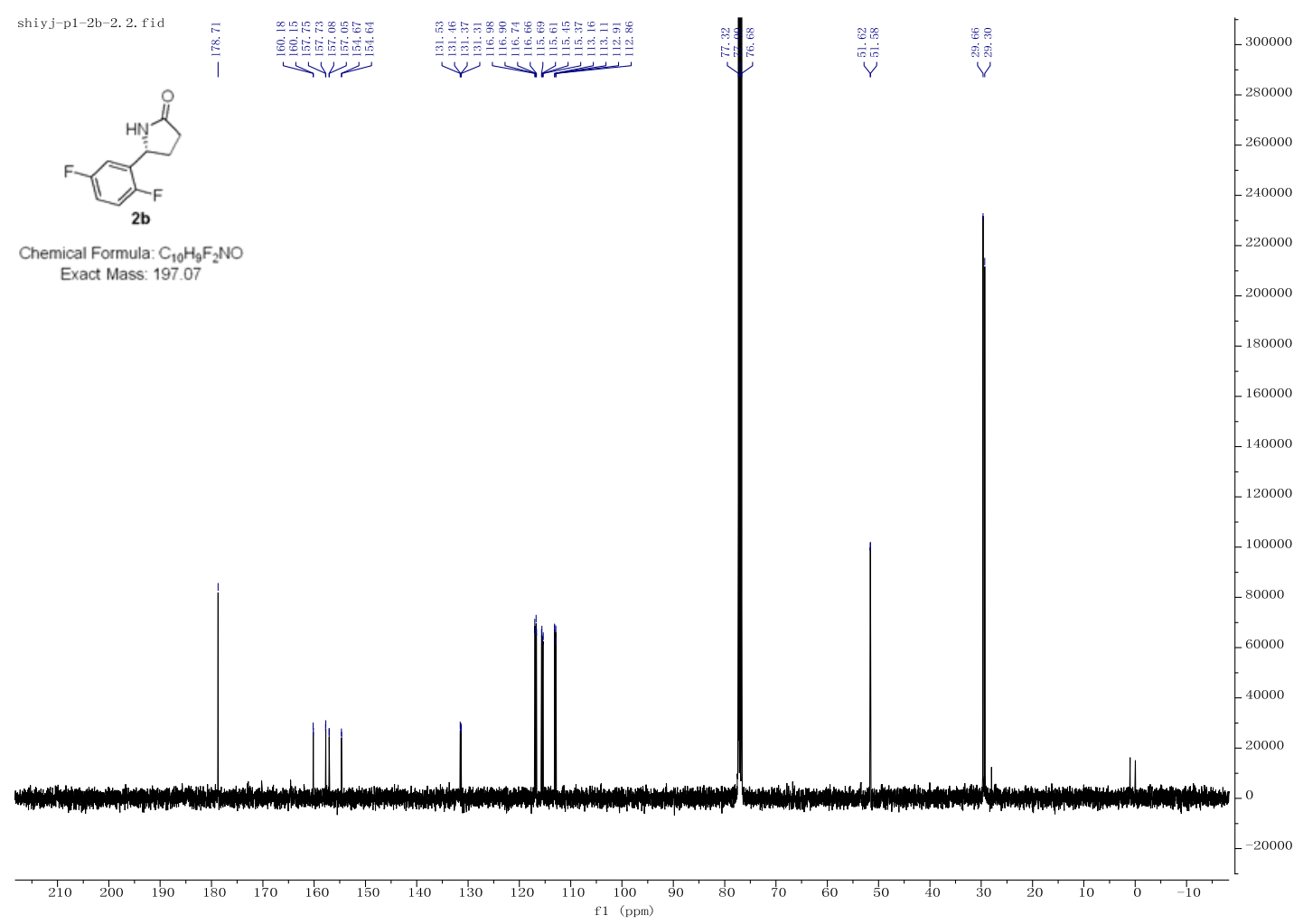


${ }^{1} \mathrm{H}$ NMR (400 MHz, Chloroform-d) of compound 2c

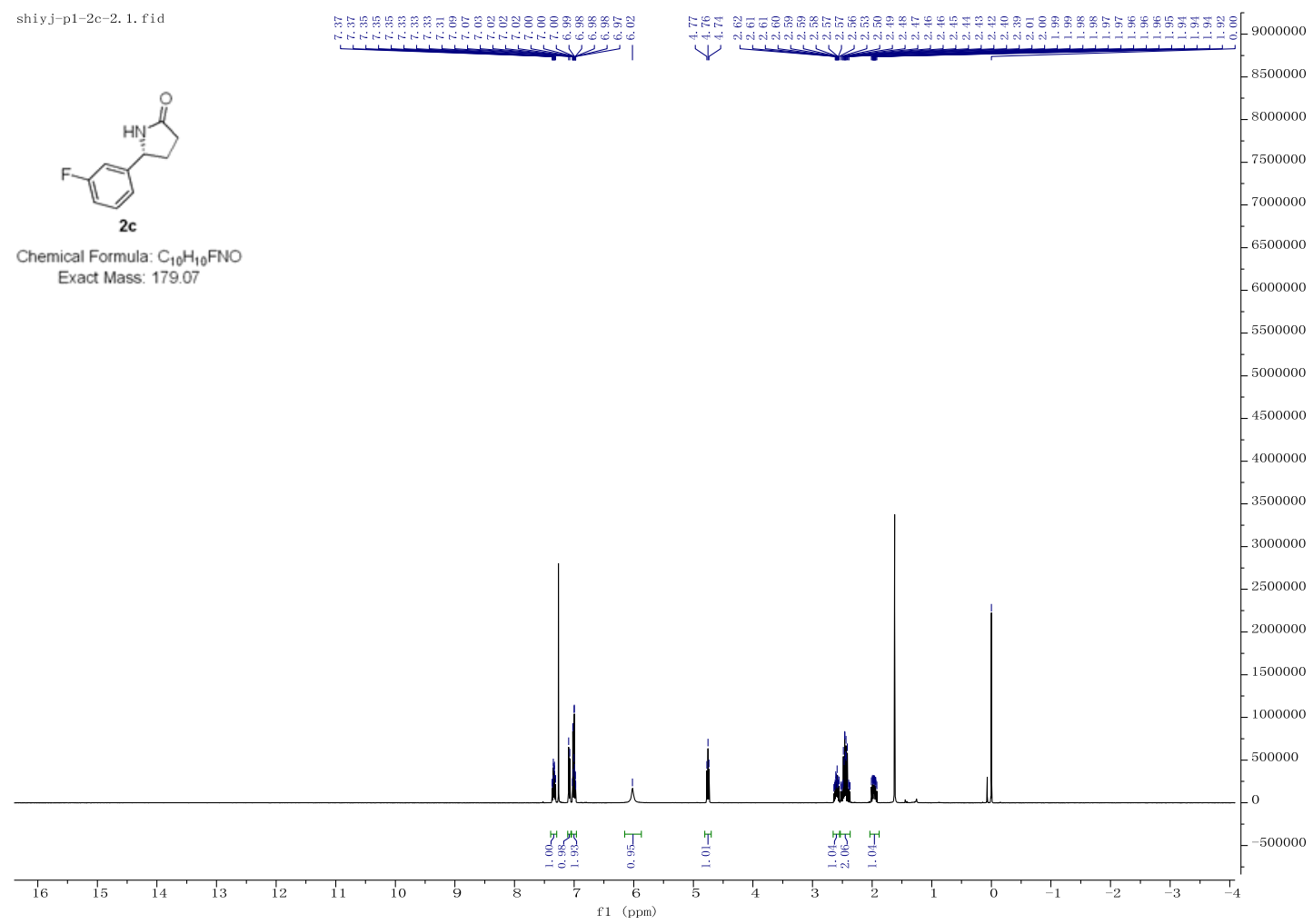

${ }^{13} \mathrm{C}\left\{{ }^{1} \mathrm{H}\right\}$ NMR (101 MHz, Chloroform-d) of compound 2c

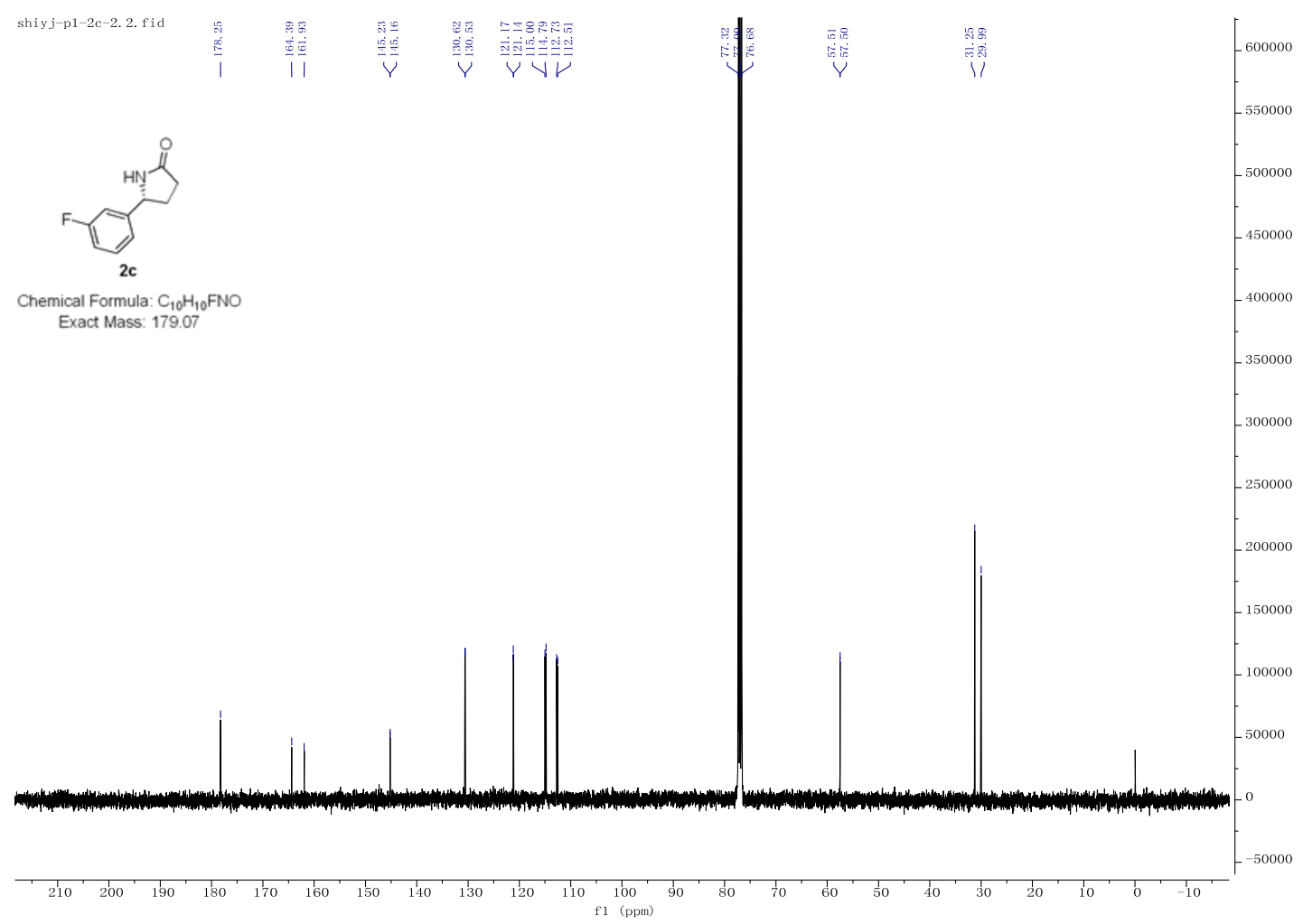


${ }^{1} \mathrm{H}$ NMR (400 MHz, Chloroform-d) of compound 2d

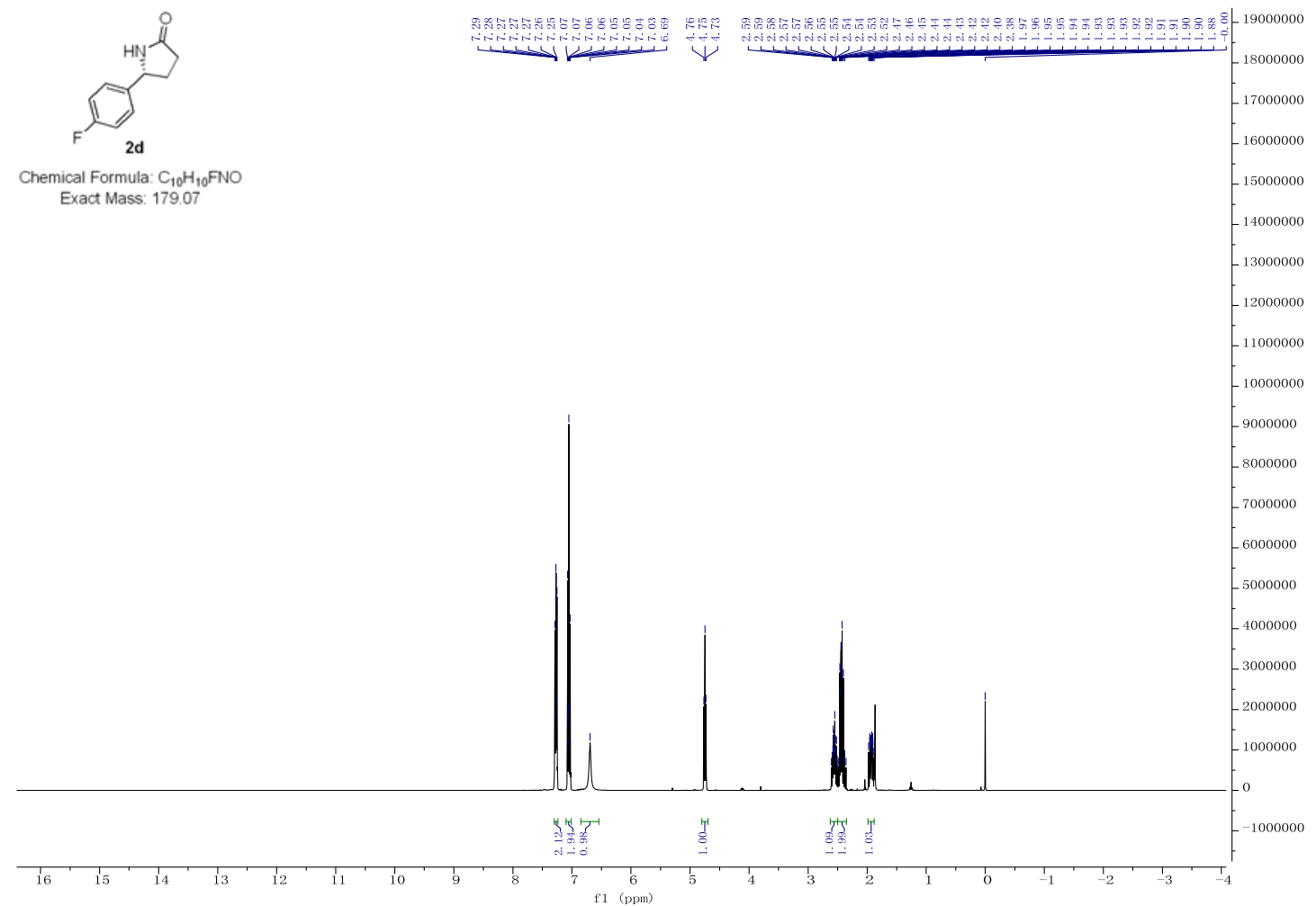

${ }^{13} \mathrm{C}\left\{{ }^{1} \mathrm{H}\right\}$ NMR (101 MHz, Chloroform-d) of compound 2d
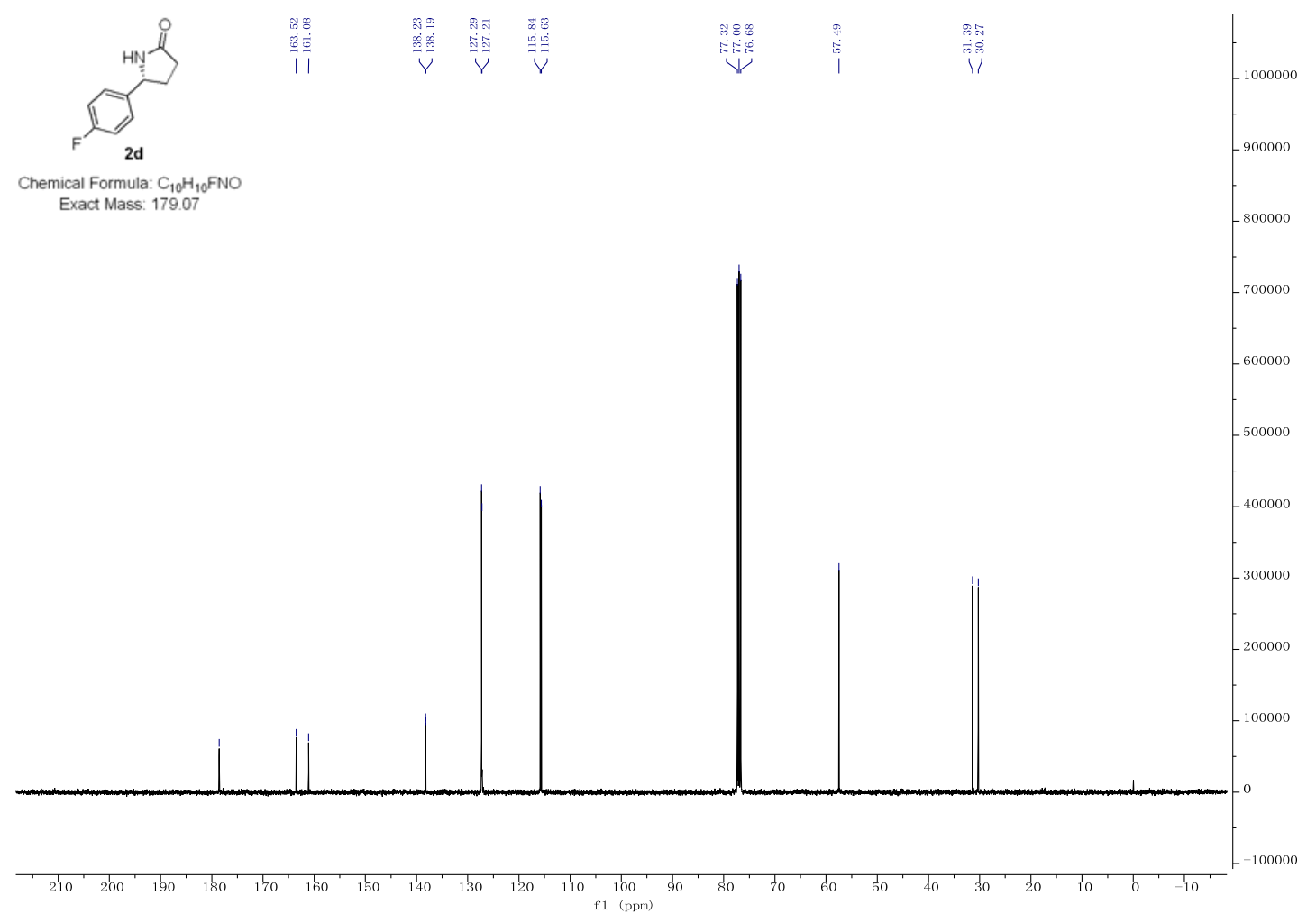
${ }^{1}$ H NMR (400 MHz, Chloroform-d) of compound $2 \mathbf{e}$

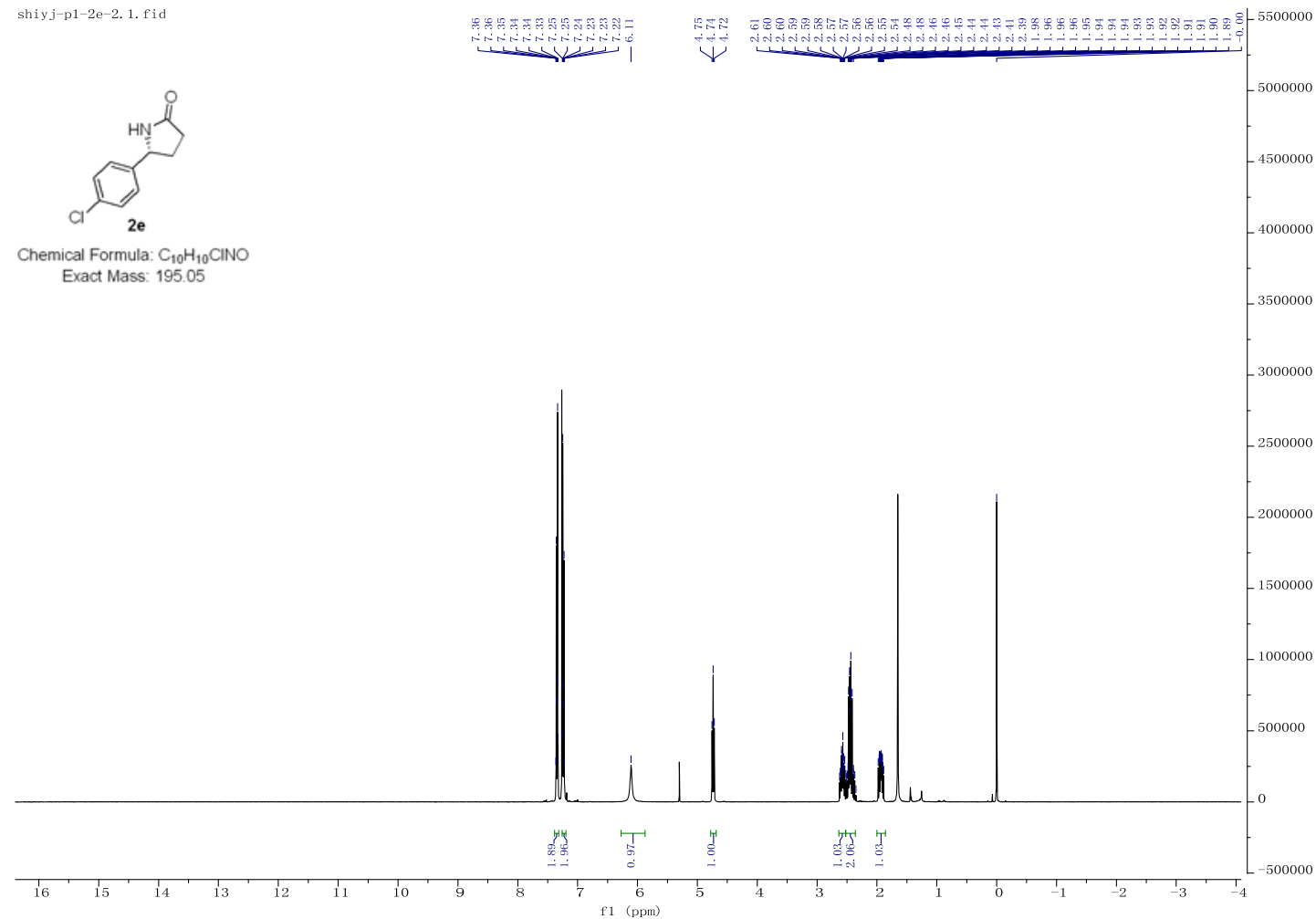

${ }^{13} \mathrm{C}\left\{{ }^{1} \mathrm{H}\right\}$ NMR (101 MHz, Chloroform-d) of compound 2e

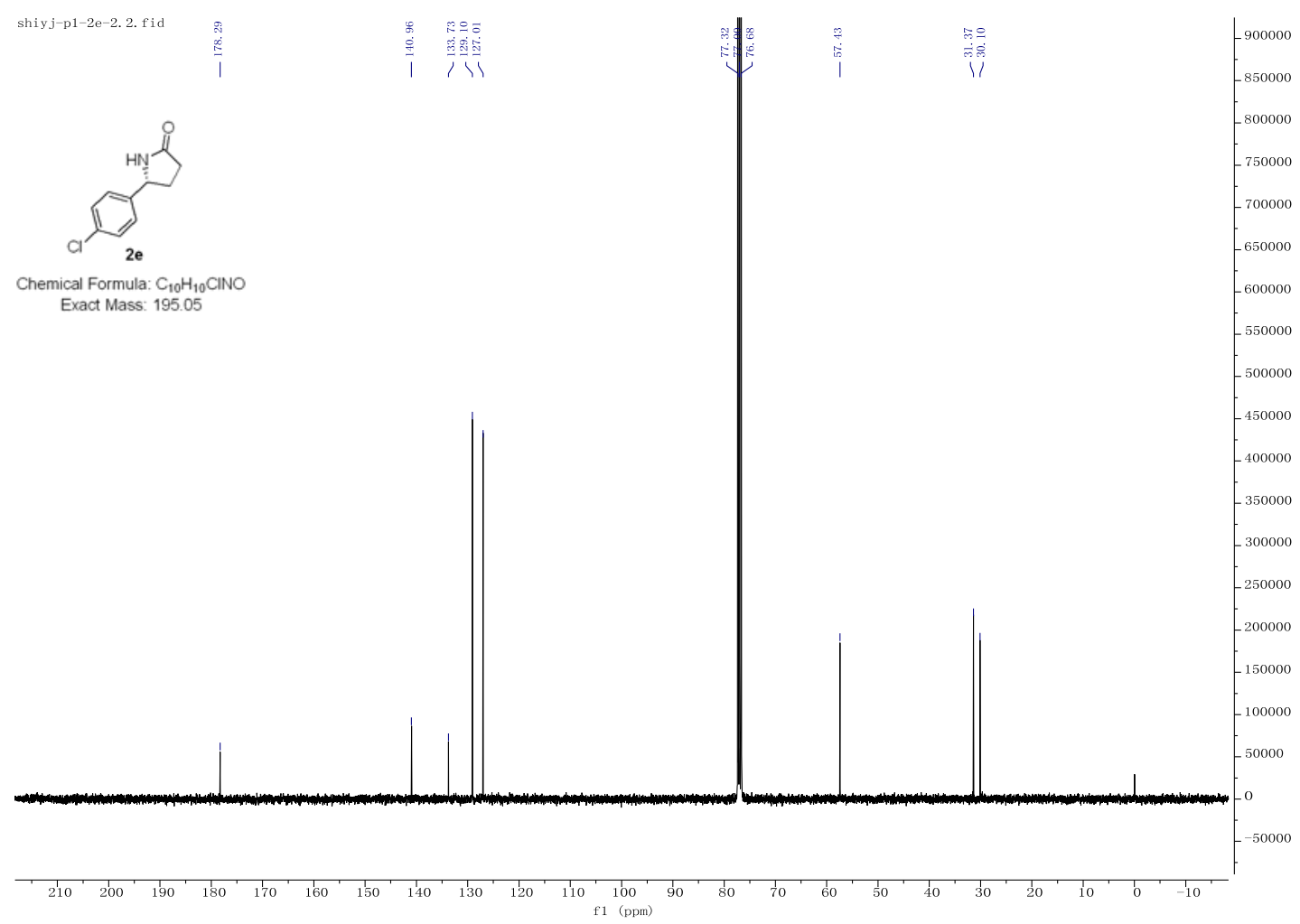


${ }^{1} \mathrm{H}$ NMR (400 MHz, Chloroform-d) of compound $2 \mathbf{f}$

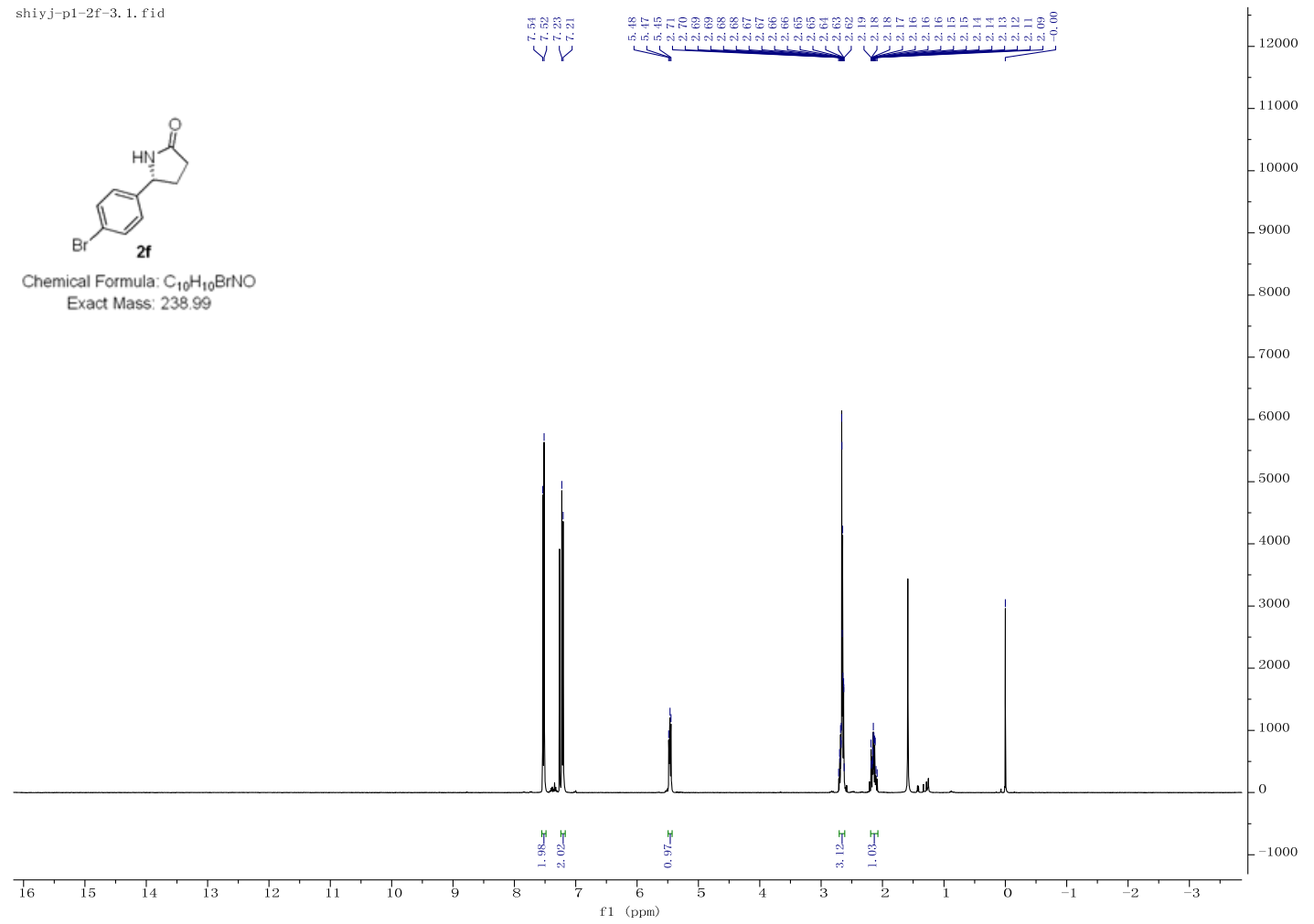

${ }^{13} \mathrm{C}\left\{{ }^{1} \mathrm{H}\right\}$ NMR (101 MHz, Chloroform-d) of compound $2 \mathrm{f}$

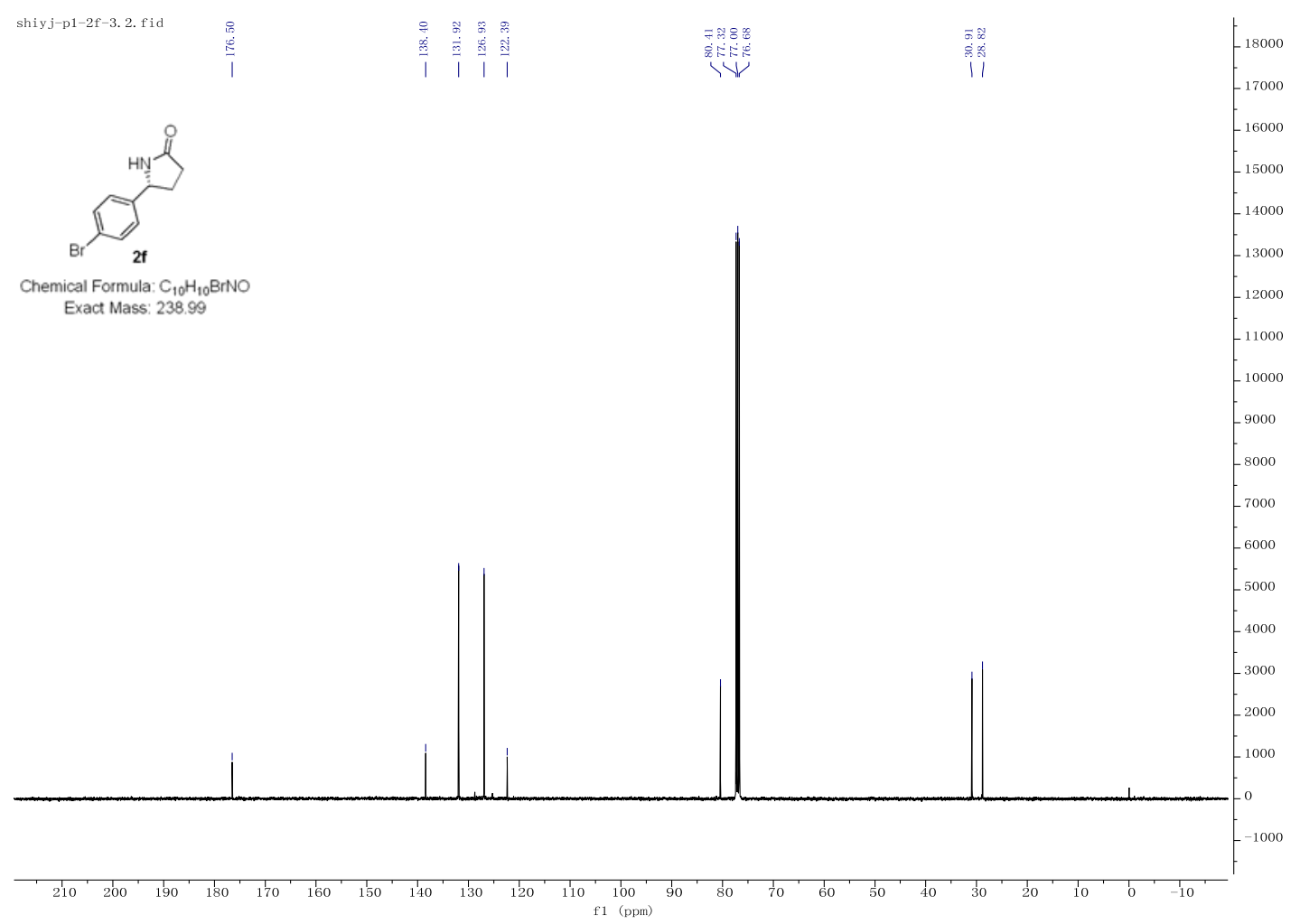


${ }^{1}$ H NMR (400 MHz, Chloroform-d) of compound $2 \mathrm{~g}$

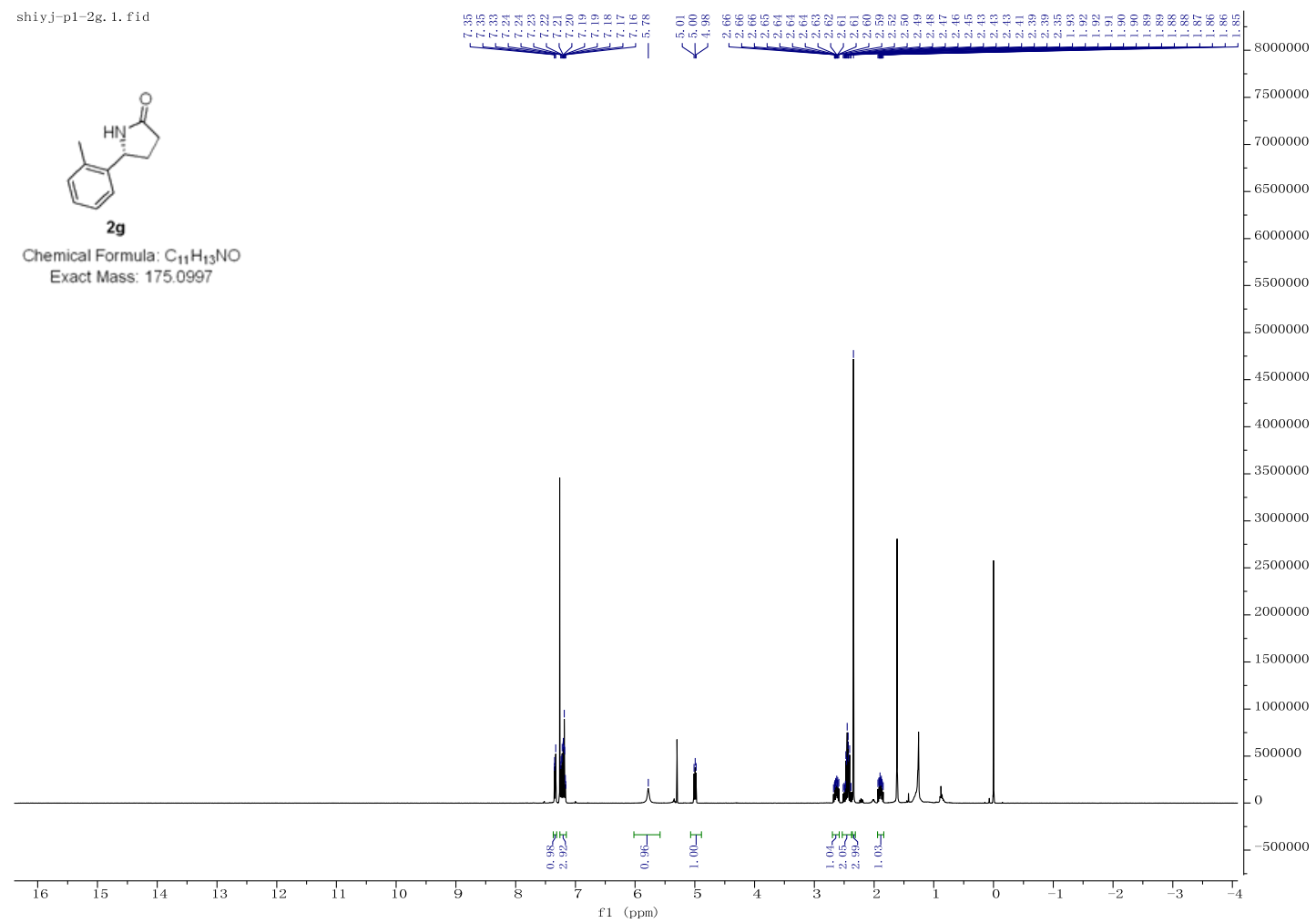

${ }^{13} \mathrm{C}\left\{{ }^{1} \mathrm{H}\right\}$ NMR (101 MHz, Chloroform-d) of compound $\mathbf{2 g}$

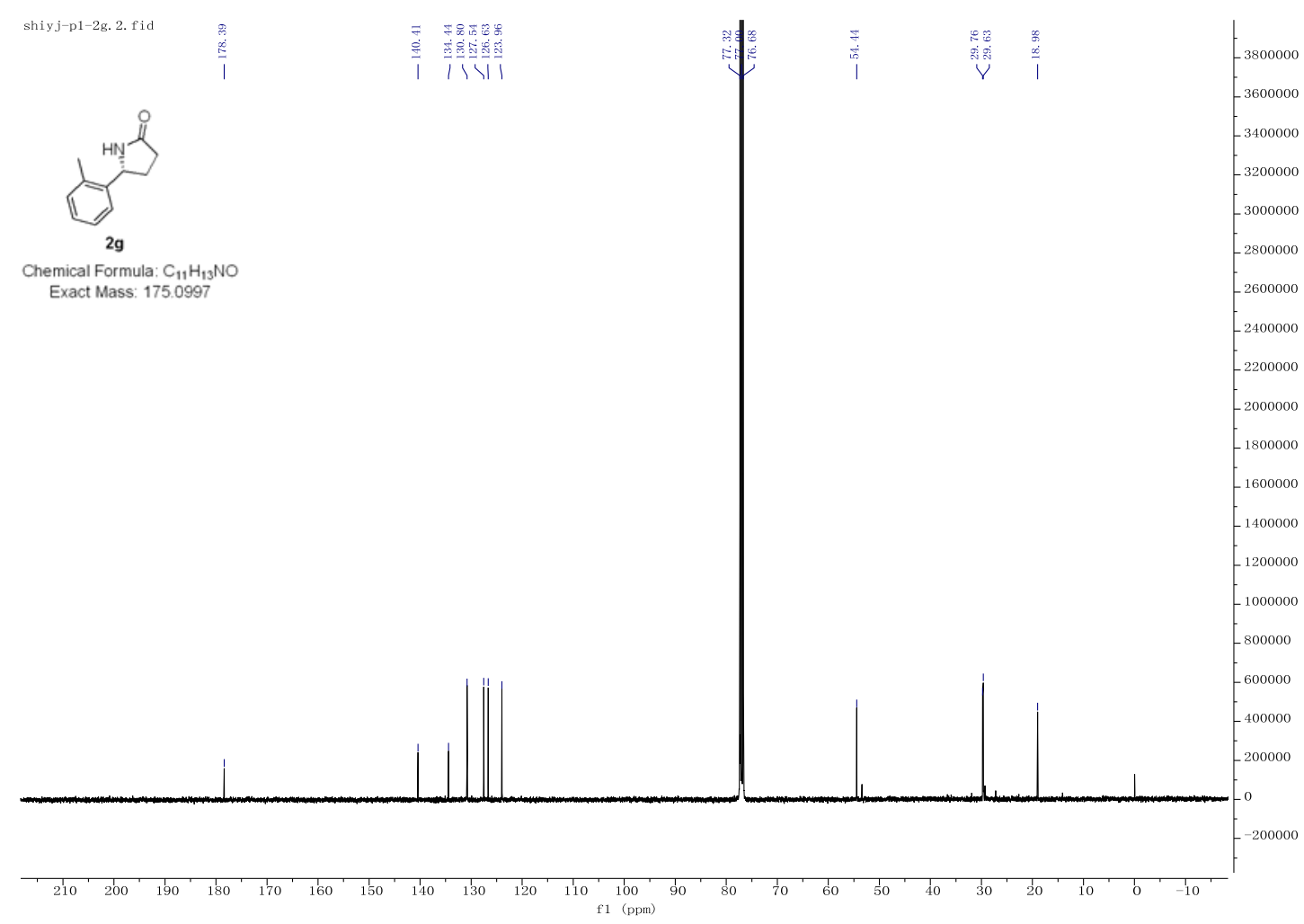


${ }^{1}$ H NMR (600 MHz, Chloroform-d) of compound $2 \mathbf{h}$

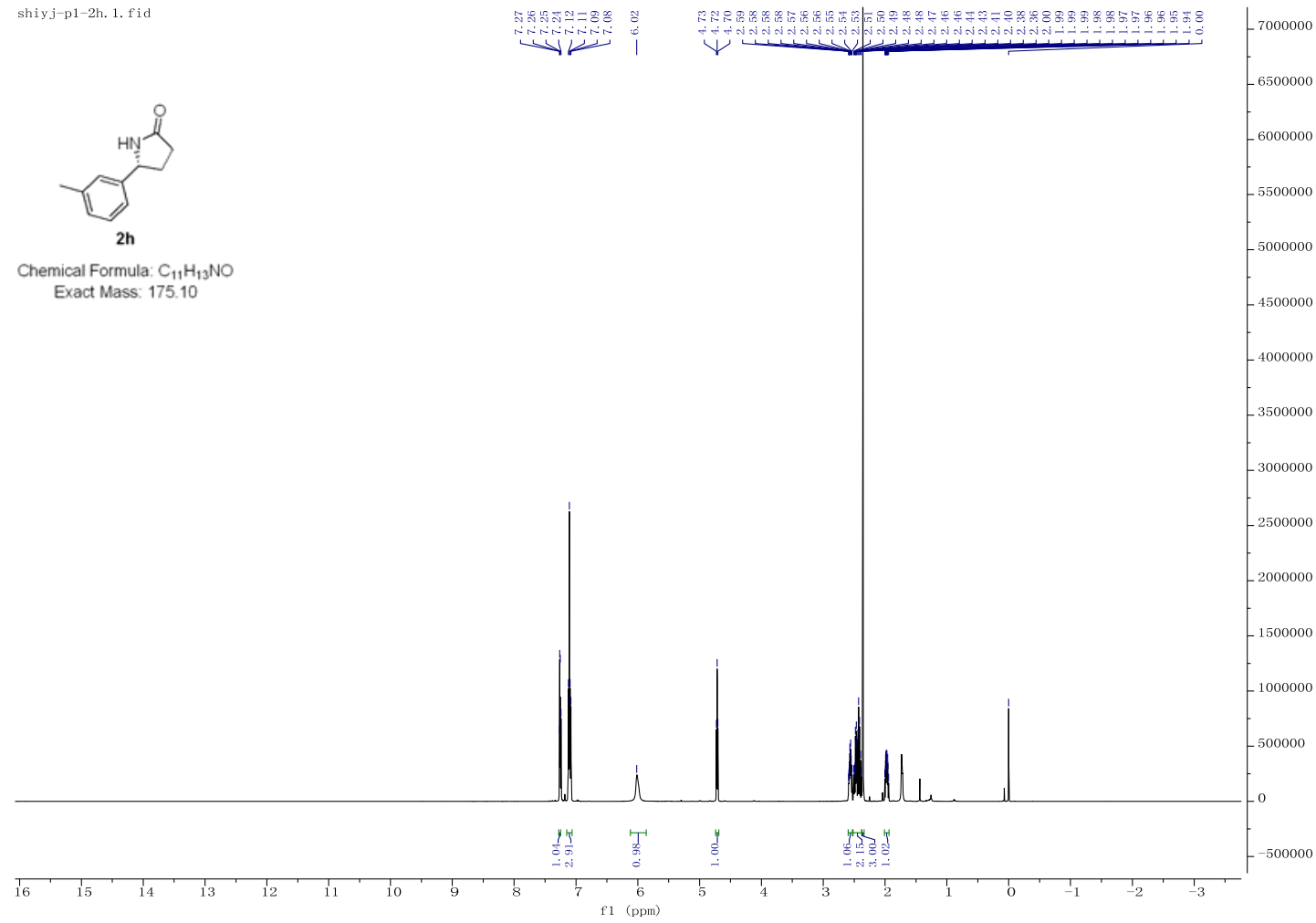

${ }^{13} \mathrm{C}\left\{{ }^{1} \mathrm{H}\right\}$ NMR (151 MHz, Chloroform-d) of compound $\mathbf{2 h}$

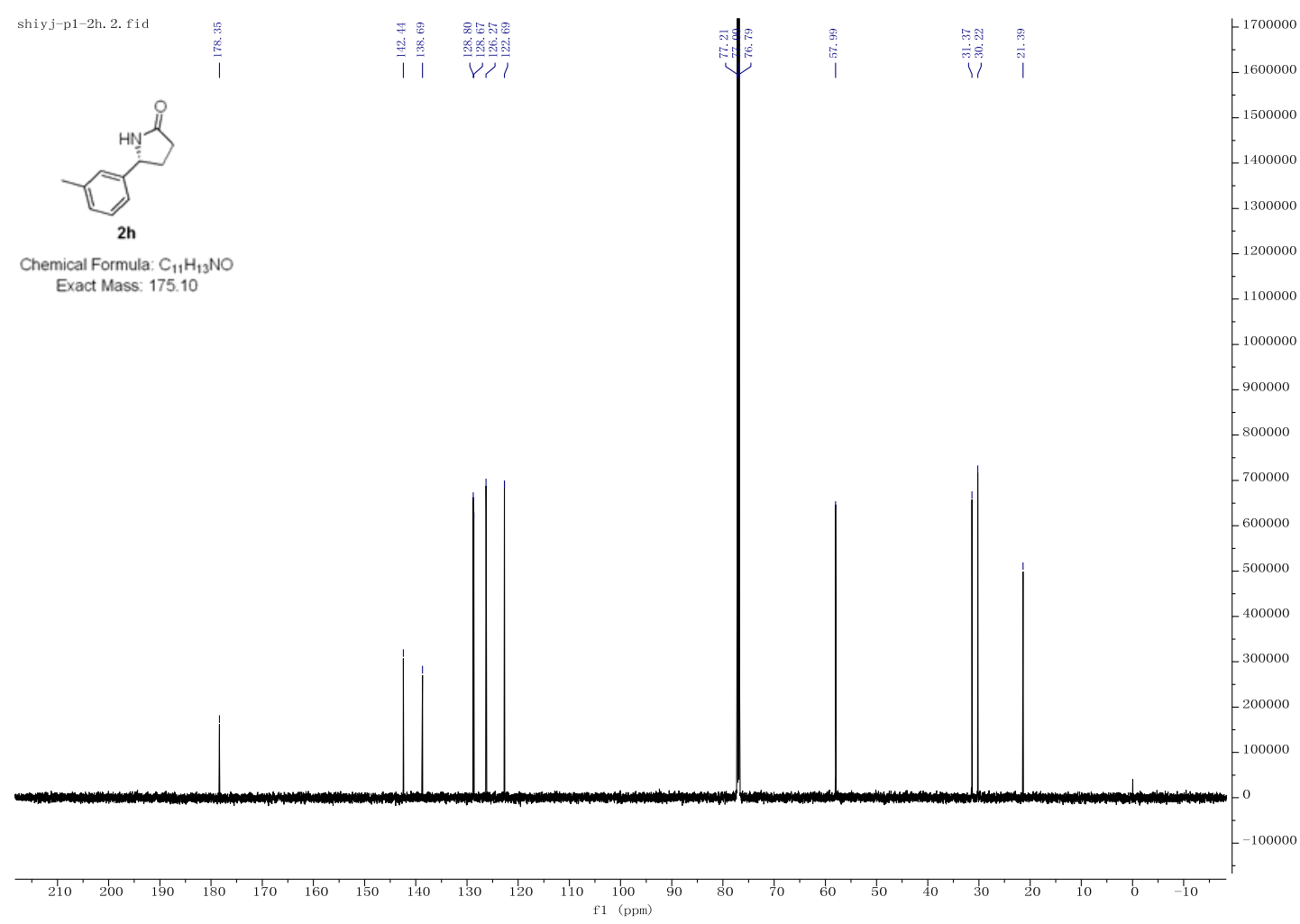


${ }^{1}$ H NMR (600 MHz, Chloroform-d) of compound 2i

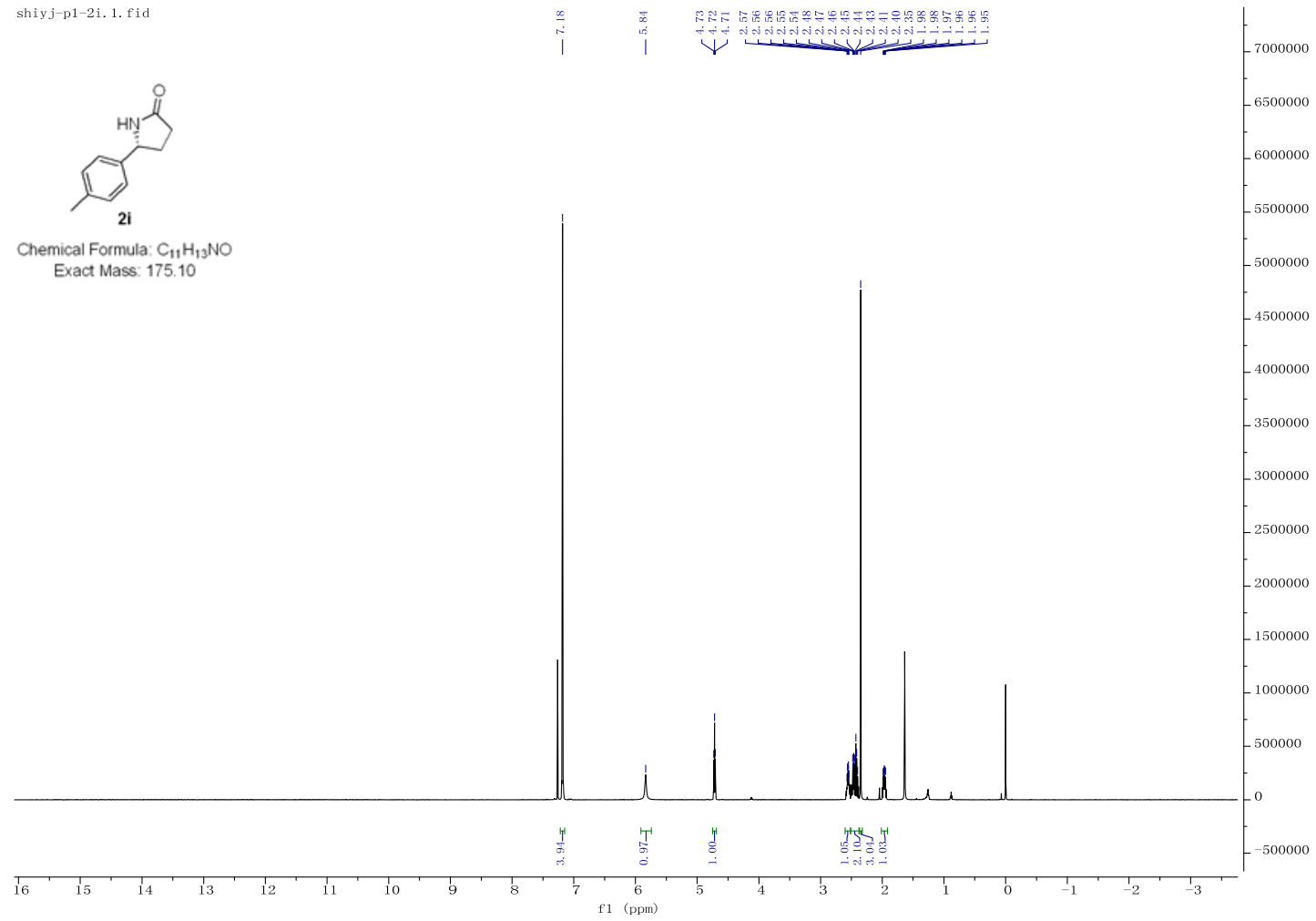

${ }^{13} \mathrm{C}\left\{{ }^{1} \mathrm{H}\right\}$ NMR (151 MHz, Chloroform-d) of compound $2 \mathbf{i}$

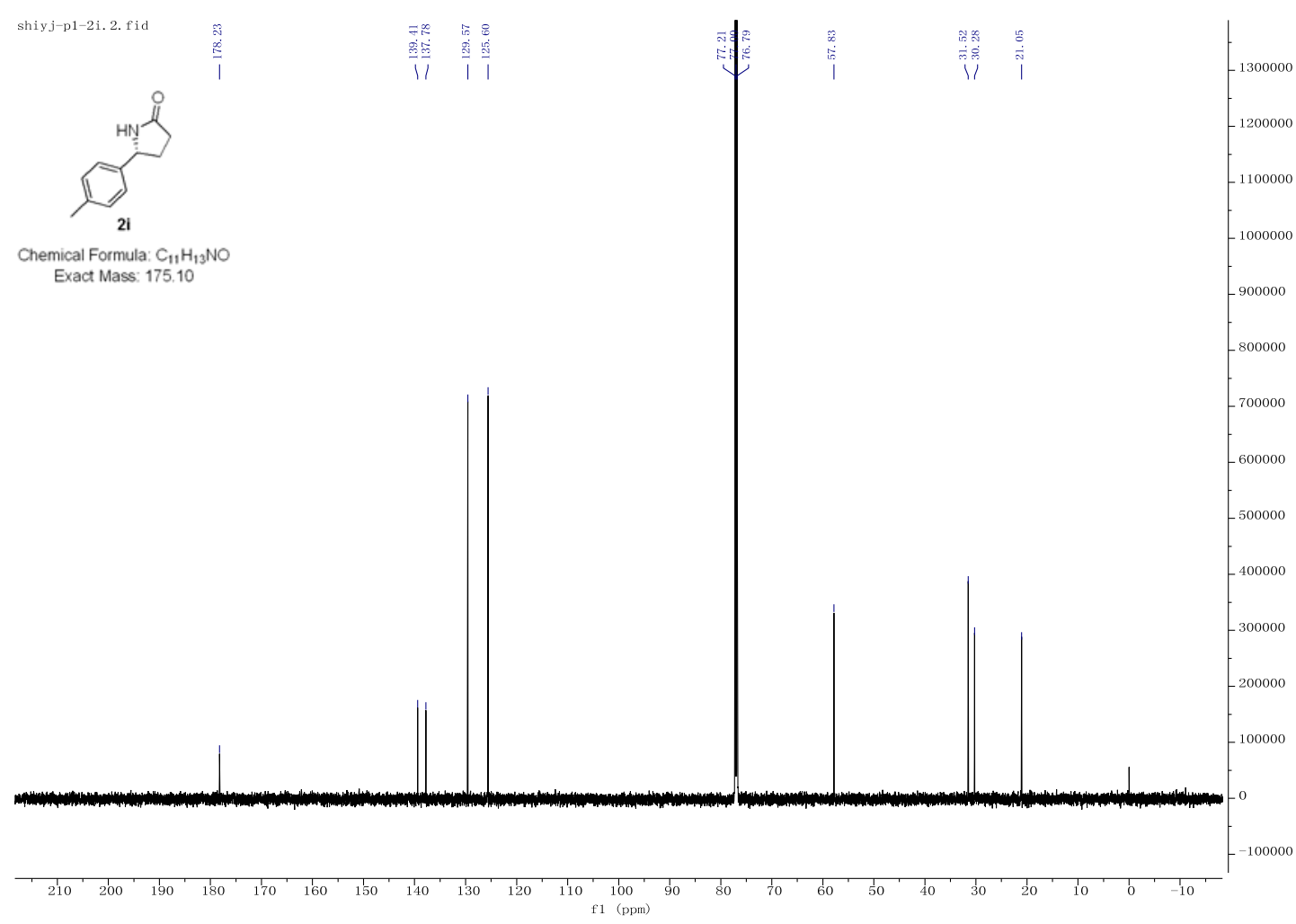


${ }^{1} \mathrm{H}$ NMR (400 MHz, Chloroform-d) of compound 2j

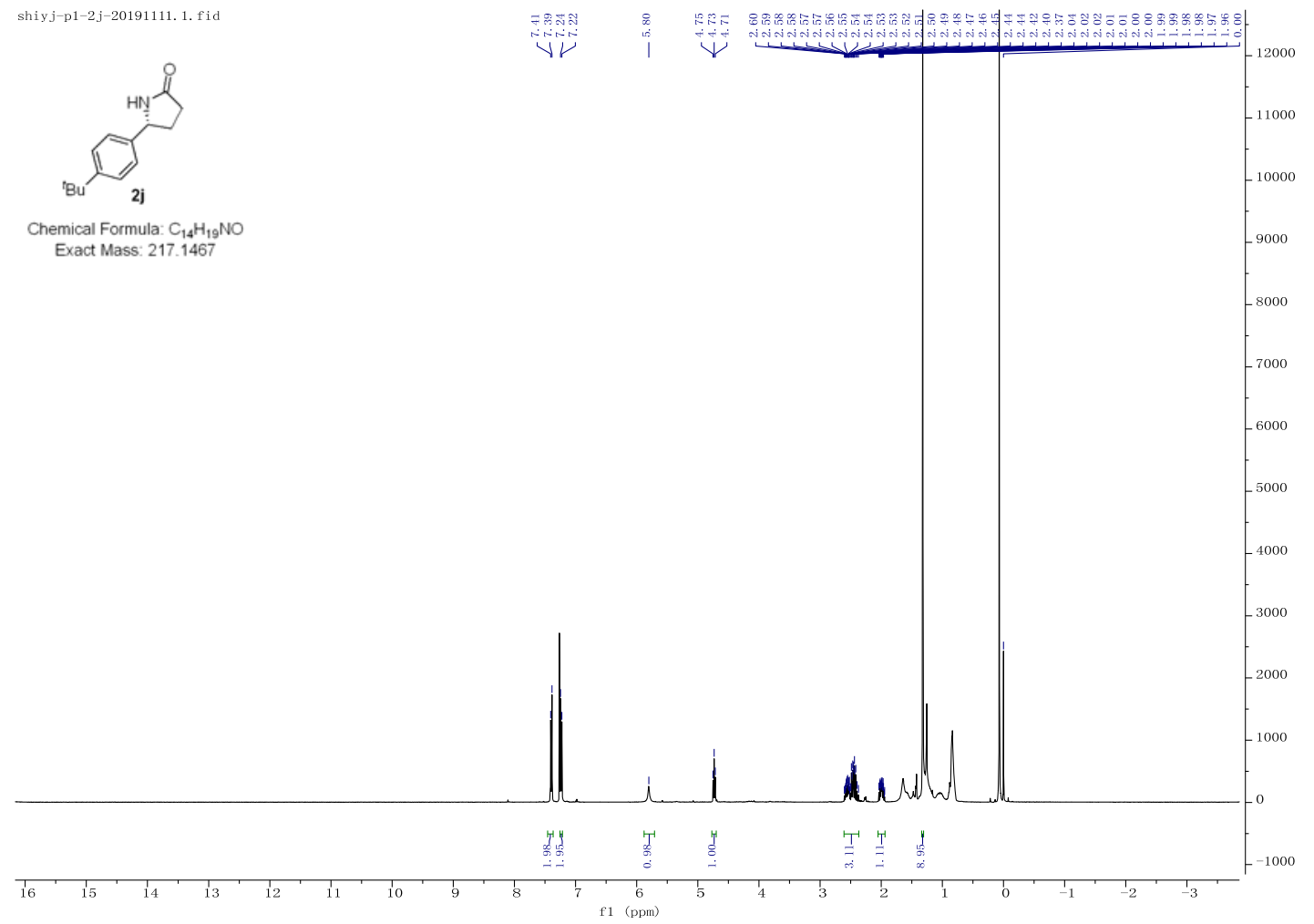

${ }^{13} \mathrm{C}\left\{{ }^{1} \mathrm{H}\right\}$ NMR (101 MHz, Chloroform-d) of compound 2j

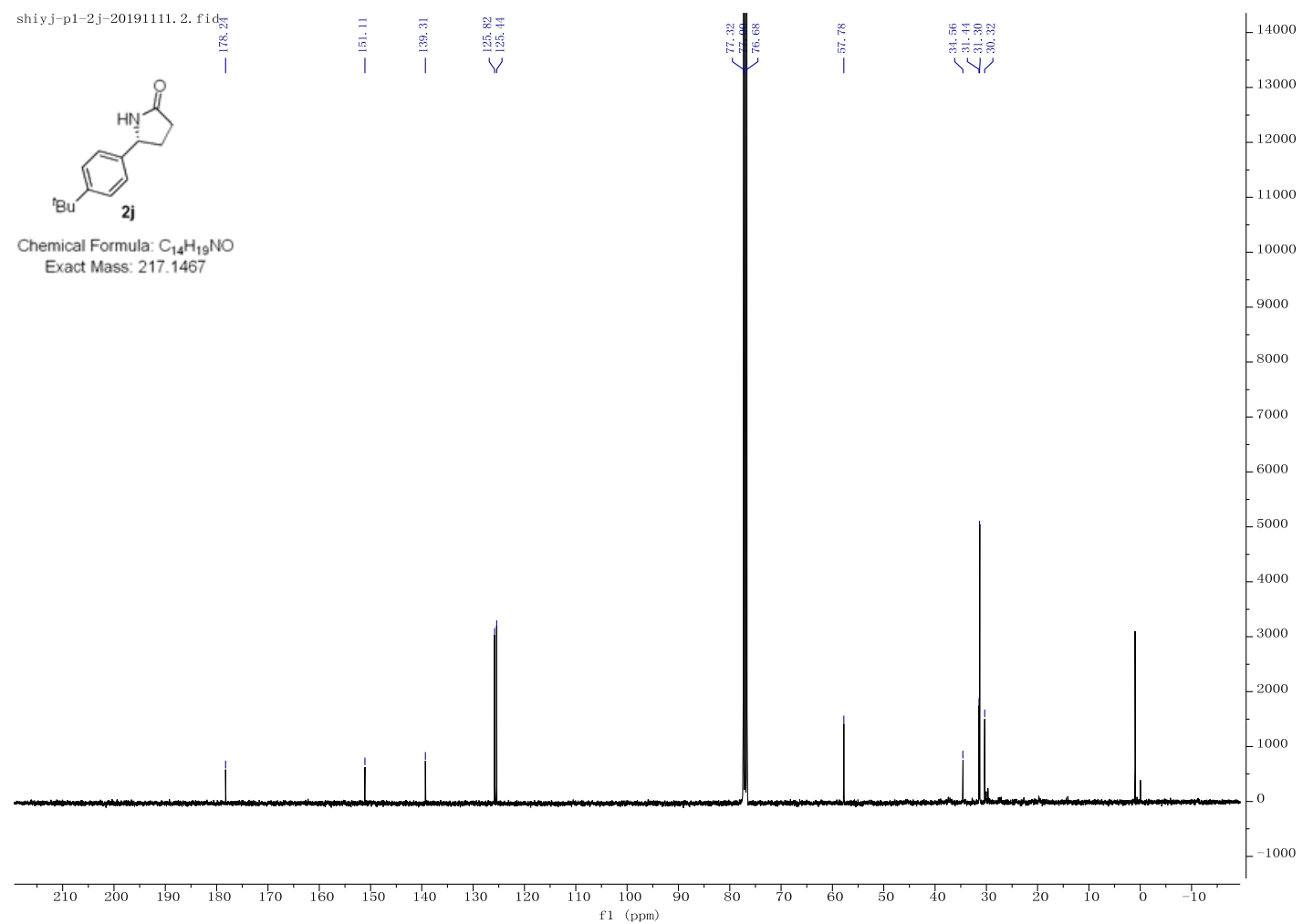


${ }^{1} \mathrm{H}$ NMR (400 MHz, Chloroform-d) of compound 2k

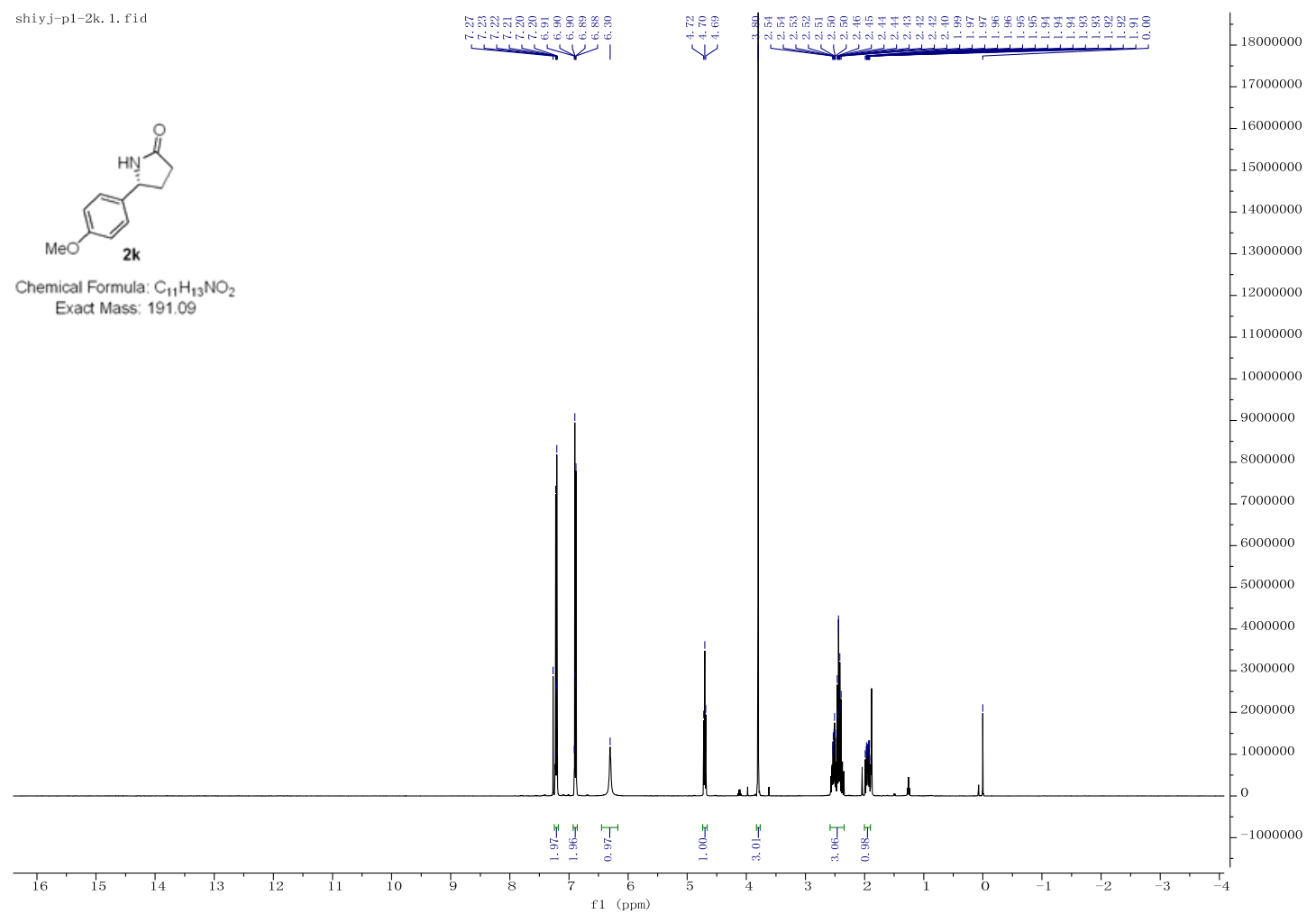

${ }^{13} \mathrm{C}\left\{{ }^{1} \mathrm{H}\right\}$ NMR (101 MHz, Chloroform-d) of compound 2k

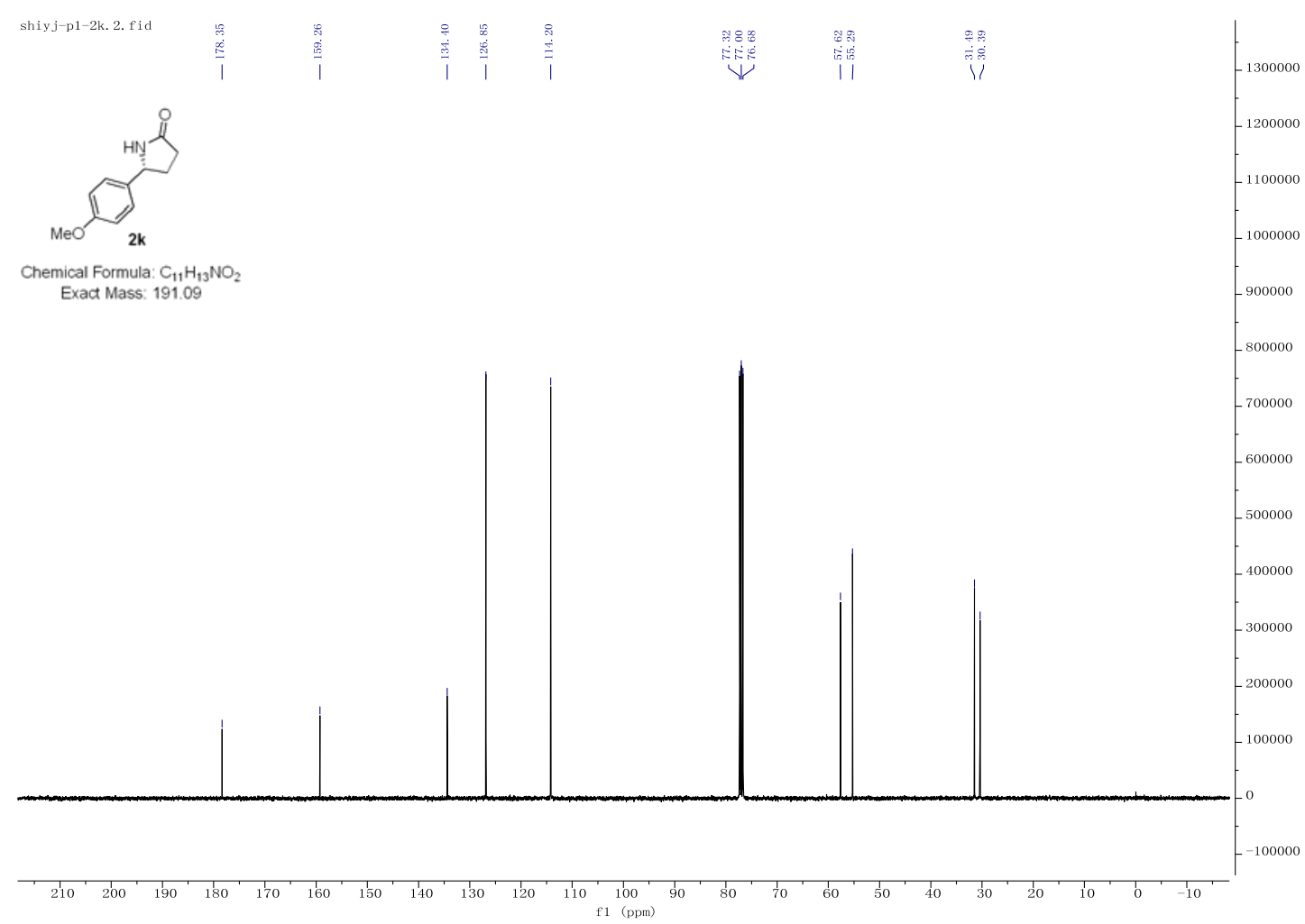


${ }^{1}$ H NMR (400 MHz, Chloroform-d) of compound 2 I

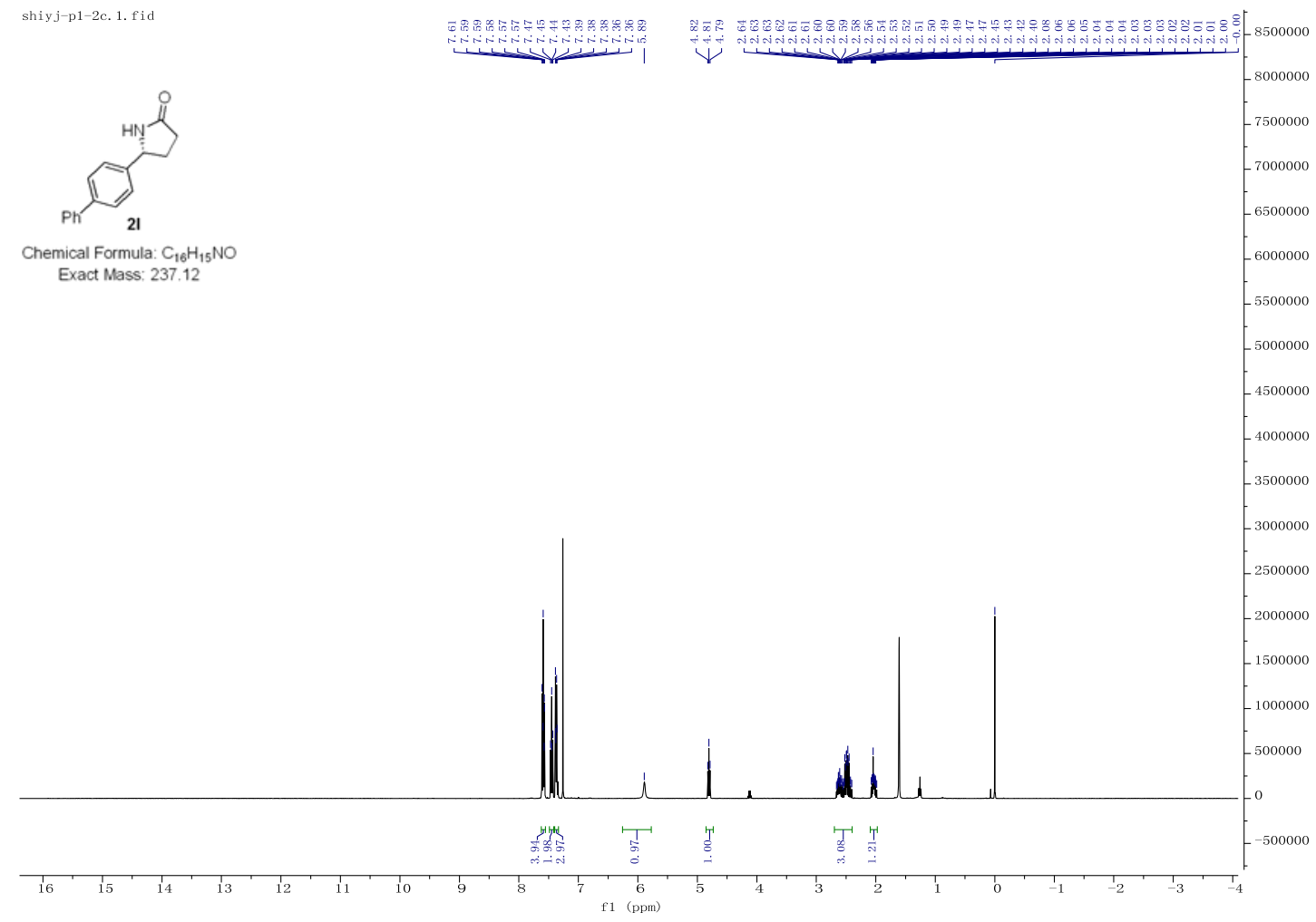

${ }^{13} \mathrm{C}\left\{{ }^{1} \mathrm{H}\right\}$ NMR (101 MHz, Chloroform-d) of compound $2 \mathbf{e}$

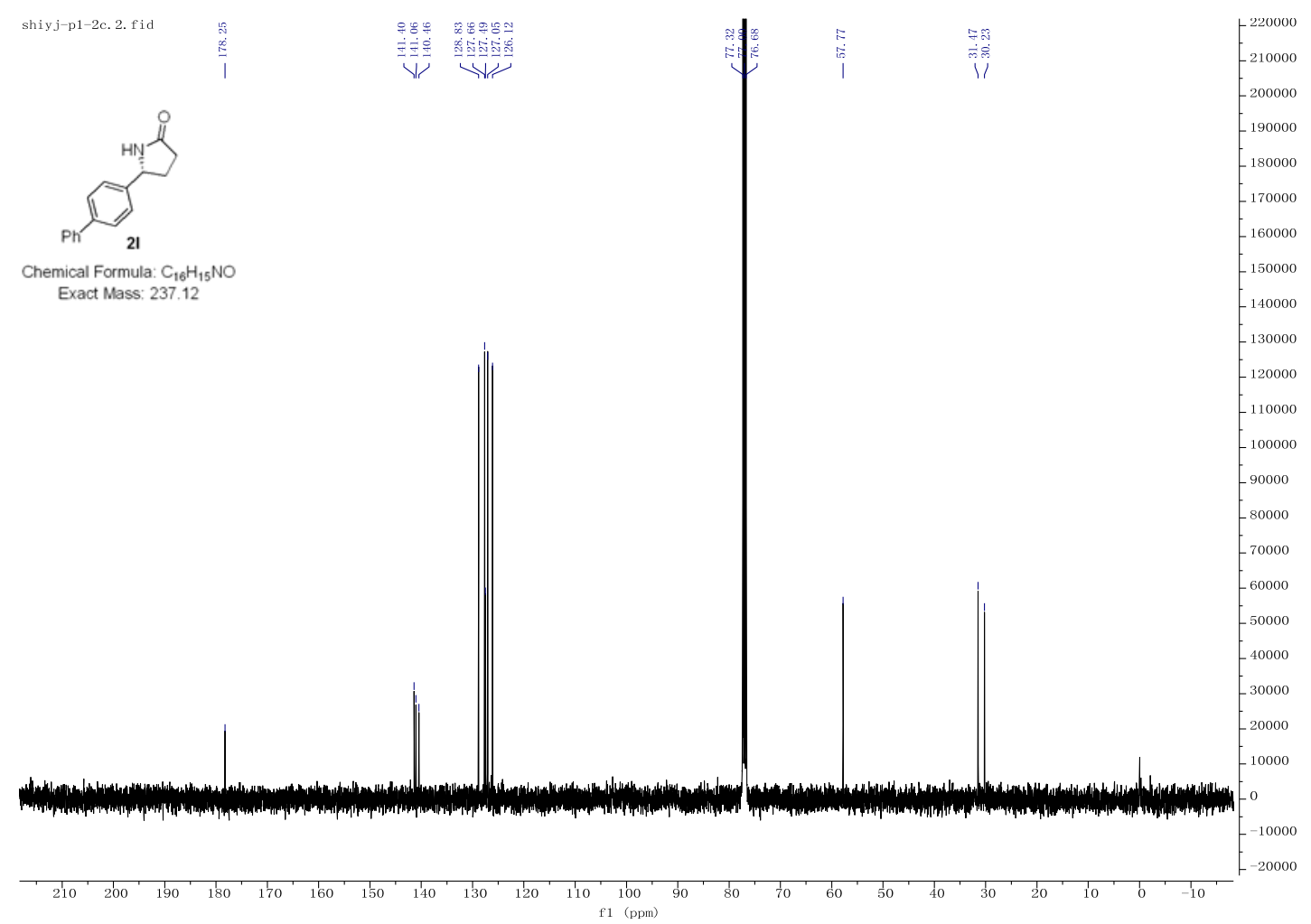


${ }^{1} \mathrm{H}$ NMR (600 MHz, Chloroform-d) of compound 2m

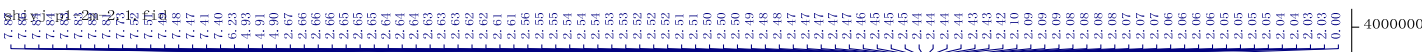

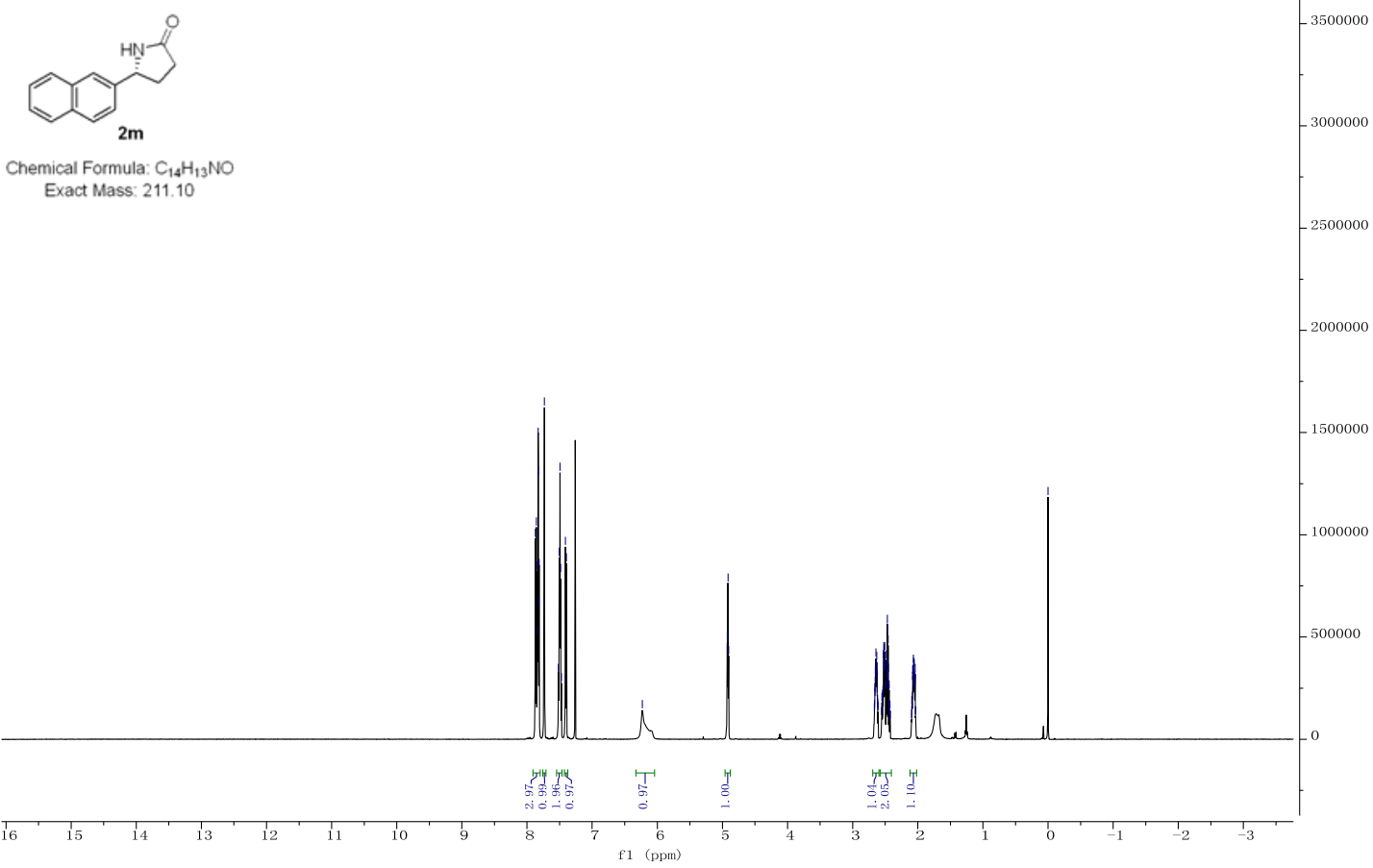

${ }^{13} \mathrm{C}\left\{{ }^{1} \mathrm{H}\right\}$ NMR (151 MHz, Chloroform-d) of compound 2m

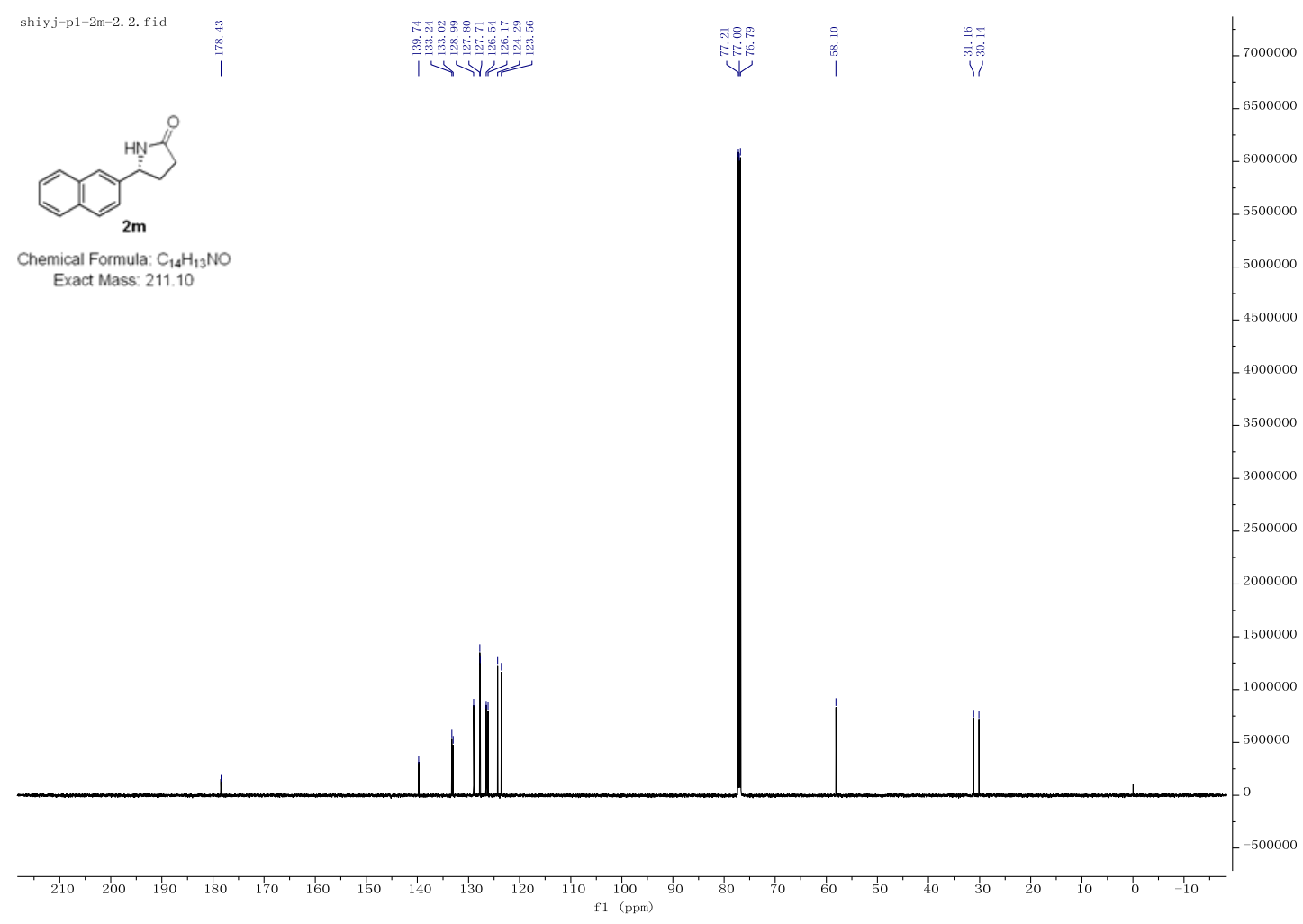


${ }^{1}$ H NMR (400 MHz, Chloroform-d) of compound 2n

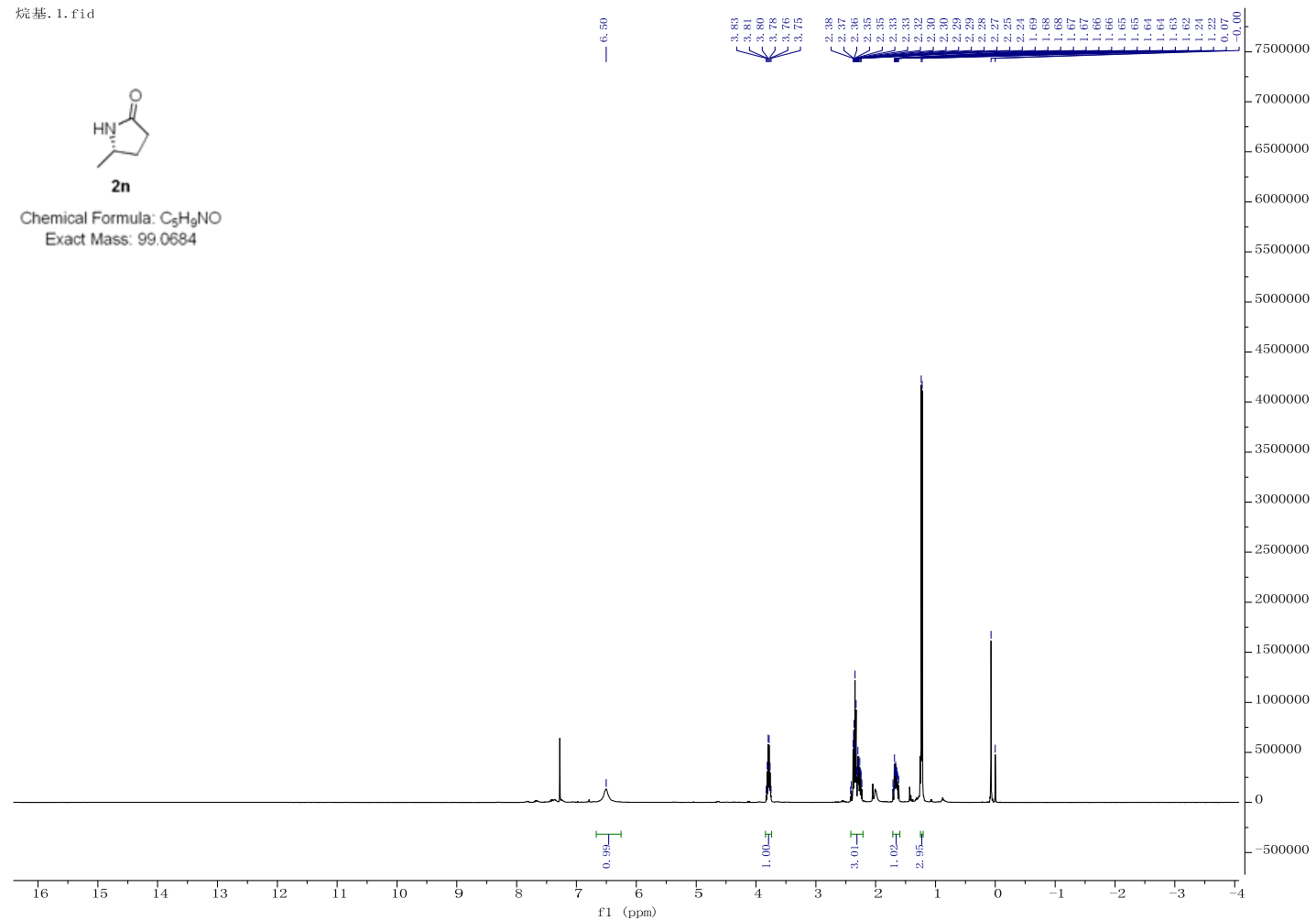

${ }^{13} \mathrm{C}\left\{{ }^{1} \mathrm{H}\right\}$ NMR (400 MHz, Chloroform-d) of compound 2n

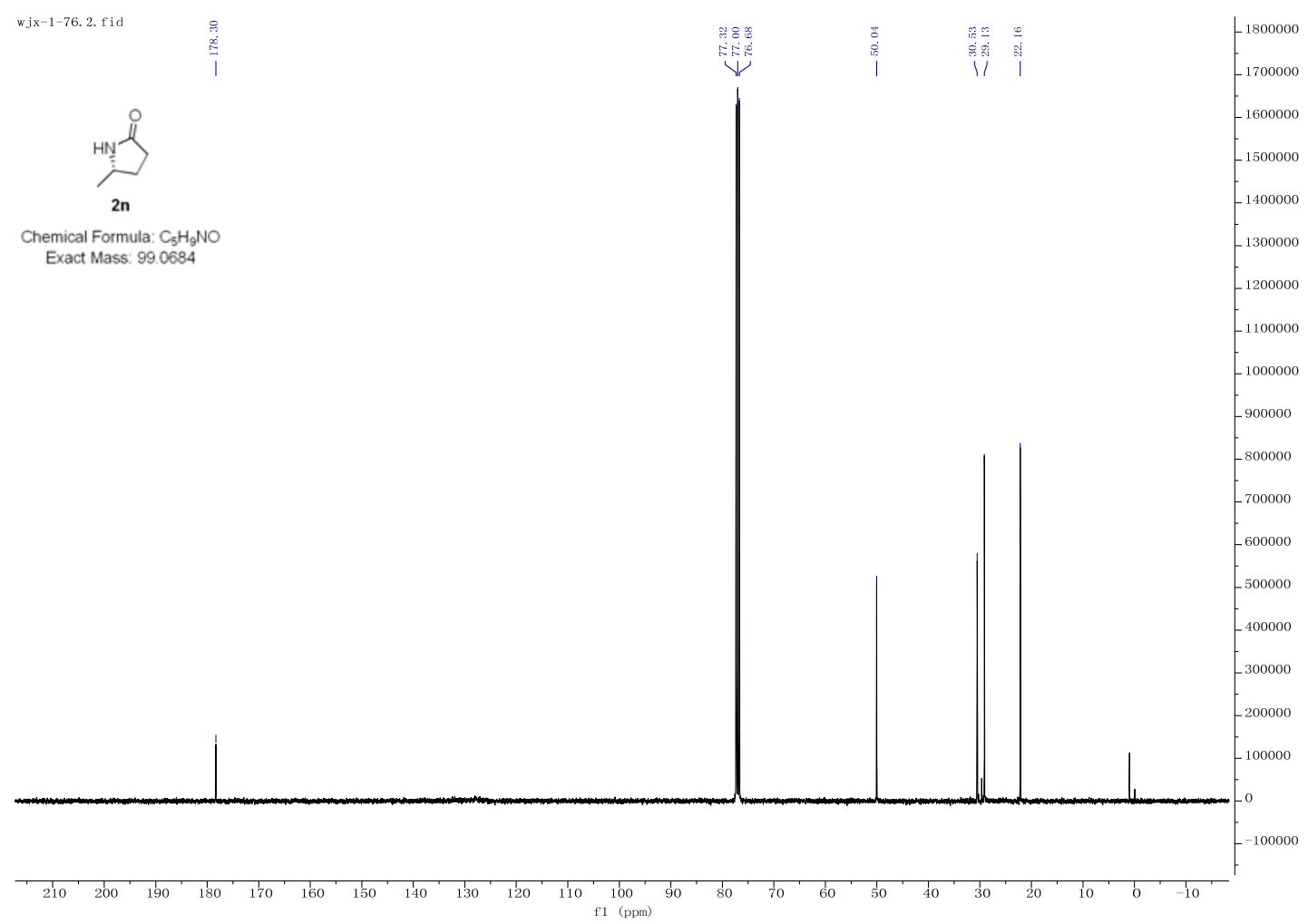


${ }^{1} \mathrm{H}$ NMR (400 MHz, Chloroform-d) of compound 4a

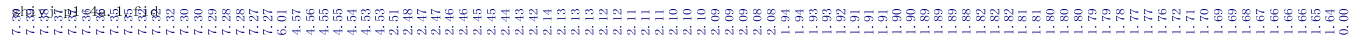

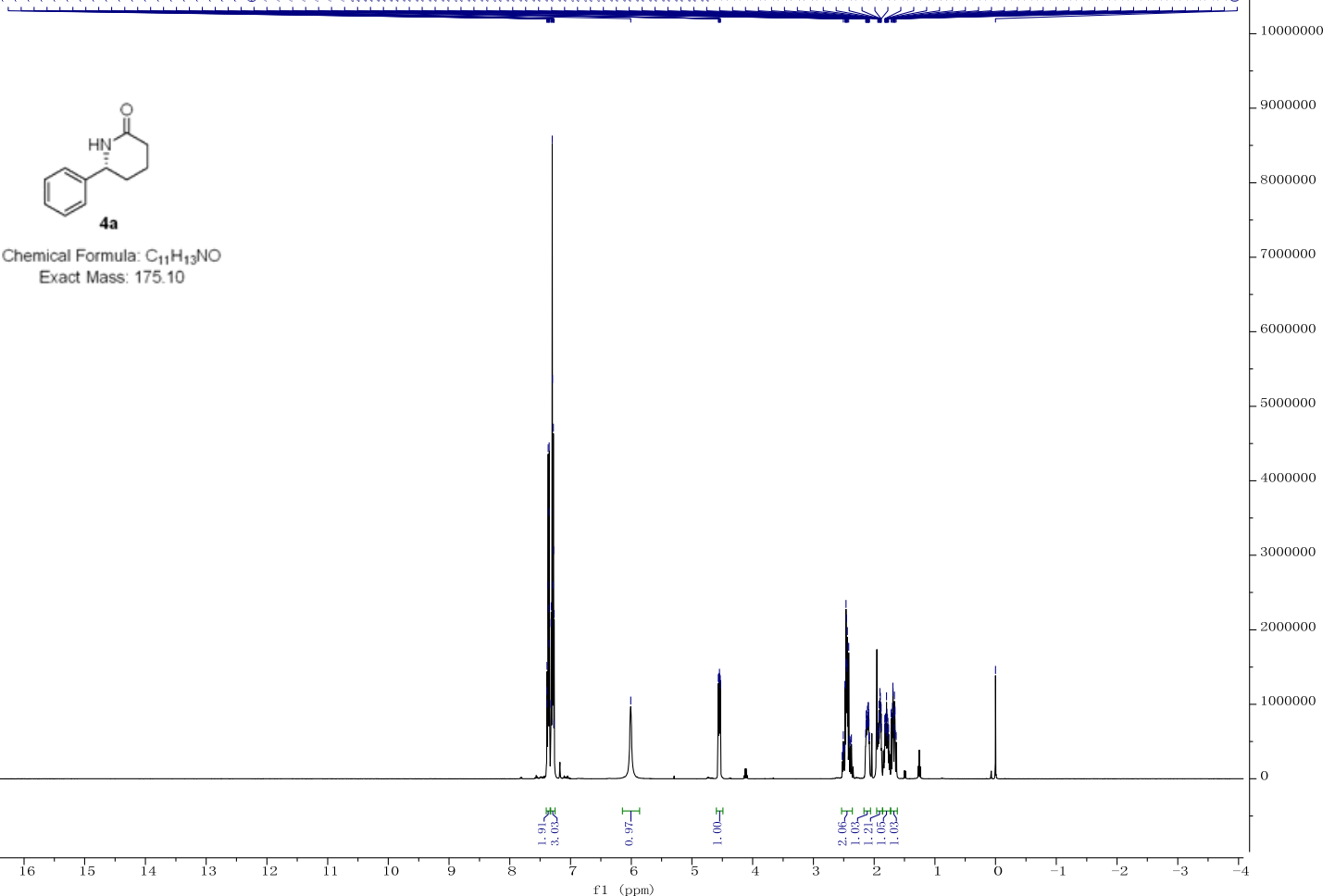

${ }^{13} \mathrm{C}\left\{{ }^{1} \mathrm{H}\right\}$ NMR (101 MHz, Chloroform-d) of compound 4a

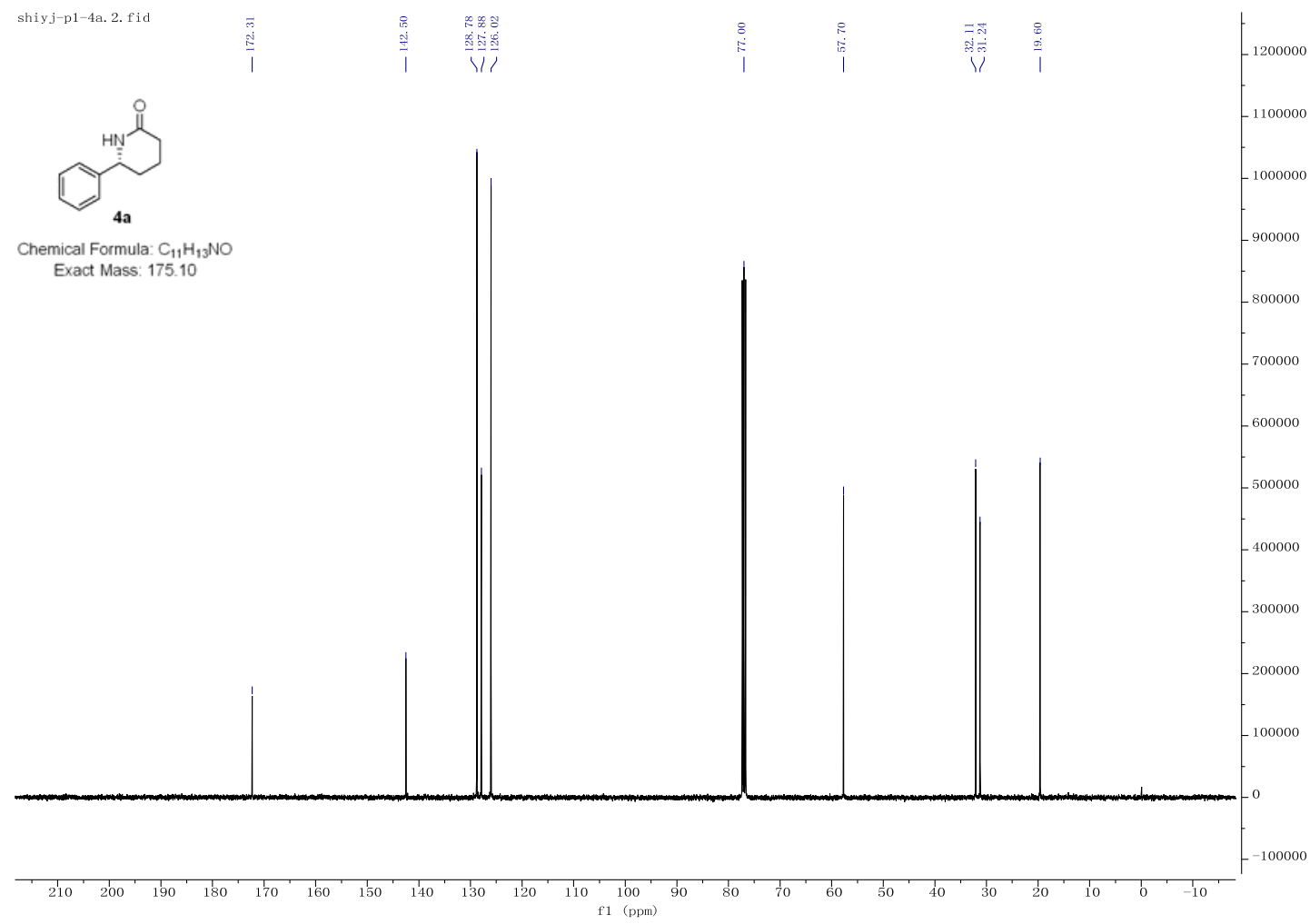


${ }^{1}$ H NMR (600 MHz, Chloroform-d) of compound $\mathbf{4 b}$

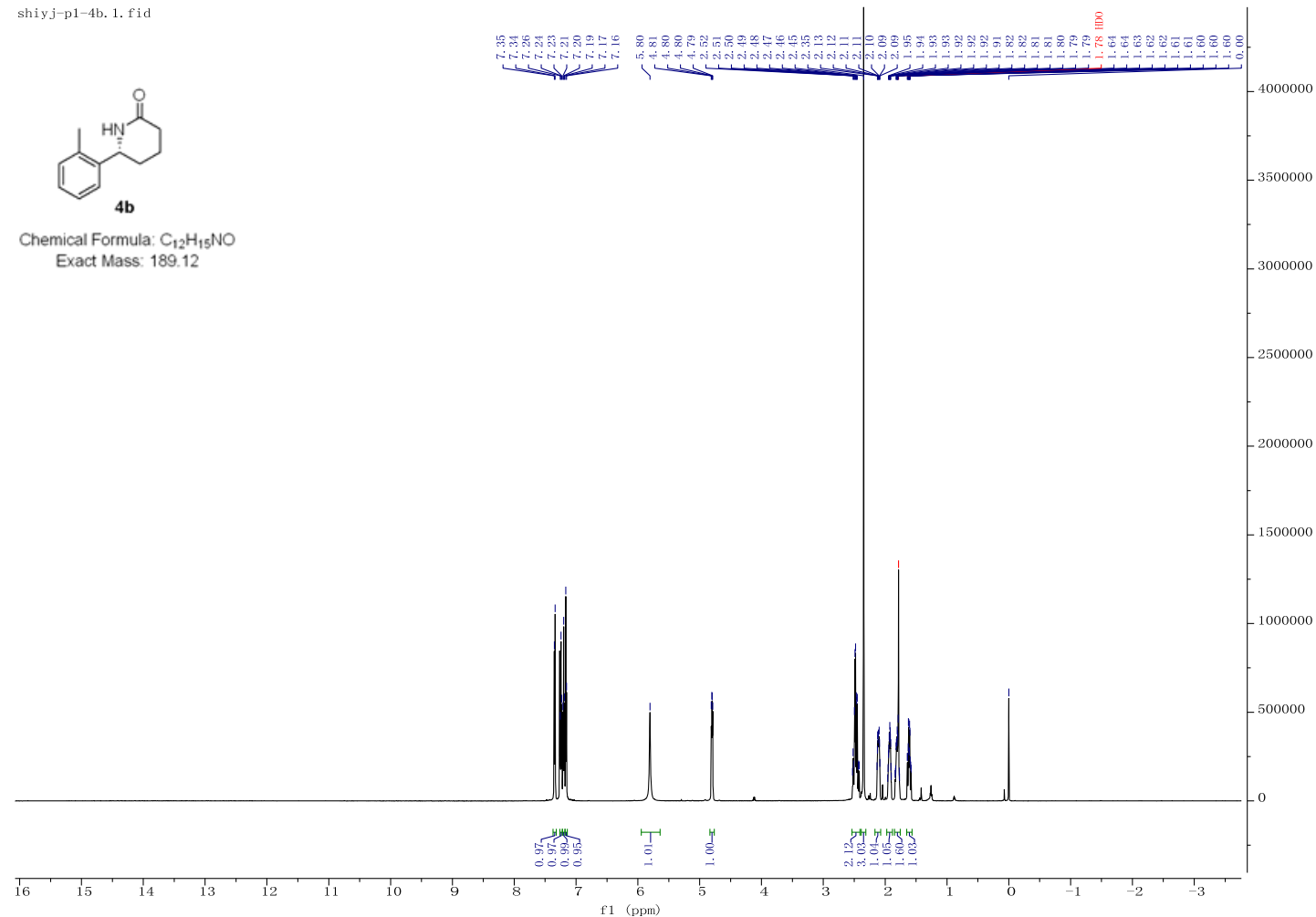

${ }^{13} \mathrm{C}\left\{{ }^{1} \mathrm{H}\right\}$ NMR (151 MHz, Chloroform-d) of compound $\mathbf{4 b}$

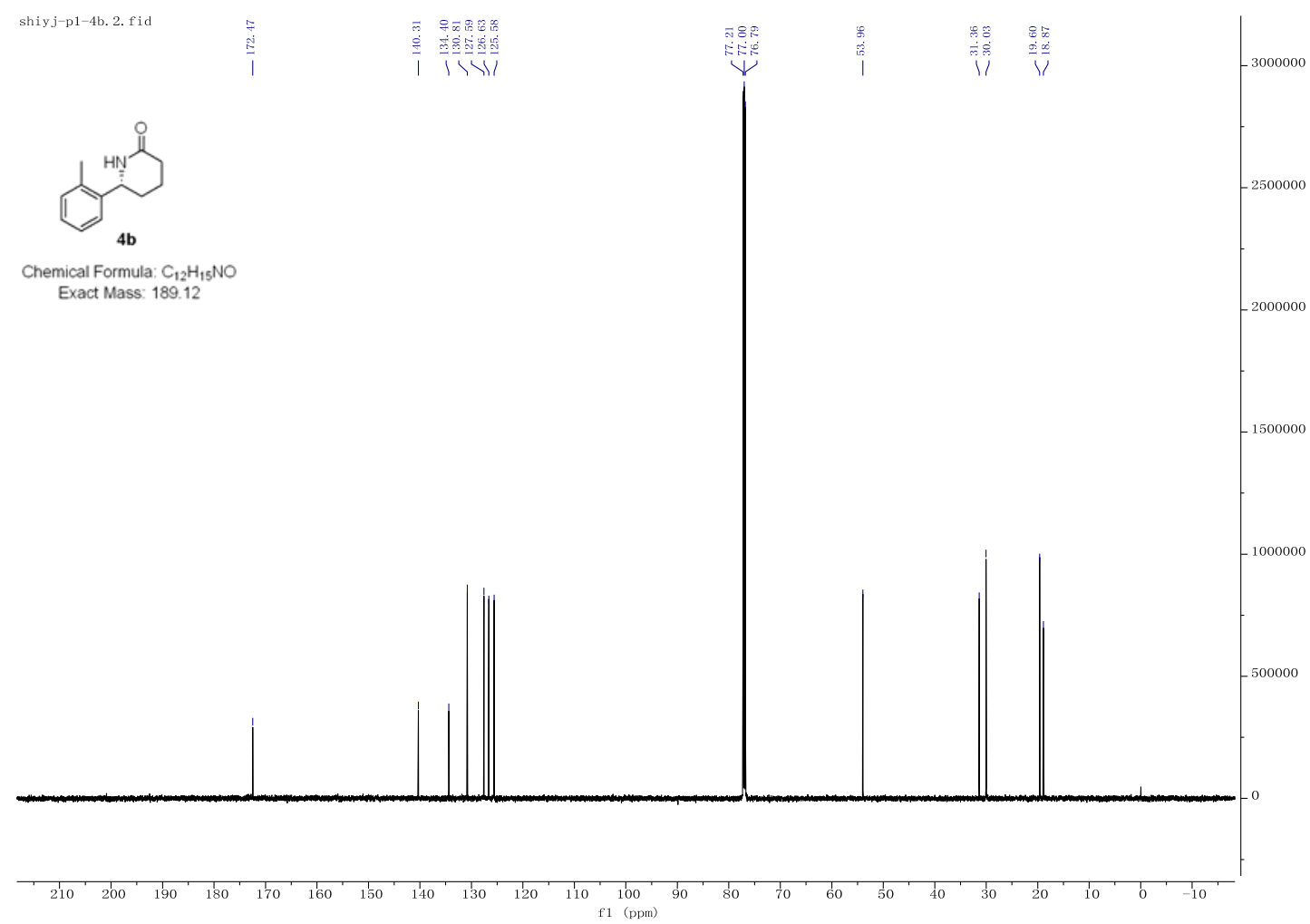


${ }^{1}$ H NMR (600 MHz, Chloroform-d) of compound 4c

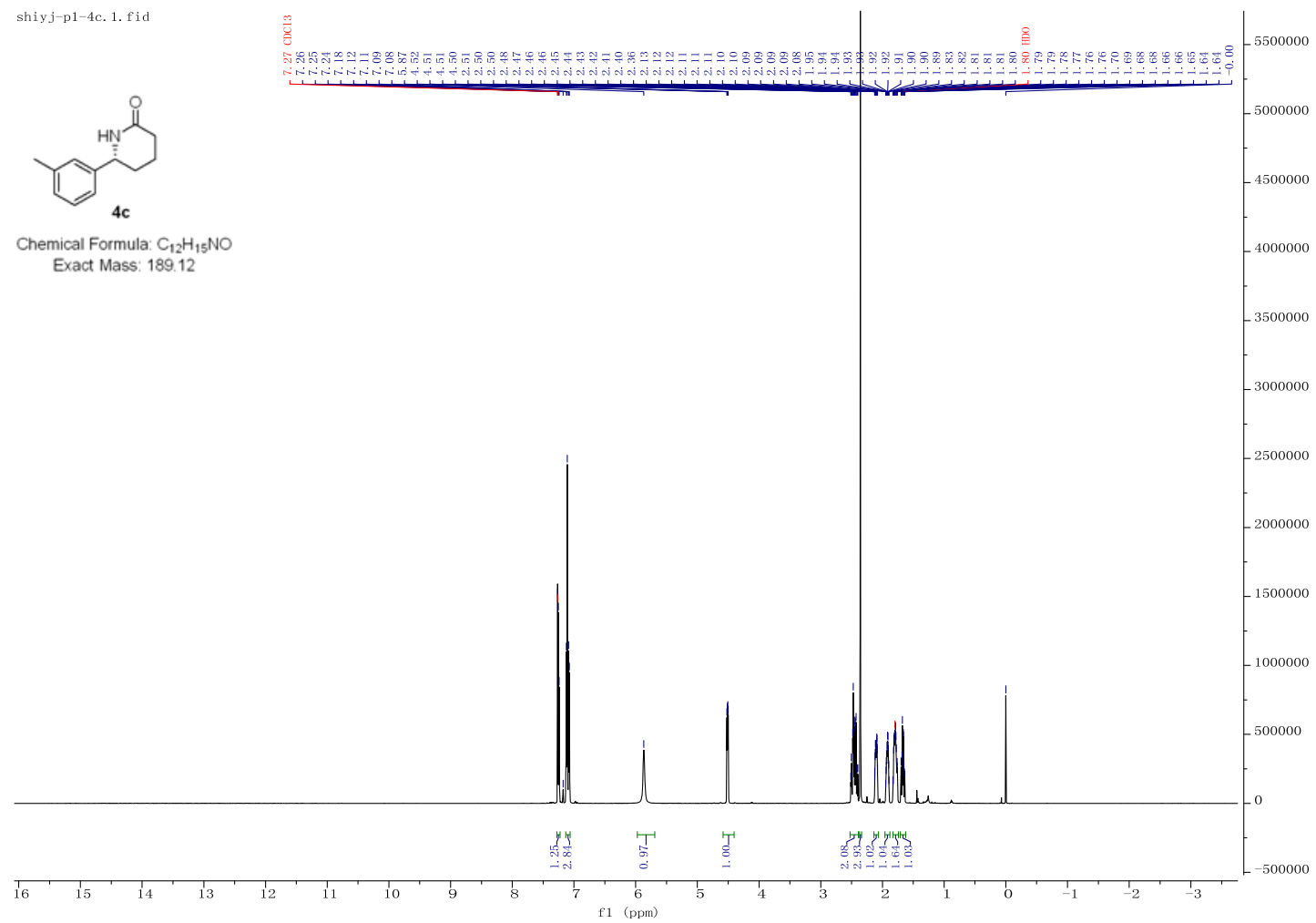

${ }^{13} \mathrm{C}\left\{{ }^{1} \mathrm{H}\right\}$ NMR (151 MHz, Chloroform-d) of compound 4c

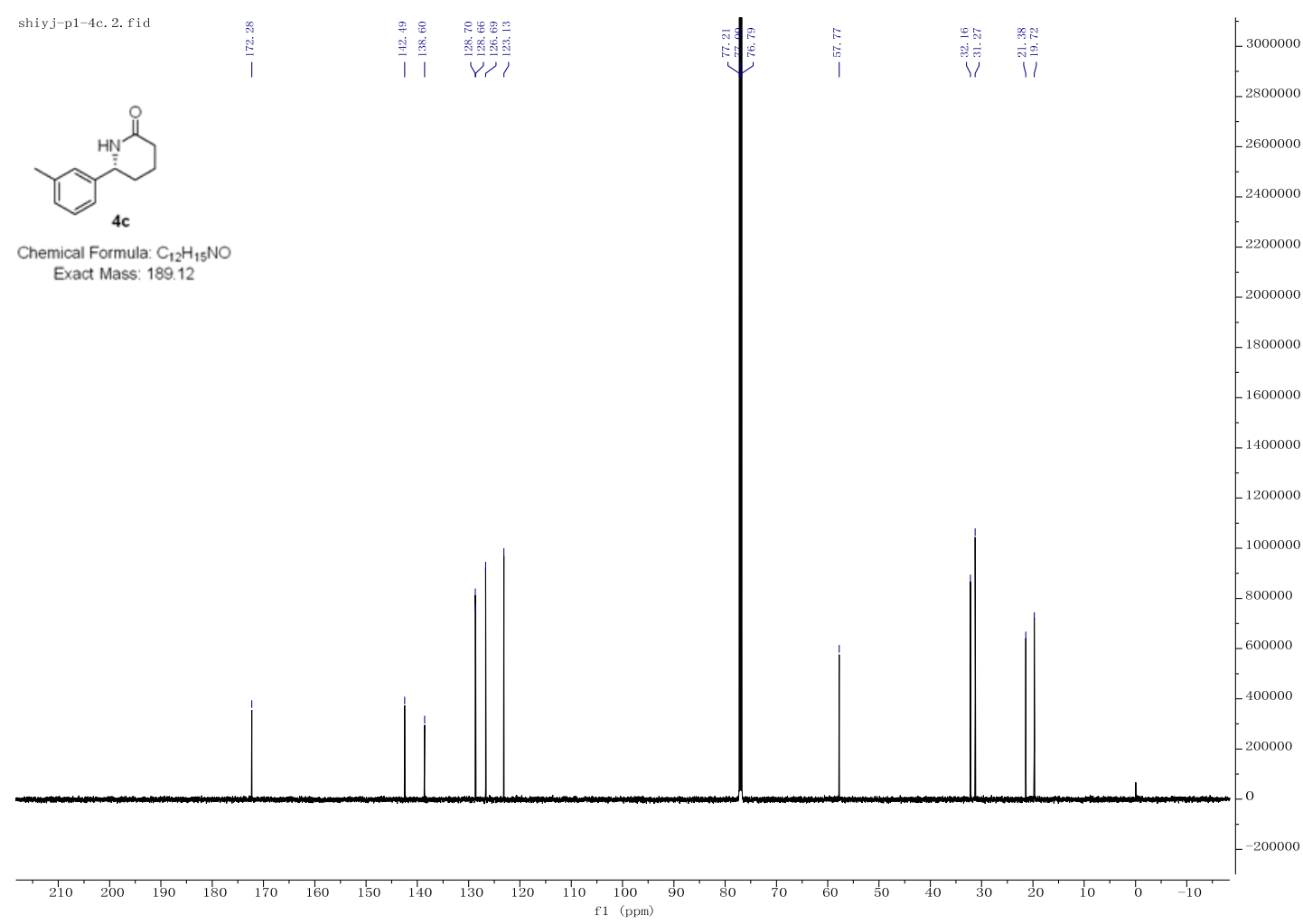


${ }^{1} \mathrm{H}$ NMR (600 MHz, Chloroform-d) of compound 4d

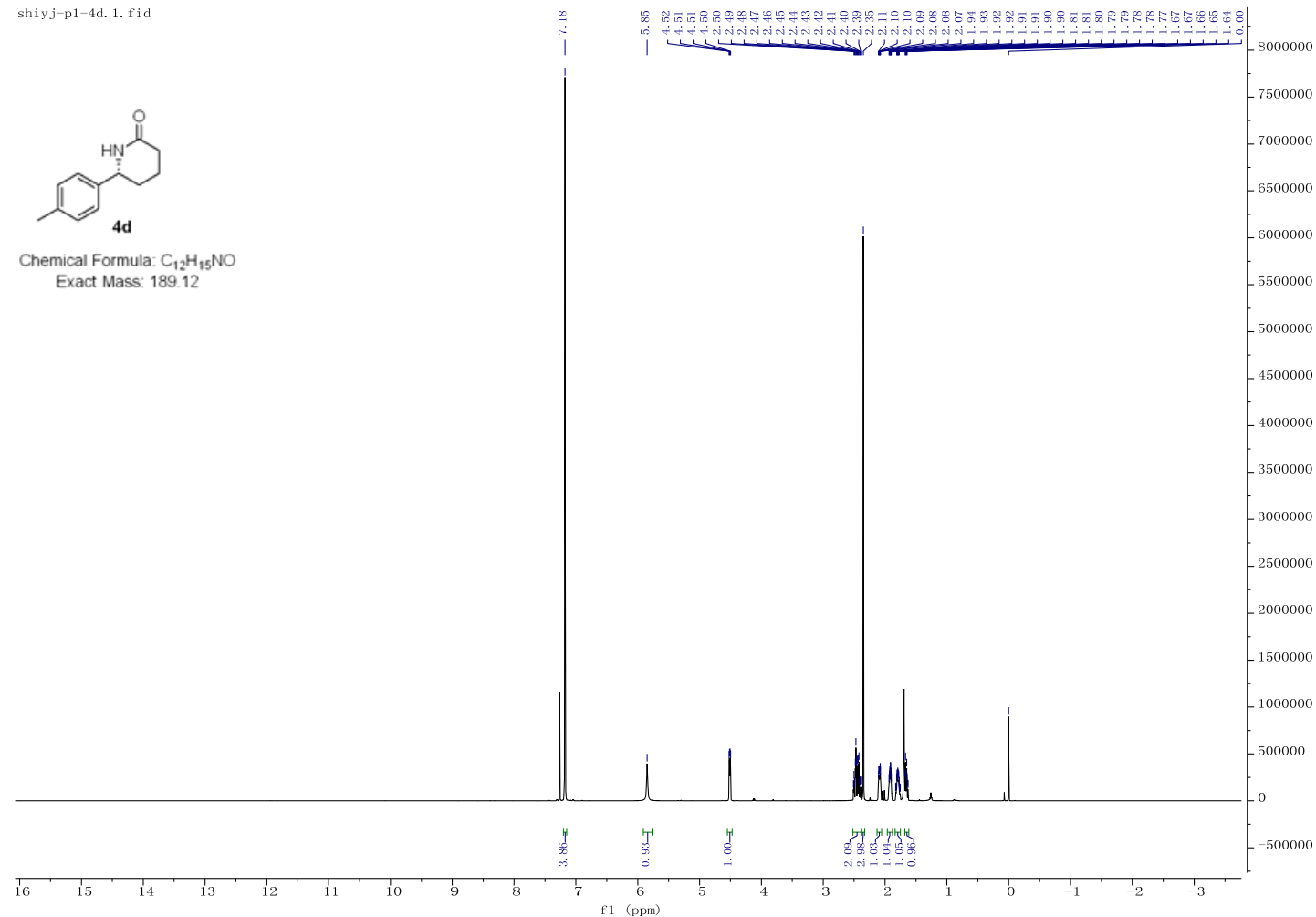

${ }^{13} \mathrm{C}\left\{{ }^{1} \mathrm{H}\right\}$ NMR (151 MHz, Chloroform-d) of compound $\mathbf{4 d}$

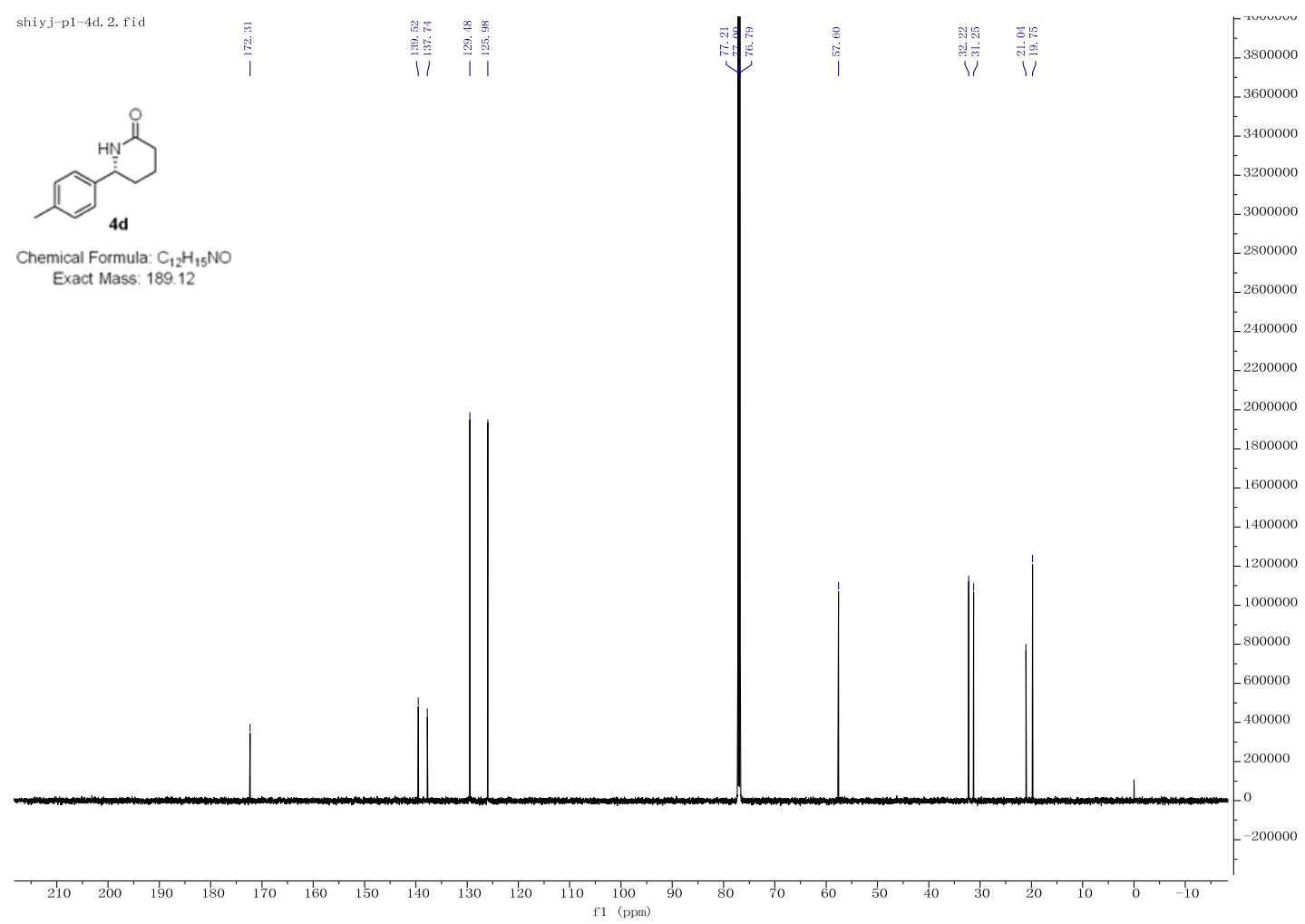


${ }^{1} \mathrm{H}$ NMR (600 MHz, Chloroform-d) of compound $\mathbf{4 e}$

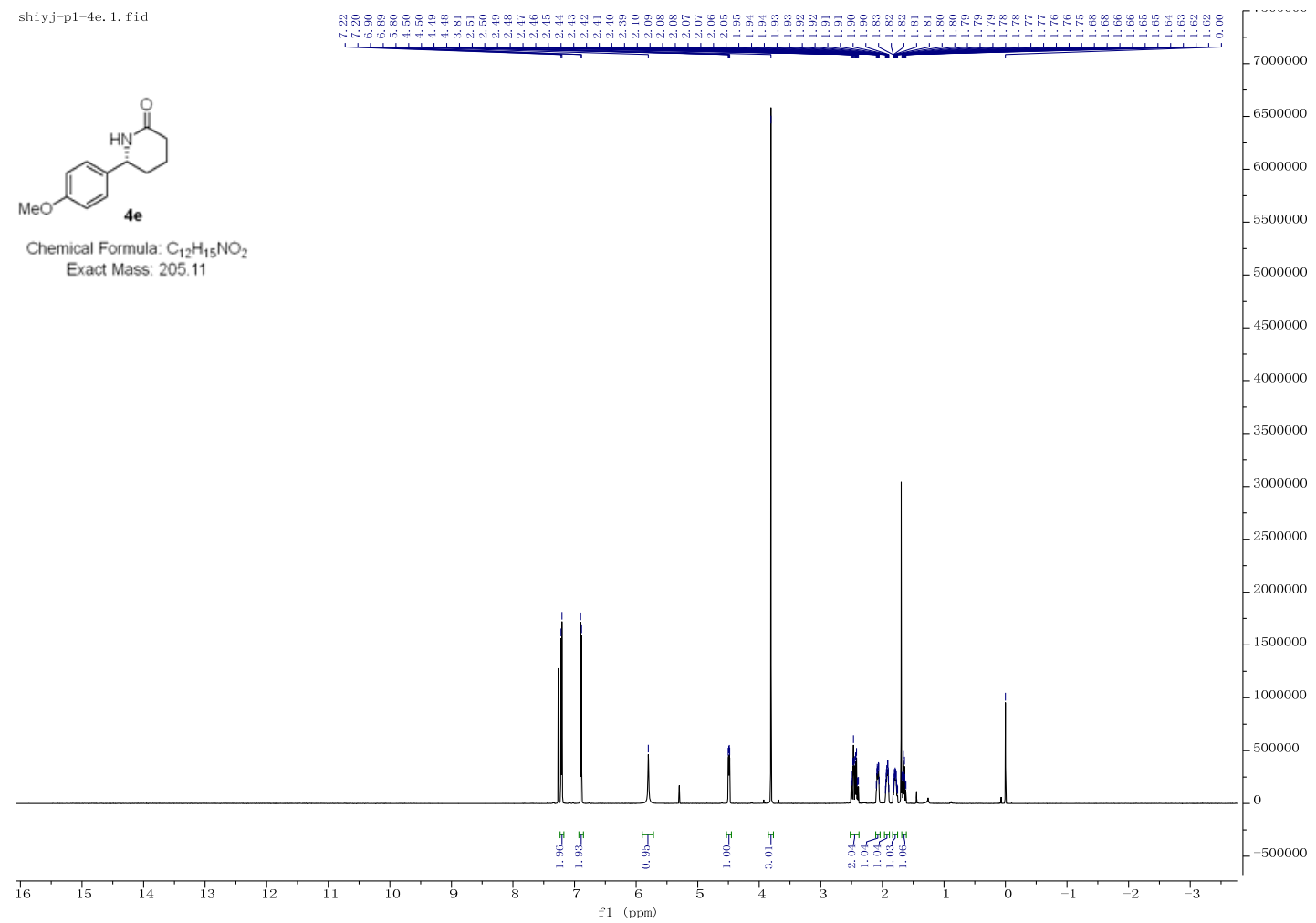

${ }^{13} \mathrm{C}\left\{{ }^{1} \mathrm{H}\right\}$ NMR (151 MHz, Chloroform-d) of compound $4 \mathbf{e}$ shiyj-p1-4e. 2. fid
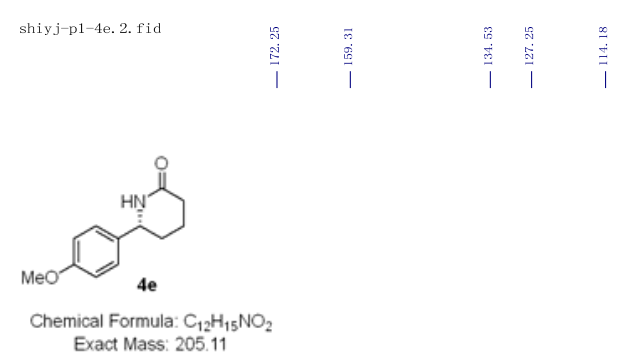

Exact Mass: 205.11
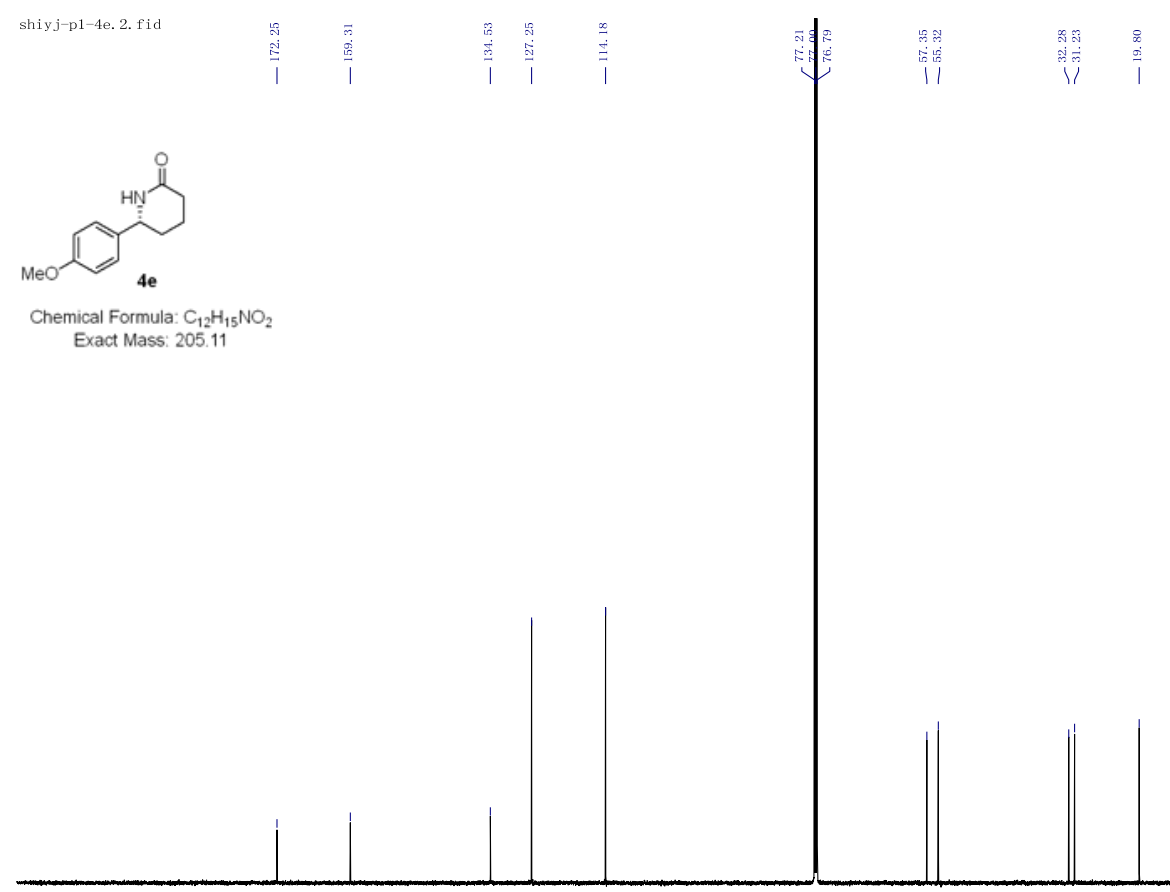

6000000

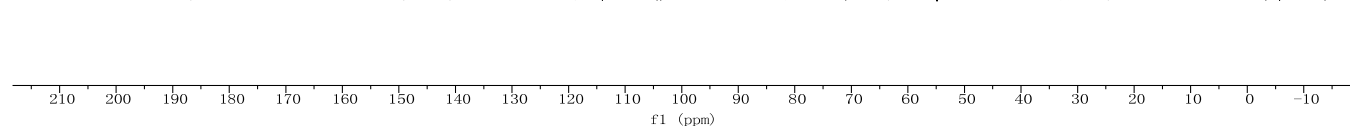


${ }^{1}$ H NMR (600 MHz, Chloroform-d) of compound $4 \mathbf{f}$

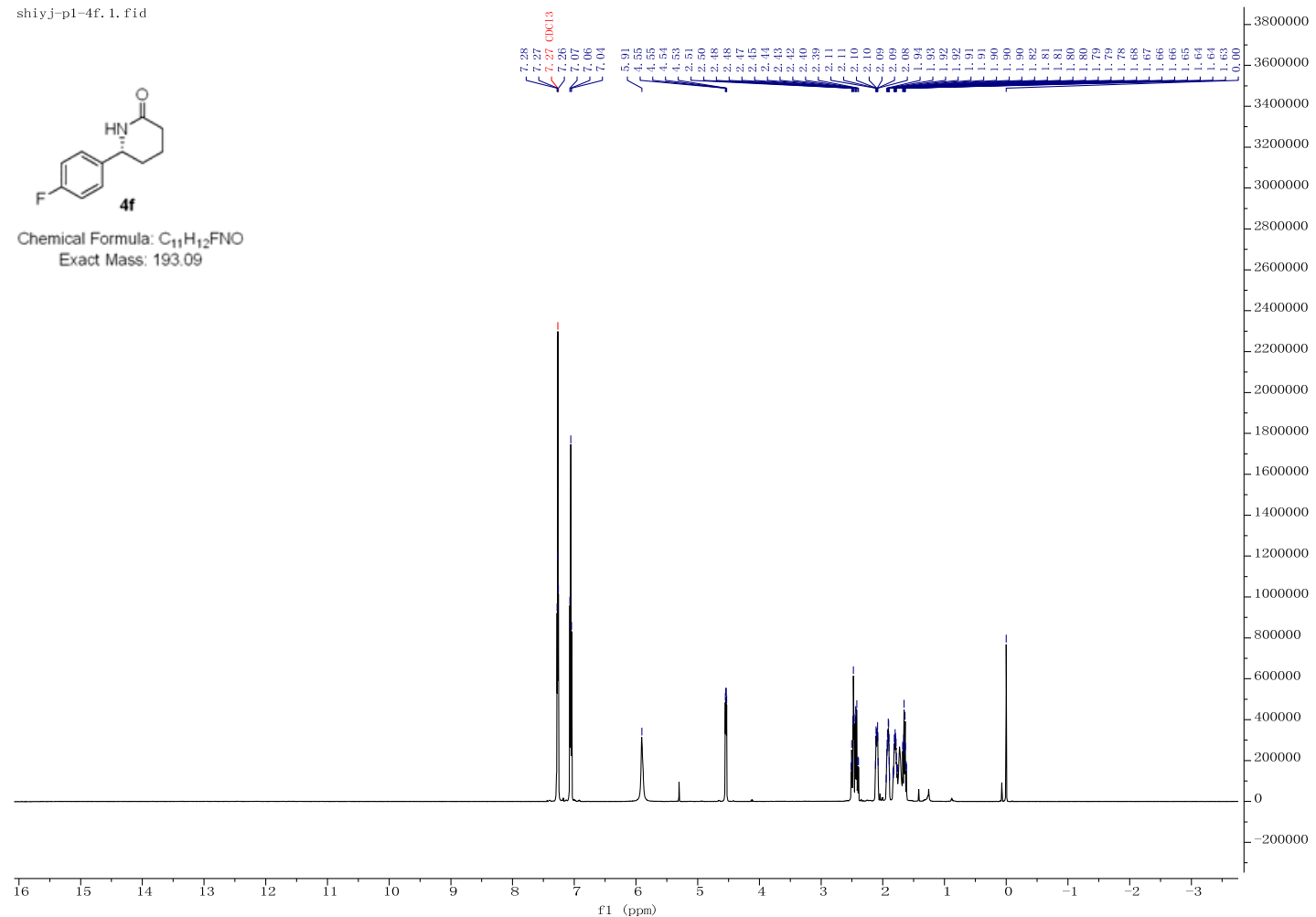

${ }^{13} \mathrm{C}\left\{{ }^{1} \mathrm{H}\right\}$ NMR (151 MHz, Chloroform-d) of compound $\mathbf{4 f}$

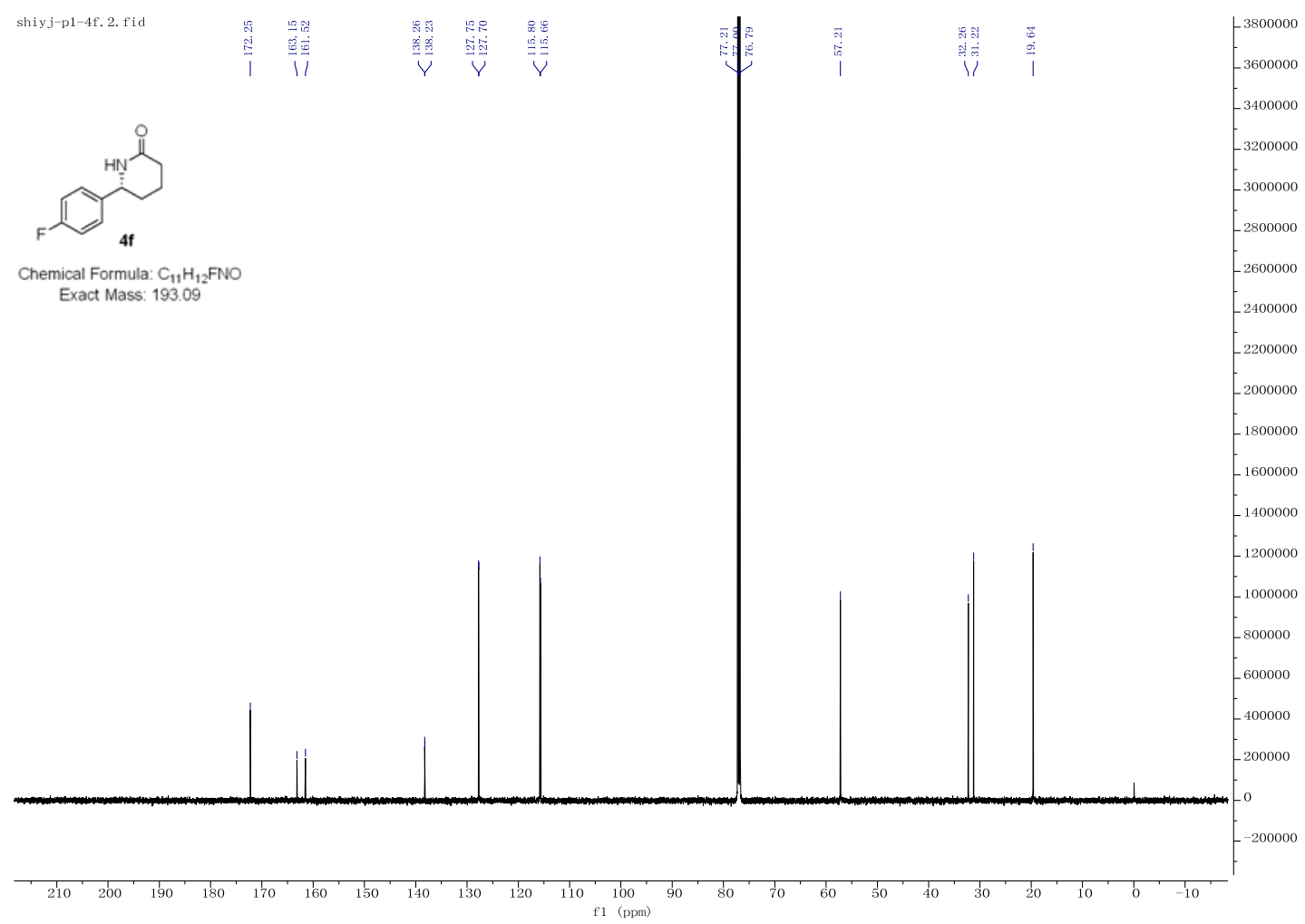


${ }^{1}$ H NMR (400 MHz, Chloroform-d) of compound $\mathbf{4 g}$

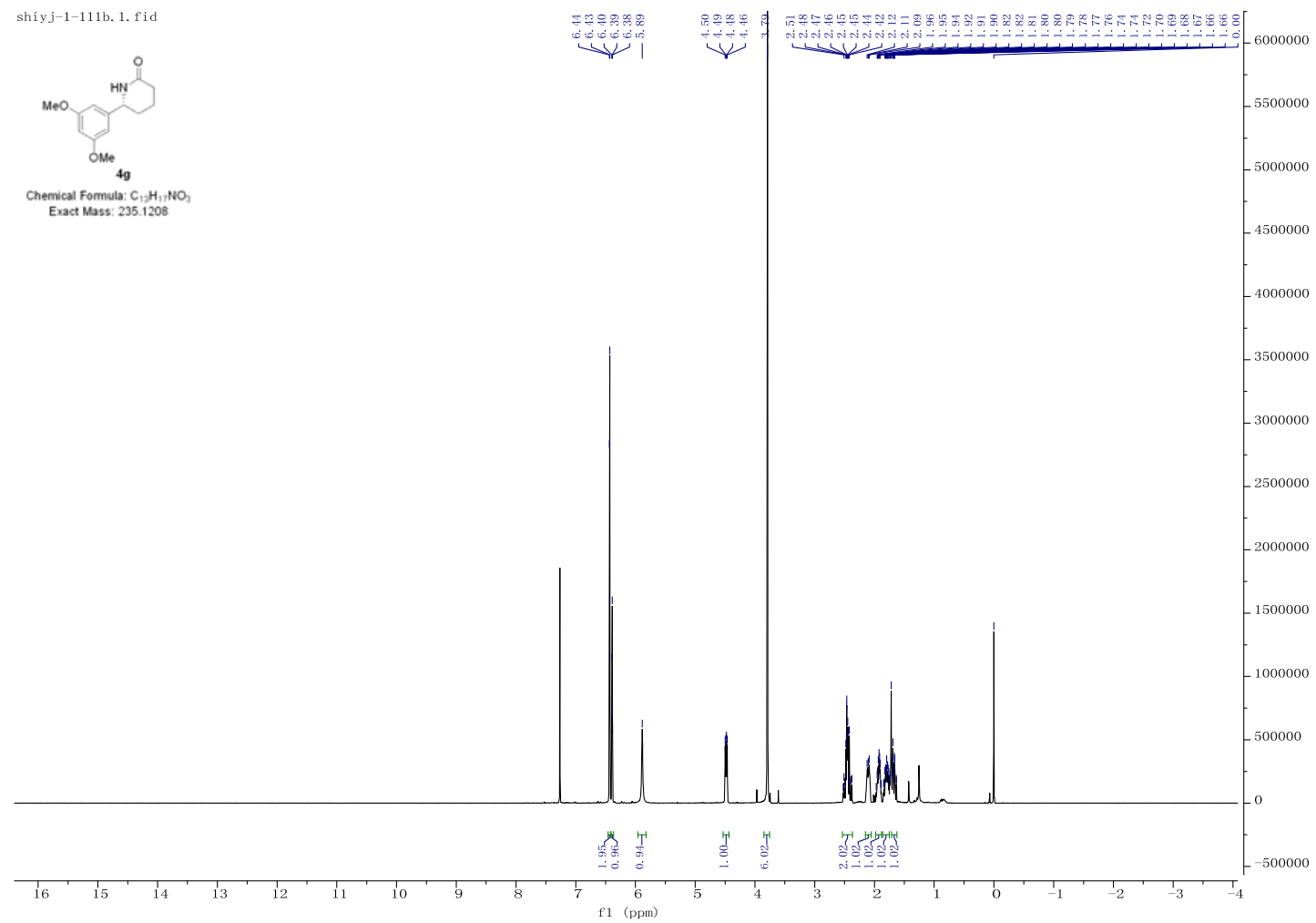

${ }^{13} \mathrm{C}\left\{{ }^{1} \mathrm{H}\right\}$ NMR (101 MHz, Chloroform-d) of compound $\mathbf{4 g}$

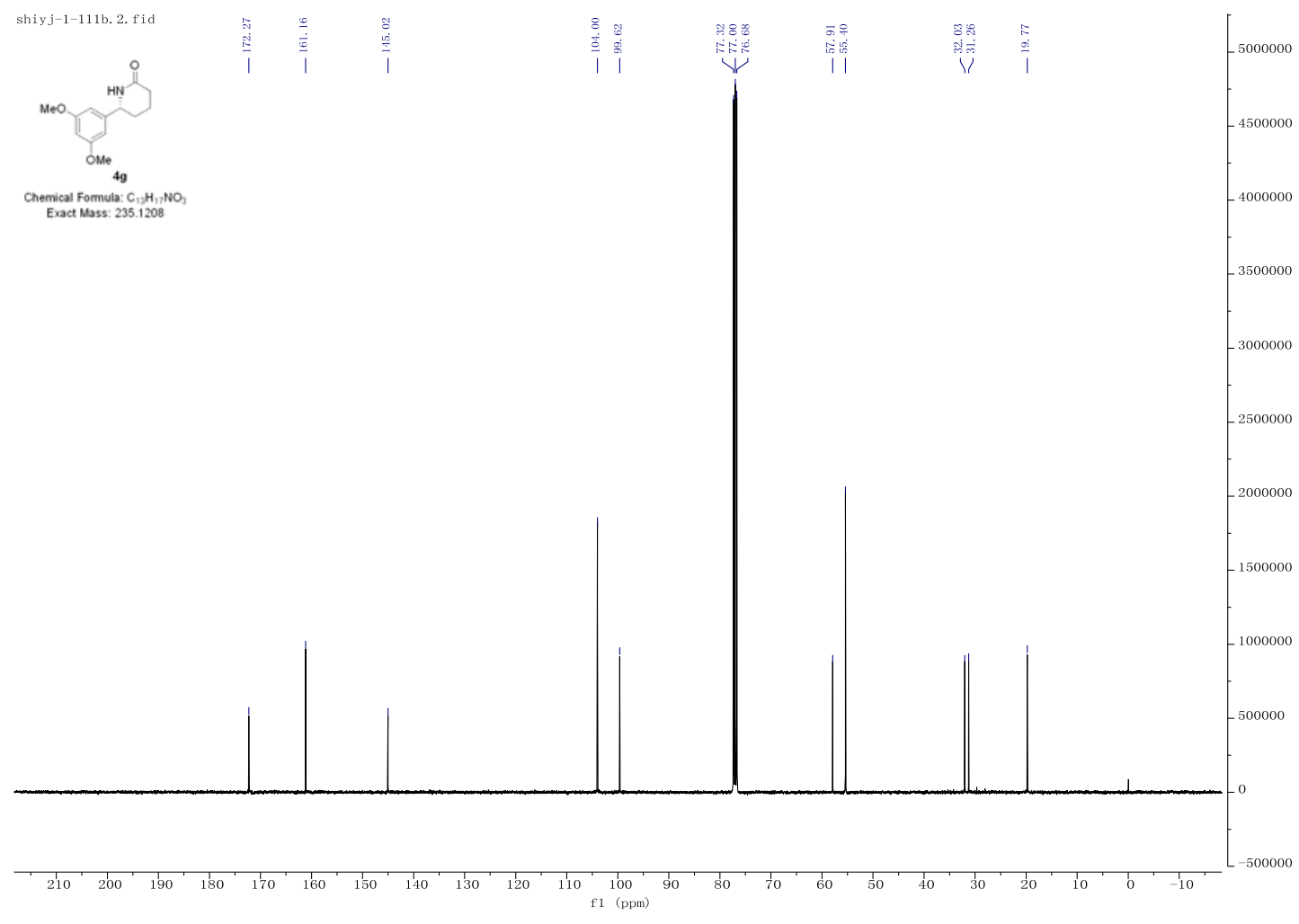


${ }^{1} \mathrm{H}$ NMR (400 MHz, Chloroform-d) of compound $\mathbf{4 h}$

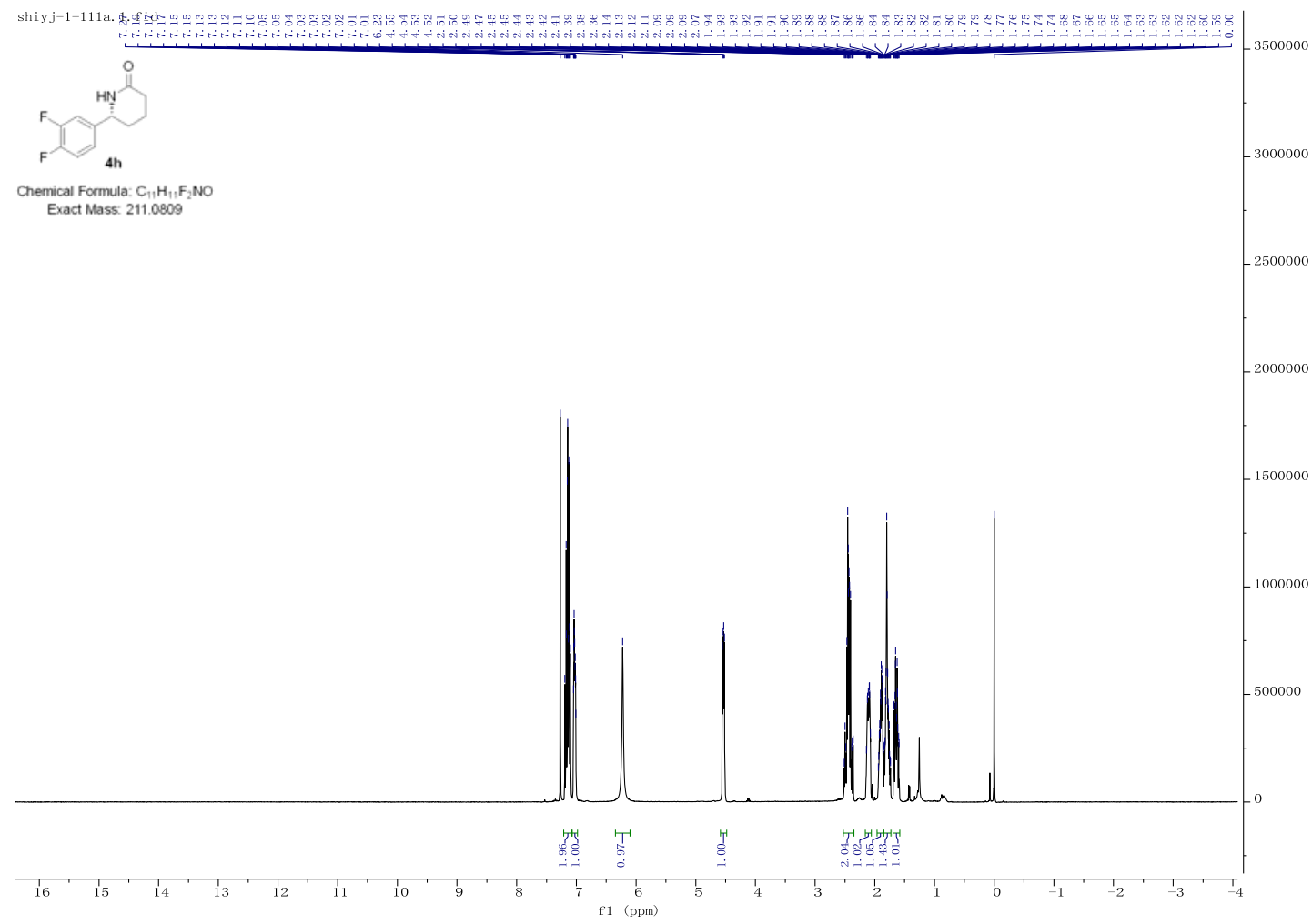

${ }^{13} \mathrm{C}\left\{{ }^{1} \mathrm{H}\right\}$ NMR (101 MHz, Chloroform-d) of compound $\mathbf{4 f}$

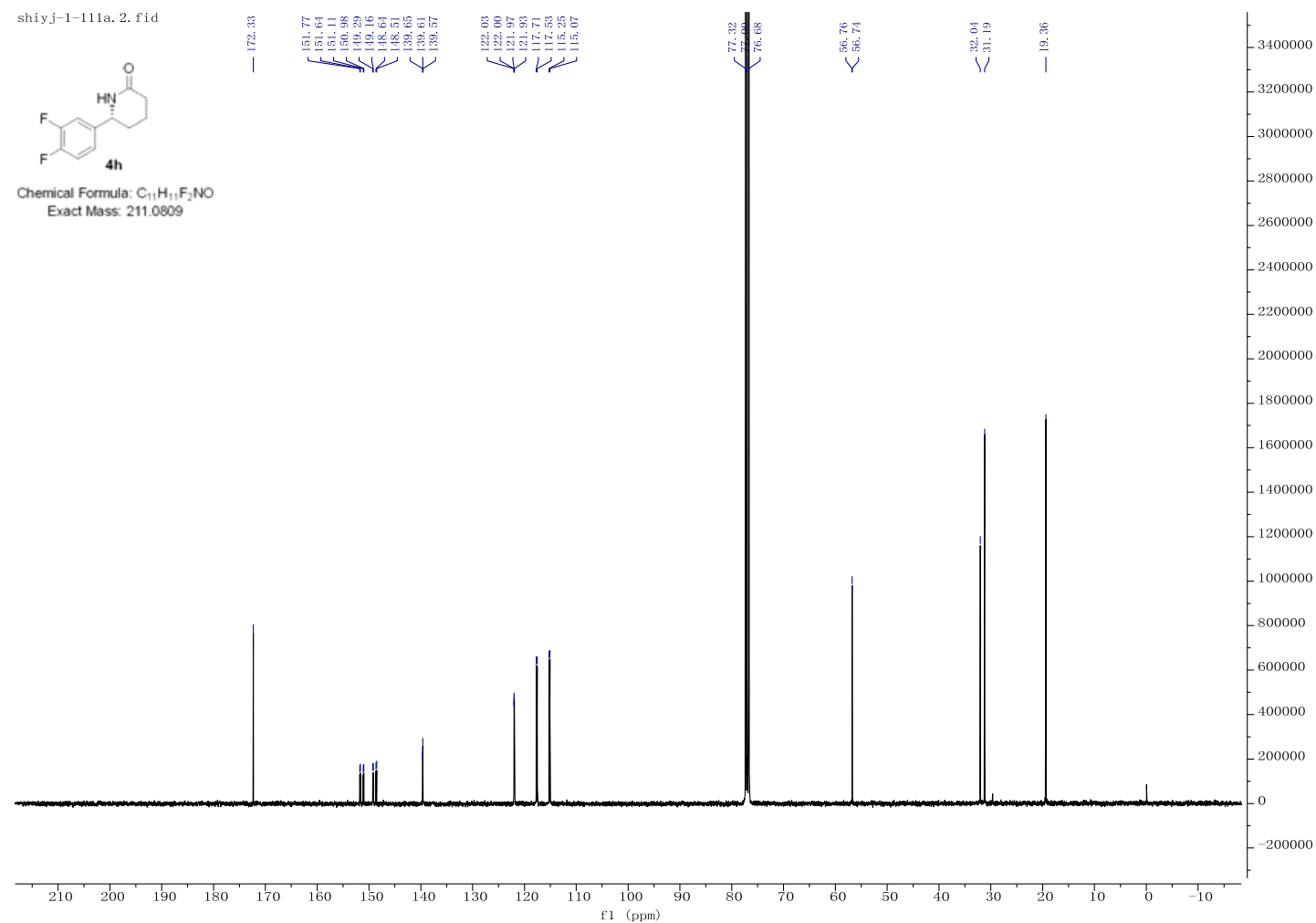


${ }^{1} \mathrm{H}$ NMR (600 MHz, Chloroform-d) of compound 4i

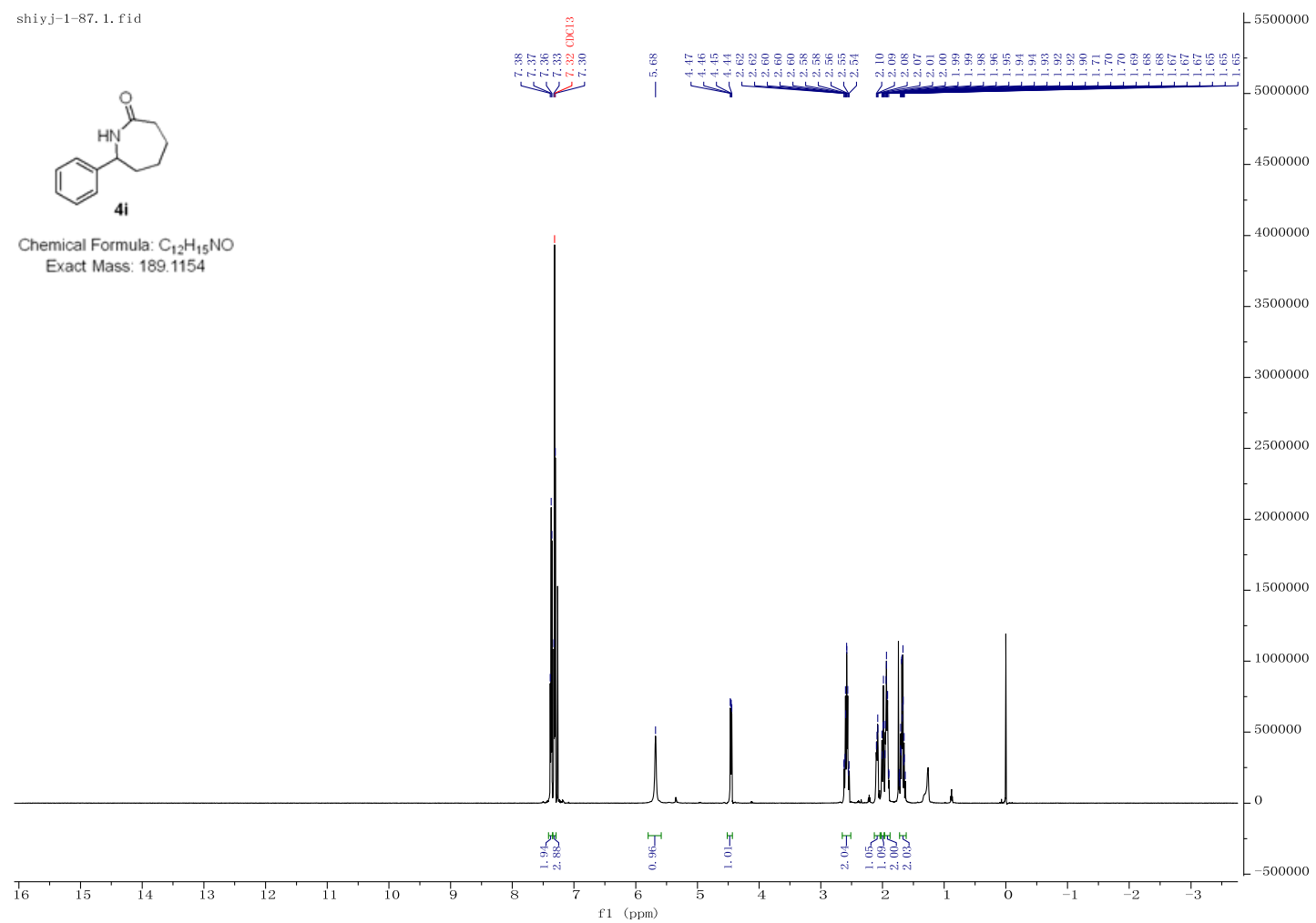

${ }^{13} \mathrm{C}\left\{{ }^{1} \mathrm{H}\right\}$ NMR (101 MHz, Chloroform-d) of compound 4i

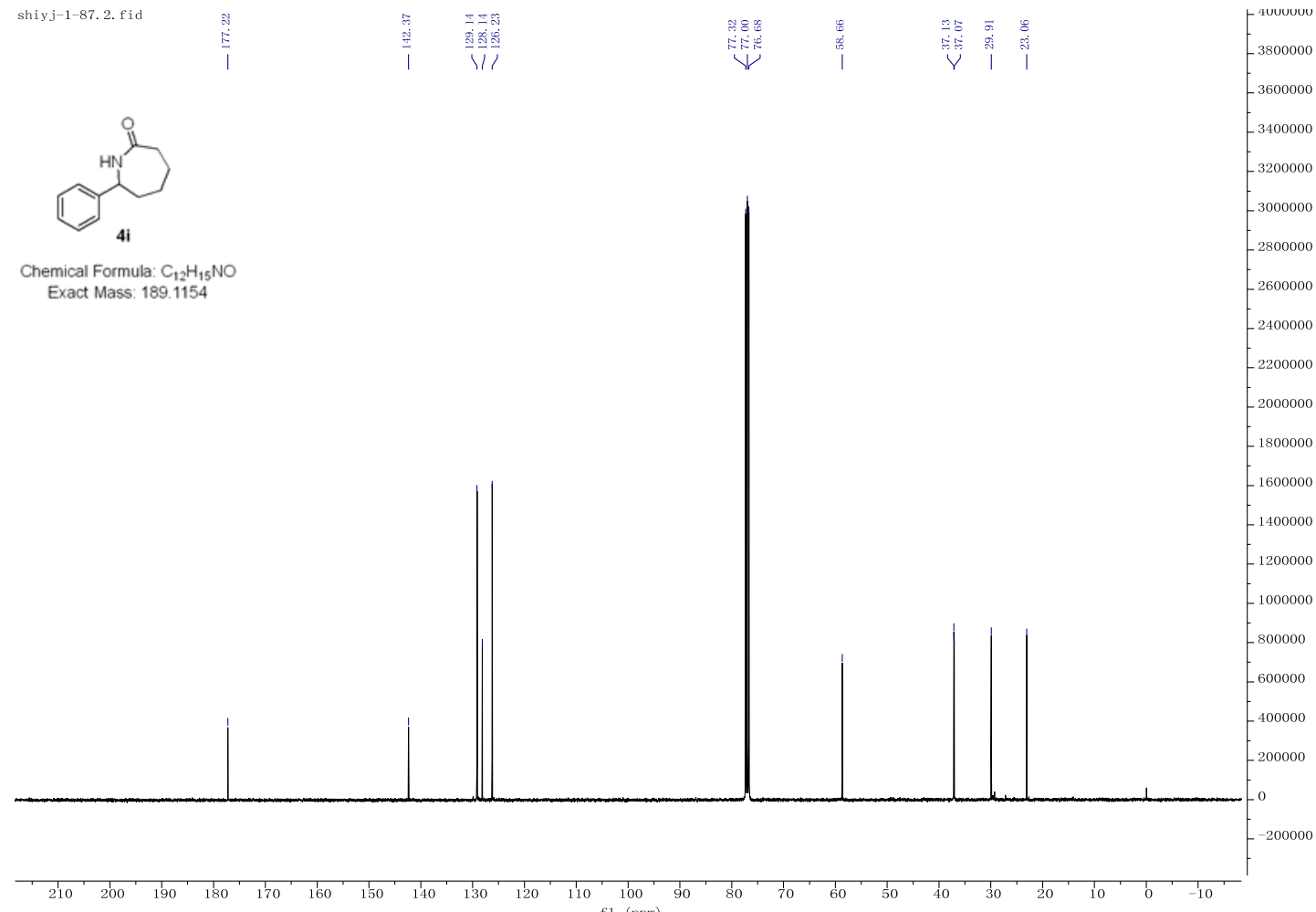


${ }^{1} \mathrm{H}$ NMR (400 MHz, Chloroform-d) of compound 6a

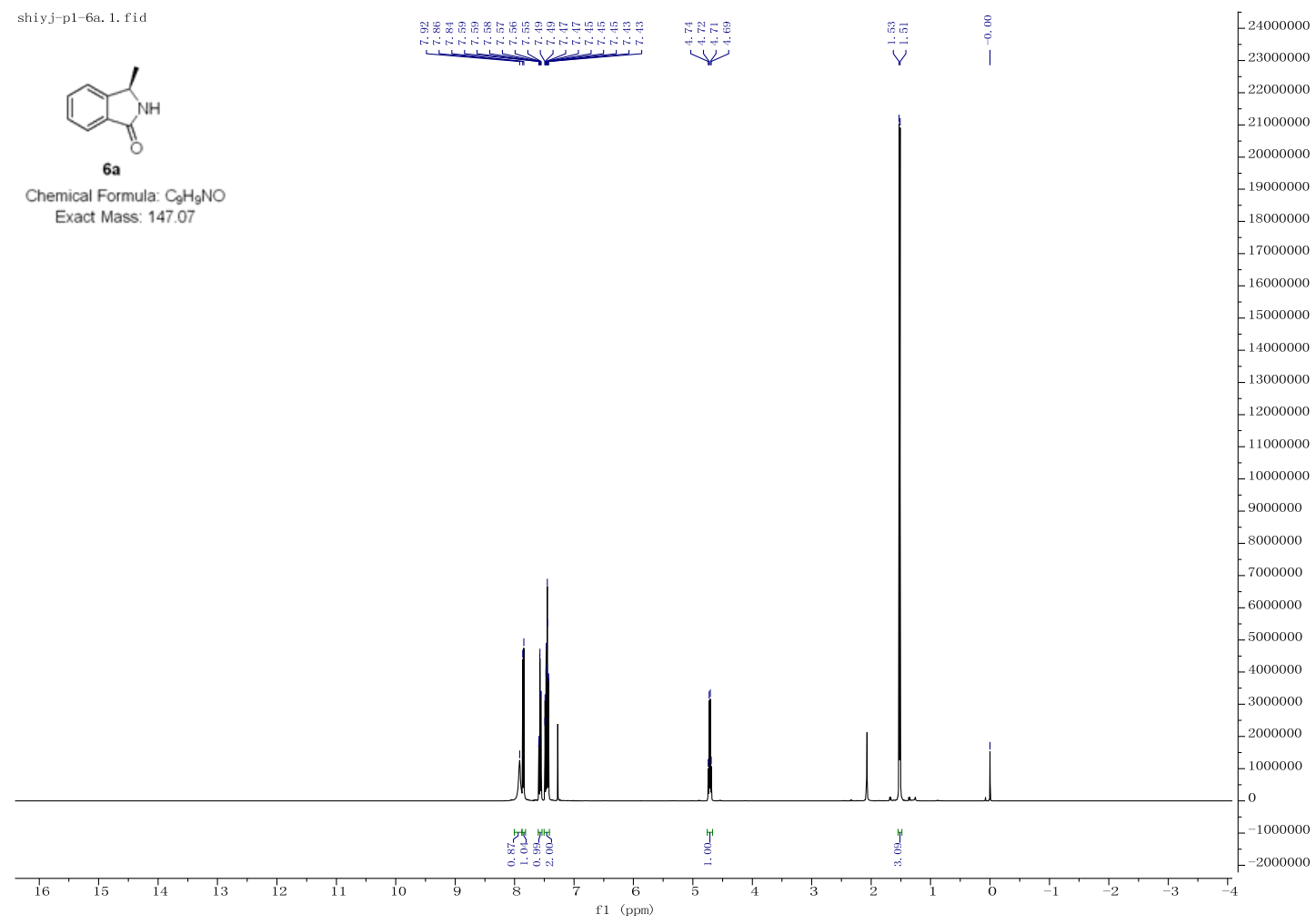

${ }^{13} \mathrm{C}\left\{{ }^{1} \mathrm{H}\right\}$ NMR (101 MHz, Chloroform-d) of compound 6a

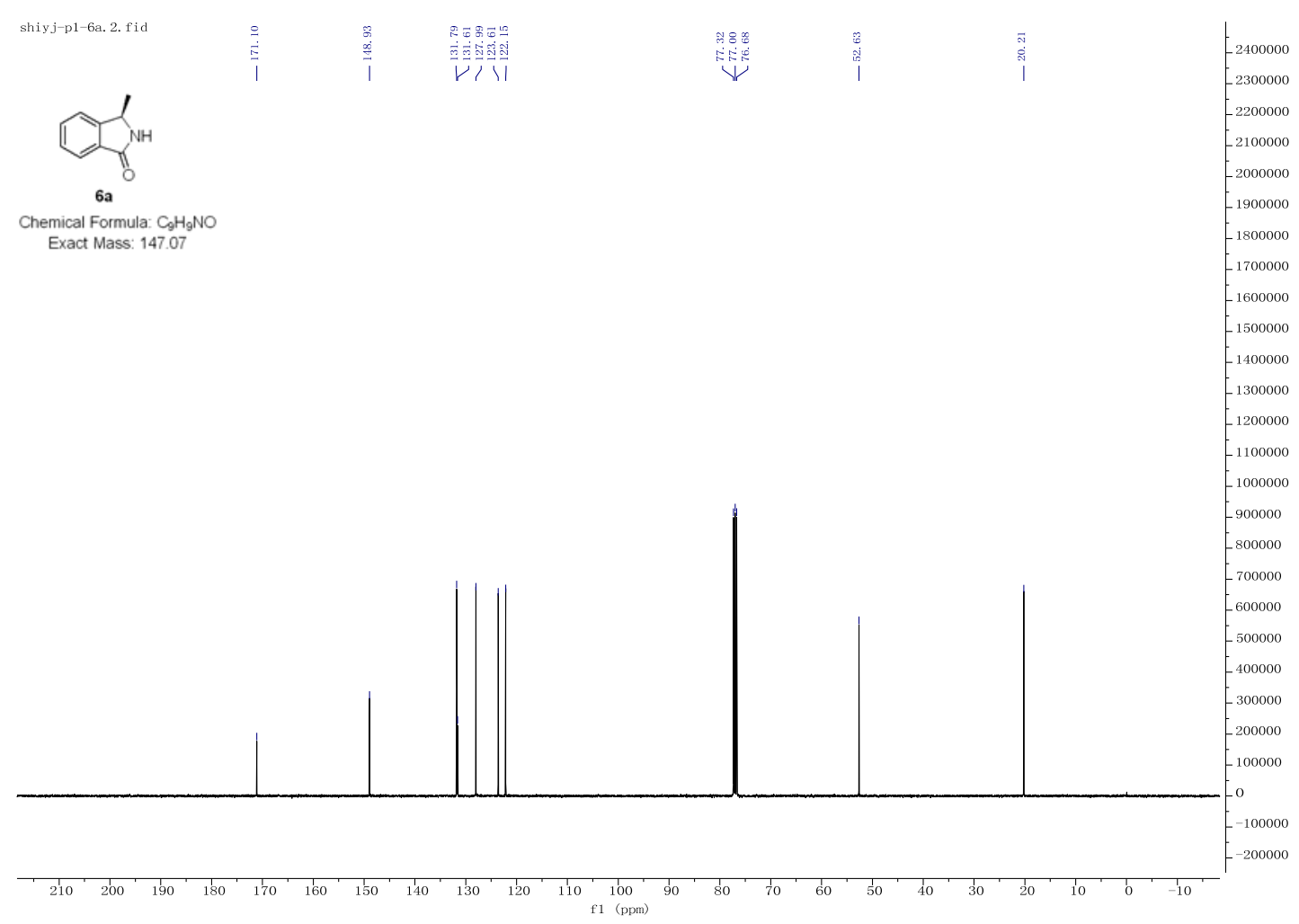


${ }^{1} \mathrm{H}$ NMR (400 MHz, Chloroform-d) of compound $\mathbf{6 b}$

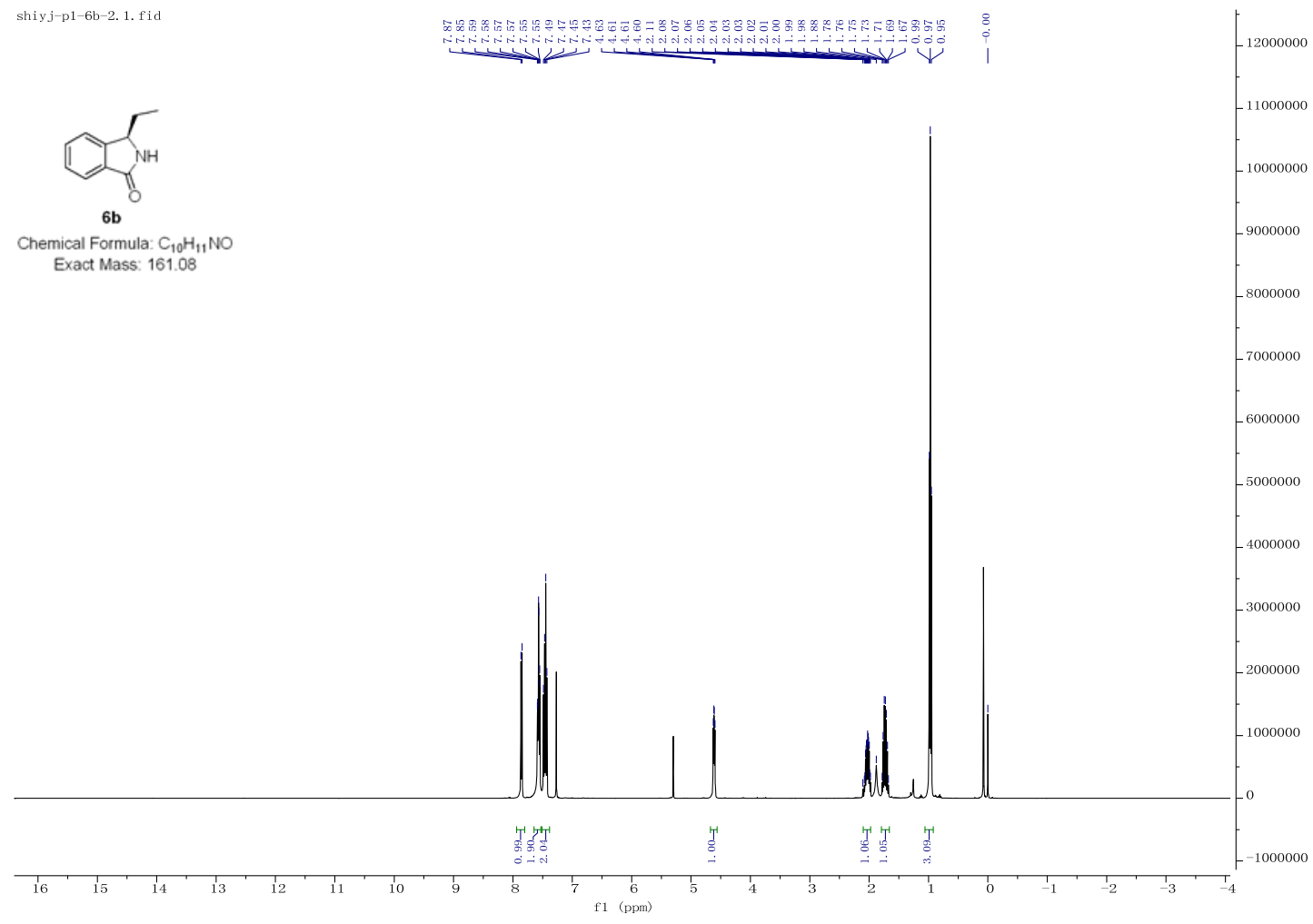

${ }^{13} \mathrm{C}\left\{{ }^{1} \mathrm{H}\right\}$ NMR (101 MHz, Chloroform-d) of compound $\mathbf{6 b}$

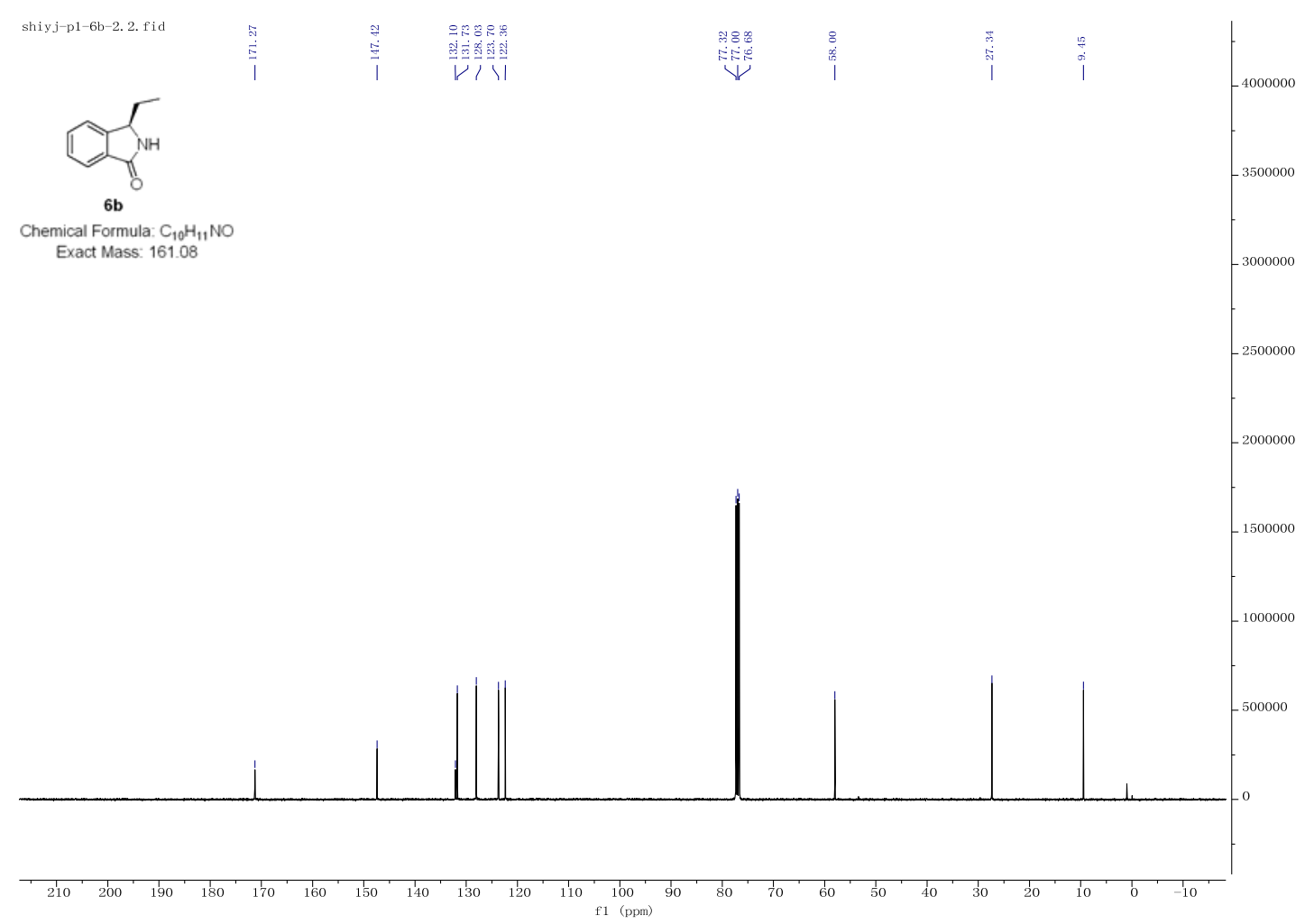


${ }^{1} \mathrm{H}$ NMR (600 MHz, Chloroform-d) of compound $\mathbf{6 c}$

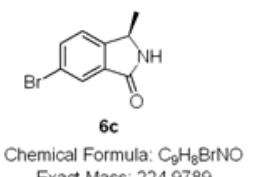

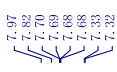

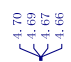

Exact Mass: 2249789

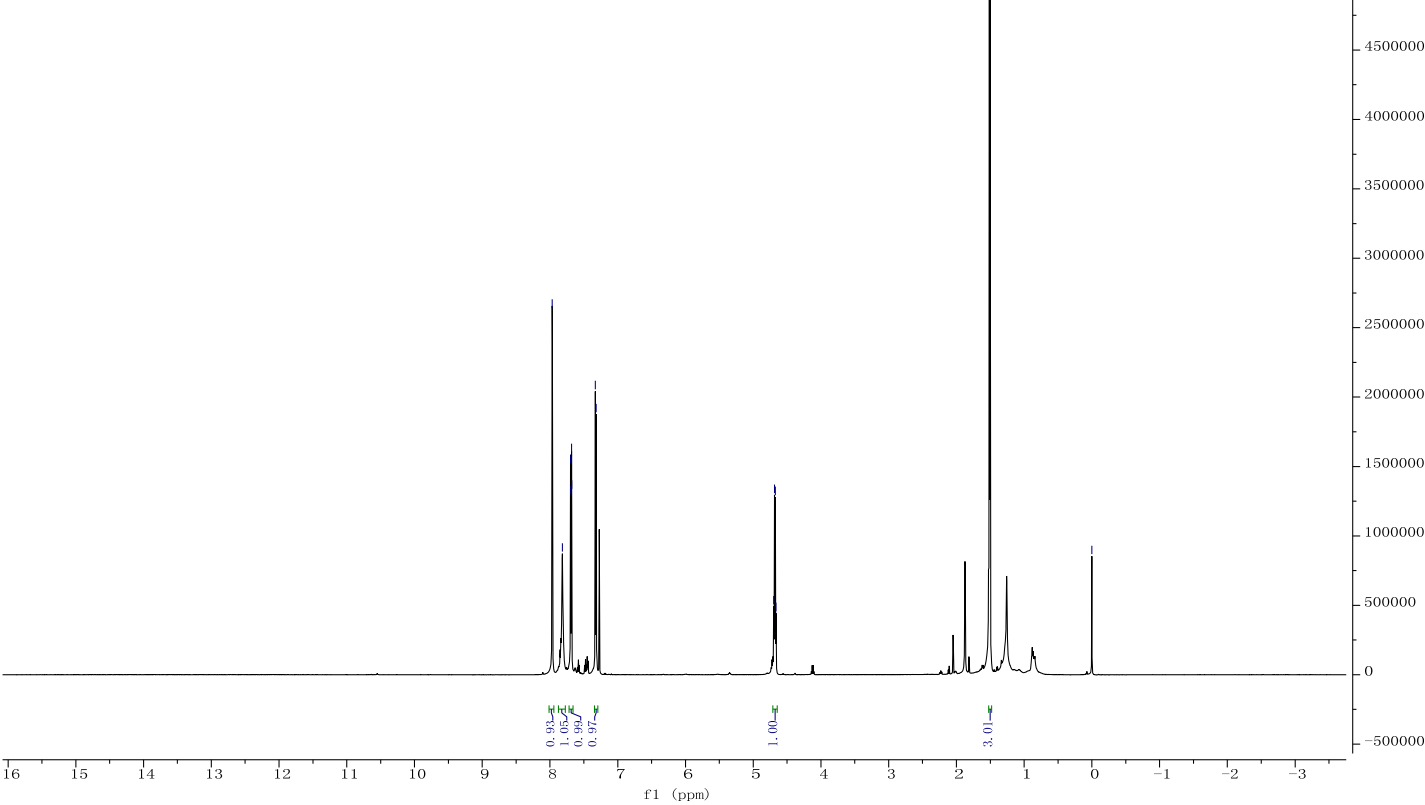

${ }^{13} \mathrm{C}\left\{{ }^{1} \mathrm{H}\right\}$ NMR (151 MHz, Chloroform-d) of compound 6c
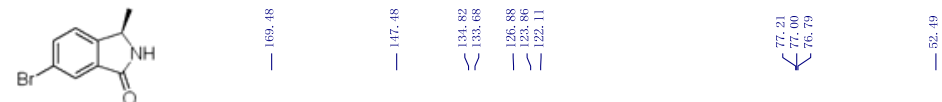

Chemical Formula: $\mathrm{C}_{9} \mathrm{H}_{8} \mathrm{BrN}$

Exact Mass: 224.9789

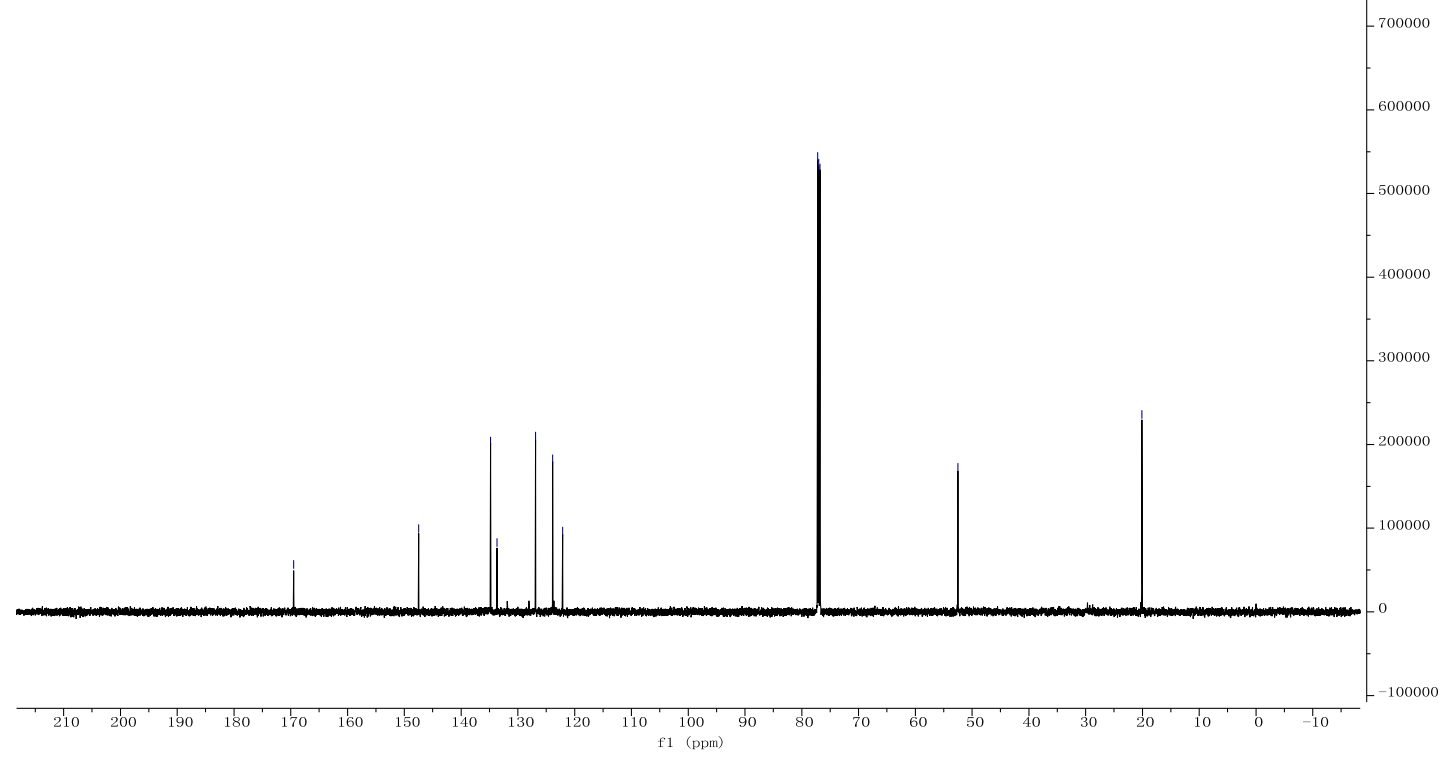


${ }^{1}$ H NMR (400 MHz, Chloroform-d) of compound $\mathbf{6 d}$

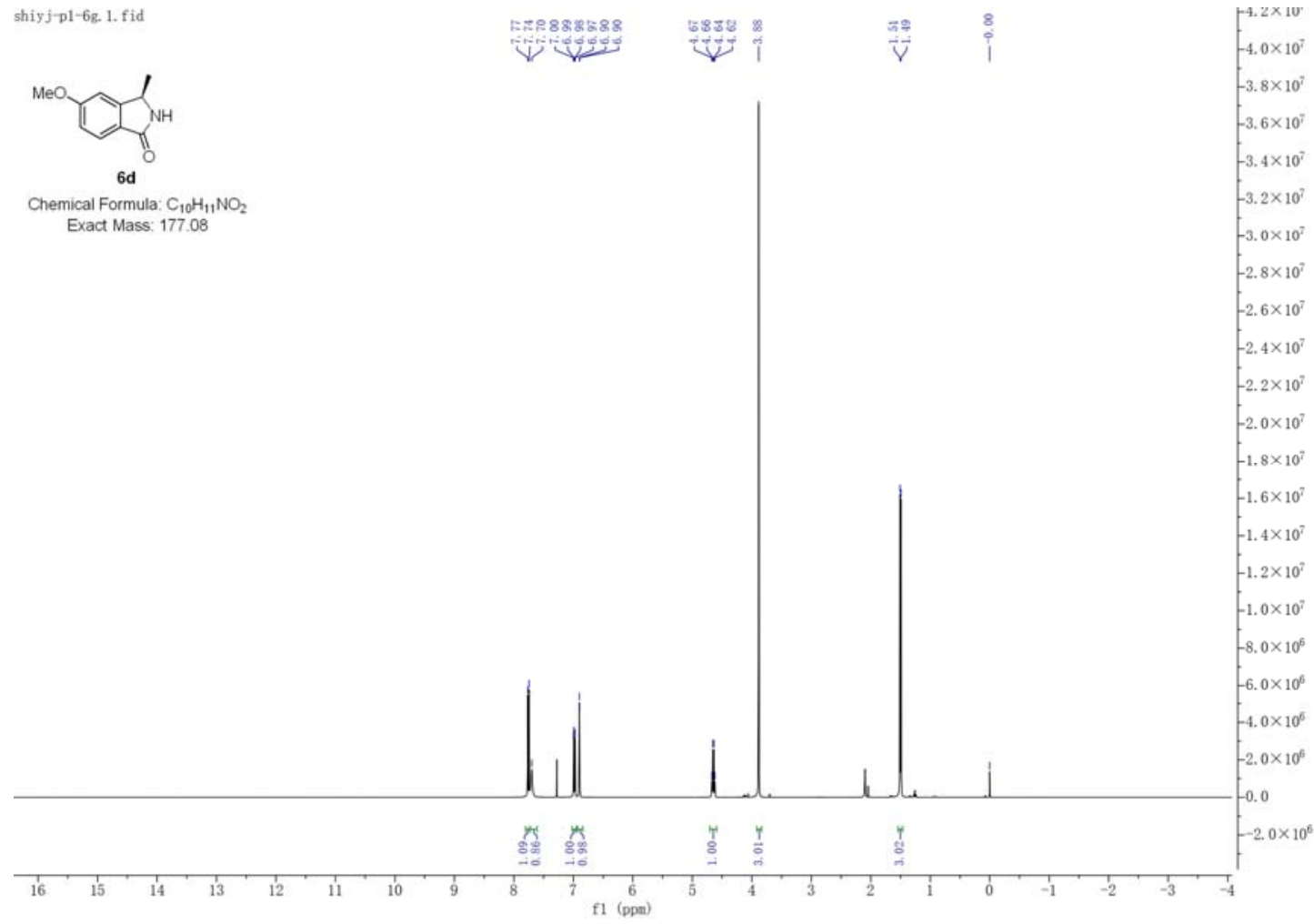

${ }^{13} \mathrm{C}\left\{{ }^{1} \mathrm{H}\right\}$ NMR (101 MHz, Chloroform-d) of compound $\mathbf{6 d}$

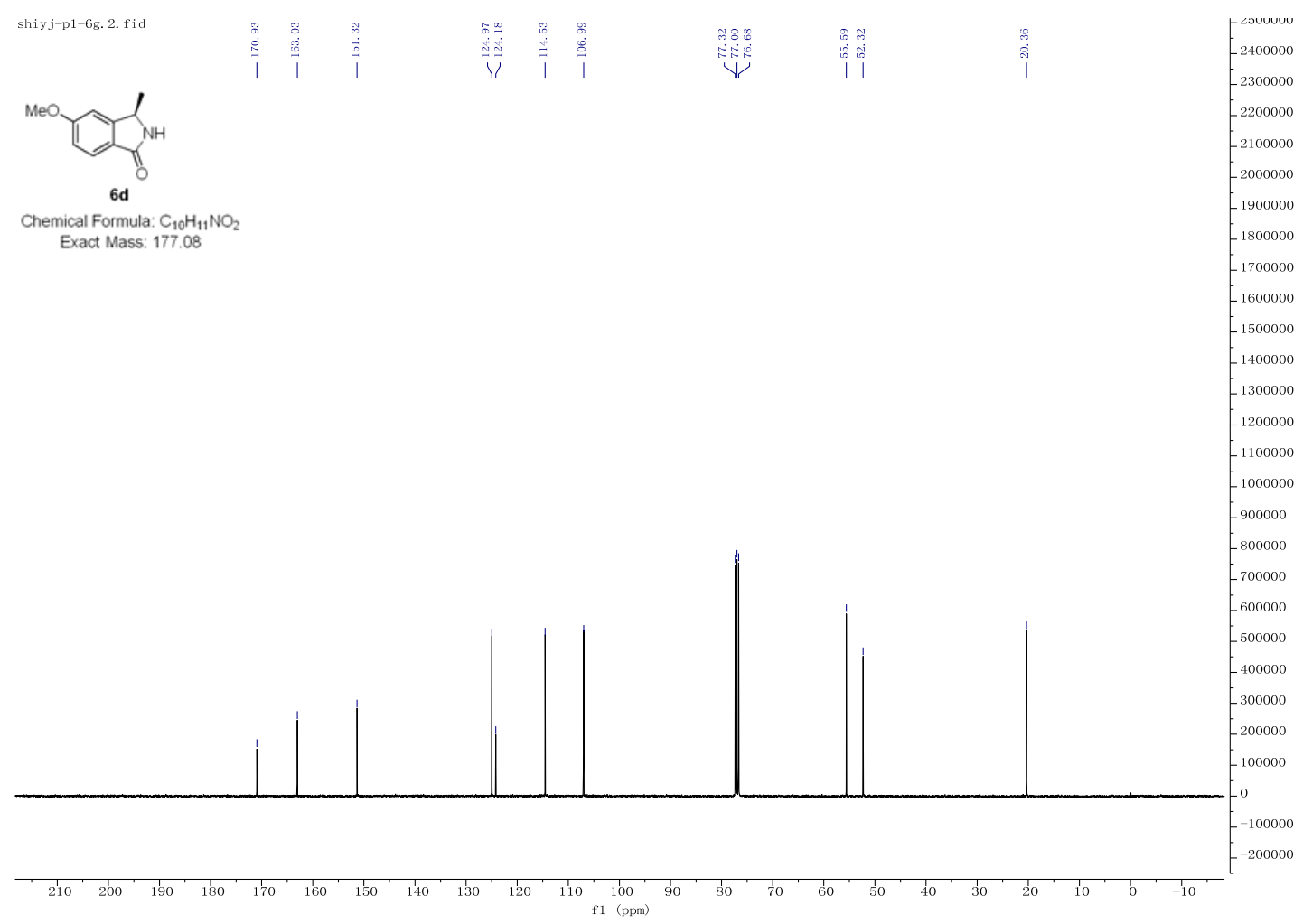


${ }^{1} \mathrm{H}$ NMR (400 MHz, Chloroform-d) of compound $6 \mathbf{e}$

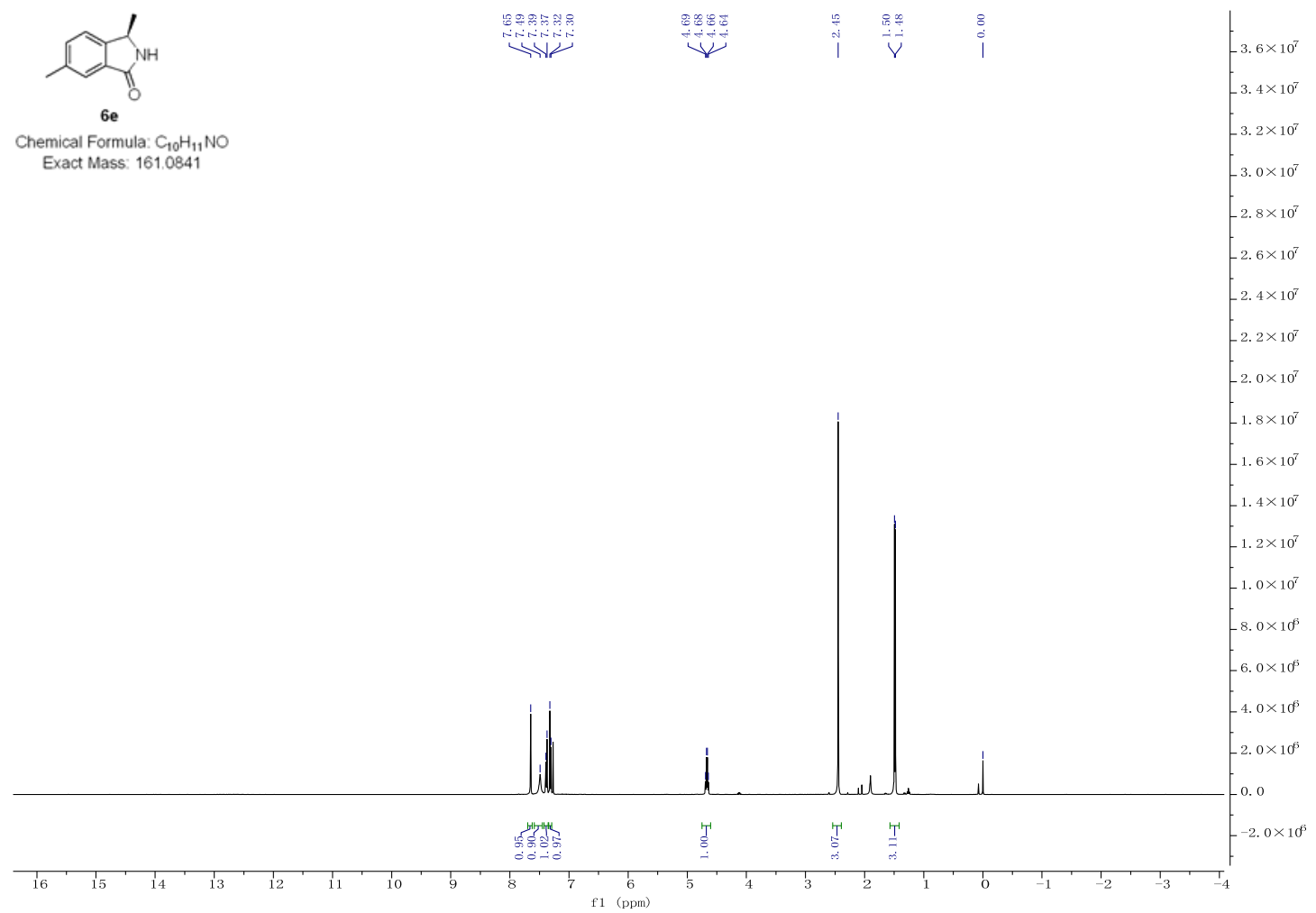

${ }^{13} \mathrm{C}\left\{{ }^{1} \mathrm{H}\right\}$ NMR (101 MHz, Chloroform-d) of compound $\mathbf{6 e}$

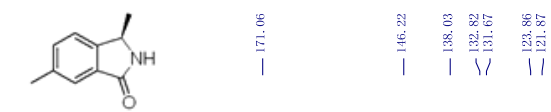

6 e

Chemical Formula: $\mathrm{C}_{10} \mathrm{H}_{11} \mathrm{NO}$

Exact Mass: 161.0841
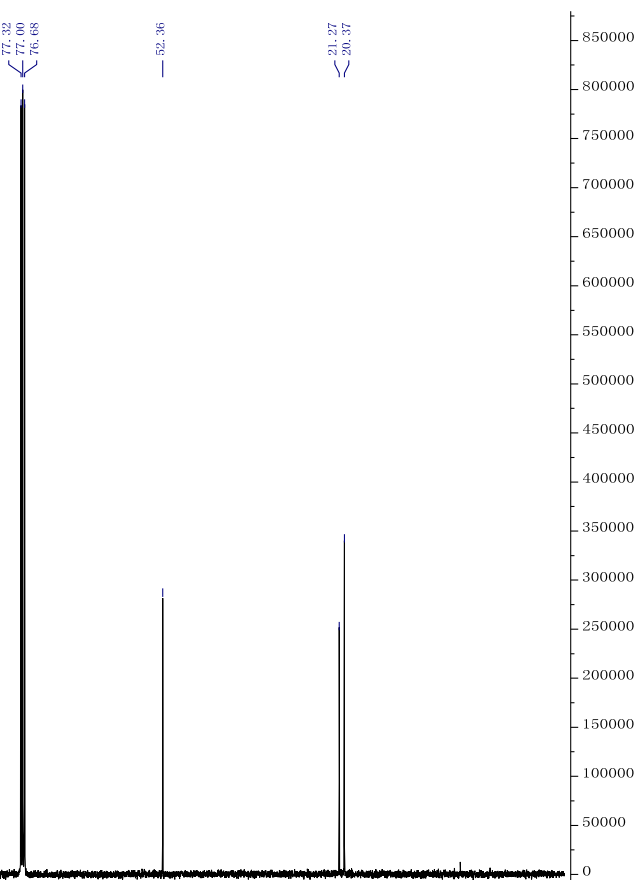

21

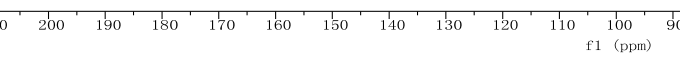


${ }^{1} \mathrm{H}$ NMR (400 MHz, Chloroform-d) of compound $\mathbf{6 f}$

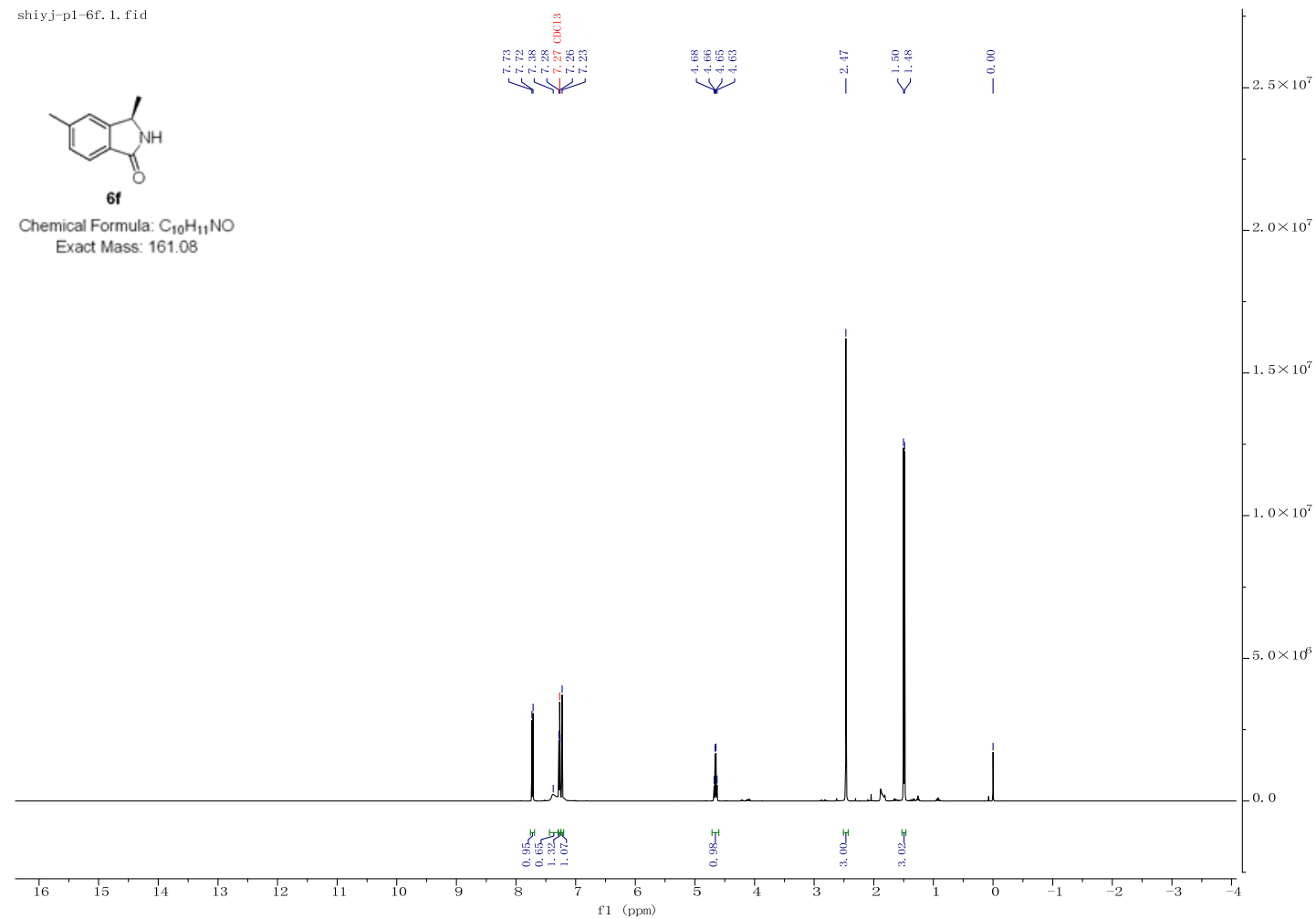

${ }^{13} \mathrm{C}\left\{{ }^{1} \mathrm{H}\right\}$ NMR (101 MHz, Chloroform-d) of compound $6 \mathbf{f}$

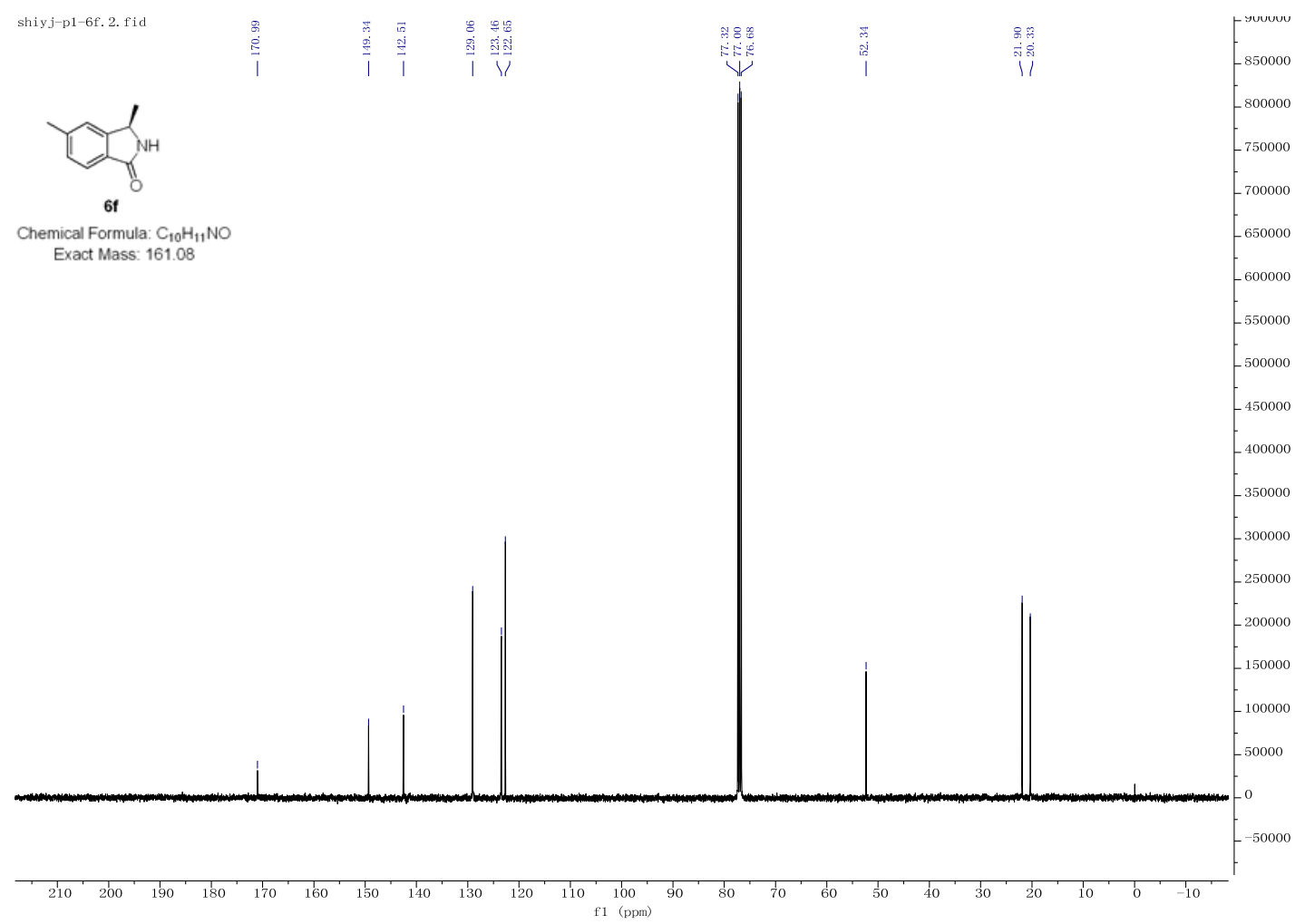


${ }^{1} \mathrm{H}$ NMR (400 MHz, Chloroform-d) of compound $\mathbf{6 g}$

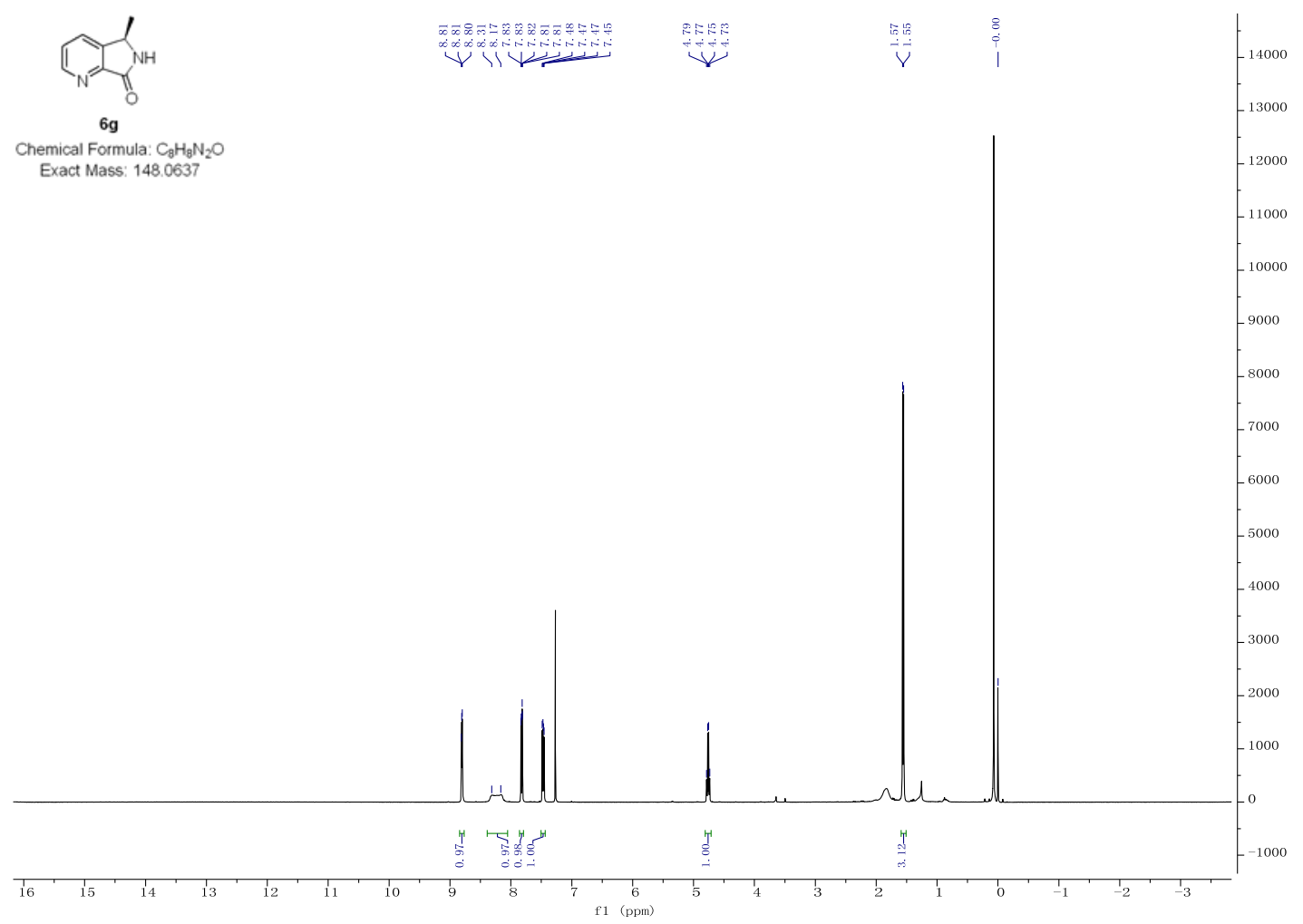

${ }^{13} \mathrm{C}\left\{{ }^{1} \mathrm{H}\right\}$ NMR (101 MHz, Chloroform-d) of compound $\mathbf{6 g}$

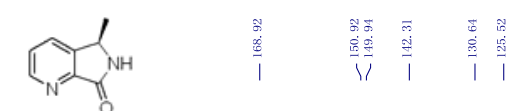

$6 \mathrm{~g}$

Chemical Formula: $\mathrm{C}_{8} \mathrm{H}_{8} \mathrm{~N}_{2} \mathrm{O}$

Exact Mass: 148.0637

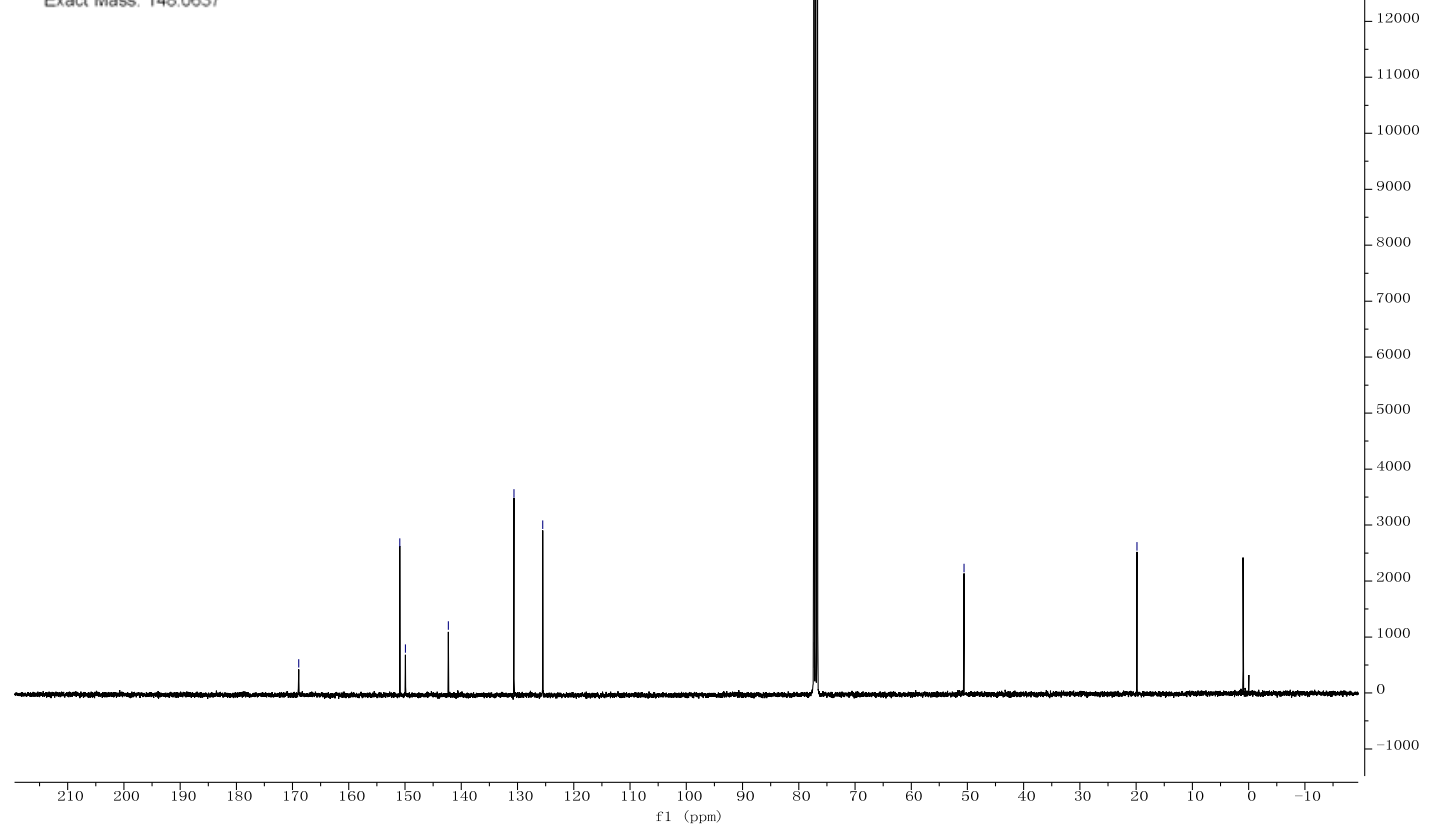


${ }^{1}$ H NMR (600 MHz, Chloroform-d) of compound $\mathbf{6 h}$

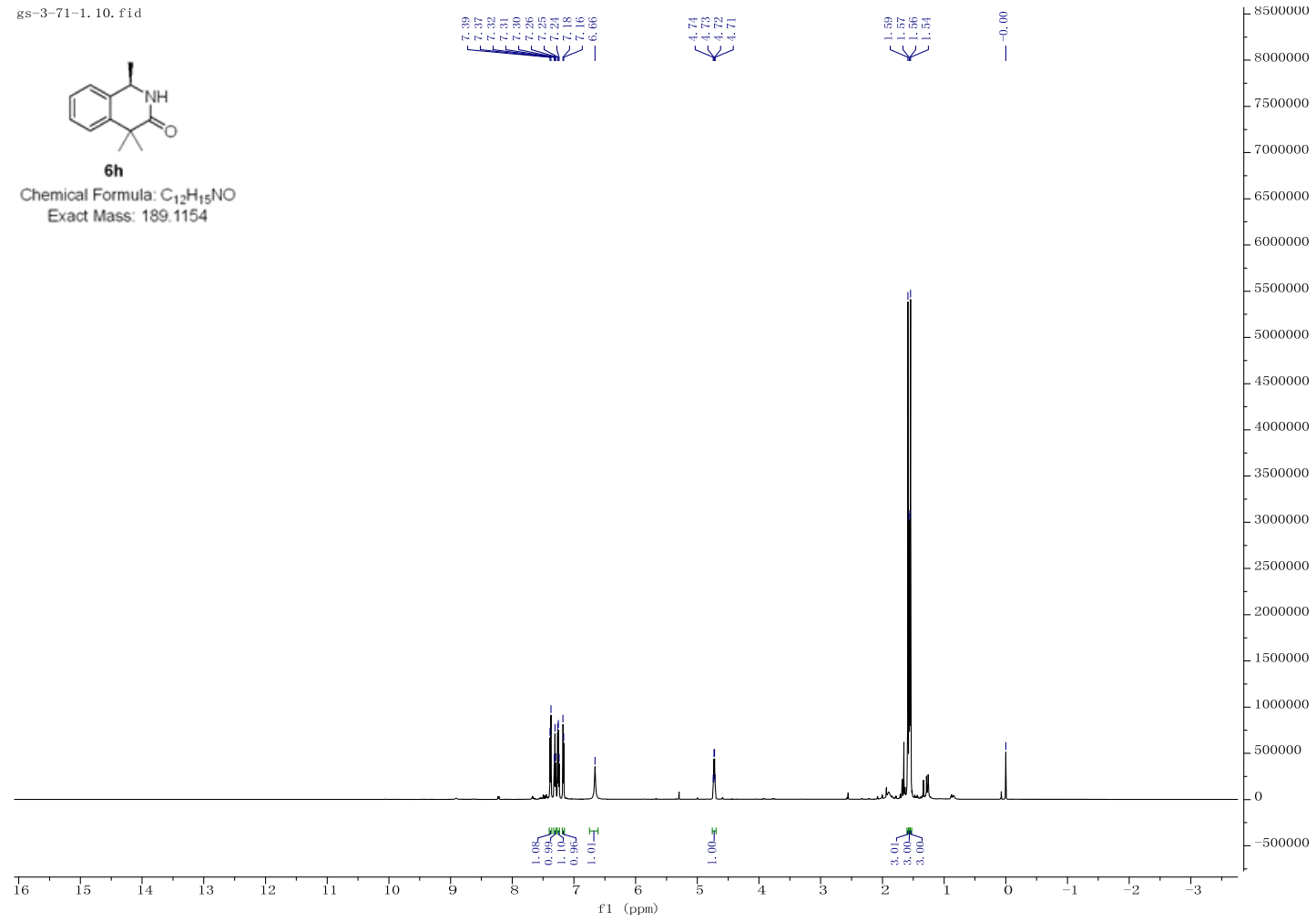

${ }^{13} \mathrm{C}\left\{{ }^{1} \mathrm{H}\right\}$ NMR (101 MHz, Chloroform-d) of compound $\mathbf{6 h}$

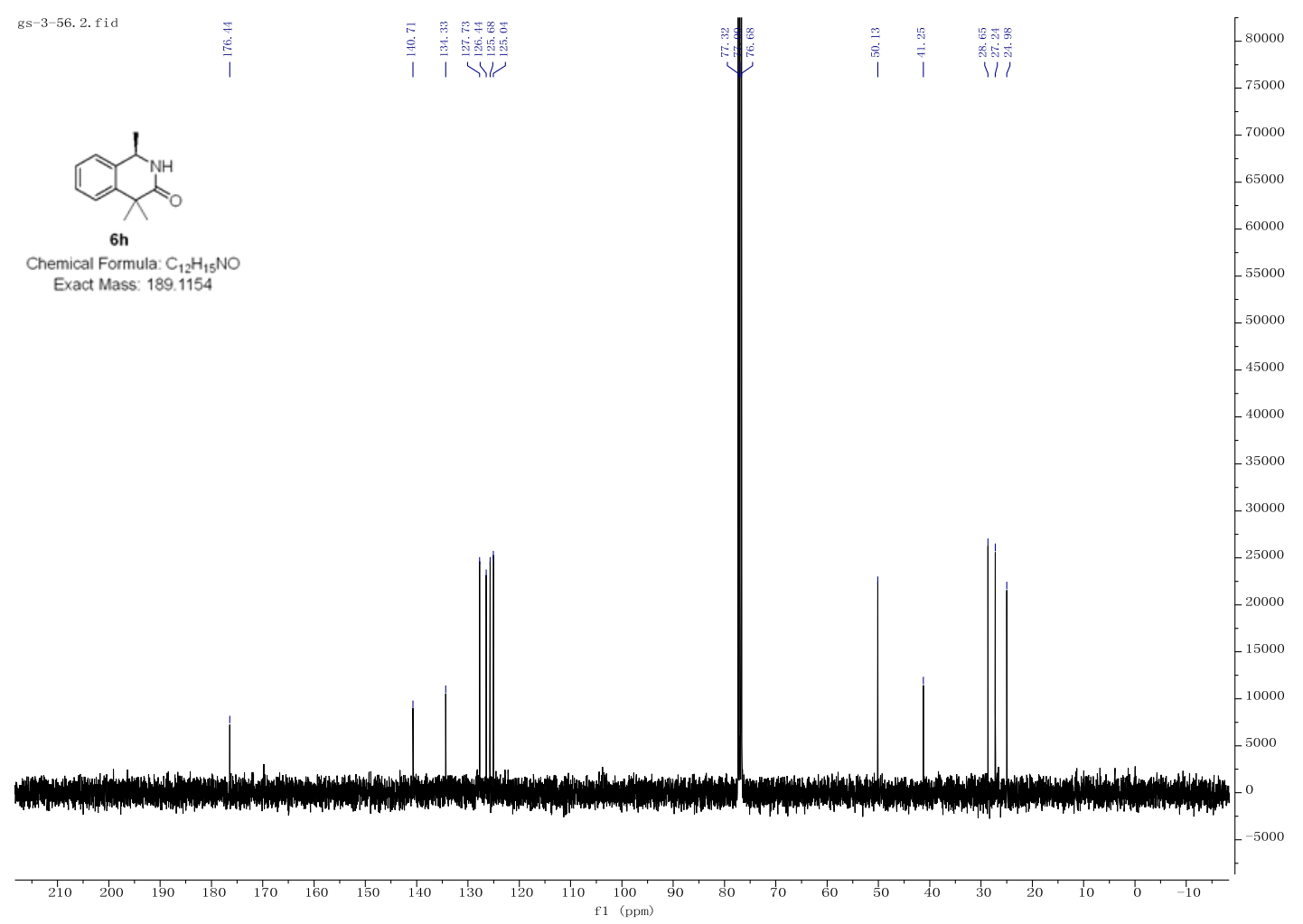


${ }^{1} \mathrm{H}$ NMR (400 MHz, Chloroform-d) of compound $\mathbf{6 i}$

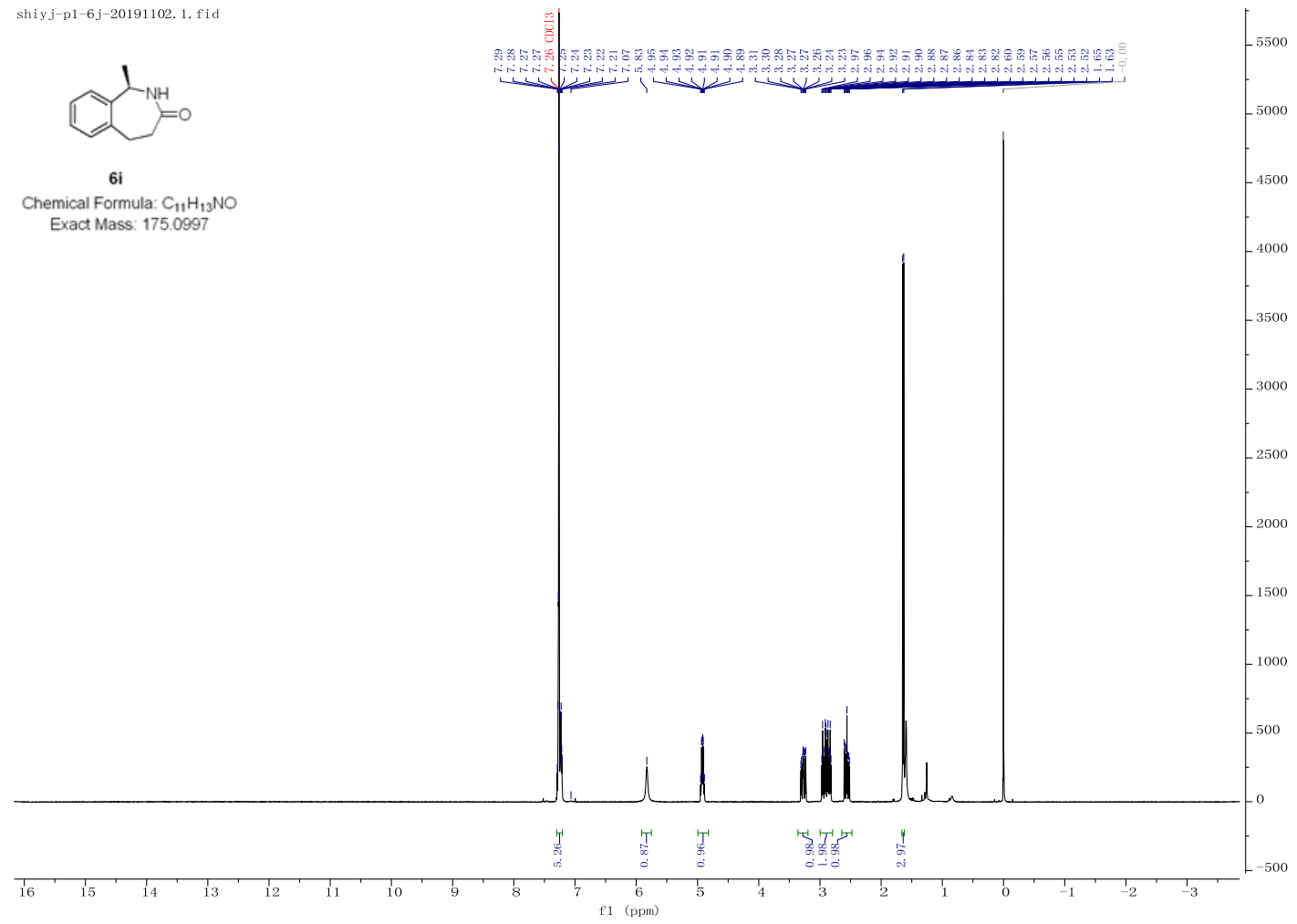

${ }^{13} \mathrm{C}\left\{{ }^{1} \mathrm{H}\right\}$ NMR (101 MHz, Chloroform-d) of compound $6 \mathbf{6}$

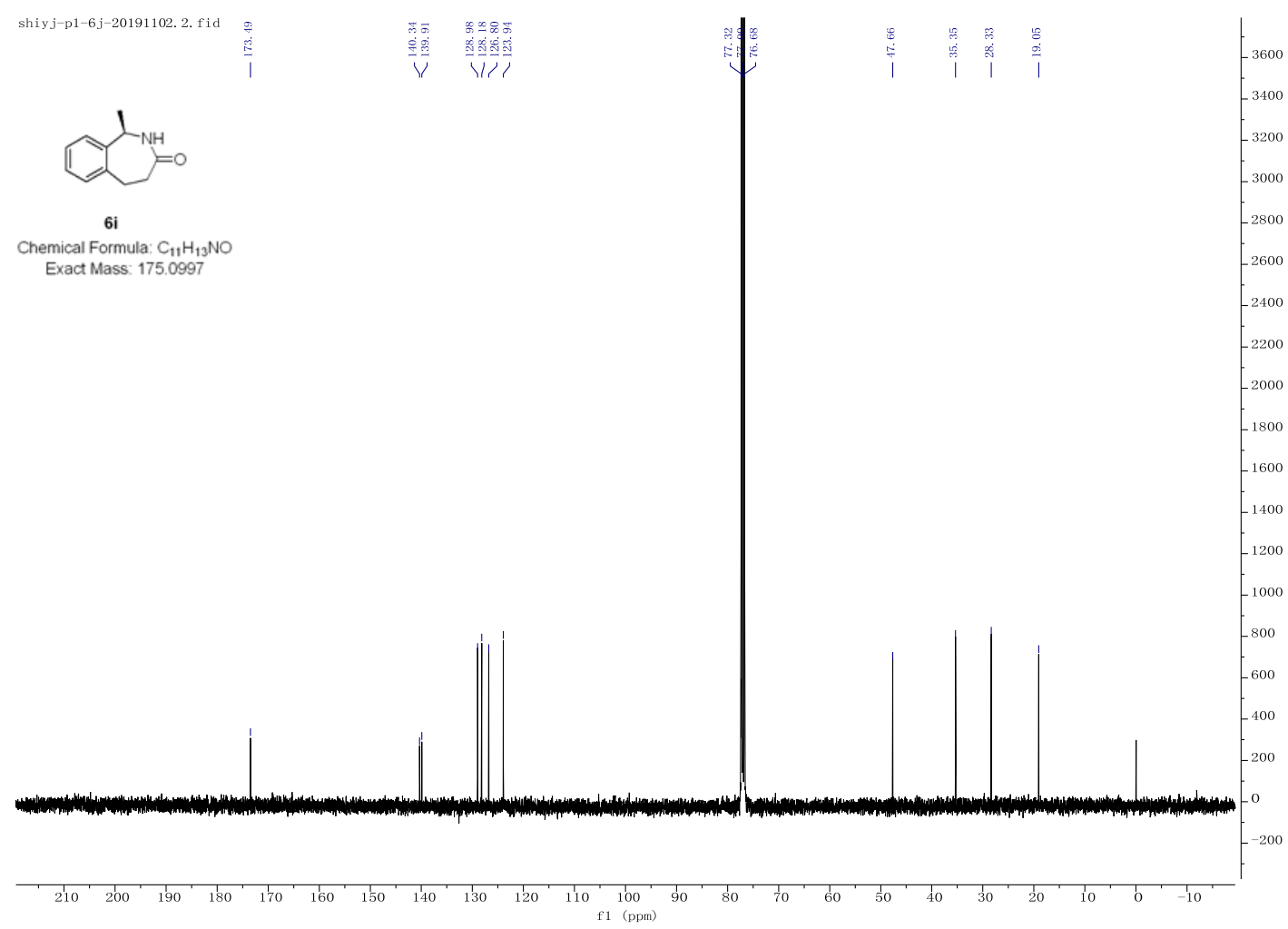


${ }^{1} \mathrm{H}$ NMR (400 MHz, DMSO-d $\mathrm{d}_{6}$ ) of compound $\mathbf{6 j}$

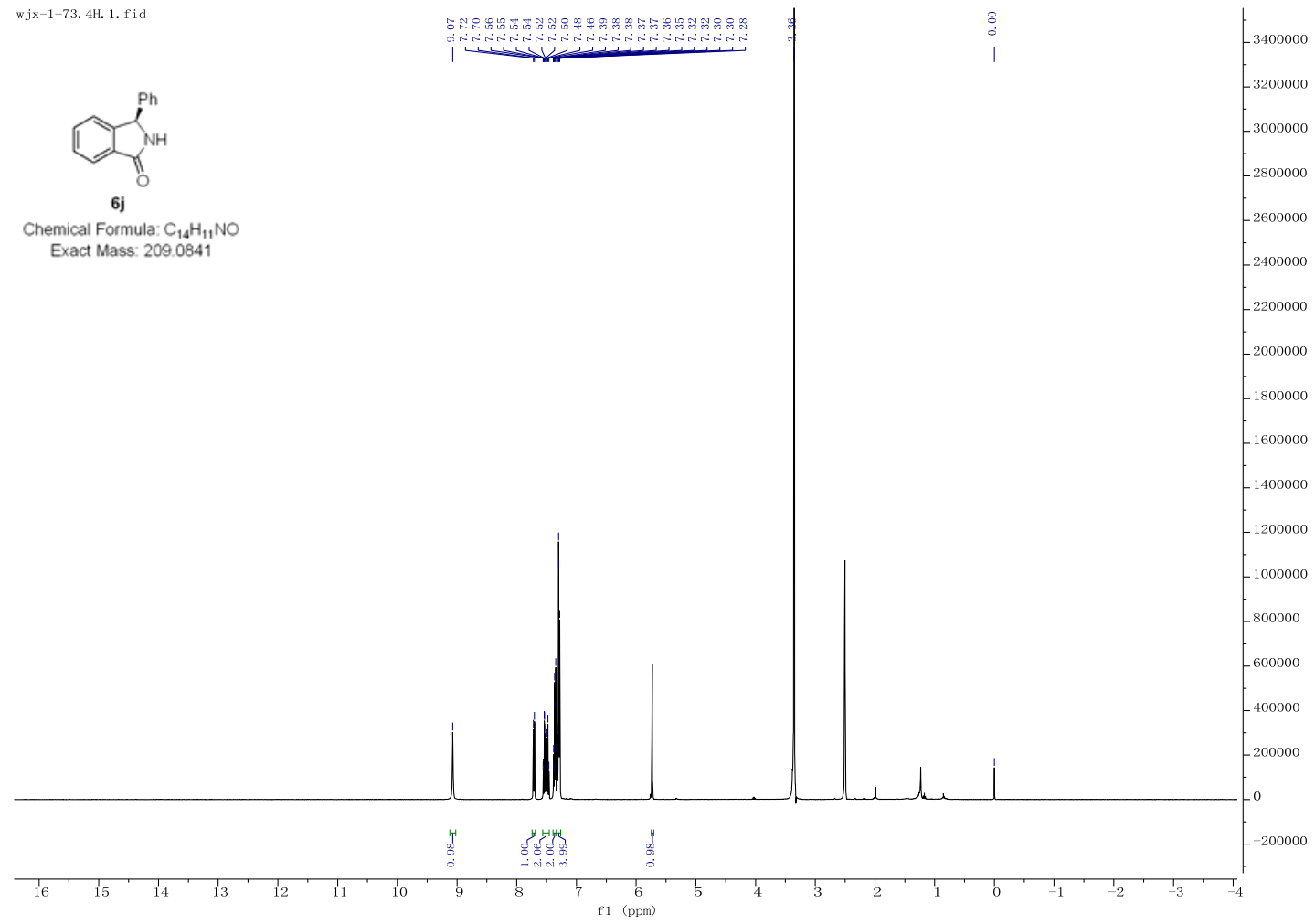

${ }^{13} \mathrm{C}\left\{{ }^{1} \mathrm{H}\right\}$ NMR (101 MHz, DMSO-d $\mathrm{d}_{6}$ ) of compound $\mathbf{6 j}$

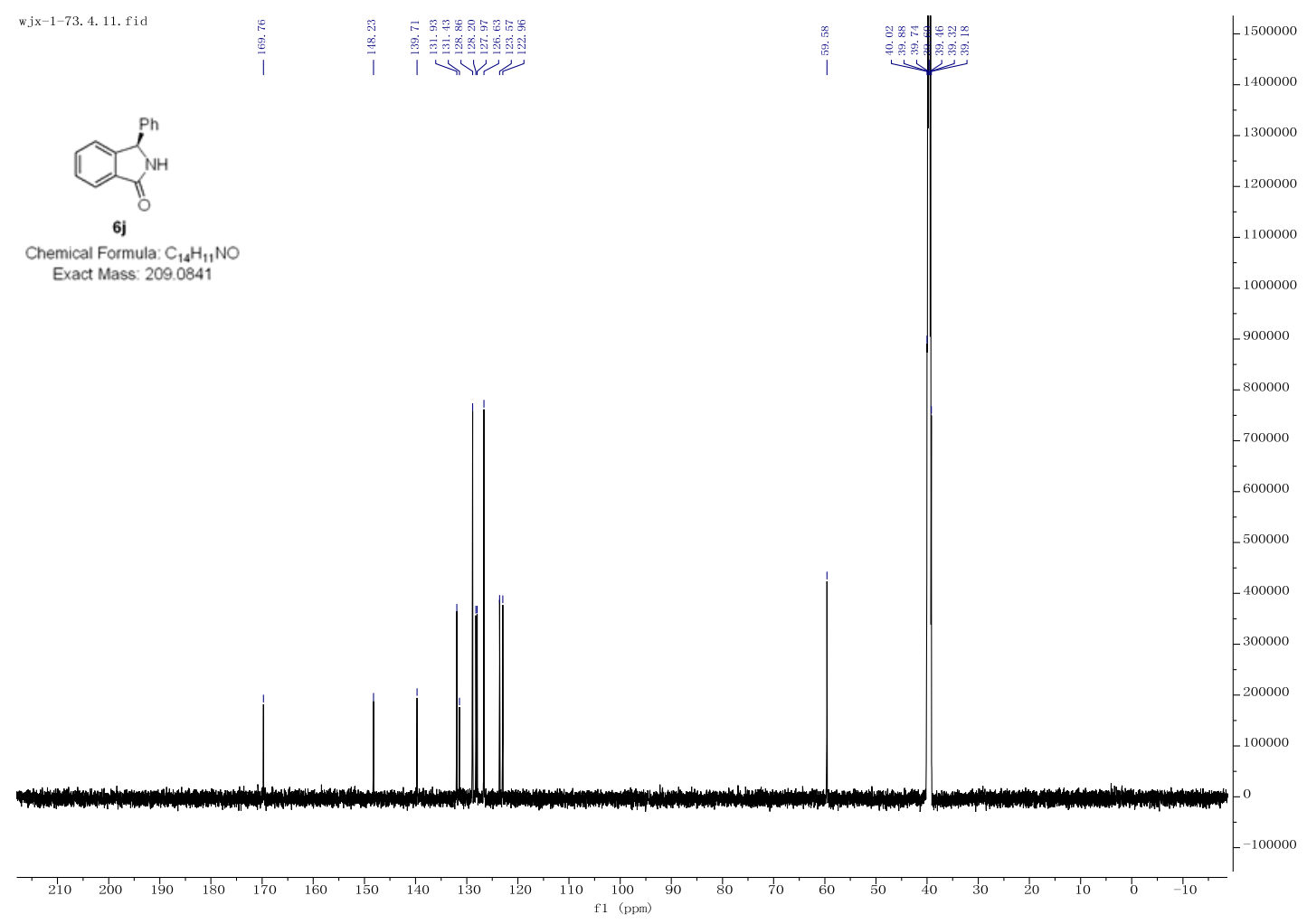


${ }^{1} \mathrm{H}$ NMR (600 MHz, Chloroform-d) of compound 7

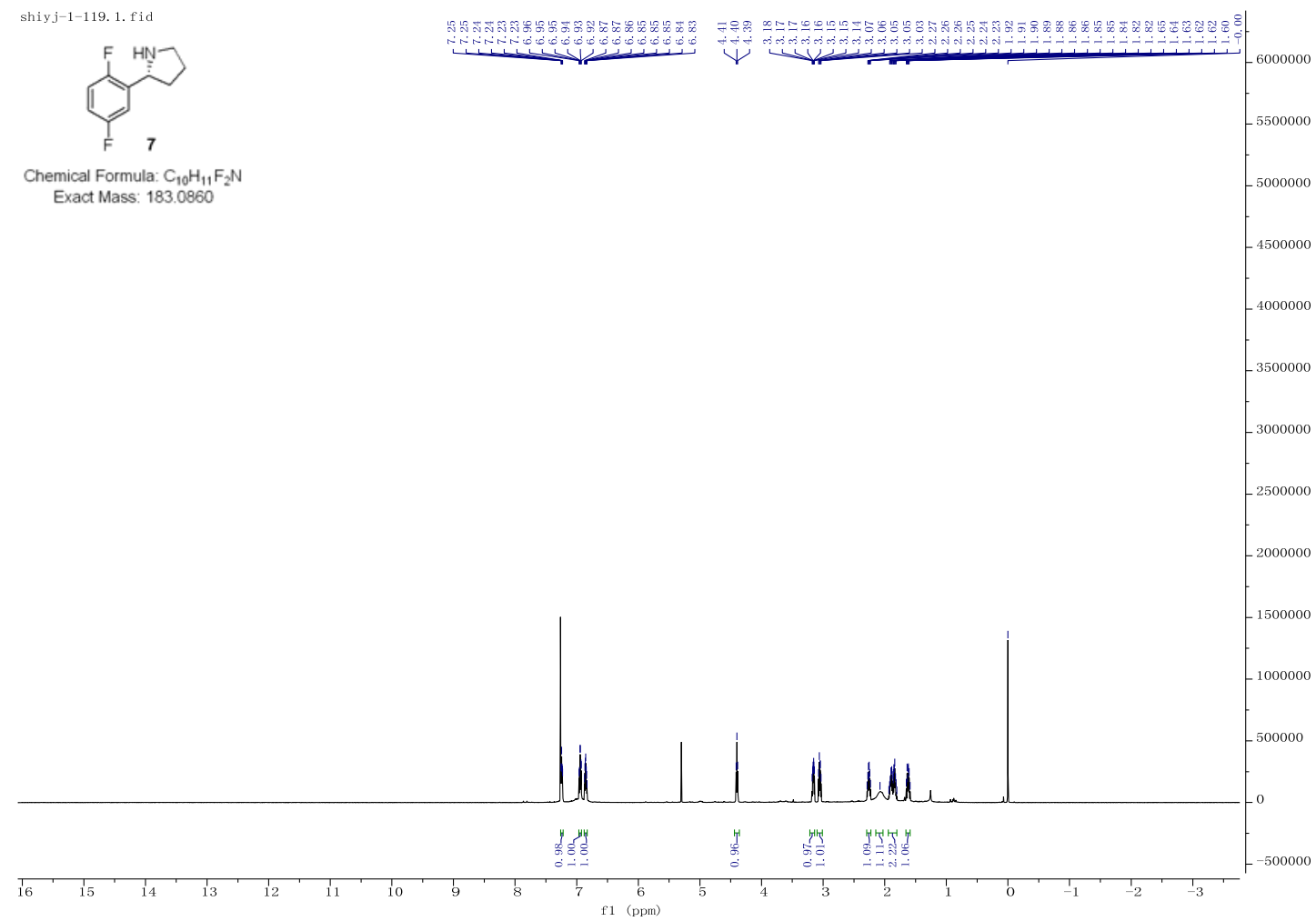


${ }^{1}$ H NMR (400 MHz, Chloroform-d) of compound C

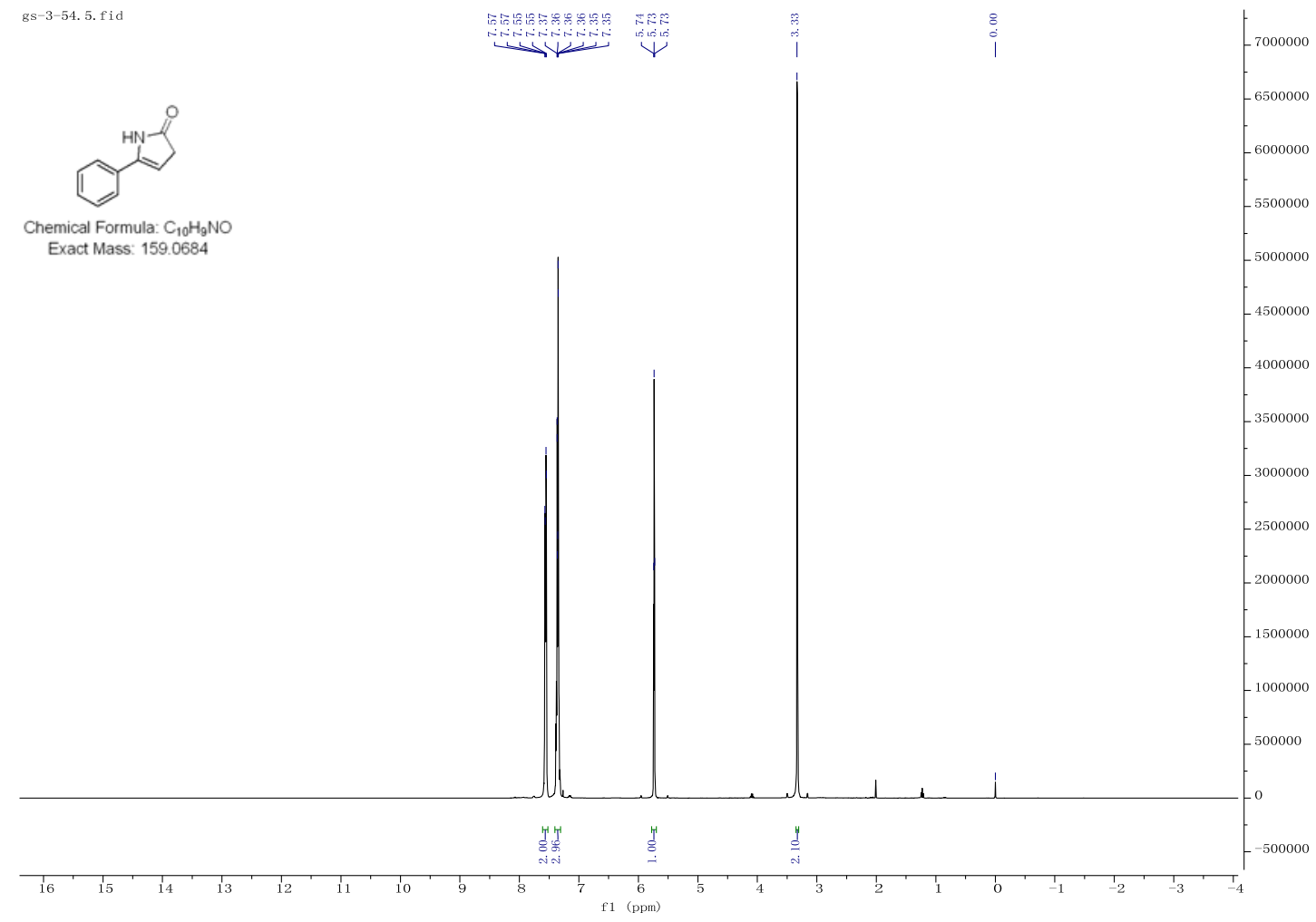

${ }^{13} \mathrm{C}\left\{{ }^{1} \mathrm{H}\right\}$ NMR (101 MHz, Chloroform-d) of compound $\mathbf{C}$

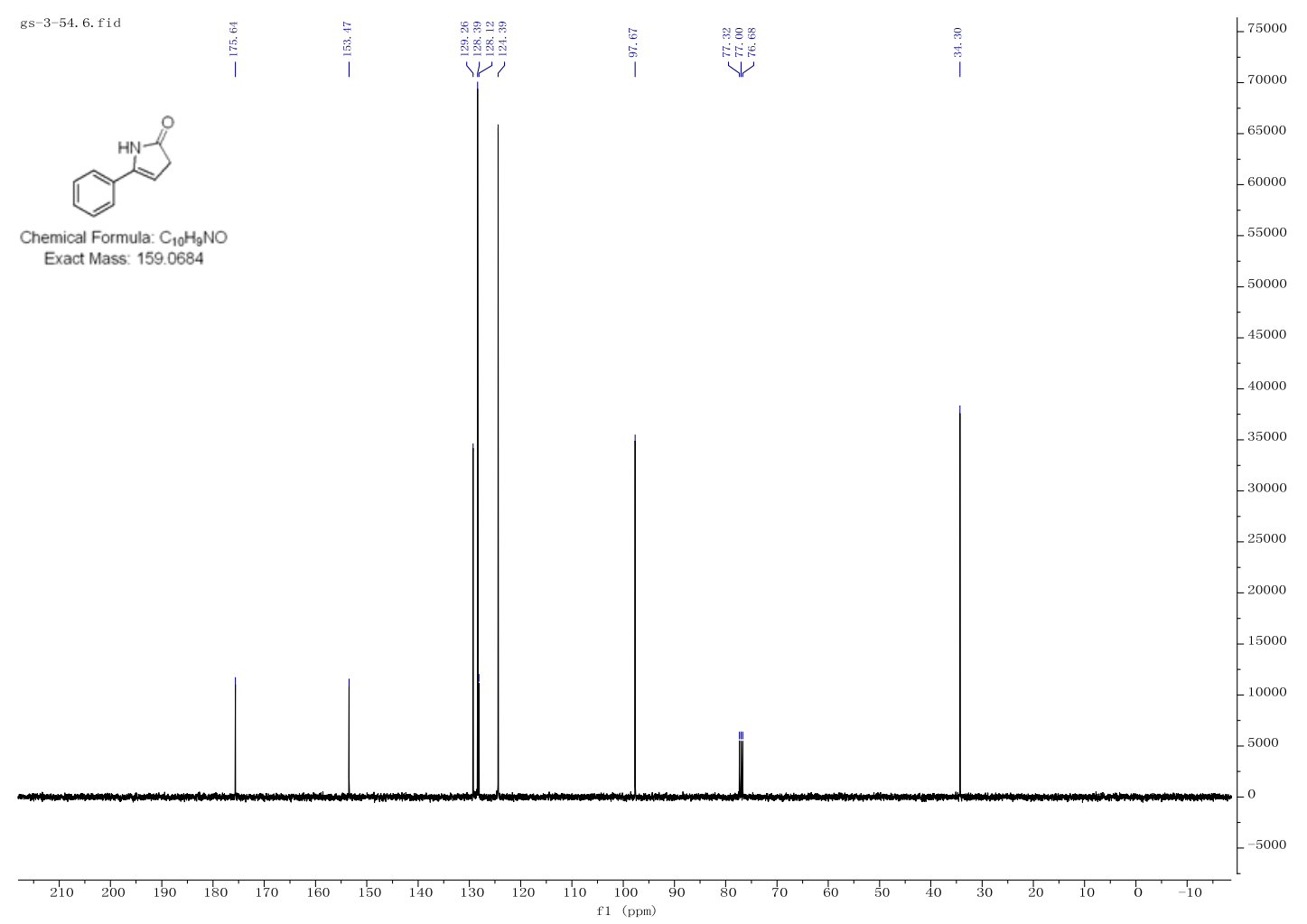


${ }^{1}$ H NMR (400 MHz, Methanol-d) of compound $\mathbf{E}$
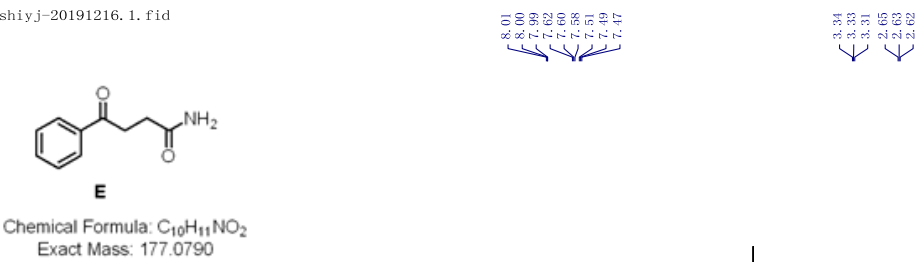

$2.6 \times 10^{2}$

Exact Mass: 177.0790

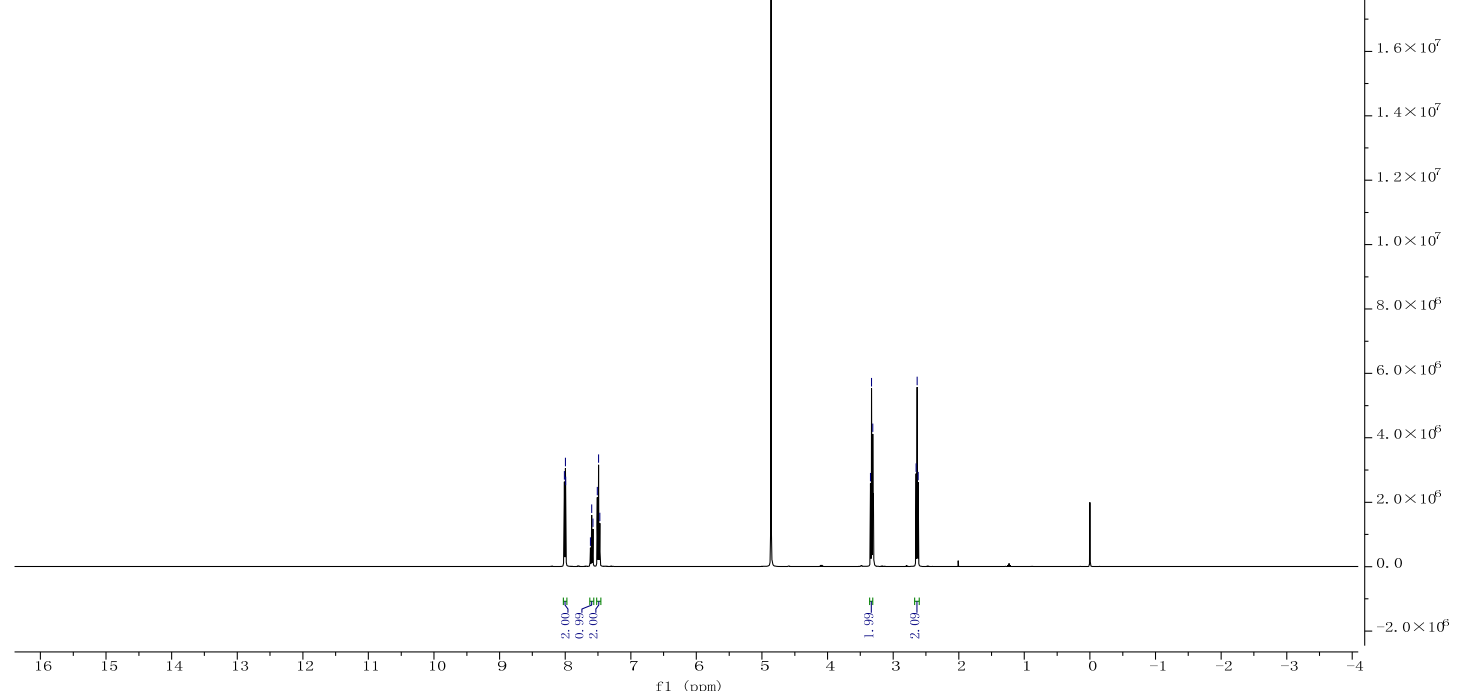




\section{HPLC Spectra}

\section{(R)-5-phenylpyrrolidin-2-one (2a)}

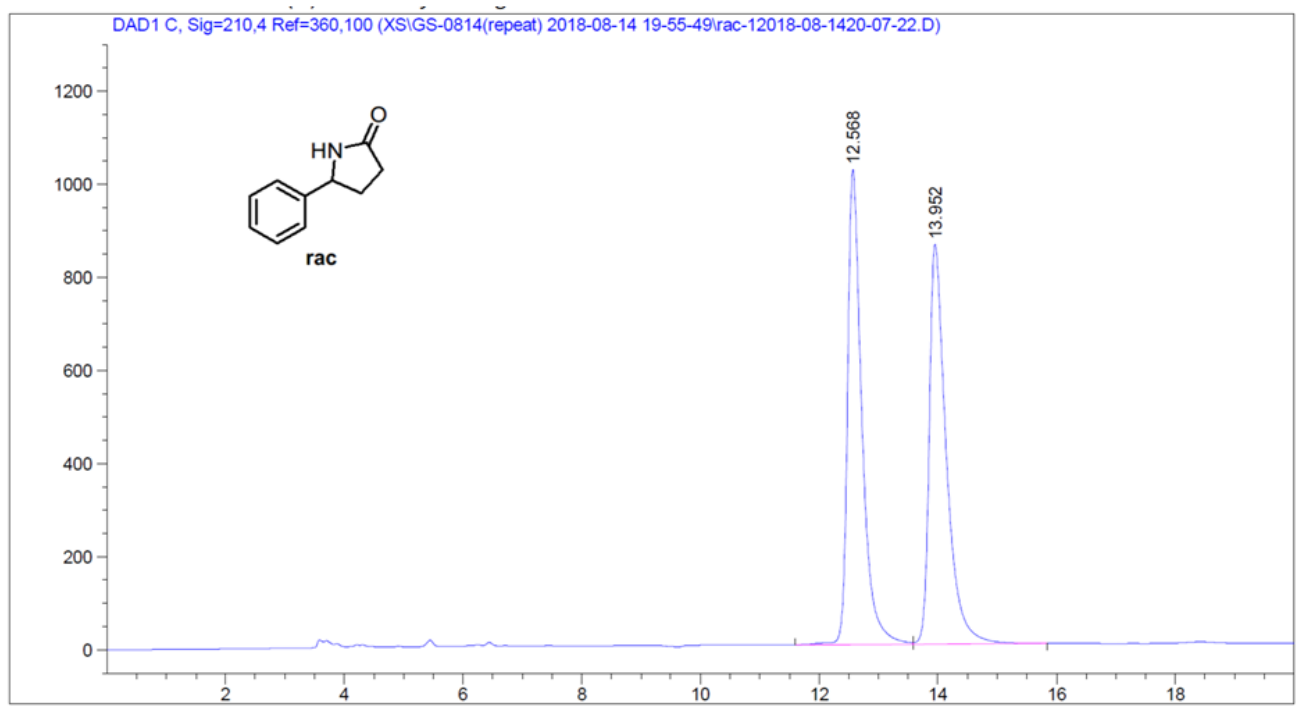

Signal 1: DAD1 C, Sig=210,4 Ref $=360,100$

\begin{tabular}{|c|c|c|c|c|c|}
\hline eak & $\begin{array}{l}\text { RetTime Type } \\
\text { [min] }\end{array}$ & $\begin{array}{l}\text { Width } \\
\text { [min] }\end{array}$ & $\begin{array}{c}\text { Area } \\
{\left[\mathrm{mAU}^{*} \mathrm{~s}\right]}\end{array}$ & $\begin{array}{l}\text { ght } \\
\text { U] }\end{array}$ & $\begin{array}{c}\text { Area } \\
\%\end{array}$ \\
\hline & & & & --- & \\
\hline 1 & & & 4 & 101 & 50 \\
\hline 2 & $13.952 \mathrm{VB}$ & 913 & $1.70178 \mathrm{e} 4$ & 858.22931 & 49.9128 \\
\hline
\end{tabular}

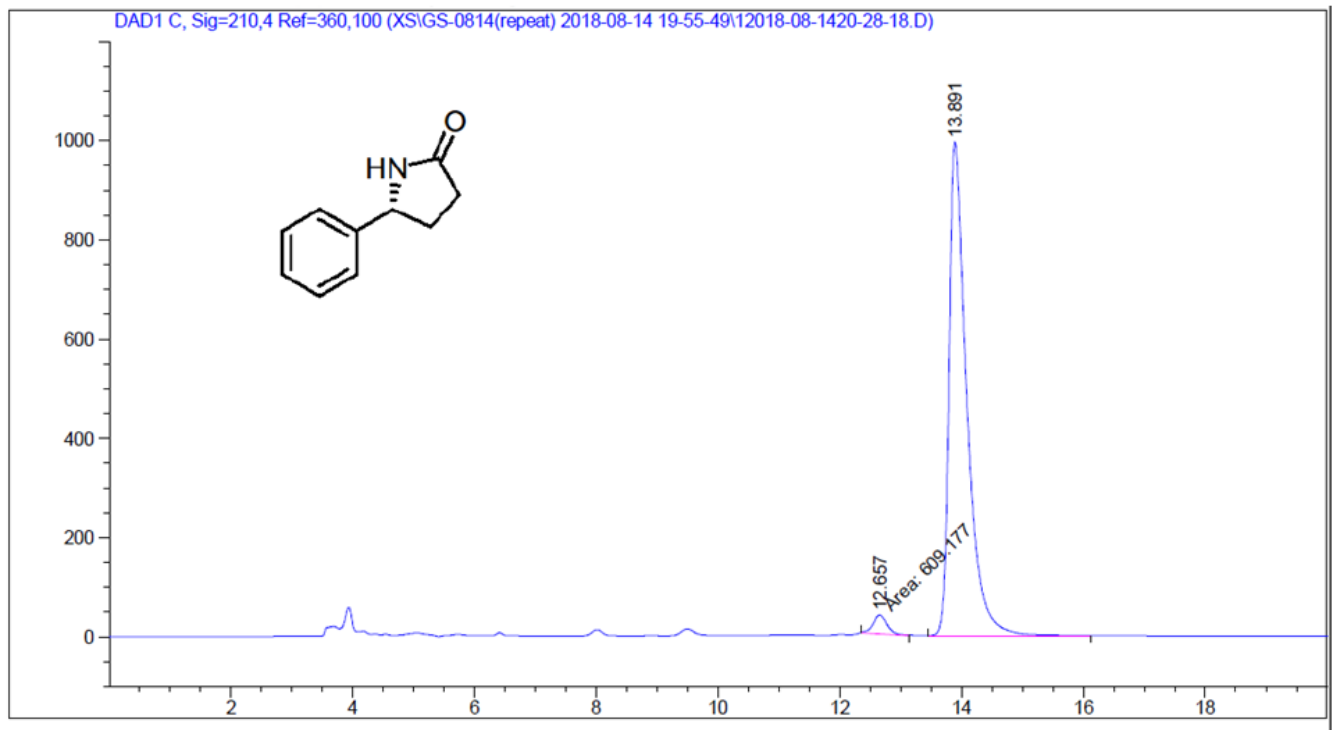

Signal 1: DAD1 C, Sig $=210,4$ Ref $=360,100$

\begin{tabular}{cccccc}
$\begin{array}{c}\text { Peak RetTime Type } \\
\text { \# } \begin{array}{c}\text { Width } \\
\text { [min] }\end{array}\end{array}$ & $\begin{array}{c}\text { Area } \\
\text { [mAU*s] }\end{array}$ & $\begin{array}{c}\text { Height } \\
\text { [mAU] }\end{array}$ & $\begin{array}{c}\text { Area } \\
\%\end{array}$ \\
\hline 1 & 12.657 MM & 0.2659 & 609.17657 & 38.18148 & 2.9473 \\
2 & 13.891 BB & 0.2952 & $2.00597 e 4$ & 994.82056 & 97.0527
\end{tabular}




\section{(R)-5-(2,5-difluorophenyl)pyrrolidin-2-one (2b)}

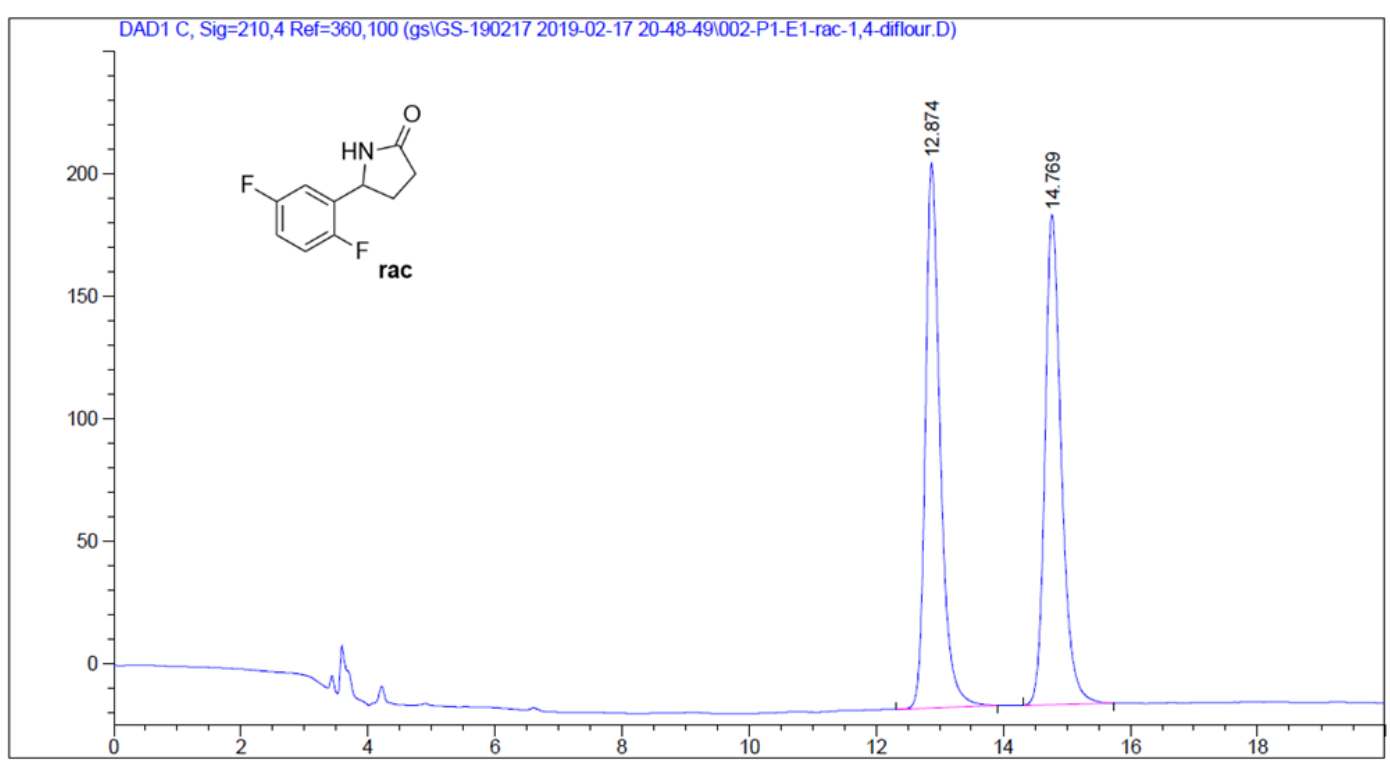

Signal 1: DAD1 C, Sig $=210,4$ Ref $=360,100$

\begin{tabular}{|c|c|c|c|c|c|c|}
\hline $\begin{array}{c}\text { eak } \\
\#\end{array}$ & $\begin{array}{c}\text { RetTime } \\
\text { [min] }\end{array}$ & Type & $\begin{array}{l}\text { Width } \\
\text { [min] }\end{array}$ & $\begin{array}{c}\text { Area } \\
{\left[\mathrm{mAU}{ }^{*} \mathrm{~s}\right]}\end{array}$ & $\begin{array}{l}\text { Height } \\
\text { [mAU] }\end{array}$ & $\begin{array}{c}\text { Area } \\
\%\end{array}$ \\
\hline & & & & & & \\
\hline 1 & 12 & BB & 0.2 & 3599.99316 & 222.31839 & 50.0929 \\
\hline 2 & 14. & B & .2693 & 3586.63940 & 199.96323 & 49.9071 \\
\hline
\end{tabular}

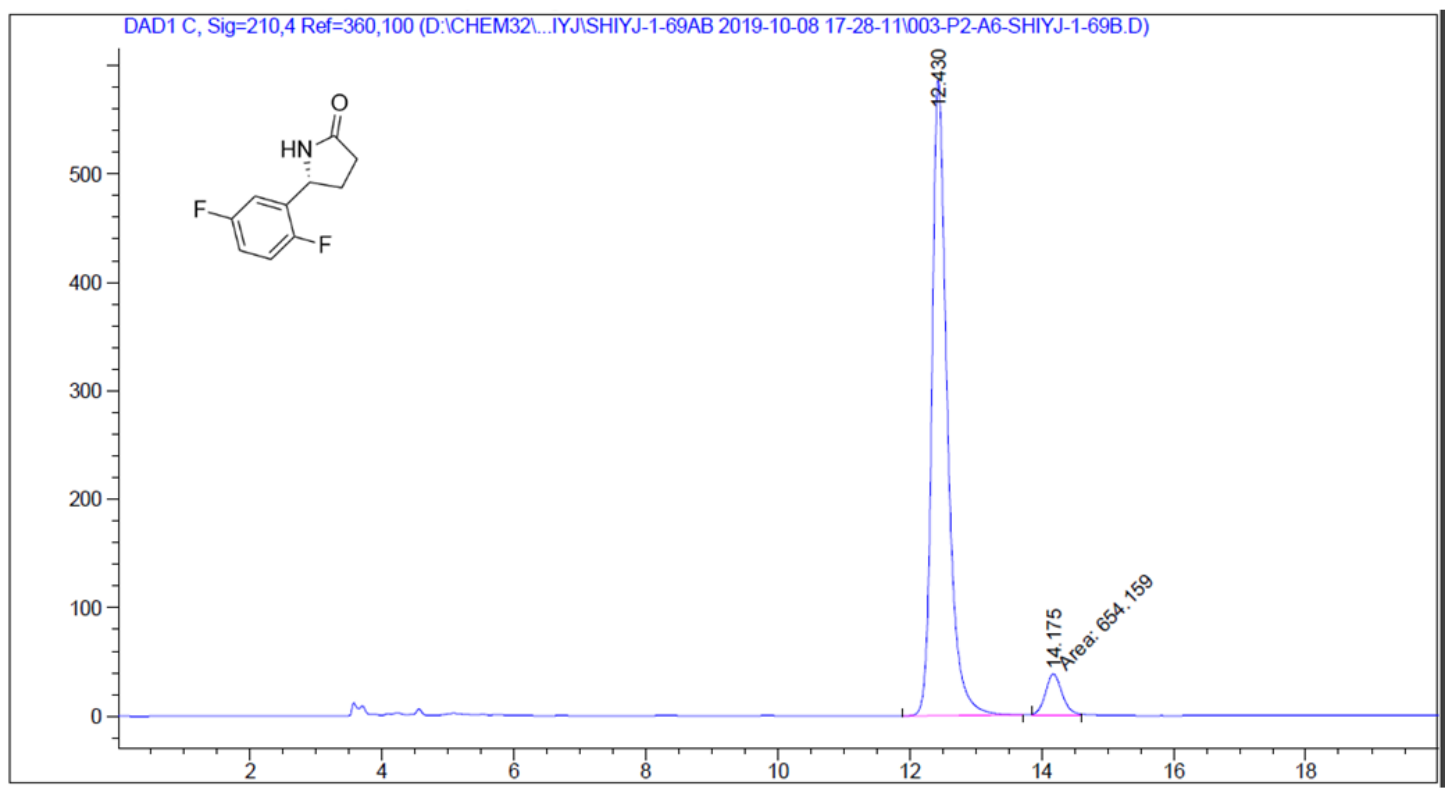

Signal 1: DAD1 C, Sig $=210,4$ Ref $=360,100$

\begin{tabular}{cccccc}
$\begin{array}{c}\text { Peak RetTime Type } \\
\text { \# }\end{array}$ [min] & $\begin{array}{c}\text { Width } \\
{[\mathrm{min}]}\end{array}$ & $\begin{array}{c}\text { Area } \\
{[\mathrm{mAU} \text { s }]}\end{array}$ & $\begin{array}{c}\text { Height } \\
{[\mathrm{mAU}]}\end{array}$ & $\begin{array}{c}\text { Area } \\
\%\end{array}$ \\
\hline 1 & 12.430 BB & 0.2408 & 9486.34668 & 586.05933 & 93.5491 \\
2 & 14.175 MM & 0.2894 & 654.15881 & 37.67200 & 6.4509
\end{tabular}


2b ( $>99 \%$ ee after once recrystallization)

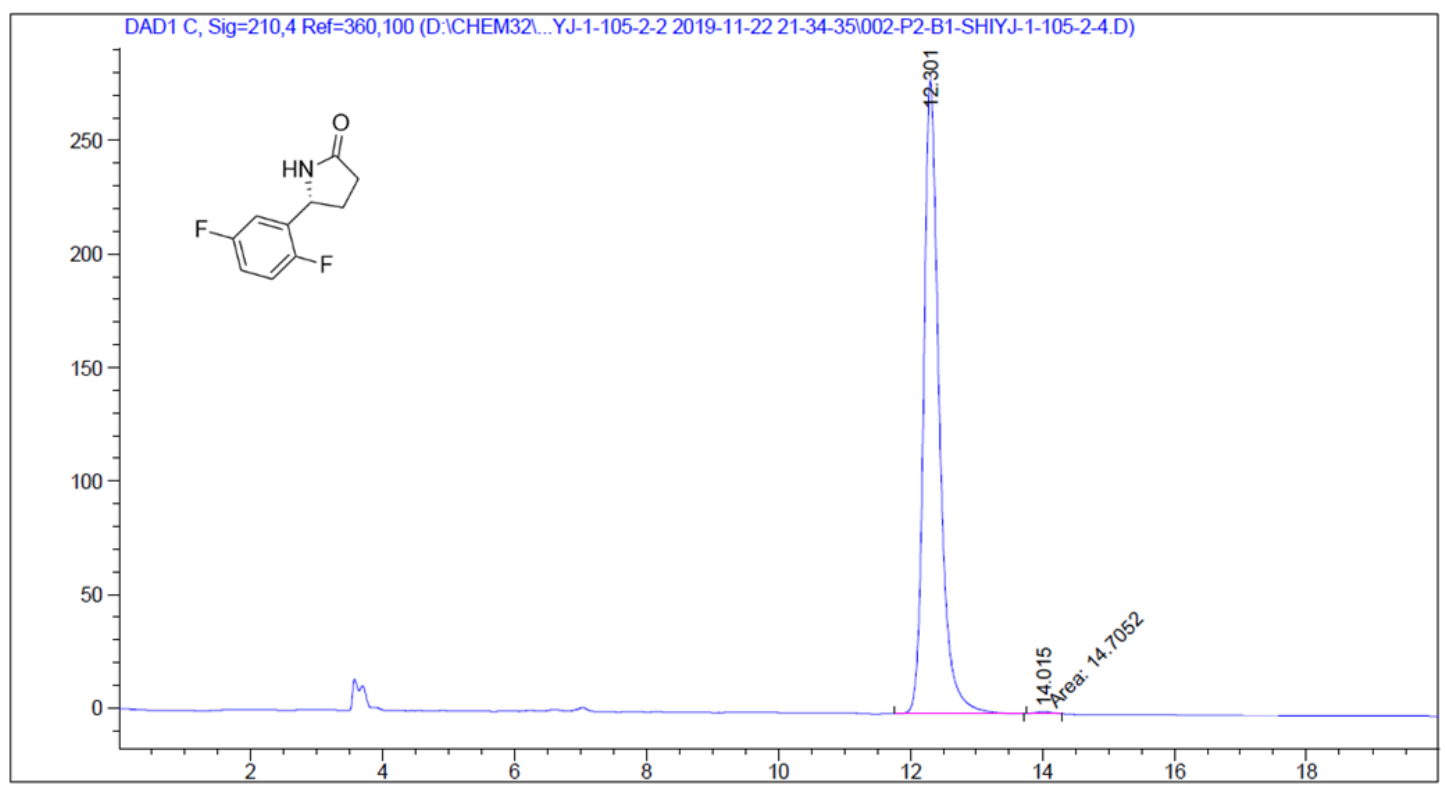

Signal 1: DAD1 C, Sig=210,4 Ref $=360,100$

\begin{tabular}{|c|c|c|c|c|c|c|}
\hline $\begin{array}{c}\text { eak } \\
\#\end{array}$ & $\begin{array}{c}\text { RetTime } \\
\text { [min] }\end{array}$ & Type & $\begin{array}{l}\text { Width } \\
\text { [min] }\end{array}$ & $\begin{array}{c}\text { Area } \\
{\left[\mathrm{mAU}^{*} \mathrm{~s}\right]}\end{array}$ & $\begin{array}{l}\text { Height } \\
{[\mathrm{mAU}]}\end{array}$ & $\begin{array}{c}\text { Area } \\
\%\end{array}$ \\
\hline & & & & & - & \\
\hline 1 & & & & 4574.27344 & 27 & 99.6796 \\
\hline 2 & & & & 14.70524 & $8.72711 \mathrm{e}-1$ & 0.3204 \\
\hline
\end{tabular}


(R)-5-(3-fluorophenyl)pyrrolidin-2-one (2c)

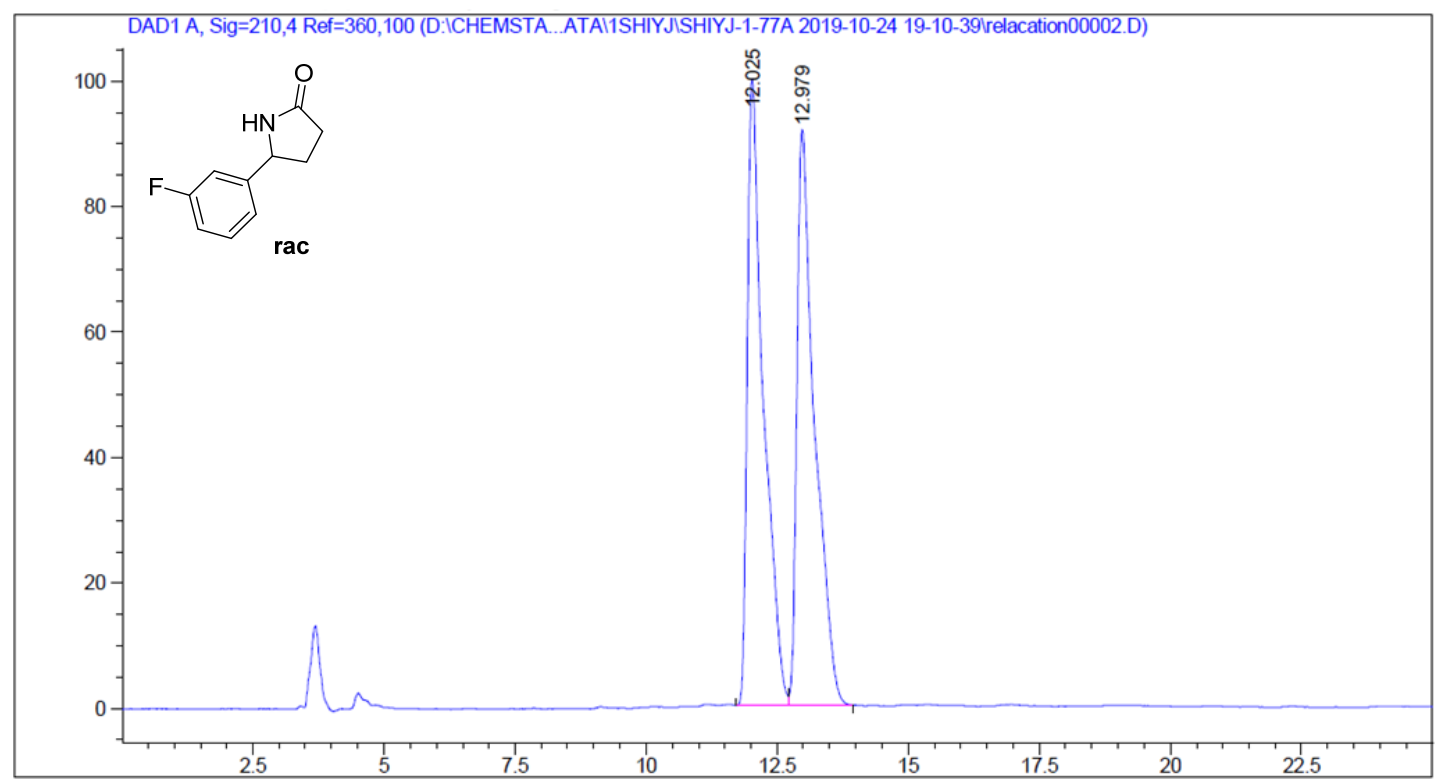

Signal 1: DAD1 A, Sig=210,4 Ref $=360,100$

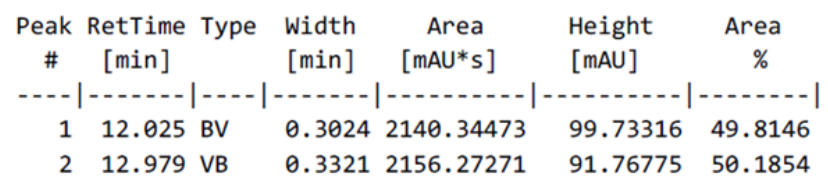

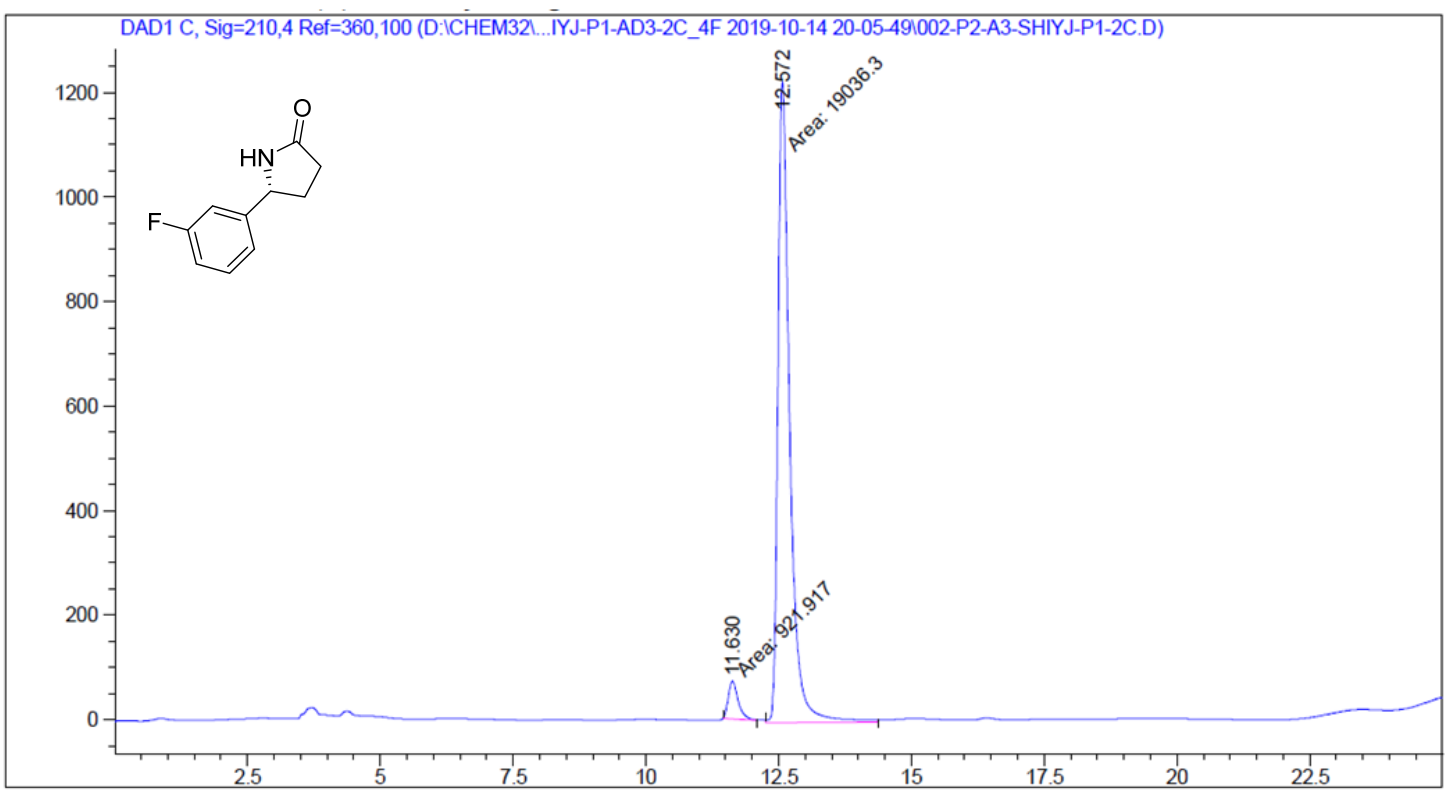

Signal 1: DAD1 C, Sig=210, 4 Ref $=360,100$

\begin{tabular}{|c|c|c|c|c|c|}
\hline $\begin{array}{c}\text { Peak } \\
\#\end{array}$ & $\begin{array}{l}\text { RetTime Type } \\
\text { [min] }\end{array}$ & $\begin{array}{l}\text { Width } \\
\text { [min] }\end{array}$ & $\begin{array}{c}\text { Area } \\
{\left[\mathrm{mAU}^{*} \mathrm{~s}\right]}\end{array}$ & $\begin{array}{l}\text { Height } \\
{[\mathrm{mAU}]}\end{array}$ & $\begin{array}{c}\text { Area } \\
\%\end{array}$ \\
\hline & & & & & 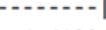 \\
\hline 1 & $11.630 \mathrm{MM}$ & 0.2140 & 921.91663 & 71.80766 & 4.6192 \\
\hline 2 & $12.572 \mathrm{MM}$ & 0.2586 & $1.90363 \mathrm{e} 4$ & 1226.66406 & 95.3808 \\
\hline
\end{tabular}


(R)-5-(4-fluorophenyl)pyrrolidin-2-one (2d)

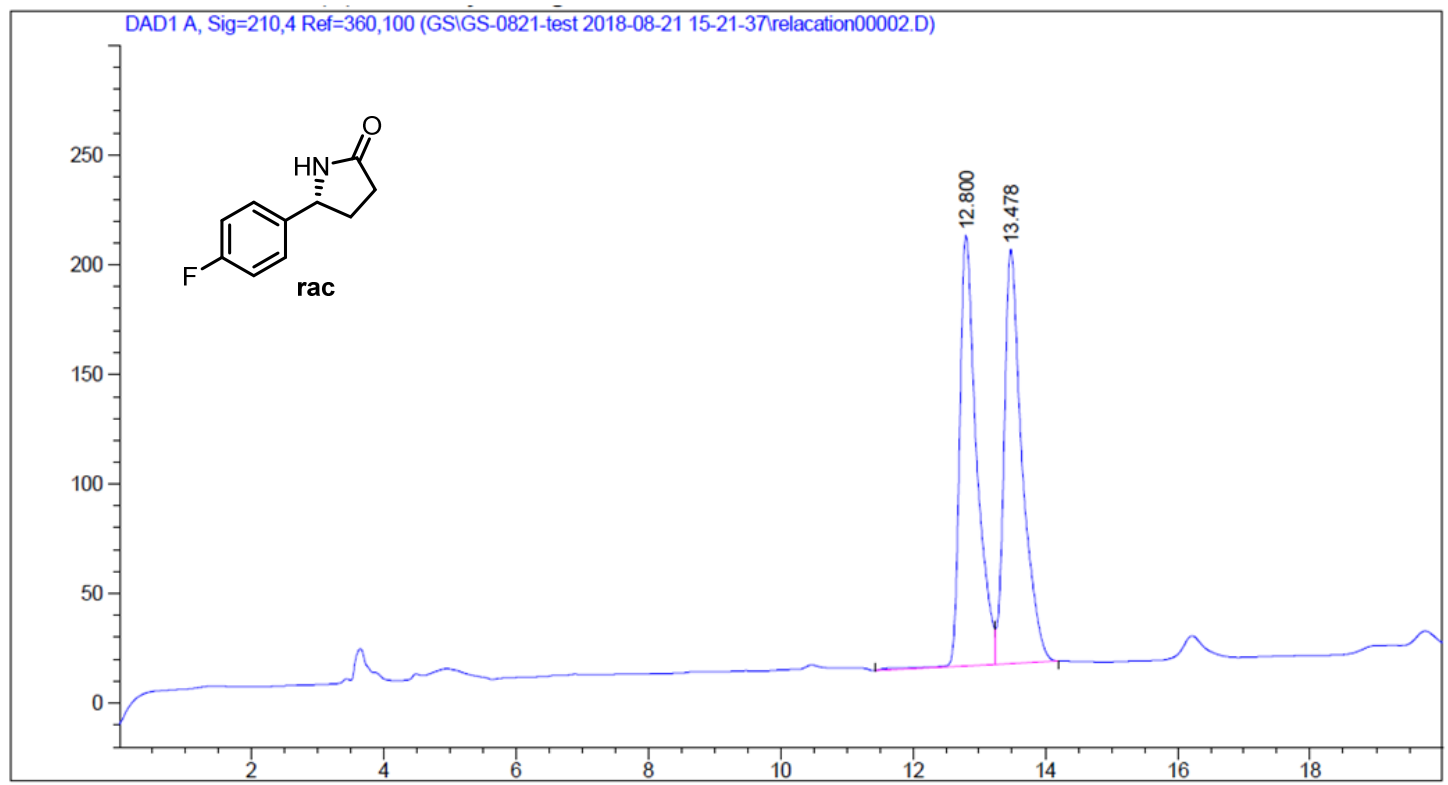

Signal 1: DAD1 A, Sig=210,4 Ref $=360,100$

\begin{tabular}{cccccc}
$\begin{array}{c}\text { Peak RetTime Type } \\
\text { \# }[\mathrm{min}]\end{array}$ & $\begin{array}{c}\text { Width } \\
\text { [min] }\end{array}$ & $\begin{array}{c}\text { Area } \\
{[\mathrm{mAU} \text { s] }}\end{array}$ & $\begin{array}{c}\text { Height } \\
{[\mathrm{mAU}]}\end{array}$ & $\begin{array}{c}\text { Area } \\
\%\end{array}$ \\
\hline 1 & 12.800 BV & 0.2597 & 3499.40088 & 196.59767 & 49.4219 \\
2 & 13.478 VB & 0.2769 & 3581.26416 & 189.15977 & 50.5781
\end{tabular}

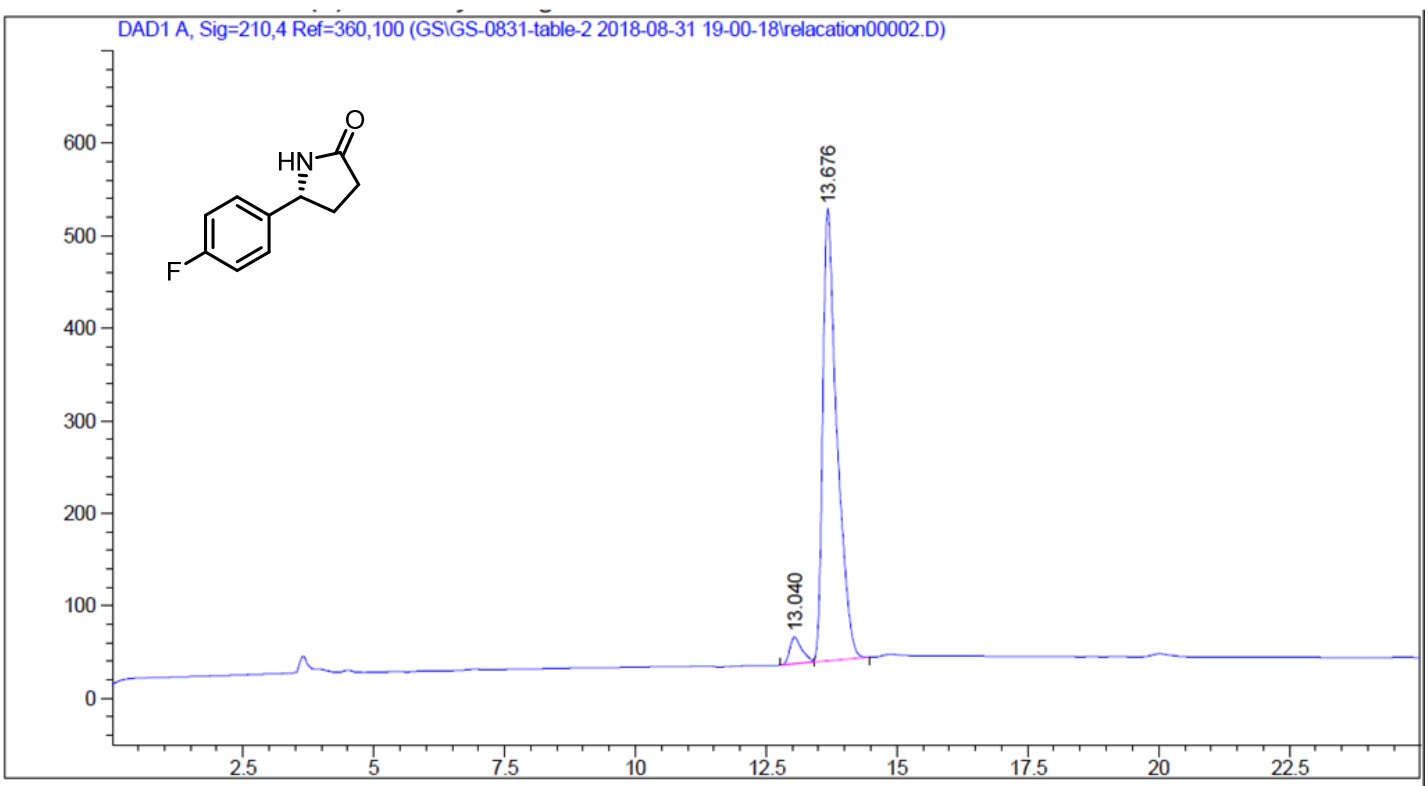
Peak RetTime Type Width Area Height Area
\# [min]
[min] [mAU*s]
[mAU] \%

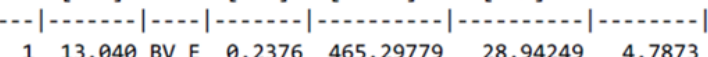
$\begin{array}{llllllll}2 & 13.676 & \text { VB } R & 0.2751 & 9254.19727 & 488.33295 & 95.2127\end{array}$ 
(R)-5-(4-chlorophenyl)pyrrolidin-2-one (2e)

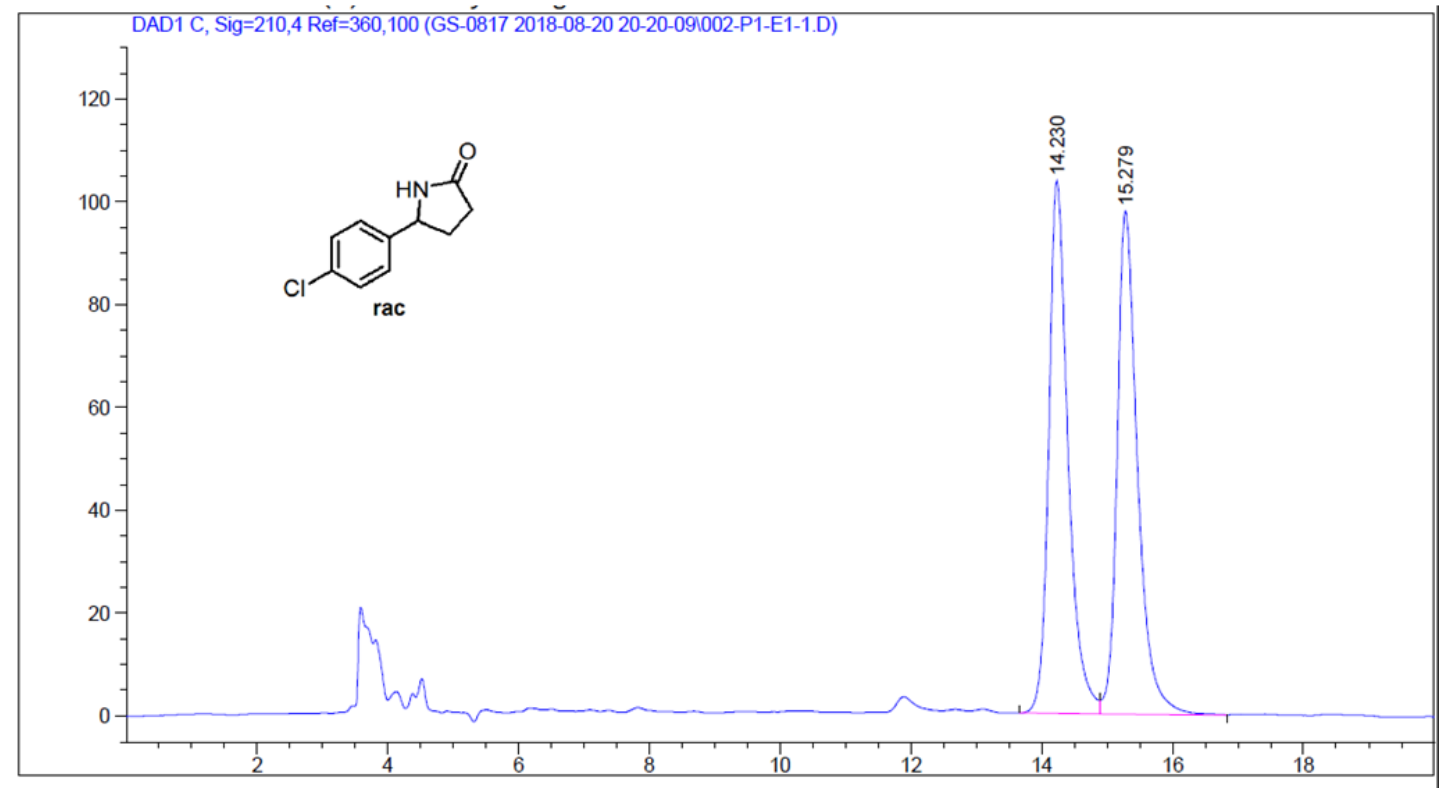

Signal 1: DAD1 C, Sig=210,4 Ref $=360,100$

\begin{tabular}{|c|c|c|c|c|c|c|}
\hline $\begin{array}{c}\text { Peak } \\
\#\end{array}$ & $\begin{array}{c}\text { RetTime } \\
\text { [min] }\end{array}$ & Type & $\begin{array}{l}\text { Width } \\
\text { [min] }\end{array}$ & $\begin{array}{c}\text { Area } \\
{\left[\mathrm{mAU}^{*} \mathrm{~s}\right]}\end{array}$ & $\begin{array}{l}\text { Height } \\
{[\mathrm{mAU}]}\end{array}$ & $\begin{array}{c}\text { Area } \\
\%\end{array}$ \\
\hline & & & & 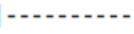 & . & - \\
\hline 1 & 14.230 & BV & 0.3052 & 2123.73120 & 103.58163 & 49.9986 \\
\hline 2 & 15.279 & VB & 0.3232 & 2123.84644 & 97.86845 & 50.0014 \\
\hline
\end{tabular}

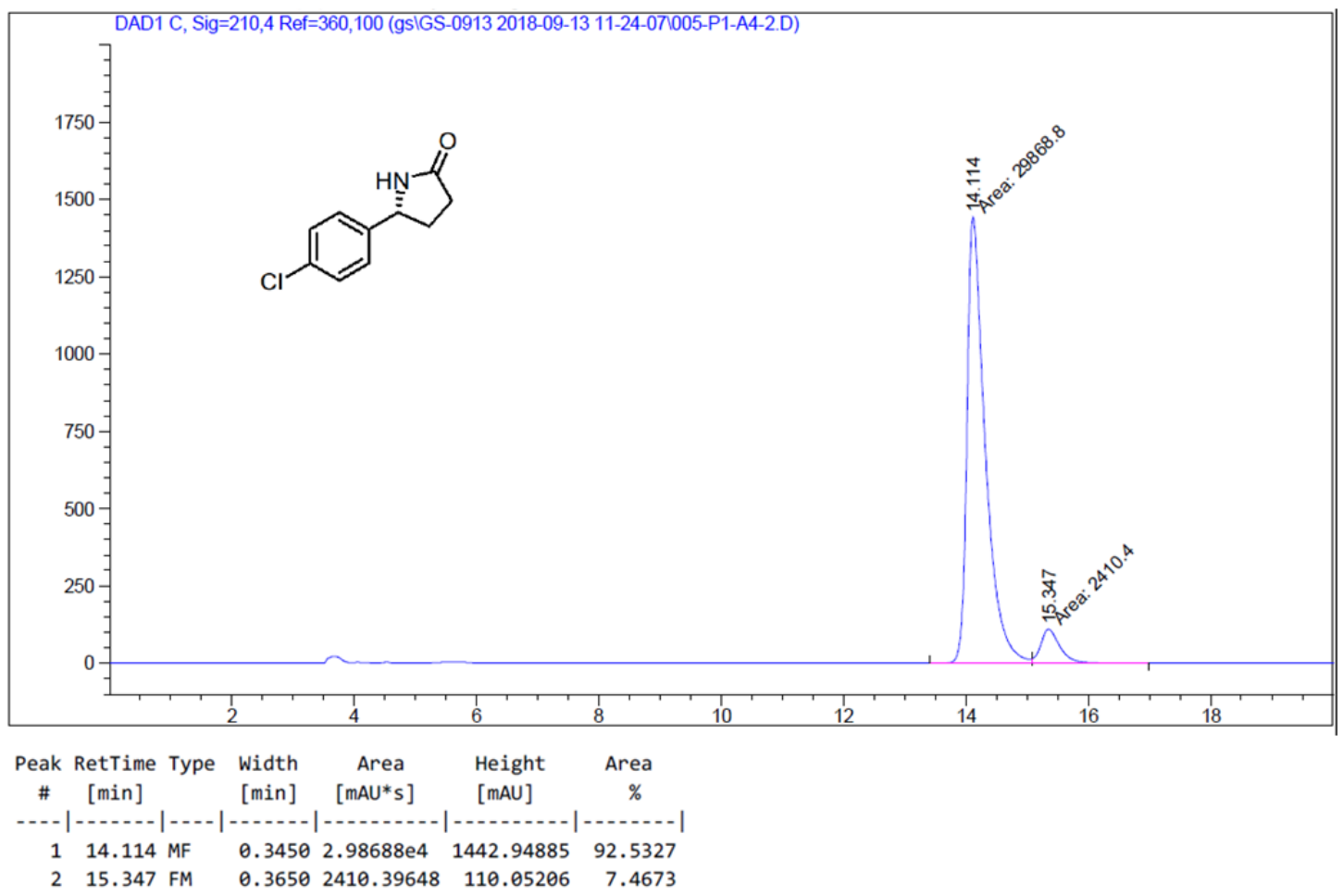


(R)-5-(4-bromophenyl)pyrrolidin-2-one (2f)

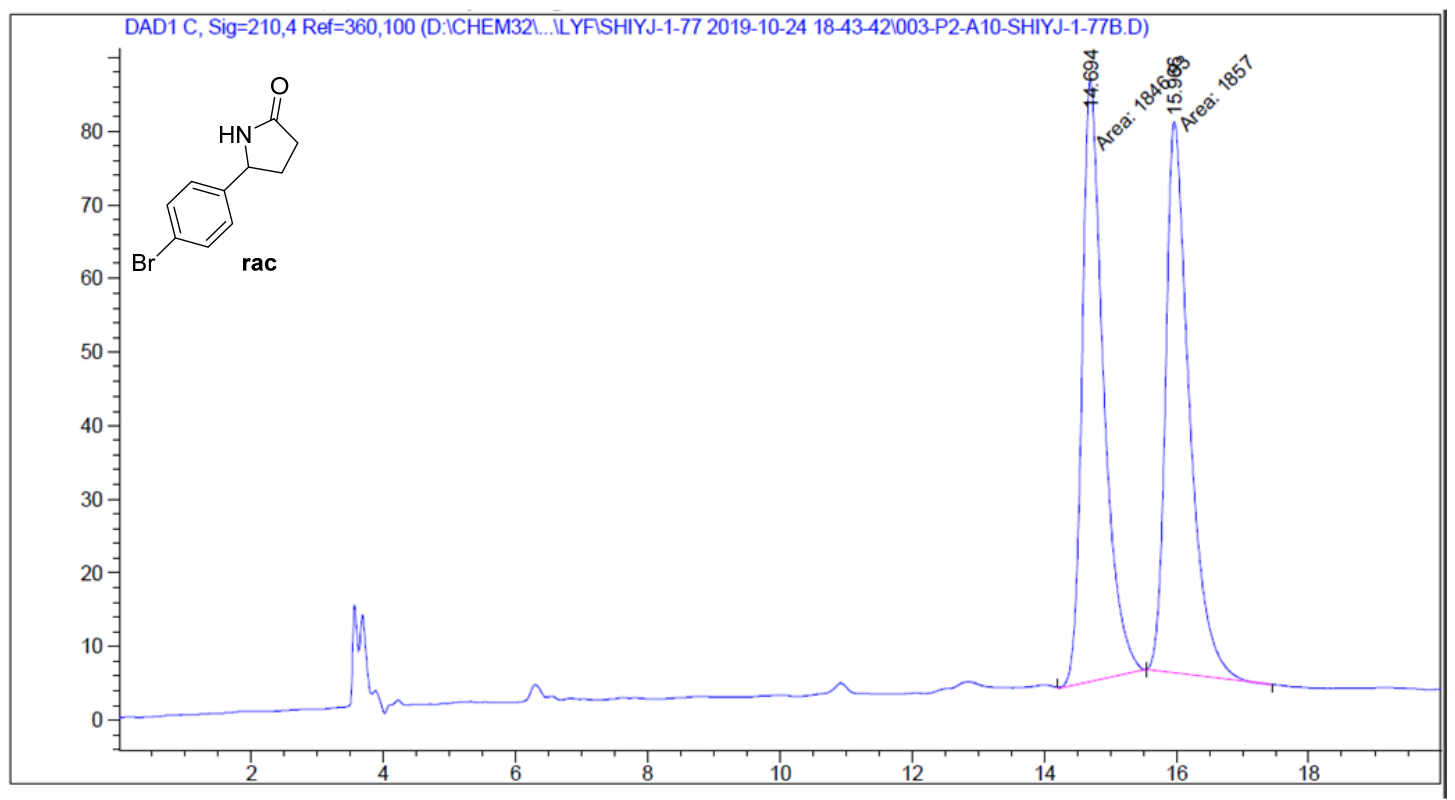

Signal 1: DAD1 C, Sig=210,4 Ref $=360,100$

\begin{tabular}{|c|c|c|c|c|c|c|}
\hline eak & $\begin{array}{c}\text { RetTime } \\
\text { [min] }\end{array}$ & Type & $\begin{array}{l}\text { Width } \\
\text { [min] }\end{array}$ & $\begin{array}{c}\text { Area } \\
{\left[\mathrm{mAU}^{*} \mathrm{~s}\right]}\end{array}$ & $\begin{array}{l}\text { Height } \\
\text { [mAU] }\end{array}$ & $\begin{array}{c}\text { Area } \\
\%\end{array}$ \\
\hline & & & & & & \\
\hline 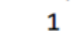 & & & & 1846 & 62 & 541 \\
\hline 2 & 15.966 & $\mathrm{MM}$ & 4132 & 1856.99963 & 74.90785 & 50.1359 \\
\hline
\end{tabular}

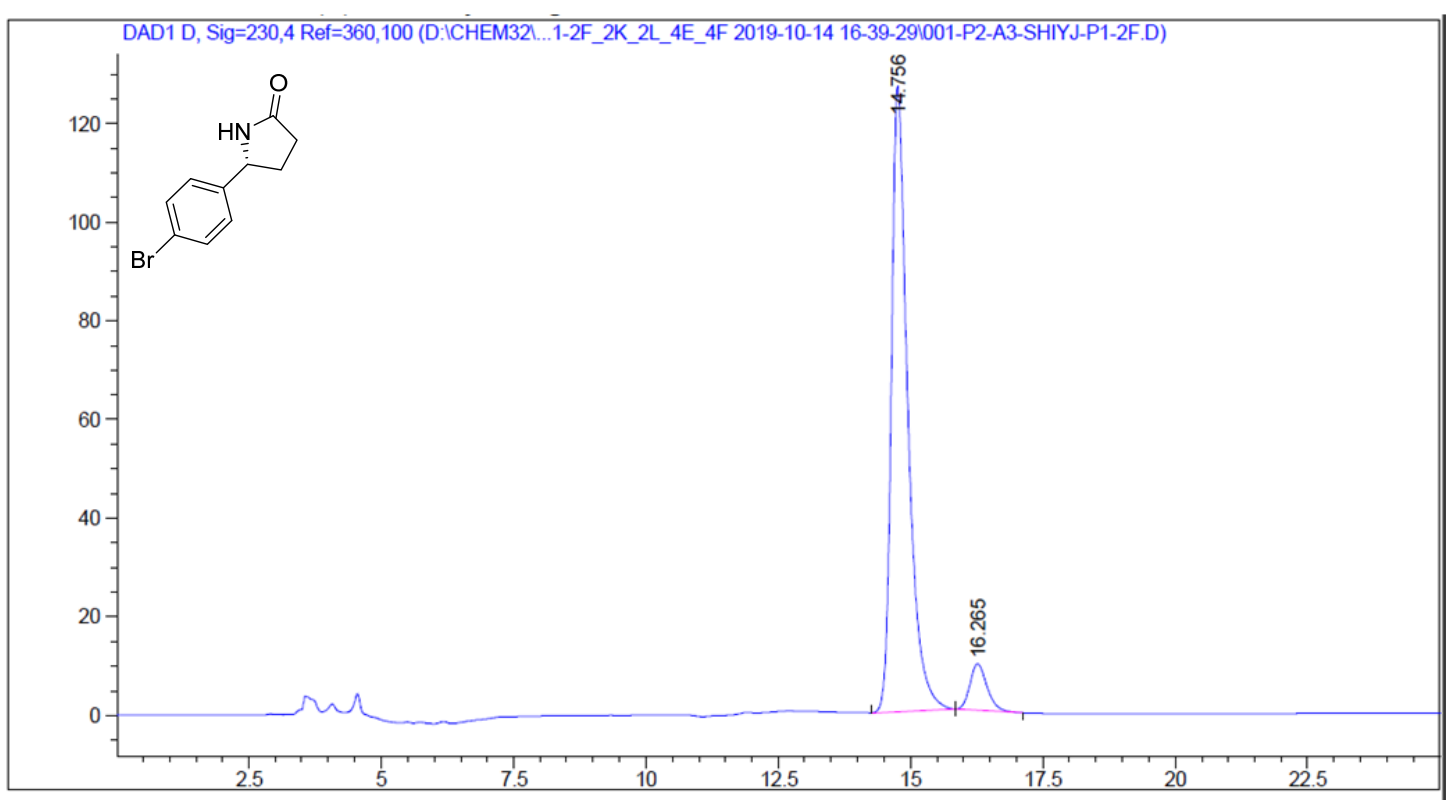

Signal 1: DAD1 D, Sig $=230,4$ Ref $=360,100$

\begin{tabular}{cccccc}
$\begin{array}{c}\text { Peak RetTime Type width } \\
\text { \# }[\text { min] }\end{array}$ & $\begin{array}{c}\text { Area } \\
\text { [min] }\end{array}$ & $\begin{array}{c}\text { Height } \\
\text { [mAU* }]\end{array}$ & \multicolumn{1}{c}{$\begin{array}{c}\text { Area } \\
\text { [mAU] }\end{array}$} & \multicolumn{1}{c}{$\%$} \\
\hline 1 & 14.756 BB & 0.3191 & 2728.09985 & 126.76987 & 92.7789 \\
2 & 16.265 BB & 0.3419 & 212.33244 & 9.46004 & 7.2211
\end{tabular}


(R)-5-(o-tolyl)pyrrolidin-2-one (2g)

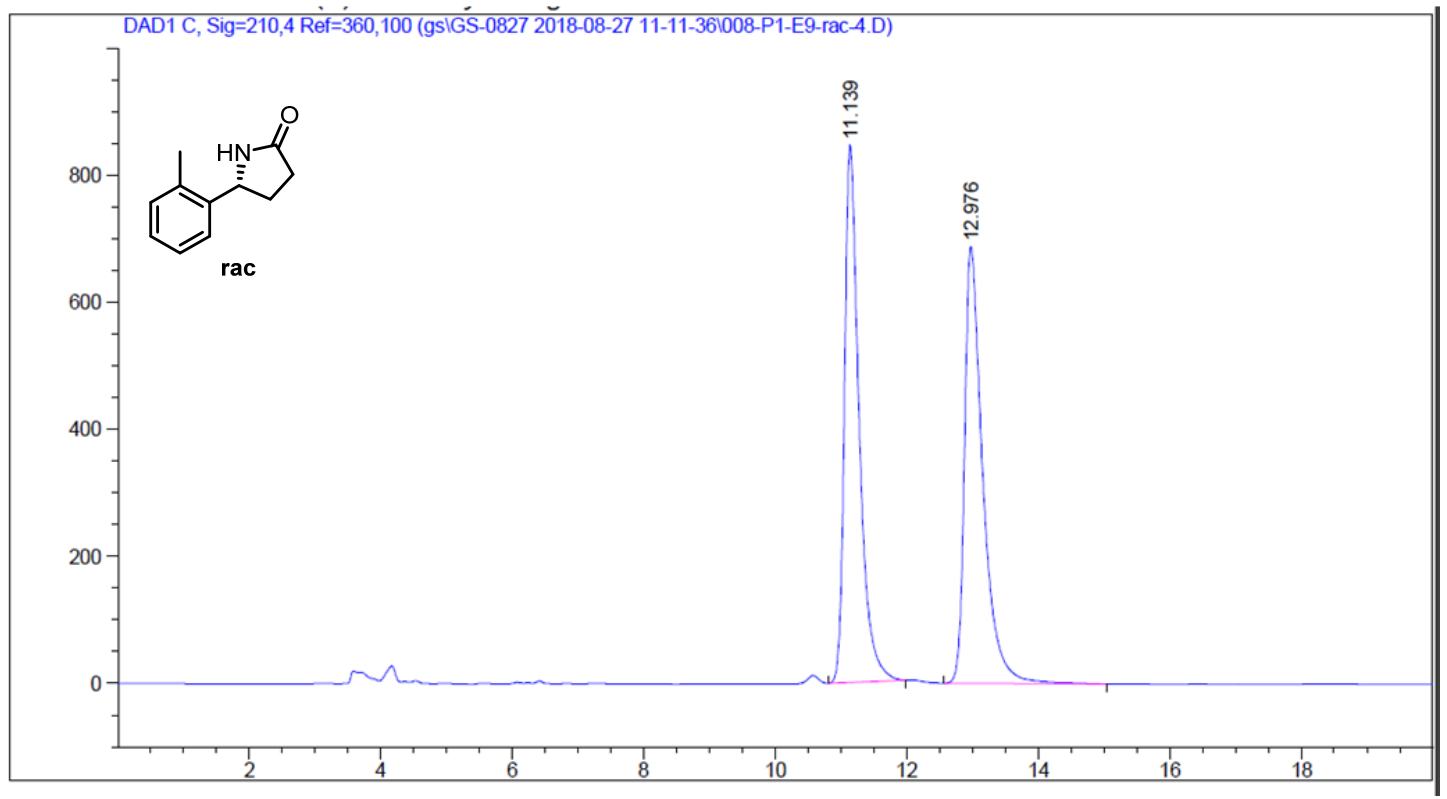

Signal 1: DAD1 C, Sig=210,4 Ref $=360,100$

\begin{tabular}{cccccc}
$\begin{array}{c}\text { Peak RetTime Type Width } \\
\text { \# }[\text { min] }\end{array}$ & $\begin{array}{c}\text { Area } \\
\text { [min] }\end{array}\left[\begin{array}{c}\text { Height } \\
{\left[\mathrm{mAU}{ }^{*} \text { ] }\right.}\end{array}\right.$ & $\begin{array}{c}\text { Area } \\
{[\mathrm{mAU}]}\end{array}$ & $\%$ \\
\hdashline 1 & 11.139 BB & 0.2304 & $1.30964 \mathrm{e} 4$ & 846.84076 & 49.7416 \\
2 & 12.976 BB & 0.2825 & $1.32324 \mathrm{e} 4$ & 687.70020 & 50.2584
\end{tabular}

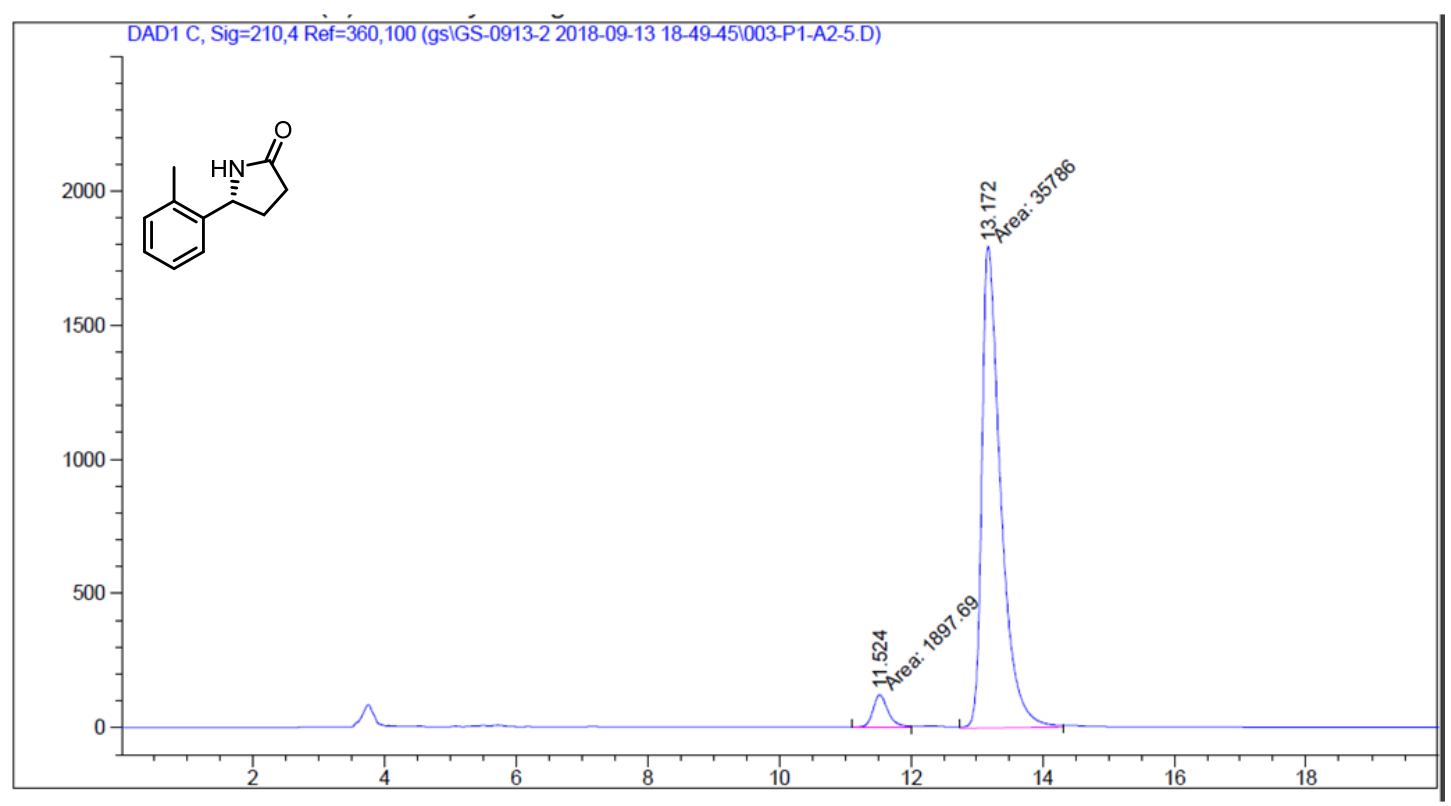

Signal 1: DAD1 C, Sig $=210,4$ Ret $=360,100$

\begin{tabular}{|c|c|c|c|c|c|c|}
\hline $\begin{array}{c}\text { Peak } \\
\#\end{array}$ & $\begin{array}{c}\text { RetTime } \\
\text { [min] }\end{array}$ & Type & $\begin{array}{l}\text { Width } \\
\text { [min] }\end{array}$ & $\begin{array}{c}\text { Area } \\
{[\mathrm{mAU} * \mathrm{~s}]}\end{array}$ & $\begin{array}{l}\text { Height } \\
{[\mathrm{mAU}]}\end{array}$ & $\begin{array}{c}\text { Area } \\
\%\end{array}$ \\
\hline & & & & & & 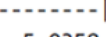 \\
\hline 1 & 11.524 & MM & 0.2636 & 1897.69067 & 119.97943 & 5.0358 \\
\hline 2 & 13.172 & MM & 0.3321 & $3.57860 \mathrm{e} 4$ & 1795.76685 & 94.9642 \\
\hline
\end{tabular}


(R)-5-(m-tolyl)pyrrolidin-2-one (2h)

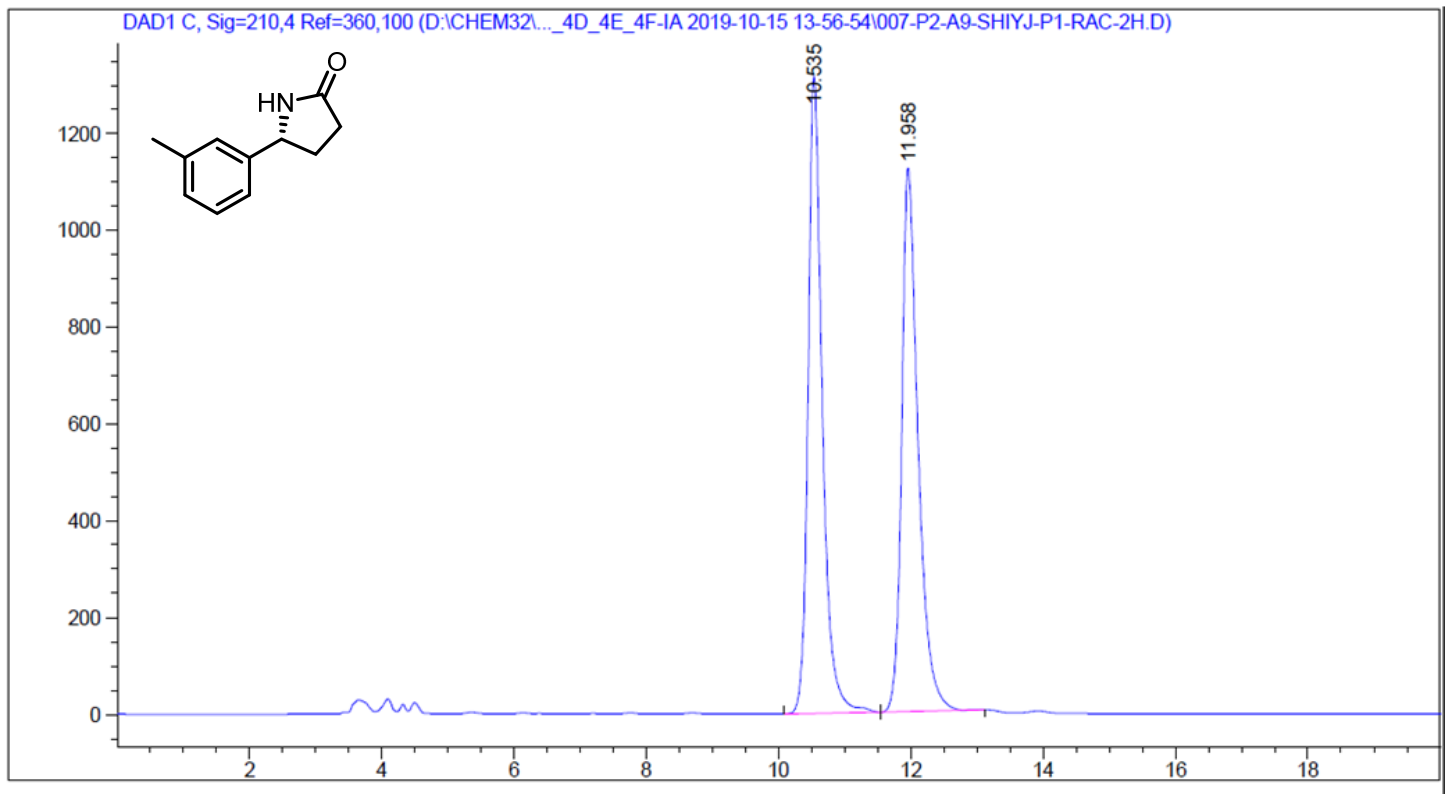

Signal 1: DAD1 C, Sig=210,4 Ref $=360,100$

\begin{tabular}{|c|c|c|c|c|c|c|}
\hline $\begin{array}{c}\text { eak } \\
\#\end{array}$ & $\begin{array}{c}\text { RetTime } \\
\text { [min] }\end{array}$ & Type & $\begin{array}{l}\text { Width } \\
\text { [min] }\end{array}$ & $\begin{array}{c}\text { Area } \\
{\left[\mathrm{mAU}^{*} \mathrm{~s}\right]}\end{array}$ & $\begin{array}{l}\mathrm{He} \\
{[\mathrm{m}}\end{array}$ & $\begin{array}{c}\text { Area } \\
\%\end{array}$ \\
\hline & & & & & & \\
\hline & & & & e4 & 131 & 705 \\
\hline 2 & 11.958 & $B V R$ & 522 & $1.93072 \mathrm{e} 4$ & 1124.34644 & 49.6295 \\
\hline
\end{tabular}

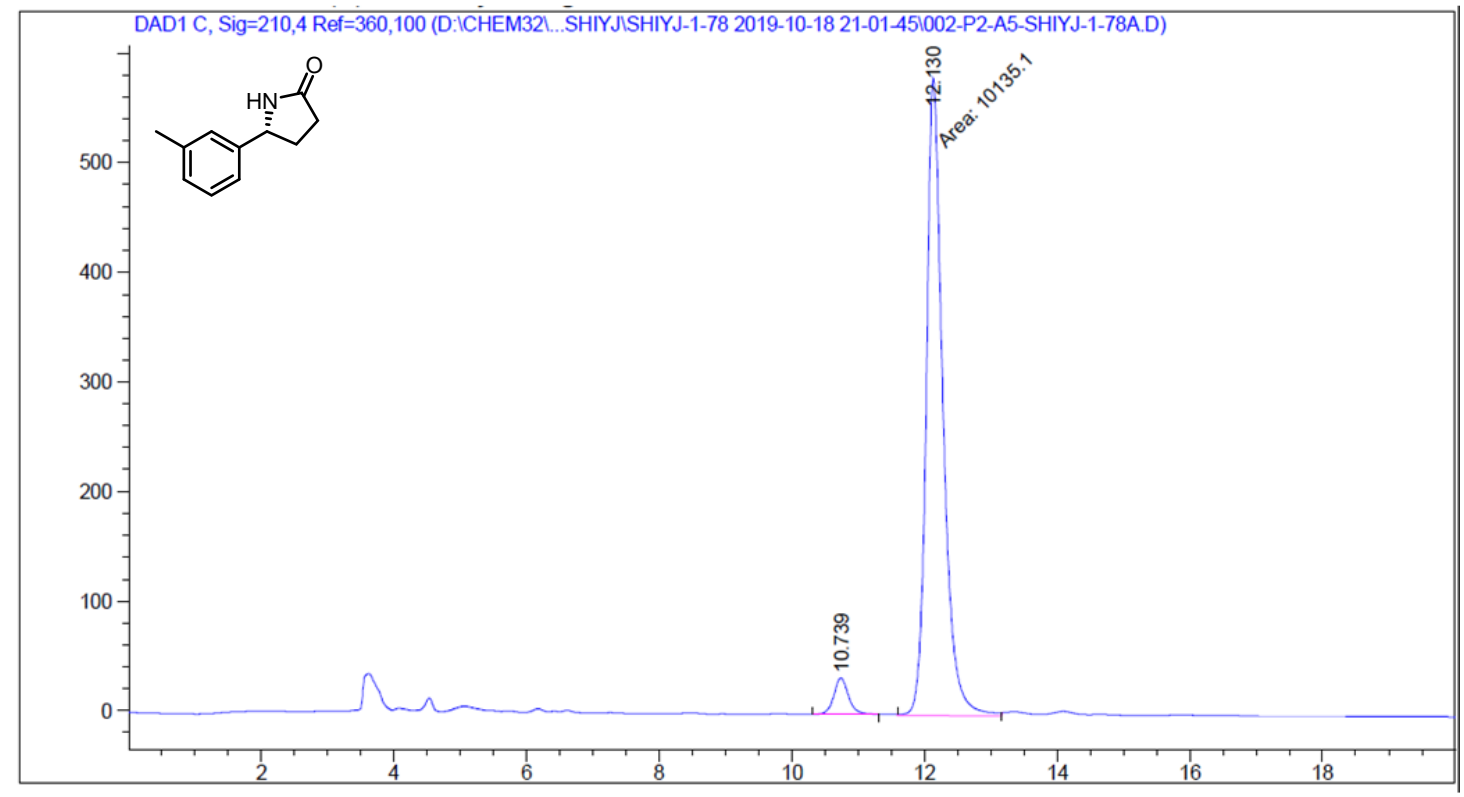

Signal 1: DAD1 C, Sig=210, 4 Ref $=360,100$

\begin{tabular}{|c|c|c|c|c|c|c|}
\hline $\begin{array}{c}\text { Peak } \\
\#\end{array}$ & $\begin{array}{c}\text { RetTime } \\
\text { [min] }\end{array}$ & Type & $\begin{array}{l}\text { Width } \\
\text { [min] }\end{array}$ & $\begin{array}{c}\text { Area } \\
{\left[\mathrm{mAU}^{*} \mathrm{~s}\right]}\end{array}$ & $\begin{array}{l}\mathrm{He} \\
{[\mathrm{m} A}\end{array}$ & $\begin{array}{c}\text { Area } \\
\%\end{array}$ \\
\hline & & & & & $-\cdots$ & \\
\hline 1 & & & & 498.71146 & 6837 & 399 \\
\hline 2 & 12 & $\mathrm{MIN}$ & .2903 & $1.01351 \mathrm{e} 4$ & 581.79559 & 95.3101 \\
\hline
\end{tabular}


(R)-5-(p-tolyl)pyrrolidin-2-one (2i)

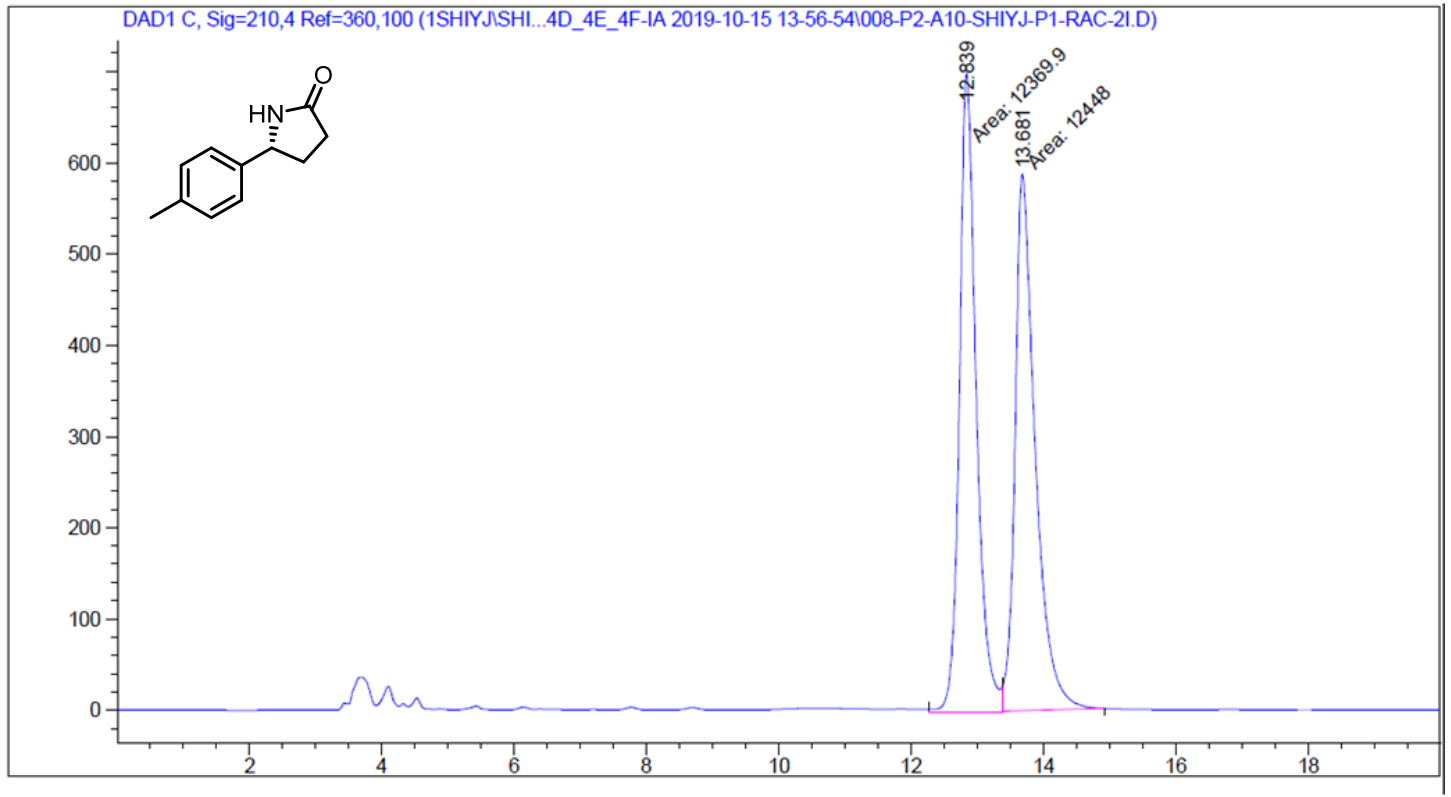

Signal 1: DAD1 C, Sig=210,4 Ref $=360,100$

\begin{tabular}{|c|c|c|c|c|c|}
\hline eak & $\begin{array}{l}\text { RetTime Type } \\
\text { [min] }\end{array}$ & $\begin{array}{l}\text { Width } \\
\text { [min] }\end{array}$ & $\begin{array}{c}\text { Area } \\
{\left[\mathrm{mAU}^{*} \mathrm{~s}\right]}\end{array}$ & $\begin{array}{l}\text { Height } \\
{[\mathrm{mAU}]}\end{array}$ & $\begin{array}{c}\text { Area } \\
\%\end{array}$ \\
\hline & & & & & \\
\hline 1 & 12 & & 4 & 700.01471 & 425 \\
\hline 2 & $13.681 \mathrm{MM}$ & .3526 & $1.24480 \mathrm{e} 4$ & 588.45490 & 50.1575 \\
\hline
\end{tabular}

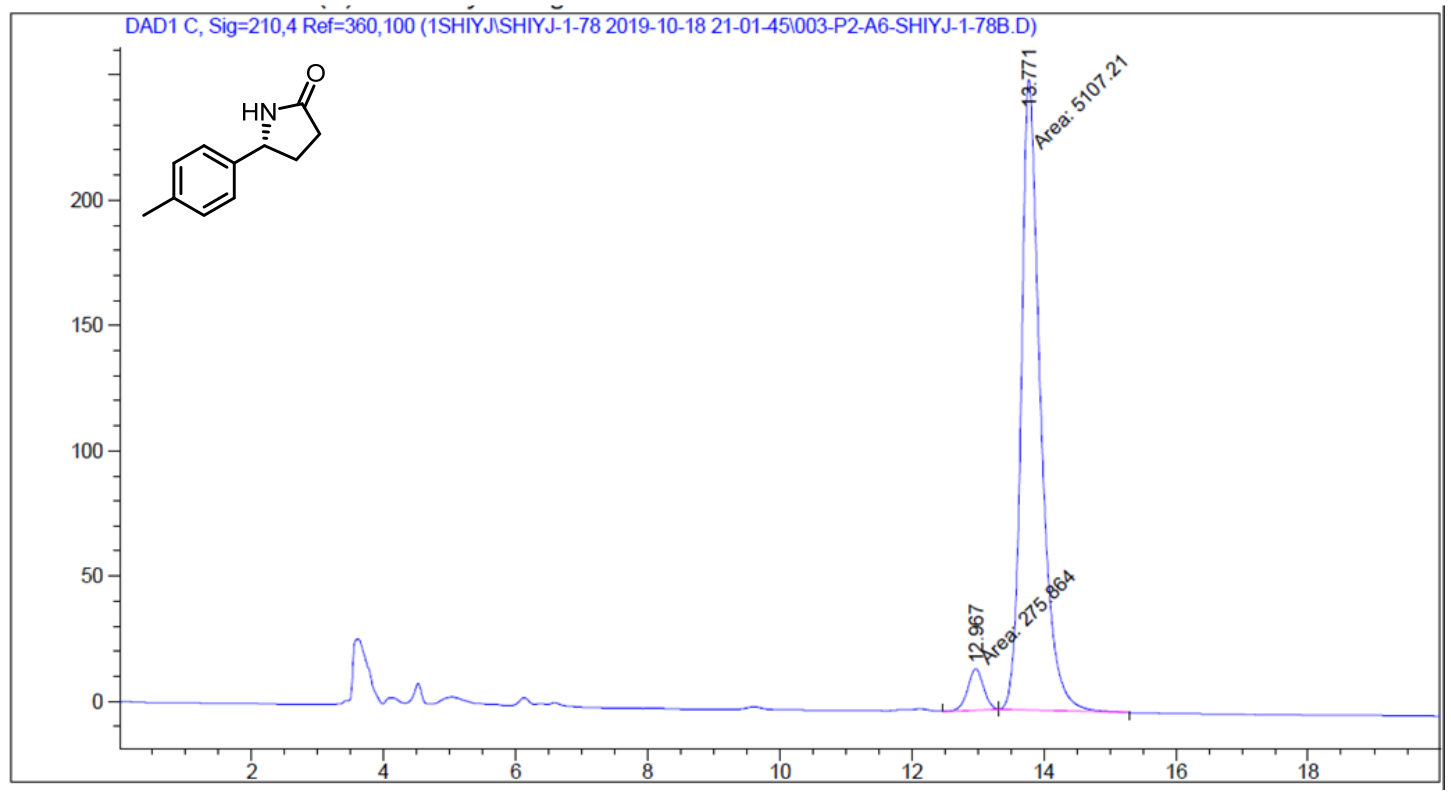

Signal 1: DAD1 C, Sig=210,4 Ref=360,100

\begin{tabular}{cccccc}
$\begin{array}{c}\text { Peak RetTime Type } \\
\text { Width } \\
\text { [min] }\end{array}$ & $\begin{array}{c}\text { Area } \\
{[\mathrm{min}]}\end{array}$ & $\begin{array}{c}\text { Height } \\
{[\mathrm{mAU} \text { s }]}\end{array}$ & $\begin{array}{c}\text { Area } \\
{[\mathrm{mAU}]}\end{array}$ & $\%$ \\
\hline 1 & $12.967 \mathrm{MM}$ & 0.2783 & 275.86435 & 16.51952 & 5.1247 \\
2 & $13.771 \mathrm{MM}$ & 0.3383 & 5107.21143 & 251.59737 & 94.8753
\end{tabular}


(R)-5-(4-(tert-butyl)phenyl)pyrrolidin-2-one (2j)

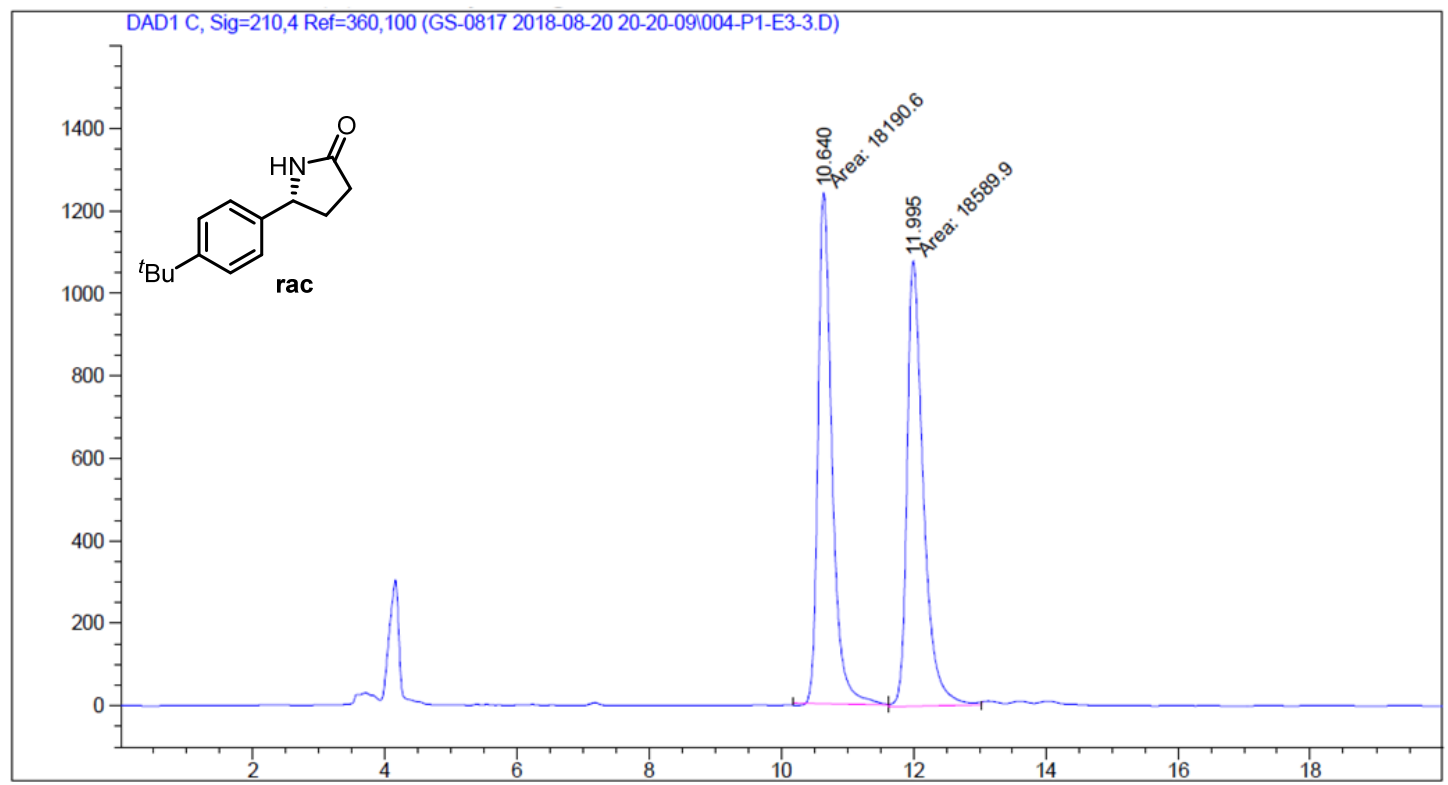

Signal 1: DAD1 C, Sig=210, 4 Ref $=360,100$

\begin{tabular}{cccccc}
$\begin{array}{c}\text { Peak RetTime Type } \\
\text { Width } \\
\text { [min] }\end{array}$ & $\begin{array}{c}\text { Area } \\
{[\mathrm{min}]}\end{array}$ & $\begin{array}{c}\text { Height } \\
{[\mathrm{mAU} \text { s }]}\end{array}$ & $\begin{array}{c}\text { Area } \\
{[\mathrm{mAU}]}\end{array}$ & $\%$ \\
\hline & $10.640 \mathrm{MM}$ & 0.2444 & $1.81906 \mathrm{e} 4$ & 1240.58313 & 49.4572 \\
2 & $11.995 \mathrm{MM}$ & 0.2868 & $1.85899 \mathrm{e} 4$ & 1080.48474 & 50.5428
\end{tabular}

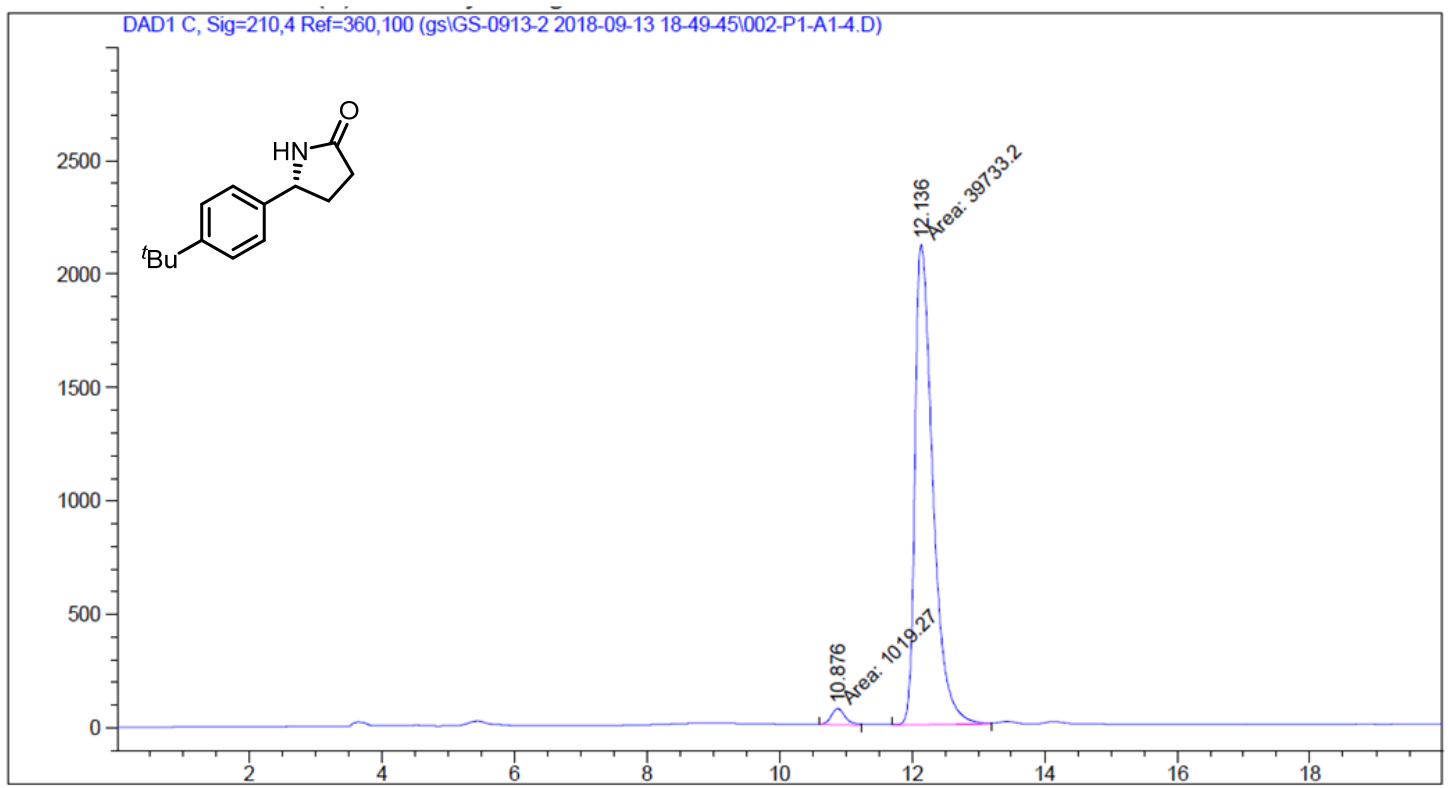

Signal 1: DAD1 C, Sig=210,4 Ref $=360,100$

\begin{tabular}{|c|c|c|c|c|c|c|}
\hline $\begin{array}{c}\text { Peak } \\
\#\end{array}$ & $\begin{array}{c}\text { RetTime } \\
\text { [min] }\end{array}$ & Type & $\begin{array}{l}\text { Width } \\
\text { [min] }\end{array}$ & $\begin{array}{c}\text { Area } \\
{\left[\mathrm{mAU}^{*} \mathrm{~s}\right]}\end{array}$ & $\begin{array}{l}\text { Height } \\
\text { [mAU] }\end{array}$ & $\begin{array}{c}\text { Area } \\
\%\end{array}$ \\
\hline & & & & & & \\
\hline 1 & & & & 1019 & 77 & 211 \\
\hline 2 & 12 & & & $3.97332 \mathrm{e} 4$ & 2118.87866 & 97.4989 \\
\hline
\end{tabular}


(R)-5-(4-methoxyphenyl)pyrrolidin-2-one (2k)

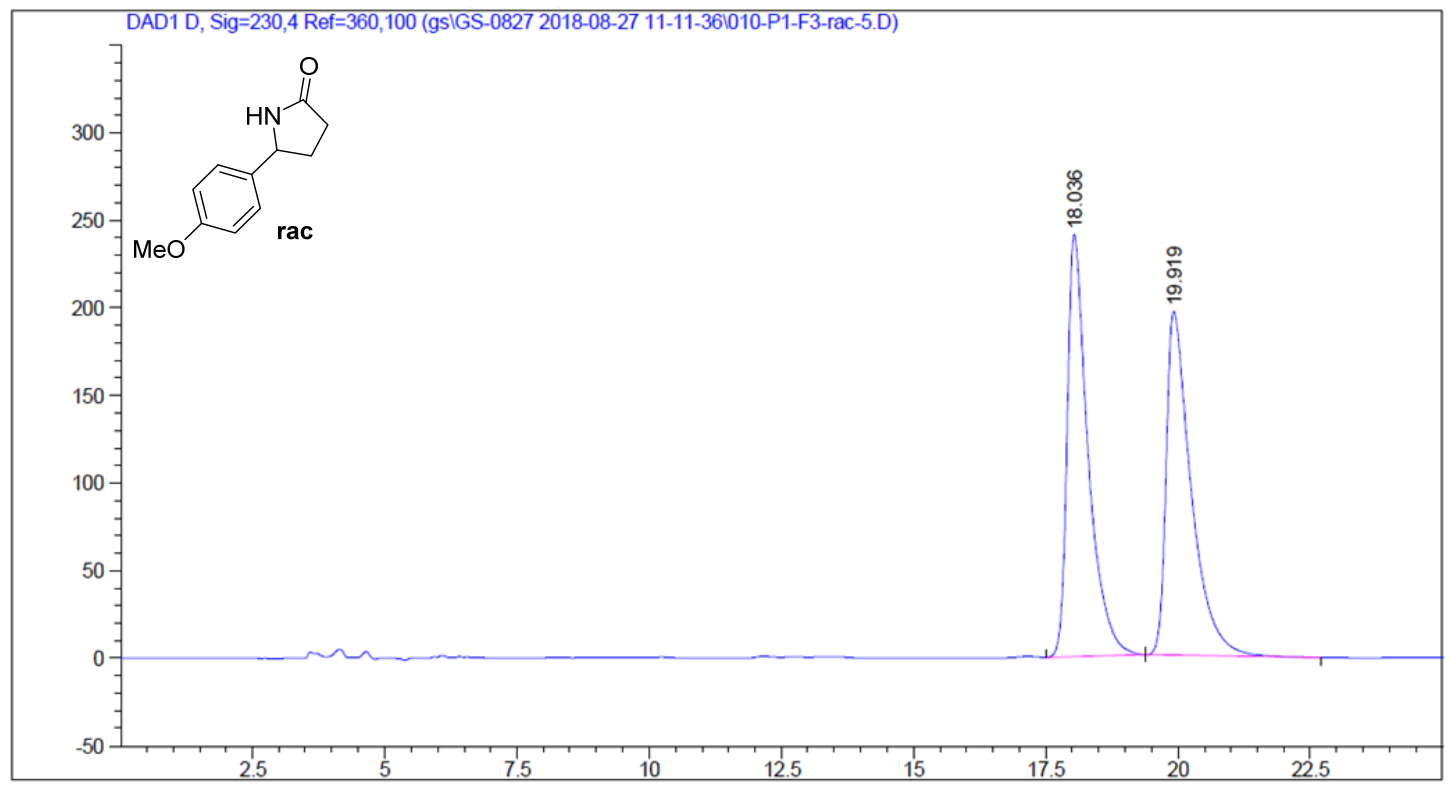

Signal 1: DAD1 D, Sig=230,4 Ref $=360,100$

\begin{tabular}{|c|c|c|c|c|c|}
\hline $\begin{array}{c}\text { Peak } \\
\#\end{array}$ & $\begin{array}{l}\text { RetTime Type } \\
\text { [min] }\end{array}$ & $\begin{array}{l}\text { Width } \\
\text { [min] }\end{array}$ & $\begin{array}{c}\text { Area } \\
{[\mathrm{mAU} * \mathrm{~s}]}\end{array}$ & $\begin{array}{l}\text { Height } \\
\text { [mAU] }\end{array}$ & $\begin{array}{c}\text { Area } \\
\%\end{array}$ \\
\hline & & & & & \\
\hline 1 & $9 \mathrm{BB}$ & & 6621. & $\begin{array}{l}8106 \\
0167\end{array}$ & 3138 \\
\hline
\end{tabular}

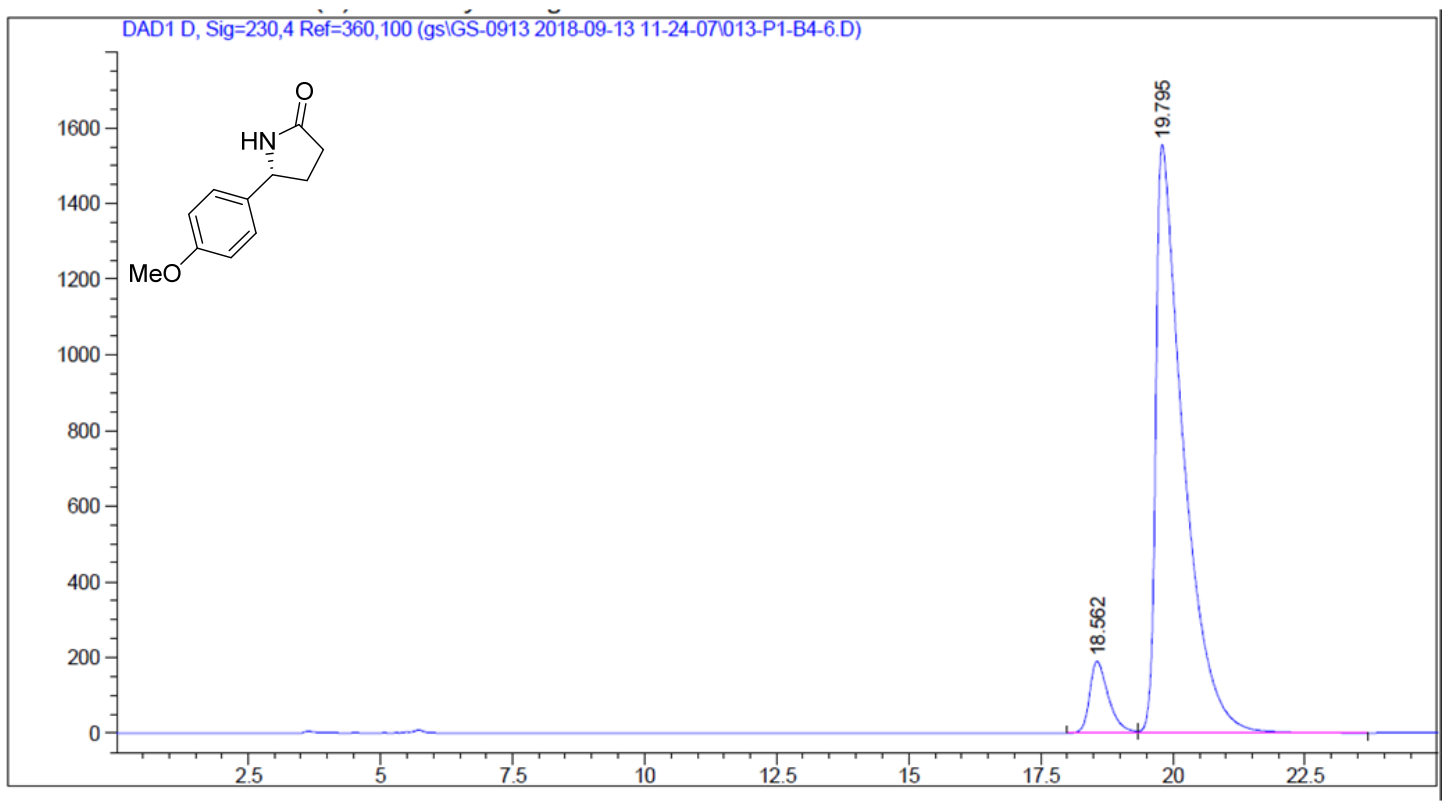

Signal 1: DAD1 D, Sig=230, 4 Ref $=360,100$

\begin{tabular}{|c|c|c|c|c|c|c|}
\hline $\begin{array}{l}\text { eak } \\
\#\end{array}$ & $\begin{array}{c}\text { RetTime } \\
\text { [min] }\end{array}$ & Type & $\begin{array}{l}\text { Width } \\
\text { [min] }\end{array}$ & $\begin{array}{c}\text { Area } \\
{\left[\mathrm{mAU}^{*} \mathrm{~s}\right]}\end{array}$ & $\begin{array}{l}\text { Height } \\
{[\mathrm{mAU}]}\end{array}$ & $\begin{array}{c}\text { Area } \\
\%\end{array}$ \\
\hline & & & & & & \\
\hline 1 & & & & 4732 & 196 & 100 \\
\hline , & & & & $5.64150 \mathrm{e} 4$ & 822 & 92.2600 \\
\hline
\end{tabular}


(R)-5-([1,1'-biphenyl]-4-yl)pyrrolidin-2-one (2l)

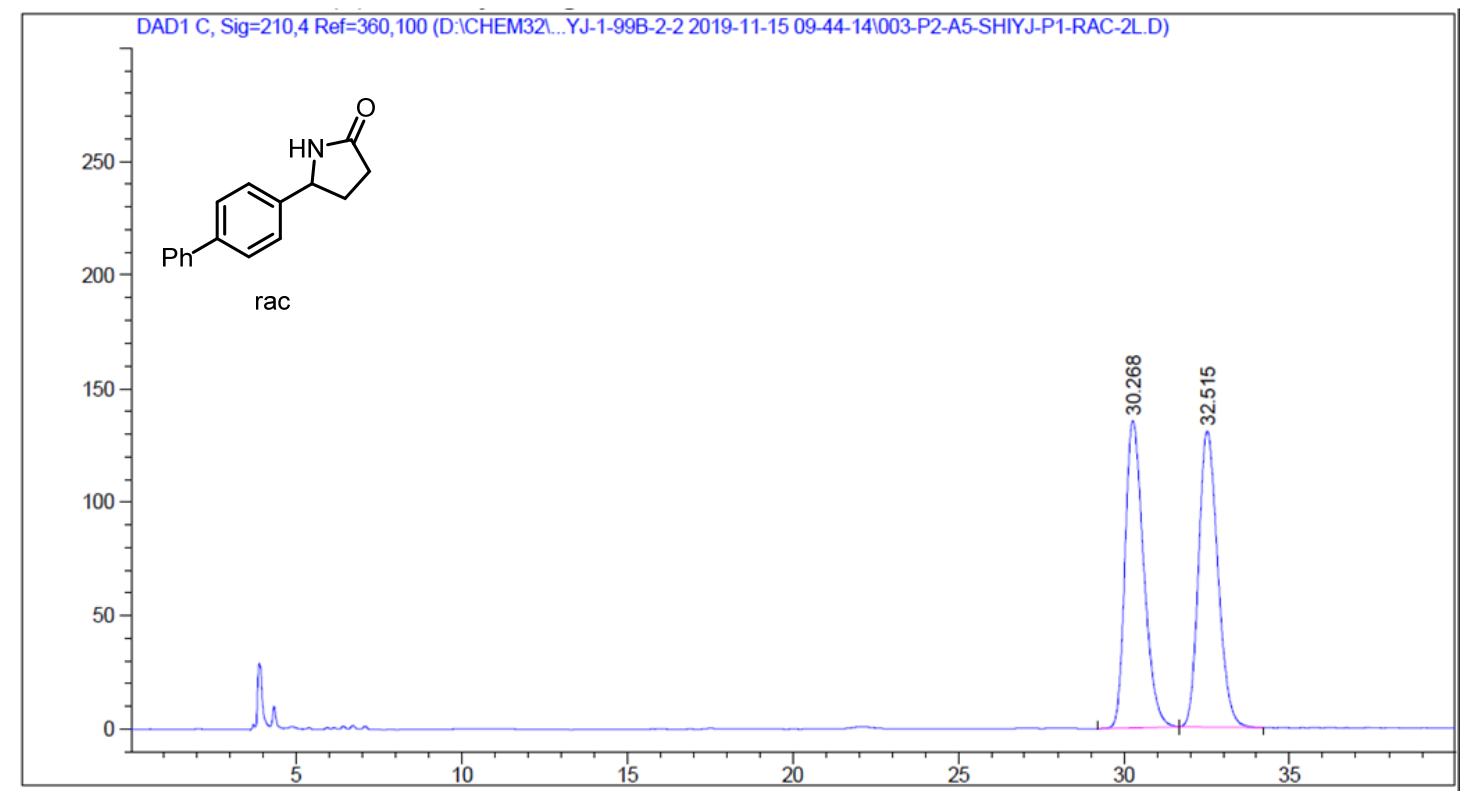

Signal 1: DAD1 C, Sig=210,4 Ref $=360,100$

\begin{tabular}{|c|c|c|c|c|c|c|}
\hline $\begin{array}{c}\text { Peak } \\
\#\end{array}$ & $\begin{array}{c}\text { RetTime } \\
\text { [min] }\end{array}$ & Type & $\begin{array}{l}\text { Width } \\
\text { [min] }\end{array}$ & $\begin{array}{c}\text { Area } \\
{\left[\mathrm{mAU}^{*} \mathrm{~s}\right]}\end{array}$ & $\begin{array}{l}\text { Height } \\
\text { [mAU] }\end{array}$ & $\begin{array}{c}\text { Area } \\
\%\end{array}$ \\
\hline & & & & & & \\
\hline 1 & 30 & & 1 & 5283 & 135 & 193 \\
\hline 2 & 32.5 & BB & .6276 & 5279.69141 & 130.60262 & 49.9807 \\
\hline
\end{tabular}

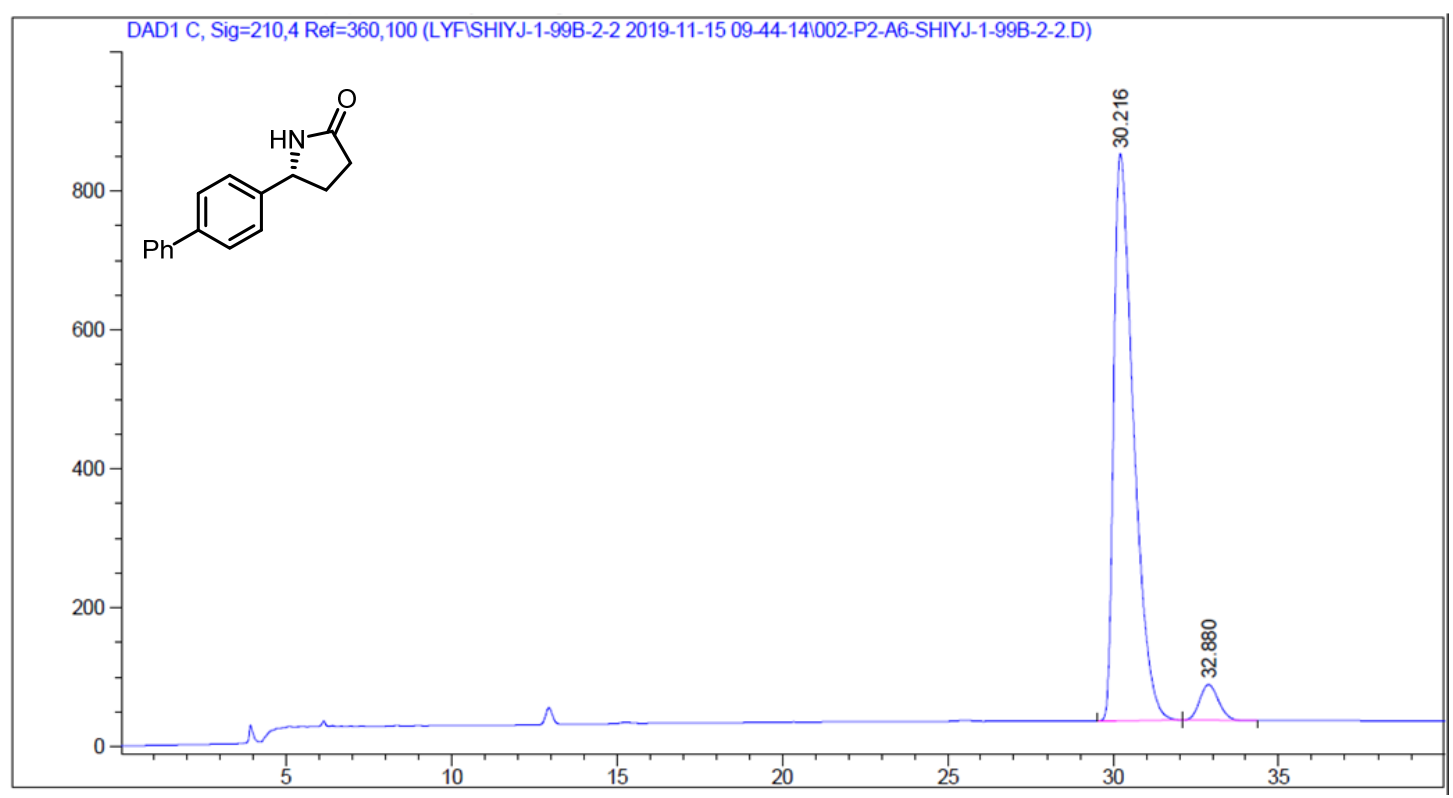

Signal 1: DAD1 C, Sig $=210,4$ Ref $=360,100$

\begin{tabular}{|c|c|c|c|c|c|c|}
\hline $\begin{array}{l}\text { eak } \\
\#\end{array}$ & $\begin{array}{c}\text { RetTime } \\
\text { [min] }\end{array}$ & Type & $\begin{array}{l}\text { Width } \\
\text { [min] }\end{array}$ & $\begin{array}{c}\text { Area } \\
{\left[\mathrm{mAU}^{*} \mathrm{~s}\right]}\end{array}$ & $\begin{array}{l}\text { Height } \\
\text { [mAU] }\end{array}$ & $\begin{array}{c}\text { Area } \\
\%\end{array}$ \\
\hline & & & & & & \\
\hline 1 & & & & .46 & 10 & 94 \\
\hline 2 & 32.880 & BB & .6153 & 2033.96411 & 51.44038 & 5.6433 \\
\hline
\end{tabular}


(R)-5-(naphthalen-2-yl)pyrrolidin-2-one (2m)

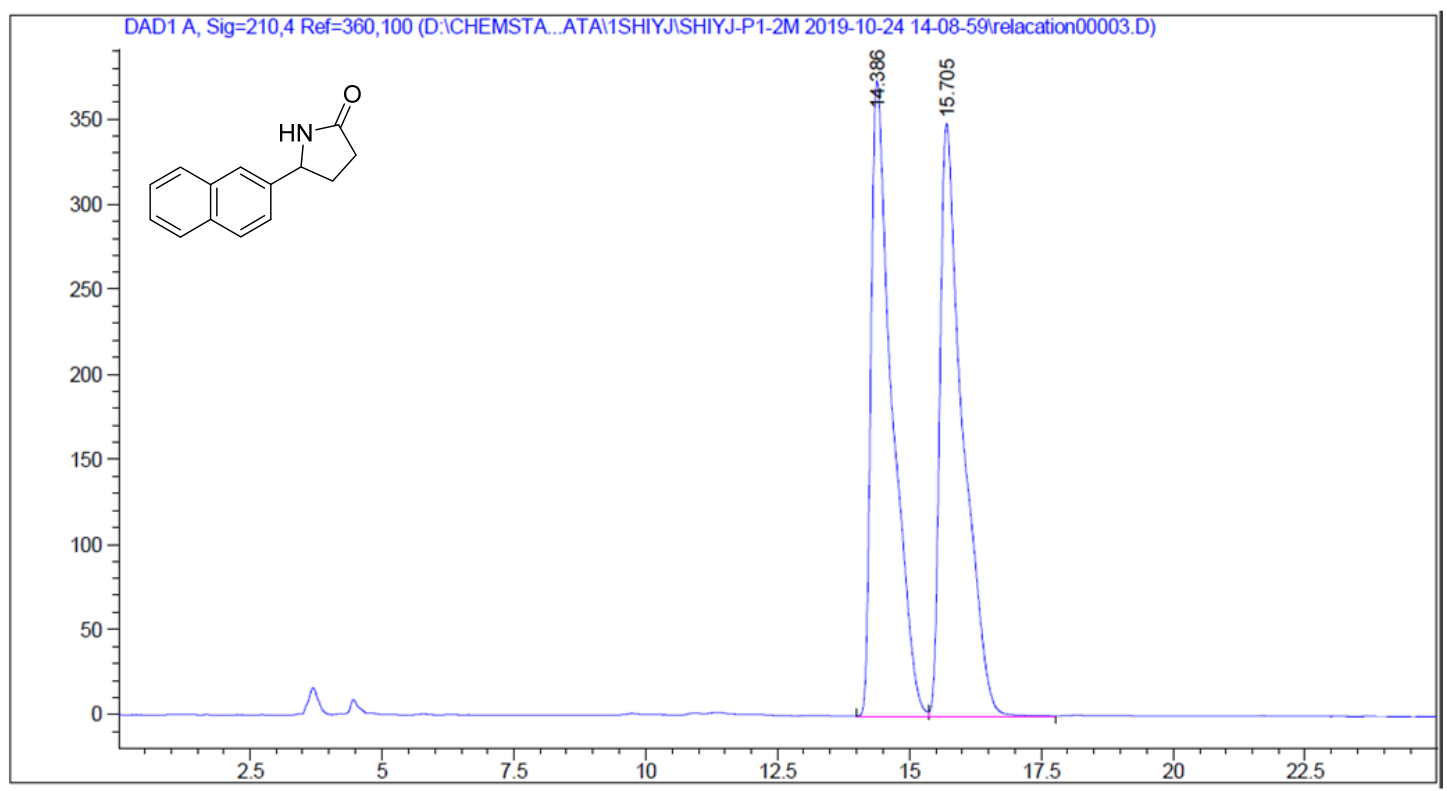

Signal 1: DAD1 A, Sig=210,4 Ref $=360,100$
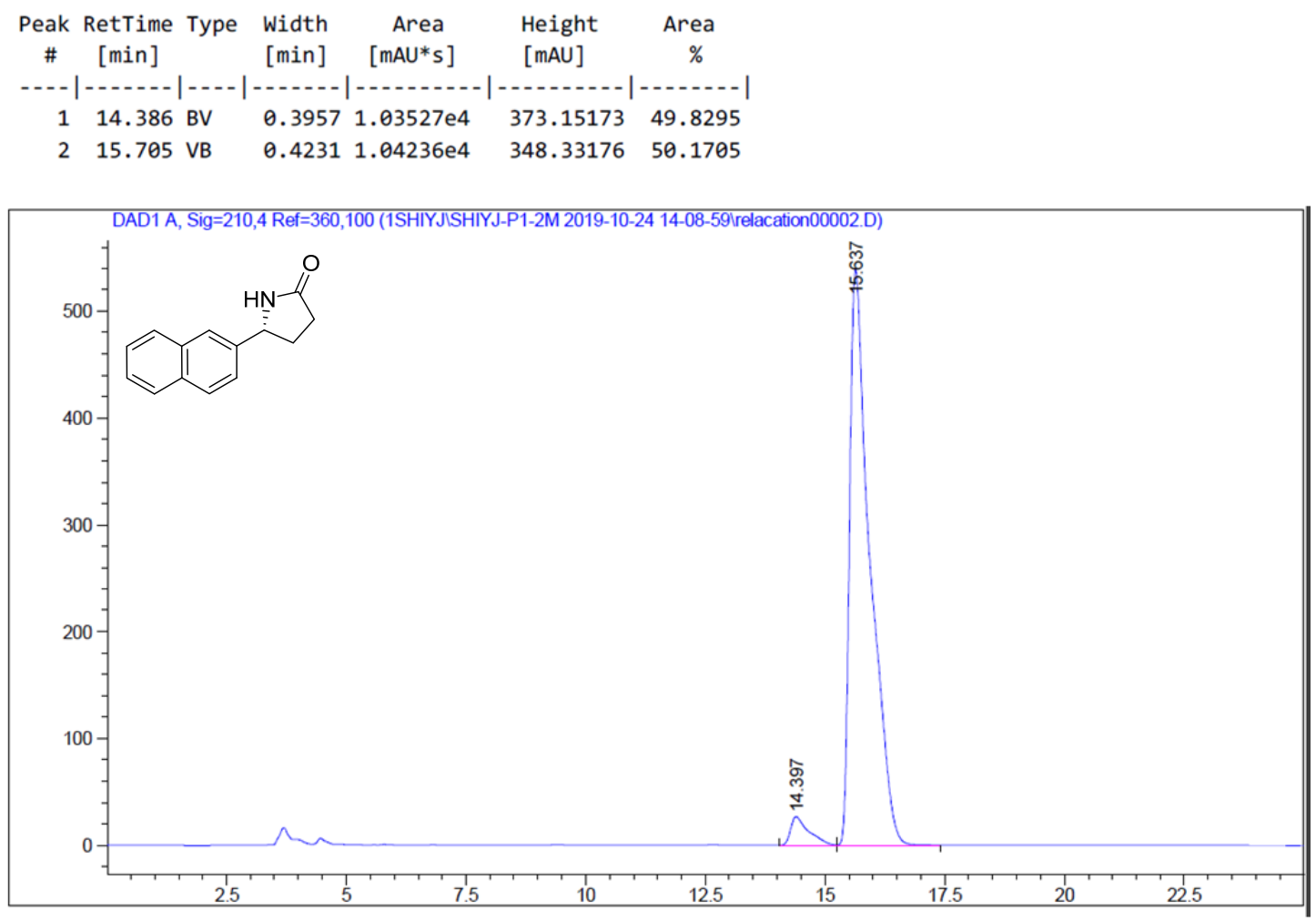

Signal 1: DAD1 A, Sig $=210,4$ Ref $=360,100$

\begin{tabular}{cccccc}
$\begin{array}{c}\text { Peak RetTime Type } \\
\text { W }\end{array}$ [min] & $\begin{array}{c}\text { Width } \\
\text { [min] }\end{array}$ & $\begin{array}{c}\text { Area } \\
{[\mathrm{mAU} \text { s }]}\end{array}$ & \multicolumn{1}{c}{$\begin{array}{c}\text { Height } \\
\text { [mAU] }\end{array}$} & \multicolumn{1}{c}{$\begin{array}{c}\text { Area } \\
\%\end{array}$} \\
\hline 1 & 14.397 BV & 0.3703 & 701.85541 & 26.73432 & 4.2162 \\
2 & 15.637 VB & 0.4210 & $1.59449 \mathrm{e} 4$ & 539.23969 & 95.7838
\end{tabular}


(S)-5-methylpyrrolidin-2-one (2n)

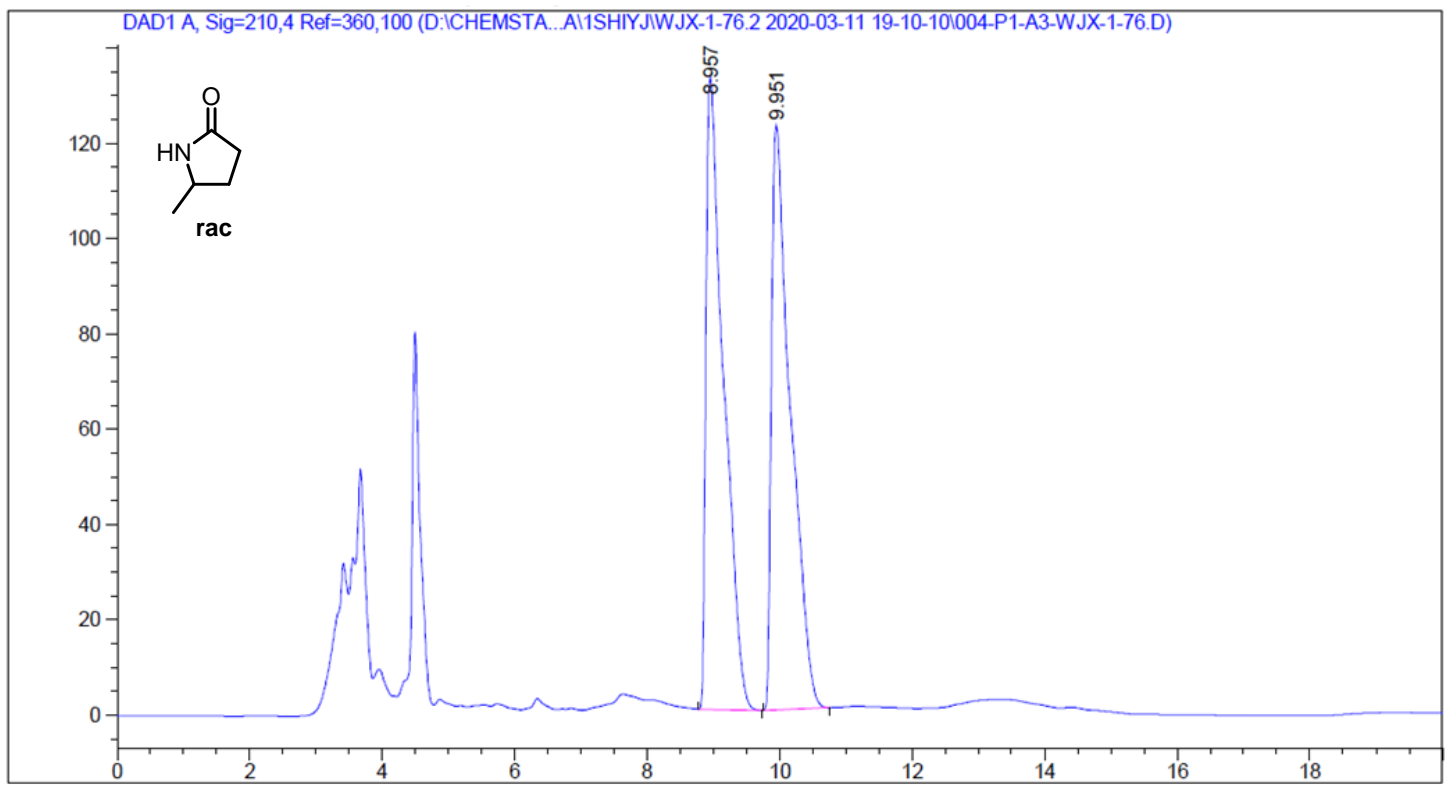

Signal 1: DAD1 A, Sig=210, 4 Ref $=360,100$

\begin{tabular}{cccccc}
$\begin{array}{c}\text { Peak RetTime Type } \\
\text { \# }\end{array}$ [min] & $\begin{array}{c}\text { Width } \\
{[\mathrm{min}]}\end{array}$ & $\begin{array}{c}\text { Area } \\
{[\mathrm{mAU} \text { s }]}\end{array}$ & $\begin{array}{c}\text { Height } \\
{[\mathrm{mAU}]}\end{array}$ & $\begin{array}{c}\text { Area } \\
\%\end{array}$ \\
\hline 1 & 8.957 BB & 0.2616 & 2451.93970 & 132.68640 & 50.0335 \\
2 & 9.951 BB & 0.2828 & 2448.65820 & 122.69194 & 49.9665
\end{tabular}

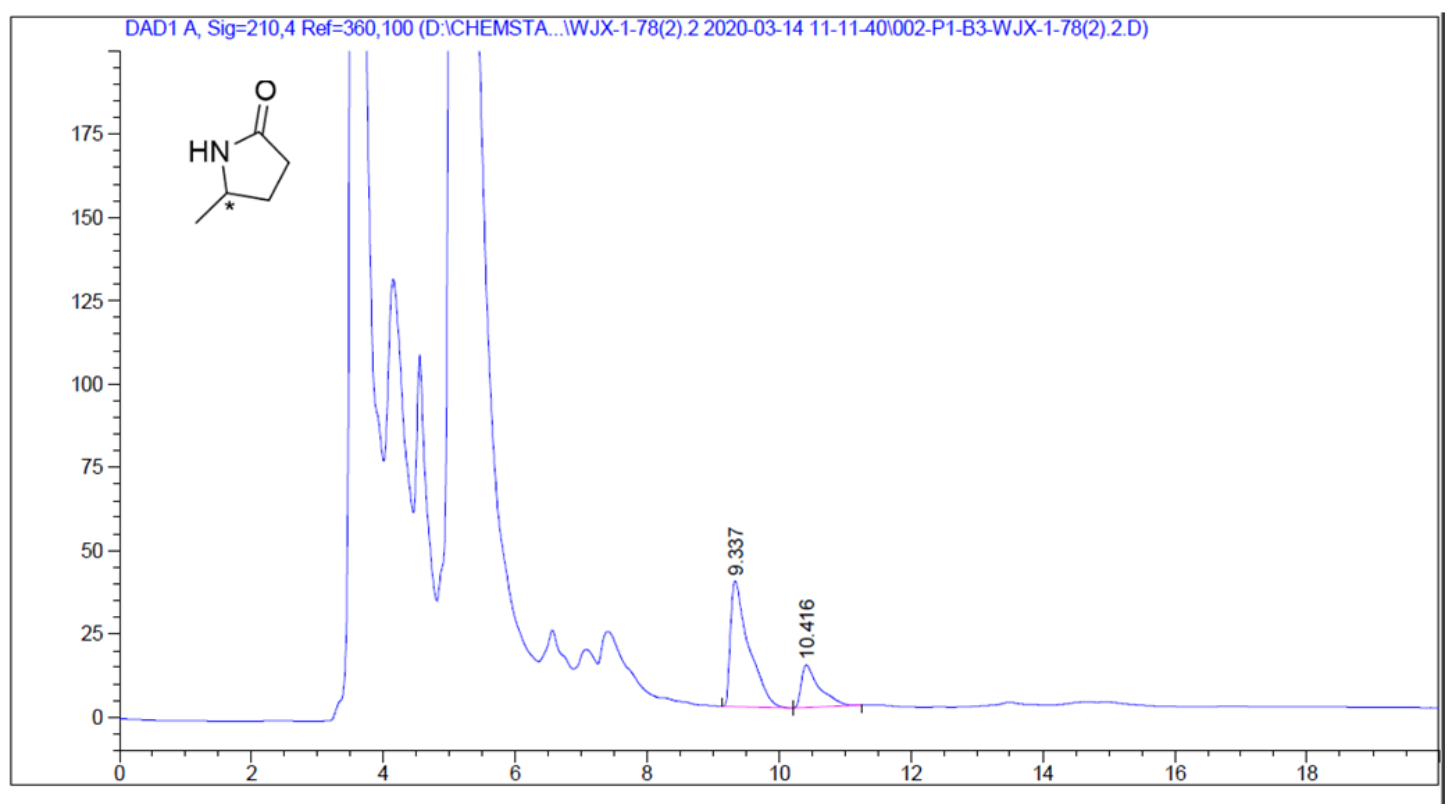

Signal 1: DAD1 A, Sig=210,4 Ref $=360,100$

\begin{tabular}{|c|c|c|c|c|c|c|}
\hline eak & $\begin{array}{c}\text { RetTime } \\
\text { [min] }\end{array}$ & Type & $\begin{array}{l}\text { Width } \\
\text { [min] }\end{array}$ & $\begin{array}{c}\text { Area } \\
{\left[\mathrm{mAU}^{*} \mathrm{~s}\right]}\end{array}$ & $\begin{array}{l}\text { Height } \\
\text { [mAU] }\end{array}$ & $\begin{array}{c}\text { Area } \\
\%\end{array}$ \\
\hline & & & & 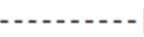 & & \\
\hline 1 & & & & 728 & 37 & 25 \\
\hline 2 & 0.416 & BB & 0.2701 & 243.84419 & 12.80784 & 25.0675 \\
\hline
\end{tabular}


(R)-6-phenylpiperidin-2-one (4a)
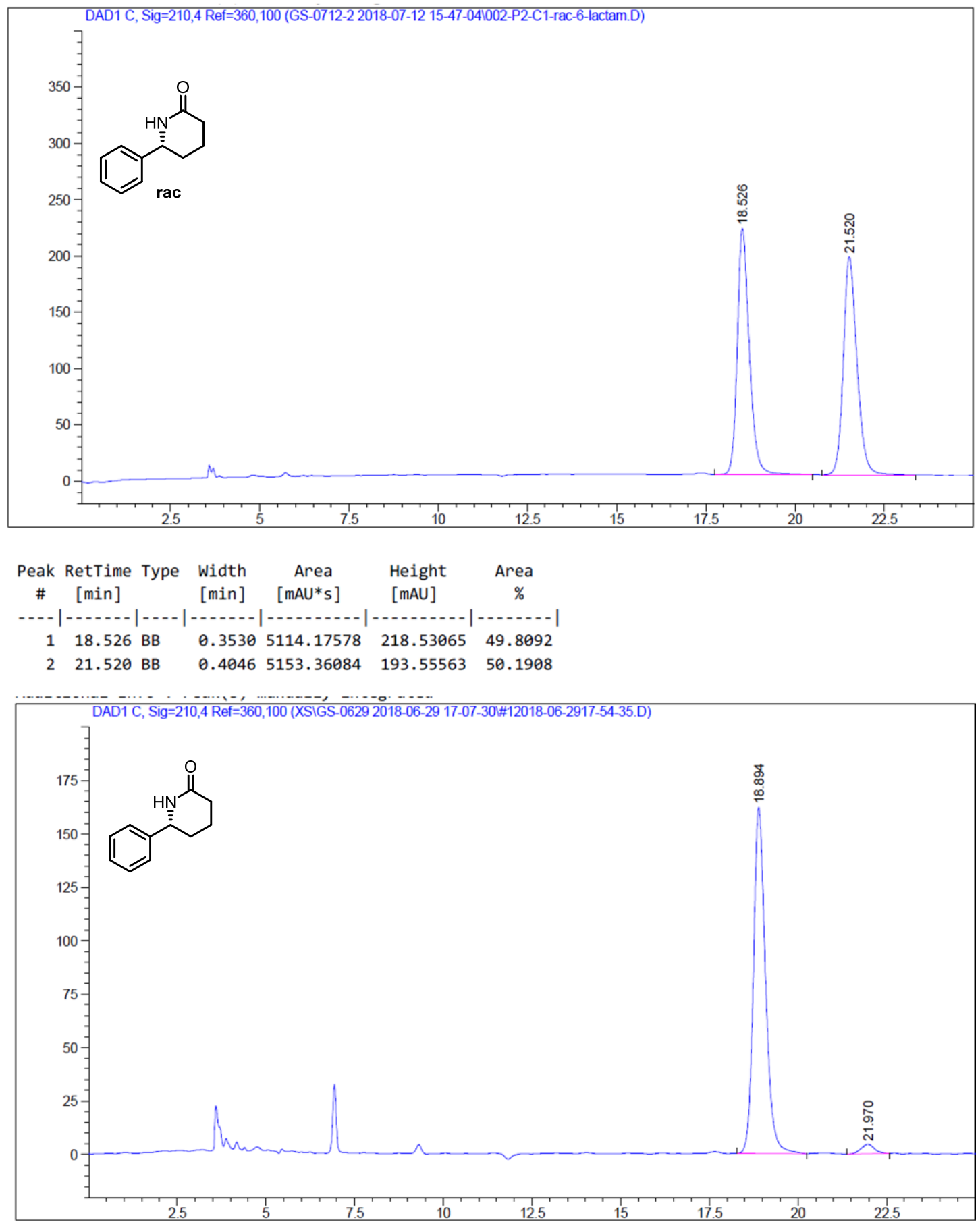

Signal 1: DAD1 C, Sig $=210,4$ Ref $=360,100$

\begin{tabular}{cccccc}
$\begin{array}{c}\text { Peak RetTime Type } \\
\text { \# } \\
\text { [min] }\end{array}$ & $\begin{array}{c}\text { Width } \\
{[\mathrm{min}]}\end{array}$ & $\begin{array}{c}\text { Area } \\
{[\mathrm{mAU} \text { s }]}\end{array}$ & $\begin{array}{c}\text { Height } \\
{[\mathrm{mAU}]}\end{array}$ & $\begin{array}{c}\text { Area } \\
\%\end{array}$ \\
\hline 1 & 18.894 BB & 0.3612 & 3844.08057 & 161.79329 & 97.0720 \\
2 & 21.970 BB & 0.3924 & 115.94972 & 4.35778 & 2.9280
\end{tabular}


(R)-6-(o-tolyl)piperidin-2-one (4b)

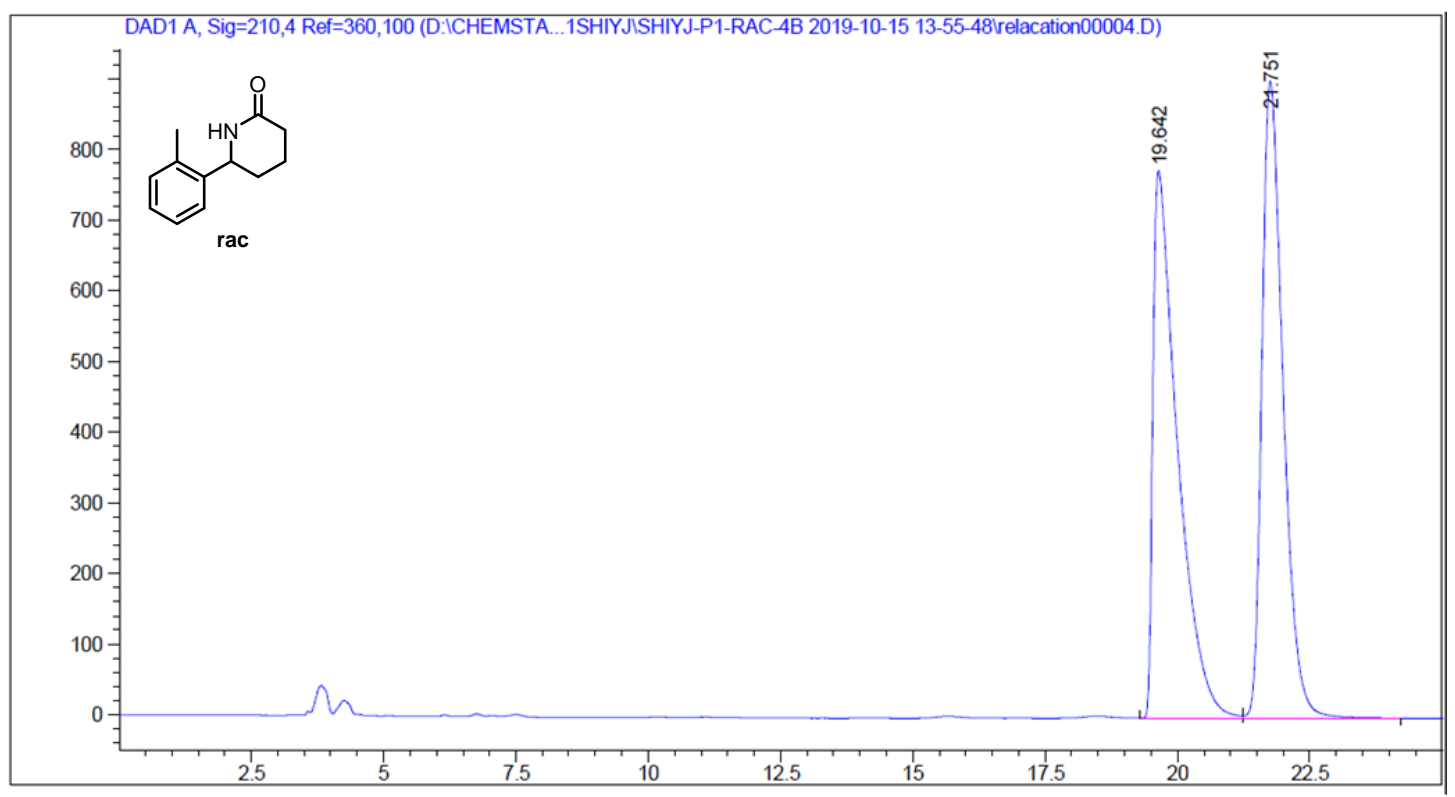

Signal 1: DAD1 A, Sig=210,4 Ref $=360,100$

\begin{tabular}{|c|c|c|c|c|c|c|}
\hline $\begin{array}{l}\text { eak } \\
\#\end{array}$ & $\begin{array}{c}\text { RetTime } \\
\text { [min] }\end{array}$ & Type & $\begin{array}{l}\text { Width } \\
\text { [min] }\end{array}$ & $\begin{array}{c}\text { Area } \\
{\left[\mathrm{mAU}^{*} \mathrm{~s}\right]}\end{array}$ & $\begin{array}{l}\text { ght } \\
\text { J] }\end{array}$ & $\begin{array}{c}\text { Area } \\
\%\end{array}$ \\
\hline & & & & & -- & $1--$ \\
\hline 1 & 19. & & 0.4 & 240 & 775. & 49. \\
\hline , & 21.751 & & .4247 & $2.50995 \mathrm{e} 4$ & 901.76825 & $50.116 e$ \\
\hline
\end{tabular}

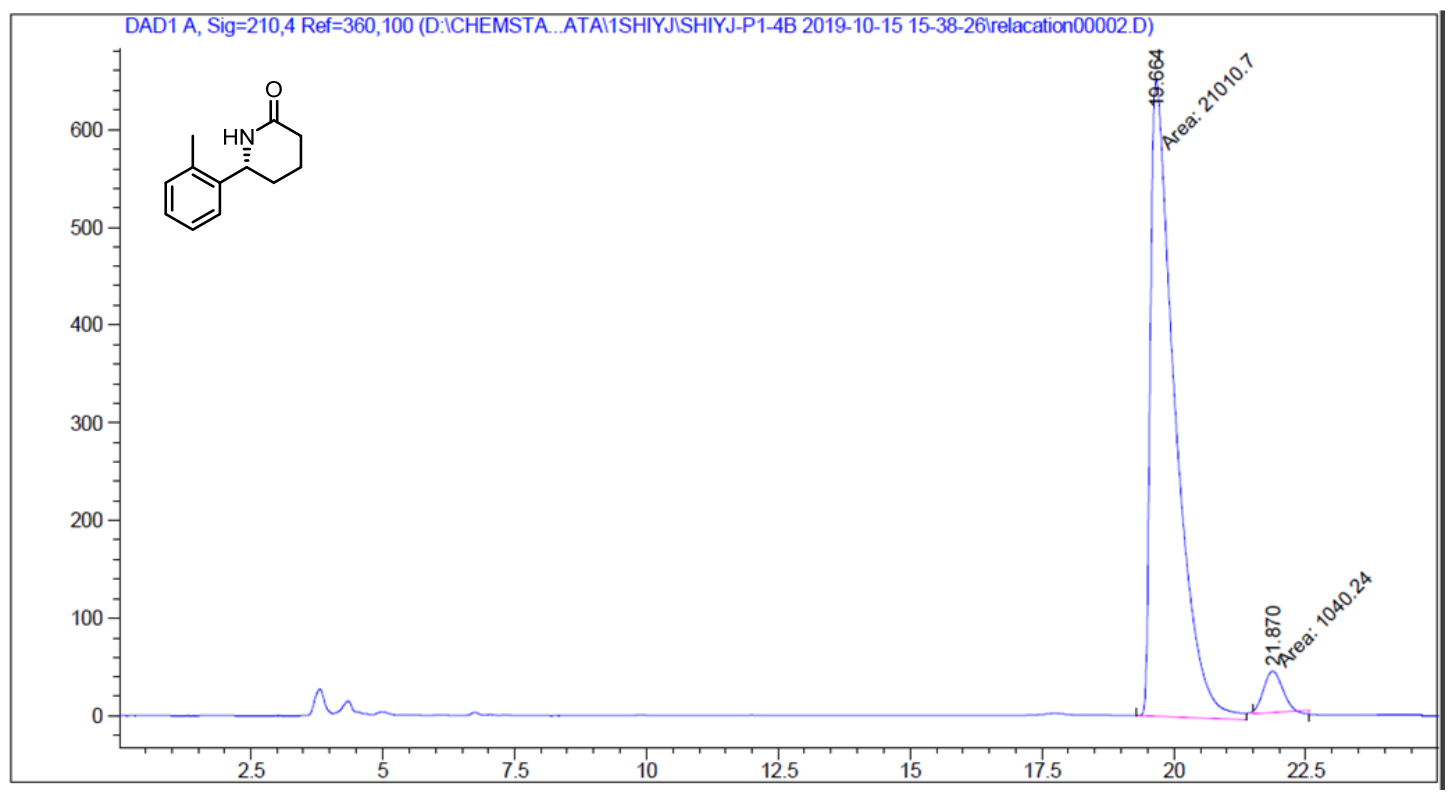

Signal 1: DAD1 A, Sig $=210,4$ Ref $=360,100$

\begin{tabular}{|c|c|c|c|c|c|c|}
\hline $\begin{array}{c}\text { Peak } \\
\#\end{array}$ & $\begin{array}{c}\text { RetTime } \\
\text { [min] }\end{array}$ & Type & $\begin{array}{l}\text { Width } \\
\text { [min] }\end{array}$ & $\begin{array}{c}\text { Area } \\
{[\mathrm{mAU} * \mathrm{~s}]}\end{array}$ & $\begin{array}{l}\text { Height } \\
{[\mathrm{mAU}]}\end{array}$ & $\begin{array}{c}\text { Area } \\
\%\end{array}$ \\
\hline & & & & & 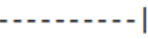 & 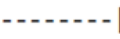 \\
\hline 1 & & & & $2.10107 \mathrm{e} 4$ & 651.40686 & 95.2826 \\
\hline 2 & 876 & & 118 & 1040.24426 & 42.09724 & .7174 \\
\hline
\end{tabular}


(R)-6-(m-tolyl)piperidin-2-one (4c)

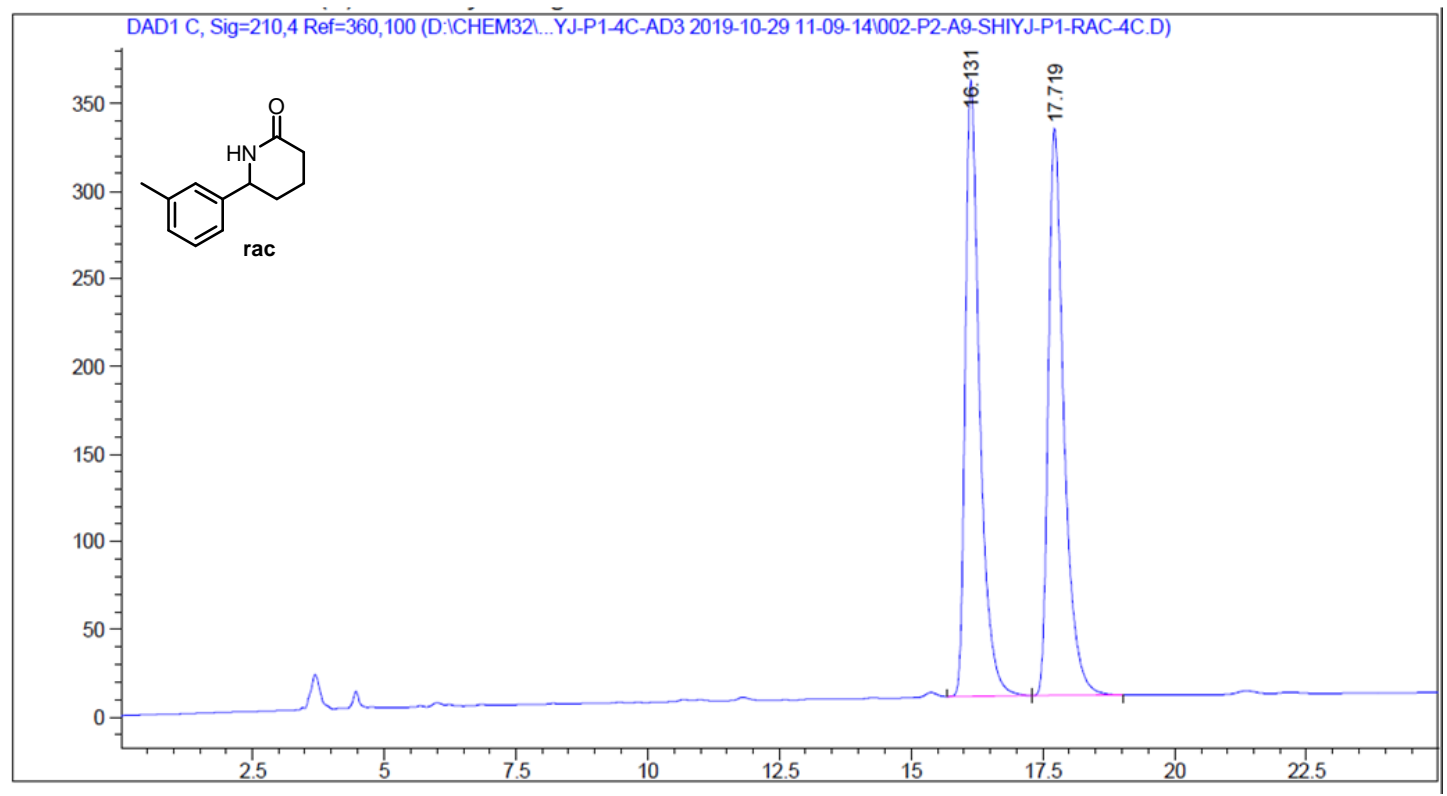

Signal 1: DAD1 C, Sig=210,4 Ref $=360,100$

\begin{tabular}{cccccc}
$\begin{array}{c}\text { Peak RetTime Type } \\
\text { \# } \\
\text { [min] }\end{array}$ & $\begin{array}{c}\text { Width } \\
{[\mathrm{min}]}\end{array}$ & $\begin{array}{c}\text { Area } \\
{[\mathrm{mAU} \text { s }]}\end{array}$ & $\begin{array}{c}\text { Height } \\
\text { [mAU] }\end{array}$ & $\begin{array}{c}\text { Area } \\
\%\end{array}$ \\
\hline 1 & 16.131 BB & 0.2829 & 6653.85791 & 351.36380 & 49.8965 \\
2 & 17.719 BB & 0.3089 & 6681.46289 & 323.54901 & 50.1035
\end{tabular}

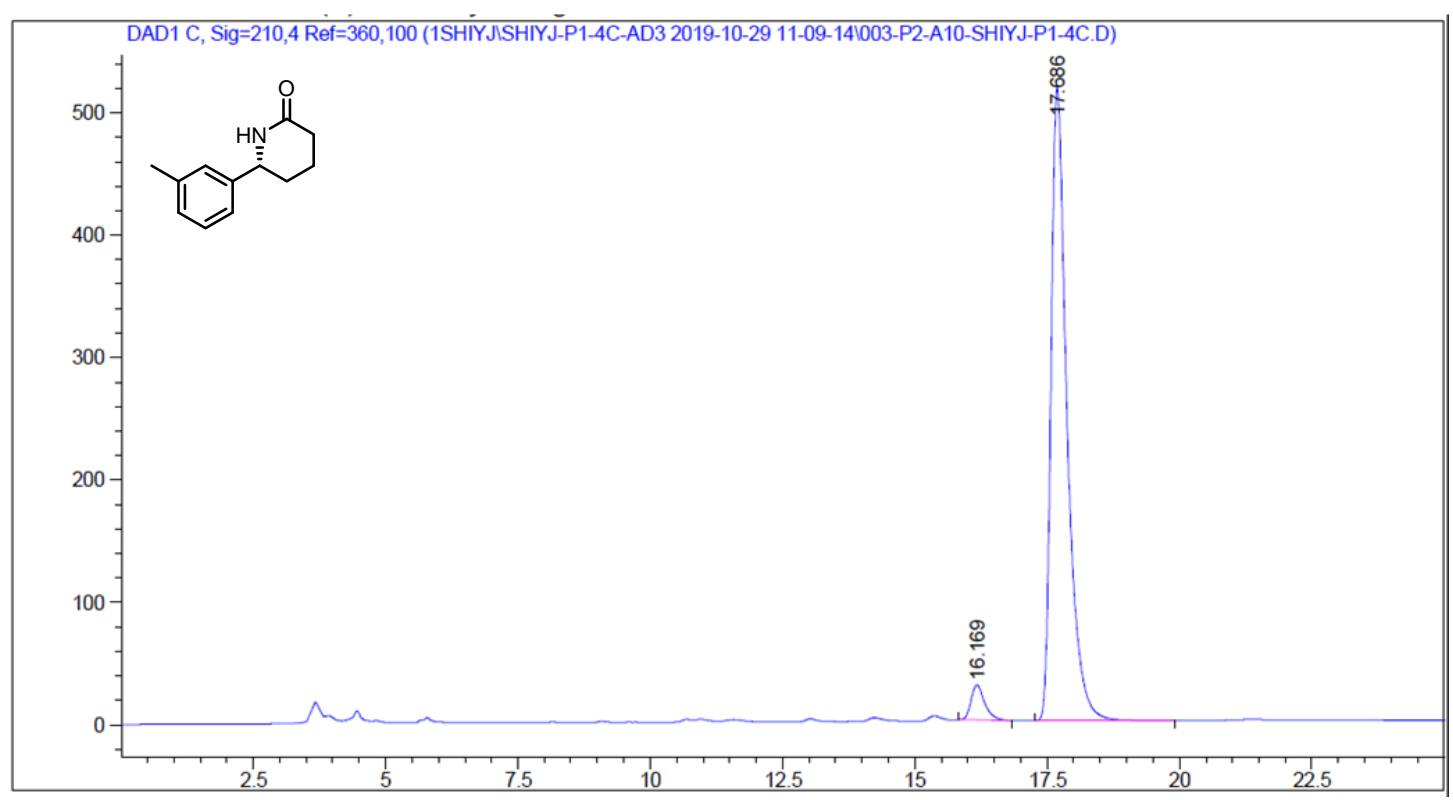

Signal 1: DAD1 C, Sig=210,4 Ref $=360,100$

\begin{tabular}{|c|c|c|c|c|c|c|}
\hline $\begin{array}{l}\text { eak } \\
\#\end{array}$ & $\begin{array}{c}\text { RetTime } \\
\text { [min] }\end{array}$ & Type & $\begin{array}{l}\text { Width } \\
\text { [min] }\end{array}$ & $\begin{array}{c}\text { Area } \\
{\left[\mathrm{mAU}^{*} \mathrm{~s}\right]}\end{array}$ & $\begin{array}{l}\text { Height } \\
\text { [mAU] }\end{array}$ & $\begin{array}{c}\text { Area } \\
\%\end{array}$ \\
\hline & & & & & & \\
\hline 1 & & & & 370 & 91 & 20 \\
\hline 2 & 7. & & 22 & $1.08338 \mathrm{e} 4$ & 517.59314 & 4780 \\
\hline
\end{tabular}




\section{(R)-6-(p-tolyl)piperidin-2-one (4d)}

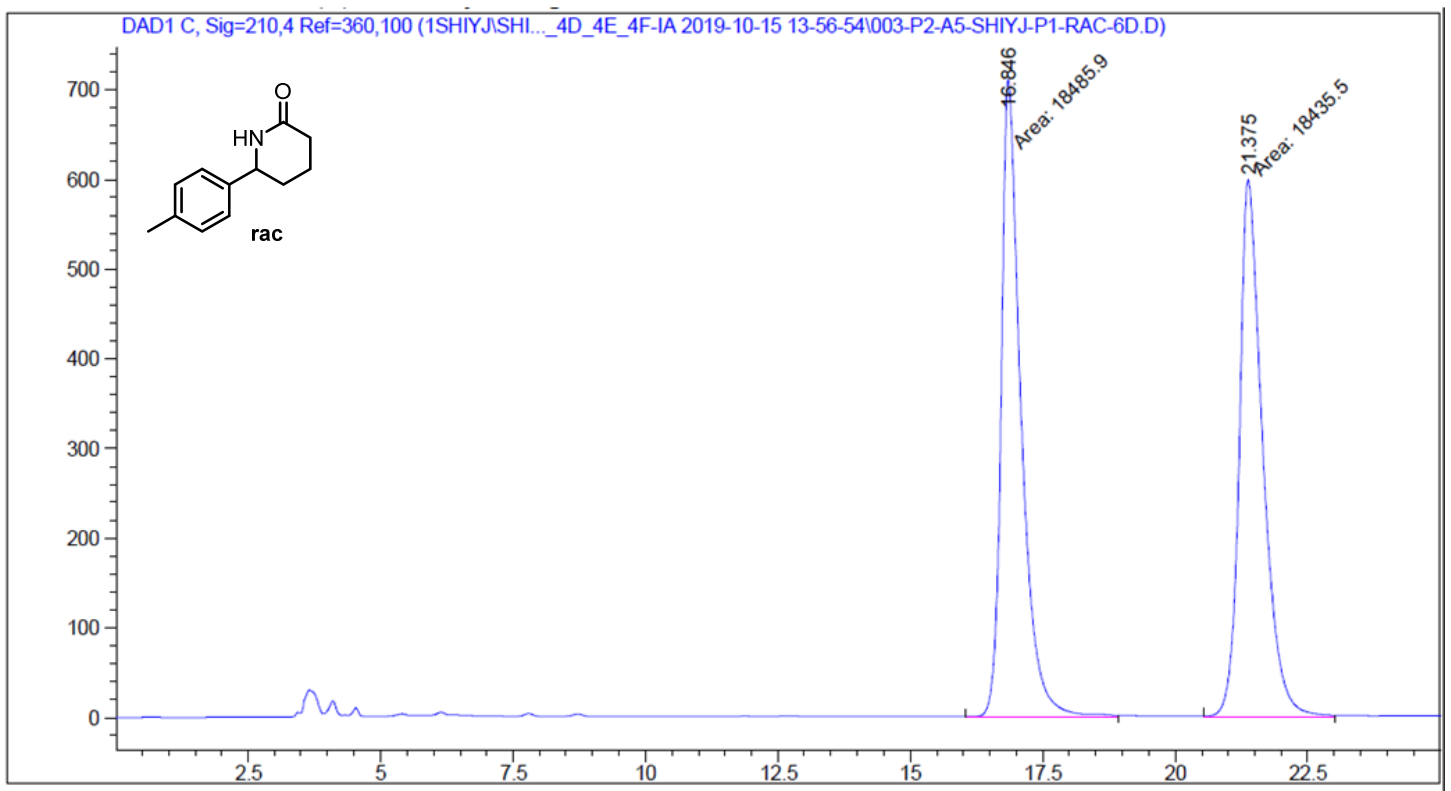

Signal 1: DAD1 C, Sig $=210,4$ Ref $=360,100$

\begin{tabular}{|c|c|c|c|c|c|c|}
\hline $\begin{array}{c}\text { Peak } \\
\#\end{array}$ & $\begin{array}{c}\text { RetTime } \\
\text { [min] }\end{array}$ & Type & $\begin{array}{l}\text { Width } \\
\text { [min] }\end{array}$ & $\begin{array}{c}\text { Area } \\
{\left[\mathrm{mAU}^{*} \mathrm{~s}\right]}\end{array}$ & $\begin{array}{l}\text { Height } \\
\text { [mAU] }\end{array}$ & $\begin{array}{c}\text { Area } \\
\%\end{array}$ \\
\hline . & & & & & & \\
\hline 1 & 16.846 & MM & 0.4 & $1.84859 \mathrm{e} 4$ & 710.69879 & 50.0682 \\
\hline 2 & 21.375 & MM & 31 & $1.84355 \mathrm{e} 4$ & 598.77026 & 49.9318 \\
\hline
\end{tabular}

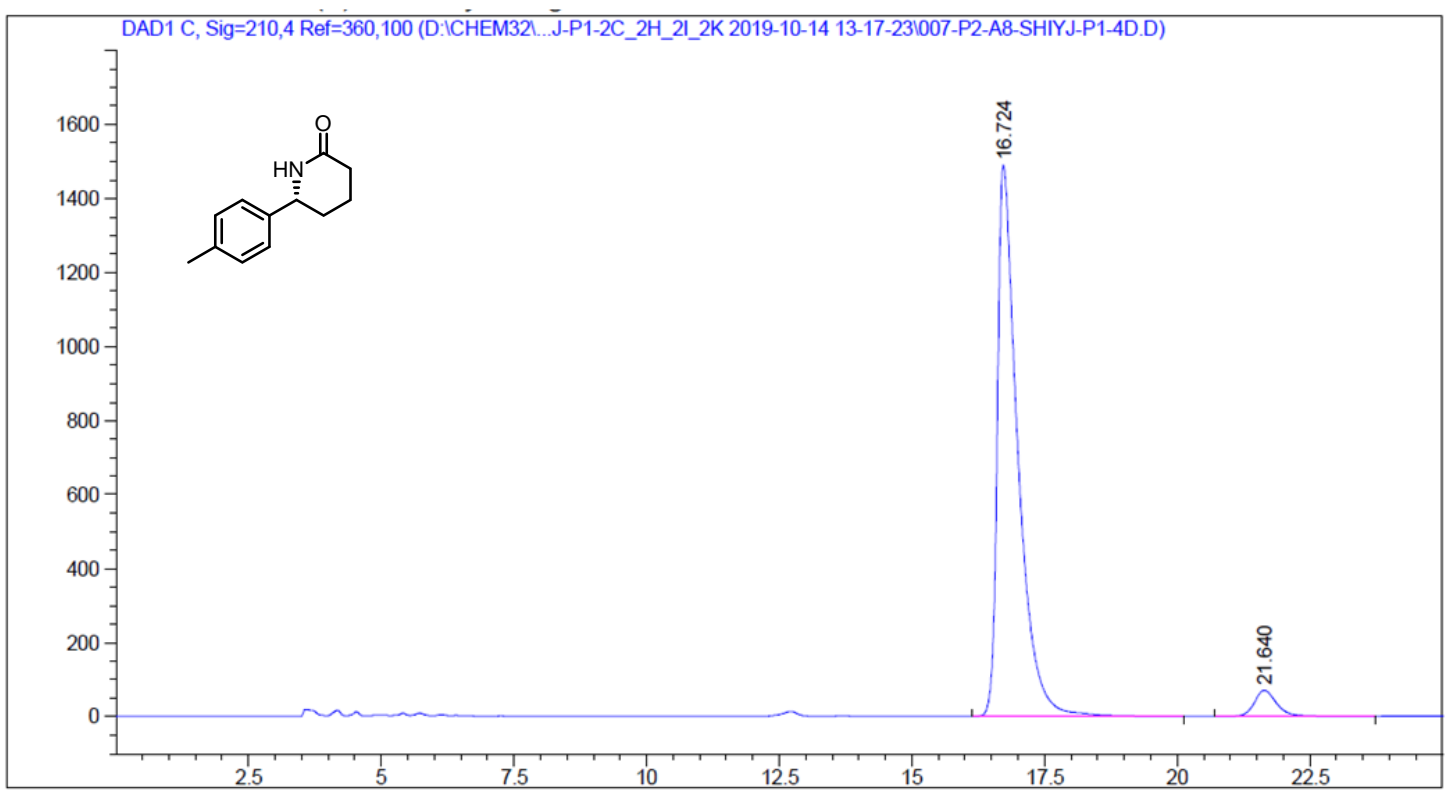

Signal 1: DAD1 C, Sig=210,4 Ref $=360,100$

\begin{tabular}{cccccc}
$\begin{array}{c}\text { Peak RetTime Type } \\
\text { Width } \\
\text { [min] }\end{array}$ & $\begin{array}{c}\text { Area } \\
\text { [min] }\end{array}$ & $\begin{array}{c}\text { Height } \\
\text { [mAU*s] }\end{array}$ & $\begin{array}{c}\text { Area } \\
\text { [mAU] }\end{array}$ & $\begin{array}{c}\% \\
1\end{array}$ \\
\hline 2 & 16.724 BB & 0.3882 & $3.98682 \mathrm{e} 4$ & 1489.70557 & 94.9630 \\
\hdashline & 21.640 BB & 0.4473 & 2114.67627 & 71.03542 & 5.0370
\end{tabular}


(R)-6-(4-methoxyphenyl)piperidin-2-one (4e)

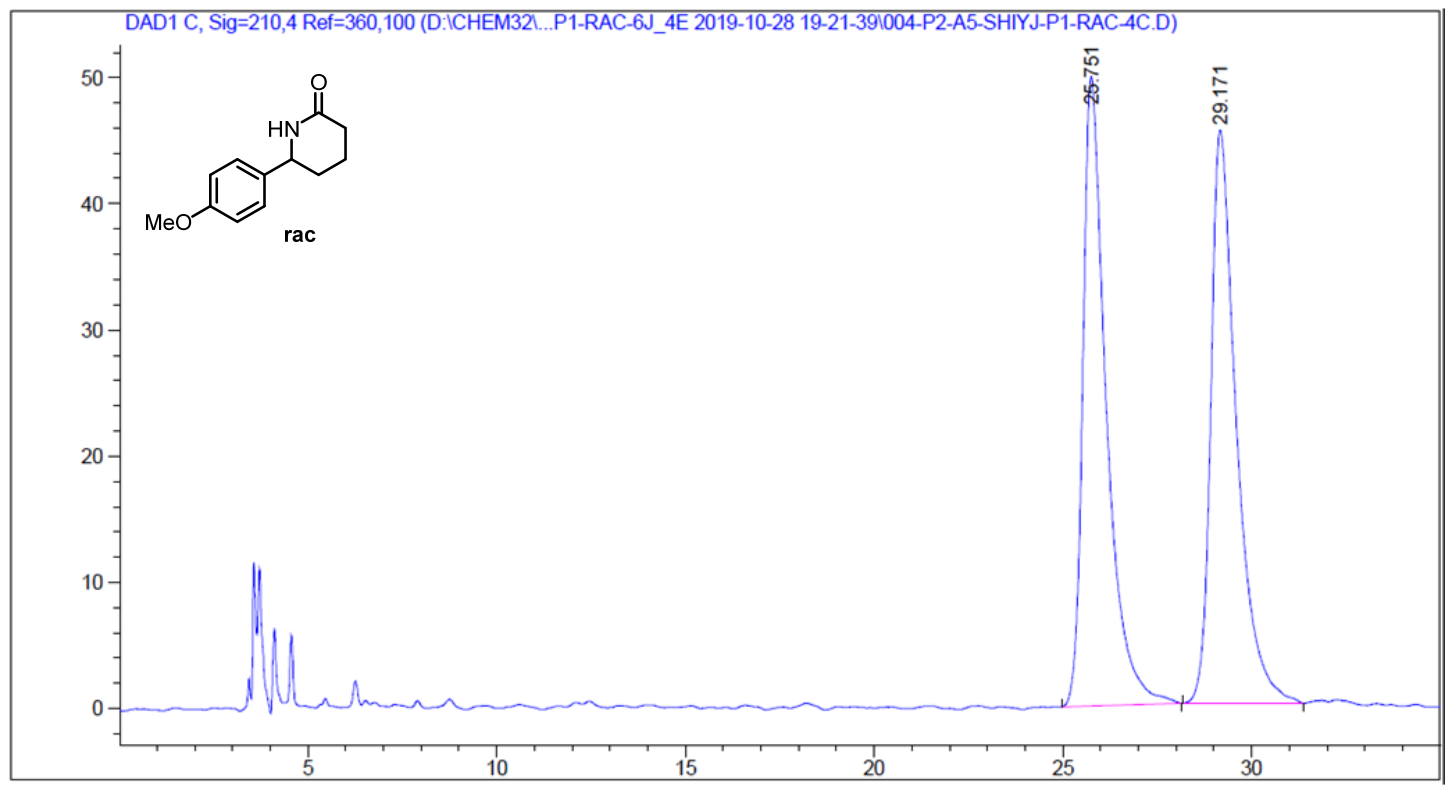

Signal 1: DAD1 C, Sig=210,4 Ref $=360,100$

\begin{tabular}{cccccc}
$\begin{array}{c}\text { Peak RetTime Type } \\
\text { \# } \\
\text { [min] }\end{array}$ & $\begin{array}{c}\text { Width } \\
{[\mathrm{min}]}\end{array}$ & $\begin{array}{c}\text { Area } \\
{[\mathrm{mAU*} \text { ] }}\end{array}$ & $\begin{array}{c}\text { Height } \\
{[\mathrm{mAU}]}\end{array}$ & $\begin{array}{c}\text { Area } \\
\%\end{array}$ \\
\hline 1 & 25.751 BB & 0.6318 & 2152.04419 & 49.83823 & 50.0838 \\
2 & 29.171 BB & 0.6873 & 2144.84131 & 45.38601 & 49.9162
\end{tabular}

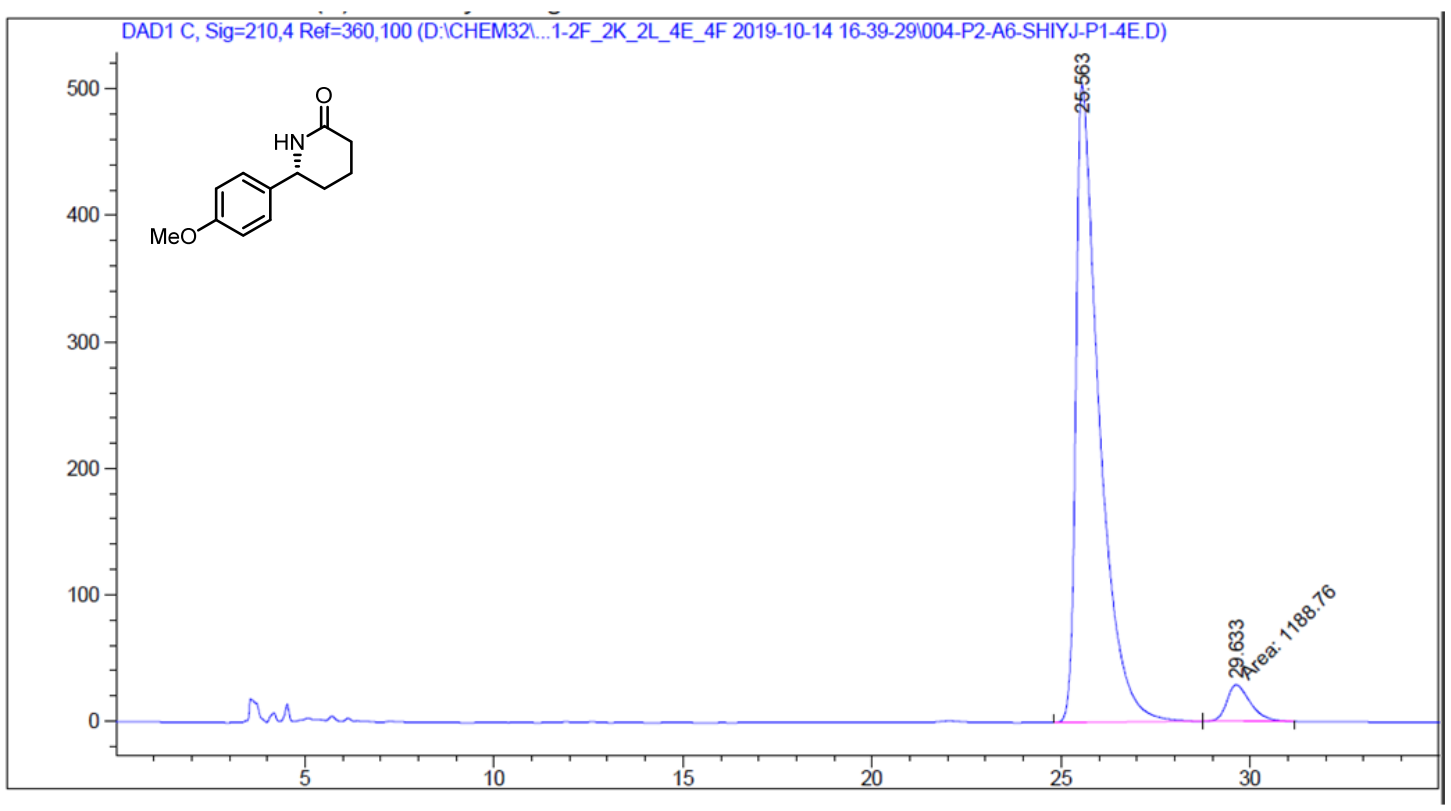

Signal 1: DAD1 C, Sig=210,4 Ref $=360,100$

\begin{tabular}{|c|c|c|c|c|c|c|}
\hline $\begin{array}{c}\text { eak } \\
\#\end{array}$ & $\begin{array}{c}\text { RetTime } \\
\text { [min] }\end{array}$ & Type & $\begin{array}{l}\text { Width } \\
\text { [min] }\end{array}$ & $\begin{array}{c}\text { Area } \\
{\left[\mathrm{mAU}^{*} \mathrm{~s}\right]}\end{array}$ & $\begin{array}{l}\text { Height } \\
\text { [mAU] }\end{array}$ & $\begin{array}{c}\text { Area } \\
\%\end{array}$ \\
\hline & & & & & & -1 \\
\hline 1 & & & & $37 e 4$ & 970 & 94.7913 \\
\hline 2 & & & 1 & 1188.76343 & 28.37964 & 5.2087 \\
\hline
\end{tabular}


(R)-6-(4-fluorophenyl)piperidin-2-one (4f)

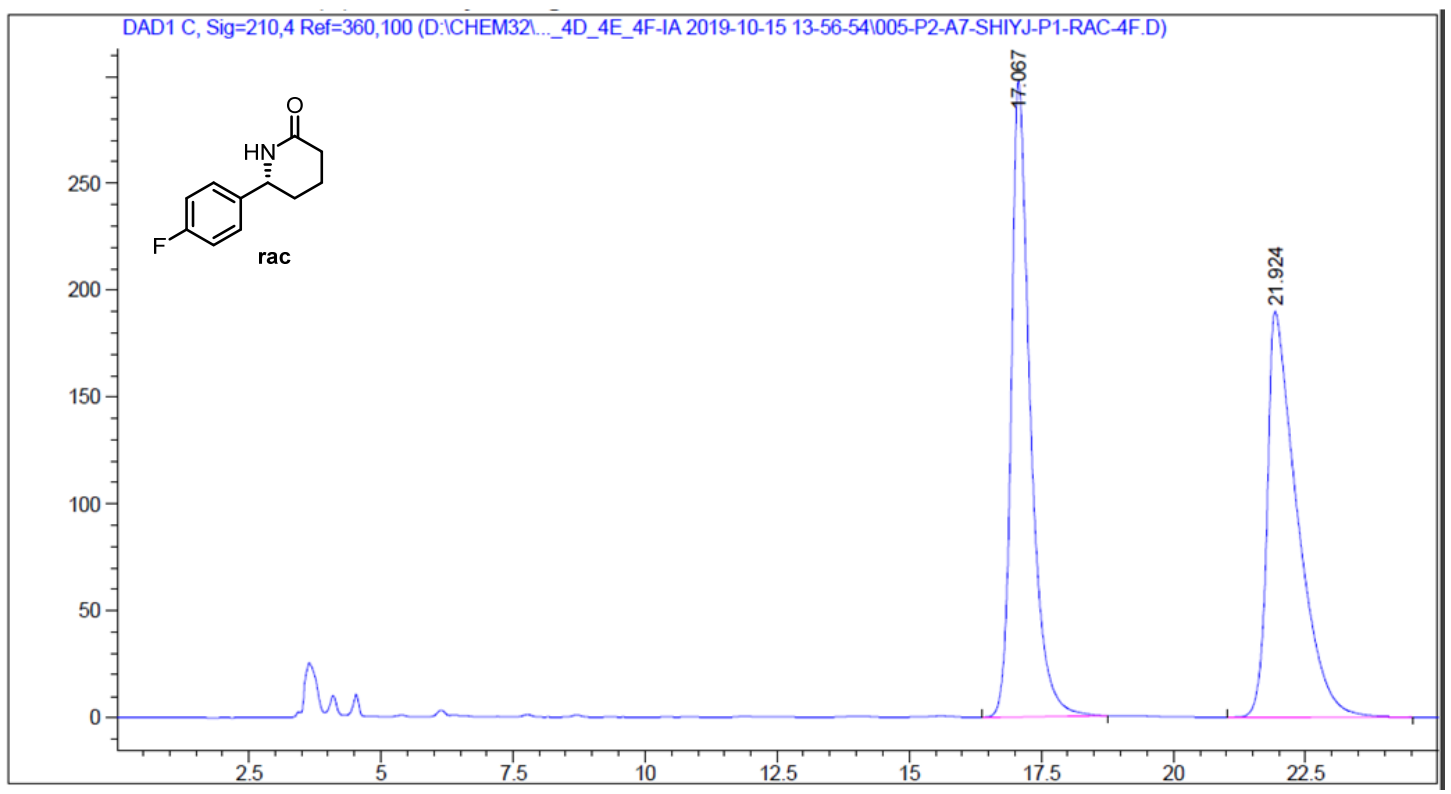

Signal 1: DAD1 C, Sig=210,4 Ref $=360,100$

\begin{tabular}{|c|c|c|c|c|c|c|}
\hline $\begin{array}{l}\text { eak } \\
\#\end{array}$ & $\begin{array}{c}\text { RetTime } \\
\text { [min] }\end{array}$ & Type & $\begin{array}{l}\text { Width } \\
\text { [min] }\end{array}$ & $\begin{array}{c}\text { Area } \\
{\left[\mathrm{mAU}{ }^{*} \mathrm{~s}\right]}\end{array}$ & $\begin{array}{l}\text { Height } \\
{[\mathrm{mAU}]}\end{array}$ & $\begin{array}{c}\text { Area } \\
\quad \%\end{array}$ \\
\hline & & & & & & \\
\hline 1 & & & & 7467.58936 & 297 & 49 \\
\hline , & 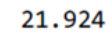 & Do & 4 & 7508.82813 & 189.86357 & 50.1377 \\
\hline
\end{tabular}

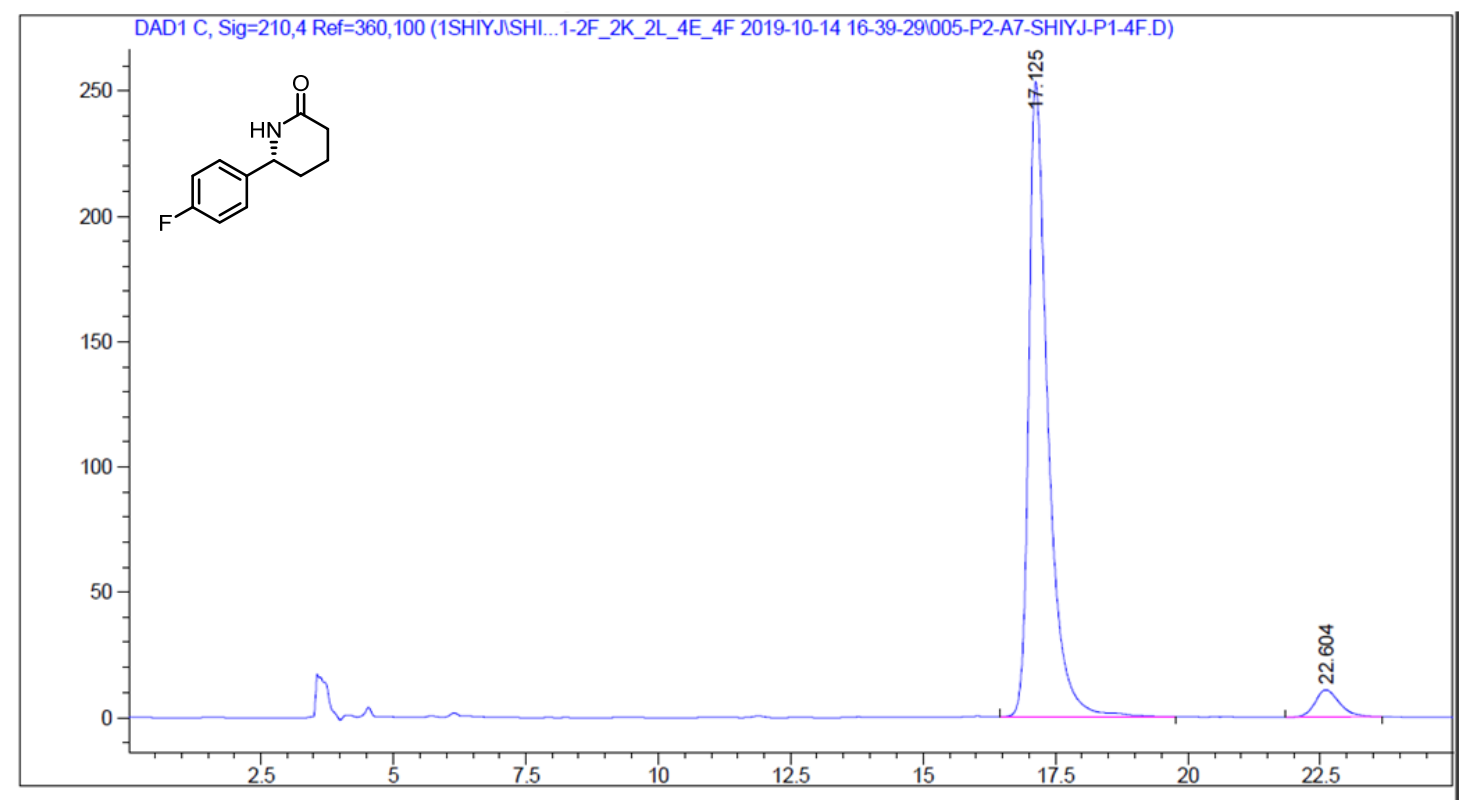

Signal 1: DAD1 C, Sig=210,4 Ref $=360,100$

\begin{tabular}{cccccc}
$\begin{array}{c}\text { Peak RetTime Type } \\
\text { \# }[\text { Width }\end{array}$ & $\begin{array}{c}\text { Area } \\
\text { [min] }\end{array}\left[\begin{array}{c}\text { Height } \\
{[\text { mAU*s] }}\end{array}\right.$ & $\begin{array}{c}\text { Area } \\
\text { [mAU] }\end{array}$ & $\%$ \\
\hline 1 & 17.125 BB & 0.3696 & 6373.56836 & 253.23863 & 94.8080 \\
2 & 22.604 BB & 0.4803 & 349.03955 & 10.87801 & 5.1920
\end{tabular}


(R)-6-(3,5-dimethoxyphenyl)piperidin-2-one (4g)

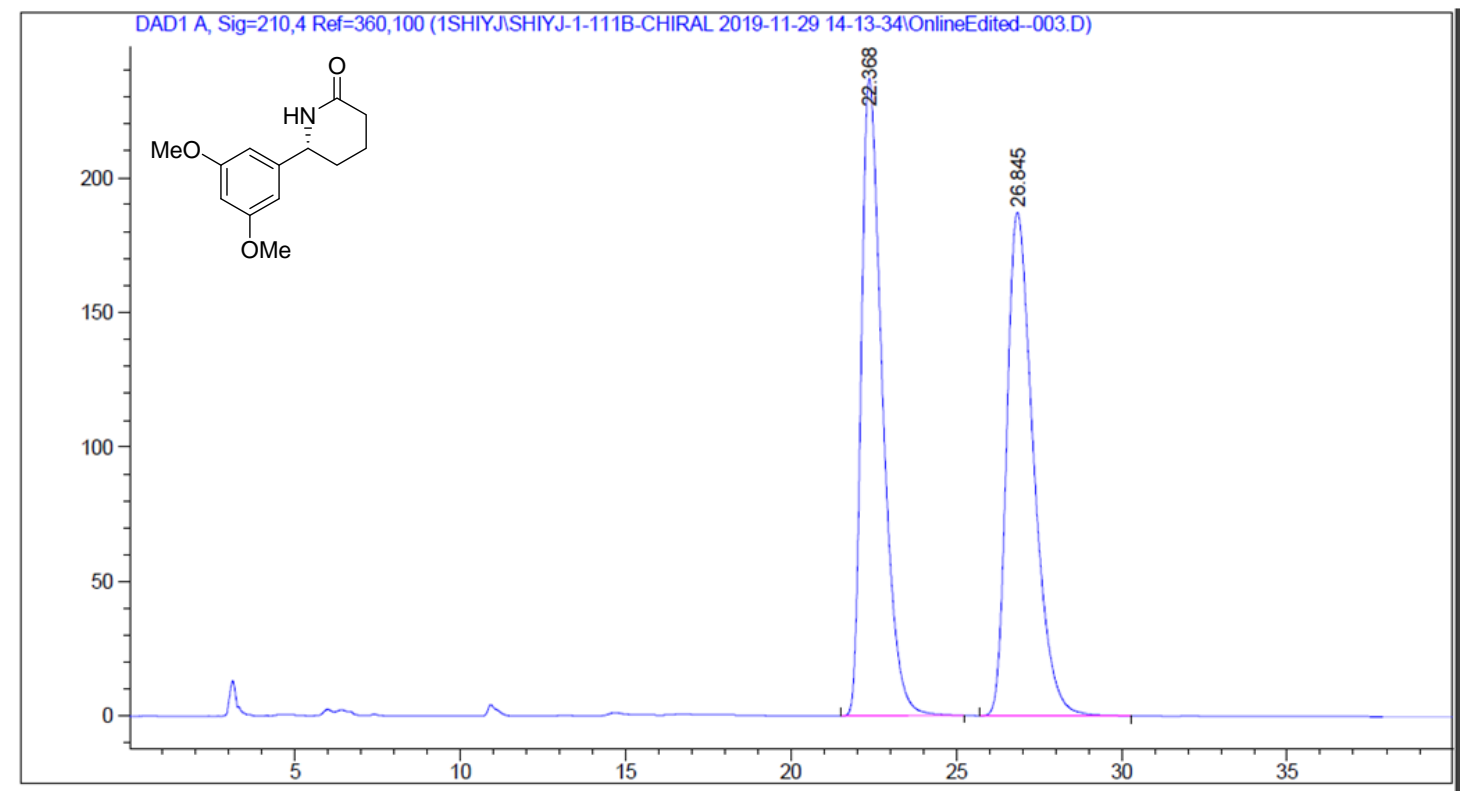

Signal 1: DAD1 A, Sig=210,4 Ref $=360,100$

\begin{tabular}{cccccc}
$\begin{array}{c}\text { Peak RetTime Type } \\
\text { [min] }\end{array}$ & $\begin{array}{c}\text { Width } \\
{[\mathrm{min}]}\end{array}$ & $\begin{array}{c}\text { Area } \\
{[\mathrm{mAU} \text { s }]}\end{array}$ & $\begin{array}{c}\text { Height } \\
{[\mathrm{mAU}]}\end{array}$ & $\begin{array}{c}\text { Area } \\
\%\end{array}$ \\
\hline 1 & 22.368 BB & 0.6718 & $1.04699 \mathrm{e} 4$ & 236.82520 & 50.0295 \\
2 & 26.845 BB & 0.8544 & $1.04575 e 4$ & 187.00970 & 49.9705
\end{tabular}

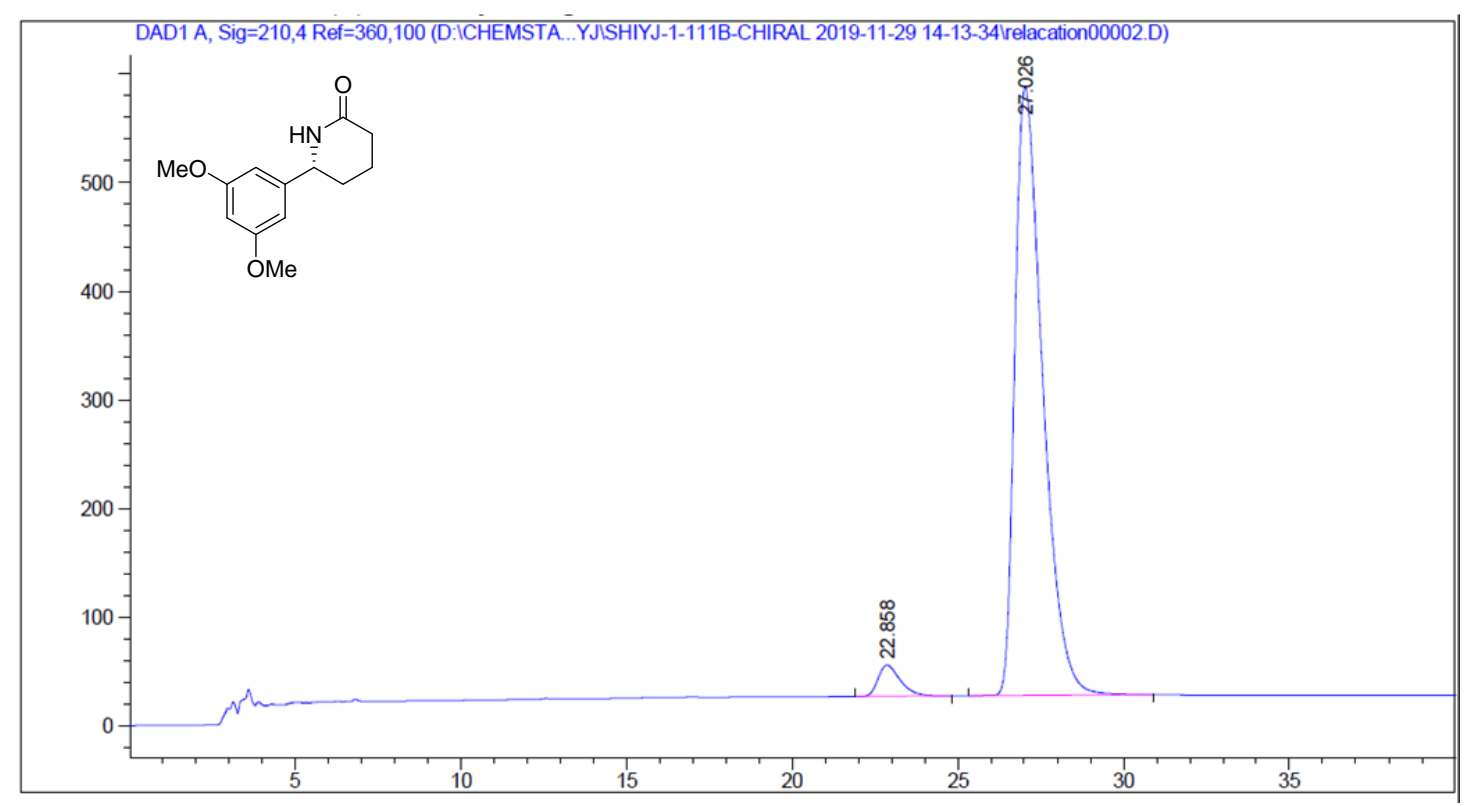

Signal 1: DAD1 A, Sig=210,4 Ref $=360,100$

\begin{tabular}{cccccc}
$\begin{array}{c}\text { Peak RetTime Type } \\
\text { \# } \\
\text { [min] }\end{array}$ & $\begin{array}{c}\text { Width } \\
{[\mathrm{min}]}\end{array}$ & $\begin{array}{c}\text { Area } \\
{[\mathrm{mAU} \text { s }]}\end{array}$ & $\begin{array}{c}\text { Height } \\
{[\mathrm{mAU}]}\end{array}$ & $\begin{array}{c}\text { Area } \\
\%\end{array}$ \\
\hline 1 & 22.858 BB & 0.7025 & 1348.48657 & 28.78746 & 3.9727 \\
2 & 27.026 BB & 0.8916 & $3.25957 e 4$ & 559.56018 & 96.0273
\end{tabular}


(R)-6-(3,4-difluorophenyl)piperidin-2-one (4h)

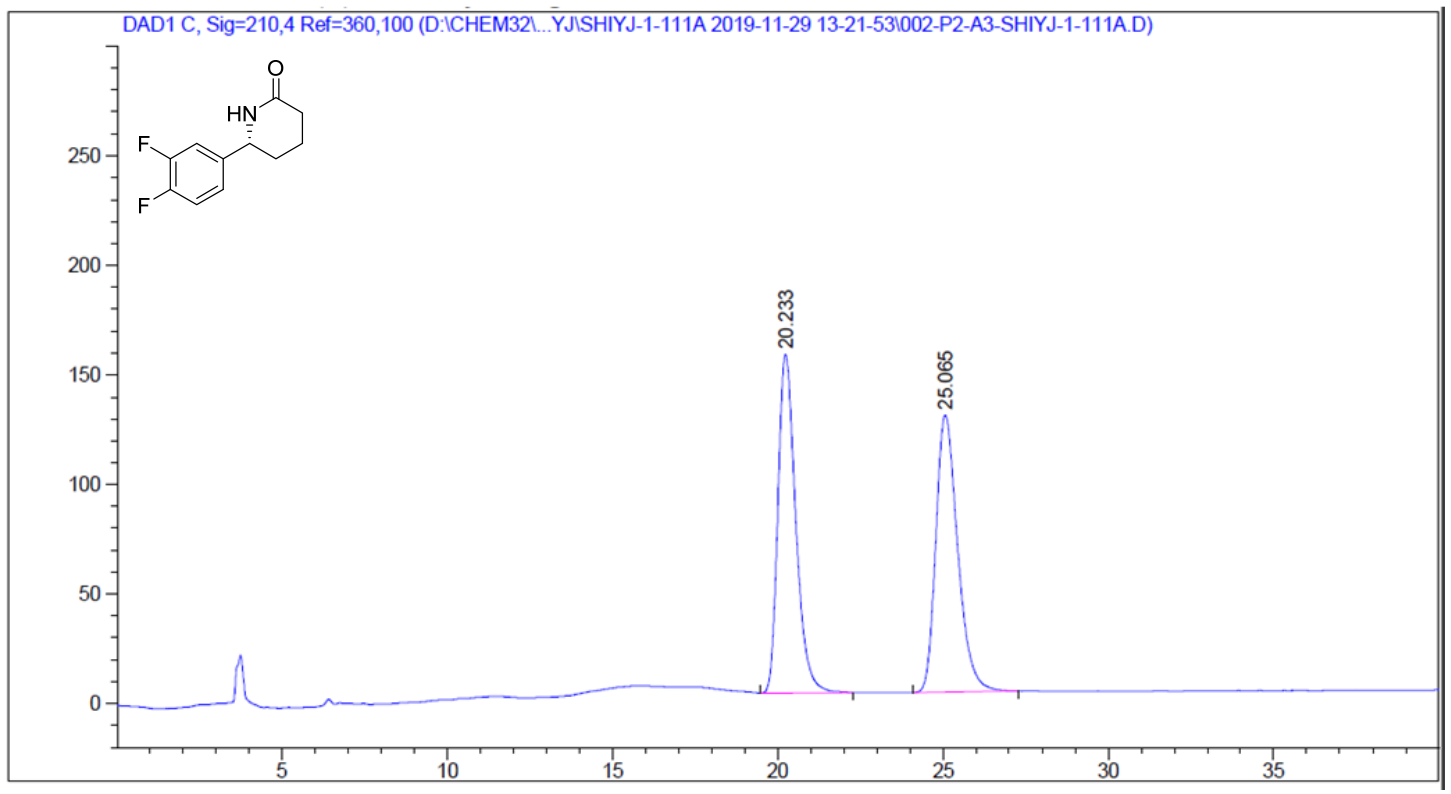

Signal 1: DAD1 C, Sig=210,4 Ref $=360,100$

\begin{tabular}{|c|c|c|c|c|c|c|}
\hline $\begin{array}{c}\text { Peak } \\
\text { \# }\end{array}$ & $\begin{array}{c}\text { RetTime } \\
\text { [min] }\end{array}$ & Type & $\begin{array}{l}\text { Width } \\
\text { [min] }\end{array}$ & $\begin{array}{c}\text { Area } \\
{\left[\mathrm{mAU}^{*} \mathrm{~s}\right]}\end{array}$ & $\begin{array}{l}\text { Height } \\
\text { [mAU] }\end{array}$ & $\begin{array}{c}\text { Area } \\
\%\end{array}$ \\
\hline & & & & & & \\
\hline & & & & 8 & & 39 \\
\hline & & & & 826.65234 & 26.56580 & 50.1761 \\
\hline
\end{tabular}

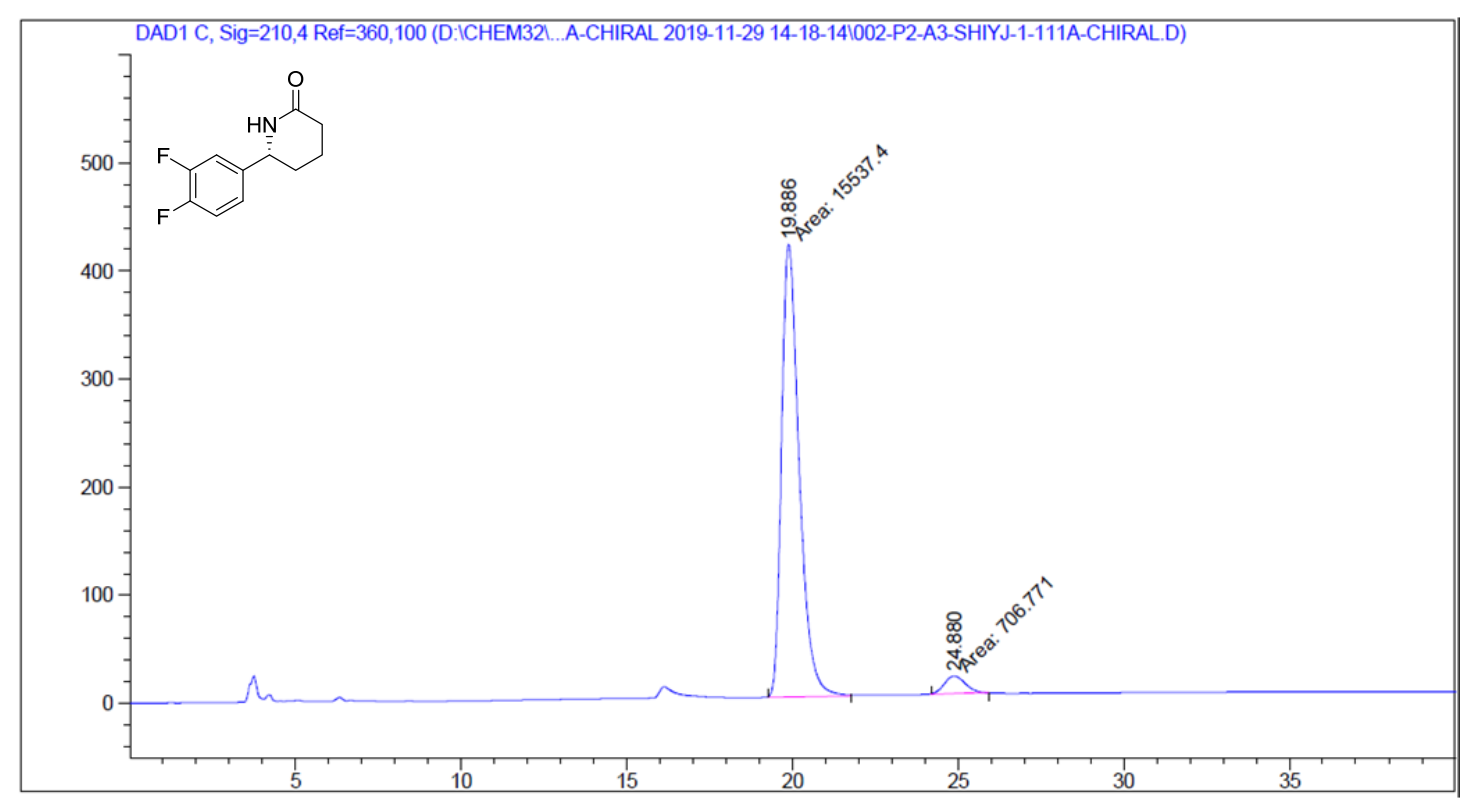

Signal 1: DAD1 C, Sig=210,4 Ref $=360,100$

\begin{tabular}{|c|c|c|c|c|c|c|}
\hline eak & $\begin{array}{c}\text { RetTime } \\
\text { [min] }\end{array}$ & Type & $\begin{array}{l}\text { Width } \\
\text { [min] }\end{array}$ & $\begin{array}{c}\text { Area } \\
{\left[\mathrm{mAU}^{*} \mathrm{~s}\right]}\end{array}$ & $\begin{array}{l}\text { Height } \\
\text { [mAU] }\end{array}$ & $\begin{array}{c}\text { Area } \\
\%\end{array}$ \\
\hline & & & & & & \\
\hline 1 & & & & 1.55 & 47 & 95. \\
\hline 2 & 0 & & 36 & 706.77112 & 27941 & 4.3509 \\
\hline
\end{tabular}




\section{7-phenylazepan-2-one (4i)}

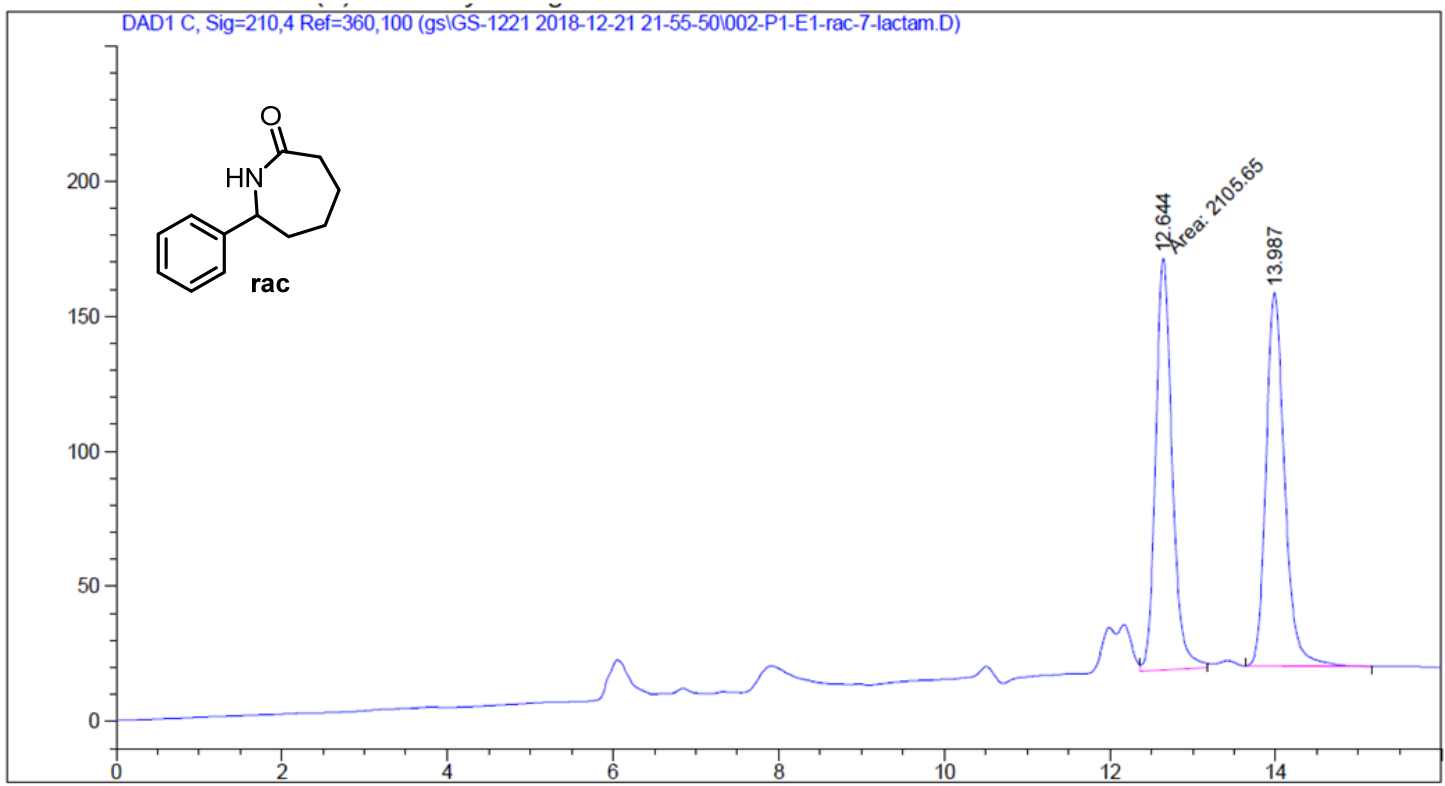

Signal 1: DAD1 C, Sig=210,4 Ref $=360,100$

\begin{tabular}{|c|c|c|c|c|c|c|}
\hline $\begin{array}{c}\text { Peak } \\
\text { \# }\end{array}$ & $\begin{array}{c}\text { RetTime } \\
\text { [min] }\end{array}$ & Type & $\begin{array}{l}\text { Width } \\
\text { [min] }\end{array}$ & $\begin{array}{c}\text { Area } \\
{\left[\mathrm{mAU}^{*} \mathrm{~s}\right]}\end{array}$ & $\begin{array}{l}\text { Height } \\
{[\mathrm{mAU}]}\end{array}$ & $\begin{array}{c}\text { Area } \\
\%\end{array}$ \\
\hline & & & & & & \\
\hline 1 & & & & 105 & 91 & 49 \\
\hline 2 & t & & & 161.34644 & 7665 & 50.6527 \\
\hline
\end{tabular}

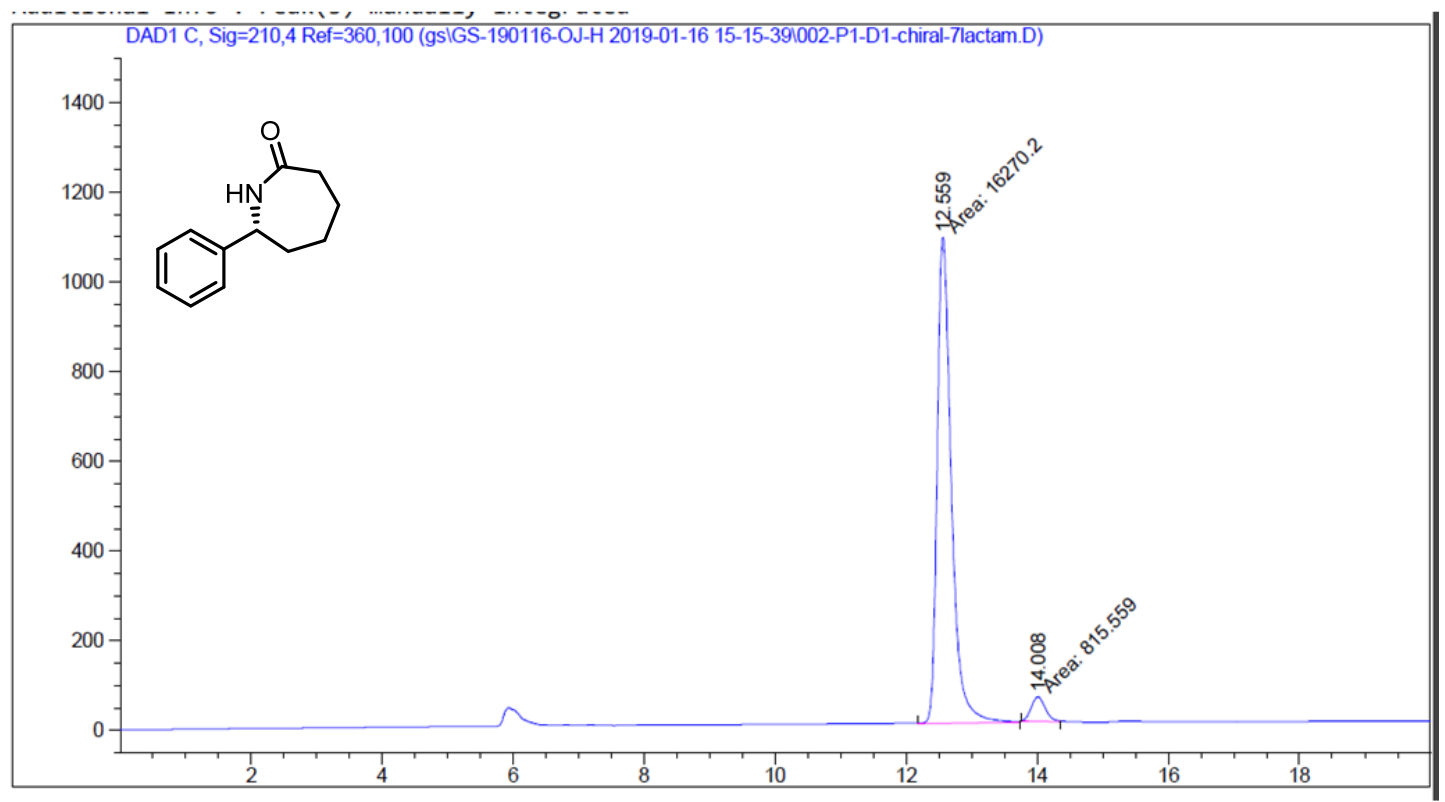

Signal 1: DAD1 C, Sig $=210,4$ Ref $=360,100$

\begin{tabular}{cccccc}
$\begin{array}{c}\text { Peak RetTime Type } \\
\text { [min] }\end{array}$ & $\begin{array}{c}\text { Width } \\
\text { [min] }\end{array}$ & $\begin{array}{c}\text { Area } \\
\text { [mAU*s] }\end{array}$ & $\begin{array}{c}\text { Height } \\
\text { [mAU] }\end{array}$ & $\begin{array}{c}\text { Area } \\
\%\end{array}$ \\
\hline 1 & $12.559 \mathrm{MM}$ & 0.2500 & $1.62702 \mathrm{e} 4$ & 1084.63574 & 95.2267 \\
2 & $14.008 \mathrm{MM}$ & 0.2495 & 815.55859 & 54.47503 & 4.7733
\end{tabular}


(R)-3-methylisoindolin-1-one (6a)

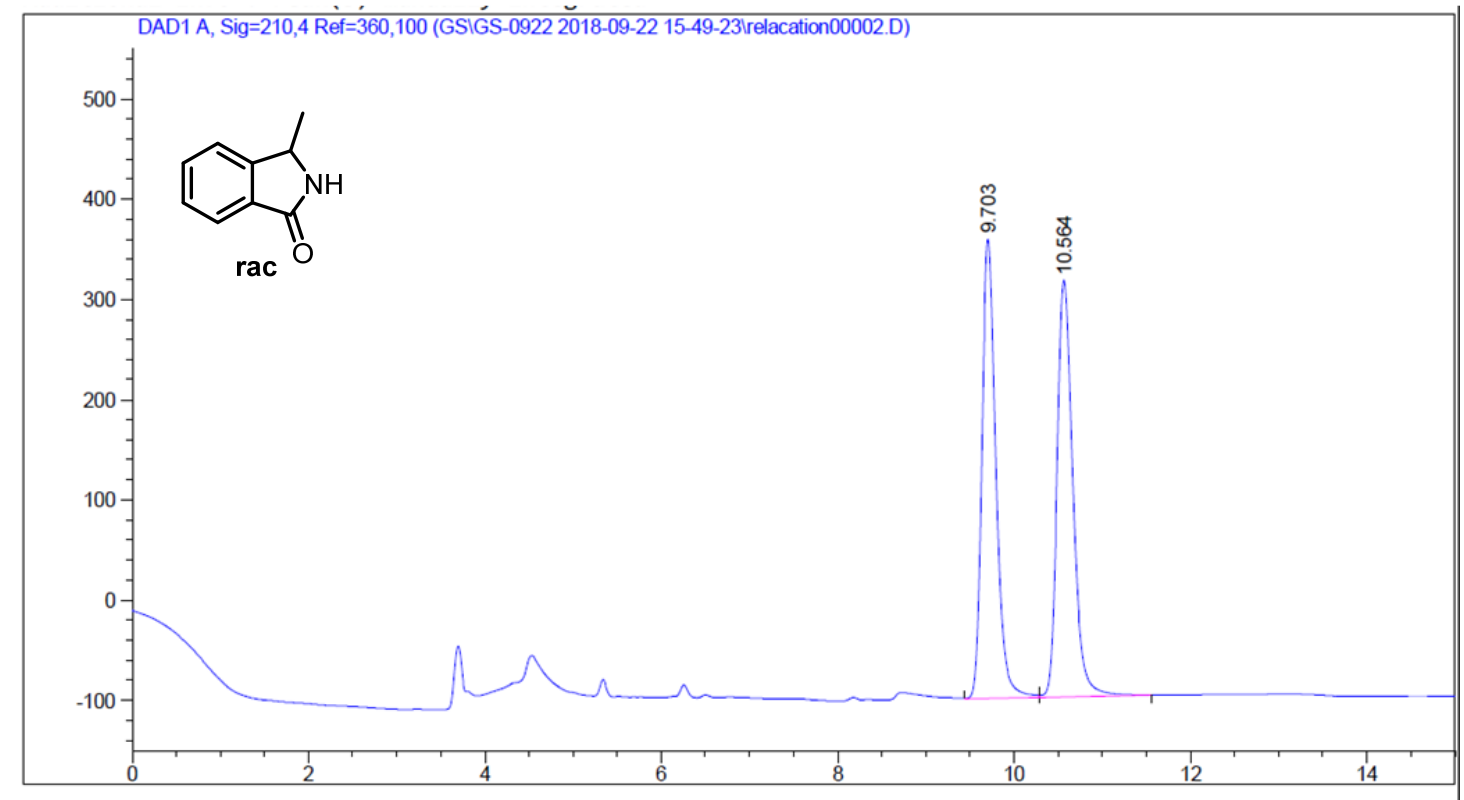

Signal 1: DAD1 A, Sig=210,4 Ref $=360,100$

\begin{tabular}{|c|c|c|c|c|c|c|}
\hline $\begin{array}{l}\text { eak } \\
\#\end{array}$ & $\begin{array}{c}\text { RetTime } \\
\text { [min] }\end{array}$ & Type & $\begin{array}{l}\text { Width } \\
\text { [min] }\end{array}$ & $\begin{array}{c}\text { Area } \\
{\left[\mathrm{mAU}^{*} \mathrm{~s}\right]}\end{array}$ & $\begin{array}{l}\mathrm{He} \\
{[\mathrm{m}}\end{array}$ & $\begin{array}{c}\text { Area } \\
\%\end{array}$ \\
\hline & & & & & & - \\
\hline 1 & & & & 5070. & 245 & 49.6472 \\
\hline & 10.564 & 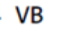 & & 5142.70947 & 415.69778 & 50.3528 \\
\hline
\end{tabular}

Totals : $\quad 1.02133 \mathrm{e} 4 \quad 873.99023$

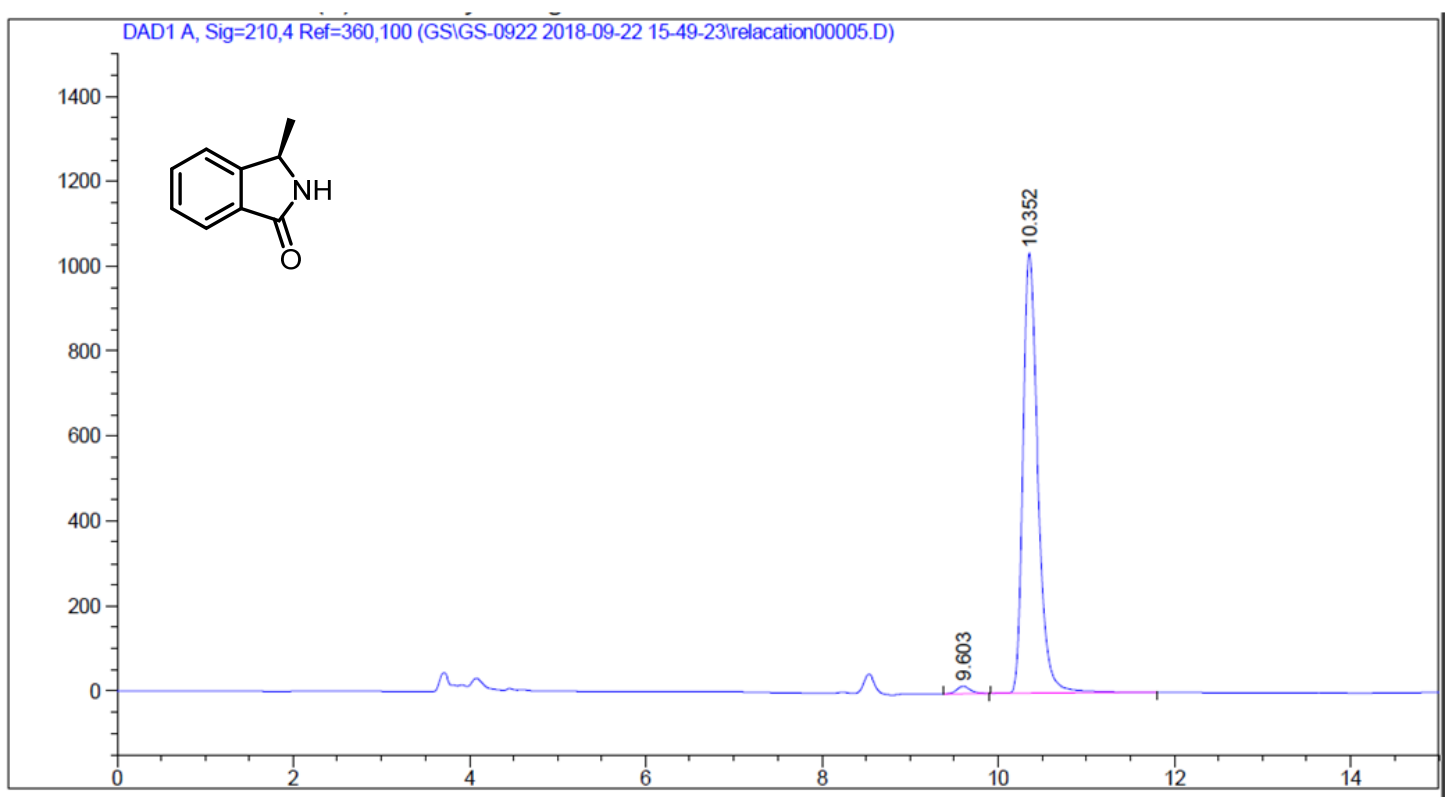

Signal 1: DAD1 A, Sig $=210,4$ Ref $=360,100$

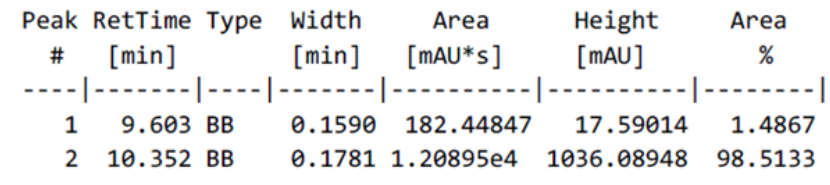

Totals :

$1.22720 \mathrm{e} 4 \quad 1053.67962$ 
(R)-3-ethylisoindolin-1-one (6b)

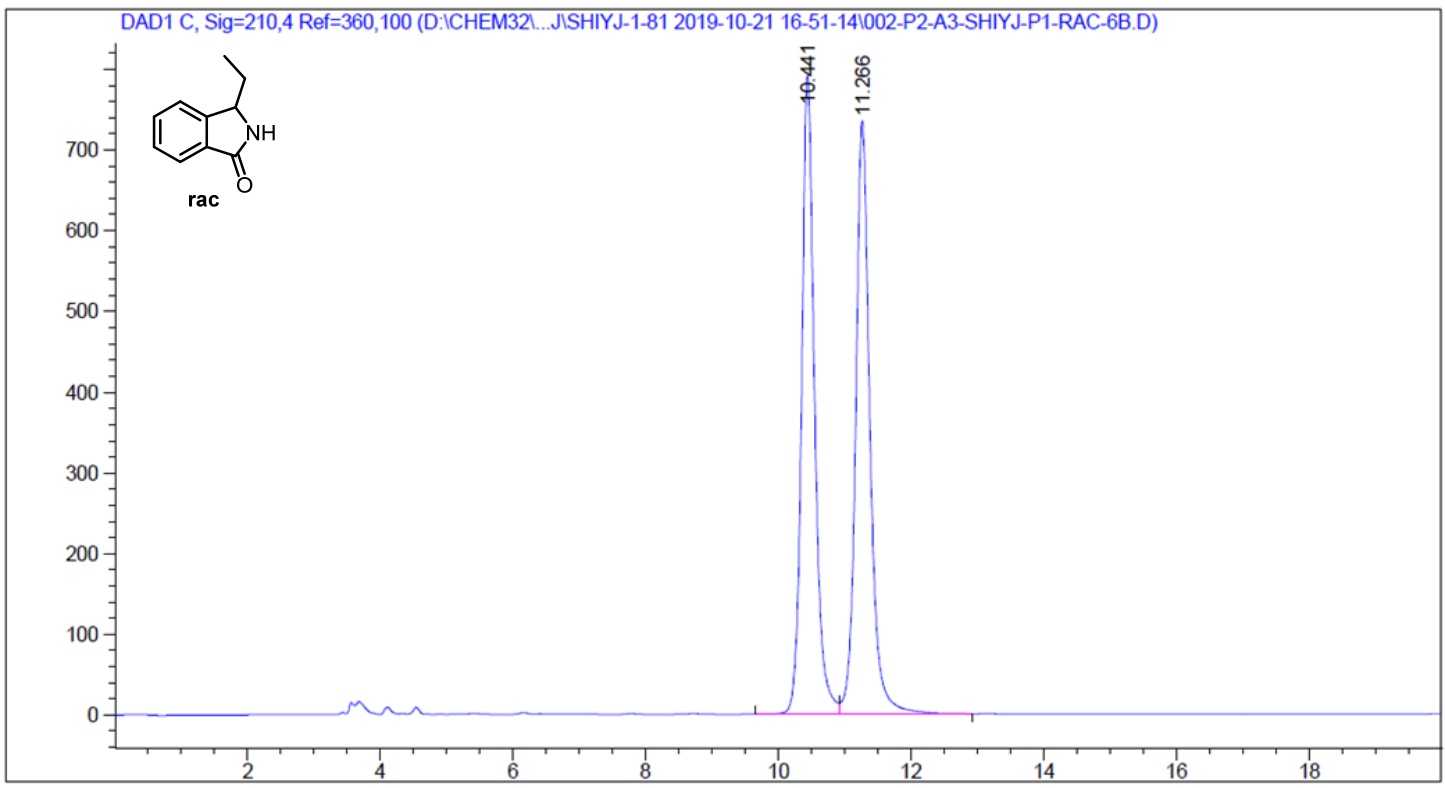

Signal 1: DAD1 C, Sig=210,4 Ref $=360,100$

\begin{tabular}{cccccc}
$\begin{array}{c}\text { Peak RetTime Type } \\
\text { [min] }\end{array}$ & $\begin{array}{c}\text { Width } \\
\text { [min] }\end{array}$ & $\begin{array}{c}\text { Area } \\
\text { [mAU*s] }\end{array}$ & $\begin{array}{c}\text { Height } \\
\text { [mAU] }\end{array}$ & $\begin{array}{c}\text { Area } \\
\%\end{array}$ \\
\hline 1 & 10.441 BV & 0.2016 & $1.06822 \mathrm{e} 4$ & 790.89142 & 49.3118 \\
2 & 11.266 VB & 0.2224 & $1.09804 \mathrm{e} 4$ & 734.57422 & 50.6882
\end{tabular}

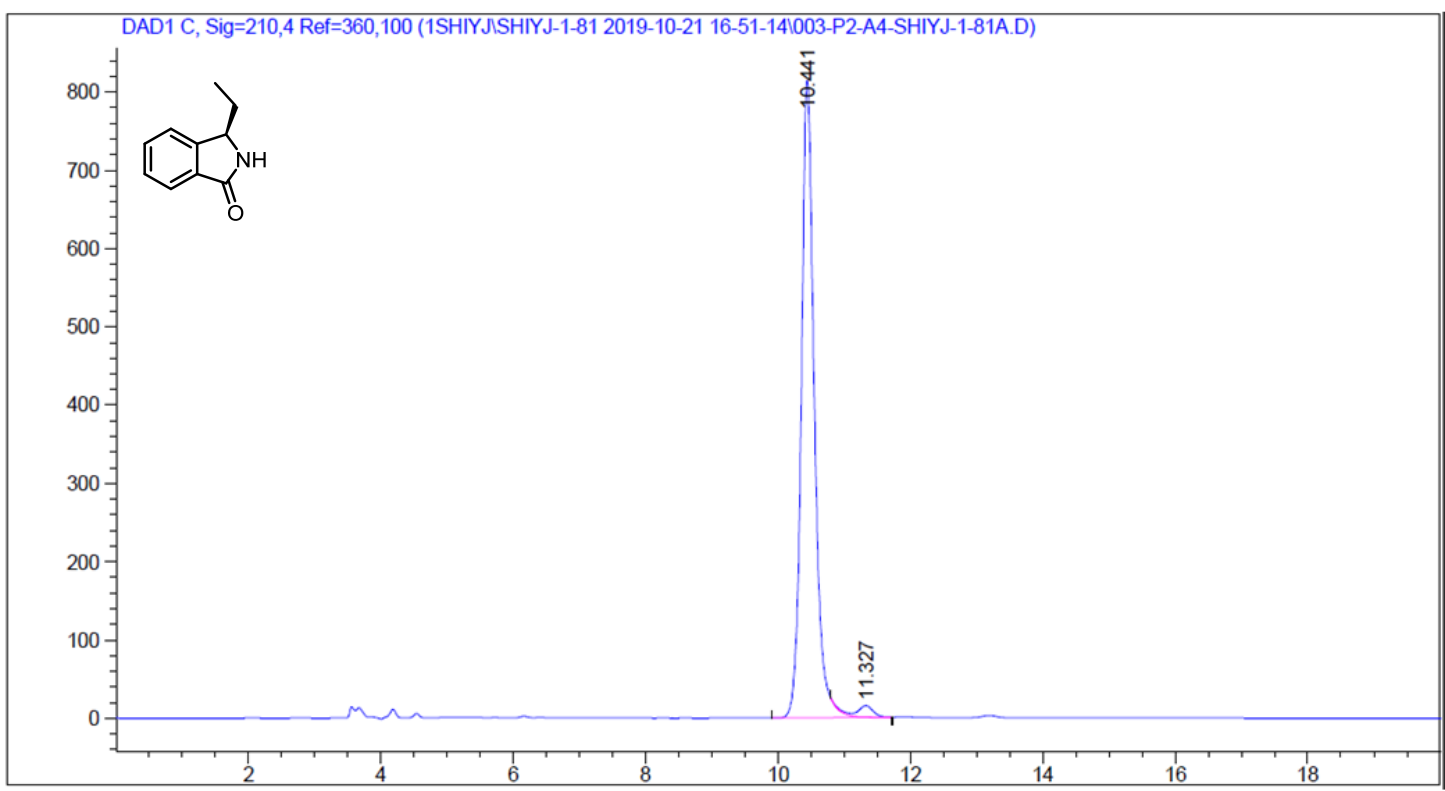

Signal 1: DAD1 C, Sig=210,4 Ref $=360,100$

\begin{tabular}{cccccc}
$\begin{array}{c}\text { Peak RetTime Type } \\
\text { \# } \\
\text { [min] }\end{array}$ & $\begin{array}{c}\text { Width } \\
\text { [min] }\end{array}$ & $\begin{array}{c}\text { Area } \\
{[\mathrm{mAU} \text { s }]}\end{array}$ & $\begin{array}{c}\text { Height } \\
\text { [mAU] }\end{array}$ & $\begin{array}{c}\text { Area } \\
\%\end{array}$ \\
\hline 1 & 10.441 BV R & 0.2056 & 1.11385 e4 & 814.33008 & 97.7905 \\
2 & 11.327 VB E & 0.2501 & 251.66557 & 14.67115 & 2.2095
\end{tabular}


(R)-6-bromo-3-methylisoindolin-1-one (6c)

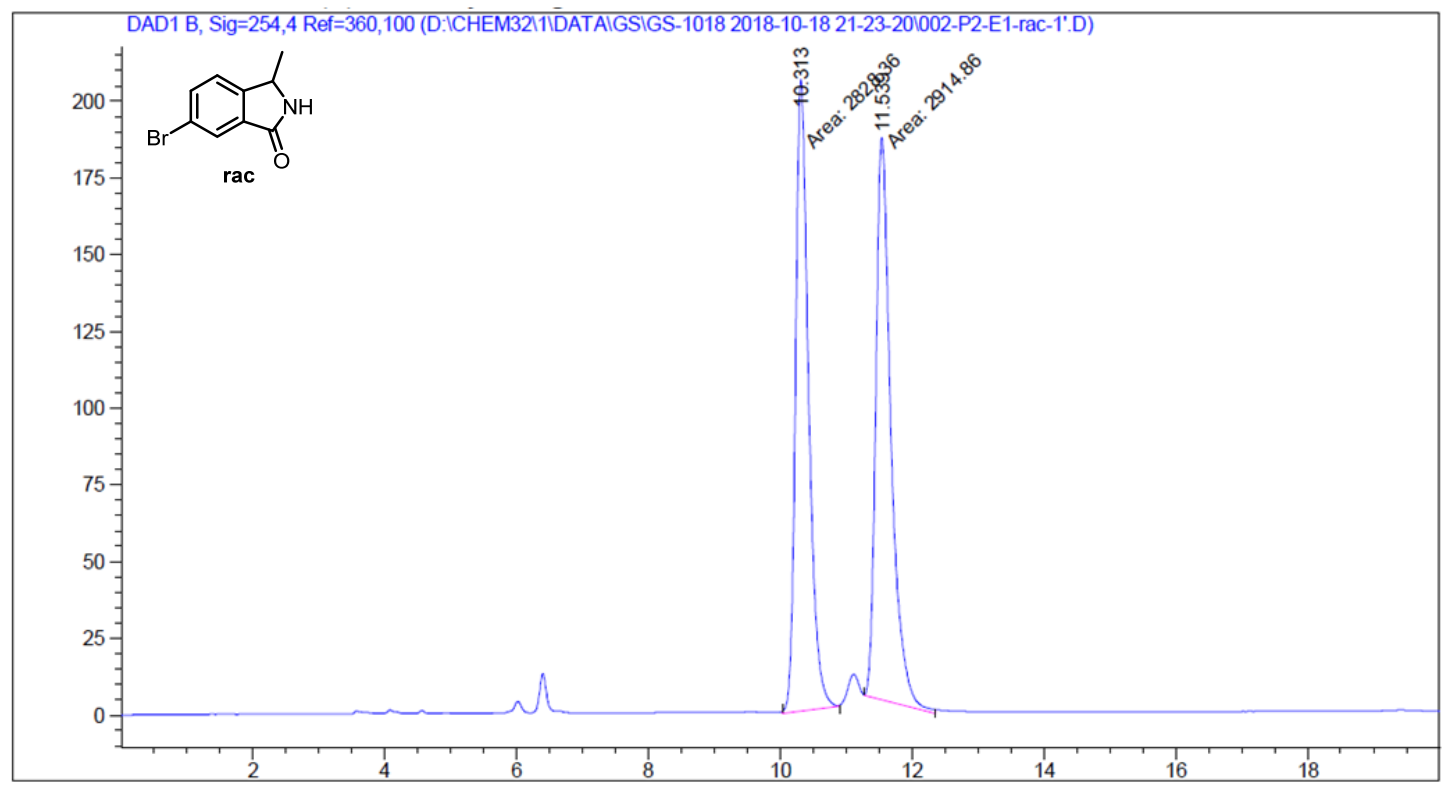

Signal 1: DAD1 B, Sig=254,4 Ref $=360,100$

\begin{tabular}{cccccc}
$\begin{array}{c}\text { Peak RetTime Type } \\
\text { W } \\
\text { [min] }\end{array}$ & $\begin{array}{c}\text { Width } \\
{[\mathrm{min}]}\end{array}$ & $\begin{array}{c}\text { Area } \\
{[\mathrm{mAU} \text { s }]}\end{array}$ & $\begin{array}{c}\text { Height } \\
{[\mathrm{mAU}]}\end{array}$ & $\begin{array}{c}\text { Area } \\
\%\end{array}$ \\
\hline $\begin{array}{c}1 \\
2\end{array}$ & $11.539 \mathrm{MM}$ & 0.2652 & 2914.86426 & 183.19814 & 50.7531
\end{tabular}

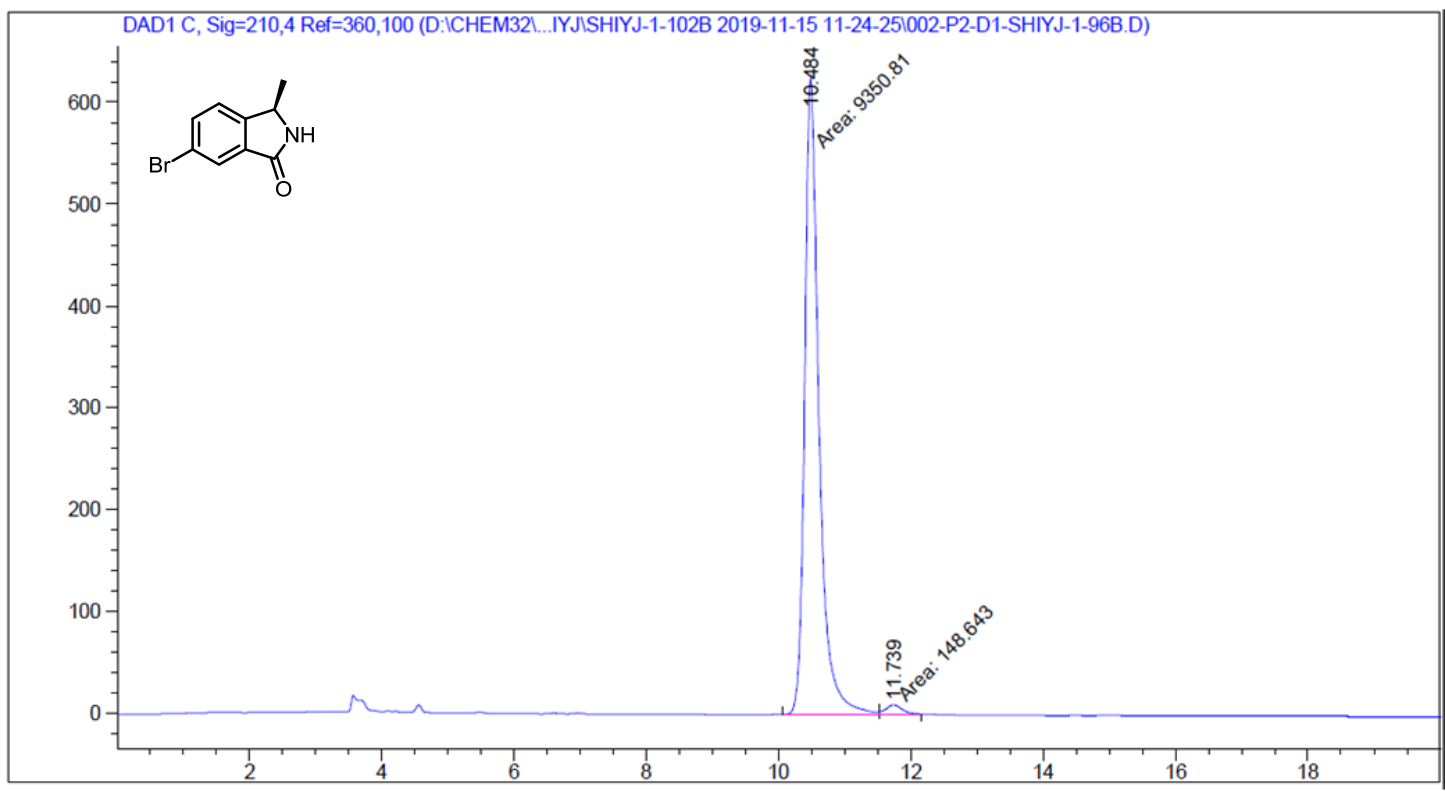

Signal 1: DAD1 C, Sig $=210,4$ Ref $=360,100$

\begin{tabular}{cccccc}
$\begin{array}{c}\text { Peak RetTime Type } \\
\text { Width } \\
\text { [min] }\end{array}$ & $\begin{array}{c}\text { Area } \\
{[\mathrm{min}]}\end{array}$ & $\begin{array}{c}\text { Height } \\
{[\mathrm{mAU} \text { s }]}\end{array}$ & $\begin{array}{c}\text { Area } \\
{[\mathrm{mAU}]}\end{array}$ & $\begin{array}{c}\% \\
1\end{array}$ \\
\hline & 10.484 MM & 0.2496 & 9350.80762 & 624.34955 & 98.4352 \\
2 & 11.739 MM & 0.2774 & 148.64319 & 8.93089 & 1.5648
\end{tabular}


(R)-5-methoxy-3-methylisoindolin-1-one (6d)

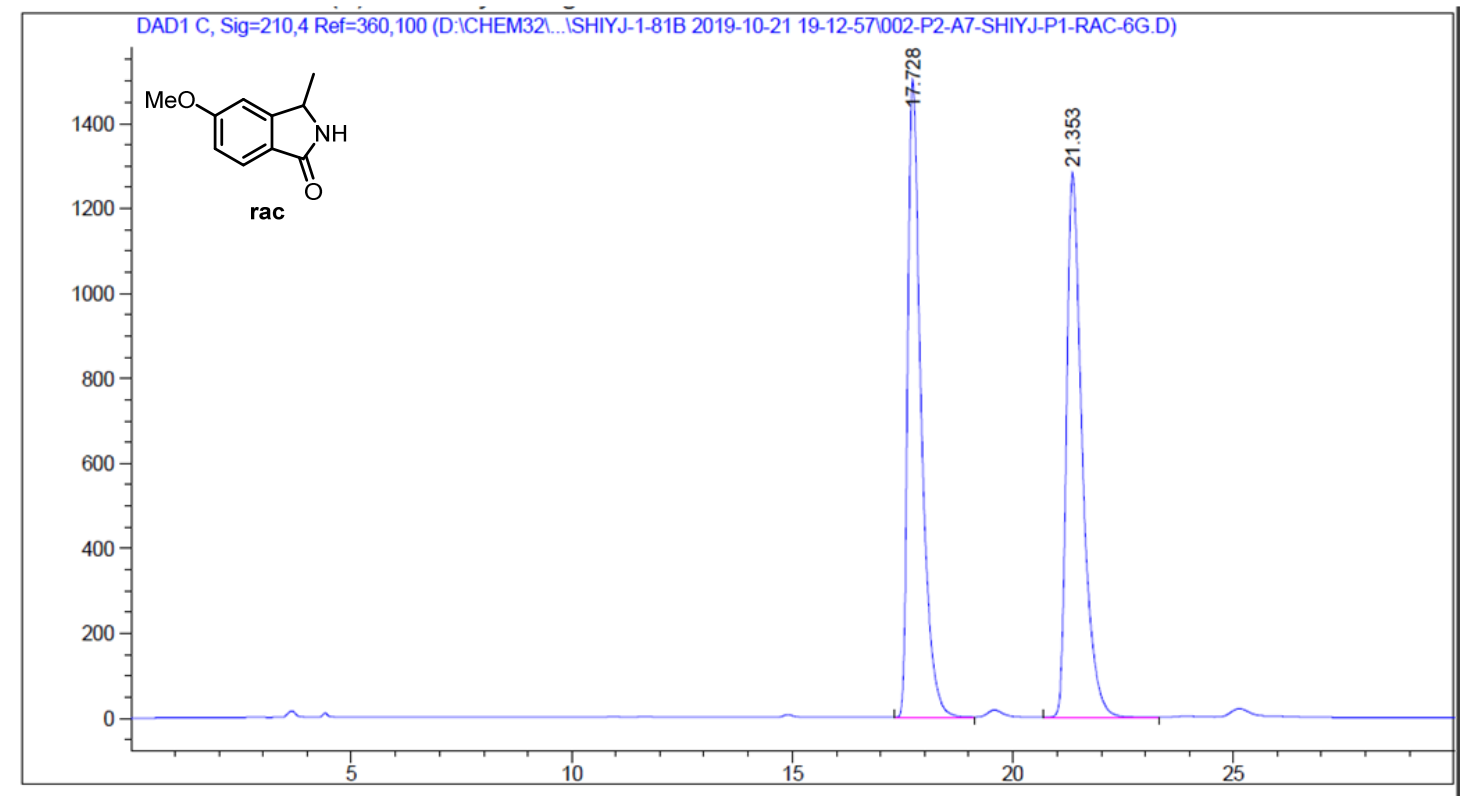

Signal 1: DAD1 C, Sig=210,4 Ref $=360,100$

\begin{tabular}{cccccc}
$\begin{array}{c}\text { Peak RetTime Type } \\
\text { \# } \\
{[\text { min] }}\end{array}$ & $\begin{array}{c}\text { Width } \\
{[\mathrm{min}]}\end{array}$ & $\begin{array}{c}\text { Area } \\
{[\mathrm{mAU} \text { s }]}\end{array}$ & $\begin{array}{c}\text { Height } \\
{[\mathrm{mAU}]}\end{array}$ & $\begin{array}{c}\text { Area } \\
\%\end{array}$ \\
\hline 1 & 17.728 BB & 0.3161 & $3.16365 \mathrm{e} 4$ & 1499.80432 & 49.9351 \\
2 & 21.353 BB & 0.3728 & $3.17188 \mathrm{e} 4$ & 1281.40247 & 50.0649
\end{tabular}

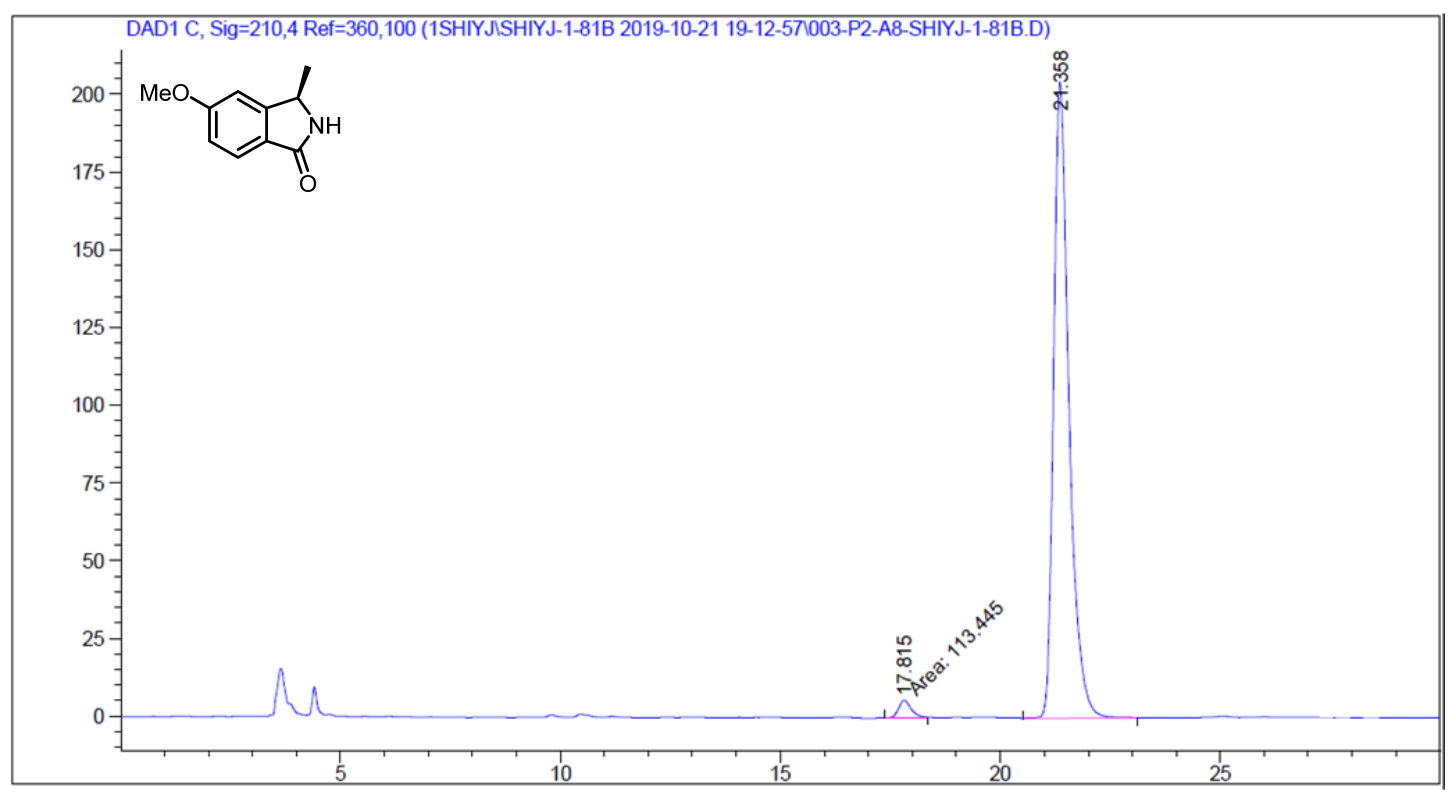

Signal 1: DAD1 C, Sig=210,4 Ref $=360,100$

\begin{tabular}{cccccc}
$\begin{array}{c}\text { Peak RetTime Type } \\
\text { W } \\
\text { [min] }\end{array}$ & $\begin{array}{c}\text { Width } \\
{[\mathrm{min}]}\end{array}$ & $\begin{array}{c}\text { Area } \\
\text { [mAU*s] }\end{array}$ & $\begin{array}{c}\text { Height } \\
{[\mathrm{mAU}]}\end{array}$ & $\begin{array}{c}\text { Area } \\
\%\end{array}$ \\
\hline 1 & 17.815 MM & 0.3356 & 113.44519 & 5.63420 & 2.2347 \\
2 & 21.358 BB & 0.3631 & 4963.10889 & 204.51950 & 97.7653
\end{tabular}


(R)-3,6-dimethylisoindolin-1-one (6e)

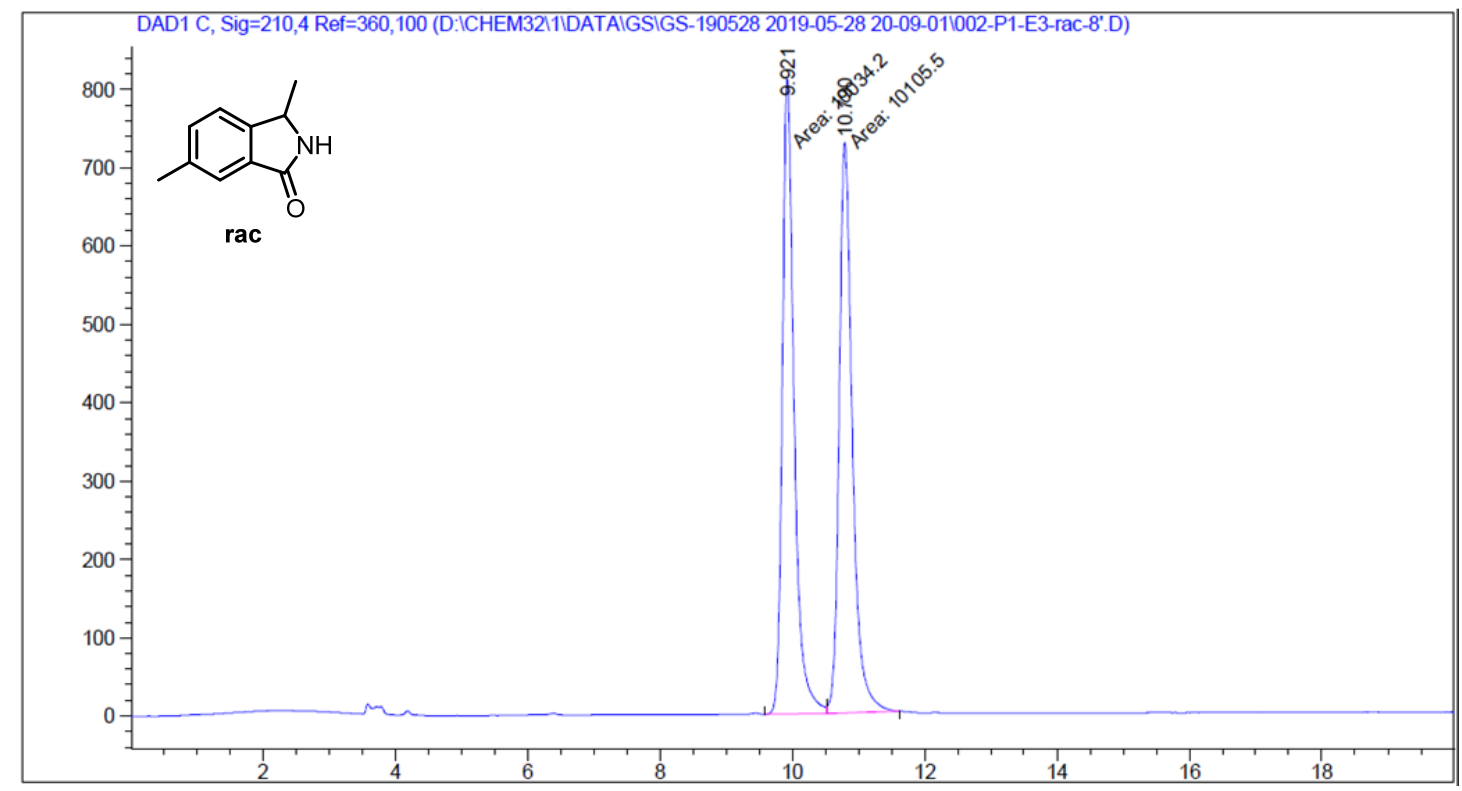

Signal 1: DAD1 C, Sig $=210,4$ Ref $=360,100$

\begin{tabular}{cccccc}
$\begin{array}{c}\text { Peak RetTime Type } \\
\text { Width } \\
\text { [min] }\end{array}$ & $\begin{array}{c}\text { Area } \\
{[\mathrm{min}]}\end{array}$ & $\begin{array}{c}\text { Height } \\
{[\mathrm{mAU} \text { s }]}\end{array}$ & $\begin{array}{c}\text { Area } \\
{[\mathrm{mAU}]}\end{array}$ & $\%$ \\
\hline 1 & $9.921 \mathrm{MM}$ & 0.2061 & $1.00342 \mathrm{e} 4$ & 811.38324 & 49.8232 \\
2 & $10.790 \mathrm{MM}$ & 0.2312 & $1.01055 \mathrm{e} 4$ & 728.55713 & 50.1768
\end{tabular}

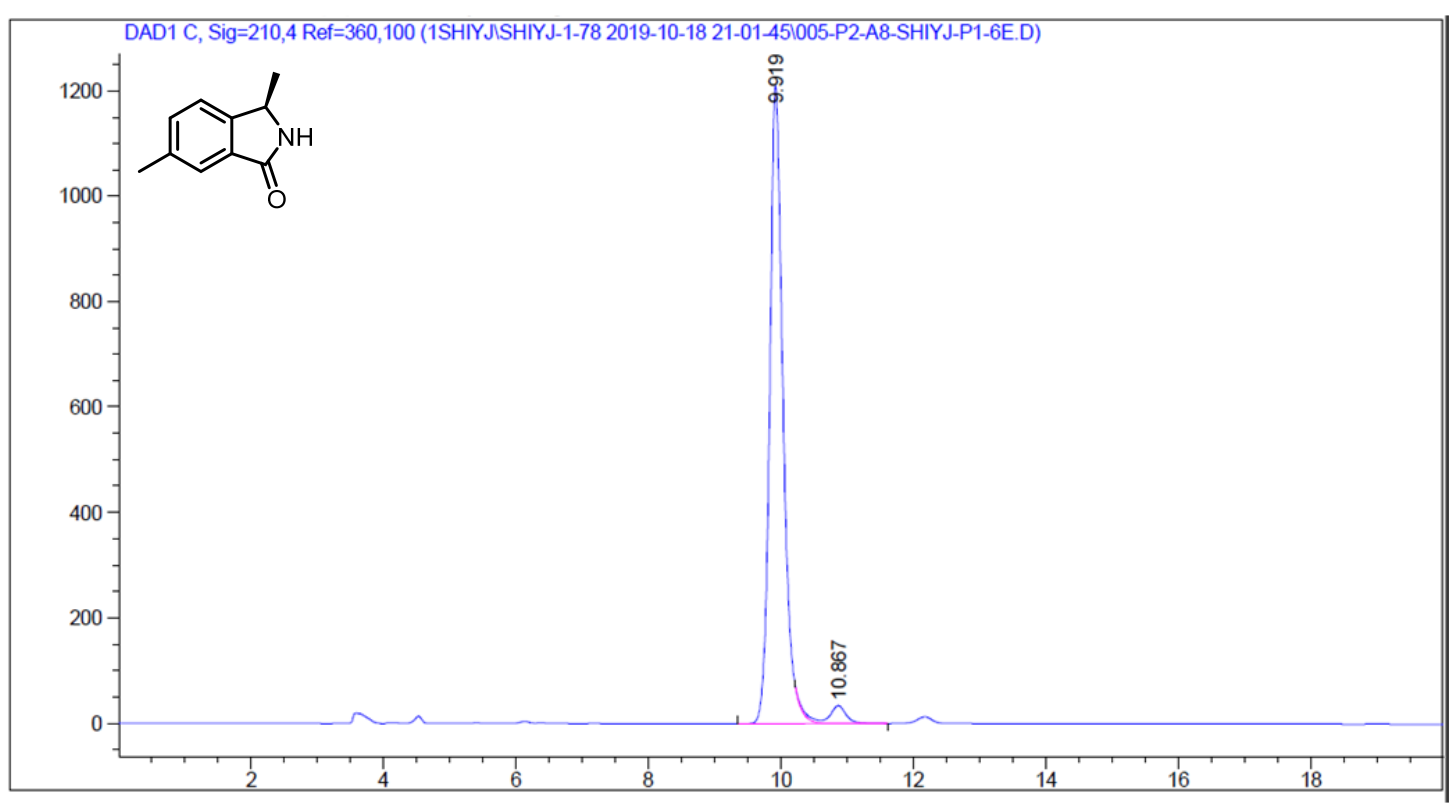

Signal 1: DAD1 C, Sig=210,4 Ref $=360,100$

\begin{tabular}{|c|c|c|c|c|c|c|}
\hline eak & $\begin{array}{c}\text { RetTime } \\
\text { [min] }\end{array}$ & Type & $\begin{array}{l}\text { Width } \\
\text { [min] }\end{array}$ & $\begin{array}{c}\text { Area } \\
{\left[\mathrm{mAU}^{*} \mathrm{~s}\right]}\end{array}$ & $\begin{array}{l}\text { Height } \\
{[\mathrm{mAU}]}\end{array}$ & $\begin{array}{c}\text { Area } \\
\%\end{array}$ \\
\hline & & & & & & \\
\hline 1 & & V & & 1.70 & 120 & 96. \\
\hline 2 & 198 & 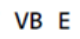 & & 678.50201 & 50324 & 3.8284 \\
\hline
\end{tabular}


(R)-3,5-dimethylisoindolin-1-one (6f)

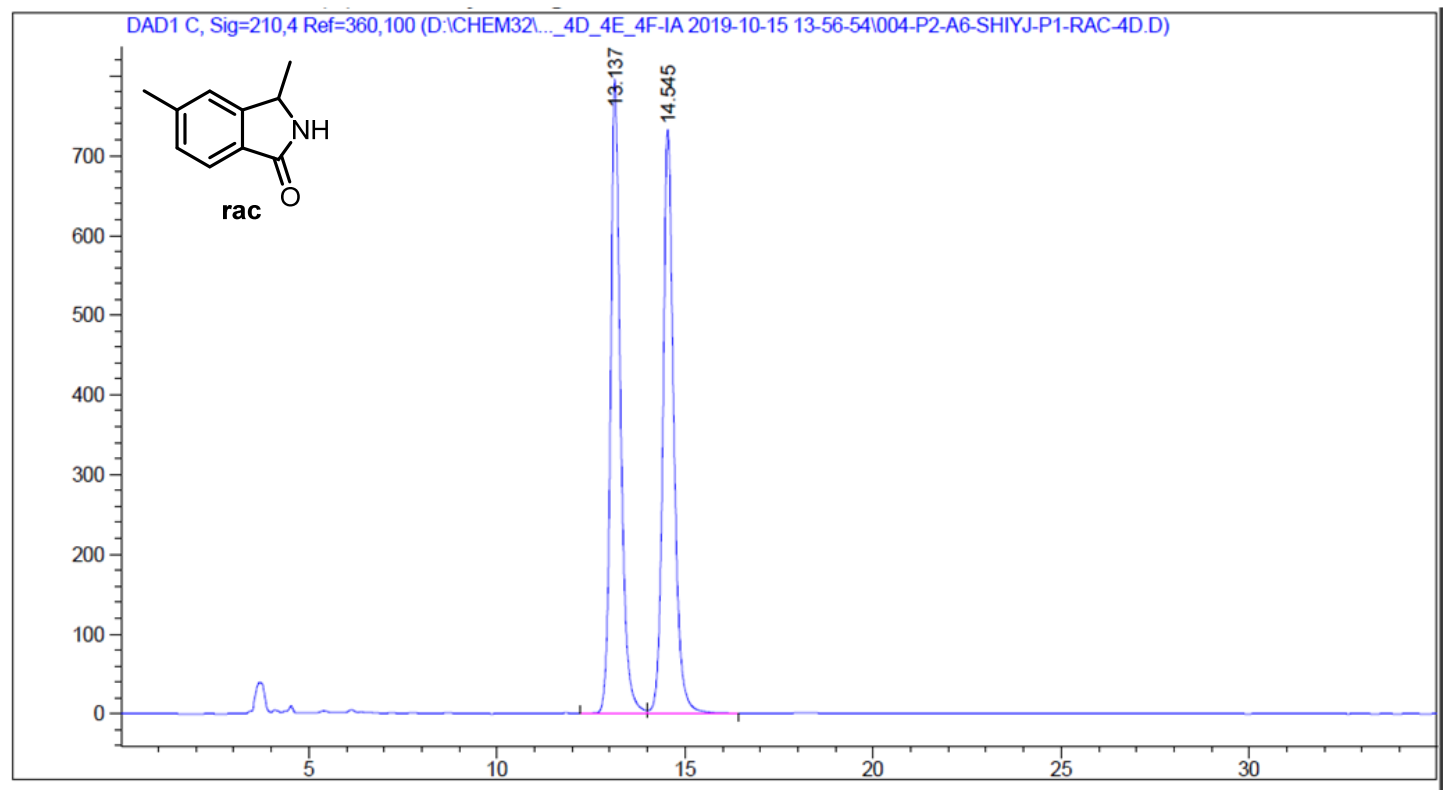

Signal 1: DAD1 C, Sig=210,4 Ref $=360,100$

\begin{tabular}{cccccc}
$\begin{array}{c}\text { Peak RetTime Type } \\
\text { Width } \\
\text { [min] }\end{array}$ & $\begin{array}{c}\text { Area } \\
{[\mathrm{min}]}\end{array}$ & $\begin{array}{c}\text { Height } \\
{[\mathrm{mAU} \text { s }]}\end{array}$ & $\begin{array}{c}\text { Area } \\
{[\mathrm{mAU}]}\end{array}$ & $\begin{array}{c}\% \\
1\end{array}$ \\
\hline 2 & 13.137 BV & 0.2730 & $1.46834 \mathrm{e} 4$ & 796.67596 & 49.8430 \\
2 & 14.545 VB & 0.2992 & $1.47759 \mathrm{e} 4$ & 732.86377 & 50.1570
\end{tabular}

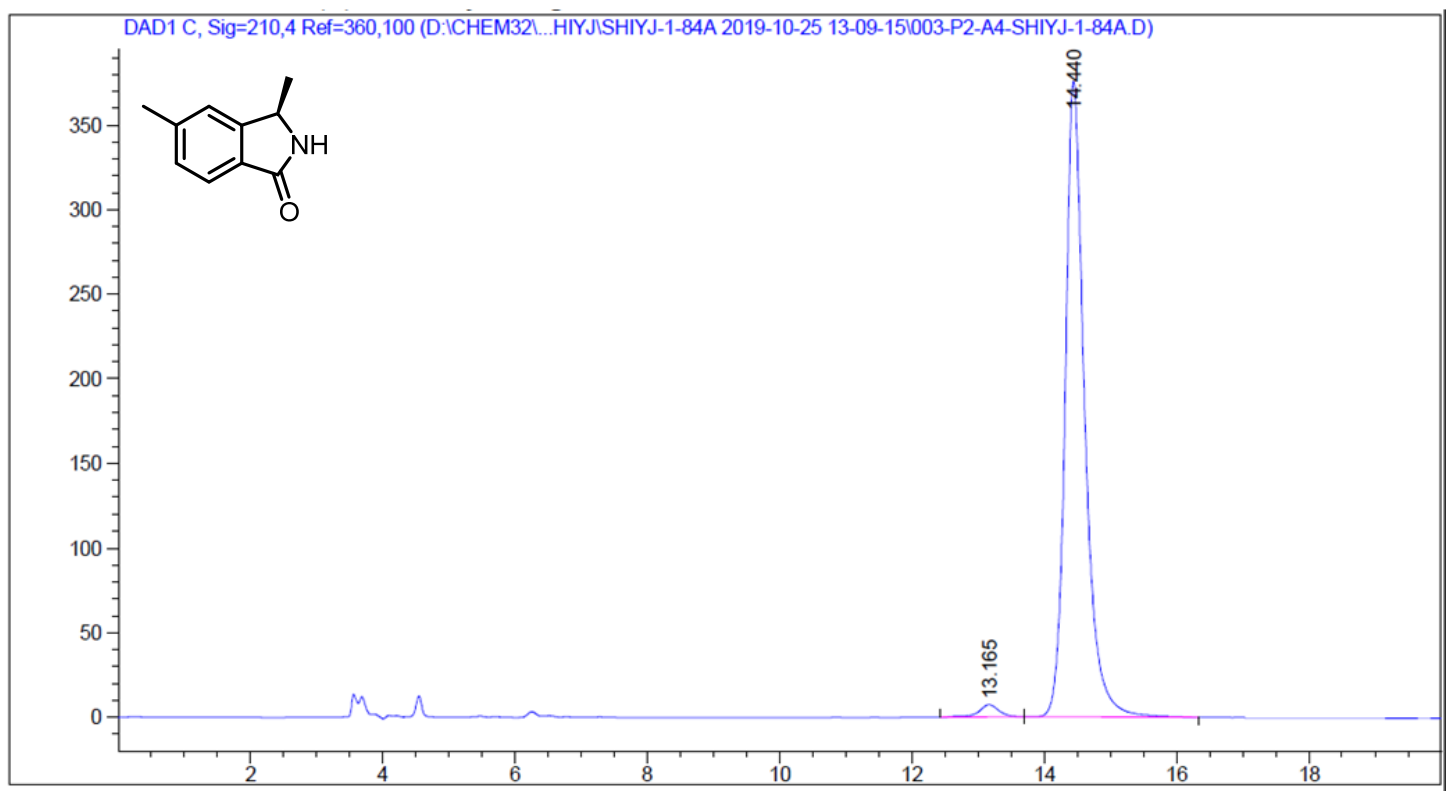

Signal 1: DAD1 C, Sig $=210,4$ Ref $=360,100$

\begin{tabular}{cccccc}
$\begin{array}{c}\text { Peak RetTime Type } \\
\text { Width } \\
\text { [min] }\end{array}$ & $\begin{array}{c}\text { Area } \\
{[\mathrm{min}]}\end{array}$ & $\begin{array}{c}\text { Height } \\
{[\mathrm{mAU} \text { s }]}\end{array}$ & $\begin{array}{c}\text { Area } \\
{[\mathrm{mAU}]}\end{array}$ & $\%$ \\
\hline 1 & 13.165 BB & 0.3179 & 159.08031 & 7.31106 & 2.0285 \\
2 & 14.440 BB & 0.3024 & 7683.27051 & 376.04767 & 97.9715
\end{tabular}


(R)-5-methyl-5,6-dihydro-7H-pyrrolo[3,4-b]pyridin-7-one (6g)

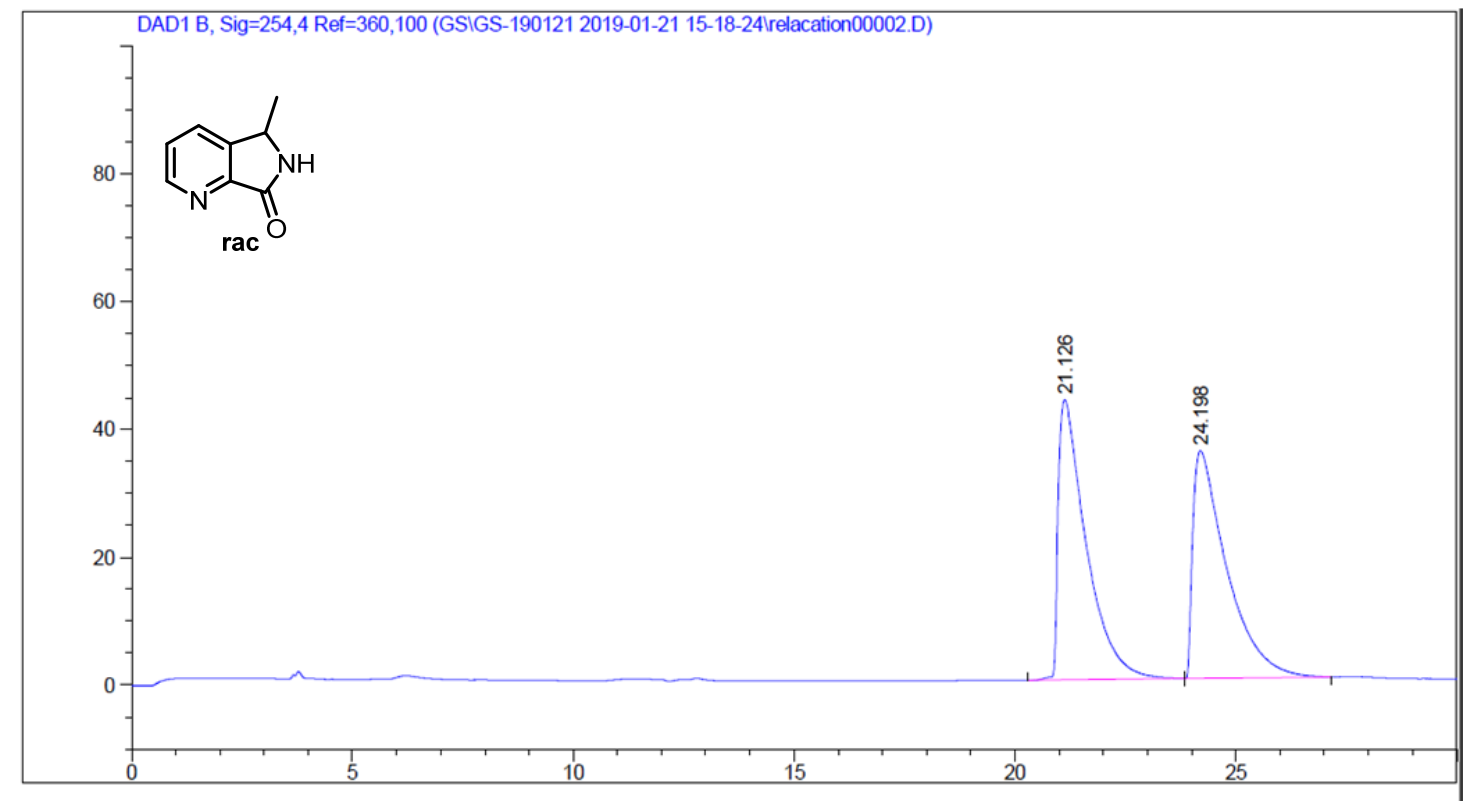

Signal 1: DAD1 B, Sig=254, 4 Ref $=360,100$

\begin{tabular}{cccccc}
$\begin{array}{c}\text { Peak RetTime Type } \\
\text { Width } \\
\text { [min] }\end{array}$ & $\begin{array}{c}\text { Area } \\
\text { [min] }\end{array}$ & $\begin{array}{c}\text { Height } \\
\text { [mAU*s] }\end{array}$ & $\begin{array}{c}\text { Area } \\
\text { [mAU] }\end{array}$ & $\begin{array}{c}\text { \% } \\
1\end{array}$ \\
\hline 2 & 21.126 BB & 0.6227 & 1927.79968 & 43.86827 & 50.4012 \\
24.198 & BB & 0.7549 & 1897.10742 & 35.64874 & 49.5988
\end{tabular}

Totals :

$3824.90710 \quad 79.51701$

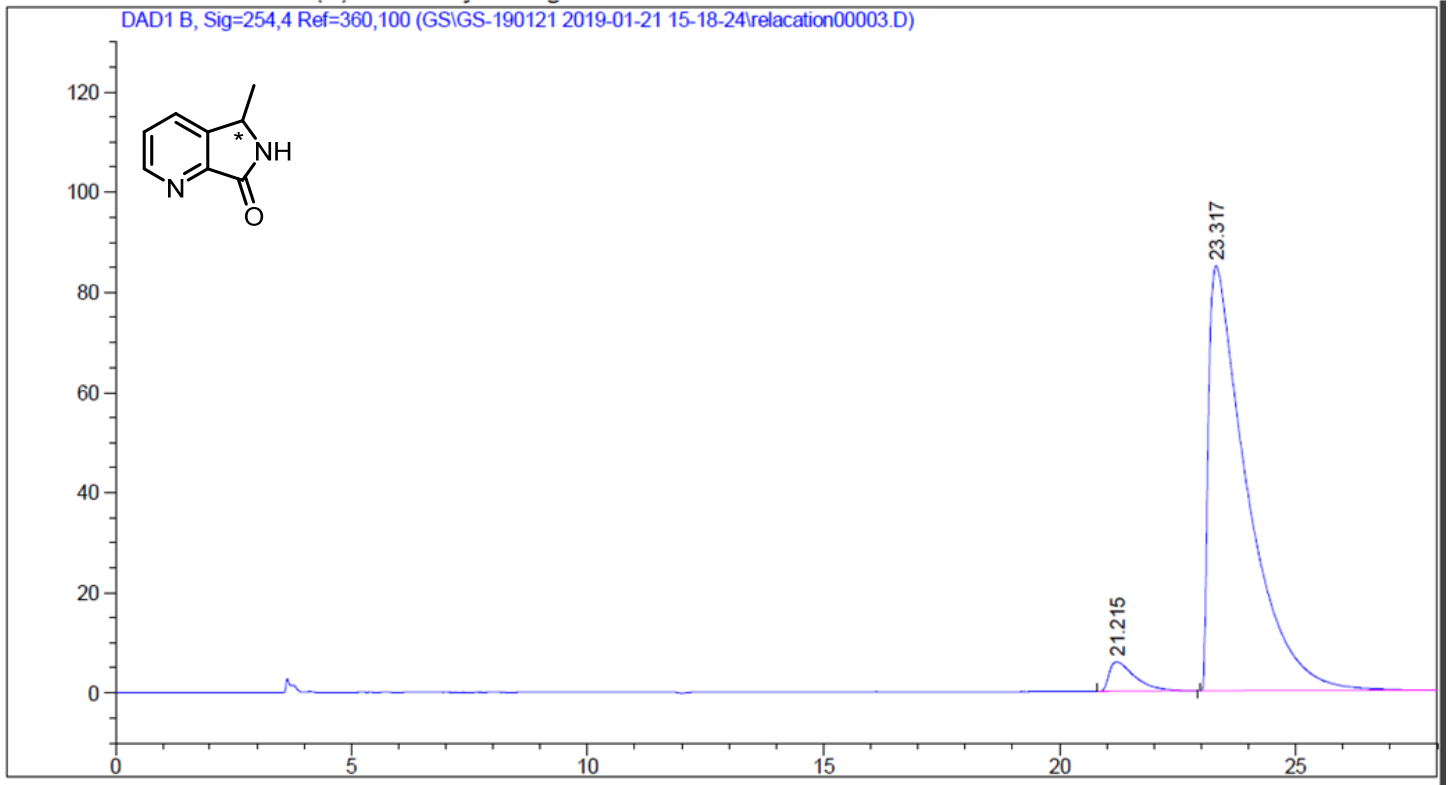

Signal 1: DAD1 B, Sig $=254,4$ Ref $=360,100$

\begin{tabular}{|c|c|c|c|c|c|c|}
\hline $\begin{array}{c}\text { eak } \\
\#\end{array}$ & $\begin{array}{c}\text { RetTime } \\
\text { [min] }\end{array}$ & Type & $\begin{array}{l}\text { Width } \\
\text { [min] }\end{array}$ & $\begin{array}{c}\text { Area } \\
{\left[\mathrm{mAU}^{*} \mathrm{~s}\right]}\end{array}$ & $\begin{array}{l}\text { Height } \\
\text { [mAU] }\end{array}$ & $\begin{array}{c}\text { Area } \\
\%\end{array}$ \\
\hline & & & & & & \\
\hline 1 & & & & 224 & & 507 \\
\hline 2 & 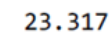 & & & 4820.53076 & 84.91447 & 95.5493 \\
\hline
\end{tabular}

Totals :

5045.0726290 .79942 
(R)-5-methyl-5,6-dihydro-7H-pyrrolo[3,4-b]pyridin-7-one (6g)

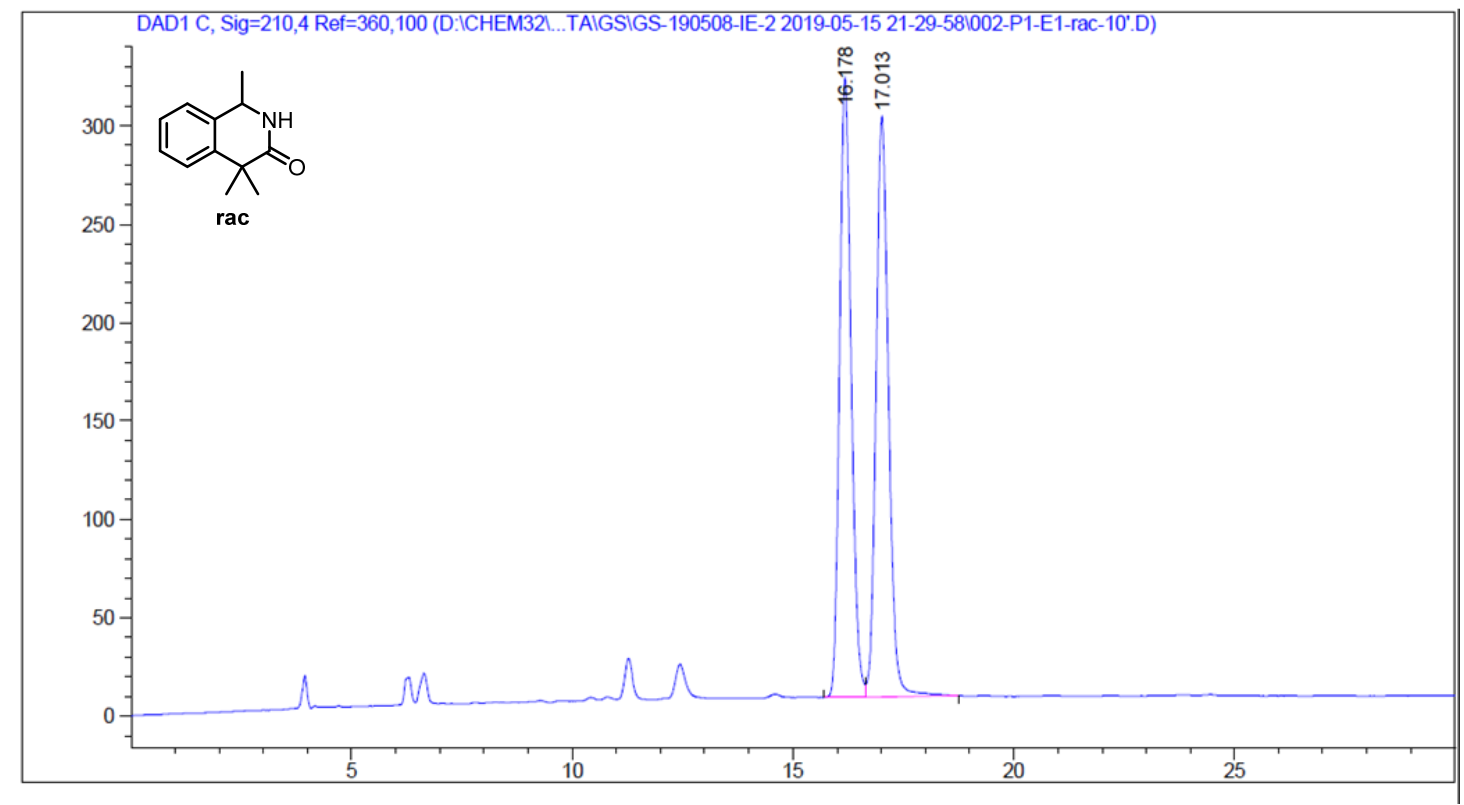

Signal 1: DAD1 C, Sig=210,4 Ref $=360,100$

\begin{tabular}{cccccc}
$\begin{array}{c}\text { Peak RetTime Type } \\
\text { Width } \\
\text { [min] }\end{array}$ & $\begin{array}{c}\text { Area } \\
{[\mathrm{min}]}\end{array}\left[\begin{array}{c}\text { Height } \\
{[\mathrm{mAU} \text { s }]}\end{array}\right.$ & $\begin{array}{c}\text { Area } \\
{[\mathrm{mAU}]}\end{array}$ & $\begin{array}{c}\% \\
1\end{array}$ \\
\hline 1 & 16.178 BV & 0.2854 & 5756.21289 & 314.72772 & 49.7236 \\
2 & 17.013 VB & 0.3022 & 5820.21680 & 294.94183 & 50.2764
\end{tabular}

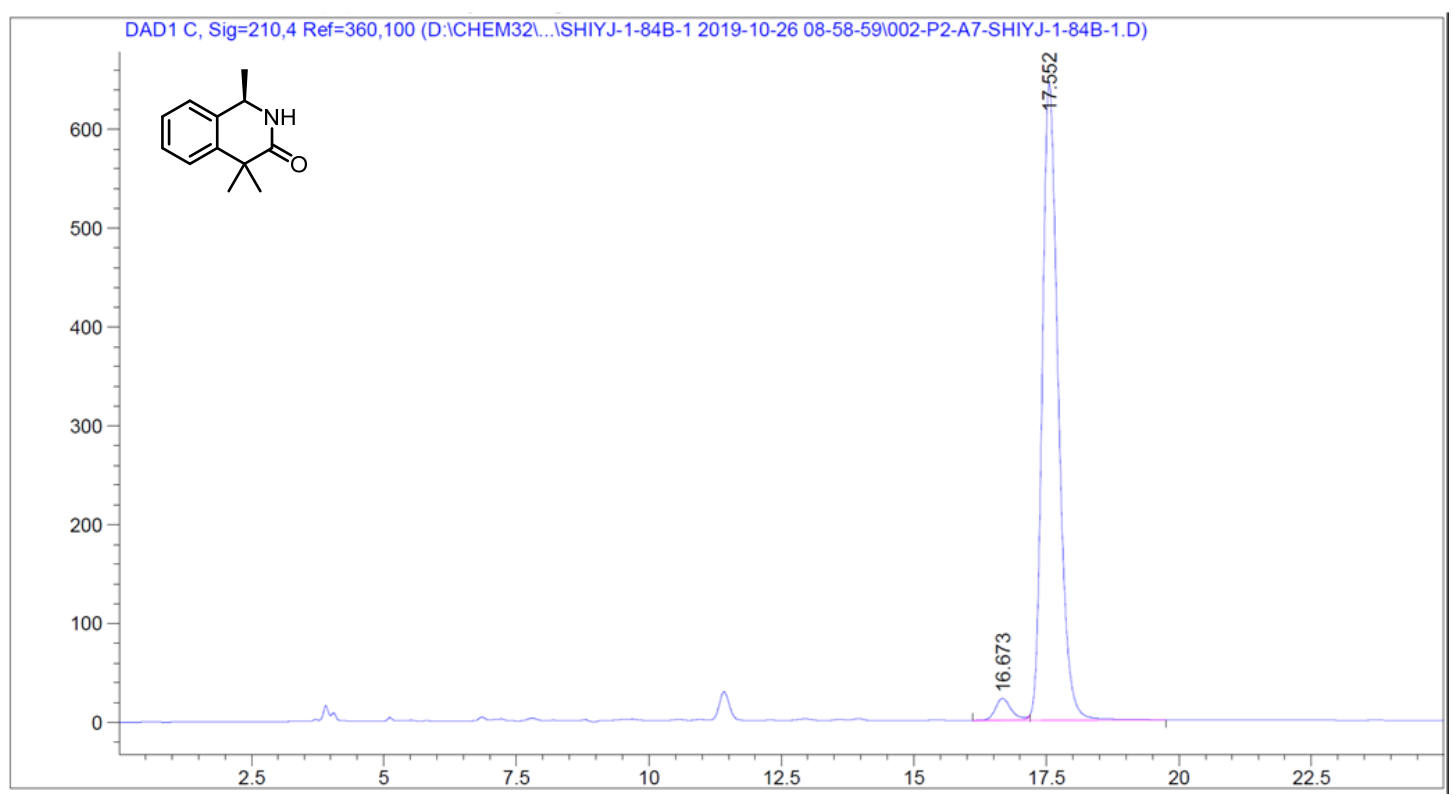

Signal 1: DAD1 C, Sig=210,4 Ref $=360,100$

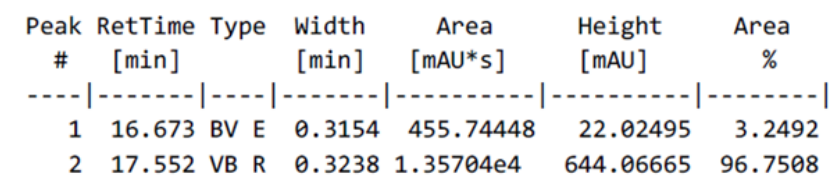


(R)-1-methyl-1,2,4,5-tetrahydro-3H-benzo[c]azepin-3-one (6i)

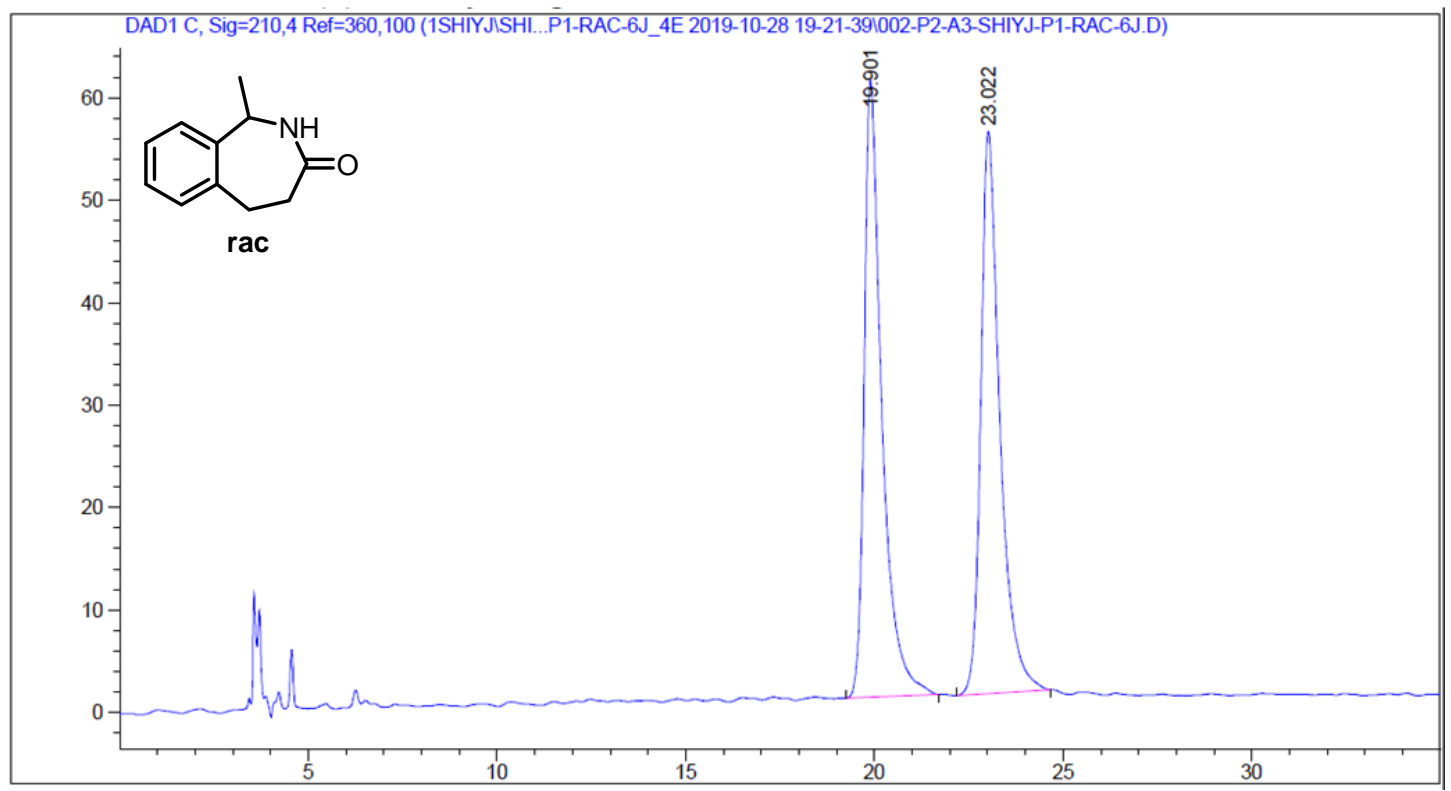

Signal 1: DAD1 C, Sig $=210,4$ Ref $=360,100$

\begin{tabular}{|c|c|c|c|c|c|c|}
\hline $\begin{array}{c}\text { Peak } \\
\#\end{array}$ & $\begin{array}{c}\text { RetTime } \\
\text { [min] }\end{array}$ & Type & $\begin{array}{l}\text { Width } \\
\text { [min] }\end{array}$ & $\begin{array}{c}\text { Area } \\
{\left[\mathrm{mAU}^{*} \mathrm{~s}\right]}\end{array}$ & $\begin{array}{l}\text { Height } \\
\text { [mAU] }\end{array}$ & $\begin{array}{c}\text { Area } \\
\%\end{array}$ \\
\hline & & & & & & \\
\hline 1 & 19. & & 0.4 & 1962 & 60.27996 & 50.3247 \\
\hline 2 & 13 & & .5219 & 1937.00854 & 54.86320 & 49.675 \\
\hline
\end{tabular}

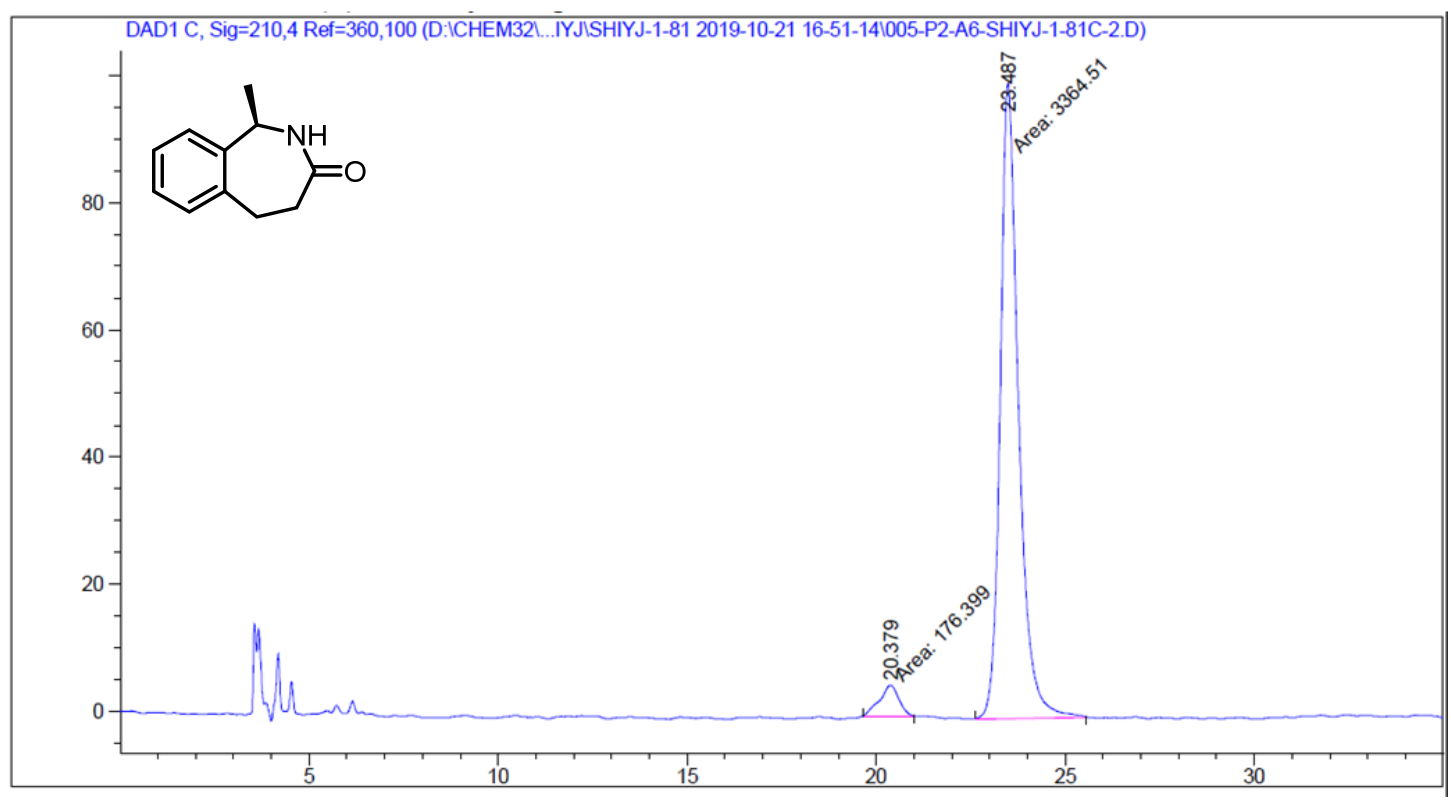

Signal 1: DAD1 C, Sig $=210,4$ Ref $=360,100$

\begin{tabular}{cccccc}
$\begin{array}{c}\text { Peak RetTime Type } \\
\text { \# }\end{array}$ [min] & $\begin{array}{c}\text { Width } \\
\text { [min] }\end{array}$ & $\begin{array}{c}\text { Area } \\
\text { [mAU*s] }\end{array}$ & $\begin{array}{c}\text { Height } \\
\text { [mAU] }\end{array}$ & $\begin{array}{c}\text { Area } \\
\%\end{array}$ \\
\hline 1 & 20.379 MM & 0.6003 & 176.39909 & 4.89720 & 4.9817 \\
2 & 23.487 MM & 0.5607 & 3364.51318 & 100.01168 & 95.0183
\end{tabular}


(R)-3-phenylisoindolin-1-one (6j)

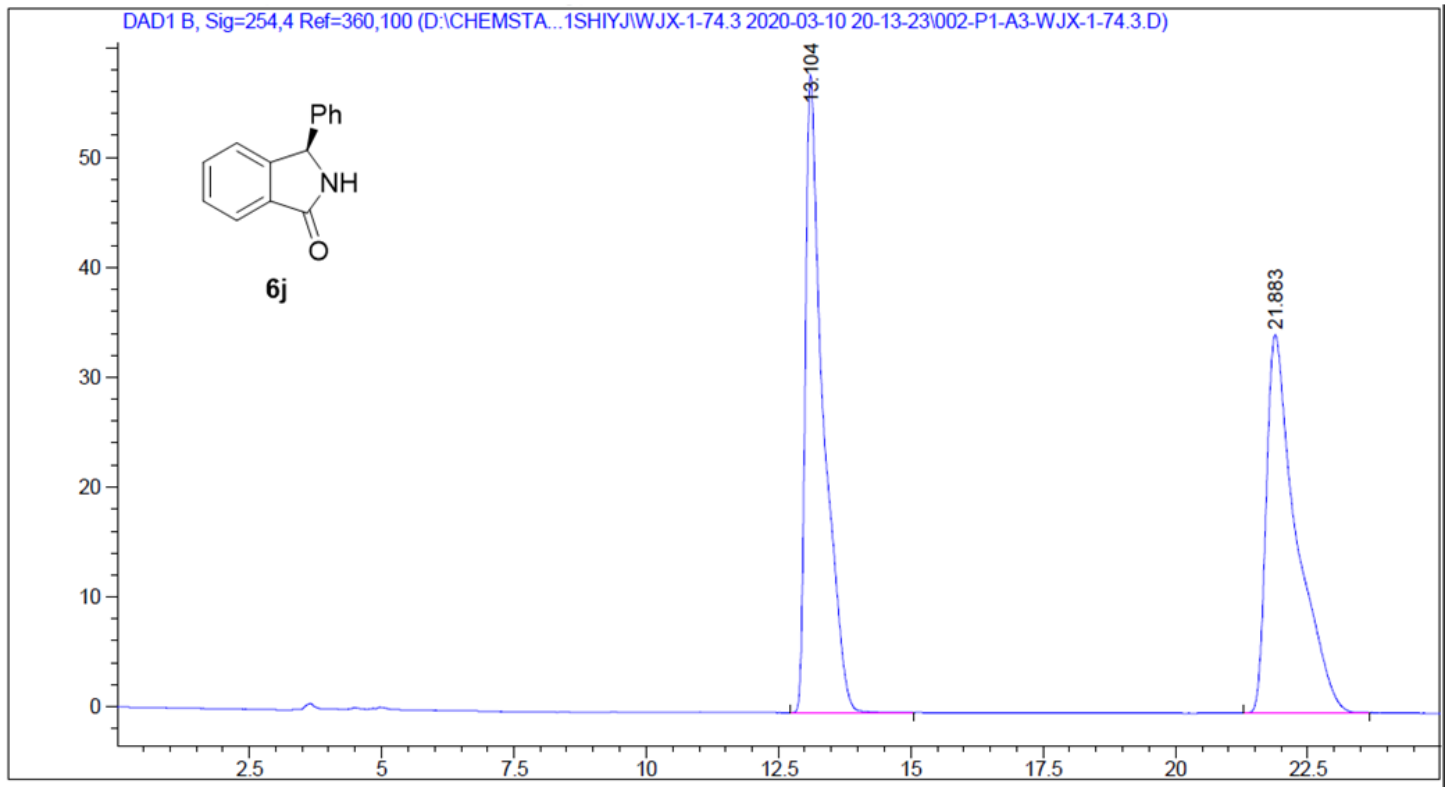

Signal 1: DAD1 B, Sig $=254,4$ Ref $=360,100$

\begin{tabular}{|c|c|c|c|c|c|}
\hline $\begin{array}{c}\text { Peak } \\
\#\end{array}$ & $\begin{array}{l}\text { RetTime Type } \\
\text { [min] }\end{array}$ & $\begin{array}{l}\text { Width } \\
\text { [min] }\end{array}$ & $\begin{array}{c}\text { Area } \\
{\left[\mathrm{mAU}^{*} \mathrm{~s}\right]}\end{array}$ & $\begin{array}{l}\text { Height } \\
{[\mathrm{mAU}]}\end{array}$ & $\begin{array}{c}\text { Area } \\
\%\end{array}$ \\
\hline--1 & $1-$ & & 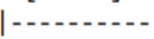 & $-\ldots$ & | \\
\hline 1 & $13.104 \mathrm{BB}$ & 0.3289 & 1368.53577 & 58.08244 & 50.1045 \\
\hline 2 & $21.883 \mathrm{BB}$ & 0.5574 & 1362.82471 & 34.45839 & 49.8955 \\
\hline Total & ls : & & 2731.36047 & 92.54082 & \\
\hline
\end{tabular}

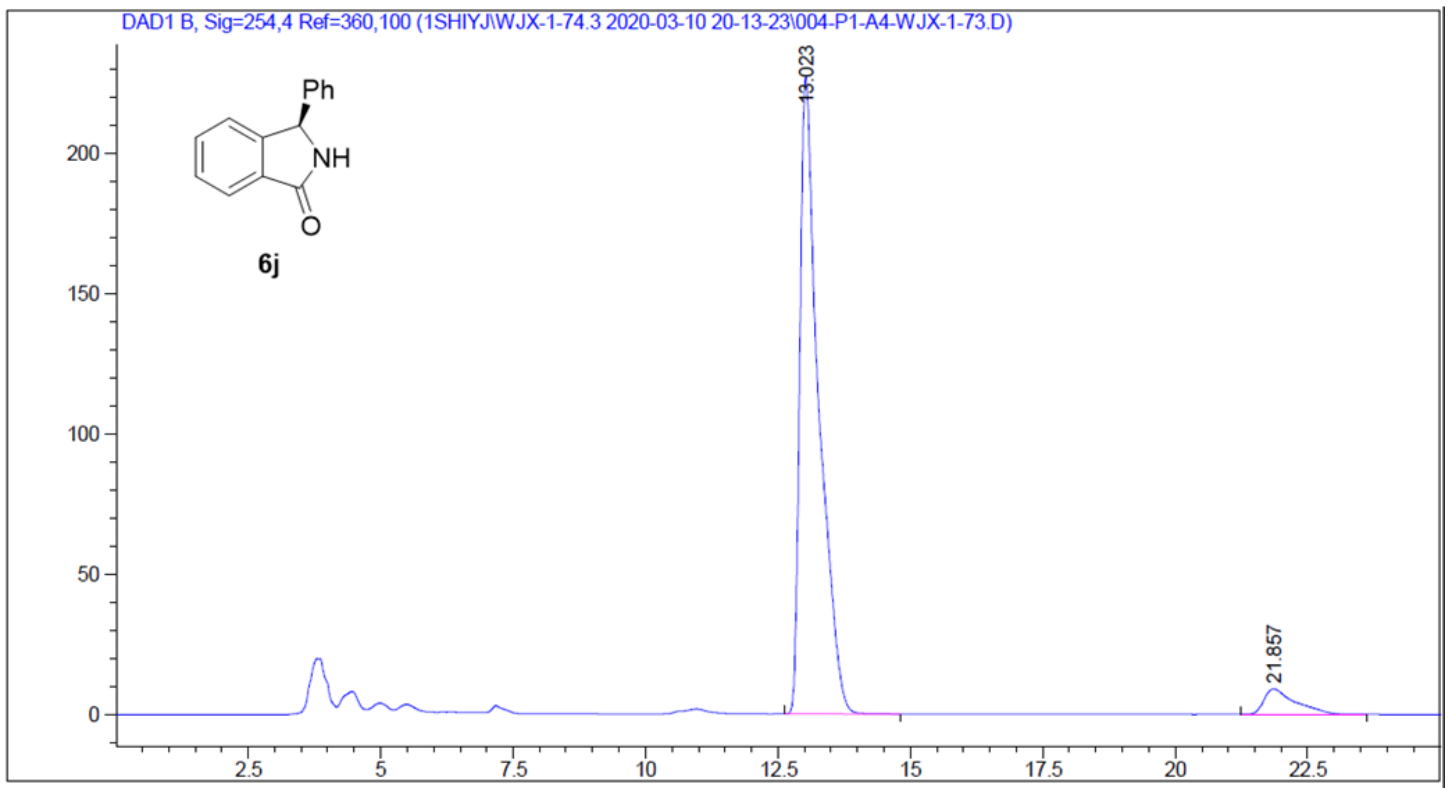

Signal 1: DAD1 B, Sig=254, 4 Ref $=360,100$

\begin{tabular}{cccccc}
$\begin{array}{c}\text { Peak RetTime Type } \\
\text { \# } \\
\text { [min] }\end{array}$ & $\begin{array}{c}\text { Width } \\
{[\mathrm{min}]}\end{array}$ & $\begin{array}{c}\text { Area } \\
{[\mathrm{mAU} \text { s }]}\end{array}$ & $\begin{array}{c}\text { Height } \\
{[\mathrm{mAU}]}\end{array}$ & $\begin{array}{c}\text { Area } \\
\%\end{array}$ \\
\hline 1 & 13.023 BB & 0.3543 & 5653.96777 & 227.23563 & 93.7530 \\
2 & 21.857 BB & 0.5816 & 376.73703 & 9.12721 & 6.2470
\end{tabular}

